Juan Camilo Ríos-Orjuela

Revisión taxonómica de una especie neotropical ampliamente distribuida: el complejo Myiozetetes cayanensis (Linnaeus, 1766) (Aves: Tyrannidae)

Taxonomic revision of a widely spread distributed neotropical species: the Myiozetetes cayanensis complex (Linnaeus, 1766) (Aves:

Tyrannidae) 

Juan Camilo Ríos-Orjuela

\section{Revisión taxonómica de una especie neotropical ampliamente distribuida: el complejo Myiozetetes cayanensis (Linnaeus, 1766) (Aves: Tyrannidae)}

Taxonomic revision of a widely spread distributed neotropical species: the Myiozetetes cayanensis complex (Linnaeus, 1766) (Aves: Tyrannidae)

Versión original

Disertación sometida al Programa de PósGraduação do Museu de Zoologia da Universidade de São Paulo en cumplimiento parcial a los requisitos para la obtención del título de Mestre em Ciências (Sistemática, Taxonomia Animal e Biodiversidade).

Orientador: Prof. Dr. Luís Fábio Silveira

SÃO PAULO 
“Autorizo la reproducción y divulgación total o parcial de este trabajo, por cualquier medio convencional o electrónico, para fines de estudio e investigación siempre que sea citada la fuente".

"I authorize the total or partial reproduction and dissemination of this work, by any conventional or electronic means, for study and research purposes, provide the source is cited".

"Autorizo a reprodução e divulgação total ou parcial deste trabalho, por qualquer meio convencional ou eletrônico, para fins de estudo e pesquisa, desde que citada a fonte".

Serviço de Biblioteca e Documentação

Museu de Zoologia da Universidade de São Paulo

Catalogação na Publicação 
RÍOS-ORJUELA, Juan Camilo

Revisión taxonómica de una especie neotropical ampliamente distribuida: el complejo Myiozetetes cayanensis (Linnaeus, 1766) (Aves: Tyrannidae)

Taxonomic revision of a widely spread distributed neotropical species: the Myiozetetes cayanensis complex (Linnaeus, 1766) (Aves: Tyrannidae)

Disertación sometida al Programa de Pós-Graduação do Museu de Zoologia da Universidade de São Paulo en cumplimiento parcial a los requisitos para la obtención del título de Mestre em Ciências (Sistemática, Taxonomia Animal e Biodiversidade).

Data de aprovação:

Instituição:

Prof. Dr.

Assinatura:

Prof. Dr.

Instituição:

Decisão:

Assinatura:

Prof. Dr.

Instituição:

Decisão:

Assinatura: 
A Martha Esperanza, con todo mi amor y gratitud por la vida. 


\section{AGRADECIMIENTOS}

Al Museu de Zoologia da Universidade de São Paulo, mi casa durante los últimos dos años y la institución que me permitió crecer como biólogo y como persona. A sus funcionarios, profesores y alumnos por hacer parte de este proyecto.

A Luís Fábio Silveira, por su confianza en mis capacidades científicas, por hacerme sentir siempre bienvenido y valioso en su laboratorio, pero sobre todo por su amistad y apoyo durante este tiempo.

A la Coordinación de la formación del personal de nivel superior (CAPES) y al Ministerio de Educación de Brasil por la beca de maestría y la financiación recibida, sin la cual este proyecto no podría haberse llevado a cabo. El presente trabajo fue realizado con el apoyo de de la Coordenação de Aperfeiçoamento de Pessoal de Nível Superior - Brasil (CAPES) - Código de Financiamento 001.

Al Instituto de Ciencias Naturales de la Universidad Nacional de Colombia, el Instituto Alexander von Humboldt, el Museu de Ciências Naturais da PUC-Minas, el Museu de Zoologia da Universidade de São, a Gary Stiles, Andrés Cuervo, Orlando Acevedo, Marcelo Vasconcelos y Luís Fábio Silveira por poner a disposición el material de colección para este estudio.

A Felipe Escobar por su ayuda en todas las etapas del proyecto, su asesoría estadística, sus consejos, por las largas jornadas de trasnoche junto a mí y sobre todo por su inmensa amistad en los últimos 10 años.

A Paulo Pachelle y Julie por recibirme, acompañarme, enseñarme, por las jornadas de descanso jugando Guitar Hero, pero sobre todo por estar siempre prestos a ayudarme con mi vida diaria en Brasil.

Alejandra Arias, Nataly Casas, Nelson Falcón, Juan Camilo Gutierrez y Rafaela Lumi por su ayuda en la etapa de colecta de datos y toma de medidas morfométricas. A Rafael Nascimento por poner a disposición su ayuda y su talento artístico en la elaboración de las ilustraciones en acuarela del plumaje de Myiozetetes cayanensis. 
A todos los miembros y colaboradores del Laboratorio de Ornitología (Fernanda Bocalini, Sergio Bolivar, Rafael Nascimento, Glaucia Del-Río, Natalia Luchetti, Thiago Vernaschi, Bianca Matinata, Vitor De Deus, Gislaine Dalazen, Bruno Fermino, José Eduardo "Pepe" Serrano, Rafaela Lumi, Francisca, y los que faltan...), por su compañía, amistad y todo el aprendizaje a lo largo de este camino. Agradezco especialmente a Fernanda, Rafaela, Sergio, Pepe y Rafael, el "crew mastornito", por su amistad, sus consejos, por distraerme en los descansos y la hora del café, y sobre todo por su compañía durante tantos días de trabajo en el Museo.

A la galera del "Futebol-MZUSP", por brindarme un espacio de despeje, ejercicio físico y sobre todo amistad dentro y fuera de la cancha.

A mi madre y mis hermanos, por creer en todos mis sueños, por el apoyo y la paciencia durante todas las etapas de este proyecto.

Por último, pero no menos importante, a E-bird y la Macaulay Library en el Cornell Lab of Ornithology, Xeno-canto.org y wikiaves.com.br, y a todos los observadores de aves y ornitólogos del mundo que alimentan estas bases de datos con su información valiosa, que sirvió como materia prima para este estudio. De la misma forma, a todos los ornitólogos y naturalistas que desde el siglo XIX han contribuido con la ornitología neotropical depositando especímenes en las colecciones científicas. Por su esfuerzo y dedicación hoy es posible para mi realizar este trabajo. Gracias. 


\section{RESUMEN}

El complejo Myiozetetes cayanensis está compuesto actualmente por cuatro subespecies: M. c. cayanensis, M. c. hellmayri, M. c. rufipennis y M. c. erythropterus. Estos taxones se distribuyen desde el sur de Costa Rica, a través del norte de Suramérica hasta el sur de Brasil, Bolivia y Paraguay, se distinguen por tener diferencias en la coloración del plumaje a nivel de las alas, el dorso, la cola y la coronilla. El presente trabajo tiene como objetivo revisar la taxonomía de este complejo de especies a través de un análisis de caracteres de coloración, morfometría y vocalizaciones. Fueron estudiados 262 especímenes, 640 archivos sonoros y 80 fotografías, depositados en colecciones ornitológicas y repositorios virtuales en Brasil, Colombia y Estados Unidos. Fueron determinados 65 caracteres (17 de plumaje, 31 morfométricos y 17 de vocalizaciones) de los cuales 26 son informativos. El estudio taxonómico de los caracteres sugiere la existencia de cuatro taxones: (1) M. hellmayri Hartert y Goodson, 1917, que ocurre desde el sur de Costa Rica y en todo el territorio transandino hasta el noroccidente de Venezuela y el noreste de Ecuador, (2) M. erythropterus (Lafresnaye, 1853), que se encuentra al oriente de Brasil en los estados de Bahía, Minas Gerais, Espirito Santo y Rio de Janeiro, (3) M. c. rufipennis Lawrence, 1870, que se distribuye al noroccidente de Venezuela y en el Orinoco hasta el amazonas en Colombia y Ecuador, y (4) M. c. cayanensis (Linnaeus, 1766), que ocurre en todo el nororiente sudamericano en la franja amazónica, el escudo guyanés y la diagonal seca hasta el norte de Bolivia y Paraguay. Las similitudes entre los taxones aquí mencionados apuntan a un proceso de variación clinal morfológica y vocal, de manera que los taxones más lejanos entre sí difieren más que con respecto a sus vecinos cercanos en distribución geográfica. Es necesaria la inclusión de análisis moleculares en futuras revisiones, que permitan complementar y dilucidar con mayor claridad los procesos evolutivos actuales del complejo M. cayanensis.

Palabras clave: Myiozetetes. Taxonomía. Distribución geográfica. Límites entre especies. 


\section{RESUMO}

O complexo Myiozetetes cayanensis é atualmente composto por quatro subespécies: M. c. cayanensis, M. c. hellmayri, M. c. rufipennis e M. c. erythropterus. Esses táxons se distribuem desde o sul da Costa Rica, passando pelo norte da América do Sul ao sul do Brasil, Bolívia e Paraguai, e se distinguem por apresentar diferenças na coloração da plumagem ao nível das asas, o dorso, cauda e a coroa. O presente trabalho tem como objetivo revisar a taxonomia desse complexo de espécies por meio de uma análise de caracteres de coloração, morfometria e vocalizações. Foram estudados 262 espécimes, 640 arquivos sonoros e 80 fotografias, depositados em coleções ornitológicas e repositórios virtuais no Brasil, Colômbia e Estados Unidos. Foram determinados 65 caracteres (17 plumagem, 31 morfométricos e 17 vocalizações) dos quais 26 são informativos. O estudo taxonômico dos caracteres sugere a existência de quatro táxons: (1) M. hellmayri Hartert e Goodson, 1917, que ocorre no sul da Costa Rica e em todo o território transandino ao noroeste da Venezuela e nordeste do Equador, ( 2) M. erythropterus (Lafresnaye, 1853), encontrada ao leste do Brasil nos estados da Bahia, Minas Gerais, Espírito Santo e Rio de Janeiro, (3) M. c. rufipennis Lawrence, 1870, que se distribui ao noroeste da Venezuela e no Orinoco até a Amazônia na Colômbia e Equador, e (4) M. c. cayanensis (Linnaeus, 1766), que ocorre em todo o nordeste da América do Sul na porção amazônica, no escudo da Guiana e na diagonal seca ao norte da Bolívia e Paraguai. As semelhanças entre os táxons aqui mencionados apontam para um processo de variação clinal morfológica e vocal, de forma que os táxons que estão mais distantes uns dos outros diferem mais do que em relação a seus vizinhos próximos na distribuição geográfica. A inclusão de análises moleculares em revisões futuras é necessária para complementar e elucidar com maior clareza os processos evolutivos atuais do complexo M. cayanensis.

Palavras-chave: Myiozetetes. Taxonomia. Distribuição geográfica. Limites entre espécies. 


\begin{abstract}
The Myiozetetes cayanensis complex is currently composed by four subspecies: M. c. cayanensis, M. c. hellmayri, M. c. rufipennis and M. c. erythropterus. These taxa are distributed from south Costa Rica, through north South America to the south of Brazil, Bolivia and Paraguay, and they are distinguished by having differences in plumage coloration in wings, back, tail and crown. This work aims to review the taxonomy of this species complex through an analysis of coloration, morphometry and vocalizations. 262 specimens, 640 sound files and 80 photographs, deposited in ornithological collections and virtual repositories in Brazil, Colombia and the United States were studied. 65 characters were determined (17 plumage, 31 morphometric and 17 vocalizations) of which 26 were informative. The taxonomic study suggests the existence of four taxa: (1) M. hellmayri Hartert and Goodson, 1917, which occurs from south Costa Rica, throughout the trans-Andean area to northwest Venezuela and northeast Ecuador, (2) M. erythropterus (Lafresnaye, 1853), extant to east Brazil in the states of Bahia, Minas Gerais, Espirito Santo and Rio de Janeiro, (3) M. c. rufipennis Lawrence, 1870, which is distributed to northwest Venezuela in the Orinoco to the Amazonia in Colombia and Ecuador, and (4) M. c. cayanensis (Linnaeus, 1766), which occurs throughout northeastern South America in the Amazon fringe, the Guyanese shield, and the dry diagonal to northern Bolivia and Paraguay. The similarities between the taxa mentioned here point to a process of morphological and vocal clinal variation, so that the taxa that are more distant from each other differ more than with respect to their close neighbors in geographic distribution. The inclusion of molecular analyzes in future reviews is necessary to complement and clarify the current evolutionary processes of the $M$. cayanensis complex.
\end{abstract}

Keywords: Myiozetetes. Taxonomy. Geographical distribution. Species limits. 


\section{LISTA DE FIGURAS Y TABLAS}

Figura 1. Descripción original del género Muscicapa y la especie Myiozetetes (Muscicapa) cayanensis en el "Systema naturæ" de Linnaeus en 1776. .86

Figura 2. Patrón de plumaje general de M. c. cayanensis y M. c. rufipennis. .87

Figura 3. Partes del ave utilizadas para evaluar coloración. .88

Figura 4. Áreas de coloración de las plumas de contorno y de vuelo. . .89

Figura 5. Muestras de color de Myiozetetes cayanensis siguiendo la guía de Smithe (1975). ..90

Figura 6. Mapa de distribución de coloración de la corona de Myiozetetes cayanensis. . .91

Figura 7. Mapa de distribución de coloración de la zona auricular de Myiozetetes cayanensis.

Figura 8. Mapa de distribución de coloración de la coronilla (basal y distal) y el pecho de Myiozetetes cayanensis. .93

Figura 9. Mapa de distribución de coloración de la coronilla (medial) de Myiozetetes cayanensis. .94

Figura 10. Mapa de distribución de coloración del dorso (basal) y las supracaudales (basal) de Myiozetetes cayanensis. . .95

Figura 11. Mapa de distribución de coloración del dorso (distal) de Myiozetetes cayanensis. .96

Figura 12. Mapa de distribución de coloración de las plumas de arrastre y rectrices de Myiozetetes cayanensis. . .97

Figura 13. Mapa de distribución de coloración del borde de las plumas primarias de Myiozetetes cayanensis. .98

Figura 14. Mapa de distribución de coloración del borde de las plumas secundarias y rectrices de Myiozetetes cayanensis. . .99

Figura 15. Mapa de distribución de coloración de las coberteras menores y mayores (basal) de Myiozetetes cayanensis. 100 
Figura 16. Mapa de distribución de coloración de las coberteras menores y mayores (distal) de Myiozetetes cayanensis.

Figura 17. Mapa de distribución de coloración de las supracaudales (distal) de Myiozetetes cayanensis. 102

Figura 18. Distribución geográfica de las UTO en el complejo Myiozetetes cayanensis. .......103

Figura 19. Medidas de tendencia central de las variables morfométricas analizadas. 104

Figura 20. Linealidad de las variables anormales identificadas con la prueba de normalidad de Shapiro-Wilks. 105

Figura 21. Matriz de correlación, linealidad y colinealidad de las variables morfométricas en el modelo 3. 106

Figura 22. Distribución de los datos morfométricos del modelo 3 explicados por UTO. 107

Figura 23. Diferencias de media en la comparación de UTO según los intervalos de confianza de Dunnet. 108

Figura 24. Agrupación supervisada de $K$-means para las variables morfométricas de las UTO..

Figura 25. Mapa de agrupación no supervisada por pares de $K$-means para las variables morfométricas de las UTO.

Figura 26. Distribución de las variables con dimorfismo sexual en la UTO 1.

Figura 27. Elemento único (D) que compone el llamado reproductivo de Myiozetetes cayanensis. 112

Figura 28. Elementos del canto de comunicación de Myiozetetes cayanensis. .113

Figura 29. Distribución de los datos de cadencia de trino, índice de diversidad y número de notas de las UTO. 114

Figura 30. Intervalos de confianza de Tukey-Kramer para los atributos del canto de comunicación de las UTO.

Figura 31. Intervalos de confianza de Tukey-Kramer para los atributos de la nota de tipo D (canto reproductivo) de las UTO. 
Figura 32. Agrupación supervisada por pares de $k$-means para los tipos de notas de las UTO.

Figura 33. Mapa de agrupación no supervisada por pares de $K$-means para la nota de tipo D de las UTO.

Figura 34. Ejemplos de sonogramas de los individuos pertenecientes a la UTO 1.

Figura 35. Ejemplos de sonogramas de los individuos pertenecientes a la UTO 2. 120

Figura 36. Ejemplos de sonogramas de los individuos pertenecientes a la UTO 3. 121

Figura 37. Ejemplos de sonogramas de los individuos pertenecientes a la UTO 4. 122

Figura 38. Myiozetetes hellmayri Hartet y Goodson, 1917. Ilustración: Rafael Nascimento. .123

Figura 39. Distribución geográfica de Myiozetetes hellmayri Hartet y Goodson, 1917. .124

Figura 40. Myiozetetes cayanensis rufipennis Lawrence, 1870. Ilustración: Rafael Nascimento. .125

Figura 41. Distribución geográfica de Myiozetetes cayanensis rufipennis Lawrence, 1870. .126

Figura 42. Myiozetetes cayanensis cayanensis (Linnaeus, 1766). Ilustración: Rafael Nascimento. .127

Figura 43. Distribución geográfica de Myiozetetes cayanensis cayanensis (Linnaeus, 1766). 128

Figura 44. Myiozetetes erythropterus (Lafresnaye, 1853). Ilustración: Rafael Nascimento. ...129

Figura 45. Distribución geográfica de Myiozetetes erythropterus (Lafresnaye, 1853). .130

Figura 46. Variación en la coloración del plumaje de las Unidades Taxonómicas Operativas.

Figura 47. Distribución geográfica de Myiozetetes hellmayri, Myiozetetes cayanensis y Myiozetetes erythropterus. 
Tabla 1. Características del plumaje en las UTO del complejo Myiozetetes cayanensis en muda reproductiva.

Tabla 2. Medidas de tendencia central de las variables morfométricas analizadas. 136

Tabla 3. Matriz de correlación de las variables morfométricas seleccionadas para el análisis.

Tabla 4. Valores de normalidad de Shapiro-Wilks para las variables morfométricas seleccionadas.

Tabla 5. Parámetros y evaluación de supuestos de análisis de MANOVA en los modelos 1, 2 y

3.

Tabla 6. Resultados de MANOVA para las variables morfométricas del modelo 3.

Tabla 7. Soporte de diferencias entre los grupos de UTO en las pruebas de comparación por pares

Tabla 8. Valores $p$ de t-student para dimorfismo sexual de las Unidades taxonómicas operativas.

Tabla 9. Resumen descriptivo de las características auditivas de las notas identificadas. .....143

Tabla 10. Valores $\mathrm{p}$ de ANOVA para los parámetros vocales de las UTO. .144

Tabla 11. Resultados de ANOVA para los atributos del canto de comunicación.

Tabla 12. Valores de $p$ de Tukey-Kramer para el índice de diversidad y el número de notas del canto de las UTO.

Tabla 13. Atributos principales del canto de comunicación de las Unidades Taxonómicas Operativas (UTO).

Tabla 14. Matriz de soporte de diferencias entre taxones. 148 


\section{TABLA DE CONTENIDO}

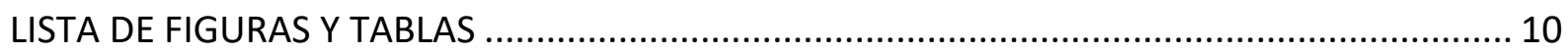

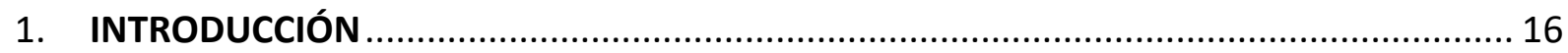

1.1. El género Myiozetetes Sclater, 1859: sistemática y taxonomía................................... 16

1.2. Histórico taxonómico del complejo Myiozetetes cayanensis Linnaeus, 1766 .............. 19

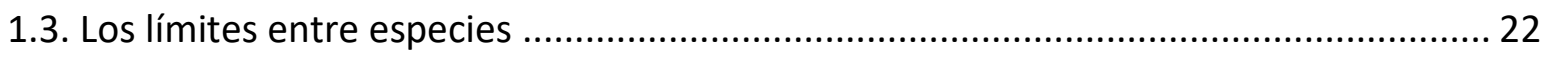

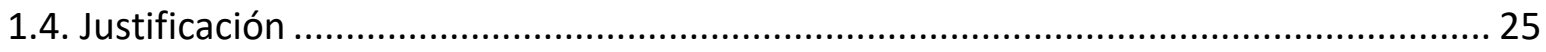

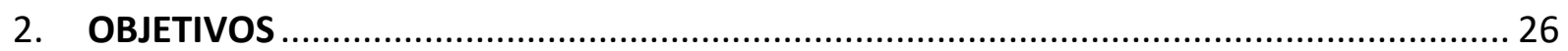

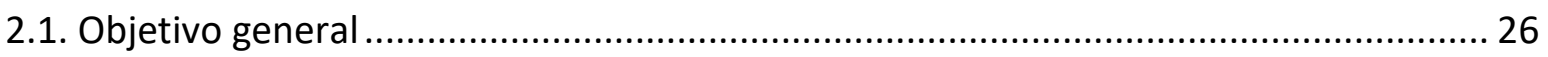

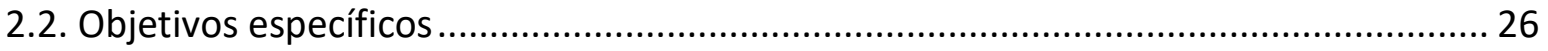

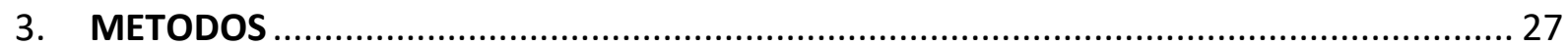

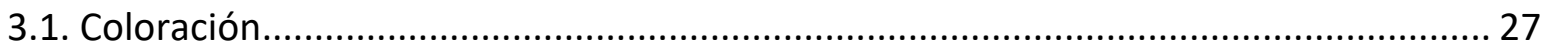

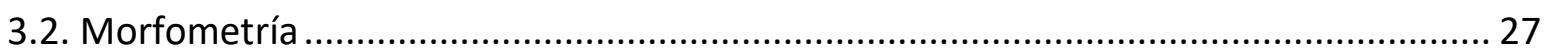

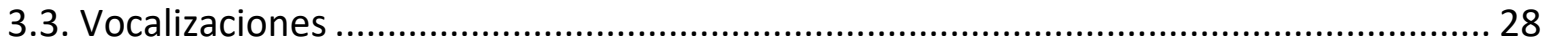

3.4. Distribución geográfica y rangos de ocurrencia ......................................................... 30

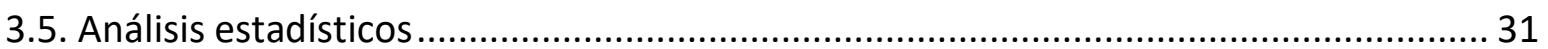

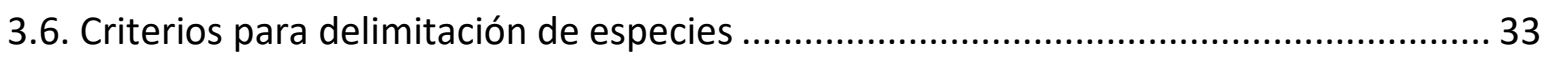

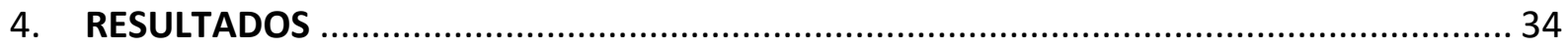

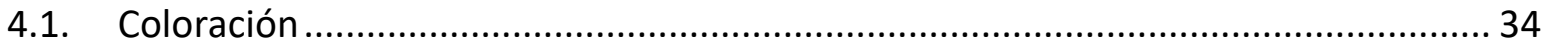

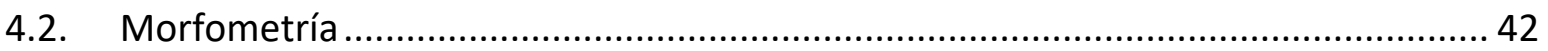

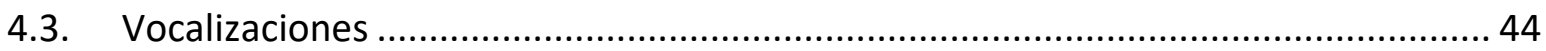

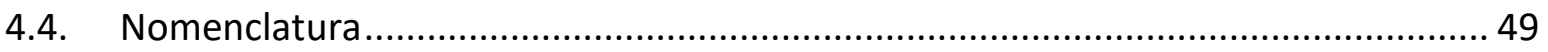

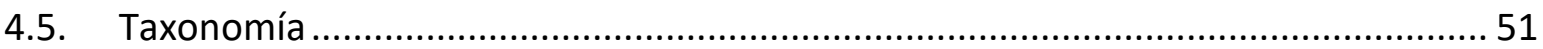

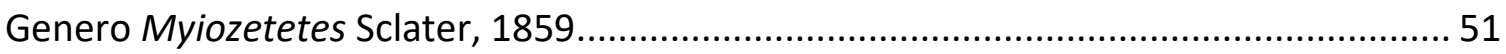


Myiozetetes hellmayri Hartert y Goodson, 1917

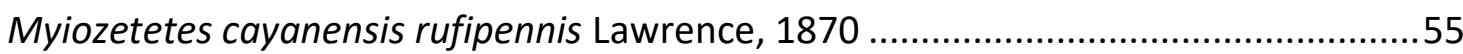

Myiozetetes cayanensis cayanensis (Linnaeus, 1766) .............................................58

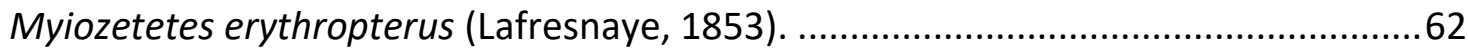

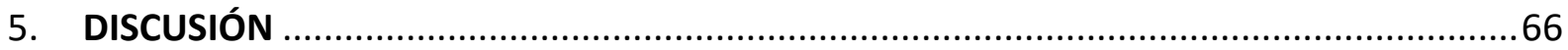

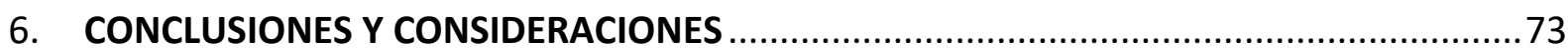

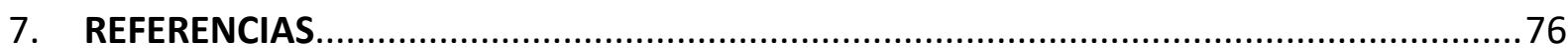

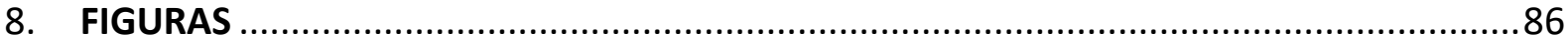

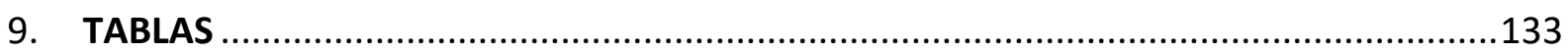

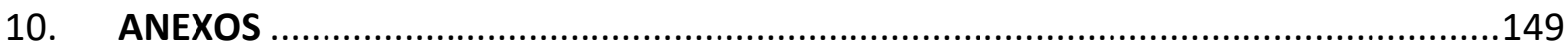




\section{INTRODUCCIÓN}

\subsection{El género Myiozetetes Sclater, 1859: sistemática y taxonomía}

La familia Tyrannidae Vigors, 1825, es una de las familias de aves más diversas del mundo, con cerca de 422 especies distribuidas en el Neotrópico (Winkler et al. 2020). Esta familia se ha adaptado a diversos tipos de hábitat y condiciones de vida, lo que ha permitido aumentar su riqueza y la colonización de nuevos nichos (Fitzpatrick 1980, 1985; Traylor y Fitzpatrick 1982; Ohlson et al. 2008; Ridgely y Tudor 2009; Fjeldså et al. 2018). El clado Tyrannoidea (Sibley y Ahlquist, 1985, 1990) ha sido sustentado tradicionalmente como un grupo conformado por las familias Tyrannidae, Cotingidae Bonaparte, 1849 y Pipridae Rafinesque, 1815 (Ohlson et al. 2008; Fitzpatrick 2020), pero los límites de estas familias han sido problemáticos a causa de las afinidades taxonómicas inciertas de algunos taxones (Johansson et al. 2002; Ericson et al. 2006; Tello et al. 2009; Ohlson et al. 2012; Harvey et al. 2020). Otros estudios han sugerido incluso dividir la familia en varios taxones, a causa de las diferencias morfológicas y moleculares (Tello y Bates 2007; Ohlson et al. 2007, 2008, 2013; Tello et al. 2009; Fjeldså et al. 2018), contribuyendo sustancialmente en la resolución del estatus filogenético del clado. En una síntesis provista por Ohlson et al. (2020), uno de los grandes cambios sugeridos para la familia radica en considerar a las subdivisiones clásicas de Tyrannidae (ej. los "flatbills" [picoplanos], "typical fly-catchers" [atrapamoscas típicos], "todytyrants" [espatulillas], etc.) como un super clado que se divide en varias familias, donde Tyrannidae y Rhynchocyclidae Berlepsch, 1907, contienen la mayoría de las especies (Ohlson et al. 2013). Los tiránidos fueron primero agrupados en subfamilias según caracteres morfológicos como los músculos de la siringe (Ames 1971) y caracteres tarsales, entre otros (Prum 1990; Chaves et al. 2008), pero estos caracteres mostraban inconsistencias en algunos grupos, creando discordancias filogenéticas (Ohlson et al. 2013; Fjeldså et al. 2018). Estudios filogenéticos han cubierto de manera más detallada algunos grupos terminales dentro de Tyrannidae, como es el caso de los géneros Muscisaxicola d'Orbigny y Lafresnaye, 1837 (Chesser 2000), Empidonax Cabanis, 1855 (Johnson y Cicero 2002), Myiophobus Reichenbach, 1850 (Ohlson et al. 2009), y Knipolegus Boie, 1826 (Hosner y Moyle 2012), encontrando aún 
preguntas por resolver en la clasificación debido a la alta diversidad en los patrones morfológicos, ecológicos y geográficos del clado (Ohlson et al. 2020).

En la actualidad, muchas propuestas filogenéticas integrativas basadas en caracteres moleculares, morfología y comportamiento han sugerido diferentes hipótesis acerca de las relaciones taxonómicas del grupo, pero aún permanecen sin establecer un consenso definitivo (Traylor-Jr 1977; Lanyon 1985, 1988a, b; Birdsley 2002; Chesser 2004; Tello y Bates 2007; Chaves et al. 2008; Tello et al. 2009; Ohlson et al. 2013, 2020; Dickinson y Christidis 2014; Fjeldså et al. 2018). Sin embargo, según Chaves et al. (2008) y Ohlson et al. (2020), la clasificación de las subfamilias y de algunos grupos dentro de Tyrannidae sigue siendo usada de manera "no oficial", ya que existen vacíos de información sobre algunos taxones, lo cual no permite construir hipótesis completas.

El género Myiozetetes Sclater, 1859, comprende cuatro especies reconocidas ( $M$. cayanensis (Linnaeus, 1766), M. similis (Spix, 1825), M. granadensis Lawrence, 1862 y $M$. luteiventris (Sclater, 1858)), las cuales están ampliamente distribuidas en el neotrópico, desde el sur de México, a través de Centroamérica y todo el norte de Sudamérica hasta el noreste de Argentina (Winkler et al. 2020); siendo uno de los más ampliamente distribuidos en las zonas bajas de esta área. Aunque estas especies son de amplia distribución y de usual registro, no se conocen a profundidad las relaciones filogenéticas dentro del grupo y su variación morfológica y ecológica, siendo necesario el estudio de sus poblaciones con el fin de aclarar su estatus taxonómico, así como la historia natural del grupo. A través de los años, algunos autores han sustentado el género Myiozetetes como un clado monofilético a partir del análisis de caracteres morfológicos, moleculares y ecológicos; por ejemplo, según McKitrick (1985), el género Myiozetetes se diferencia de otros tiránidos por la ausencia del M. latissimus dorsi caudalis. Posteriormente, revisiones taxonómicas de la familia Tyrannidae han ubicado al género Myiozetetes como un grupo monofilético asociado usualmente al género Legatus Sclater, 1859, junto con otros "Tyrant flycatchers" como Pitangus Swainson, 1827, Philohydor Lanyon, 1984 y Phelpsia Lanyon, 1984, quienes conforman un grupo natural (Birdsley 2002; Chesser 2004; Tello et al. 2009; Ohlson et al. 2013). Sin embargo, en algunas clasificaciones alternas se sugiere su cercanía con Phelpsia debido a la composición similar de los músculos de la siringe (Birdsley 2002). Así pues, las relaciones filogenéticas del género no son claras en la actualidad, siendo necesario la inclusión de análisis más detallados. Del mismo modo, las 
relaciones filogenéticas dentro del género no han sido esclarecidas todavía, si bien los datos moleculares indican un fuerte apoyo para el clado conformado por $M$. cayanensis y $M$. similis como grupo hermano de $M$. granadensis y $M$. luteiventris, los taxones infraespecíficos de este grupo requieren un escrutinio más detallado.

Myiozetetes cayanensis corresponde a una de las especies más comunes y mayormente distribuidas dentro del género, teniendo poblaciones aisladas por fuertes barreras geográficas como la cordillera andina, que separa las poblaciones de centro américa, la región occidental de Colombia y Ecuador, de las poblaciones presentes en los llanos orientales, el escudo guayanés y la Amazonía. Esto puede significar una diversificación en las poblaciones aisladas, lo cual podría derivar en eventos de especiación (Davis y Nixon 1992). Así pues, en la actualidad el complejo $M$. cayanensis está compuesto por cuatro subespecies reconocidas (M. c. hellmayri Hartert y Goodson, 1917, M. c. rufipennis Lawrence, 1870, M. c. cayanensis (Linnaeus, 1766) y M. c. erythropterus (Lafresnaye, 1853)), de las cuales no se conocen muchos datos sobre su estatus filogenético; por ejemplo, anteriormente se ha sugerido separar poblaciones de $M$. cayanensis en el este de Panamá como $M$. c. harterti Bangs y Penard, 1921, argumentando un tamaño más pequeño, pero que hasta ahora, es inseparable de M. c. hellmayri (Mobley y Kirwan 2020). De la misma forma, M. c. erythropterus fue originalmente descrito por Lafresnaye (1853) como Tyrannula erythroptera, y ubicado posteriormente como Myiozetetes erythropterus por Sclater (1871), confiriéndole así el estatus de especie, pero no fue ubicado como subespecie sino hasta 1906 por Hellmayr, quien manifiesta que este taxón está estrechamente relacionado con $M$. c. cayanensis, pero se diferencia por ser más grande y por algunos caracteres sutiles en el color de las alas.

Por su parte, el taxón tipo M. c. cayanensis, ha sido objeto de diversas controversias y sinonimias, ha sido descrito tres veces distintas como Muscicapa cayanensis (descripción original por Linnaeus, 1776), Myiozetetes guianensis Cabanis y Heine, 1859, y Myiozetetes columbianus Pelzeln, 1868, siendo los dos últimos sinónimos de la primera descripción (Hellmayr, 1927). Así mismo, la subespecie M. c. rufipennis se mantiene con el estatus de especie durante 33 años después de su descripción como Myiozetetes rufipennis (Lawrence, 1870), y es hasta 1902 que Berlepsch y Hartert la ubican como subespecie a partir de nuevo material colectado en el este de Venezuela. Tradicionalmente, los límites entre estos taxones se han basado en el tamaño y en las diferencias sutiles que presentan sus plumajes, como la 
presencia o ausencia del color rufo a lo largo del borde de las plumas primarias, y el tono negro-oliváceo de la región superior de la cabeza, siendo necesaria una revisión detallada de la morfología, el plumaje y el comportamiento que permita un mejor acercamiento al análisis de las relaciones filogenéticas que existen dentro del grupo.

\subsection{Histórico taxonómico del complejo Myiozetetes cayanensis Linnaeus, 1766}

Muscicapa cayanensis, descrito únicamente en relato en el texto del "Systema naturæ" por Linnaeus en 1776 (Linnaeus 1766, Tomo 1, parte 1, p327), basado en la plancha de François-Nicolas Martinet en la obra de Brisson (P. 38, Fig. 4, 1760) de un individuo de Cayena en la actual Guyana Francesa, es el taxón tipo con el que se describe la especie originalmente. Linnaeus describió el género Muscicapa como aquellas aves Passeres con la siguiente combinación de caracteres: "Rostrum subtrigonum, utrinque emarginatum, apice incurvo, vibrissae patentes verfus fauces. Nares subrotundae.", que traduce: "Pico subtriangular, un poco alargado, ápice curvo, vibrisas frente a la garganta, culmen redondeado." (Figura 1). Compuesto originalmente por 21 especies, el género Muscicapa contenía varios atrapamoscas del nuevo mundo, hoy reclasificados en géneros como Tyrannus Lacépède, 1799, Contopus Cabanis, 1855, y Elaenia Sundevall, 1836, e incluso algunas especies del viejo mundo hoy clasificadas en otras familias, como el caso de Muscicapa mutata (Terpsiphone mutata (Linnaeus, 1766)), perteneciente a la familia Monarchidae Bonaparte, 1854, en la actualidad.

La descripción de la especie provista por Linnaeus se basa únicamente en la coloración de las áreas primarias del individuo, con los siguientes caracteres: " $M$. fusca, subtus lutea, supercilis albis, vertice sub-aurantio. Corporis pennae supra fuscae margine olivaceo. Rectrices marginibus rufus. Remiges margine exteriore rufo. Verticis pennae bafi aurantiae. Muscicapa cayanensis. Briff. av. 2. p. 404. 1. 38. f. 4. Habitat in cayana.", que traduce "Mandíbula oscura, amarillo por debajo, ceja blanca, coronilla sub-amarilla (el color está escondido). Las plumas superiores del cuerpo son oscuras con márgenes oliva. Rectrices con bordes rojos, remeras con márgenes externas rojas. La base de las plumas de la coronilla es naranja. Muscicapa 
cayanensis, que habita en Cayana" (Figura 1). Los registros más antiguos en colecciones biológicas datan de 1815, con 3 especímenes provenientes de Brasil (localidad desconocida) depositados en la Colección Zoológica de la Universidad de Lund (MZLU, números L815/3234, L815/3235 y L815/3234 [Ekström 2020]), en Suecia.

Durante casi 100 años este taxón permanece intacto, hasta que en 1859 es incluido dentro del género Myiozetetes por Sclater. Posteriormente en el mismo año Cabanis y Heine describen a Myiozetetes guianensis basados en individuos de Guyana y Surinam, pero este resulta ser un sinónimo de M. c. cayanensis. En 1837, Lafresnaye y D’Orbigny basados en un espécimen examinado de la provincia de Mojos, Bolivia, proponen que Muscicapa cayanensis (en ese momento considerada con el estatus de especie), debería incluirse dentro del género Muscipeta, como Muscipeta cayennensis, diferenciado de las otras especies del género Muscicapa teniendo en cuenta caracteres de coloración. En 1848, Cabanis propone incluir esta especie dentro del género Elaenea, como E. cayanensis usando como referencia un espécimen observado en Guyana. En 1867, Sclater y Salvin ubican la especie como Myiozetetes cayennensis después de analizar amplio material de localidades en Brasil y Guyana Francesa. Es hasta 1906 que este taxón es considerado como una subespecie de M. cayanensis, Hellmayr y colaboradores proponen este cambio basados en la revisión de amplio material de localidades en Brasil, Guyana, Guyana Francesa y Bolivia, dando como resultado la subespecie M. cayanensis cayanenesis. Esta subespecie incluye como criterio de identificación a los individuos distribuidos en las zonas planas al oriente de la cordillera oriental (es decir, toda la distribución conocida hasta la fecha, excepto las poblaciones de Bogotá, el occidente de Ecuador y el pie de monte andino en Colombia, Venezuela, las Guyanas y el occidente de Brasil). En 1868, Pelzeln describe a Myiozetetes columbianus, que resultó ser un sinónimo de M. c. cayanensis (Figura 2).

Myiozetetes cayanensis erythropterus fue descrito por Lafresnaye en 1853 como Tyrannula erythroptera con material proveniente de Rio de Janeiro en Brasil, como una especie similar en coloración a $M$. cayanensis, pero de mayor tamaño corporal $(48 \mathrm{~cm}$ de longitud en promedio), con un color pardo opaco que cubre todas las rectrices y primarias formando una amplia banda longitudinal, y con un color rufo intenso de en las márgenes de las primarias (Lafresnaye 1853). Posteriormente es ubicado dentro del género Myiozetetes por Sclater en 1871, después de revisar especímenes del estado de Minas Gerais. Hellmayr 
sugiere el estatus de subespecie en 1906, basado en individuos observados en Rio de Janeiro y Minas Gerais, dando como resultado el nombre Myiozetetes cayanensis erythropterus, para las poblaciones que habitan estos estados en Brasil.

Myiozetetes cayanensis rufipennis es descrito originalmente como Myiozetetes rufipennis por Lawrence en 1870 con base en material recolectado en Valencia, Venezuela. Además de la descripción general de coloración, similar en todos los aspectos a la coloración de $M$. c. cayanensis, Lawrence resalta que este taxón se diferencia de los demás por poseer los márgenes de las plumas rufo-canela en las alas y la cola, y por poseer un pico más grande y robusto. También resalta especialmente el color rufo en la región distal de las plumas coberteras, el cual se diferencia de otros taxones similares como $M$. c. cayanensis y $M$. . . erythropterus, que los presentan de tonos oliva o marrón (Lawrence 1870). Posteriormente, con muestras adicionales de Venezuela, Taylor en 1864 reporta esta especie como Myiozetetes cayanensis, con base en la similitud de coloración. Sclater y Salvin en 1868 proponen este taxón como Myiozetetes guianensis con individuos colectados en otras localidades de Venezuela. Es hasta 1902 que se propone como subespecie por Berlepsch y Hartert, como M. cayanensis rufipennis (Figura 2), basados en material proveniente del estado de Bolívar, Venezuela, donde también se describe el nido y los huevos de esta población. En 1906 Hellmayr adopta esta clasificación y ratifica su estatus de subespecie después de revisar material de la misma localidad.

Myiozetetes cayanensis hellmayri es descrita por Hartert y Goodson en 1917 con base en especímenes del occidente de Ecuador y el Valle del Cauca en Colombia. Según los autores, esta difiere de M. c. cayanensis por poseer más rufo en las márgenes externas de las primarias y la región superior del dorso no tan oscura y más olivácea (Hartert y Goodson 1917). Fue considerada como parte de Myiozetetes guianensis por Sclater en 1871 y como M. cayanensis por Sclater en 1883, en ambas ocasiones a partir de un análisis de coloración. En 1879 Sclater y Salvin la describen como Myiozetetes texensis a partir de muestras de los municipios de Envigado y Medellín en Colombia. En 1901 es considerada como M. c. cayanensis por Ridgway, quien consideraba que la diferencia en la coloración rufa en las alas era incipiente y no representaba un carácter suficiente para la diferenciación de las poblaciones. 
Myiozetetes cayanensis harterti es descrita originalmente por Bangs y Penard en 1921 con muestras del Darién en el chocó biogeográfico. Según los autores, esta subespecie es cercana a M. c. hellmayri, pero se diferencia por tener un tamaño menor y una coloración rufa más oscura en el borde de las plumas primarias (Bangs y Penard 1921). Aunque los autores que proponen esta diferencia argumentan tener una serie amplia de material recolectado para considerar los taxones como distintos, posteriormente Hellmayr discute que los individuos analizados no son suficientes para considerar la diferencia. Lawrence la ubica como M. cayanensis en 1862 y Ridgway la describe como M. c. cayanensis en 1918, ambos basados en la coloración del plumaje.

De esta forma, el histórico taxonómico del complejo $M$. cayanensis ha sido discutido principalmente a partir de la coloración, siendo el color del borde las rectrices y las primarias, así como la coloración de las plumas coberteras y el dorso, los caracteres más utilizados para la separación de taxones. Desde la última revisión del estatus taxonómico del género (Amadon et al. 1979), el complejo $M$. cayanensis está compuesto por cuatro subespecies vigentes ( $M$. c. cayanensis, M. c. hellmayri, M. c. rufipennis y M. c. erythropterus), estructura que se ha mantenido hasta la actualidad (Mobley y Kirwan 2020; Remsen et al. 2021). Aunque $M$. cayanensis es una de las especies más ampliamente distribuidas en todo el norte de Sudamérica, durante los últimos cien años han sido pocos o nulos los trabajos taxonómicos y filogenéticos que incluyen el complejo a nivel específico (Tello et al. 2009; Ohlson et al. 2013, 2020; Harvey et al. 2020), y ninguno a nivel infraespecífico.

\subsection{Los límites entre especies}

El concepto biológico de especie de Mayr (1942, 1963) ha prevalecido tradicionalmente en la ornitología (McKitrick y Zink 1988), y aunque a través de los años se han propuesto diversos conceptos de especie (Zink y McKitrick 1995; Cracraft 1997; Helbig et al. 2002; De Queiroz 2007; Zachos 2018) la mayor parte de las especies de aves están organizadas según este concepto, donde los taxones se clasifican por especies y subespecies dependiendo del grado de aislamiento reproductivo observado entre dos taxones hermanos 
(De Queiroz 2007). Por otro lado, otros conceptos de especie como el filogenético (Nixon y Wheeler 1990; Davis y Nixon 1992), aceptan la idea de taxones infraespecíficos, solo teniendo en cuenta que, contrario a las especies, las subespecies no exhiben caracteres fijos diferenciales con respecto a otros conespecíficos (Alström et al. 2008). Siguiendo esta idea, es posible utilizar el concepto filogenético de especie para proponer límites entre los taxones presentes en los complejos de especies de interés (Zink et al. 2016). Sin embargo, el histórico taxonómico clásico del complejo $M$. cayanensis, basa sus descripciones en relatos de caracteres fenotípicos, asociados principalmente a la coloración del plumaje y sin estar afiliados a ningún concepto de especie en particular. Si bien, la ausencia de un concepto de especie dificulta el posicionamiento del complejo en un plano evolutivo (Aleixo 2007), estas descripciones tradicionales sientan una base fundamental para el análisis de las poblaciones reconocidas en la actualidad, que son, de hecho, el punto de partida de este trabajo taxonómico.

Los taxones del complexo M. cayanensis se encuentran actualmente en la categoría de subespecie, definida como un grupo poblacional diagnosticable por diferencias morfológicas evidentes, pero que no se encuentra aislado reproductivamente de otras poblaciones (Vinarski 2015). El concepto Biológico de especie de Mayr no alcanza criterios objetivos que permitan verificar el estatus de las poblaciones alopátricas conespecíficas (Cracraft 1983; McKitrick y Zink 1988), y estas poblaciones son susceptibles a hibridación, ya que puede darse la existencia de un flujo génico consistente entre las mismas (Silveira y Olmos 2007). En las últimas décadas los estudios de caracterización de fauna han derivado en la descripción de múltiples subespecies, lo cual se torna en un ejercicio indiscriminado en el marco de la ausencia de estudios intensivos sobre la variación geográfica (Barrowclough 1982). Un abordaje más moderno de este problema, en que una especie es definida como el menor grupo de individuos diagnosticables (Cracraft 1983), surge con la adición del criterio de ancestralidad y descendencia, con lo cual la diagnosticabilidad de las poblaciones que pueden ser reconocidas como dos especies filogenéticas distintas, deriva en la invalidación implícita de la categoría de subespecie (McKitrick y Zink 1988). Según Raposo (2001), la eliminación de la categoría de subespecie permite un conocimiento más refinado sobre la biodiversidad, proporcionando una base más sólida para estudios sistemáticos, biogeográficos y de conservación. 
Aunque la delimitación de especies es motivo usual de debate en la ornitología (McKitrick y Zink 1988; Haffer 1992; Yésou 2002; Collinson et al. 2006; Aleixo 2007; Alström et al. 2008; Hausdorf 2011; Barrowclough et al. 2016), los métodos basados en morfología son usuales para llevar a cabo este proceso. Sin embargo, las aves son conocidas dentro de los vertebrados por tener relaciones complejas, y una alta variabilidad en aspectos como la distribución, la morfología, las vocalizaciones, el comportamiento, la ecología, etc.; lo que permite separar grupos, en buena medida, gracias al análisis de varias de estas características. Aunque los caracteres moleculares y morfológicos han sido los más utilizados tradicionalmente para hacer inferencias filogenéticas en ornitología, las vocalizaciones han permitido diferenciar taxones antes indiferenciables por los métodos clásicos dada su gran similaridad (Alström y Ranft 2003; Van Doren et al. 2018; Gjershaug et al. 2020), lo cual posibilita el descubrimiento de nuevas especies. Así pues, numerosos ejemplos muestran cómo en algunos géneros de amplia distribución y presencia de especies alopátricas ha sido posible "ascender" algunas subespecies al estatus de especie por las diferencias en sus vocalizaciones (Alström y Ranft 2003; Bocalini 2013; Van Doren et al. 2018). Por supuesto, estos caracteres han sido usados por diversos autores y estudios (Bretagnolle 1995; Helbig et al. 1996; Isler et al. 1997, 2013; Andersson 1999; Saitoh et al. 2008; Carneiro et al. 2012; Maurício et al. 2014; Bolaños-Sittler et al. 2020; Gjershaug et al. 2020; Lima et al. 2020), sustentando su validez para inferir límites entre especies y relaciones filogenéticas. Tradicionalmente el uso de información morfológica y vocal obtenido a través del material depositado en colecciones biológicas como herramienta para establecer criterios filogenéticos, ha permitido discernir entre complejos de especies (Martens et al. 2003) lo cual establece un parámetro de uso de datos, y junto a otros usos, es un ejemplo de la gran utilidad de las colecciones biológicas para el entendimiento de la historia evolutiva de los organismos vivos. 


\subsection{Justificación}

Aunque la familia Tyrannidae es una de las más diversas en el neotrópico, es usual encontrar una morfología muy similar dentro de este grupo, incluso en especies distantes filogenéticamente (Chaves et al. 2008). Por lo tanto, se hace necesario un mayor esfuerzo en el reconocimiento y la efectiva identificación de los taxones de este grupo, del cual se sabe que ha radiado en cientos de especies desde el mioceno (Futuyma 1997; Chaves et al. 2008; Harvey et al. 2020). Por su parte, el complejo M. cayanensis ha sido estudiado en diversas áreas de la biología como la ecología reproductiva, estrategias de forrajeo, anidamiento, morfología general, comportamiento agonístico, ectoparásitos, uso del hábitat, historia natural y distribución, entre otros (Haverschmidt 1971; McKitrick 1985; Cruz y Andrews 1989; Dyrcz 1991, 2002; Davis 1993; Rowlett 2003; Price et al. 2005; Screnci-Ribeiro y Duca 2010; Watson 2012; Smith et al. 2014b; Sandoval et al. 2018), pero poco se conoce sobre el estatus taxonómico de sus poblaciones actuales. Debido a su gran similitud, $M$. cayanensis es usualmente confundido con $M$. similis en las listas de registro, lo cual crea ruido en los registros actuales de distribución y abundancia. Este patrón de error es usual dentro del grupo, ya que normalmente la identificación de los tiranidos presenta inconsistencias debido a la semejanza que presentan muchas de las especies, incluso con aquellas que son distantes filogenéticamente (Chaves et al. 2008; Ohlson et al. 2020). Por esta razón, se hace necesaria una revisión exhaustiva de los registros disponibles con la intención de decantar al máximo posible la información de cada especie y corroborar sus rangos de distribución. Es necesario estudiar estos complejos de especie en las aves "comunes" que pueden servir como base para estudios en especies menos frecuentes. Adicionalmente algunas de las subespecies dentro de estos complejos pueden presentar un estado de conservación crítico o tener preferencias de hábitat no conocidas, al considerar la distribución y densidad poblacional de la especie generalizada (ej. Leptodon forbesi en Dénes et al. [2011]; Grallaria saltuensis y Grallaria spatiator en Isler et al. [2020]). 


\section{OBJETIVOS}

\subsection{Objetivo general}

Determinar las especies válidas del complejo Myiozetetes cayanensis (Linnaeus, 1766).

\subsection{Objetivos específicos}

- Establecer una propuesta taxonómica actualizada que refleje la historia evolutiva del complejo Myiozetetes cayanensis.

- Revisar los límites de especie a partir de un análisis integrado de variación morfológica y vocal en el complejo M. cayanensis.

- Definir la distribución geográfica de los taxones analizados. 


\section{METODOS}

\subsection{Coloración}

Para la descripción de la coloración del plumaje de los individuos analizados se utilizó la guía de colores naturales de Smithe (1975), diagnosticando el color en las siguientes partes del cuerpo: corona (color general del fondo), zona auricular, basal de la coronilla, medial de la coronilla, distal de la coronilla; pecho, basal de las coberteras dorsales, distal de las coberteras dorsales; dorsal de las plumas primarias, borde externo de las primarias, borde externo de las secundarias; basal de las coberteras mayores, distal de las coberteras mayores; basal de las supracaudales, distal de las supracaudales; dorsal de las plumas rectrices y borde externo de las rectrices. La Figura 3 y la Figura 4 muestran las partes del ave utilizadas para evaluar la coloración y la descripción de las áreas de coloración de las plumas de contorno y de vuelo respectivamente. Como se describió anteriormente en el histórico taxonómico del complejo M. cayanensis, las diferencias en coloración de las áreas externas han sido la base para la diagnosis y comparación entre taxones (Cory y Hellmayr 1927), razón por la cual un muestreo de la coloración de las partes externas es vital para el desarrollo de este trabajo.

Las mediciones de coloración se realizaron bajo luz contralada usando un iluminador de luz fría LEIKA KL 1500 LCD, observando los colores con luz superior y lateral a $3200^{\circ} \mathrm{K}$ (tungsteno), con el fin de eliminar el error causado por la variación de la luz ambiental. Los colores fueron determinados como diferentes cuando no existía un patrón sucesivo entre las pieles de la misma localidad.

\subsection{Morfometría}

Se revisaron 262 pieles de M. cayanensis (119 machos, 98 hembras y 45 individuos sin sexo determinado) disponibles en las colecciones ornitológicas del Museu de Zoologia da 
Universidade de Sao Paulo (MZUSP) y el Museu de Ciencias Naturais PUC-Minas (MCN) en Brasil; el Instituto de Ciencias Naturales de la Universidad Nacional de Colombia (ICN) y el Instituto Alexander von Humboldt (IAvH) en Colombia. Además, se incluyeron los datos obtenidos de seis pieles colectadas en noviembre de 2019 durante una campaña de campo en estado de Pará (PA), que fueron depositadas en la colección de ornitología del MZUSP (números de catálogo pendientes).

Siguiendo los protocolos de Baldwin et al. (1931), Borras et al. (2000) y Alström et al. (2008), se tomaron 18 medidas morfométricas básicas así: longitud del culmen desde la base del cráneo (culmen total), longitud del culmen desde el borde anterior de la narina (culmen (n)), alto del pico a nivel de la narina, ancho del pico a nivel de la narina, ancho de la comisura; longitud del tarso, ancho distal del tarso, longitud del hallux; longitud de la cola, ancho de la rectriz central; longitud máxima del ala en su curvatura natural (cuerda alar), la longitud de las primarias 1 a 4 y la primaria 10, el ancho de la primaria 1 a nivel medio; y el peso del individuo (datos en la etiqueta). Así mismo, en este trabajo se incluyeron las siguientes 14 medidas adicionales: la longitud de las rectrices 1 y 6 (la más externa y la central); la longitud de la secundaria 1 , el ancho de la secundaria 1 a nivel medio, la distancia entre la primaria más larga y la secundaria 1, y la diferencia entre la punta de las primarias 2 a 10 con respecto a la primaria 1. (distal distances; Baldwin et al. 2010). Las medidas de las aves vivas fueron tomadas de la misma forma que en los especímenes de museo.

Todas las medidas fueron realizadas con un calibrador digital (precisión $\pm 0,01 \mathrm{~mm}$ ), procurando siempre el ala izquierda del individuo. Se anotaron también datos de muda y edad (basado en tamaño y plumaje) de los individuos analizados.

\subsection{Vocalizaciones}

Se analizaron todas las grabaciones (640 archivos) de vocalizaciones de $M$. cayanensis disponibles en la colección de sonidos naturales del Instituto de Recursos Biológicos Alexander von Humboldt (IAvH) y las bases de datos en línea de Macaulay Library del Cornell Lab of Ornitology (macaulaylibrary.org), xeno-canto (xeno-canto.org) y Wikiaves (wikiaves.com.br). 
Para el análisis se tomaron en cuenta las grabaciones de calidad suficiente para la identificación de los parámetros vocales de la especie de interés. Una vez eliminados los archivos duplicados entre bases de datos, fueron considerados como registros negativos y descartados aquellos cantos que contenían ruido de fondo (estática del micrófono, golpes en el receptor u otros elementos, cantos de otras aves, cantos de otros grupos de fauna), sonidos no medibles (reclamos y otros sonidos sin patrón aparente) y errores técnicos (archivos corruptos o con problemas para la lectura en el software), que no permitían la identificación adecuada de la información de interés. Los elementos independientes de las vocalizaciones se definieron siguiendo la terminología propuesta por Van Doren et al. (2018) como: elemento - una unidad discreta e indivisible de un sonograma; nota - el sonido de uno o más elementos; y estrofa - serie de notas separadas por pausas.

Para establecer una medida de cambio de la estructura de las vocalizaciones se calculó un índice de diversidad iD. Este toma en cuenta la composición de notas de la serie y su proporción, usando como referencia el porcentaje de notas del trino (notas de tipo $\mathrm{C}$ en secuencia repetitiva), dividido entre el número de tipos de nota -1. A esta ecuación se debe restar un tipo de nota debido a que es la nota identificadora (es decir, la que determina el inicio del canto) y, por tanto, todas las vocalizaciones evaluadas la poseen. De esta forma, se describe el índice de diversidad como:

$$
\boldsymbol{i D}=1-\frac{C / N}{E-1}
$$

Donde:

$\mathrm{C}=$ Número de notas de tipo $\mathrm{C}$.

$\mathrm{N}=$ Número de notas totales de la vocalización.

$E=$ Número de tipos de notas de la vocalización.

El iD busca representar la diversidad del canto en un espectro comparativo entre los individuos evaluados. Al igual que el número de notas por canto y la cadencia del trino, este índice se utilizó para evaluar la diversidad vocal entre las unidades taxonómicas operativas (UTO), a través de un análisis de varianza ANOVA. 
Siguiendo los protocolos establecidos por Van Doren et al. (2018) y Alström et al. (2008), utilizando el software Raven Pro 1.5 (Bioacustics Research Program 2017), para cada canto se identificaron y seleccionaron manualmente las notas dibujando cajas de selección para medir las siguientes propiedades acústicas de las vocalizaciones: frecuencia del pico de poder, frecuencia mínima y máxima del contorno de la frecuencia pico, la pendiente promedio del contorno de la frecuencia pico, la duración conteniendo el $90 \%$ de la energía dela nota y el ancho de banda conteniendo el $90 \%$ de la energía dela nota, seleccionando un canto por individuo en cada caso. Adicionalmente, se midió la variación delta de la frecuencia, la variación delta del tiempo, la frecuencia central, la máxima frecuencia, el número de notas por canto, la frecuencia más alta y la frecuencia más baja. Estas medidas fueron tomadas usando una resolución de frecuencia de $46.9 \mathrm{~Hz}$ y un tiempo de resolución de 1,06 milisegundos. Los valores obtenidos de las notas se organizaron en las siguientes nueve medidas para cada canto: (1) frecuencia pico media, (2) ancho de banda medio, $(3,4)$ máxima y mínima frecuencia media, (5) número de notas por canto, (6) duración del canto, (7) duración media de cada nota, $(8,9)$ frecuencia máxima y mínima del canto.

Para el tratamiento de los datos se promediaron los valores obtenidos por cada tipo de nota identificada en cada canto. Con esto, para aquellos cantos con registros de dos o más notas se unificó el valor como promedio de los dos valores obtenidos para cada característica.

\subsection{Distribución geográfica y rangos de ocurrencia}

Los mapas de distribución de los especímenes analizados fueron construidos usando las coordenadas de las localidades depositadas en las etiquetas y en las bases de datos de vocalizaciones. Cuando las coordenadas no estuvieron disponibles, estas fueron consultadas en los Gazetteers ornitológicos de centro y sur américa (Paynter 1982, 1992, 1993, 1997; Stephen y Traylor 1983, 1985; Paynter y Traylor 1991; Vanzolini 1992) y las bases de datos geográficos en línea Fallin grain (fallingrain.com) y Google Earth Pro (versión 7.3.2.5776, 2019). Adicionalmente se utilizaron los bancos de imágenes de Macaulay Library (una fotografía) y Wikiaves (80 fotografías) para corroborar los posibles rangos obtenidos, así como 
para establecer los posibles rangos de distribución de las unidades taxonómicas operativas analizadas. Los mapas fueron elaborados usando el software libre Quantum GIS 3.10.2 (QGIS Development Team 2019, disponible en qgis.org).

El mapa de localización, la lista completa de pieles, archivos de audio, fotografías analizadas y sus respectivos números de catálogo se encuentran disponibles en el Anexo A y el Anexo B. En el siguiente enlace se encuentra disponible un mapa interactivo con la información descrita anteriormente: jcrioso.github.io/Mapa myiozetetes cayanensis/.

\subsection{Análisis estadísticos}

A fin de conocer si las variables morfológicas y vocales soportan las diferencias en la agrupación por unidades taxonómicas operativas (UTO), se realizó un análisis multivariante de varianza (MANOVA), utilizando la función "Manova” del paquete CAR de R (Fox y Weisberg 2019). Se definieron las medidas morfológicas y vocales como variables de respuesta múltiples (dependientes) y la agrupación por UTO obtenida en la clasificación por coloración del plumaje como variable explicativa (independiente). Se calculó la significancia de los coeficientes obtenidos a partir de los valores de $\mathrm{p}(\mathrm{p}<0,05)$, usando el estadístico de Pillai para diseños muestrales desbalanceados (debido a la diferencia en el tamaño de los grupos de UTO).

Previo al análisis de MANOVA, se realizó una prueba de correlación de Pearson a los datos para evaluar la posible colinealidad de las variables dependientes y eliminar posibles sesgos en el modelo, utilizando la función "rcorr" del paquete HMISC (Harrell 2017), que evalúa el nivel de certeza en la correlación al calcular un valor de $\mathrm{p}$, que fue considerado como criterio final de selección. De la misma forma, se evaluó la linealidad entre las variables de respuesta usando el método gráfico de matrices de correlación de la función "ggpairs" del paquete GGALLY (Schloerke et al. 2018). Adicionalmente se analizaron los supuestos de ausencia de outliers univariados a partir de los extremos de los rangos intercuartílicos y de outliers multivariados usando las distancias de Mahalanobis (Mahalanobis 1936). Los valores identificados como "outlier" fueron reemplazados por el valor de la media de la variable en cada caso. Siguiendo la metodología propuesta por Sokal y Rohlf (1995), los datos fueron 
sometidos a un test de Shapiro-Wilks para evaluar normalidad y a un test de Levene para evaluar homocedasticidad. De la misma forma, se evaluó la homogeneidad de covarianza siguiendo el método propuesto por Box (1949).

Una vez obtenidos los resultados de MANOVA, se realizó una serie de análisis ANOVA para cada variable dependiente con el fin de identificar cuales variables dependientes específicas contribuyen al efecto significativo global. Estos se realizaron usando el estadístico de welch, ya que este no asume homogeneidad en las varianzas y es más conservador para el análisis de grupos con distintos tamaños muestrales (Moder 2010). Los valores p obtenidos en las pruebas univariadas fueron corregidos usando el método de Bonferroni (1936), para evitar falsos positivos por la múltiple comparación de los valores. Para determinar cuáles grupos de UTO son diferentes se calcularon múltiples comparaciones por pares usando los métodos de Tukey-Kramer (1949), Games-Howell (1976) y Dunnett (1980). Los pares de UTO obtenidos fueron agrupados y posteriormente comparados a fin de conocer las diferencias encontradas.

Con el fin de conocer posibles agrupaciones independientes de las UTO identificadas por coloración (agrupación no supervisada), a partir de las observaciones morfométricas se realizó una prueba de agrupamiento de K-means (MacQueen 1967; Kanungo et al. 2002) usando las funciones kmenas y fviz_cluster del paquete FACTOEXTRA (Kassambara y Mundt 2020). Previo al análisis, se corrieron los estadísticos de Elbow y Silhouette para encontrar el número óptimo de agrupaciones supuestas y con base en esta información se realizó el análisis de agrupamiento en dos, cuatro y siete grupos.

Adicionalmente, se realizó una prueba de t-student para evidenciar la presencia de dimorfismo sexual morfométrico en cada variable analizada para cada una de las UTO.

El análisis de los datos fue realizado en el software R 3.6.1 (R Core Team 2019). El código script reproducible completo se encuentra disponible como material suplementario en el enlace: https://github.com/jcrioso/Mapa myiozetetes cayanensis. 


\subsection{Criterios para delimitación de especies}

Tomando como referencia el trabajo de Marcondes (2013), para la delimitación de especies en este trabajo se adoptó el criterio de diagnosticabilidad de las poblaciones. Es decir, que los caracteres cuyos estados ocurren con frecuencias distintas entre dos taxones indican grupos diferentes por acumulación de divergencias a lo largo del tiempo (Helbig et al. 2002).

Según la definición filogenética de especie, donde los grupos validos son linajes de meta poblaciones que evolucionan de forma separada (Cracraft 1983), a lo largo del tiempo la acumulación de variaciones de caracteres deriva en la formación de nuevos taxones independientes, lo cual está de acuerdo con el criterio de diagnosticabilidad.

En trabajos taxonómicos previos basados exclusivamente en caracteres fenotípicos, la diagnosticabilidad de poblaciones es el método más usual de delimitación de especies, dado que es capaz de diferenciar los agrupamientos de individuos con patrón de ancestro y descendencia, así como otras propiedades propias de los taxones a nivel especifico, tales como la monofilia y el aislamiento reproductivo (Cracraft 1983, 1997; Coyne y Orr 2004).

De esta forma, en este trabajo se consideran especies distintas aquellas poblaciones con un patrón geográfico especifico que son diagnosticables entre sí por (1) la combinación de tres o más diferencias en los parámetros individuales del comportamiento vocal, en conjunto con (2) al menos una diferencia morfológica y (3) una diferencia en la coloración del plumaje. 


\section{RESULTADOS}

\subsection{Coloración}

Se identificaron 21 colores asociados al plumaje de $M$. cayanensis, que abarcan toda la variación de los individuos evaluados. Una vez definidos los colores principales, aquellos que corresponden a la mayoría de los individuos en cada carácter (es decir, la moda independiente de cada carácter), se obtuvo una paleta de 18 colores finales, compuestos por tonos marrones, amarillos y verdes. La Figura 5 muestra la paleta de colores identificados, así como sus nombres y códigos de referencia, los cuales son mencionados recurrentemente en este documento.

\subsubsection{Descripción de coloración}

Los individuos evaluados presentan un patrón morfológico general estable, con una predominancia de colores marrones en el fondo (dorso, cabeza y plumas de vuelo), amarillos en el pecho y la coronilla; y una franja superciliar blanca conspicua. No se identificaron caracteres diferenciales en el plumaje (ej. manchas, listas, barras u otras) asociadas a ninguno de los individuos y/o zonas geográficas. Sin embargo, en una vista más detallada, la variación de tonos de color asociados a las zonas específicas del plumaje (Figura 3) es en general amplia, con lo cual se pueden identificar varios morfotipos.

Corona: en este carácter se identificaron dos colores principales, el fusco (NCS21), que es uniforme en los individuos examinados en casi toda la distribución; y el marrón grisáceo oscuro (NCS20) propio de las poblaciones que ocurren en el estado de Minas Gerais en Brasil (Figura 6). Este color es ligeramente más pálido en las mudas pre-reproductivas y con frecuencia es grisáceo en individuos juveniles.

Zona auricular: se identificó solamente un color asociado a la zona auricular en los individuos analizados (Figura 7), el fusco (NCS21) es constante a lo largo de toda la distribución 
de la especie y es un carácter diagnóstico del complejo $M$. cayanensis como unidad taxonómica. Al igual que el color de la corona, este carácter puede ser más pálido en mudas pre-reproductivas, pero usualmente es más oscuro que la corona y es contrastante con otras áreas de colores similares en las primeras etapas del plumaje.

Coronilla (basal y distal): al igual que la zona auricular, en este carácter solo se identificó un color principal asociado a los individuos examinados; el amarillo intenso (NCS55), es un carácter diagnóstico de M. cayanensis y está presente en toda su distribución (Figura 8). Sin embargo, en algunos individuos provenientes del valle del Magdalena (departamentos de Caldas y Antioquia en Colombia), este color puede ser ligeramente más oscuro, contrastando menos con el color medial de la coronilla. Este carácter está ausente en mudas prereproductivas.

Coronilla (medial): se identificaron dos estados de coloración principal asociados a las poblaciones de la zona oriental y occidental de la cordillera oriental de los andes (Figura 9). Los individuos con distribución transandina (zona occidental de la cordillera oriental) muestran este carácter de color amarillo naranja (NCS18), mientras que los individuos con distribución cisandina (zona oriental de la cordillera oriental) muestran este carácter de color naranja intenso (NCS17). Por lo general contrasta con la coloración basal y medial de la coronilla, siendo esta más clara. Este carácter está ausente en mudas pre-reproductivas.

Pecho: este carácter se comporta de forma similar al color basal y distal de la coronilla, presentando un solo estado principal en los individuos analizados. El color amarillo intenso (NCS55) ocurre a lo largo de toda su distribución (Figura 8) y al igual que la zona auricular y el color basal y distal de la coronilla es diagnostico para el complejo M. cayanensis. En individuos juveniles y mudas pre-reproductivas es usualmente más pálido.

Dorso (basal): se identificaron dos estados de coloración principales para este carácter, el marrón oliva (NCS28) que es uniforme en los individuos que ocurren en la región transandina y el escudo guyanés hasta el límite norte del Río Orinoco en Venezuela (Figura 10); y el marrón acanelado (NCS33) que se presenta en las poblaciones que ocurren al oriente de la Amazonía y el resto de la distribución del complejo. Este carácter puede ser grisáceo en individuos juveniles a lo largo de toda su distribución. 
Dorso (distal): para este carácter se identificaron tres estados principales de coloración, el citrino (NCS51) que está presente en los individuos de la región transandina y el escudo guyanés hasta el límite norte del Río Orinoco en Venezuela; el oliva verdoso (NCS49), presente en las poblaciones de la amazonia, la diagonal seca y la mata atlántica hasta el sur de Brasil; y el verde oliva (NCS48), presente en las poblaciones del estado de Minas Gerais en Brasil (Figura 11). Este carácter puede ser rojizo o estar ausente en mudas pre-reproductivas.

Plumas de arrastre: las plumas de arrastre se definen como el conjunto de las plumas primarias y secundarias. En este carácter se identificaron dos estados de coloración principal, el marrón oliva (NCS28) que está presente en los individuos de la región transandina, el escudo guyanés hasta el límite norte del Río Orinoco en Venezuela y el estado de Minas Gerais en Brasil; y el fusco (NCS21), presente en las poblaciones de la amazonia, la diagonal seca y la mata atlántica hasta el sur de Brasil (Figura 12). Este carácter es constante a lo largo de las mudas y suele tener poca variación entre individuos.

Borde de las plumas primarias: se identificaron tres caracteres principales de coloración, el marrón antiguo (NCS37), presente en los individuos distribuidos en la región transandina, la Amazonía, la diagonal seca y la mata atlántica hasta el sur de Brasil; el leonado (NCS38), presente en las poblaciones del escudo guyanés; y el ámbar (NCS36), presente en las poblaciones del estado de Minas Gerais (Figura 13). Este carácter puede ser canela (NCS39) en las poblaciones del noroccidente de Brasil (Amazonas y Roraima). Puede ser más pálido, anteado o rojizo en individuos juveniles y mudas pre-reproductivas.

Borde de las plumas secundarias y rectrices: en este carácter se identificaron tres colores principales, el amarillo-anteado (NCS53), presente en los individuos transandinos; el canela (NCS39), presente en los individuos del escudo guyanés y el estado de Minas Gerais; y el arcilla (NCS26), en el resto de la distribución (Figura 14). Al igual que el borde de las plumas primarias, este carácter puede ser más pálido, anteado o rojizo en individuos juveniles y mudas pre-reproductivas.

Coberteras menores y mayores (basal): este carácter presenta una distribución similar al color de las plumas de arrastre, con dos estados de coloración principal, el marrón oliva (NCS28) que está presente en los individuos de la región transandina, el escudo guyanés hasta 
el límite norte del Río Orinoco en Venezuela y el estado de Minas Gerais en Brasil; y el marrón acanelado (NCS33), presente en las poblaciones de la amazonia, la diagonal seca y la mata atlántica hasta el sur de Brasil (Figura 15). Puede ser más pálido o grisáceo en mudas prereproductivas.

Coberteras menores y mayores (distal): para este carácter se identificaron cuatro estados de coloración principales, siendo el más variable junto con el color distal de las plumas supracaudales. El color arcilla (NCS123B), que ocurre en los individuos transandinos; el canela (NCS123A), presente en los individuos del escudo guyanés; el citrino (NCS51), es el de distribución más amplia y ocurre en los individuos de la Amazonía, la diagonal seca y la mata atlántica hasta el sur de Brasil; y el marrón oliva (NCS28), presente en los individuos del estado de Minas Gerais (Figura 16). Este carácter puede ser más pálido, rojizo, abarcar una mayor proporción de la pluma o estar ausente en individuos juveniles. Es muy variable a través de la edad y puede tener tonos distintos a lo largo de las regiones.

Supracaudales (basal): este carácter sigue un patrón de distribución similar al del color basal del dorso, donde se identificaron dos estados de coloración principales, el marrón oliva (NCS28) que es uniforme en los individuos que ocurren en la región transandina y el escudo guyanés hasta el límite norte del Río Orinoco en Venezuela; y el marrón acanelado (NCS33) que se presenta en las poblaciones que ocurren al oriente de la Amazonía y el resto de la distribución del complejo (Figura 10). Este carácter puede ser grisáceo en individuos juveniles a lo largo de toda su distribución.

Supracaudales (distal): se identificaron cuatro estados de coloración principal, el color arcilla (NCS123B), presente en los individuos transandinos; el leonado (NCS38), presente en los individuos del escudo guyanés; el oliva verdoso (NCS49), en el oriente de la Amazonía, la diagonal seca y la mata atlántica hasta el sur de Brasil; y el verde oliva (NCS48), presente en los individuos del estado de Minas Gerais (Figura 17). Este carácter es muy variable a lo largo de su distribución y aunque esta descripción sigue el patrón general, es posible encontrar otras variaciones dentro de todo su rango de ocurrencia. Suele ser más pálido o está ausente en mudas pre-reproductivas. 
Rectrices: este carácter sigue el mismo patrón de cloración de las plumas de arrastre (Figura 12). Puede ser ligeramente más pálido en mudas pre-reproductivas.

Los caracteres aquí presentados son apenas una descripción cualitativa de los morfotipos de plumaje más representativos dentro de la muestra de individuos analizados. Esta información fue utilizada como soporte para la definición y diagnosis de las unidades taxonómicas operativas (UTO).

\subsubsection{Definición de Unidades Taxonómicas Operativas (UTO)}

Tomando como referencia las similitudes y diferencias en la coloración del plumaje, es posible identificar cuatro agrupaciones (poblaciones) definidas por la coloración distal de las plumas coberteras y el color distal de las supracaudales (Tabla 1). En contraste, el color de la zona auricular (fusco, NCS21), el color basal y distal de la coronilla (amarillo intenso, NCS51) y el color del pecho (amarillo intenso, NCS51) son caracteres estables a lo largo de toda la distribución de los individuos evaluados, siendo un carácter diagnóstico del complejo $M$. cayanensis. Estas poblaciones son definidas como Unidades Taxonómicas Operativas (UTO), las cuales son usadas como base fundamental en los análisis de morfología y vocalizaciones.

\section{UTO 1}

Su distribución está delimitada principalmente por la cordillera oriental de los andes (Figura 18). Se extiende desde el sur de Costa Rica, a través de Panamá; el noroccidente de Venezuela alrededor de la cuenca de Maracaibo en los estados de Zulia y Falcón, en la porción occidental de los estados de Lara y Táchira; y en todo el territorio transandino que comprende el occidente de la cordillera oriental de los andes en Colombia y Ecuador hasta el noreste de la provincia de Loja. Para esta población se han atribuido los nombres Myiozetetes guianensis (Sclater, 1871), Myiozetetes texensis (Sclater y Salvin, 1879), Myiozetetes cayanensis (Ridgway, 1901) y Myiozetetes cayanensis hellmayri (Hartert y Goodson, 1917). Una subpoblación del chochó biogeográfico fue considerada como Myiozetetes cayanensis harterti, que fue posteriormente discutida por Hellmayr por no contar con suficiente material 
que soportara las diferencias con otros individuos del resto de la distribución de la población.

Presenta como caracteres generalizados el color amarillo-anteado (NCS53) en el borde de las plumas secundarias y las rectrices; el color arcilla (NCS123B) en la región distal de las coberteras menores, mayores y las supracaudales; y la coloración amarillo naranja (NCS18) en la zona media de la coronilla. Sin embargo, aunque estos estados de carácter ocurren en la gran mayoría de los individuos analizados, se encontró que puede haber variaciones asociadas a estados de plumaje pre-reproductivos en toda la distribución.

El color de la corona es fusco uniforme (NCS21), el cual comparte con las UTO 2 y 3 . El área basal del dorso es marrón oliva (NCS28) al igual que la UTO 2, siendo este carácter más canela/rojizo en las UTO 3 y 4, este patrón se repite para el área basal de las supracaudales. Del mismo modo, el color distal del dorso (citrino, NCS51) es un carácter compartido con la UTO 2, que es más pálido con respecto a las demás UTO. Las plumas de arreste comparten un patrón general de coloración marrón oliva (NCS28) con las UTO 2 y 4, mientras que se diferencia del color de la misma zona en UTO 3 al ser más claro, este patrón se repite con el color de las rectrices y el color basal de las coberteras. El borde de las primarias es marrón antiguo (NCS37), que comparte con la UTO 3 y es más oscuro con respecto a las demás UTO.

\section{UTO 2}

Esta población se distribuye desde el noroccidente de Venezuela en la margen izquierda del Río Orinoco, a través del oriente de los andes hasta el norte de los departamentos de Amazonas y Vaupés en Colombia y hasta el norte de las provincias de Napo y Orellana en Ecuador (Figura 18). Debido a la falta de muestras de colección de individuos provenientes de la porción noroccidental de la cuenca amazónica en Colombia, Ecuador y Brasil, los limites surorientales de esta población son difíciles de definir con claridad, pero es probable que pueda extenderse hasta el sur de la provincia de Morona Santiago en Ecuador y hasta la margen izquierda del Río Negro en Brasil. A esta población se le han atribuido los nombres Myiozetetes cayanensis (Taylor, 1864), Myiozetetes guianensis (Sclater y Salvin, 1868), Myiozetetes rufipennis (Lawrence, 1870) y Myiozetetes cayanensis rufipennis (Berlepsch y Hartert, 1902; ratificado por Hellmayr, 1906). 
Se caracteriza por tener el borde de las plumas primarias y la porción distal de las supracaudales de color leonado (NCS38); el color distal de las coberteras es canela (NCS123A) y estos tres caracteres ocupan una proporción mayor de la pluma que las demás UTO (>30\%), dando una apariencia rufa al fondo y el dorso de las alas. Por lo general, estos caracteres son más rojizos y amplios en mudas pre-reproductivas.

El color de la corona es fusco uniforme (NCS21), al igual que en las UTO 1 y UTO 3. EI color medial de la coronilla es naranja intenso (NCS17), aunque en los individuos juveniles puede ser amarillo y con frecuencia la intensidad del color aumenta en las mudas subsecuentes. La porción basal del dorso y las supracaudales, así como las plumas de arrastre son color marrón oliva (NCS28), que comparte con la UTO 1 y contrastan con las UTO 3 y 4. Del mismo modo, la UTO 2 comparte el color distal del dorso con la UTO 1, al ser citrino (NCS51), es más pálido/brillante con respecto a las UTO 3 y 4 . El borde de las plumas secundarias y rectrices es canela (NCS39), al igual que la UTO 4 y el color basal de las coberteras y las rectrices es marrón oliva (NCS28), que es uniforme al igual que en las UTO 1 y 4, siendo más pálidos que el color presente en la UTO 3.

\section{UTO 3}

La UTO 3 es la de más amplia distribución en el complejo $M$. cayanensis, ocurre desde el oriente de Venezuela (margen derecha del Río Orinoco en Amazonas, Bolívar y Delta Amacuro), al nororiente de Suramérica en Guyana, Guyana Francesa y Surinam. Al norte y centro de Brasil hasta el noroccidente de Ceará y Pernambuco, hacia el sur del Río San Francisco en Sergipe y Bahía hasta el norte del Río Contas. En la diagonal seca y la porción occidental de Minas Gerais hasta el norte de Santa Catarina, norte de la provincia de Misiones en Argentina y en Paraguay al oriente del rio Paraná. Hacia el occidente hasta la mitad norte de Bolivia, la porción cisandina de Perú (Puno, Madre de Dios, Uyucali y Loreto) y el departamento del amazonas en Colombia (Figura 18). Los límites entre las UTO 2 y 3 son difíciles de establecer con claridad, por lo cual es necesario revisar material adicional proveniente del occidente de la cuenca amazónica. A esta población se le han atribuido los nombres de Muscicapa cayanensis (Linnaeus, 1776; retomado por Sclater y Salvin, 1867), Muscipeta cayanensis (Lafresnaye y D'Orbigny, 1837), Elaenea cayanensis (Cabanis, 1848), 
Myiozetetes guianensis (Cabanis y Heine, 1859), Myiozetetes columbianus (Pelzeln, 1868) y Myiozetetes cayanensis (Hellmayr, 1906).

Los caracteres principales de esta UTO incluyen la porción distal del dorso y las supracaudales color oliva verdoso (NCS49), las plumas de arrastre y rectrices fusco uniforme (NCS21), el borde las plumas secundarias y rectrices color arcilla (NCS26) y las porciones basal y distal de las coberteras marrón acanelado (NCS33) y citrino (NCS51) respectivamente. Aunque estos colores representan la tendencia general de los individuos analizados, debido a su amplio rango de distribución, en la UTO 3 se encontró una mayor variabilidad en los tonos y colores asociados a las partes del plumaje aquí descritas.

Al igual que las UTO 1 y 2, la corona en la UTO 3 es color fusco (NCS21). El color medial de la coronilla es naranja intenso (NCS17), el cual comparte con las UTO 2 y 4 . La porción basal del dorso y las supracaudales es marrón acanelado (NCS33) al igual que la UTO 4, que contrasta con las UTO 1 y 2 al ser más canela/rojizo. El borde de las primarias es marrón antiguo (NCS37), carácter que comparte con la UTO 1, siendo este más oscuro con respecto a las UTO 2 y 4.

\section{UTO 4}

La distribución de esta UTO ocurre en el oriente de Brasil al sur del Río Contas y el estado de Bahía, al oriente de Minas Gerais, Espírito Santo y Río de Janeiro hasta la margen izquierda del Río Grande y el norte de la Serra da Mantiqueira (Figura 18). Se le han atribuido los nombres Tyrannula erythroptera (Lafresnaye, 1853), Myiozetetes erythropterus (Sclater, 1871) y Myiozetetes cayanensis erythropterus (Hellmayr, 1906).

Se distingue de las demás UTO por tener el borde de las primarias color ámbar (NCS36), el cual es intenso y se corta de manera conspicua con la porción distal de las plumas primarias formando un semi triangulo en vista lateral. Adicionalmente, el color de la corona es marrón grisáceo (NCS20), el color distal del dorso y las supracaudales es verde oliva (NCS48) y la porción distal de las coberteras es marrón oliva (NCS28), aunque puede tender hacia los tonos rufos en las mudas pre-reproductivas. 
La corona es más oscura con respecto a las demás UTO, con lo cual la zona auricular parece más clara en contraste. Al igual que las UTO 2 y 3 , el color medial de la coronilla es naranja intenso (NCS17). El área basal del dorso y las supracaudales es marrón acanelado (NCS33), que comparte con la UTO 3 y se diferencia de las UTO 1 y 2 al ser más canela/rojizo. Las plumas de arrastre, las rectrices y la porción basal de las coberteras son marrón oliva (NCS28), al igual que las UTO 1 y 2. En la UTO 3, las plumas de arrastre y las rectrices son más oscuras, mientras que el área basal de las coberteras es más canela/rojizo. El borde de las secundarias y rectrices es canela (NCS39), carácter que comparte con la UTO 2, y se diferencia de las UTO 1 y 3 al ser tonos rojizos y no anteados. Sin embargo, los tonos anteados pueden aparecer en algunos individuos juveniles a lo largo de la distribución.

\subsection{Morfometría}

Myiozetetes cayanensis es un ave de tamaño mediano ( 165 mm, Hilty y Brown [1986]), con un pico de tamaño mediano a pequeño (culmen $(n)=9,58 \pm 0,58 \mathrm{~mm}$; alto del pico al nivel de la narina $=5,04 \pm 0,37 \mathrm{~mm})$. El tarso tiene una longitud de $18,46 \mathrm{~mm}( \pm 0,86$ $\mathrm{mm})$, la cola de 73,7 $\mathrm{mm}( \pm 4,39 \mathrm{~mm})$ y la cuerda alar de $86,82( \pm 4,38 \mathrm{~mm}$ ) (Figura 19, Tabla 2). Debido al bajo número de registros pertenecientes a la UTO 4 (5 machos, 4 hembras, 2 sexo indeterminado), para cumplir el supuesto del tamaño de muestra adecuada, donde el número de registros en cada agrupación debe ser mayor al número de variables de salida (Warne 2014; Uttley 2019), se seleccionaron diez variables morfométricas para el análisis (modelo 1), tomando como referencia su representatividad en estudios ornitológicos (culmen (n), alto del pico, tarso, cola, cuerda alar) y el potencial de variación especifica del complejo M. cayanensis (ancho distal del tarso, ancho de la rectriz central, largo de P10, largo de P1 y largo de S1). Ninguna de las variables mostró correlación o colinealidad según la prueba de Pearson (todos Pearson < 0,9; Tabla 3).

Una vez identificados los valores extremos (outliers univariados), estos fueron tratados como NA (ausencia de datos para la variable especifica) y posteriormente fueron reemplazados por el valor de la media corregida de la variable. Se eliminó el registro completo 
de un individuo que presentó valores extremos multivariados (MZUSP-64451), identificados siguiendo el método de Mahalanobis (Mahalanobis 1936). Las variables de alto de pico y ancho distal del tarso no fueron normales según la prueba de normalidad de Shapiro-Wilks para la UTO 1 (alto de pico Shapiro-Wilks = 0,0043; ancho distal del tarso Shapiro-Wilks = 0,0398; Tabla 4). En una mirada grafica el desvió de los datos no muestra una asimetría pronunciada (Figura 20) y la anormalidad de estas variables esta explicada por oblicuidad y no por valores extremos, con lo cual el análisis de varianza es consistente y no pierde robustez (Tiku 1971; Khan y Rayner 2003). Sin embargo, la prueba de normalidad multivariada de M Shapiro, mostro una alta anormalidad de los datos ( $\mathrm{M}$ Shapiro $=1,18 \mathrm{E}-07$ ) y el método de Box's M mostro que los datos no presentan homogeneidad de covarianza (Box's $M=0,0206$ ).

Tomando en cuenta lo anterior, se seleccionaron dos modelos adicionales con siete (modelo 2: culmen total, culmen ( $\mathrm{n}$ ), alto del pico, tarso, cola, cuerda alar y largo de S1) y cuatro variables (modelo 3: culmen ( $n$ ), tarso, cola y cuerda alar). Al igual que el modelo 1, el modelo 2 presenta anormalidad multivariada y heterogeneidad en la covarianza ( $\mathrm{M}$ Shapiro = 1,18E-07; Box's $M=0,0218)$, mientras que el modelo 3 resultó ser consistente con los supuestos de análisis de MANOVA (M Shapiro = 0,232; Box's M =0,108; Figura 21, Tabla 5), razón por la cual fue seleccionado para el análisis del modelo lineal. El MANOVA mostró que existe una diferencia multivariada significativa entre las variables de las agrupaciones de UTO (Pillai $\mathrm{p}=2.769 \mathrm{e}-13$; test stat $=0.421 ; \mathrm{Df}=848 ;$ Tabla 6) este se realizó utilizando el estadístico de Pillai, ya que no asume homocedasticidad de las variables ni homogeneidad en la covarianza, lo cual permite un tratamiento más adecuado para muestras con tamaño disímil entre el número de datos de las agrupaciones (ej. 117 registros en UTO 1 con respecto a 11 registros en UTO 4).

En las pruebas post hoc, el estimador de Welch arrojó valores de $\mathrm{p}$ significativos para todas las variables morfométricas (Welch culmen $(n)=4,06 \mathrm{E}-07$; tarso $=7,04 \mathrm{E}-08$; cola $=$ 0,000317; cuerda alar =0,003), con lo cual todas difieren entre las agrupaciones de UTO en al menos un parámetro (Figura 22). En cuanto a las pruebas de agrupación por pares, TukeyKramer y Dunnet (Figura 23) soportaron la diferencia entre todos los pares de agrupaciones en al menos un parámetro, excepto entre la agrupación de UTO 2 - 3 (Tabla 7), mientras que Games-Howell solamente soportó la diferencia entre las agrupaciones de UTO $1-2$ y UTO 3 -4 . 
Las pruebas de agrupación supervisada de $k$-means mostraron una tendencia a agrupar la mayoría de los elementos de la UTO 4. De la misma forma, la mayoría de los elementos de la UTO 1 fueron incluidos en una de las cuatro agrupaciones sugeridas (Figura 24).

El mapa de agrupación no supervisada por k-means, muestra una matriz mixta de agrupación en todo el rango de distribución conocido para las cuatro UTO. Sin embargo, se hace notoria la agrupación 1, cuyos elementos se concentran únicamente en cercanías a la cordillera de los Andes en las vertientes oriental y occidental (Figura 25).

Los resultados de $t$-student revelaron que existe dimorfismo sexual en la longitud del tarso $(p=3,824 E-03, D f=109,1)$, cuerda alar $(p=1,480 E-13, D f=109,4)$, largo P1 $(p=2,380 E-$ 03, Df = 109,9), largo P10 ( $p=2,964 E-09, D f=109,5)$ y largo S1 $(p=2,196 E-07, D f=107,3$; Figura 26) entre machos y hembras de la UTO 1. En la UTO 3 se encontró dimorfismo sexual en el largo de P10 ( $p=9,720 \mathrm{E}-03, \mathrm{Df}=414,5)$. En contraste no se encontraron diferencias morfométricas entre los machos y las hembras de las UTO 2 y 4 (Tabla 8).

\subsection{Vocalizaciones}

Fue posible la inclusión de 435 grabaciones de audio, una vez descartados 209 archivos que no cumplían con las especificaciones técnicas, de información mínima para el análisis o que contaban con registros duplicados en otras bases de datos. El Anexo C, muestra el resumen de los archivos descartados, así como el tipo de error presentado y algunas observaciones.

En el análisis de sonogramas se evidenció una amplia variabilidad de vocalizaciones de los individuos en toda la distribución. Fue posible identificar dos tipos principales de vocalizaciones, (1) el llamado reproductivo, compuesto por un único elemento repetitivo con forma de "paracaídas aplanado" y sonido "wiiiiiii" (Frecuencia pico $(\mathrm{Hz})=2902,21 \pm 309,33$, Ancho de banda al 90\% $(\mathrm{Hz})=385,02 \pm 178,44$, Duración $(s)=1,11 \pm 0,27$; elemento D, Figura 27), y (2) el canto de comunicación, compuesto por cinco elementos definidos según las 
categorías propuestas por Van Doren et al. (2018) como: (A) es el silbido inicial de la vocalización, tiene forma de " $\mathrm{S}$ " alargada lateralmente y es más largo que los demás elementos del canto (Frecuencia pico $(\mathrm{Hz})=3715,21 \pm 896,88$, Ancho de banda al $90 \%(\mathrm{~Hz})=$ 1022,58 \pm 476,89, Duración (s) = 0,87 $\pm 0,2$; Figura $28-A$, Tabla 9); (B) es un silbido secundario, tiene forma de " $M$ " plana, es más corto que el silbido inicial y por lo general de frecuencia más alta (Frecuencia pico $(\mathrm{Hz})=4951,41 \pm 1452,23$, Ancho de banda al 90\% $(\mathrm{Hz})=910,25 \pm 264,42$, Duración $(s)=0,56 \pm 0,08$; Figura 28 - B); (C) es un trino que suele presentarse como estrofa de tres a once elementos, tiene forma de " $U$ " y suele estar acompañado de armónicos muy marcados que son claramente diferenciables en el espectrograma (Frecuencia pico $(\mathrm{Hz})=$ 4094,86 \pm 712,36, Ancho de banda al 90\% $(\mathrm{Hz})=1803,19 \pm 514,12$, Duración $(\mathrm{s})=0,32 \pm 0,19$;

Figura 28 - C); (F) es un silbido corto, tiene forma de "paracaídas convexo" y generalmente está ubicado al final del canto de comunicación, posterior a los elementos de tipo $C$ (Frecuencia pico $(\mathrm{Hz})=4986,05 \pm 108,12$, Ancho de banda al $90 \%(\mathrm{~Hz})=399 \pm 166,59$, Duración $(s)=0,29 \pm 0,06$; Figura $28-F)$ y (Z) es el canto de comunicación completo, una estrofa que agrupa los elementos A, B, C y F (Duración (s) = 3,38 \pm 1,23; Figura 28 - Z).

\subsubsection{Análisis cuantitativo}

El ANOVA mostró que existen diferencias significativas en la distribución de la media de las UTO para la nota de tipo $D$ (canto reproductivo), en los parámetros de Frecuencia más baja ( $p=1,27 E-06)$, Frecuencia más alta $(p=4,90 E-12)$, Frecuencia pico $(p=4,00 E-11)$, PFC de la máxima frecuencia ( $p=1,21 E-11)$, PFC de la mínima frecuencia $(p=3,21 E-06)$, Frecuencia central ( $p=9,30 E-13)$ y Máxima frecuencia $(p=4,00 E-11)$. Ninguno de los otros tipos de nota mostró diferencias entras las UTO en los parámetros evaluados (Tabla 10).

Adicionalmente, los resultados de ANOVA mostraron una diferencia en el índice de diversidad (iD) ( $p=0,0412, \mathrm{Df}=3$ ) y el número de notas $(p=0,0440, D f=3$; Figura 29, Tabla 11) para las UTO previamente establecidas. Por el contrario, la cadencia del trino no mostro una diferencia significativa para este parámetro $(p=0,0609, \mathrm{Df}=3)$.

La prueba post hoc de Tukey-Kramer mostró la diferencia entre las UTO 1 y 4 en el índice de diversidad (iD) ( $p=0,0214$, Diff $=0,0900$, Figura 30, Tabla 12) y entre las UTO 1 y 2 
en el número de notas del canto ( $p=0,0243$, Diff = 2,6389). Así mismo, los valores de TukeyKramer soportan la diferencia en la nota de tipo D entre las UTO 3 - 1, 2 - 4, 2 - 1 en la Frecuencia central; entre las UTO 3-4, 3-2, 1 - 4 en el delta de la frecuencia; entre las UTO $3-1,2-1$ en la duración; entre las UTO $3-4,3-1,2-4$ y $2-1$ en la frecuencia más alta; entre las UTO 4-1, 3-1 y 2-1 en la frecuencia más baja; entre las UTO 3-4, 3-1, 2-1 en la Máxima frecuencia, la Frecuencia pico y la PFC de la máxima frecuencia; y entre las UTO 4 -1, 3-1 y 2-1 en la PFC de la frecuencia mínima (Figura 31).

El método de determinación del codo ("Elbow method", Thorndike 1953), encontró como número de agrupaciones óptimas cuatro o cinco agrupaciones. Los análisis de agrupación en cuatro elementos mostraron una dispersión de los individuos de forma heterogénea. No obstante, para la nota de tipo $D$ (canto reproductivo), la agrupación muestra una tendencia a agrupar los elementos de las UTO 1 y 3 en agrupaciones diferentes, que comparten determinada cantidad de elementos en una tercera agrupación intermedia (Figura 32- D).

El mapa de agrupación no supervisada por $k$-means, muestra una matriz mixta de presencia de las cuatro agrupaciones sugeridas a lo largo de toda la distribución de las UTO. Sin embargo, los individuos pertenecientes a la agrupación 4 se concentran únicamente en la porción cisandina de la distribución, al oriente de la cordillera oriental de los Andes hasta el Río Paraná (Figura 33).

\subsubsection{Análisis cualitativo}

Los caracteres cualitativos permiten hacer una comparación minuciosa del formato de los sonogramas y la composición de las frases entre las unidades taxonómicas operativas previamente establecidas. Fue posible recolectar 63 vocalizaciones (UTO $3=31$; UTO $1=12$; UTO 4 = 11; UTO 2 = 9) completas y en condiciones técnicas adecuadas para el análisis cualitativo. Para este análisis se tomó en cuenta únicamente el canto de comunicación y se definieron los siguientes términos y características: 
Composición: Hace referencia a los tipos (y número de tipos) de notas diferentes presentes en una vocalización. En el caso de las vocalizaciones analizadas se identificaron dos notas (de tipo A y C; ver numeral 4.3) como componentes estructurales principales que aparecen en el $98 \%$ de las vocalizaciones a lo largo de toda la distribución. Las notas de tipo F aparecen en el $27 \%$ de las vocalizaciones, mientras que las de tipo $B$ y $D$ en el $13 \%$ y el $3 \%$ respectivamente. Adicionalmente, las notas de tipo $B$ no se registraron en las vocalizaciones de los individuos contenidos en la UTO 4.

Longitud: Es el número de notas presentes en una vocalización. Este carácter es consistente entre las UTO, con una variación de entre 6 a 8 notas por vocalización, siendo la UTO 1 la que presenta vocalizaciones con menos elementos $(6,25)$ y la UTO 2 la que presenta las vocalizaciones más largas (8,9 notas en promedio) (Tabla 13).

Índice de diversidad (iD): En el índice de diversidad los valores cercanos a uno indican vocalizaciones más diversas, mientras que los valores cercanos a cero indican un comportamiento contrario, es decir, vocalizaciones poco diversas. Este carácter mostro un gradiente de diversificación vocal en las UTO, siendo la UTO 1 la que presenta vocalizaciones más diversas ( $\mathrm{iD}=0,52 \pm 0,22$ ), seguida por las UTO 2 ( $\mathrm{iD}=0,46 \pm 0,25$ ) y UTO 3 ( $\mathrm{iD}=0,32 \pm$ 0,24). La UTO 4 presentó el menor valor de diversidad en las vocalizaciones, siendo este cercano a cero (iD =0,23 $\pm 0,21$; Tabla 13).

4.3.4. Caracterización de las vocalizaciones de las Unidades Taxonómicas Operativas (UTO)

\section{UTO 1}

La UTO 1 está compuesta por notas de tipo A, B, C y F (Figura 34). El 58\% de las vocalizaciones analizadas finalizó con notas de tipo $\mathrm{C}$ como último elemento, mientras que el restante $42 \%$ finalizó con notas de tipo F. Los individuos de esta UTO se caracterizan por presentar las vocalizaciones más cortas, con una media de 6,25 notas, que también son entregadas en un tiempo más corto que las demás UTO $(2,89 \pm 0,82$ segundos). El valor de índice de diversidad (iD) para esta UTO es el más alto ( $\mathrm{iD}=0,52 \pm 0,22$ ), con una estructura de 
trinos más cortos ( 4,41 $\pm 2,10$, hasta siete elementos) y una mayor variabilidad en el recambio de notas dentro del canto. Sin embargo, a pesar de que el trino es más corto, este tiene una menor cadencia, con lo cual el tiempo que existe entre la entrega de los elementos de este es mayor, lo que produce un sonido más espaciado con respecto a las demás UTO $(2,90 \pm 0,69$ notas/s).

\section{UTO 2}

Esta UTO se compone por notas de tipo A, B, C y F (Figura 35). El porcentaje de vocalizaciones terminadas en nota de tipo $\mathrm{C}$ es del $77 \%$, mientras que el $22 \%$ restante finaliza en notas de tipo F y B ( $11 \%$ cada una). Los individuos de esta UTO presentan las vocalizaciones más largas ( 8,88 notas por vocalización) y la mayor longitud de trino ( 7,22 $\pm 3,15$, hasta once elementos) entre las UTO. El índice de diversidad (iD) para esta UTO es medio (iD = 0,46 $\pm 0,25)$, debido a que, aunque el canto posee un recambio alto de elementos, la longitud del trino domina la mayor parte de la estructura. La cadencia del trino es de $2,78 \pm 0,72$ notas por segundo y el canto tiene una duración promedio de 3,5 segundos, similar al tiempo registrado por las UTO 3 y 4.

\section{UTO 3}

La UTO 3 está compuesta por notas de tipo A, B, C, F y D (Figura 36), siendo la estructura más compleja de todas las UTO. El 64\% de las vocalizaciones analizadas poseen terminal de tipo $\mathrm{C}$, el $26 \%$ de tipo $\mathrm{F}$, el $7 \%$ de tipo $\mathrm{D}$ y el $3 \%$ de tipo B. Esta UTO posee vocalizaciones de 7,9 notas en promedio, con una duración de $~ 3,54 \pm 0,82$ segundos. La longitud del trino es alta ( 6,45 $\pm 1,63$, hasta diez elementos), con una cadencia de $2,48 \pm$ 0,28 notas por segundo. Aunque su composición es compleja, los valores del índice de diversidad (iD) son bajos $(0,32 \pm 0,24)$, con una estructura dominada en su mayoría por los elementos del trino y con un recambio de notas bajo dentro del canto. 


\section{UTO 4}

Las notas que componen la UTO 4 son de tipo A, C y F (Figura 37). Esta UTO se caracteriza por poseer la estructura menos completa y por la ausencia total de notas de tipo B. El $81 \%$ de las vocalizaciones poseen terminal de tipo C, mientras que el restante $18 \%$ poseen terminal de tipo F. La UTO 4 posee valores de longitud del canto y del trino medias ( 7, 36 notas totales; 6,18 $\pm 1,60$, hasta nueve elementos), con una duración promedio de 3,35 \pm 0,88 segundos y una cadencia de trino de 2,69 $\pm 0,50$ notas por segundo. El índice de diversidad (iD) de la UTO 4 es el más bajo entre las UTO $(0,23 \pm 0,21)$, principalmente debido a su estructura poco compleja, compuesta por tres tipos de notas con un recambio muy bajo o nulo dentro del canto.

\subsection{Nomenclatura}

Todas las unidades taxonómicas operativas definidas en este trabajo poseen nombres previos en literatura, así como sinónimos y propuestas acordes con el código Internacional de Nomenclatura Zoológica (Comisión Internacional de Nomenclatura Zoológica 2000).

\section{UTO 1}

Myiozetetes hellmayri Hartert y Goodson, 1917

Esta unidad es reconocida actualmente con el nombre de Myiozetetes cayanensis hellmayri Hartert y Goodson, 1917, el nombre con el que fue descrita originalmente con base en individuos del occidente de Ecuador, el Valle del Cauca y Bogotá en Colombia. Con base en la revisión taxonómica realizada en el presente estudio, aquí se reconoce como taxón independiente con el estatus de especie, bajo el nombre de Myiozetetes hellmayri Hartert y Goodson, 1917. Los nombres Myiozetetes guianensis Sclater, 1871, Myiozetetes cayennensis Sclater, 1883, Myiozetetes texensis Sclater y Salvin, 1879 y Myiozetetes cayanensis harterti 
Bangs y Penard, 1921, son considerados como sinónimos junior de Myiozetetes hellmayri, ya que no fue posible establecer una diferencia de estas diagnosis en los análisis.

\section{UTO 2}

Myiozetetes cayanensis rufipennis Lawrence, 1870

El taxón representado por la UTO 2 se conoce actualmente como Myiozetetes cayanensis rufipennis, descrito originalmente como Myiozetetes rufipennis Lawrence, 1870. Aquí se corrobora su estatus actual como subespecie propuesto por Berlepsch y Hartert (1902) y soportado por Hellmayr (1906), ya que este no pudo ser diferenciado morfológicamente de la UTO 3. Los nombres Myiozetetes cayennensis Traylor, 1864 y Myiozetetes guianensis Sclater y Salvin, 1868, son sinónimos junior de este taxón.

\section{UTO 3}

Myiozetetes cayanensis cayanensis (Linnaeus, 1766)

La unidad evolutiva definida como UTO 3 es actualmente conocida como Myiozetetes cayanensis cayanensis (Linnaeus, 1766). Descrita originalmente dentro del género Muscicapa por Linnaeus (1766), basado en la plancha de François-Nicolas Martinet en la obra de Brisson (P. 38, Fig. 4, 1760) de un individuo de Cayena en la actual Guyana Francesa, es el taxón con el que se describe la especie tipo del complejo Myiozetetes cayanensis. Se corrobora su estatus de subespecie propuesto por Hellmayr (1906), ya que este no pudo ser diferenciado morfológicamente de la UTO 2. Los nombres Myiozetetes guianensis Cabanis y Heine, 1859, Muscipeta cayennensis Lafresnaye y D’Orbigny, 1837, Elaenea cayanensis Cabanis, 1848, Myiozetetes cayennensis Sclater y Salvin, 1867 y Myiozetetes columbianus Pelzeln, 1868 son sinónimos de este taxón. 


\section{UTO 4}

Myiozetetes erythropterus (Lafresnaye, 1853).

El nombre utilizado en la actualidad para esta unidad es Myiozetetes cayanensis erythropterus (Lafresnaye, 1853). Anteriormente descrito como Tyrannula erythroptera por Lafresnaye en 1853 con material del estado de Rio de Janeiro en Brasil. La presente revisión taxonómica permite el reconocimiento de este taxón con el estatus de especie plena bajo el nombre de Myiozetetes erythropterus (Lafresnaye, 1853), soportado previamente por Sclater (1871). El nombre Myiozetetes cayanensis erythroptera Hellmayr, 1906 es sinónimo de este taxón.

\subsection{Taxonomía}

Genero Myiozetetes Sclater, 1859

Myiozetetes Sclater, 1859. Lond., 27, p. 46. Localidad tipo: origen de designación de "Elainia cayennensis" = Muscicapa cayanensis (Linnaeus, 1766)

Myiozetetes hellmayri Hartert y Goodson, 1917

Myiozetetes cayanensis hellmayri Hartert y Goodson, 1917. Nov. Zool., 24, p. 412. Localidad: Cachavi, Provincia Esmeraldas, Ecuador; Todd y Carriker, 1922. Ann. Carnegie Mus., 14, p. 360. Localidad: Trojas de Cataca, Tucurinca y Fundación, Santa Marta.

Myiozetetes guianensis Sclater, 1860. (diferente de Cabanis y Heine). Lond., 28, p. 283. Localidad: Babahoyo, Ecuador; Wyatt, 1871. Ibis, p. 333. Localidad: entre Canta y el Magdalena, Colombia.

Myiozetetes cayennensis Sclater, 1871. (diferente de Linnaeus). Lond., 1, p. 752. Localidad: Babahoyo, 
Ecuador; Berlepsch y Taczanowski, 1883. 1.c., p. 556. Localidad: Guayaquil, Ecuador; Berlepsch, 1884. Journ. Orn., 32, p. 302. Localidad: Bucaramanga, Colombia; Sclater, 1888. Cat. B. Brit. Mus., 14, p. 160. part, spec, d-p. Localidad: Valle del Magdalena, Medellin, Bucaramanga, Bogotá (Colombia), Babahoyo, Quito, Balzar, Santa Rita (Ecuador); Robinson, 1895. Flying Trip, p. 160. Localidad: Barranquilla a Honda, y Guaduas, Colombia; Hartert, 1898. Nov. Zool., 5, p. 488. Localidad: Cachavi, Ecuador; Salvadori y Festa, 1899. Boll. Mus. Zool. Torino, 14, No. 362, p. 9. Localidad: Vinces y Río Peripa, Ecuador; Goodfellow, 1901. Ibis, p. 705. Localidad: Santo Domingo, Ecuador; Menegaux, 1911. Miss. Mes. Arc Me"rid. Equat., 9, p. 650. Localidad: Santo Domingo, Ecuador.

Myiozetetes texensis Sclater y Salvin, 1879. (diferente de Giraud). Lond., p. 513. Localidad: Envigado y Medellin, Colombia.

Myiozetetes cayanensis cayanensis Ridgway, 1901. Bull. U. S. Nat. Mus., 50, Part 4, p. 444. Localidad: part, Colombia y Ecuador; Hellmayr, 1906. Abhandl. 2. KI.Bayr. Ak. Wiss., 22, No. 3, p. 649. Localidad: part, Bogota y Ecuador; Hellmayr, 1911. Lond., p. 1134. Localidad: Novita y Boca de Calima, Colombia; Chapman, 1917. Bull. Amer. Mus. Nat. Hist., 36, p. 460, Localidad: numerosas localidades en Colombia.

Myiozetetes cayanensis harterti Bangs y Penard, 1921. Bull. Mus. Comp. Zool., 64, p. 374. Localidad: Loma del León, Panamá; Bangs y Barbour, 1922. I.e., 65, p. 217. Localidad: Jesusito, Darien.

Myiozetetes cayennensis Lawrence, 1862. (diferente de Linnaeus). Ann. Lye. Nat. Hist.N. Y., 7, p. 295. Localidad: Lion Hill, Panamá; Sclater, 1888. Cat. B. Brit. Mus., 14, p. 160. Localidad: part, spec, a, b, Estación Paraiso y Panamá; Salvin y God-Man, 1889. Biol. Centr.-Amer., Aves, 2, p. 40. Localidad: part, Panamá; Bangs, 1900. Proc.New Engl. Zool. Cl., 2, p. 21. Localidad: Loma del León; Bangs y Thayer, 1906. Bull. Mus. Comp. Zool., 46, p. 218. Localidad: Sabana of Panamá.

Myiozetetes cayanensis cayanensis Ridgway, 1907. Bull. U. S. Nat. Mus., 50, Part 4, p. 444. Localidad: part, Panamá; Stone, 1918. Proc. Ac. Nat. Sci. Phila., p. 265. Localidad: Gatun, Panamá; Hallinan, 1924. Auk, 41, p. 318. Localidad: Mindi y Nueva Culebra, Panamá.

Myiozetetes cayanensis hellmayri Cory y Hellmayr, 1927. Catalogue of birds of the Americas and the adjacent Islands in Field Museum of Natural History: Part V Tyrannidae. Zool. Series. Vol. 13, p. 139. 
Myiozetetes cayanensis hellmayri Amadon, D., Mayr, E., Snow, D. W., Traylor, M. A., y Zimmer, J. T. 1979. En: Check-List of the Birds of the World. Traylor Jr. M. (ed). Museum of Comparative Zoology, Harvard University. Cambridge, Massachusetts. Vol. 8, P. 210.

Myiozetetes cayanensis hellmayri Clements, J. F., Schulenberg, T. S., Iliff, M. J., Billerman, S. M., Fredericks, T. A., Sullivan, B. L., y Wood, C. L. 2019. The eBird/Clements Checklist of Birds of the World: v2019.

Myiozetetes cayanensis hellmayri Mobley y Kirwan, 2020. Rusty-margined Flycatcher (Myiozetetes cayanensis), Version 1.0. En: Birds of the World (J. del Hoyo, A. Elliott, J. Sargatal, D. A. Christie, and E. de Juana, Editors). Cornell Lab of Ornithology, Ithaca, NY, USA. https://doi.org/10.2173/bow.rumfly1.01

\section{Holotipo}

Macho adulto depositado en el Museo Americano de Historia Natural (AMNH Skin496721 [Trombone 2013]), Nueva York, Estados unidos.

\section{Localidad tipo}

Cachabí, provincia de Esmeraldas, Ecuador.

\section{Descripción}

No presenta dimorfismo sexual en el plumaje. El borde de las plumas secundarias y las rectrices es color amarillo-anteado (NCS53); la región distal de las coberteras menores, mayores y las supracaudales es color arcilla (NCS123B); la zona media de la coronilla es amarillo naranja (NCS18); el color de la corona es fusco uniforme (NCS21); el área basal del dorso y las supracaudales es marrón oliva (NCS28); el color distal del dorso es citrino (NCS51); las plumas de arreste, las rectrices y el área basal de las coberteras poseen un patrón general de coloración marrón oliva (NCS28); el borde de las primarias es marrón antiguo (NCS37); resto del plumaje igual que Myiozetetes cayanensis adultos sensu Brisson (1760) (Figura 38). Variación individual: presente en la coloración de la coronilla, que puede ser más pálida (amarilla); la coloración basal del dorso, que puede ser más acanelada (marrón acanelado) y el borde de las primarias, que puede ser más pálido (leonado). Juveniles: plumaje similar al de los individuos adultos, pero el color basal, medial y distal de la coronilla es marrón a grisáceo; 
el color distal y basal del dorso es uniforme marrón a grisáceo o en ocasiones se pueden presentar los bordes dorsales acanelados en primeras etapas de muda de vuelo.

Presenta dimorfismo sexual en el tamaño. Hembras (media \pm desviación estándar; $\mathrm{n}=$ 52): culmen 9,59 $\pm 0,59 \mathrm{~mm}$, alto de pico 5,08 $\pm 0,41 \mathrm{~mm}$, ancho de pico 5,89 $\pm 0,45 \mathrm{~mm}$, cuerda ala $86,71 \pm 4,49 \mathrm{~mm}$, tarso $18,44 \pm 0,85 \mathrm{~mm}$, cola 73,69 $\pm 4,33 \mathrm{~mm}$. Machos (media \pm desviación estándar; $\mathrm{n}=60$ ): culmen 9,58 $\pm 0,59 \mathrm{~mm}$, alto de pico 5,05 $\pm 0,41 \mathrm{~mm}$, ancho de pico $5,90 \pm 0,45 \mathrm{~mm}$, cuerda alar $86,73 \pm 4,35 \mathrm{~mm}$, tarso $18,45 \pm 0,83 \mathrm{~mm}$, cola $73,57 \pm 4,27$ $\mathrm{mm}$. Aunque esta especie presenta una amplia variación en el comportamiento vocal, es posible describir patrones generales como su composición (notas de tipo A, B, C y F), longitud (6,25 notas en promedio), duración ( $2,89 \pm 0,82$ segundos) y cadencia $(2,90 \pm 0,69$ notas/s) promedio. Presenta un índice de diversidad alto ( $\mathrm{iD}=0,52 \pm 0,22$ ), con una estructura de trinos cortos ( 4,41 $\pm 2,10$, hasta siete elementos) y una alta variabilidad en el recambio de notas dentro del canto.

\section{Diagnosis}

El plumaje es similar a los individuos de Myiozetetes cayanensis adultos sensu Brisson (1760), pero difiere en la coloración distal de las plumas coberteras y supracaudales, que son color arcilla (NCS123B, más pálido y amplio en individuos pre-reproductivos), en combinación con el color del borde de las secundarias y las rectrices (más amarillo-anteado) y en el color medial de la coronilla (más claro, amarillo naranja). También difiere de los demás taxones en el PFC de la mínima frecuencia y la frecuencia más baja de la nota $D$, al ser más bajos.

Adicionalmente, difiere con M. erythropterus en el color de la corona (más pálida); la longitud de la cola y el tarso (más cortos); el índice de diversidad vocal (más alto) y el delta de frecuencia de la nota $D$ (menor). Difiere de M. c. rufipennis en el número de notas del canto (canto más corto). Difiere de M. c. cayanensis el color de las plumas de arrastre, el color basal de las coberteras y el color de las rectrices (más pálido-oliva). Difiere de $M$. c. rufipennis y $M$. c. cayanensis en la longitud del culmen (mayor) y en los parámetros vocales de la nota de tipo D en la frecuencia central, duración, frecuencia más alta, máxima frecuencia, frecuencia pico y PFC de la máxima frecuencia. Difiere de M. c. cayanensis y M. erythropterus en el color basal y distal del dorso y las supracaudales (menos acanelado y más pálido citrino, respectivamente) 
y en la longitud de la cuerda alar (menor). Difiere de M. c. rufipennis y M. erythropterus en el color del borde de las primarias, secundaria y rectrices (más pálido y anteado-arcilla).

\section{Distribución geográfica}

Su distribución está delimitada principalmente por la cordillera oriental de los andes. Se extiende desde el sur de la provincia de Puntarenas en Costa Rica, a través de Panamá; el noroccidente de Venezuela alrededor de la cuenca de Maracaibo en los estados de Zulia y Falcón, en la porción occidental de los estados de Lara y Táchira; y en todo el territorio transandino que comprende el occidente de la cordillera oriental de los andes en Panamá, Colombia y Ecuador hasta el noreste de la provincia de Loja (Figura 39).

Myiozetetes cayanensis rufipennis Lawrence, 1870

Myiozetetes rufipennis Lawrence, 1870. Ann. Lye. Nat. Hist. N. Y., 9, p. 267, 1869-1870. Localidad: Valencia, Venezuela.

Myiozetetes cayennensis Taylor, 1864. (diferente de Linnaeus). Ibis, p. 86. Localidad: cerca de Barcelona, Venezuela; Sclater y Salvin, 1868. Lond., p. 628. Localidad: San Esteban, Venezuela; Sclater, 1871. 1.c., p. 752 (crit.); Sclater, 1888. Cat. B. Brit. Mus., 14, p. 160. Localidad: part, spec, q, r, "Trinidad", Venezuela; Phelps, 1897. Auk, 14, p. 365. Localidad: San Antonio, Bermúdez, Venezuela.

Myiozetetes guianensis Sclater y Salvin, 1868. (diferente de Cabanis y Heine). Lond., p. 168. Localidad: Carupano, Bermúdez, Venezuela.

Myiozetetes cayennensis rufipennis Berlepsch y Hartert, 1902. Nov. Zool., 9, p. 45. Localidad: Quiribana de Caicara, Altagracia, Caicara, y Ciudad Bolivar, Río Orinoco (descripción de nido y huevos).

Myiozetetes cayanensis rufipennis Hellmayr, 1906. Abhandl. 2. KI. Bayr. Ak. Wiss., 22, No. 3, p. 649. Localidad: costa norte de Venezuela (crit.); Hellmayr y Seilern, 1912. Arch. Naturg., 78, A, Heft 5, p. 165. Localidad: San Esteban y Puerto Cabello, Venezuela; Cherrie, 1916. Mus. Brookl. Inst., Sci. Bull., 2, p. 233. Localidad: Valle del Orinoco, Venezuela. 
Myiozetetes cayanensis rufipennis Cory y Hellmayr, 1927. Catalogue of birds of the Americas and the adjacent Islands in Field Museum of Natural History: Part V Tyrannidae. Zool. Series. Vol. 13, p. 139.

Myiozetetes cayanensis rufipennis Amadon, D., Mayr, E., Snow, D. W., Traylor, M. A., y Zimmer, J. T. 1979. En: Check-List of the Birds of the World. Traylor Jr. M. (ed). Museum of Comparative Zoology, Harvard University. Cambridge, Massachusetts. Vol. 8, P. 211.

Myiozetetes cayanensis rufipennis Clements, J. F., Schulenberg, T. S., Iliff, M. J., Billerman, S. M., Fredericks, T. A., Sullivan, B. L., y Wood, C. L. 2019. The eBird/Clements Checklist of Birds of the World: v2019.

Myiozetetes cayanensis rufipennis Mobley y Kirwan, 2020. Rusty-margined Flycatcher (Myiozetetes cayanensis), Version 1.0. En: Birds of the World (J. del Hoyo, A. Elliott, J. Sargatal, D. A. Christie, and E. de Juana, Editors). Cornell Lab of Ornithology, Ithaca, NY, USA. https://doi.org/10.2173/bow.rumfly1.01

\section{Holotipo}

Adulto (sexo indeterminado) depositado en el Museo Americano de Historia Natural (AMNH Skin-42614 [Trombone 2013b]), Nueva York, Estados unidos.

\section{Localidad tipo}

Estado de Valencia, Venezuela.

\section{Descripción}

No presenta dimorfismo sexual en el plumaje. El color de la corona es fusco uniforme (NCS21); el color medial de la coronilla es naranja intenso (NCS17); la porción basal del dorso y las supracaudales, así como las plumas de arrastre son color marrón oliva (NCS28); el color distal del dorso es citrino (NCS51); el borde de las plumas secundarias y rectrices es canela (NCS39) y el color basal de las coberteras y las rectrices es marrón oliva (NCS28); resto del plumaje igual que Myiozetetes cayanensis adultos sensu Brisson (1760) (Figura 40). Variación individual: presente en la coloración basal del dorso, que puede ser más rojizo (marrón acanelado); la coloración del borde de las primarias, que puede ser más oscuro (marrón antiguo); el borde de las secundarias, que puede ser más pálido (amarillo-anteado) y el borde 
de la cola, que puede ser más oscuro (leonado). Juveniles: plumaje similar al de los individuos adultos, pero el color basal, medial y distal de la coronilla es marrón a grisáceo; el color basal del dorso es uniforme marrón a grisáceo y recurrentemente presentan los bordes dorsales y de las coberteras acanelados en primeras etapas de muda de vuelo. Los individuos juveniles pre-reproductivos pueden presentar el área medial de la coronilla más clara (amarilla).

No presenta dimorfismo sexual en el tamaño. Hembras (media \pm desviación estándar; $\mathrm{n}=15$ ): culmen $9,73 \pm 0,39 \mathrm{~mm}$, alto de pico 5,00 $\pm 0,36 \mathrm{~mm}$, ancho de pico 6,05 $\pm 0,40 \mathrm{~mm}$, cuerda alar 85,28 $\pm 3,53 \mathrm{~mm}$, tarso 18,39 $\pm 1,02 \mathrm{~mm}$, cola 71,68 $\pm 3,87 \mathrm{~mm}$. Machos (media \pm desviación estándar; $\mathrm{n}=15$ ): culmen $9,76 \pm 0,57 \mathrm{~mm}$, alto de pico 5,17 $\pm 0,44 \mathrm{~mm}$, ancho de pico 5,86 \pm 0,44, cuerda alar 87,97 $\pm 4,92$, tarso $18,52 \pm 0,90 \mathrm{~mm}$, cola 74,08 $\pm 4,84 \mathrm{~mm}$. Es posible describir patrones generales en el patrón vocal en la composición (notas de tipo A, B, C y F); longitud ( 8,88 notas por vocalización) y longitud de trino $(\sim 7,22 \pm 3,15$, hasta once elementos). El índice de diversidad es medio ( $\mathrm{iD}=0,46 \pm 0,25$ ) y el trino domina la mayor parte de la estructura del canto. La cadencia del trino es de 2,78 $\pm 0,72$ notas por segundo y el canto tiene una duración promedio de 3,5 segundos.

\section{Diagnosis}

Se caracteriza por tener el borde de las plumas primarias y la porción distal de las supracaudales de color leonado (NCS38); el color distal de las coberteras es canela (NCS123A) y estos tres caracteres ocupan una proporción > 30\% del área de la pluma, dando una apariencia rufa-rojiza al fondo y el dorso de las alas. Por lo general, estos caracteres son más rojizos y amplios en mudas pre-reproductivas. Adicionalmente, difiere de los demás taxones en el color del borde de las primarias, el color de las supracaudales (más rojizo, leonado) y el color distal de las coberteras (más rojizo, canela).

Difiere de M. hellmayri en el color medial de la coronilla (más oscuro, naranja intenso); la longitud del culmen (más corto); la longitud del canto (más largo); y en los parámetros vocales de la nota de tipo D de duración (mayor), frecuencia más baja (mayor), máxima frecuencia (menor), frecuencia pico (menor) y PFC de la máxima y mínima frecuencia (mayor y menor respectivamente). Difiere de M. c. cayanensis en el color de las plumas de arrastre y las rectrices (más claro, fusco); el color basal de las coberteras (más oscuro, marrón) y el delta 
de frecuencia de la nota $D$ (mayor). Difiere de Myiozetetes erythropterus en el color de la corona (más clara, fusca); la longitud de la cola, la cuerda alar y el tarso (más cortos). Difiere de M. c. cayanensis y M. erythropterus en el color basal y distal del dorso y el color basal de las supracaudales (más oscuro, oliva y más citrino, respectivamente). Difiere de $M$. hellmayri y M. c. cayanensis en el color del borde de las secundarias y rectrices (canela, rojizo). Difiere de $M$. hellmayri y $M$. erythropterus en la frecuencia central (menor en ambos casos) y la frecuencia más alta (menor y mayor respectivamente) de la nota D.

\section{Distribución geográfica}

Se distribuye desde el noroccidente de Venezuela en la margen izquierda del Río Orinoco, a través del oriente de los andes hasta el norte de los departamentos de Amazonas y Vaupés en Colombia y hasta el norte de las provincias de Napo y Orellana en Ecuador. Debido a la falta de muestras de colección de individuos provenientes de la porción noroccidental de la cuenca amazónica en Colombia, Ecuador y Brasil, los limites surorientales de esta población son difíciles de definir con claridad, pero es probable que pueda extenderse hasta el sur de la provincia de Morona Santiago en Ecuador y hasta la margen izquierda del Río Negro en Brasil (Figura 41).

Myiozetetes cayanensis cayanensis (Linnaeus, 1766)

Muscicapa cayanensis Linnaeus, 1766. Syst. Nat., 12 (i), p. 327. Basado en: Brisson, 1760. Orn., 2, p. 404, pi. 38, fig. 4. Localidad: Cayena, Guyana Francesa.

Muscicapa similis Spix, 1825. Av. Bras., 2, p. 18, pi. 25. Localidad: Río Amazonas.

Muscipeta cayennensis Lafresnaye y D'Orbigny, 1837. Syn. Av., i, in: Mag. Zool., 7, cl. 2, p. 47. Localidad: Mojos, Bolivia (espécimen examinado); D'Orbigny, 1839. Voyage Amér. mérid., Ois., p. 317. Localidad: Río Blanco y Río Itonama, Provincia de Mojos, Bolivia.

Elaenea cayanensis Cabanis, 1848. En: Schomburgk, Reisen Brit. Guiana, 3, p. 701. Localidad: Guyana. Myiozetetes guianensis Cabanis y Heine, 1859. Mus. Hein., 2, p. 61. Localidad: Guyana y Surinam. 
Myiozetetes cayennensis (cayanensis) Sclater y Salvin, 1867. Lond., p. 577. Localidad: Pará, Brasil; Pelzeln, 1868. Orn. Bras., 2, p. 109. Localidad: Engenho do Gama, Mato Grosso (espécimen examinado); Salvin, 1885. Ibis, p. 295. Localidad: Bartica (Guyana) y Roraima (Brasil); Sclater, 1888. Cat. B. Brit. Mus., 14, p. 160. Localidad: part, Roraima, Bartica, Cayena, Pará (especimenes examinados); Riker y Chapman, 1890. Auk, 7, p. 270. Localidad: Diamantina, Santarem, Brasil; Allen, 1982. Bull. Amer. Mus. Nat. Hist., 4 P. 337. Localidad: Chapada, Mato Grosso, Brasil; Menegaux, 1904. Bull. Mus. Paris, to, p. 118. Localidad: Mahury, Guyana Francesa; Hellmayr, 1905. Nov. Zool., 12, p. 293. Localidad: Igarapé-Assú, Pará, Brasil; Berlepsch, 1908. I.e., 15, p. 127. Localidad: Cayena y Roche-Marie, Guyana Francesa; Snethlage, 1914. Bol. Mus. Goeldi, 8, p. 386. Localidad: Pará, Providência, Quatipuru, Rio Moju, Arumanduba; Chubb, 1921. Birds Brit. Guiana, 2, p. 196. Localidad: numerosas localidades en Guyana.

Myiozetetes columbianus Pelzeln, 1868. (diferente de Cabanis y Heine). Orn. Bras., 2, p. 109. Localidad: Rio Muria, cerca de Pará (espécimen examinado).

Myiozetetes cayanensis cayanensis Hellmayr, 1906. Abhdl. 2. Kl. Bayr. Ak. Wiss., 22, No. 3, p. 649. Locaidad: part, excl. Bogotá y occidente de Ecuador (crit.); Hellmayr, 1906. Nov. Zool., 13, p. 361. Localidad: Sao Antonio do Prata, Pará, Brasil; Hellmayr, 1908. I.e., 15, p. 48. Localidad: Goyaz, Rio Araguaya, y Fazenda Esperanca, Goyaz, y Rio Jordao, Araguaya, Minas Gerais (crit.); Ridgway, 1907. Bull. U. S. Nat. Mus., 50, Part 4, p. 444. Localidad: part, Guyanas, Bolivia y Brasil; Beebe, 1909. Zoologica (N. Y.), i, p. 97. Localidad: Río Guarapiche, delta del Orinoco; Hellmayr, 1912. Abhandl. math. phys. Kl. Bayr. Ak. Wiss., 26, No. 2, p. 24, 89. Localidad: Pará, Rio Muria, Igarapé-Assú, Sao Antonio, Ipitinga; Hellmayr, 1912. I.e., p. 108. Localidad: Mexiana; Bangs y Penard, 1918. Bull. Mus. Comp. Zool., 62, p. 77. Localidad: vecindad de Paramaribo y Lelydorp, Surinam; Hellmayr, 1925. Nov. Zool., 32, p. 27. Localidad: Bolivia.

Myiozetetes cayanensis cayanensis Cory y Hellmayr, 1927. Catalogue of birds of the Americas and the adjacent Islands in Field Museum of Natural History: Part V Tyrannidae. Zool. Series. Vol. 13, p. 138.

Myiozetetes cayanensis cayanensis. Pinto, 1944. Catálogo das aves do Brasil: e lista dos exemplares existentes na coleção do Departamento de Zoologia. 2a Parte: Ordem PASSERIFORMES (continuação): Superfamilia Tyrannoidea e Subordem PASSERES. Vol. 2, p. 155.

Myiozetetes cayanensis cayanensis Amadon, D., Mayr, E., Snow, D. W., Traylor, M. A., y Zimmer, J. T. 1979. En: Check-List of the Birds of the World. Traylor Jr. M. (ed). Museum of Comparative 
Zoology, Harvard University. Cambridge, Massachusetts. Vol. 8, P. 211.

Myiozetetes cayanensis cayanensis Pollock, A. W. III. 2015. Binomial and Trinomial Index to The Auk, 1884-1940. Also including the Nuttall Bulletin, 1876-1883 and The A.O.U. Check-List of North American Birds (All Seven Editions). Vol. 7, p. 6528.

Myiozetetes cayanensis cayanensis Clements, J. F., Schulenberg, T. S., lliff, M. J., Billerman, S. M., Fredericks, T. A., Sullivan, B. L., y Wood, C. L. 2019. The eBird/Clements Checklist of Birds of the World: v2019.

Myiozetetes cayanensis cayanensis Mobley y Kirwan, 2020. Rusty-margined Flycatcher (Myiozetetes cayanensis), Version 1.0. En: Birds of the World (J. del Hoyo, A. Elliott, J. Sargatal, D. A. Christie, and E. de Juana, Editors). Cornell Lab of Ornithology, Ithaca, NY, USA. https://doi.org/10.2173/bow.rumfly1.01

\section{Iconotipo}

"Le Gobe-Mouche de Cayenne", François-Nicolas Martinet. En: Brisson. 1760. Ornithologie, ou, Méthode contenant la division desoiseaux en ordres, sections, genres, especes \& leursvariétés : a laquelle on a joint une description exacte dechaque espece, avec les citations des auteurs qui en onttraité, les noms quils leur ont donné, Tomo 2, P. 38, Fig. 4, pp 404.

\section{Localidad tipo}

Cayena, Guyana Francesa.

\section{Descripción}

No presenta dimorfismo sexual en el plumaje. La corona es color fusco (NCS21); el color medial de la coronilla es naranja intenso (NCS17). La porción basal del dorso y las supracaudales son marrón acanelado (NCS33); el borde de las primarias es marrón antiguo (NCS37); resto del plumaje igual que Myiozetetes cayanensis adultos sensu Brisson (1760) (Figura 42). Variación individual: presente la coloración del borde de las primarias, que puede ser más pálido (canela); el color distal de las coberteras, que puede ser más oscuro (acanelado) y el borde de la cola, que puede ser oscuro (fusco). Juveniles: plumaje similar al de los individuos adultos, pero el color basal, medial y distal de la coronilla es marrón a grisáceo 
(amarillo-naranja en mudas pre-reproductivas); el color basal del dorso es uniforme marrón a grisáceo.

No presenta dimorfismo sexual en el tamaño. Hembras (media \pm desviación estándar; $\mathrm{n}=22$ ): culmen $9,80 \pm 0,60 \mathrm{~mm}$, alto de pico 5,24 $\pm 0,46 \mathrm{~mm}$, ancho de pico 5,81 $\pm 0,43 \mathrm{~mm}$, cuerda alar $87,50 \pm 4,88 \mathrm{~mm}$, tarso $18,50 \pm 0,77 \mathrm{~mm}$, cola 73,10 $\pm 4,32 \mathrm{~mm}$. Machos (media \pm desviación estándar; $\mathrm{n}=29$ ): culmen 9,76 $\pm 0,59 \mathrm{~mm}$, alto de pico 5,23 $\pm 0,61 \mathrm{~mm}$, ancho de pico 5,94 \pm 0,58 mm, cuerda alar $88,83 \pm 4,88 \mathrm{~mm}$, tarso $18,64 \pm 0,91 \mathrm{~mm}$, cola 75,04 $\pm 5,71$ $\mathrm{mm}$. Este taxón presenta una amplia variabilidad en el comportamiento vocal, pero es posible establecer un patrón en la composición (notas de tipo A, B, C, Fy D, la estructura más compleja de todos los taxones evaluados); la longitud (7,9 notas en promedio); la duración ( 3,54 \pm 0,82 segundos); la longitud del trino ( 6,45 $\pm 1,63$, hasta diez elementos) y la cadencia $(2,48$ $\pm 0,28$ notas por segundo). Aunque su composición es compleja, los valores del índice de diversidad (iD) son bajos $(0,32 \pm 0,24)$, con una estructura dominada en su mayoría por los elementos del trino y con un recambio de notas bajo dentro del canto.

\section{Diagnosis}

Los caracteres principales de este taxon incluyen la porción distal del dorso y las supracaudales color oliva verdoso (NCS49), las plumas de arrastre y rectrices fusco uniforme (NCS21), el borde de las plumas secundarias y rectrices color arcilla (NCS26) y las porciones basal y distal de las coberteras marrón acanelado (NCS33) y citrino (NCS51) respectivamente. Se distingue de $M$. hellmayri en el color medial de la coronilla (más oscuro, naranja intenso); la longitud del culmen (más corto); la frecuencia central; la duración; la frecuencia más baja y el PFC de la mínima frecuencia (todos mayores) de la nota D. Difiere de $M$. erythropterus en el color de la corona (más claro, fusco); la longitud de la cola y la longitud del tarso (más cortas). Se distingue de M. hellmayri y M. c. rufipennis en el color basal del dorso y las supracaudales (más canela-rojizo). Difiere de M. c. rufipennis y M. erythropterus en el borde de las primarias (más oscuro, marrón) y el delta de frecuencia de la nota $D$ (menor y mayor, respectivamente). Se distingue de $M$. hellmayri y $M$. erythropterus en la longitud de la cuerda alar (mayor y menor, respectivamente); la frecuencia más alta y el PFC de la máxima frecuencia (mayor); la máxima frecuencia y la frecuencia pico (mayor y menor en ambos casos, respectivamente) de la nota $D$. 


\section{Distribución geográfica}

Es el taxón de más amplia distribución, ocurre desde el oriente de Venezuela (margen derecha del Río Orinoco en Amazonas, Bolívar y Delta Amacuro), al nororiente de Suramérica en Guyana, Guyana Francesa y Surinam. Al norte y centro de Brasil hasta el noroccidente de Ceará y Pernambuco, hacia el sur del Río San Francisco en Sergipe y Bahía hasta el norte del Río Contas. En la diagonal seca y la porción occidental de Minas Gerais hasta el norte de Santa Catarina, norte de la provincia de Misiones en Argentina y en Paraguay al oriente del rio Paraná. Hacia el occidente hasta la mitad norte de Bolivia, la porción cisandina de Perú (Puno, Madre de Dios, Uyucali y Loreto) y el departamento del amazonas en Colombia (Figura 43). Los límites entre $M$. c. cayanensis y M. c. rufipennis son difíciles de establecer con claridad, por lo cual es necesario revisar material adicional proveniente del occidente de la cuenca amazónica en Brasil, Colombia y Ecuador.

Myiozetetes erythropterus (Lafresnaye, 1853).

Tyrannula erythroptera Lafresnaye, 1853. Rev. Mag. Zool., (2) 5, p. 56. Localidad: Brasil.

Myiozetetes erythropterus Sclater, 1888. Lond., 1871, p. 751. Localidad: Minas Gerais; Sclater, 1888.

Cat. B. Brit. Mus., 14, p. 159. Localidad: Brasil.

Myiozetetes cayanensis erythroptera Hellmayr, 1906. Abhandl. 2. KI. Bayr. Ak. Wiss.,22, No. 3, p. 650. Localidad: Brasil; Hellmayr, 1908. Nov. Zool., 15, p. 49. Localidad: Rio de Janeiro Y Santa Fé, Minas Gerais, Brasil.

Myiozetetes cayanensis erythropterus Cory y Hellmayr, 1927. Catalogue of birds of the Americas and the adjacent Islands in Field Museum of Natural History: Part V Tyrannidae. Zool. Series. Vol. 13, p. 137.

Myiozetetes cayanensis erythropterus. Pinto, 1944. Catálogo das aves do Brasil: e lista dos exemplares existentes na coleção do Departamento de Zoologia. 2a Parte: Ordem PASSERIFORMES (continuação): Superfamilia Tyrannoidea e Subordem PASSERES. Vol. 2, p. 156. 
Myiozetetes cayanensis erythropterus Amadon, D., Mayr, E., Snow, D. W., Traylor, M. A., y Zimmer, J. T. 1979. En: Check-List of the Birds of the World. Traylor Jr. M. (ed). Museum of Comparative Zoology, Harvard University. Cambridge, Massachusetts. Vol. 8, P. 211.

Myiozetetes cayanensis erythropterus Clements, J. F., Schulenberg, T. S., Iliff, M. J., Billerman, S. M., Fredericks, T. A., Sullivan, B. L., y Wood, C. L. 2019. The eBird/Clements Checklist of Birds of the World: v2019.

Myiozetetes cayanensis erythropterus Mobley y Kirwan, 2020. Rusty-margined Flycatcher (Myiozetetes cayanensis), Version 1.0. En: Birds of the World (J. del Hoyo, A. Elliott, J. Sargatal, D. A. Christie, and E. de Juana, Editors). Cornell Lab of Ornithology, Ithaca, NY, USA. https://doi.org/10.2173/bow.rumfly1.01

\section{Iconotipo}

Sin tipo designado. Descripción basada en espécimen de museo analizado por Lafresnaye en 1853 . No se especifica el registro, número de colecta del espécimen o colección en el relato u otras partes del texto (Lafresnaye 1853).

\section{Localidad tipo}

Brasil.

\section{Descripción}

No presenta dimorfismo sexual en el plumaje. La corona es más oscura con respecto a los demás taxones, con lo cual la zona auricular parece más clara en contraste; el color medial de la coronilla es naranja intenso (NCS17); el área basal del dorso y las supracaudales es marrón acanelado (NCS33); las plumas de arrastre, las rectrices y la porción basal de las coberteras son marrón oliva (NCS28); el borde de las secundarias y rectrices es canela (NCS39); resto del plumaje igual que Myiozetetes cayanensis adultos sensu Brisson (1760) (Figura 44). Variación individual: presenta variación en el color medial de la coronilla, que puede ser más claro (amarillo-naranja) en algunos individuos. El resto de la coloración del plumaje es bastante consistente entre individuos. Juveniles: plumaje similar al de los individuos adultos, pero el color basal, medial y distal de la coronilla es marrón a grisáceo (amarillo-naranja en mudas pre-reproductivas); el color basal del dorso es uniforme marrón a grisáceo. Los tonos 
anteados pueden aparecer en las áreas claras en algunos individuos juveniles a lo largo de la distribución.

No presenta dimorfismo sexual en el tamaño. Hembras (media \pm desviación estándar; $\mathrm{n}=4$ ): culmen $9,49 \pm 0,54 \mathrm{~mm}$, alto de pico $5,36 \pm 0,97 \mathrm{~mm}$, ancho de pico $6,14 \pm 0,46 \mathrm{~mm}$, cuerda alar 90,63 $\pm 6,02 \mathrm{~mm}$, tarso 18,85 $\pm 1,23 \mathrm{~mm}$, cola 77,75 $\pm 7,68 \mathrm{~mm}$. Machos (media \pm desviación estándar; $n=5$ ): culmen 9,56 $\pm 0,56 \mathrm{~mm}$, alto de pico 5,37 $\pm 0,91 \mathrm{~mm}$, ancho de pico 6,15 \pm 0,49 mm, cuerda alar $91,06 \pm 5,82 \mathrm{~mm}$, tarso $18,86 \pm 1,16 \mathrm{~mm}$, cola 78,38 $\pm 7,55$ $\mathrm{mm}$. La estructura de su canto es de notas de tipo A, C y F; esta especie se caracteriza por poseer la estructura menos completa y por la ausencia total de notas de tipo B. Posee valores de longitud del canto y del trino medias ( 7, 36 notas totales; 6,18 $\pm 1,60$, hasta nueve elementos), con una duración promedio de 3,35 $\pm 0,88$ segundos y una cadencia de trino de $2,69 \pm 0,50$ notas por segundo. El índice de diversidad (iD) es el más bajo entre los taxones analizados $(0,23 \pm 0,21)$, con un recambio de notas muy bajo o nulo dentro del canto.

\section{Diagnosis}

Se distingue por tener el borde de las primarias color ámbar (NCS36), el cual es intenso y se corta de manera conspicua con la porción distal de las plumas primarias formando un semi triangulo en vista lateral. El color de la corona es marrón grisáceo (NCS20); el color distal del dorso y las supracaudales es verde oliva (NCS48) y la porción distal de las coberteras es marrón oliva (NCS28), aunque puede tender hacia los tonos rufos en las mudas prereproductivas. Adicionalmente difiere de los demás taxones en todas las medidas morfológicas, a excepción de la longitud del culmen, siendo en general de tamaño mayor. Difiere de M. hellmayri en el color medial de la coronilla (más oscuro, naranja intenso); el índice de diversidad vocal (menor); la frecuencia más baja y el PFC de la mínima frecuencia (mayor en ambos casos) de la nota D. Se distingue de M. c. rufipennis en la frecuencia central de la nota $D$, teniendo un valor es mayor. Difiere de $M$. c. cayanensis en el color de las plumas de arrastre y las rectrices (más claro, marrón); el color basal de las coberteras (más oscuro, oliváceo); la máxima frecuencia (mayor); la frecuencia pico (mayor) y el PFC de la máxima frecuencia (menor) de la nota D. Se distingue de $M$. hellmayri y $M$. c. rufipennis en el color basal del dorso y las supracaudales (más rojizo-canela). Difiere de $M$. hellmayri y $M$. c. cayanensis en el color del borde de las secundarias y rectrices (más pálido, canela) y en el delta 
de frecuencia de la nota $D$ (menor en ambos casos). Se distingue de $M$. c. rufipennis y $M$. . $c$. cayanensis en el valor de la frecuencia más alta de la nota $D$, al tener un valor menor en ambos casos.

\section{Distribución geográfica}

Ocurre en el oriente de Brasil al sur del Río Contas y el estado de Bahía, al oriente de Minas Gerais, Espírito Santo y Río de Janeiro hasta la margen izquierda del Río Grande y el norte de la Serra da Mantiqueira (Figura 45). Es necesaria la revisión de más material proveniente del área occidental de la distribución para determinar con más exactitud los límites de esta. 


\section{DISCUSIÓN}

Aunque gran parte de la controversia en la cladística entre los años 60 y 80 radica en la dualidad que presenta la inclusión de la "teoría de la clasificación" (Hennig 1950, 1966; Rieppel 2010; Olsson et al. 2017), con los cladistas argumentando la diferencia entre los clados en un árbol y los grupos en una clasificación, y los oponentes abogando por mantener el statu quo de la clasificación evolutiva, que defiende la clasificación basada en la fenética; son estos caracteres fenotípicos (expresados en caracteres físicos como el plumaje o la forma corporal, así como en expresiones ecológicas tales como el canto y el comportamiento) los que soportan en la actualidad muchos grupos de biológicos (Levit et al. 2004; Rieppel 2011), como el complejo M. cayanensis.

A pesar de su amplia distribución y abundancia en el neotrópico (Restall et al. 2007), el complejo $M$. cayanensis nunca fue objeto de estudio en trabajos taxonómicos o filogenéticos a nivel infraespecífico. Siguiendo las normas taxonómicas y nomenclaturales de la época, desde Linnaeus (1766) hasta Bangs y Penard (1921), los taxones del complejo fueron agrupados y descritos como un grupo de subespecies compuesto por cuatro poblaciones, que presentaban distinción a nivel de coloración y tamaño corporal. De esta forma, la utilización de la categoría subespecífica en el complejo M. cayanensis se estableció en los textos durante casi un siglo, prevaleciendo como decisión taxonómica, incluso después de que Hellmayr (1927) llamara la atención sobre la necesidad de revisar series mayores para comprobar la validez de los taxones propuestos (Cory y Hellmayr 1927).

En este trabajo es posible reconocer la diferencia entre las UTO 1 (M. c. hellmayri), UTO 2 (M. c. rufipennis), UTO 3 (M. c. cayanensis) y UTO 4 (M. c. erythropterus) por medio de dos caracteres en la coloración del plumaje: (1) el color distal de las plumas coberteras y (2) el color distal de las plumas supracaudales (Figura 46). Sin embargo, aunque existen diferencias en las tendencias de coloración de los individuos a lo largo de toda su distribución, estos caracteres suelen presentar una amplia variabilidad, lo cual dificulta su uso como carácter diagnóstico de cualquiera de las poblaciones. Por otro lado, es posible reconocer los taxones propuestos por la combinación de algunos caracteres de coloración, descritos inicialmente por Lafresnaye (1853), Lawrence (1870), Hartert y Goodson (1917) y Bangs y 
Penard (1921), como el color pardo opaco que cubre todas las rectrices y primarias formando una amplia banda longitudinal y el mayor tamaño corporal en $M$. c. erythropterus; el color rufo-canela en las márgenes de las alas y la cola en $M$. c. rufipennis; y la región del dorso más olivácea en M. c. hellmayri con respecto a las demás subespecies. Estos caracteres son consistentes dentro de las Unidades Taxonómicas Operativas establecidas a lo largo de su distribución geográfica y permiten la identificación de las subespecies actualmente reconocidas.

Además de esto, en este trabajo fue posible reconocer diferencias en la media de los valores morfométricos y los parámetros vocales evaluados en las Unidades Taxonómicas Operativas analizadas. Las diferencias entre las UTO están soportadas por diferentes parámetros en cada comparación (ej. la longitud del culmen difiere entre las UTO 1 - 2 y 1 3 , mientras que la frecuencia central del canto reproductivo difiere entre las UTO $3-1,2-4$ y $2-1$ ). Aunque ningún parámetro especifico es diagnóstico y permite diferenciar por si solo los cuatro taxones (lo cual podría suponer un conflicto a la luz de los sistemas de clasificación actuales, ya que la falta de definición de los taxones como entes discretos dificulta su inclusión en un esquema filogenético evolutivo [Levit et al. 2004; Olsson et al. 2017]), existe un soporte de variación entre estas unidades evolutivas, siendo posible establecer límites entre las mismas (Tabla 14).

El color de la zona auricular, el color basal y distal de la coronilla y el color del pecho son caracteres conservados a lo largo de toda la distribución de los individuos evaluados, siendo un carácter diagnóstico del complejo $M$. cayanensis. Por el contrario, el color distal de las coberteras mayores y menores, y el color distal de las plumas supracaudales, varia consistentemente entre las Unidades Taxonómicas Operativas, siendo caracteres útiles para la diferenciación de taxones. Sin embargo, al igual que en otros grupos de aves, la variación en coloración del plumaje es amplia a lo largo de la distribución geográfica y de los ciclos de vida de los individuos (Zimmer 1955; Traylor y Fitzpatrick 1982; Winger y Bates 2015; Marcondes y Brumfield 2020), con lo cual existe la posibilidad de que las diferencias observadas en la coloración (que son, en general sutiles), puedan ser producto de las muestras específicas que fueron analizadas. 
un tema de mayor complejidad en la biología evolutiva (Mayr 1963; Mayr y Diamond 2001; Marcondes y Brumfield 2020), y aunque la muestra de individuos analizados es representativa para tres de las cuatro UTO (dando información suficiente para observar un indicio sobre la variación del plumaje), las variaciones poblacionales e individuales podrían no aplicar de manera estricta a todos los individuos de la especie o UTO en cada caso.

Myiozetetes cayanensis erythropterus es diferente en casi todas las variables morfométricas de $M$. c. hellmayri y $M$. c. rufipennis, que son los taxones más lejanos geográficamente. La longitud del culmen (n) es la única variable que no difiere entre $M . c$. erythropterus y las demás subespecies, sugiriendo que el tamaño del pico es un carácter muy conservado, lo cual se ha documentado previamente (Schoener 1965; Graham et al. 2012; Ghosh-Harihar 2014) y está ligado con las estrategias alimenticias, la especialización del microhábitat y el uso de recursos (Zhang et al. 2019).

Las pruebas de comparación morfométrica por pares de Tukey-Kramer y Dunnet tienen un alto grado de soporte en la diferenciación de todos los grupos (Tabla 7), mientras que la de Games-Howell solamente soporta la diferencia entre las UTO 3 - 4 y 1 - 2. Esto puede darse debido a que la prueba de Games-Howell no parte del supuesto de igualdad de varianzas (Games y Howell 1976), corrigiendo los grados de libertad en el cálculo y arrojando resultados mucho más conservadores (Casas y Veitía 2008). Sin embargo, las agrupaciones soportan la diferencia morfológica entre todos los grupos, excepto entre las UTO 2 (M. c. rufipennis) y 3 (M. c. cayanensis), cuyos límites de distribución geográfica no son posibles de establecer con exactitud usando la información disponible para este estudio, dado que no es evidente la presencia de una posible barrera geográfica entre las dos poblaciones. Aunque, tradicionalmente, las barreras geográficas han servido como condicionantes de especiación y diferenciación poblacional (Ericson et al. 2003; Fjeldså et al. 2012; Fine 2015; Machac 2020), y en general, la especiación alopátrica es uno de los mecanismos más comunes de formación de nuevas especies (Coyne y Orr 2004; Phillimore et al. 2008), es posible que se den procesos de diferenciación simpátrica determinados por la oferta de recursos, el uso del hábitat o las condiciones climáticas, entre otros (Phillimore et al. 2008). 
Por su parte, las pruebas de agrupación morfométrica supervisada de $k$-means mostraron una tendencia a agrupar la mayoría de los elementos de la UTO 4 (Figura 24), así como la mayoría de los elementos de la UTO 1 fueron incluidos en una de las cuatro agrupaciones sugeridas. Cuando se superponen las cuatro agrupaciones individuales de $K$ means con las UTO, hay una tendencia lineal en el cambio de proporciones de las UTO entre los grupos, con la mayor cantidad de elementos de la UTO 4 en el grupo 1, y la mayor cantidad de elementos de la UTO 1 en el grupo 4. Esto puede dar indicios de una variación clinal en la morfología de las UTO analizadas, donde los individuos de las UTO 1 y 4 difieren entre sí en mayor proporción con respecto a la diferencia entre las UTO $1-3,2-4,1-2,2-3$ y $3-4$ en orden descendiente. Lo anterior sugiere que existe un gradiente geográfico de diferenciación morfológica entre los individuos, toda vez que las poblaciones más lejanas geográficamente entre sí (caso M. c. hellmayri y M. c. erythropterus), presentan un mayor grado de disimilitud morfológica que aquellas poblaciones vecinas (ej. M. c. rufipennis y M. c. cayanensis). Este comportamiento podría indicar un proceso de especiación en fase temprana, en el que la diferenciación morfológica se encuentra en dirección de cambio (Smith et al. 2014a; Reaney et al. 2020).

Con excepción de la longitud del tarso, todas las variables que presentan dimorfismo sexual en M. c. hellmayri corresponden a medidas del ala (cuerda alar, largo P1, largo P10 y largo S1 [Figura 26]). Esta subespecie cubre la mayor parte de su distribución en las zonas montañosas de los andes y los valles interandinos, teniendo una mayor distribución altitudinal y más acceso a variabilidad de hábitats y condiciones climáticas (Ruggiero y Hawkins 2008; Graham et al. 2010) con respecto a las demás subespecies. Las topografías complejas usualmente definen gradientes climáticos complejos y esto puede promover la divergencia fenotípica o ecológica en las poblaciones (Davidar et al. 2007; Ruggiero y Hawkins 2008). Es posible que las interacciones bióticas y climáticas propias de las zonas montañosas, como una mayor competencia (dada por una mayor riqueza en los andes [Kattan et al. 2004; Machac 2020]) y la necesidad de lidiar con vientos rápidos estén modulando un cambio morfológico más profundo entre los individuos de esta población (Ruggiero y Hawkins 2008). Aunque no se conocen diferencias entre el comportamiento de machos y hembras de esta población con respecto a las otras subespecies (Zimmer 1955; Haverschmidt 1971; Traylor y Fitzpatrick 1982; Dyrcz 1991, 2002; Screnci-Ribeiro y Duca 2010), es posible que las interacciones antes 
mencionadas estén afectando a ambos sexos de forma diferencial, permitiendo a su vez el cambio en el fenotipo (Davidar et al. 2007).

El canto reproductivo (nota tipo D) permite reconocer diferencias entre las Unidades Taxonómicas Operativas en nueve parámetros (Frecuencia más baja, Frecuencia más alta, Frecuencia pico, PFC de la máxima frecuencia, PFC de la mínima frecuencia, Frecuencia central, Máxima frecuencia, Delta de frecuencia y Duración [Tabla 10]). A pesar de que muchas variables acústicas mostraron superposición, la variabilidad en los parámetros vocales parece ser alta en el complejo M. cayanensis, siendo la Frecuencia (en casi todos sus parámetros), la que tiende a diferir entre las poblaciones (Tabla 14). Anteriormente se ha sugerido como criterio de delimitación de especies en tamnofílidos (Thamnophilidae, [Isler et al. 1998, 2008, 2009]) y en tororoíes (Grallariidae, [Donegan 2008]), que las poblaciones alopátricas pueden considerarse como especies distintas si se tiene una diferencia diagnosticable en al menos tres parámetros independientes de las características vocales. No en tanto, los parámetros de definición pueden ser flexibles cuando los taxones involucrados son parapátricos o muestran diferencias morfológicas (Isler et al. 2008, 2012), como es el caso del complejo M. cayanensis. Adicionalmente, este criterio de delimitación no toma en cuenta la posible interacción de la variabilidad de los caracteres entre las poblaciones, siendo más conservador (pues la definición esta principalmente basada en especies alopátricas [Isler et al. 1998]), lo cual puede soportar de forma más consistente las diferencias entre taxones, como por ejemplo M. c. erythropterus, que difiere en parámetros vocales y en el tamaño corporal, siendo claramente distinguible de los otros taxones. De hecho, la variación en los caracteres vocales suele estar ligada a los cambios en las dimensiones del cuerpo (Ryan y Brenowitz 1985, Martin et al. 2011), siendo caracteres que pueden divergir simultáneamente.

Fue posible documentar la diferenciación vocal de M. c. hellmayri y M. c. rufipennis en el número de notas del canto de comunicación, con los individuos de distribución transandina presentando cantos más cortos $(6,25$, dos notas menos en promedio) con respecto a los demás. Por otro lado, el índice de diversidad (iD) permite la diferenciación entre los cuatro taxones y presenta un aparente comportamiento de variación clinal, siendo progresivamente menor de la UTO 1 hacia la UTO 4. Lo anterior quiere decir que M. c. erythropterus tiene un índice de diversidad más bajo con respecto a los demás taxones, seguido por M. c. cayanensis y M. c. rufipennis. El valor de diversidad vocal de M. c. erythropterus es la mitad que el de $M$. 
c. hellmayri, lo cual sugiere que la variación de notas en el canto está siendo progresivamente perdida (o menor), cuanto más se avanza hacia el oriente en la distribución geográfica. Esto puede significar que la plasticidad vocal no desempeña un papel tan importante para las poblaciones del oriente, especialmente para M. c. erythropterus. A pesar de que se ha documentado previamente la variación clinal en caracteres morfológicos en las aves neotropicales (Graves 1991; este trabajo), son raros los reportes de variación clinal en el comportamiento vocal en el neotrópico (Isler et al. 2005; Van Doren et al. 2018), ya que los parámetros de vocalización suelen ser innatos en suboscines (Van Doren et al. 2018), y estos cambios en el canto suelen estar ligados a variaciones genéticas (Isler et al. 2005), lo cual debe ser investigado con más precisión.

Aunque las notas de tipo B no se registraron en $M$. c. erythropterus, esta UTO solamente contó con 11 cantos completos, siendo la UTO con menos datos disponibles para análisis. Adicionalmente, las notas de tipo B solamente aparecen en el $13 \%$ de las vocalizaciones alrededor de la distribución del complejo M. cayanensis, con lo cual es difícil saber si su ausencia en los registros vocales de $M$. c. erythropterus obedece a un sesgo muestral. Del mismo modo, las notas de tipo A y C, que aparecen en el $99 \%$ de las vocalizaciones, están condicionadas a la definición del canto de comunicación, que es aquel que comienza con una nota en forma de $S$ (nota de tipo $A$, ver numeral 4.2) y que tiene más componentes secundarios, en su mayoría elementos del trino (notas de tipo C). Esto puede crear un sesgo derivado del error del observador. De forma similar, el canto de $M$. c. cayanensis presenta la estructura más compleja, con 5 tipos de notas diferentes. No obstante, lo anterior puede deberse a que (1) esta subespecie corresponde a la UTO con más datos disponibles para análisis (a mayor muestra, más probabilidad de encontrar variación) y (2) es la población con el rango de distribución más amplio, lo cual aumenta la probabilidad de variación en el canto condicionada por el mayor número de individuos y hábitats.

El presente estudio muestra una marcada variación de los parámetros del llamado reproductivo y la ausencia de esta en los elementos del canto de comunicación. Se ha evidenciado en otros vertebrados que los caracteres vocales pueden divergir de forma independiente (Rivera-Correa et al. 2017) (ej. el llamado reproductivo puede divergir a una tasa más elevada que el canto de comunicación), con lo cual la inclusión de caracteres moleculares en próximos análisis puede dar mayor información sobre los tiempos de 
divergencia y los patrones de especiación actuales del complejo.

La definición de los factores geográficos y ambientales que pueden limitar los rangos de ocurrencia es crítica para entender la distribución de la biodiversidad (Graham et al. 2010). Los límites de distribución coinciden más a menudo con las discontinuidades ambientales que con las geográficas, promoviendo un equilibrio entre el ambiente y las especies que lo habitan (Graham et al. 2010). Lo anterior podría darse en el complejo M. cayanensis, donde los límites en la distribución de las subespecies puede ocurrir por las condiciones ambientales (Graham et al. 2004, 2010; Munguía et al. 2008). Al igual que en otros trabajos (Gutiérrez-Pinto et al. 2012; Winger y Bates 2015), los resultados soportan el papel de los andes como modulador de la divergencia y la estructura poblacional en la avifauna (ej. la cordillera oriental andina condicionando la diferenciación de M. c. hellmayri como taxón independiente).

Los límites surorientales de M. c. rufipennis son difíciles de definir con claridad debido a la falta de muestras de colección de individuos provenientes de la porción amazónica colombiana, ecuatoriana y brasilera. No obstante, es probable que pueda extenderse hasta el sur de la provincia de Morona Santiago en Ecuador y hasta la margen izquierda de la cuenca alta del río Negro en Brasil. Para resolver esta hipótesis se debe examinar más material proveniente de estas localidades, e intentar dilucidar las posibles barreras que promueven la especiación a nivel local (Hernandez et al. 2006; Smith et al. 2014a). Tradicionalmente, para muchos grupos de aves los ríos suelen ser barreras importantes (Capparella 1990; Gascon et al. 2000; Hayes y Sewlal 2004; Kopuchian et al. 2020; Maximiano et al. 2020), y aunque en este caso el rio Negro puede estar actuando como un limitante en la distribución de la población, deben considerarse también otras alternativas como las barreras ambientales (Davidar et al. 2007), o incluso procesos de especiación simpátrica. Si es el caso, podrían estar ocurriendo simultáneamente dos procesos de especiación distintos en el complejo $M$. cayanensis: (1) el curso marcado por dos poblaciones alopátricas separadas por los andes orientales (especiación con barrera clásica, M. c. hellmayri con respecto a los demás taxones), y (2) el proceso asociado a las poblaciones sin barrera geográfica aparente entre las áreas bajas del piedemonte de la vertiente oriental en la cordillera oriental de los andes, la amazonia y el suroriente de la mata atlántica en el estado de Minas Gerais (M. c. cayanensis con respecto a $M$. c. rufipennis y $M$. c. erythropterus). Sin embargo, es necesaria una aproximación molecular para dilucidar con exactitud el estado actual de estos procesos. 


\section{CONCLUSIONES Y CONSIDERACIONES}

En resumen, este trabajo muestra el soporte de diferencia que existe entre los taxones que conforman el complejo M. cayanensis. Las poblaciones transandinas (M. c. hellmayri) y aquellas con distribución restringida al oriente de los estados de Rio de Janeiro, Espirito Santo y Minas Gerais en Brasil (M. c. erythropterus), divergen fuertemente entre sí y de sus taxones

hermanos en (1) coloración (2) morfología y (3) en los parámetros vocales (particularmente en las variables de frecuencia del llamado reproductivo). Por tanto, estos dos grupos ameritan la clasificación como especies biológicas independientes.

Este trabajo resume la variación de cada grupo, proporcionando información para futuras evaluaciones taxonómicas. Siguiendo las recomendaciones de Van Doren et al. (2018), se debe incorporar en próximos trabajos una evaluación genética y considerar el uso de playback en la experimentación para comprobar si las diferencias en los parámetros de vocalización son suficientes para generar un aislamiento reproductivo pre cópula de las poblaciones, ya que las vocalizaciones han mostrado ser un indicador importante de las relaciones evolutivas en la familia Tyrannidae (Rheindt et al. 2008, 2015; Cuervo et al. 2014).

Aunque las diferencias en los parámetros vocales del llamado reproductivo sugieren la presencia de cuatro especies distintas (siguiendo el criterio de delimitación de especies basado en la divergencia de al menos tres parámetros individuales [Isler et al. 1998, 2008, 2012]), una mirada más conservadora tomando como referencia la interacción en la variabilidad de los parámetros vocales y la morfología (la cual no soporta la diferencia entre M. c. rufipennis y M. c. cayanensis), es probablemente la visión más prudente, teniendo en cuenta que la amplia distribución y la alta variabilidad dentro del complejo podrían estar super estimando la variación poblacional típica. Así pues, se recomienda tratar el complejo $M$. cayanensis (Figura 47) como un grupo de tres especies y dos subespecies así: 


\section{Especie Myiozetetes cayanensis (Linnaeus, 1776)}

Subespecie Myiozetetes c. cayanensis (Linnaeus, 1766)

Subespecie Myiozetetes c. rufipennis Lawrence, 1870

Especie Myiozetetes hellmayri Hartert y Goodson, 1917

Especie Myiozetetes erythropterus (Lafresnaye, 1853)

Myiozetetes hellmayri es una especie abundante de amplia distribución, que ocurre desde el oriente de Costa Rica en Panamá, Colombia, Venezuela y Ecuador. Aunque este trabajo valida su estatus como especie, se recomiendan futuros análisis a nivel regional que permitan conocer con mejor profundidad la variación local de esta especie en todo su rango de distribución.

Myiozetetes erythropterus es una especie endémica del oriente de Brasil al sur del Río Contas y el estado de Bahía, al oriente de Minas Gerais, Espírito Santo y Río de Janeiro hasta la margen izquierda del Río Grande y el norte de la Serra da Mantiqueira. Su distribución reducida podría significar presiones de selección sobre las poblaciones actuales, con lo cual es prioritario realizar una evaluación y categorización de amenazas que contribuya al mantenimiento y cuidado de esta población. Tradicionalmente, los esfuerzos de conservación se han aplicado a nivel de especies (Phillimore y Owens 2006), pero los taxones infraespecíficos representan una diversidad ecológica y genética considerable, ofreciendo un mayor pool de conservación a corto y largo plazo (Vinarski 2015). El no establecer medidas prioritarias de conservación en estas poblaciones de distribución reducida puede derivar en la perdida de estas, incluyendo la diversidad genética y funcional que representa (Haig et al. 2006; Phillimore y Owens 2006). Así pues, tomando como referencia los mayores esfuerzos de conservación en las jerarquías de especie, los cambios taxonómicos afectan directamente a los taxones involucrados, al visibilizar posibles amenazas antes ocultas al considerar el estatus de amenaza de la especie como un conjunto. De esta forma, la validación de especies influencia la ubicación de estas en planes concretos de conservación.

A pesar de que el complejo M. cayanensis es común y abundante a lo largo de toda su distribución, los datos analizados muestran que existe poca información disponible. Del 
mismo modo, este trabajo evidencia los problemas metodológicos típicos que suponen este tipo de evaluaciones, aun cuando se utilizan modelos de especies comunes y relativamente fáciles de registrar, grabar y fotografiar. Por esta razón, se resalta la importancia de las colecciones zoológicas como información de soporte en este y otros tipos de estudios.

El trabajo de campo continuo, la colecta de especímenes y datos de historia natural son vitales para la conservación de las poblaciones potencialmente amenazadas. Para el caso, de $M$. hellmayri y $M$. erythropterus es necesario reunir más información que permita asignar tamaños poblacionales y conocer el estado actual de las mismas. Del mismo modo, existe incertidumbre en la definición exacta del rango de ocurrencia de M. c. erythropterus al occidente de su distribución, donde registros adicionales de imágenes, vocalizaciones y especímenes de colección podrían contribuir enormemente al cierre de esta brecha en el conocimiento de la especie. 


\section{REFERENCIAS}

Aleixo A (2007) Conceitos de espécie e o eterno conflito entre continuidade e operacionalidade: uma proposta de normatização de critérios para o reconhecimento de espécies pelo Comitê Brasileiro de Registros Ornitológicos. Rev Bras Ornitol 15:297-310

Alström P, Ranft R (2003) The use of sounds in avian systematics and the importance of bird sound archives. Bull Br Ornithol Club 123:114-135

Alström P, Rasmussen PC, Olsson U, Sundberg P (2008) Species delimitation based on multiple criteria: The Spotted Bush Warbler Bradypterus thoracicus complex (Aves: Megaluridae). Zool J Linn Soc 154:291-307. https://doi.org/10.1111/j.1096-3642.2008.00418.x

Amadon D, Mayr E, Snow DW, et al (1979) Check-List of the Birds of the World, VIII. Museum of Comparative Zoology, Harvard University, Cambridge, Massachusetts

Ames PL (1971) The morphology of the syrinx in passerine birds. Bull Peabody Museum Nat Hist 37:194

Andersson M (1999) Phylogeny, Behaviour, Plumage Evolution and Neoteny in Skuas Stercorariidae. J Avian Biol 30:205-215. https://doi.org/10.2307/3677130

Baldwin MW, Winkler H, Organ CL, Helm B (2010) Wing pointedness associated with migratory distance in common-garden and comparative studies of stonechats (Saxicola torquata). J Evol Biol 23:1050-1063. https://doi.org/10.1111/j.1420-9101.2010.01975.x

Baldwin PS, Oberholser HC, Worley LG (1931) Measurements of birds. Sci Publ Clevel Museum Nat Hist 2:1-165

Bangs O, Penard TE (1921) A new subspecies of Myiozetetes cayanensis (Linné). Bull Museum Comp Zool 64:374-376

Barrowclough GF (1982) Geographic variation, predictiveness, and subspecies. Auk 99:601-603

Barrowclough GF, Cracraft J, Klicka J, Zink RM (2016) How Many Kinds of Birds Are There and Why Does It Matter? PLoS One 11:e0166307. https://doi.org/10.1371/journal.pone.0166307

Bioacustics Research Program (2017) Raven Pro: Interactive Sound Analysis Software

Birdsley JS (2002) Phylogeny of the Tyrant Flycatchers (Tyrannidae) Based on Morphology and Behavior. Auk 119:715-734. https://doi.org/10.1093/auk/119.3.715

Bocalini F (2013) Revisão taxonômica e filogenia do complexo Cyphorhinus arada (Hermann, 1783) (Aves : Troglodytidae). Universidade de Sao Paulo

Bolaños-Sittler P, Sueur J, Fuchs J, Aubin T (2020) Vocalisation of the rare and flagship species Pharomachrus mocinno (Aves: Trogonidae): implications for its taxonomy, evolution and conservation. Bioacoustics 29:654-669. https://doi.org/10.1080/09524622.2019.1647877

Bonferroni CE (1936) Teoria statistica delle classi e calcolo delle probabilità. Pubbl del R Ist Super di Sci Econ e Commer di Firenze 8:3-62

Borras A, Pascual J, Senar JC (2000) What do different bill measures measure and what is the best method to use in granivorous birds? J F Ornithol 71:606-611. https://doi.org/10.1648/02738570-71.4.606

Box GEP (1949) A general distribution theory for a class of likelihood criteria. Biometrika 36:317-346. https://doi.org/10.1093/biomet/36.3-4.317 
Bretagnolle V (1995) Systematics of the Soft-plumaged Petrel Pterodroma mollis (Procellariidae): new insight from the study of vocalizations. Ibis (Lond 1859) 137:207-218. https://doi.org/10.1111/j.1474-919X.1995.tb03241.x

Brisson MJ (1760) Ornithologie, ou, Méthode contenant la division desoiseaux en ordres, sections, genres, especes \& leursvariétés : a laquelle on a joint une description exacte dechaque espece, avec les citations des auteurs qui en onttraité, les noms quils leur ont donnés, 2 nd edn. Ad Ripam Augustinorum, apud $\mathrm{Cl}$. Joannem-Baptistam Bauche, bibliopolam, ad Insigne S. Genovesae, \& S. Joannis in Deserto, 1760 , Paris

Cabanis J, Heine F (1859) Museum Heineanum : Verzeichniss der ornithologischen Sammlung des Oberamtmann Ferdinand Heine, auf Gut St. Burchard vor Halberstadt. Museum Heineanum II:61

Capparella AP (1990) Neotropical avian diversity and riverine barriers. Proc Int Ornithol Congr 20:307316

Carneiro LS, Gonzaga LP, Rêgo PS, et al (2012) Systematic revision of the Spotted Antpitta (Grallariidae: Hylopezus macularius ), with description of a cryptic new species from Brazilian Amazonia. Auk 129:338-351. https://doi.org/10.1525/auk.2012.11157

Casas G, Veitía N (2008) Aplicación de métodos de comparaciones múltiples en Biotecnología Vegetal. Biotecnol Veg 8:67-71

Chaves A V., Clozato CL, Lacerda DR, et al (2008) Molecular taxonomy of Brazilian tyrant-flycatchers (Passeriformes: Tyrannidae). Mol Ecol Resour 8:1169-1177. https://doi.org/10.1111/j.17550998.2008.02218.x

Chesser RT (2004) Molecular systematics of New World suboscine birds. Mol Phylogenet Evol 32:1124. https://doi.org/10.1016/j.ympev.2003.11.015

Chesser RT (2000) Evolution in the High Andes: The Phylogenetics of Muscisaxicola Ground-Tyrants. Mol Phylogenet Evol 15:369-380. https://doi.org/10.1006/mpev.1999.0774

Collinson JM, Parkin DT, Knox GA, et al (2006) Species limits within the genus Melanitta, the scoters. Br Birds 99:183-201

Comisión Internacional de Nomenclatura Zoológica (2000) Código Internacional de Nomenclatura Zoológica, 4th edn. The Natural History Museum, London, UK.

Cory BC, Hellmayr CE (1927) Catalogue of birds of the Americas and the adjacent Islands in Field Museum of Natural History: Part V Tyrannidae. Zool Ser 13:242

Coyne JA, Orr HA (2004) Speciation. Sinauer Associates, Sunderland, MA.

Cracraft J (1983) Species concept and speciation analysis. In: Johnston RF (ed) Current Ornithology, 1st edn. Plenum Press, New York, USA, pp 159-187

Cracraft J (1997) Species concepts in systematics and conservation biology - an ornithological viewpoint. In: Claridge MF, Dawah HA, Wilson MR (eds) Species: the units of biodiversity. Chapman \& Hall, London, UK., pp 325-339

Cruz A, Andrews RW (1989) Observations on the breeding biology of Passerines in a seasonally flooded savanna in Venezuela. Wilson Bull 101:62-76

Cuervo AM, Stiles FG, Lentino M, et al (2014) Geographic variation and phylogenetic relationships of Myiopagis olallai (Aves: Passeriformes; Tyrannidae), with the description of two new taxa from the Northern Andes. Zootaxa 3873:1-24. https://doi.org/10.11646/zootaxa.3873.1.1

Davidar P, Rajagopal B, Mohandass D, et al (2007) The effect of climatic gradients, topographic variation and species traits on the beta diversity of rain forest trees. Glob Ecol Biogeogr 16:510518. https://doi.org/10.1111/j.1466-8238.2007.00307.x 
Davis JI, Nixon KC (1992) Populations, genetic variation, and the delimitation of phylogentic species. Syst Biol 41:421-435

Davis SE (1993) Seasonal Status, Relative Abundance, and Behavior of the Birds of Concepción, Departamento Santa Cruz, Bolivia. Fieldiana Zool 71:1-33

De Queiroz K (2007) Species Concepts and Species Delimitation. Syst Biol 56:879-886. https://doi.org/10.1080/10635150701701083

Dénes FV, Silveira LF, Seipke S, et al (2011) The White-collared Kite (Leptodon forbesi Swann, 1922) and a Review of the Taxonomy of the Grey-headed Kite (Leptodon cayanensis Latham, 1790). Wilson J Ornithol 123:323-331. https://doi.org/10.1676/10-081.1

Dickinson E., Christidis L (2014) The Howard and Moore complete checklist of the birds of the World, 2nd edn. Aves Press, Eastbourne, U.K.

Donegan T (2008) Geographical variation in Slate-crowned Antpitta Grallaricula nana, with two new subspecies, from Colombia and Venezuela. Bull Br Ornithol 128:150-178

Dunnett CW (1980) Pairwise Multiple Comparisons in the Unequal Variance Case. J Am Stat Assoc 75:796-800. https://doi.org/10.1080/01621459.1980.10477552

Dyrcz A (2002) Breeding ecology of the social (Myiozetetes similis) and rusty-margined (M. cayanensis) flycatchers at Barro Colorado Island, Republic of Panama. Ornithol Neotrop 13:143-151

Dyrcz A (1991) Observations on nesting and nestling growth in the Rusty-margined Flycatcher Myiozetetes cayanensis in Southeastern Peru. Bull Br Ornithol Club 111:33-35

Ekström J (2020) Lund Museum of Zoology (MZLU). In: GBIF-Sweden. https://www.gbif.org/occurrence/80049525. Accessed 7 Jan 2021

Ericson PGP, Irestedt M, Johansson US (2003) Evolution, biogeography, and patterns of diversification in passerine birds. J Avian Biol 34:3-15. https://doi.org/10.1034/j.1600-048X.2003.03121.x

Ericson PGP, Zuccon D, Ohlson JI, et al (2006) Higher-level phylogeny and morphological evolution of tyrant flycatchers, cotingas, manakins, and their allies (Aves: Tyrannida). Mol Phylogenet Evol 40:471-483. https://doi.org/10.1016/j.ympev.2006.03.031

Fine PVA (2015) Ecological and Evolutionary Drivers of Geographic Variation in Species Diversity. Annu Rev Ecol Evol Syst 46:369-392. https://doi.org/10.1146/annurev-ecolsys-112414-054102

Fitzpatrick J (2020) Tyrant-flycatchers (Tyrannidae). In: del Hoyo J, Elliott A, Sargatal J, et al. (eds) Handbook of the Birds of the World Alive. Lynx Edicions, Barcelona

Fitzpatrick JW (1980) Foraging Behavior of Neotropical Tyrant Flycatchers. Condor 82:43-57. https://doi.org/10.2307/1366784

Fitzpatrick JW (1985) Form, foraging behavior, and adaptive radiation in the Tyrannidae. Ornithol Monogr 36:447-470

Fjeldså J, Bowie RCK, Rahbek C (2012) The Role of Mountain Ranges in the Diversification of Birds. Annu Rev Ecol Evol Syst 43:249-265. https://doi.org/10.1146/annurev-ecolsys-102710-145113

Fjeldså J, Ohlson JI, Batalha-Filho $\mathrm{H}$, et al (2018) Rapid expansion and diversification into new niche space by fluvicoline flycatchers. J Avian Biol 49:jav-01661. https://doi.org/10.1111/jav.01661

Fox J, Weisberg S (2019) An R Companion to Applied Regression, Third. Sage, Thousand Oaks CA

Futuyma DJ (1997) Evolutionary Biology, 3rd edn. Sinauer Associates, Sunderland, M. A

Games PA, Howell JF (1976) Pairwise Multiple Comparison Procedures with Unequal N's and/or Variances: A Monte Carlo Study. J Educ Stat 1:113. https://doi.org/10.2307/1164979 
Gascon C, Malcolm JR, Patton JL, et al (2000) Riverine barriers and the geographic distribution of Amazonian species. Proc Natl Acad Sci U S A 97:13672-13677. https://doi.org/10.1073/pnas.230136397

Ghosh-Harihar M (2014) Phylogenetic and ecomorphological structure of assemblages of breeding leaf warblers (Phylloscopidae) along Himalayan elevational gradients. J Biogeogr 41:1193-1203. https://doi.org/10.1111/jbi.12281

Gjershaug JO, Diserud OH, Kleven O, et al (2020) Integrative taxonomy of the Changeable Hawk-Eagle Nisaetus cirrhatus complex (Accipitriformes: Accipitridae) in India. Zootaxa 4789:554-574. https://doi.org/10.11646/zootaxa.4789.2.10

Graham CH, Parra JL, Tinoco BA, et al (2012) Untangling the influence of ecological and evolutionary factors on trait variation across hummingbird assemblages. Ecology 93:S99-S111. https://doi.org/10.1890/11-0493.1

Graham CH, Ron SR, Santos JC, et al (2004) Integrating Phylogenetics and Environmental Niche Models to Explore Speciation Mechanisms in Dendrobatid Frogs. Evolution (N Y) 58:1781-1793. https://doi.org/10.1111/j.0014-3820.2004.tb00461.x

Graham CH, Silva N, Velásquez-Tibatá J (2010) Evaluating the potential causes of range limits of birds of the Colombian Andes. J Biogeogr 37:1863-1875. https://doi.org/10.1111/j.13652699.2010.02356.x

Graves GR (1991) Bergmann's rule near the equator: latitudinal clines in body size of an Andean passerine bird. Proc Natl Acad Sci 88:2322-2325. https://doi.org/10.1073/pnas.88.6.2322

Gutiérrez-Pinto N, Cuervo AM, Miranda J, et al (2012) Non-monophyly and deep genetic differentiation across low-elevation barriers in a Neotropical montane bird (Basileuterus tristriatus; Aves: Parulidae). Mol Phylogenet Evol 64:156-165. https://doi.org/10.1016/j.ympev.2012.03.011

Haffer J (1992) The History of Species Concepts and Species Limits in Ornithology. Bull Br Ornithol Club 112:107-158

Haig SM, Beever EA, Chambers SM, et al (2006) Taxonomic considerations in listing subspecies under the U.S. Endangered Species Act. Conserv Biol 20:1584-1594. https://doi.org/10.1111/j.15231739.2006.00530.x

Harrell FEJ (2017) Hmisc: Harrell Miscellaneous. R package

Hartert E, Goodson A (1917) Myiozetetes cayanensis hellmayri subsp. nov. Novit Zool 24:412

Harvey MG, Bravo GA, Claramunt S, et al (2020) The evolution of a tropical biodiversity hotspot. Science (80- ) 370:1343-1348. https://doi.org/10.1126/science.aaz6970

Hausdorf B (2011) Progress toward a general species concept. Evolution (N Y) 65:923-931. https://doi.org/10.1111/j.1558-5646.2011.01231.x

Haverschmidt $F$ (1971) Notes on the life history of the Rusty-margined Flycatcher in Surinam. Wilson Bull 83:124-128

Hayes FE, Sewlal J-AN (2004) The Amazon River as a dispersal barrier to passerine birds: effects of river width, habitat and taxonomy. J Biogeogr 31:1809-1818. https://doi.org/10.1111/j.13652699.2004.01139.x

Helbig AJ, Knox AG, Parkin DT, et al (2002) Guidelines for assigning species rank. Ibis (Lond 1859) 144:518-525

Helbig AJ, Martens J, Seibold I, et al (1996) Phylogeny and species limits in the Palaearctic chiffchaff Phylloscopus collybita complex: mitochondrial genetic differentiation and bioacoustic evidence. Ibis (Lond 1859) 138:650-666. https://doi.org/10.1111/j.1474-919X.1996.tb04767.x 
Hennig W (1950) Grundziige einer Theorie der phylogenetischen Systematik. Deutscher Zentralverlag, Berlin, Germany.

Hennig W (1966) Phylogenetic Systematics. University of Illinois Press, Urbana, IL, USA.

Hernandez PA, Graham CH, Master LL, Albert DL (2006) The effect of sample size and species characteristics on performance of different species distribution modeling methods. Ecography (Cop) 29:773-785. https://doi.org/10.1111/j.0906-7590.2006.04700.x

Hilty SL, Brown WL (1986) A Guide to the Birds of Colombia, Illustrate. Princeton University Press, Princeton, New Jersey

Hollander M, Wolfe DA (1973) Nonparametric Statistical Methods. Wiley Online Library, New York

Hosner PA, Moyle RG (2012) A molecular phylogeny of black-tyrants (Tyrannidae: Knipolegus) reveals strong geographic patterns and homoplasy in plumage and display behavior. Auk 129:156-167. https://doi.org/10.1525/auk.2012.11101

Isler ML, Bravo GA, Brumfield RT (2013) Taxonomic revision of Myrmeciza (Aves: Passeriformes: Thamnophilidae) into 12 genera based on phylogenetic, morphological, behavioral, and ecological data. Zootaxa 3717:469. https://doi.org/10.11646/zootaxa.3717.4.3

Isler ML, Chesser RT, Robbins MB, et al (2020) Taxonomic evaluation of the Grallaria rufula (Rufous Antpitta) complex (Aves: Passeriformes: Grallariidae) distinguishes sixteen species. Zootaxa 4817:1-74. https://doi.org/10.11646/zootaxa.4817.1.1

Isler ML, Cuervo AM, Bravo GA, Brumfield RT (2012) An Integrative Approach to Species-Level Systematics Reveals the Depth of Diversification in an Andean Thamnophilid, the Long-tailed Antbird. Condor 114:571-583. https://doi.org/10.1525/cond.2012.120012

Isler ML, Isler PR, Brumfield RT (2005) Clinal variation in vocalizations of an antbird (Thamnophilidae) and implications for defining species limits. Auk 122:433-444. https://doi.org/10.1642/00048038(2005)122[0433:CVIVOA]2.0.CO;2

Isler ML, Isler PR, Whitney BM (1997) Biogeography and Systematics of the Thamnophilus punctatus (Thamnophilidae) Complex. Ornithol Monogr 355-381. https://doi.org/10.2307/40157543

Isler ML, Isler PR, Whitney BM (1998) Use of Vocalizations to Establish Species Limits in Antbirds (Passeriformes: Thamnophilidae). Auk 115:577-590. https://doi.org/10.2307/4089407

Isler ML, Isler PR, Whitney BM, et al (2009) Species limits in antbirds (Aves: Passeriformes: Thamnophilidae): an evaluation of Frederickena unduligera (Undulated Antshrike) based on vocalizations. Zootaxa 2305:61-68. https://doi.org/10.11646/zootaxa.2305.1.5

Isler ML, Isler PR, Whitney BM (2008) Species limits in Antbirds (Aves: Passeriformes: Thamnophilidae): an evaluation of Plumbeous Antvireo (Dysithamnus plumbeus) based on vocalizations. Zootaxa 68:60-68. https://doi.org/10.11646/zootaxa.1726.1.5

Johansson US, Irestedt M, Parsons TJ (2002) Basal Phylogeny of the Tyrannoidea Based on Comparisons of Cytochrome b and Exons of Nuclear c-myc and RAG-1 Genes. Auk 119:984-995. https://doi.org/10.2307/4090228

Johnson NK, Cicero C (2002) The role of ecologic diversification in sibling speciation of Empidonax flycatchers (Tyrannidae): multigene evidence from mtDNA. Mol Ecol 11:2065-2081. https://doi.org/10.1046/j.1365-294X.2002.01588.x

Kanungo T, Mount DM, Netanyahu NS, et al (2002) An efficient $k$-means clustering algorithm: analysis and implementation. IEEE Trans Pattern Anal Mach Intell 24:881-892. https://doi.org/10.1109/TPAMI.2002.1017616

Kassambara A, Mundt F (2020) Factoextra: Extract and Visualize the Results of Multivariate Data 
Analyses

Kattan GH, Franco P, Rojas V, Morales G (2004) Biological diversification in a complex region: a spatial analysis of faunistic diversity and biogeography of the Andes of Colombia. J Biogeogr 31:18291839. https://doi.org/10.1111/j.1365-2699.2004.01109.x

Khan A, Rayner G (2003) Robustness to Non-Normality of Common Tests for the Many-Sample Location Problem. J Appl Math Decis Sci 7:187-206. https://doi.org/10.1207/s15327612jamd0704_1

Kopuchian C, Campagna L, Lijtmaer DA, et al (2020) A test of the riverine barrier hypothesis in the largest subtropical river basin in the Neotropics. Mol Ecol 29:2137-2149. https://doi.org/10.1111/mec.15384

Lafresnaye (1853) Tyrannula erythroptera. Rev Mag Zool Pure Appl 2:56

Lanyon SM (1985) Molecular Perspective on Higher-Level Relationships in the Tyrannoidea (Aves). Syst Zool 34:404. https://doi.org/10.2307/2413205

Lanyon WE (1988a) A Phylogeny of the Thirty-two Genera in the Elaenia Assemblage of Tyrant Flycathers. Novitates 2914:1-26

Lanyon WE (1988b) A Phylogeny of the Flatbill and Tody-tyrant Assemblage of Tyrant Flycatchers. Novitates 2923:1-41

Lawrence GN (1870) Myiozetetes rufipennis. Ann Lyceum Nat Hist New York 267

Levit GS, Hoßfeld U, Olsson L (2004) The integration of Darwinism and evolutionary morphology: Alexej Nikolajevich Sewertzoff (1866-1936) and the developmental basis of evolutionary change. J Exp Zool 302B:343-354. https://doi.org/10.1002/jez.b.20026

Lima RD, Tomotani BM, Silveira LF (2020) Colour variation and taxonomy of Picumnus limae Snethlage, 1924 and P. fulvescens Stager, 1961 (Piciformes: Picidae). J Ornithol 161:491-501. https://doi.org/10.1007/s10336-020-01745-0

Linnaeus C von (1766) Systema naturæ: per regna tria natura, secundum classes, ordines, genera, species, cum characteribus, differentiis, synonymis, locis, 1st, 12th edn. L. Salvii, Holmiæ (Estocolmo):

Machac A (2020) The Dynamics of Bird Diversity in the New World. Syst Biol 69:1180-1199. https://doi.org/10.1093/sysbio/syaa028

MacQueen J (1967) Some methods for classification and analysis of multivariate observations. Proc 5th Berkeley Symp Math Stat Probab 1:281-297

Mahalanobis PC (1936) On the Generalized Distance in Statistics. Proc Natl Inst Sci 2:49-55

Marcondes RS (2013) Taxonomia e filogenia do gênero Aramides Pucheran, 1845 (Aves: Gruiformes: Rallidae). Universidade de São Paulo

Marcondes RS, Brumfield RT (2020) A simple index to quantify and compare the magnitude of intraspecific geographic plumage colour variation in typical antbirds (Aves: Passeriformes: Thamnophilidae). Biol J Linn Soc 130:239-246. https://doi.org/10.1093/biolinnean/blaa041

Martens J, Eck S, Päckert M, Sun Y-H (2003) Methods of Systematic and Taxonomic Research on Passerine Birds: the Timely Example of the Seicercus burkii Complex (Sylviidae). Bonner Zool Beiträge 51:109-118

Maximiano MF de A, D'Horta FM, Tuomisto $\mathrm{H}$, et al (2020) The relative role of rivers, environmental heterogeneity and species traits in driving compositional changes in southeastern Amazonian bird assemblages. Biotropica 52:946-962. https://doi.org/10.1111/btp.12793

Mayr E (1963) Animal species and evolution, 1st edn. The Belknap Press of Harvard University Press., 
Cambridge, Massachusetts

Mayr E, Diamond J (2001) The Birds of Northern Melanesia: Speciation, Ecology, and Biogeography. Oxford University Press UK, Oxford

McKitrick MC (1985) Monophyly of the Tyrannidae (Aves): Comparison of Morphology and DNA. Syst Zool 34:35. https://doi.org/10.2307/2413343

McKitrick MC, Zink RM (1988) Species concepts in ornithology. Condor 90:1-14

Mobley J, Kirwan GM (2020) Rusty-margined Flycatcher (Myiozetetes cayanensis). In: del Hoyo J, Elliott A, Sargatal J, et al. (eds) Birds of the World. Cornell Lab of Ornithology, Ithaca, NY, USA.

Moder K (2010) Alternatives to F-Test in One Way ANOVA in case of heterogeneity of variances (a simulation study). Psychol Test Assess Model 52:343-353

Munguía M, Townsend Peterson A, Sánchez-Cordero V (2008) Dispersal limitation and geographical distributions of mammal species. J Biogeogr 35:1879-1887. https://doi.org/10.1111/j.13652699.2008.01921.x

Nixon KC, Wheeler QD (1990) An amplification of the phylogenetic species concept. Cladistics 6:211223. https://doi.org/10.1111/j.1096-0031.1990.tb00541.x

Ohlson J, Fjeldså J, Ericson PGP (2008) Tyrant flycatchers coming out in the open: Phylogeny and ecological radiation of Tyrannidae (Aves, Passeriformes). Zool Scr 37:315-335. https://doi.org/10.1111/j.1463-6409.2008.00325.x

Ohlson JI, Fjeldså J, Ericson PGP (2009) A new genus for three species of tyrant flycatchers (Passeriformes: Tyrannidae), formerly placed in Myiophobus. Zootaxa 40:36-40. https://doi.org/10.11646/zootaxa.2290.1.3

Ohlson Jl, Irestedt M, Ericson PGP, Fjeldså J (2013) Phylogeny and classification of the New World suboscines (Aves, Passeriformes). Zootaxa 3613:1-35. https://doi.org/10.11646/zootaxa.3613.1.1

Ohlson JI, Irestedt M, Filho HB, Fjeldså J (2020) A revised classification of the fluvicoline tyrant flycatchers (Passeriformes, Tyrannidae, Fluvicolinae). Zootaxa 4747:167-176. https://doi.org/10.11646/zootaxa.4747.1.7

Ohlson JI, Irestedt M, Fjeldså J, Ericson PGP (2012) Nuclear DNA from a 180-year-old study skin reveals the phylogenetic position of the Kinglet Calyptura Calyptura cristata (Passeriformes: Tyrannides). Ibis (Lond 1859) 154:533-541. https://doi.org/10.1111/j.1474-919X.2012.01243.x

Ohlson JI, Prum RO, Ericson PGP (2007) A molecular phylogeny of the cotingas (Aves: Cotingidae). Mol Phylogenet Evol 42:25-37. https://doi.org/10.1016/j.ympev.2006.05.041

Olsson L, Levit GS, Hoßfeld U (2017) Phylogenetic Systematics: Haeckel to Hennig. Acta Zool 99:415420. https://doi.org/10.1111/azo.12221

Paynter RA (1982) Ornithological Gazetteer of Venezuela

Paynter RA (1992) Ornithological Gazetteer of Bolivia

Paynter RA (1993) Ornithological Gazetteer of Ecuador

Paynter RA (1997) Ornithological Gazetteer of Colombia

Paynter RA, Traylor MA (1991) Ornithological gazetteer of Brazil

Pelzeln A (1868) Myiozetetes columbianus. Ornithol Bras 109

Phillimore AB, Orme CDL, Thomas GH, et al (2008) Sympatric Speciation in Birds Is Rare: Insights from Range Data and Simulations. Am Nat 171:646-657. https://doi.org/10.1086/587074 
Phillimore AB, Owens IPF (2006) Are subspecies useful in evolutionary and conservation biology? Proc R Soc B Biol Sci 273:1049-1053. https://doi.org/10.1098/rspb.2005.3425

Pinto OM (1944) Catalogo das aves do Brasil: e lista dos exemplares existentes na colecao do Departamento de Zoologia. 2a Parte: Ordem PASSERIFORMES (continuacao): Superfamilia Tyrannoidea e Subordem PASSERES, 2nd edn. Departamento de Zoologia, Secretaria da Agricultura, Industria e Comercio, Sao Paulo, Brasil.

Pollock AWI (2015) Binomial and Trinomial Index to The Auk, 1884-1940. Also including the Nuttall Bulletin, 1876-1883 and The A.O.U. Check-List of North American Birds (All Seven Editions), 7th edn. The A.O.U. Check-List of North American Birds

Price RD, Hellenthal RA, Dalgleish RC (2005) The genus Myrsidea Waterston (Phthiraptera: Menoponidae) from tyrant-flycatchers (Passeriformes: Tyrannidae), with descriptions of 13 new species. Zootaxa 1048:1. https://doi.org/10.11646/zootaxa.1048.1.1

Prum RO (1990) A test of the monophyly of the manakins (Pipridae) and of the cotingas (Cotingidae) based on morphology. Occassional Pap Museum Zool Univ Michigan 723:1-44

QGIS Development Team (2019) QGIS Geographic Information System

R Core Team (2019) R: A language and environment for statistical computing

Raposo MA (2001) Taxonomia alfa de aves neotropicais. In: Albuquerque JLB, Cândido JF, Straube FC, Roos AL (eds) Ornitologia e conservação: da ciência às estratégias. Tubarão: Editora Unisul, pp 249-260

Reaney AM, Bouchenak-Khelladi Y, Tobias JA, Abzhanov A (2020) Ecological and morphological determinants of evolutionary diversification in Darwin's finches and their relatives. Ecol Evol 10:14020-14032. https://doi.org/10.1002/ece3.6994

Remsen JVJ, Areta JI, Bonaccorso E, et al (2021) A classification of the bird species of South America. In: Am. Ornithol. Soc. https://www.museum.Isu.edu/ Remsen/SACCBaseline.htm. Accessed 20 Jan 2021

Restall R, Rodner C, Lentino M (2007) Birds of Northern South America, 1st edn. Yale University Press

Rheindt FE, Krabbe N, Wee AKS, Christidis L (2015) Cryptic speciation in the lesser elaenia elaenia chiriquensis (Aves: Passeriformes: Tyrannidae). Zootaxa 4032:251-263. https://doi.org/10.11646/zootaxa.4032.3.1

Rheindt FE, Norman JA, Christidis L (2008) DNA evidence shows vocalizations to be a better indicator of taxonomic limits than plumage patterns in Zimmerius tyrant-flycatchers. Mol Phylogenet Evol 48:150-156. https://doi.org/10.1016/j.ympev.2008.04.016

Ridgely R, Tudor G (2009) Tyrannidae. In: Field guide to the songbirds of South America: the passerines, 1 (Mildred. University of Texas Press, Austin, Texas., pp 401-480

Rieppel O (2010) Ernst Haeckel (1834-1919) and the monophyly of life. J Zool Syst Evol Res 49:1-5. https://doi.org/10.1111/j.1439-0469.2010.00580.x

Rieppel O (2011) Adolf Naef (1883-1949), systematic morphology and phylogenetics. J Zool Syst Evol Res 50:2-13. https://doi.org/10.1111/j.1439-0469.2011.00635.x

Rivera-Correa M, Jiménez-Rivillas C, Daza JM (2017) Phylogenetic analysis of the Neotropical Pristimantis leptolophus species group (Anura: Craugastoridae): molecular approach and description of a new polymorphic species. Zootaxa 4242:313-344. https://doi.org/10.11646/zootaxa.4242.2.6

Rowlett J (2003) The Birds of Ecuador, Volume I: Status, Distribution, and Taxonomy. Auk 120:562568. https://doi.org/10.1676/0043-5643(2002)114[0418:tboevi]2.0.co;2 
Ruggiero A, Hawkins BA (2008) Why do mountains support so many species of birds? Ecography (Cop) 31:306-315. https://doi.org/10.1111/j.0906-7590.2008.05333.x

Saitoh T, Shigeta Y, Ueda K (2008) Morphological differences among populations of the Arctic Warbler with some intraspecific taxonomic notes. Ornithol Sci 7:135-142. https://doi.org/10.2326/13470558-7.2.135

Sandoval L, Martínez D, Ocampo D, et al (2018) Range expansion and noteworthy records of Costa Rican birds (Aves). Check List 14:141-151. https://doi.org/10.15560/14.1.141

Schloerke B, Crowley J, Cook D, et al (2018) GGally: Extension to "ggplot2"

Schoener TW (1965) The Evolution of Bill Size Differences Among Sympatric Congeneric Species of Birds. Evolution (N Y) 19:189-213. https://doi.org/10.1111/j.1558-5646.1965.tb01707.x

Screnci-Ribeiro R, Duca C (2010) Táticas de forrageamento de Myiozetetes cayanensis (Linnaeus, 1766) (Passeriformes: Tyrannidae). Rev Bras Ornitol 18:113-117

Sibley CG, Ahlquist JE (1985) Phylogeny and Classification of New World Suboscine Passerine Birds (Passeriformes: Oligomyodi: Tyrannides). Ornithol Monogr 396-428. https://doi.org/10.2307/40168296

Silveira LF, Olmos F (2007) Quantas espécies de aves existem no Brasil? Conceitos de espécie, conservação e o que falta descobrir. Rev Bras Ornitol 15:289-296

Smith BT, McCormack JE, Cuervo AM, et al (2014a) The drivers of tropical speciation. Nature 515:406409. https://doi.org/10.1038/nature13687

Smith P, Pérez N, Derna R, et al (2014b) On the presence of Rusty-margined Flycatcher Myiozetetes cayanensis in Paraguay. Alauda 82:71-72

Smithe FB (1975) Naturalist's color guide. The American Museum of Natural History, New York, USA

Sokal RR, Rohlf FJ (1995) Biometry: The Principles and Practice of Statistics in Biological Research, 3rd edn. .H. Freeman and Co, New York, USA

Stephen L, Traylor MA (1983) Ornithological Gazetteer of Peru

Stephen L, Traylor MA (1985) Ornithological Gazetteer of the Guianas

Tello JG, Bates JM (2007) Molecular Phylogenetics of The Tody-Tyrant and Flatbill Assemblage of Tyrant Flycatchers (Tyrannidae). Auk 124:134. https://doi.org/10.1642/00048038(2007)124[134:mpotta]2.0.co;2

Tello JG, Moyle RG, Marchese DJ, Cracraft J (2009) Phylogeny and phylogenetic classification of the tyrant flycatchers, cotingas, manakins, and their allies (Aves: Tyrannides). Cladistics 25:429-467. https://doi.org/10.1111/j.1096-0031.2009.00254.x

Thorndike RL (1953) Who belongs in the family? Psychometrika 18:267-276. https://doi.org/10.1007/BF02289263

Tiku ML (1971) Power Function of the F-Test Under Non-Normal Situations. J Am Stat Assoc 66:913916. https://doi.org/10.2307/2284254

Traylor-Jr MA (1977) A classification of the tyrant flycatchers (Tyrannidae). Bull Museum Comp Zool 148:129-184

Traylor MA, Fitzpatrick JW (1982) A survey of the tyrant flycatchers. living bird 19:7-50

Trombone T (2013a) AMNH Bird Collection. American Museum of Natural History. In: GBIF.org. https://www.gbif.org/occurrence/858452550. Accessed 5 Jan 2021

Trombone T (2013b) AMNH Bird Collection. American Museum of Natural History. In: GBIF.org. 
https://www.gbif.org/occurrence/858908088. Accessed 20 Jan 2021

Tukey JW (1949) Comparing Individual Means in the Analysis of Variance. Biometrics 5:99. https://doi.org/10.2307/3001913

Uttley J (2019) Power Analysis, Sample Size, and Assessment of Statistical Assumptions-Improving the Evidential Value of Lighting Research. LEUKOS - J Illum Eng Soc North Am 15:143-162. https://doi.org/10.1080/15502724.2018.1533851

Van Doren BM, Freeman BG, Aristizabal N, et al (2018) Species limits in the Rusty-breasted Antpitta (Grallaricula ferrugineipectus) complex. Wilson J Ornithol 130:152-167. https://doi.org/10.1676/16-126.1

Vanzolini PE (1992) A Supplement to the Ornithological Gazetteer of Brazil

Vinarski M V. (2015) The fate of subspecies category in Zoological systematics. 1. the history. Biol Bull Rev 5:395-404. https://doi.org/10.1134/S2079086415050060

Warne RT (2014) A primer on multivariate analysis of variance (MANOVA) for behavioral scientists. Pract Assessment, Res Eval 19:1-10

Watson DM (2012) The Relative Contribution of Specialists and Generalists to Mistletoe Dispersal: Insights from a Neotropical Rain Forest. Biotropica 45:195-202. https://doi.org/10.1111/j.17447429.2012.00905.x

Winger BM, Bates JM (2015) The tempo of trait divergence in geographic isolation: Avian speciation across the Marañon Valley of Peru. Evolution (N Y) 69:772-787. https://doi.org/10.1111/evo.12607

Winkler DW, Billerman SM, Lovette IJ (2020) Tyrant Flycatchers (Tyrannidae). In: Billerman SM, Keeney BK, Rodewald PG, Schulenberg TS (eds) Birds of the World. Cornell Lab of Ornithology

Yésou P (2002) Trends in systematics: systematics of Larus argentatus-cachinnans-fuscus complex revisited. Dutch Bird 24:271-298

Zachos FE (2018) (New) Species concepts, species delimitation and the inherent limitations of taxonomy. J Genet 97:811-815. https://doi.org/10.1007/s12041-018-0965-1

Zhang Q, Holyoak M, Chen C, et al (2019) Trait-mediated filtering drives contrasting patterns of species richness and functional diversity across montane bird assemblages. J Biogeogr 47:301-312. https://doi.org/10.1111/jbi.13738

Zimmer JT (1955) Further notes on tyrant flycatchers (Tyrannidae). Am Museum Novit 1-24

Zink RM, Groth JG, Vázquez-Miranda H, Barrowclough GF (2016) Geographic variation, null hypotheses, and subspecies limits in the California Gnatcatcher: A response to McCormack and Maley. Auk 133:59-68. https://doi.org/10.1642/AUK-15-63.1

Zink RM, McKitrick MC (1995) The debate over species concepts and its implications for ornithology. Auk 112:701-719. https://doi.org/10.1093/auk/112.3.701 


\section{FIGURAS}

\section{ri3. MUSCICAPA. Roftrum fubtrigonum, utrin- que emarginatum, apice incurvo; vibriffe patentes verfus fauces. Nares fubrotundix.}

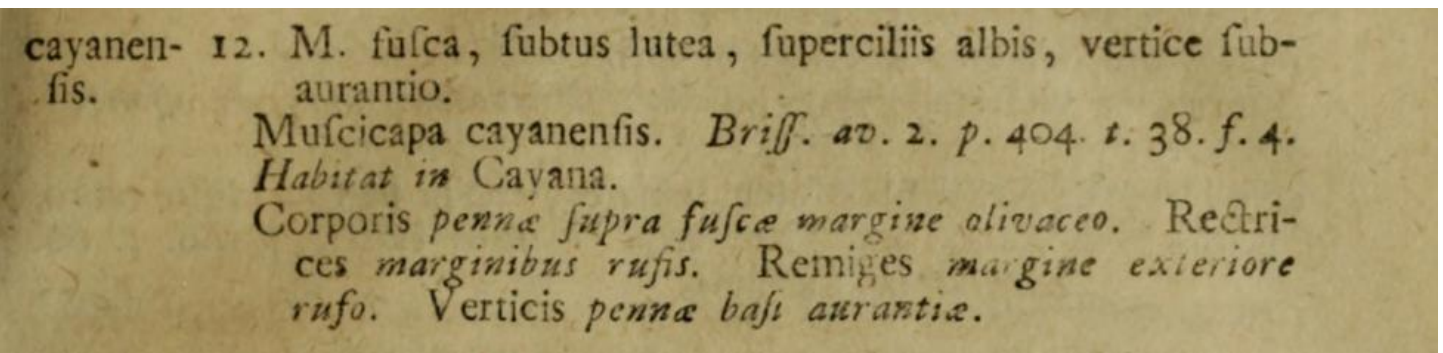

Figura 1. Descripción original del género Muscicapa y la especie Myiozetetes (Muscicapa) cayanensis en el "Systema naturæ" de Linnaeus en 1776. Se muestran fragmentos de las páginas 324 (arriba) y 327 (abajo), del tomo 1, parte 1 del "Systema naturæ: per regna tria natura, secundum classes, ordines, genera, species, cum characteribus, differentiis, synonymis, locis" por Linnaeus en 1776. 


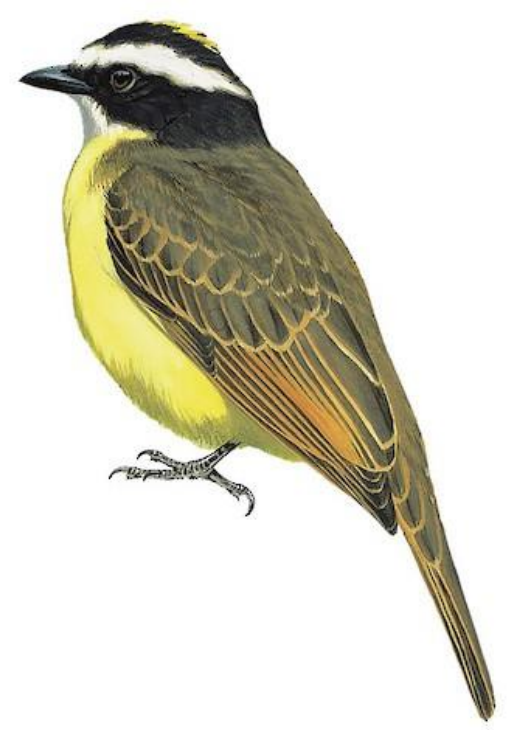

Myiozetetes cayanensis cayanensis

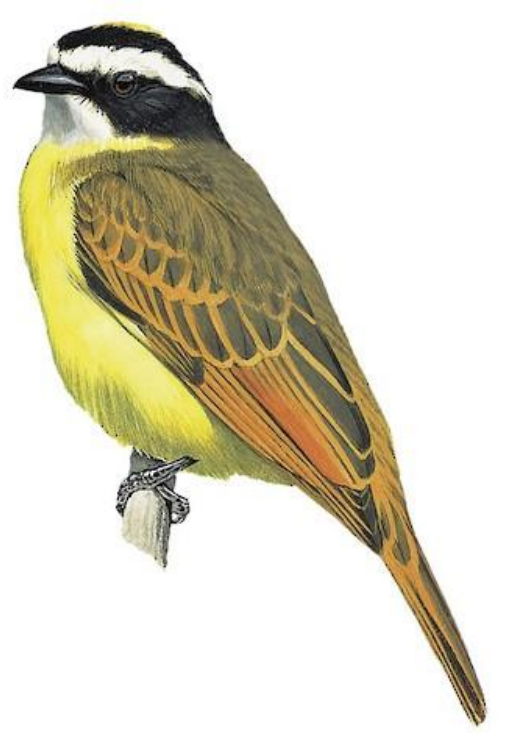

Myiozetetes cayanensis rufipennis

Figura 2. Patrón de plumaje general de $M$. c. cayanensis y $M$. c. rufipennis. Se muestra el patrón de plumaje típico de M. c. cayanensis (izquierda) y M. c. rufipennis (derecha). Ilustraciones de lan Lewington, tomadas de Mobley y Kirwan (2020). 


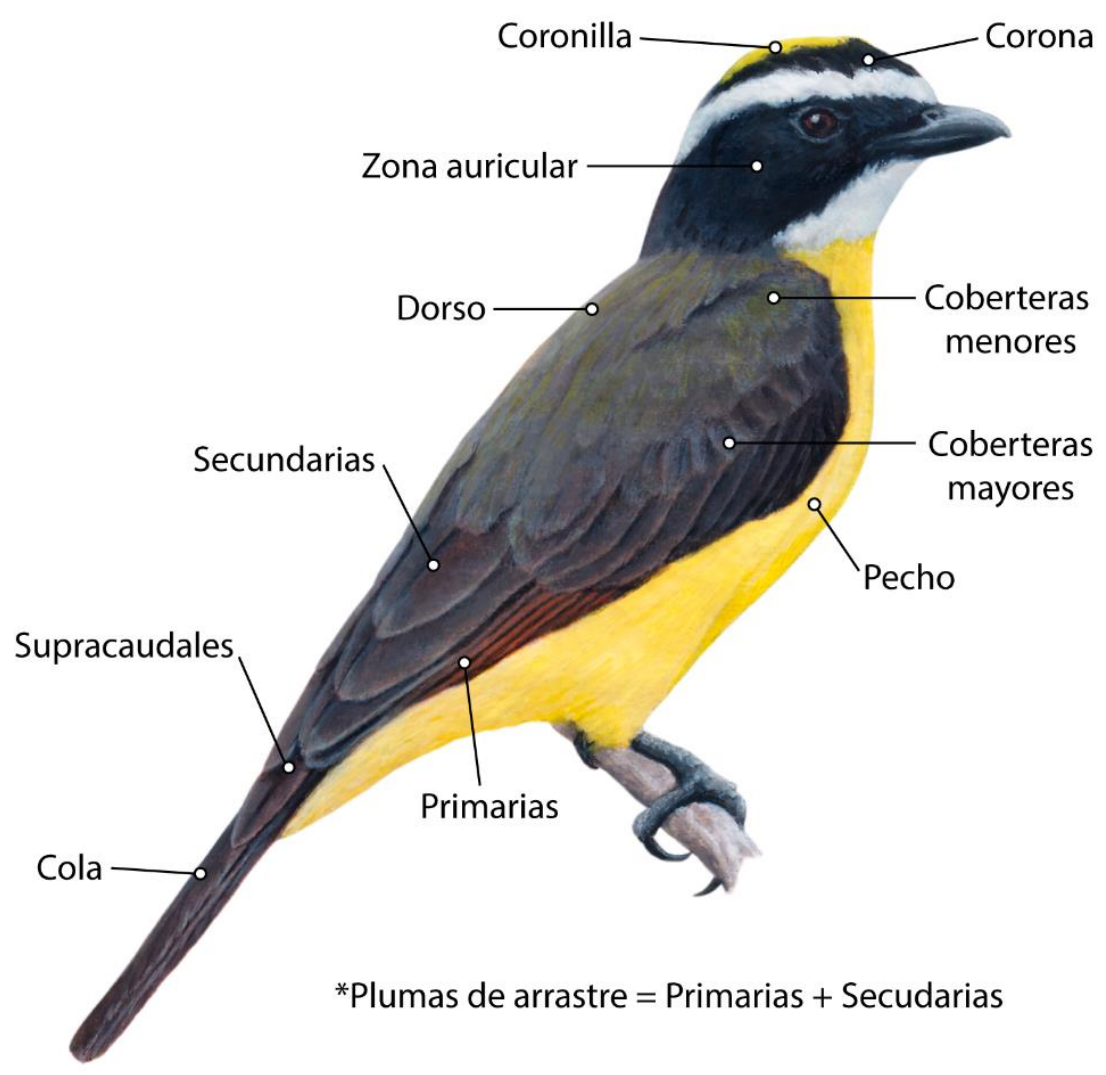

Figura 3. Partes del ave utilizadas para evaluar coloración. Se muestran las partes del plumaje tomadas como referencia para la medición de coloración. Ilustración: Rafael Nascimento. 


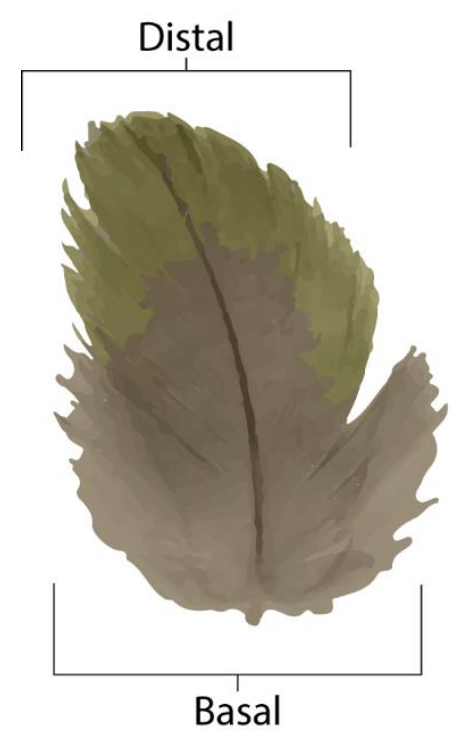

Pluma de contorno

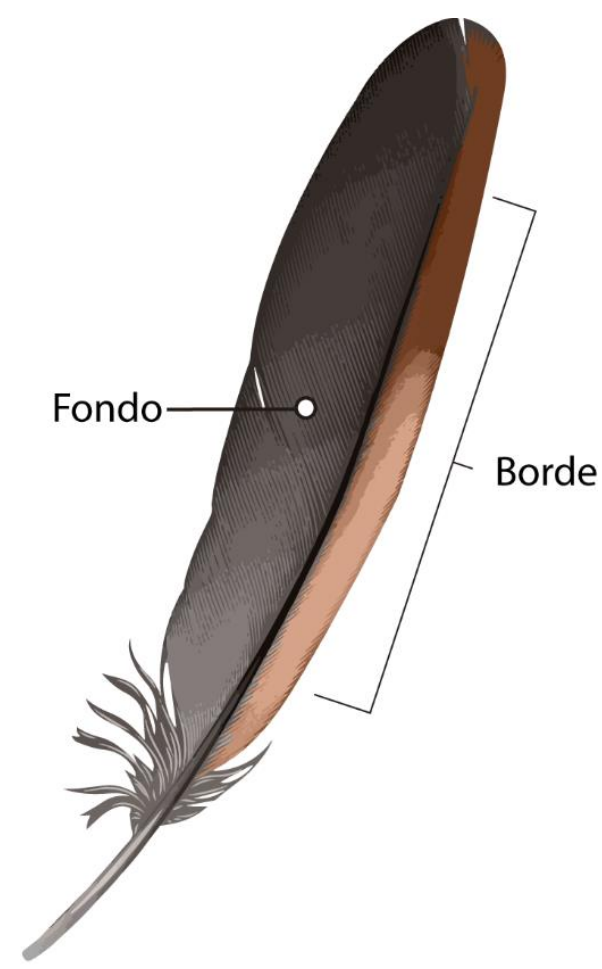

Pluma de vuelo

Figura 4. Áreas de coloración de las plumas de contorno y de vuelo. Se muestra el patrón morfológico general de las plumas de contorno (izquierda) y de vuelo (derecha) con las áreas de coloración evaluadas. Las plumas de vuelo corresponden a la combinación de las plumas primarias, secundarias y rectrices. 


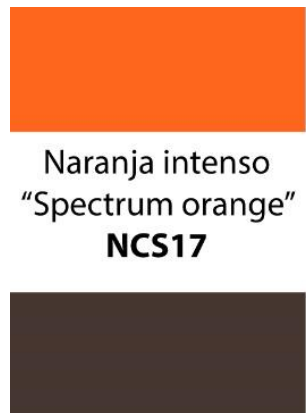

Fusco

"Fuscous"

NCS21

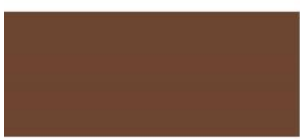

Marrón acanelado

"Cinnamon-Brown"

NCS33

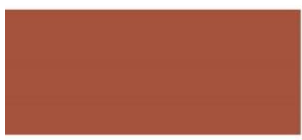

Leonado

"Tawny"

NCS38

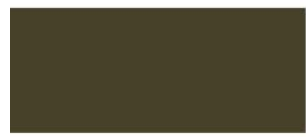

Oliva verdoso

"Greenish Olive"

NCS49

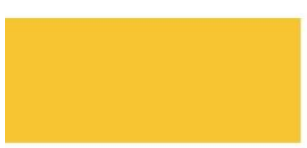

Amarillo intenso "Spectrum Yellow" NCS55

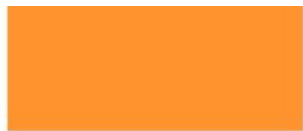

Amarillo naranja

"Orange yellow" NCS18

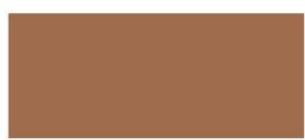

Arcilla

"Clay"

NCS26

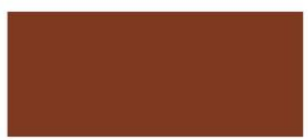

Ámbar

"Amber"

NCS36

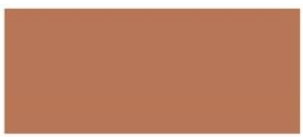

Canela

"Cinnamon"

NCS39

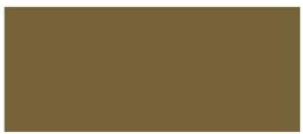

Citrino

"Citrine"

NCS51

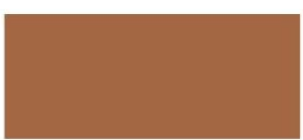

Canela

"Cinnamon"

NCS123A

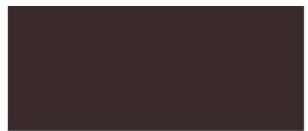

Marrón grisaceo oscuro

"Dark Grayish Brown"

NCS20

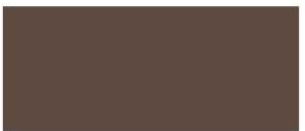

Marrón oliva

"Olive-Brown"

NCS28

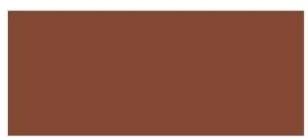

Marrón antiguo

"Antique Brown" NCS37

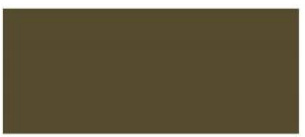

Verde oliva

"Olive-green (Auxiliary)"

NCS48

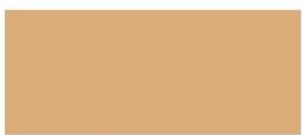

Amarillo-anteado

"Buff-Yellow"

NCS53

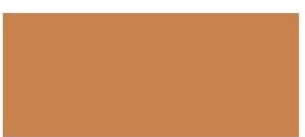

Color arcilla

"Clay color"

NCS123B

Figura 5. Muestras de color de Myiozetetes cayanensis siguiendo la guía de Smithe (1975).

Se muestra la paleta de 18 colores identificados en el plumaje de los individuos de $M$. cayanensis examinados, así como sus códigos de identificación. Los colores siguen la Guía de Colores del Naturalista de Smithe (1975). 


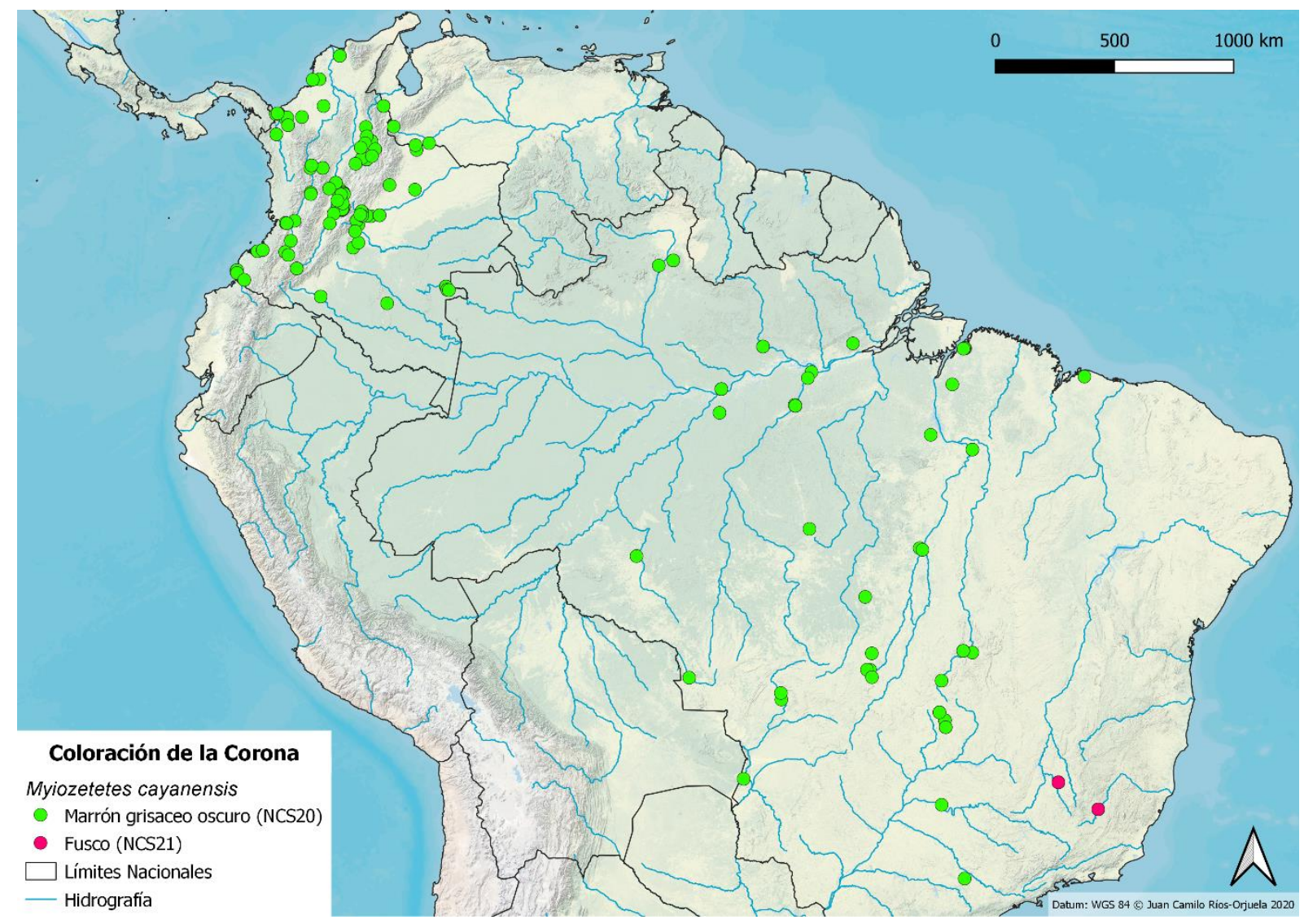

Figura 6. Mapa de distribución de coloración de la corona de Myiozetetes cayanensis. Se muestra la distribución de ocurrencia de estados del carácter en los individuos examinados para la descripción de coloración. 


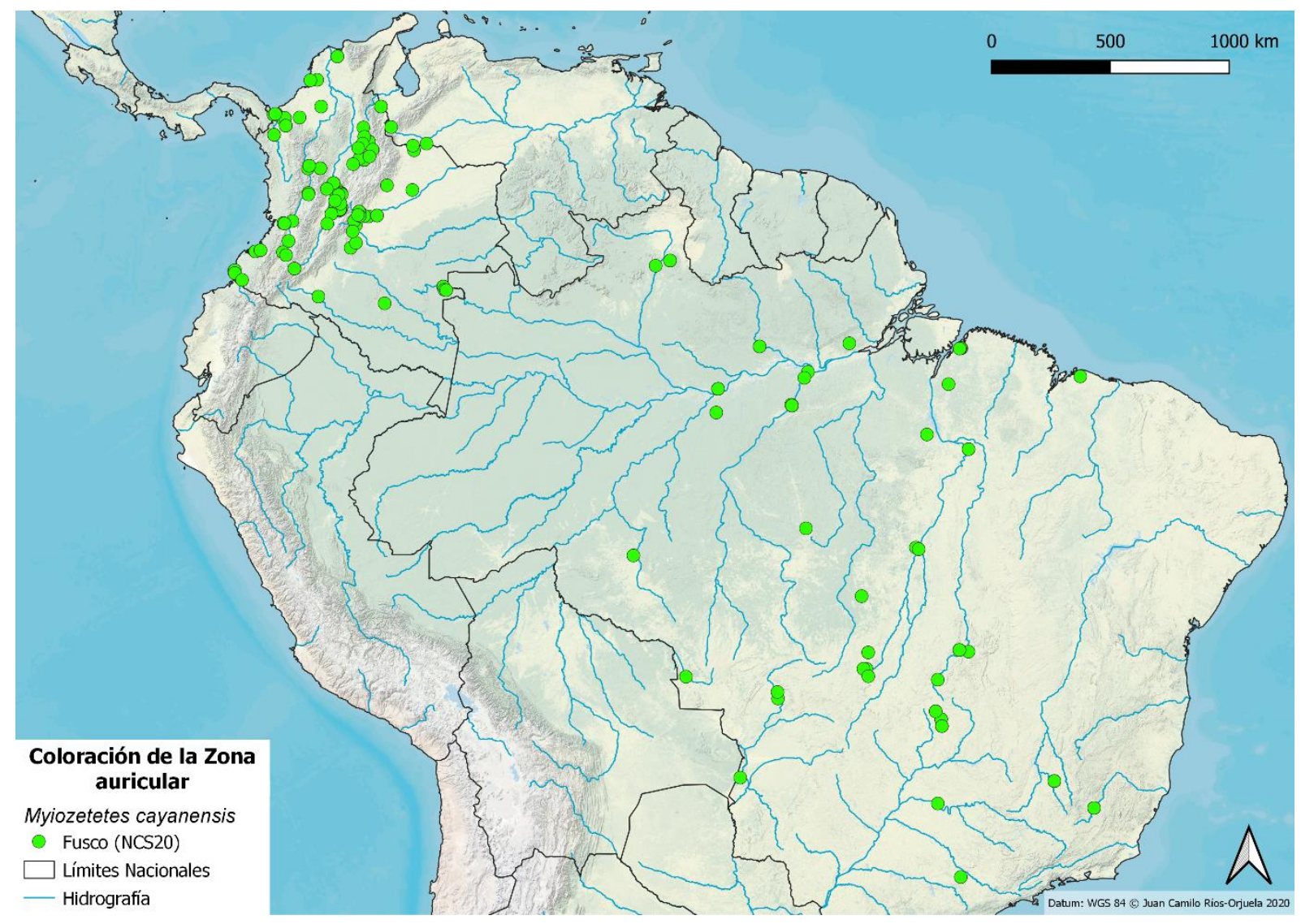

Figura 7. Mapa de distribución de coloración de la zona auricular de Myiozetetes cayanensis.

Se muestra la distribución de ocurrencia de estados del carácter en los individuos examinados para la descripción de coloración. 


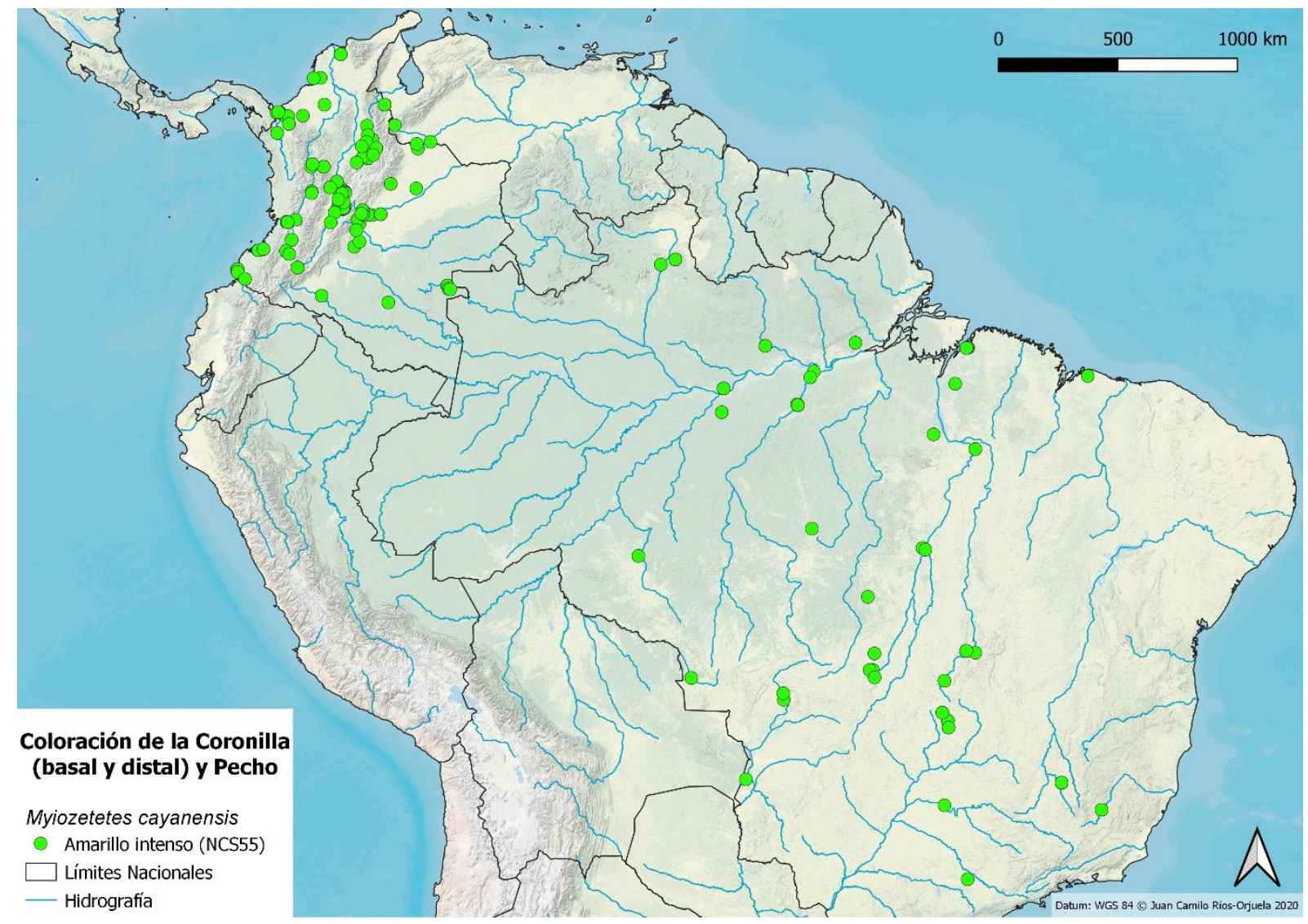

Figura 8. Mapa de distribución de coloración de la coronilla (basal y distal) y el pecho de Myiozetetes cayanensis. Se muestra la distribución de ocurrencia de estados del carácter en los individuos examinados para la descripción de coloración. 


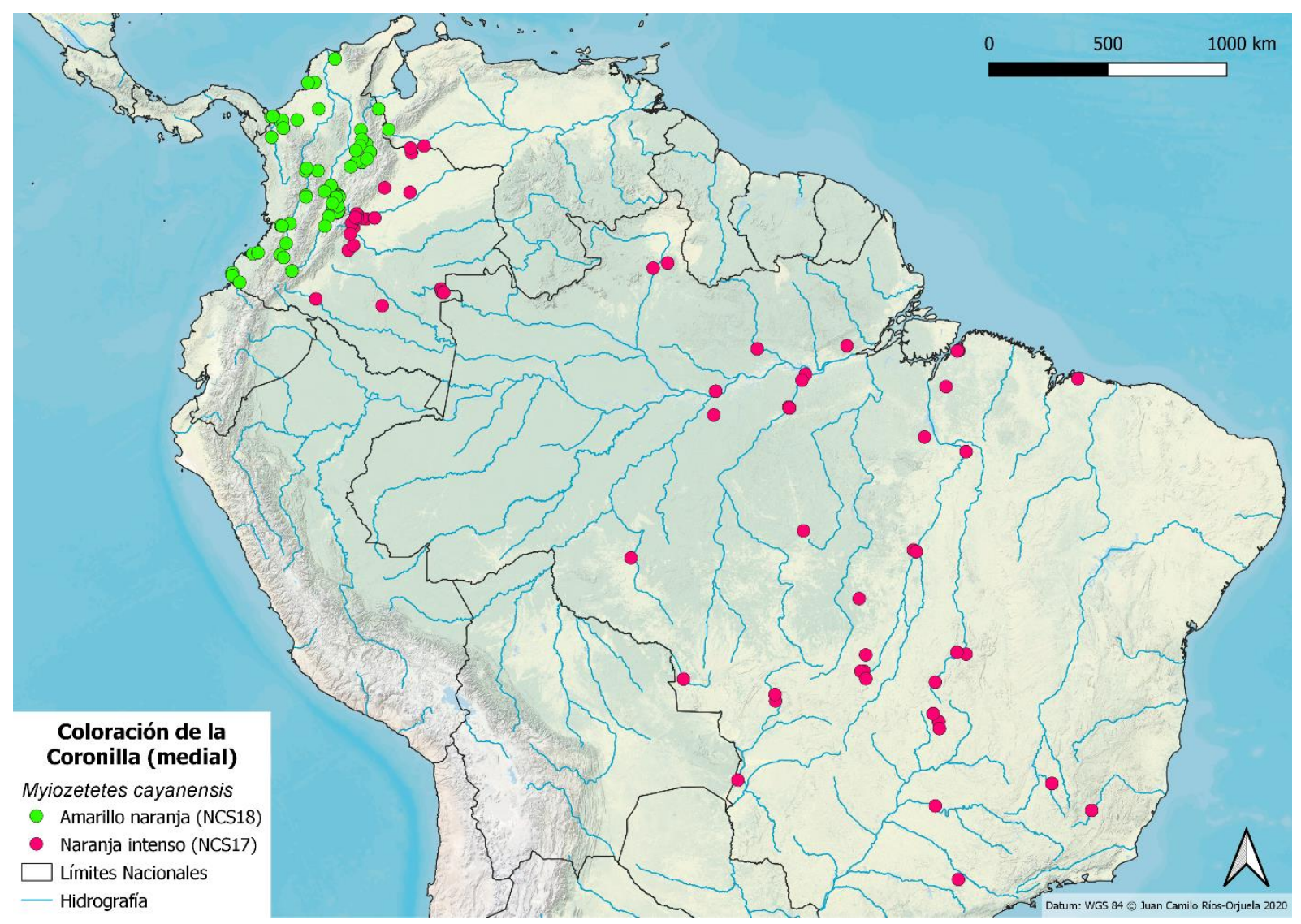

Figura 9. Mapa de distribución de coloración de la coronilla (medial) de Myiozetetes cayanensis. Se muestra la distribución de ocurrencia de estados del carácter en los individuos examinados para la descripción de coloración. 


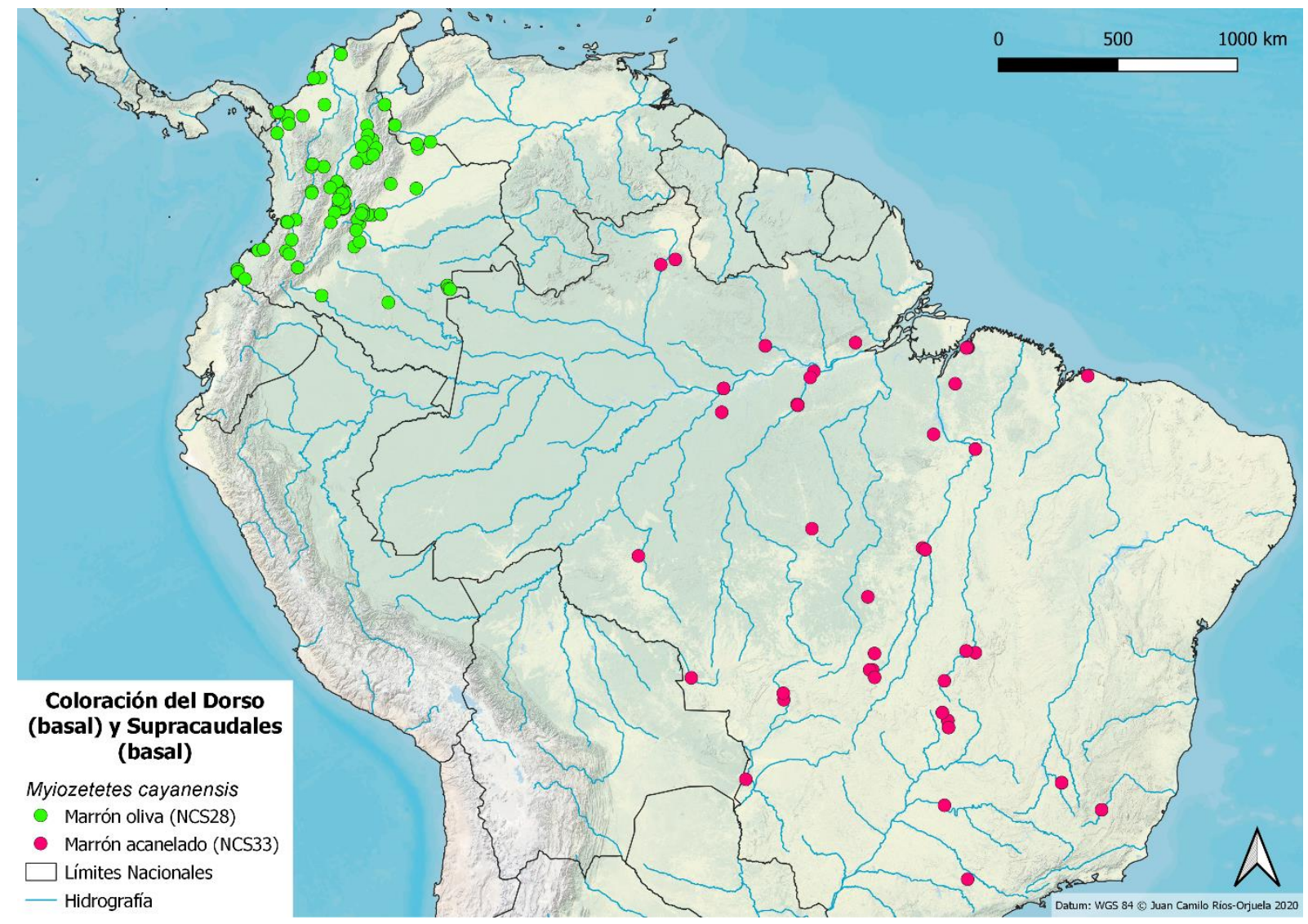

Figura 10. Mapa de distribución de coloración del dorso (basal) y las supracaudales (basal) de Myiozetetes cayanensis. Se muestra la distribución de ocurrencia de estados del carácter en los individuos examinados para la descripción de coloración. 


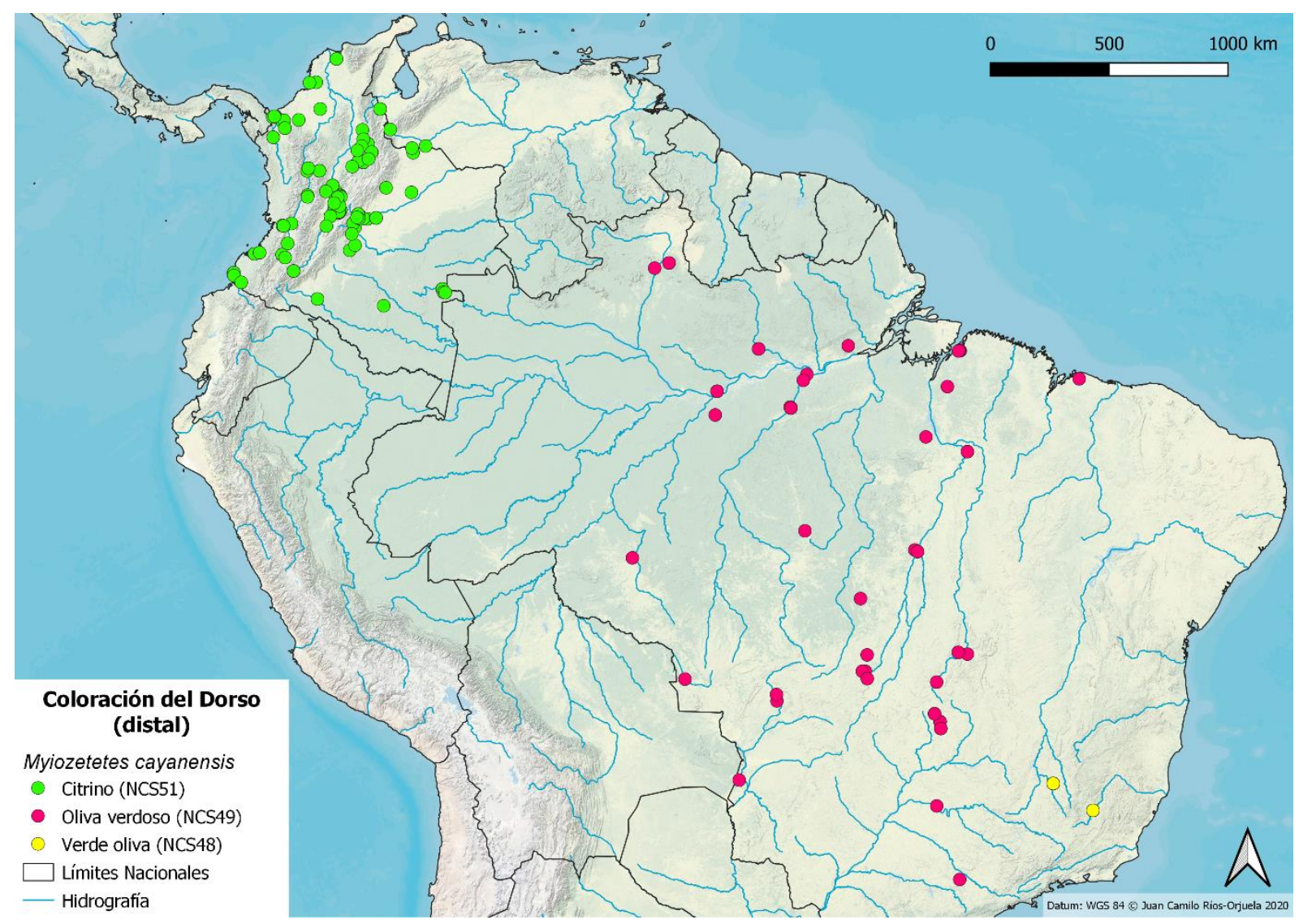

Figura 11. Mapa de distribución de coloración del dorso (distal) de Myiozetetes cayanensis.

Se muestra la distribución de ocurrencia de estados del carácter en los individuos examinados para la descripción de coloración. 


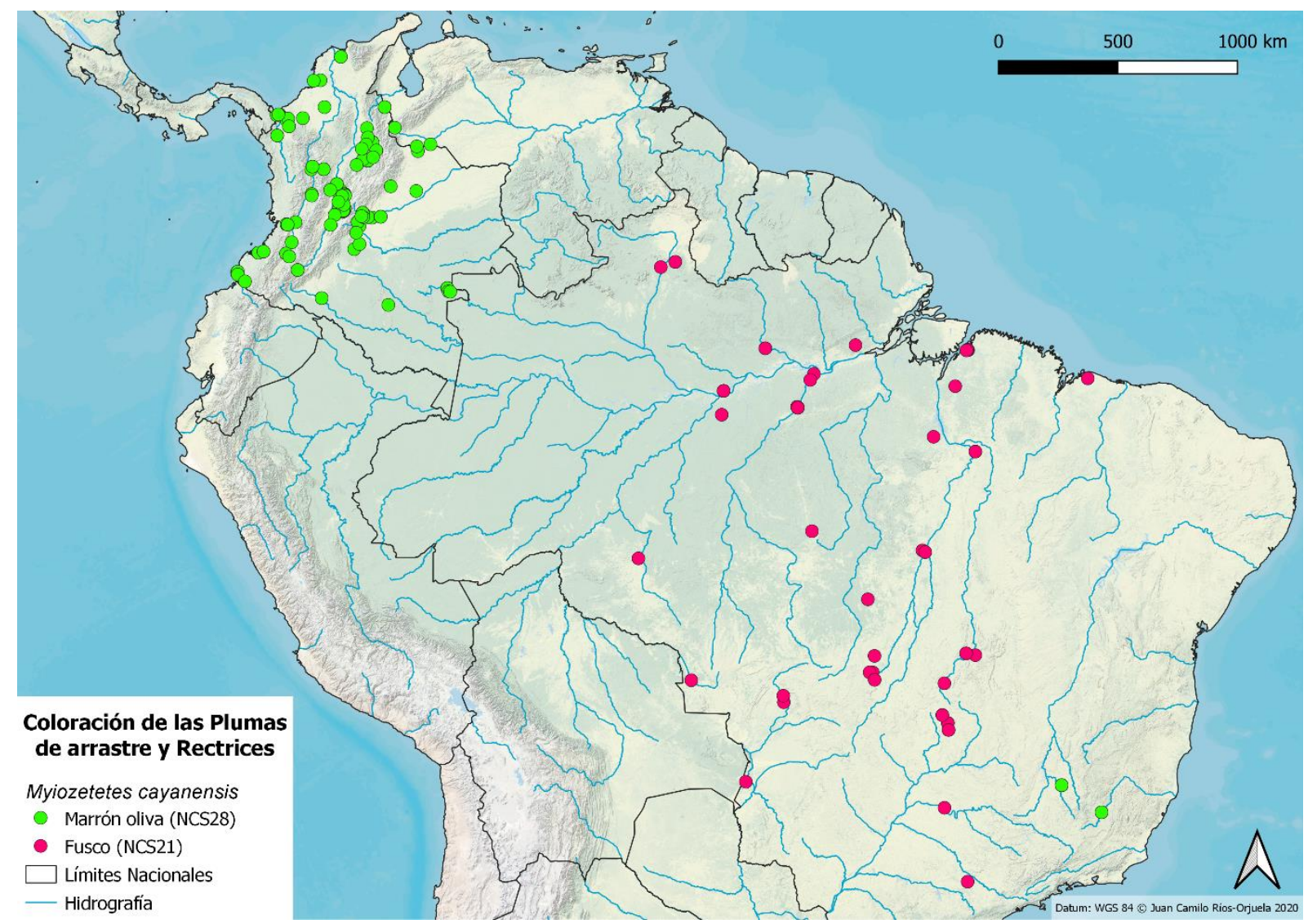

Figura 12. Mapa de distribución de coloración de las plumas de arrastre y rectrices de Myiozetetes cayanensis. Se muestra la distribución de ocurrencia de estados del carácter en los individuos examinados para la descripción de coloración. 


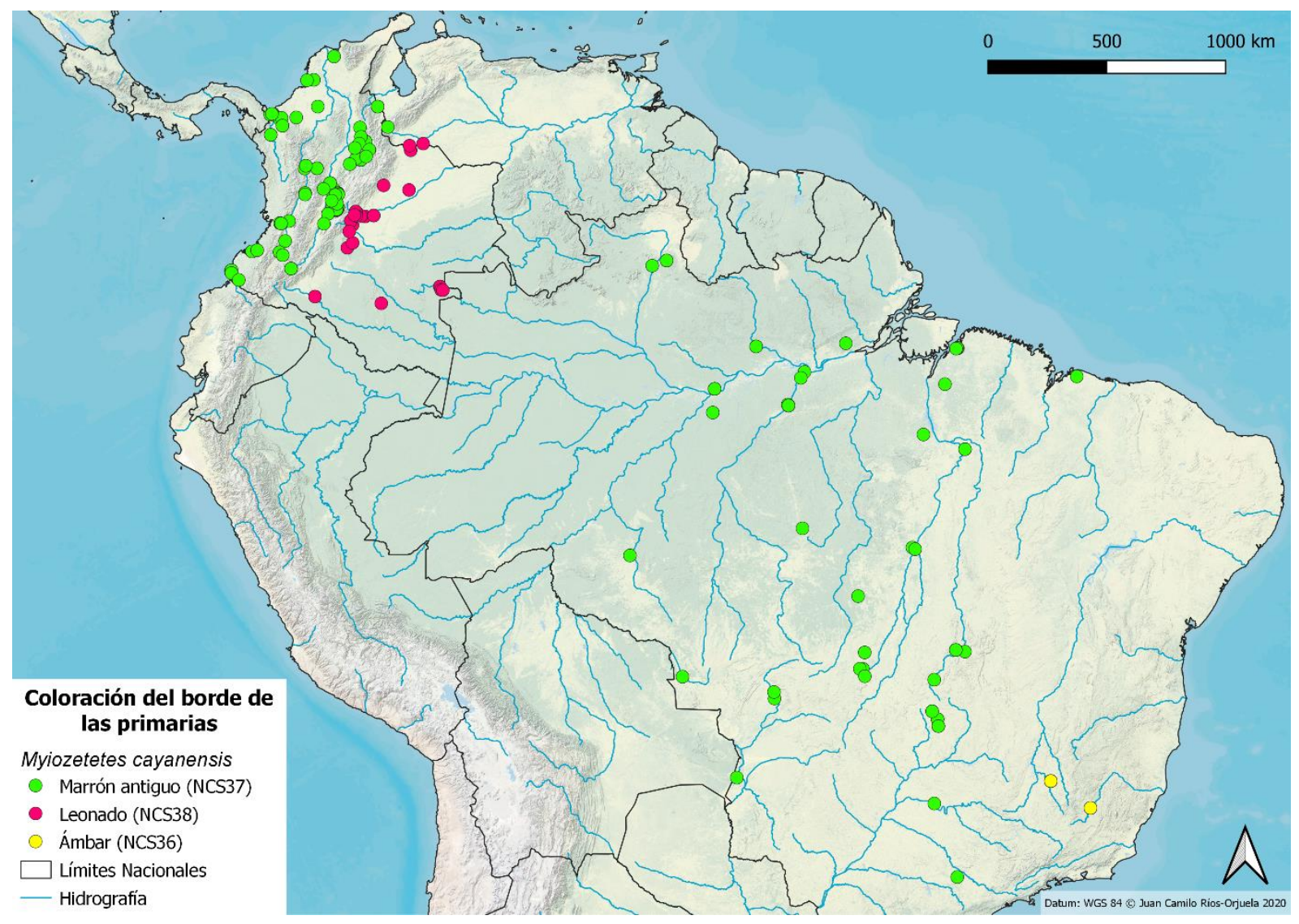

Figura 13. Mapa de distribución de coloración del borde de las plumas primarias de

Myiozetetes cayanensis. Se muestra la distribución de ocurrencia de estados del carácter en los individuos examinados para la descripción de coloración. 


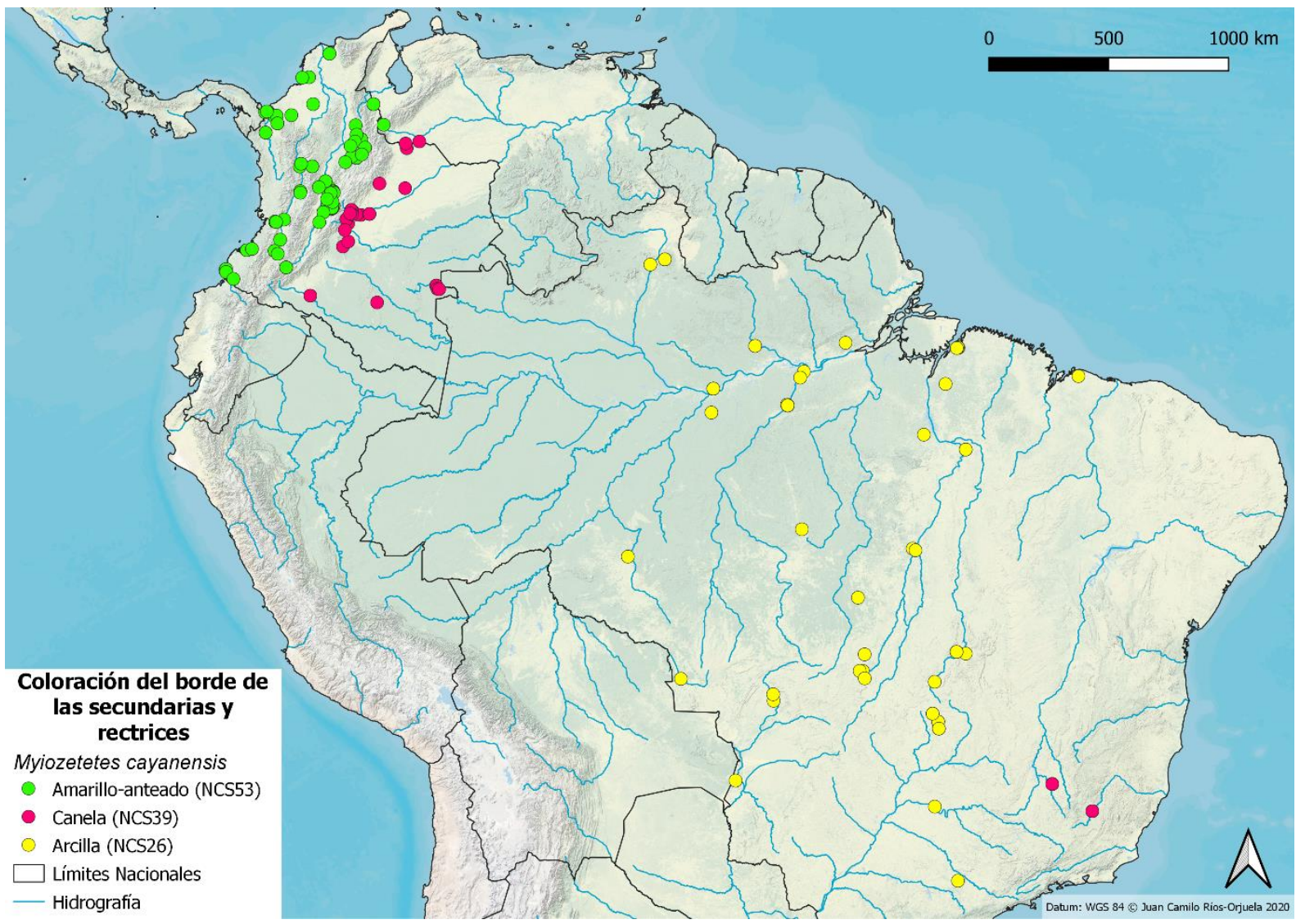

Figura 14. Mapa de distribución de coloración del borde de las plumas secundarias y rectrices de Myiozetetes cayanensis. Se muestra la distribución de ocurrencia de estados del carácter en los individuos examinados para la descripción de coloración. 


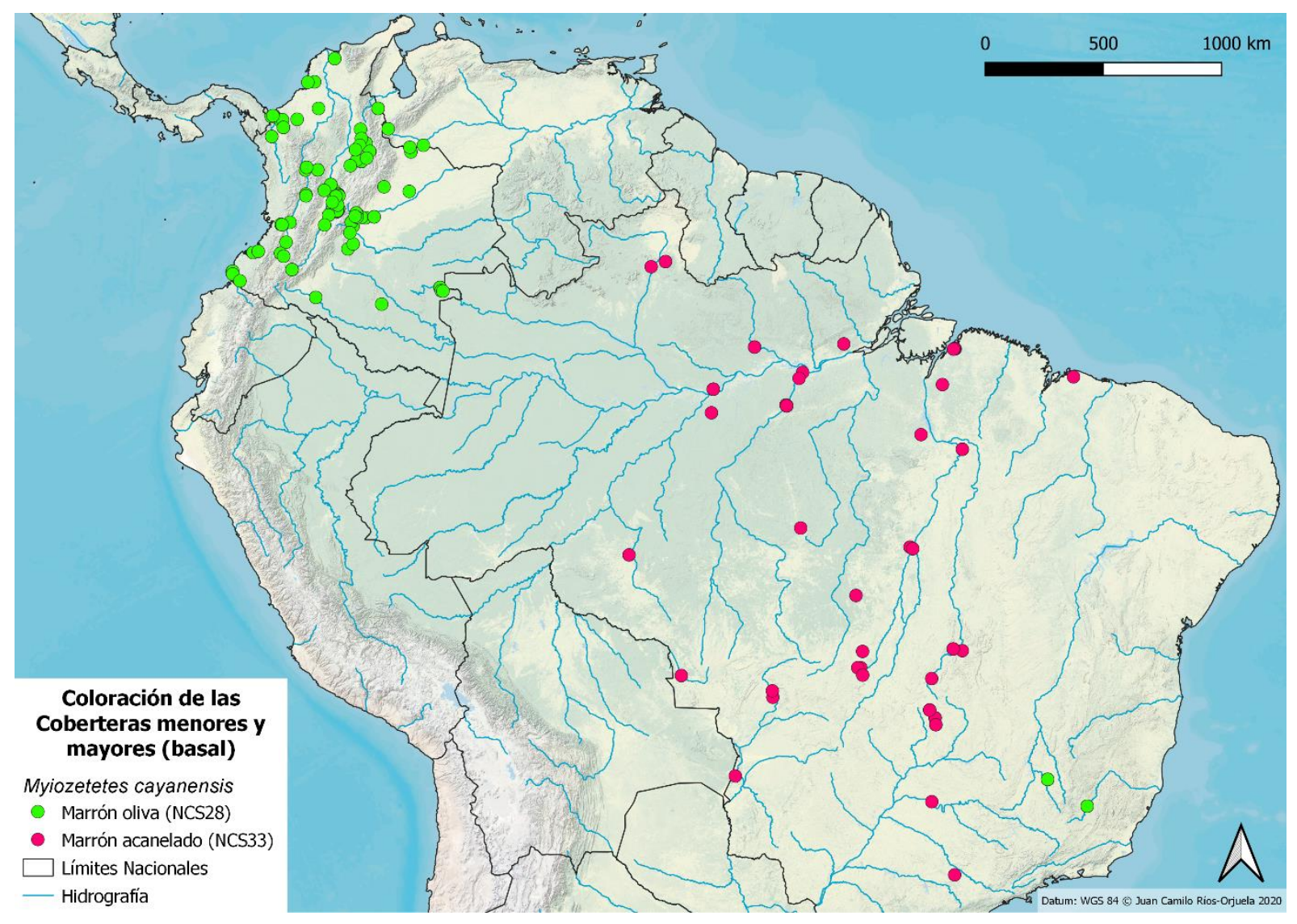

Figura 15. Mapa de distribución de coloración de las coberteras menores y mayores (basal) de Myiozetetes cayanensis. Se muestra la distribución de ocurrencia de estados del carácter en los individuos examinados para la descripción de coloración. 


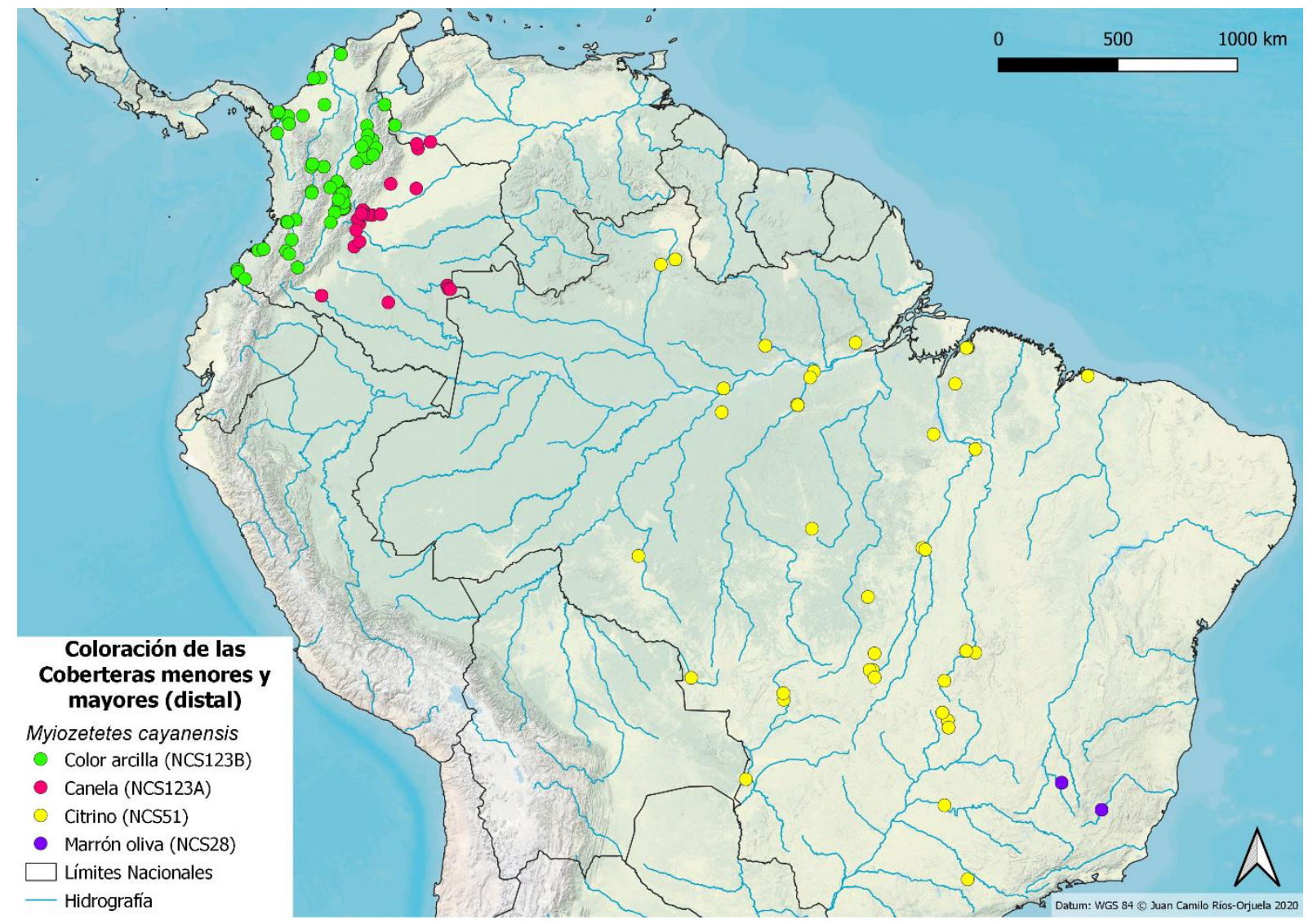

Figura 16. Mapa de distribución de coloración de las coberteras menores y mayores (distal)

de Myiozetetes cayanensis. Se muestra la distribución de ocurrencia de estados del carácter en los individuos examinados para la descripción de coloración. 


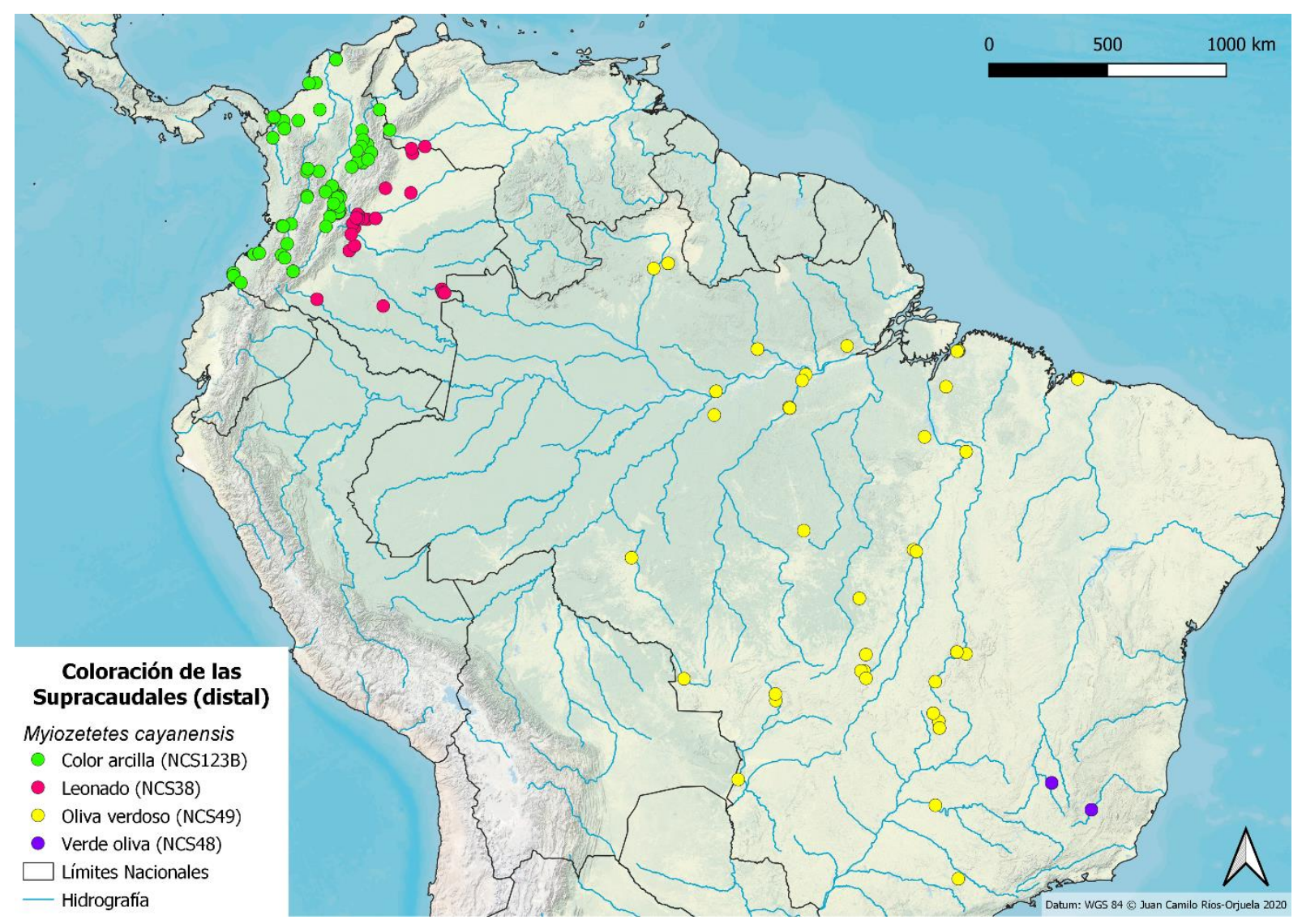

Figura 17. Mapa de distribución de coloración de las supracaudales (distal) de Myiozetetes cayanensis. Se muestra la distribución de ocurrencia de estados del carácter en los individuos examinados para la descripción de coloración. 


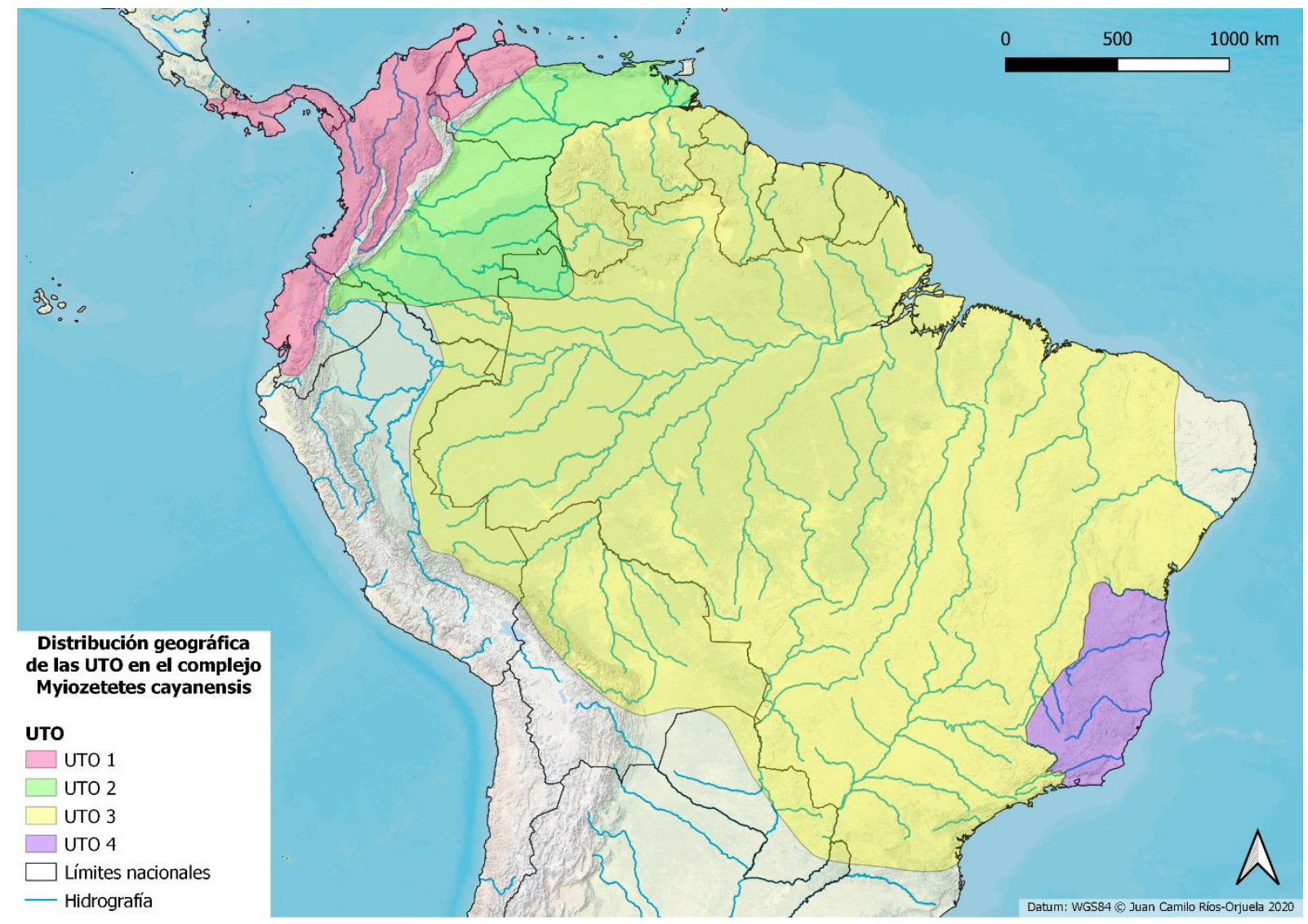

Figura 18. Distribución geográfica de las UTO en el complejo Myiozetetes cayanensis. Se muestra el rango de ocurrencia geográfica de las Unidades Taxonómicas operativas (UTO) identificadas en el complejo M. cayanensis. 

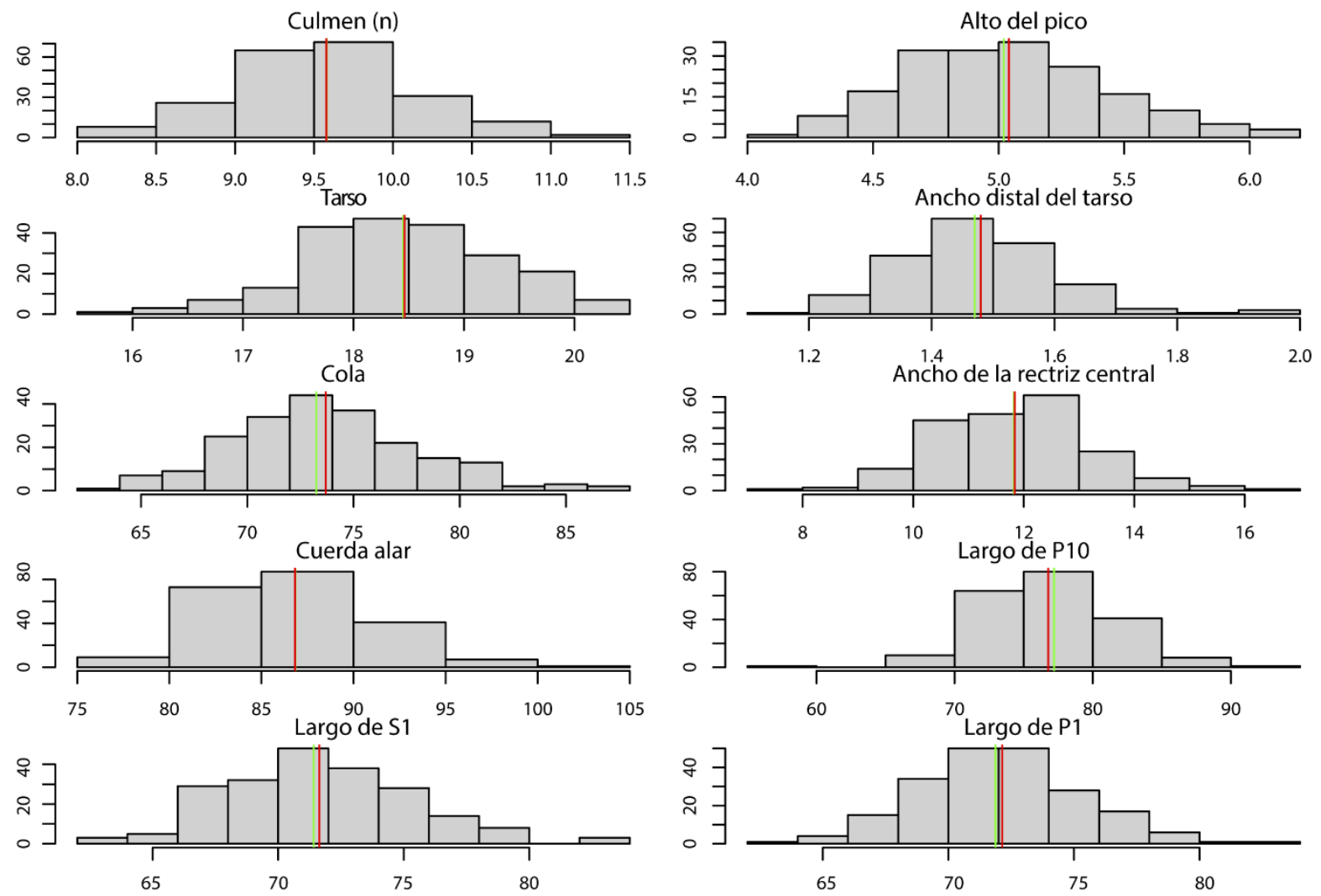

Figura 19. Medidas de tendencia central de las variables morfométricas analizadas. Se muestra la distribución de los datos, la media (línea roja) y la mediana (línea verde) de las variables morfométricas propuestas para análisis. 

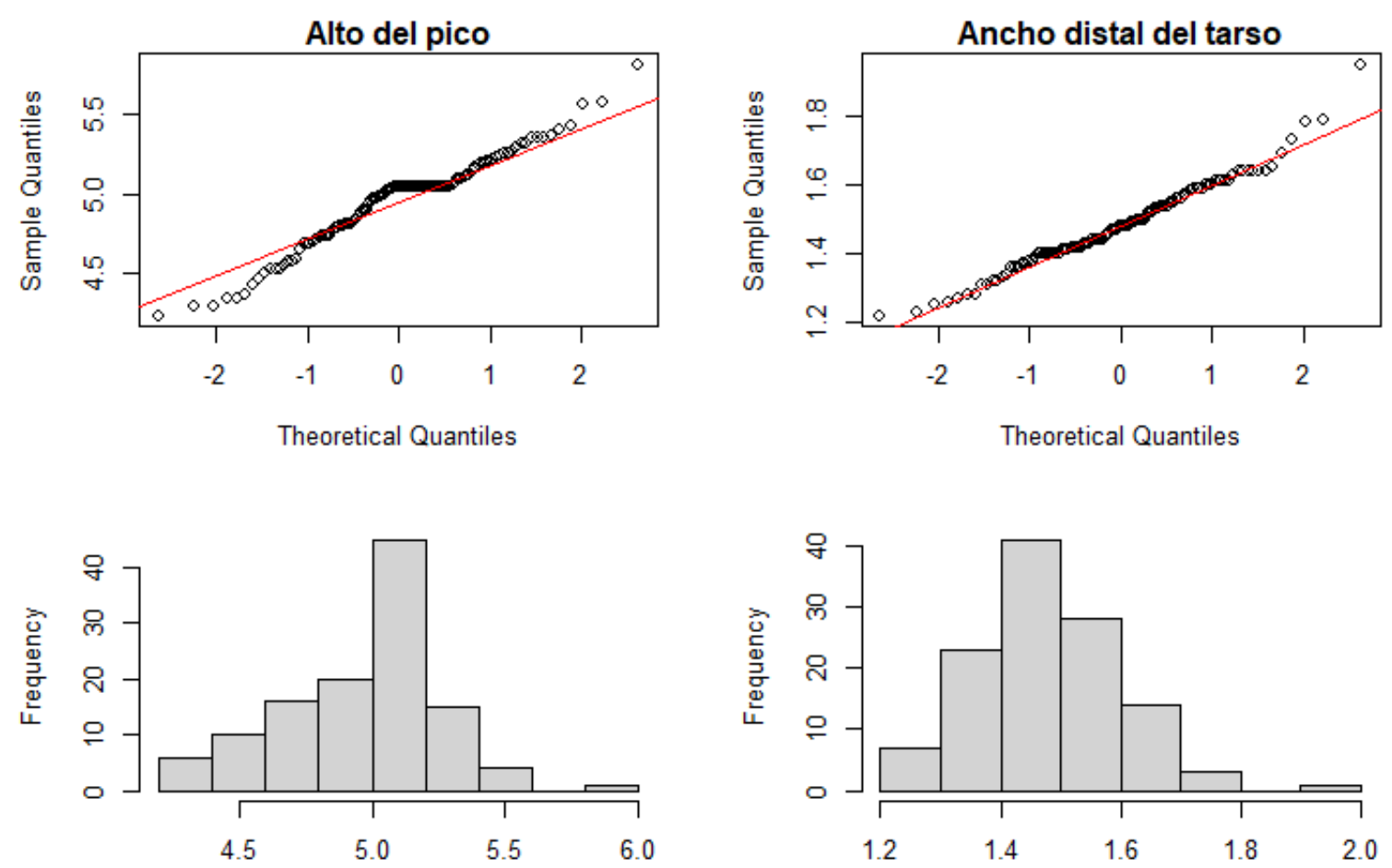

Figura 20. Linealidad de las variables anormales identificadas con la prueba de normalidad de Shapiro-Wilks. 


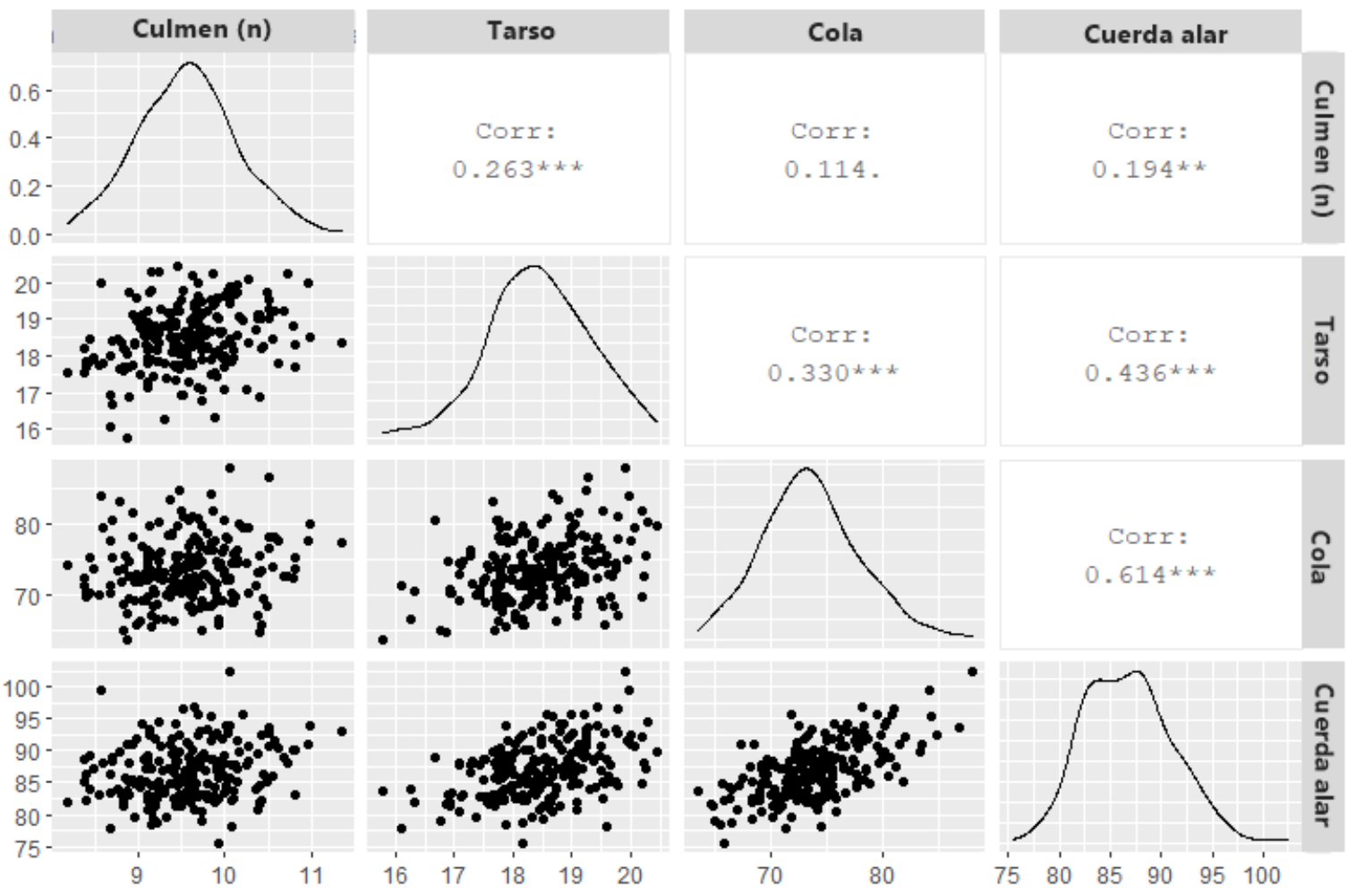

Figura 21. Matriz de correlación, linealidad y colinealidad de las variables morfométricas en el modelo 3. Se muestra el índice de correlación (porción superior), la linealidad (transversal) y la dispersión de colinealidad (porción inferior) de las variables morfométricas en el modelo 3. 


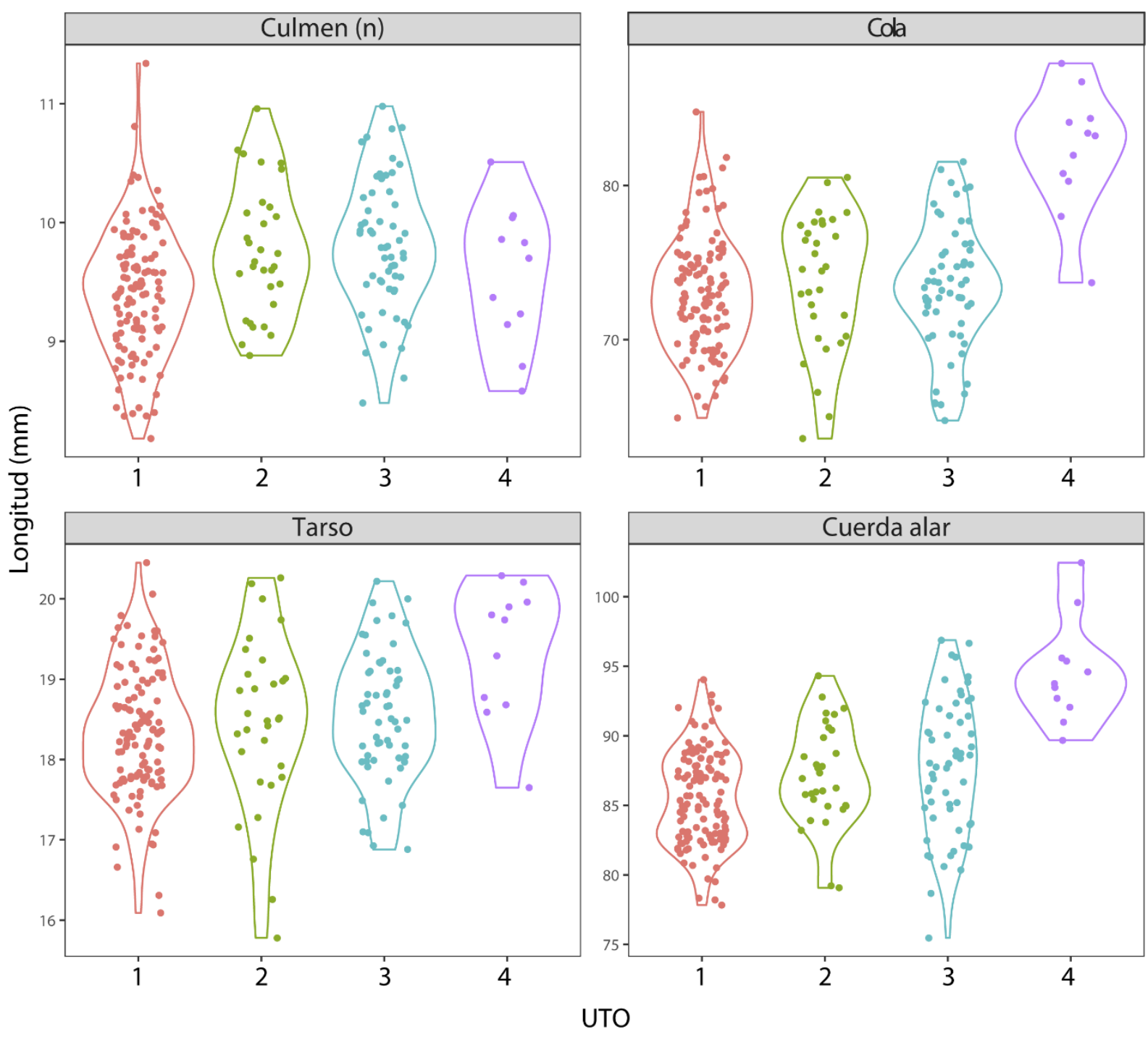

Figura 22. Distribución de los datos morfométricos del modelo 3 explicados por UTO. Se muestra la distribución de los datos morfométricos de culmen (n), cola, tarso y cuerda alar por UTO. 


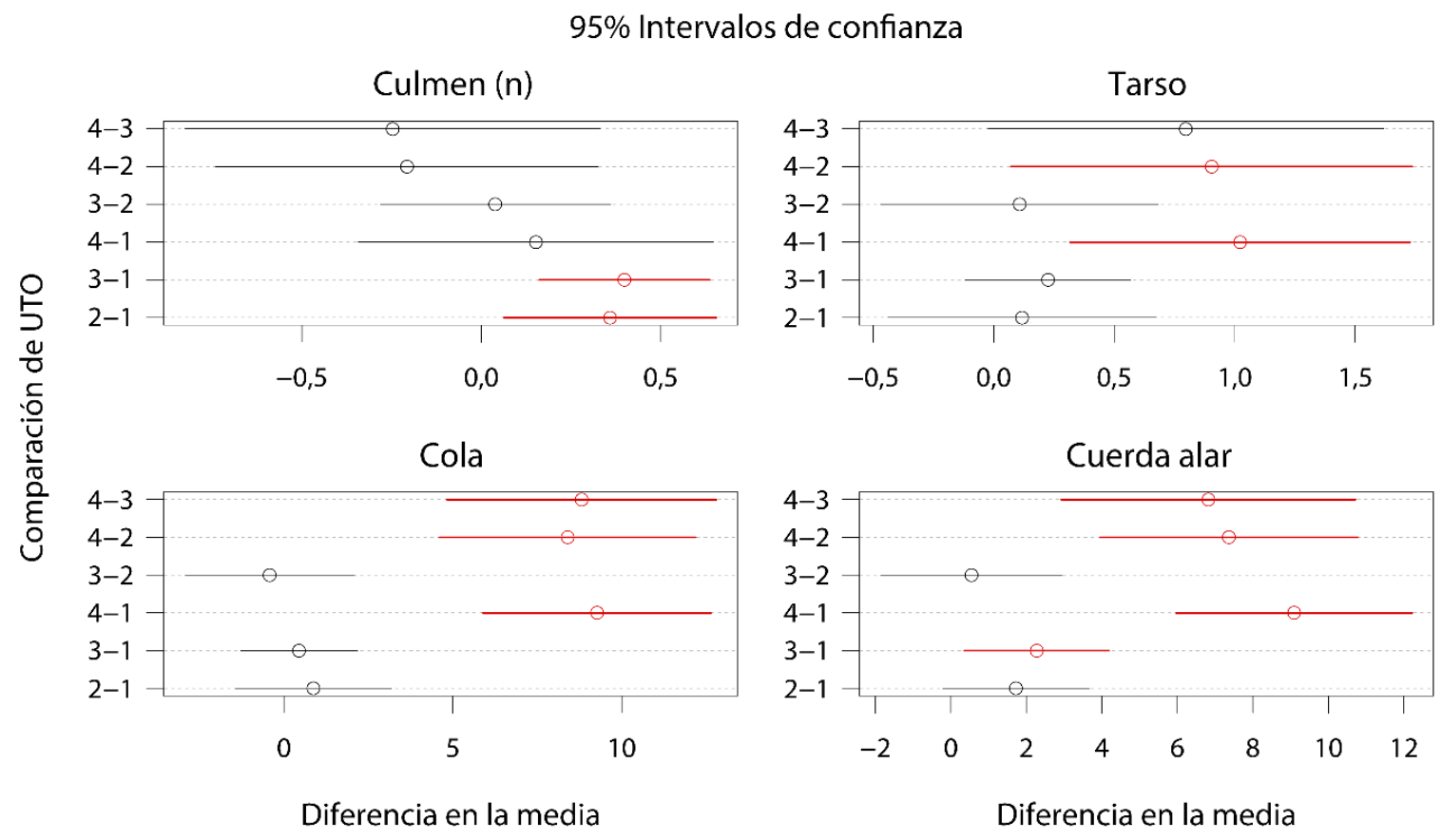

Figura 23. Diferencias de media en la comparación de UTO según los intervalos de confianza de Dunnet. Se muestran los intervalos de confianza en la diferenciación de las UTO 1 a 4, obtenidos en la prueba de comparación por pares de Dunnet. Los intervalos rojos representan diferencias significativas (intervalo $\neq 0$ ). 


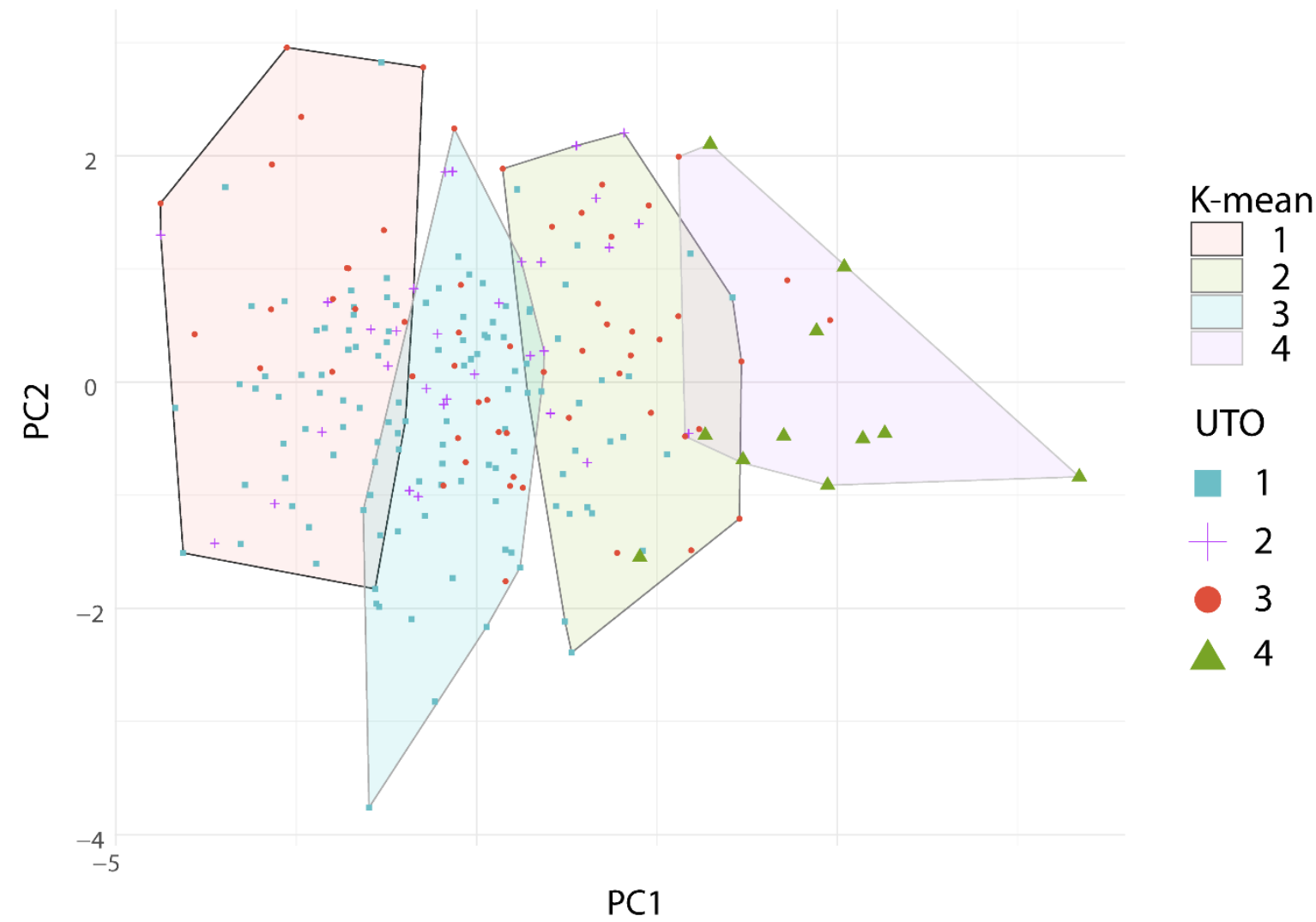

Figura 24. Agrupación supervisada de $\boldsymbol{K}$-means para las variables morfométricas de las UTO. Se muestra la distribución de los elementos de las UTO dentro de las agrupaciones supervisadas de $K$-means para las variables morfométricas. 


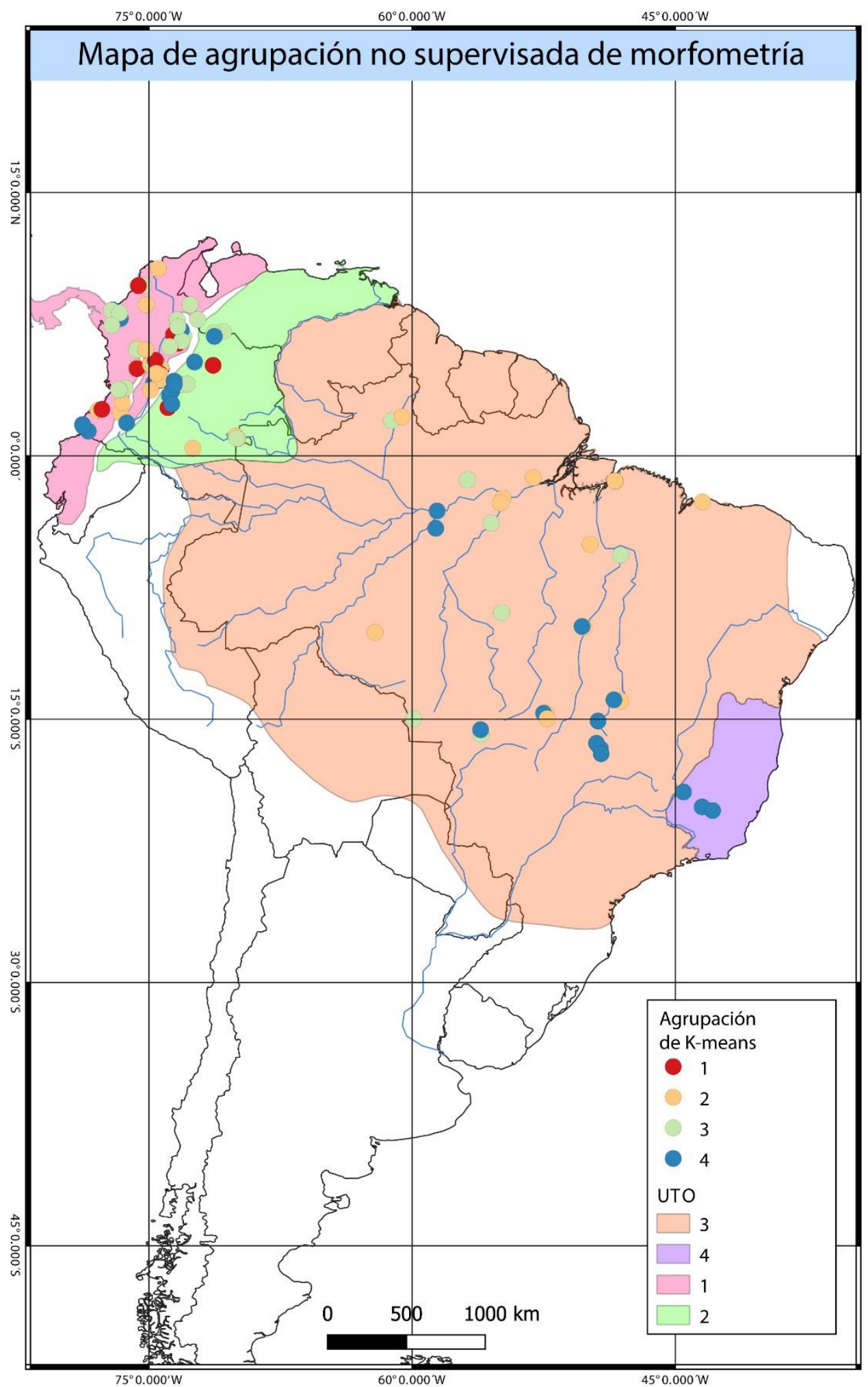

Figura 25. Mapa de agrupación no supervisada por pares de $K$-means para las variables morfométricas de las UTO. Se muestran las agrupaciones de $K$-means para las variables morfométricas y la distribución de las Unidades Taxonómicas Operativas (UTO). 


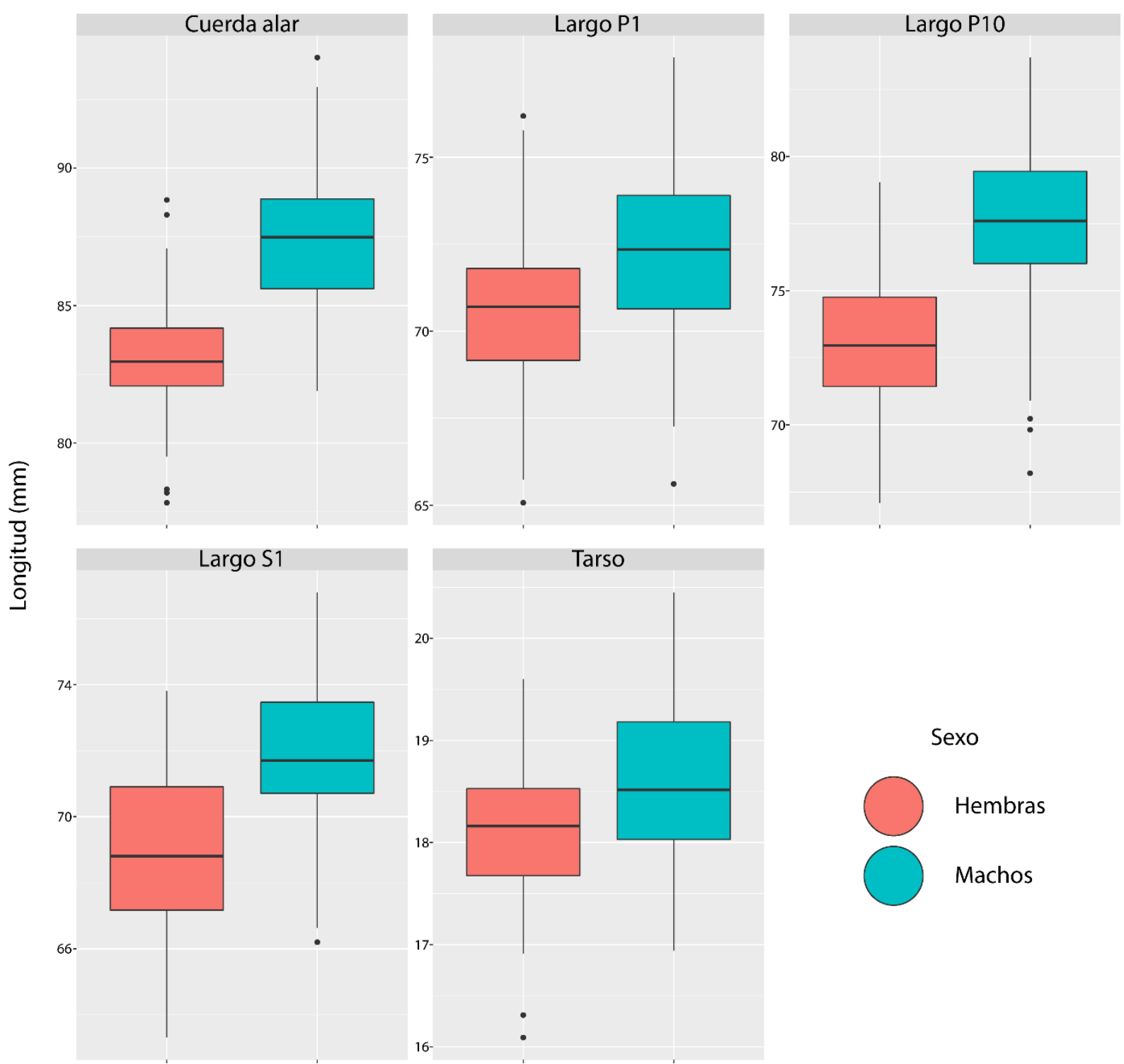

Figura 26. Distribución de las variables con dimorfismo sexual en la UTO 1. Se muestra la distribución de las variables que presentan dimorfismo sexual en la UTO 1 para hembras (rosado) y machos (azul). 


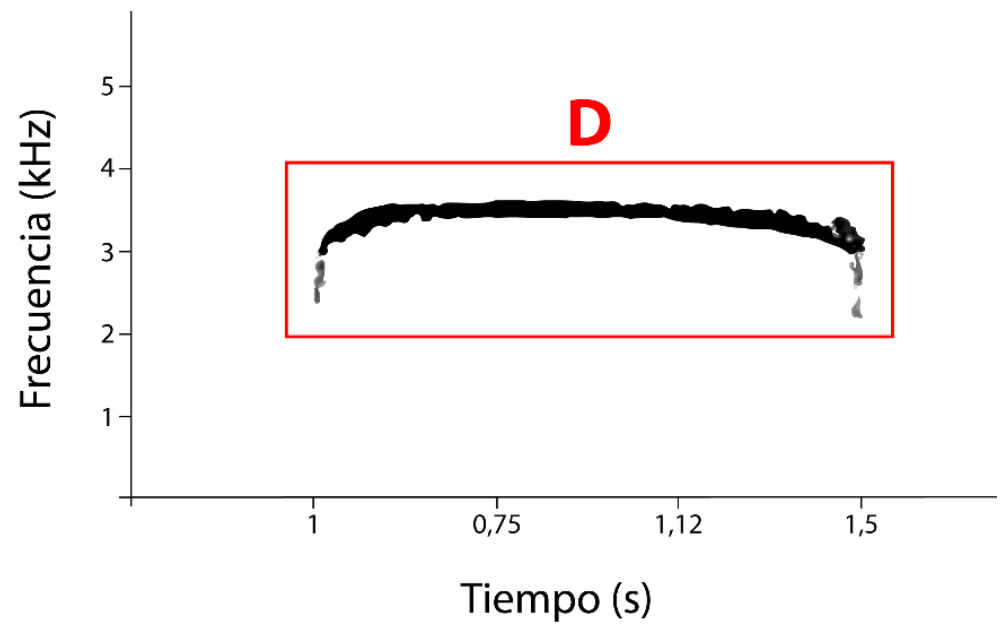

Figura 27. Elemento único (D) que compone el llamado reproductivo de Myiozetetes cayanensis. Se muestra el espectrograma del elemento D del llamado reproductivo de $M$. cayanensis. 


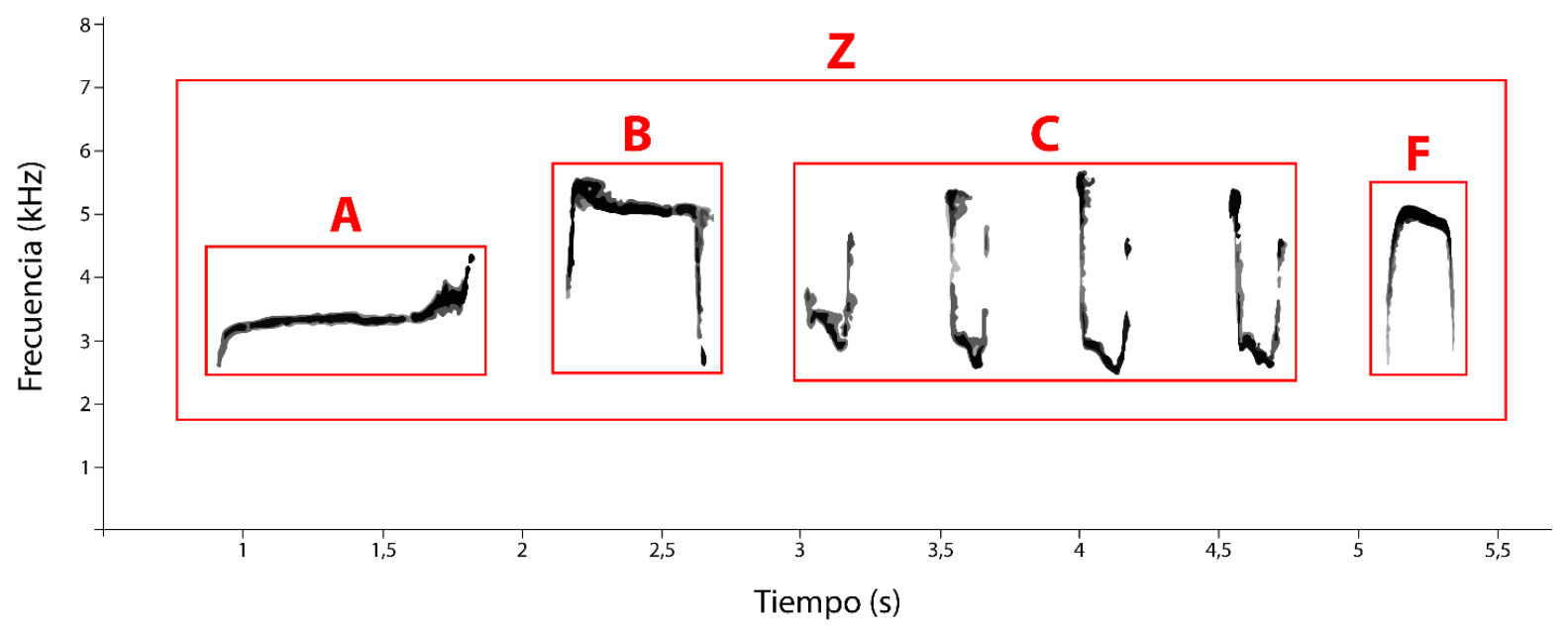

Figura 28. Elementos del canto de comunicación de Myiozetetes cayanensis. Se muestra el espectrograma de los elementos sonoros (A, B, C, F y Z) que componen el canto de comunicación de M. cayanensis. 


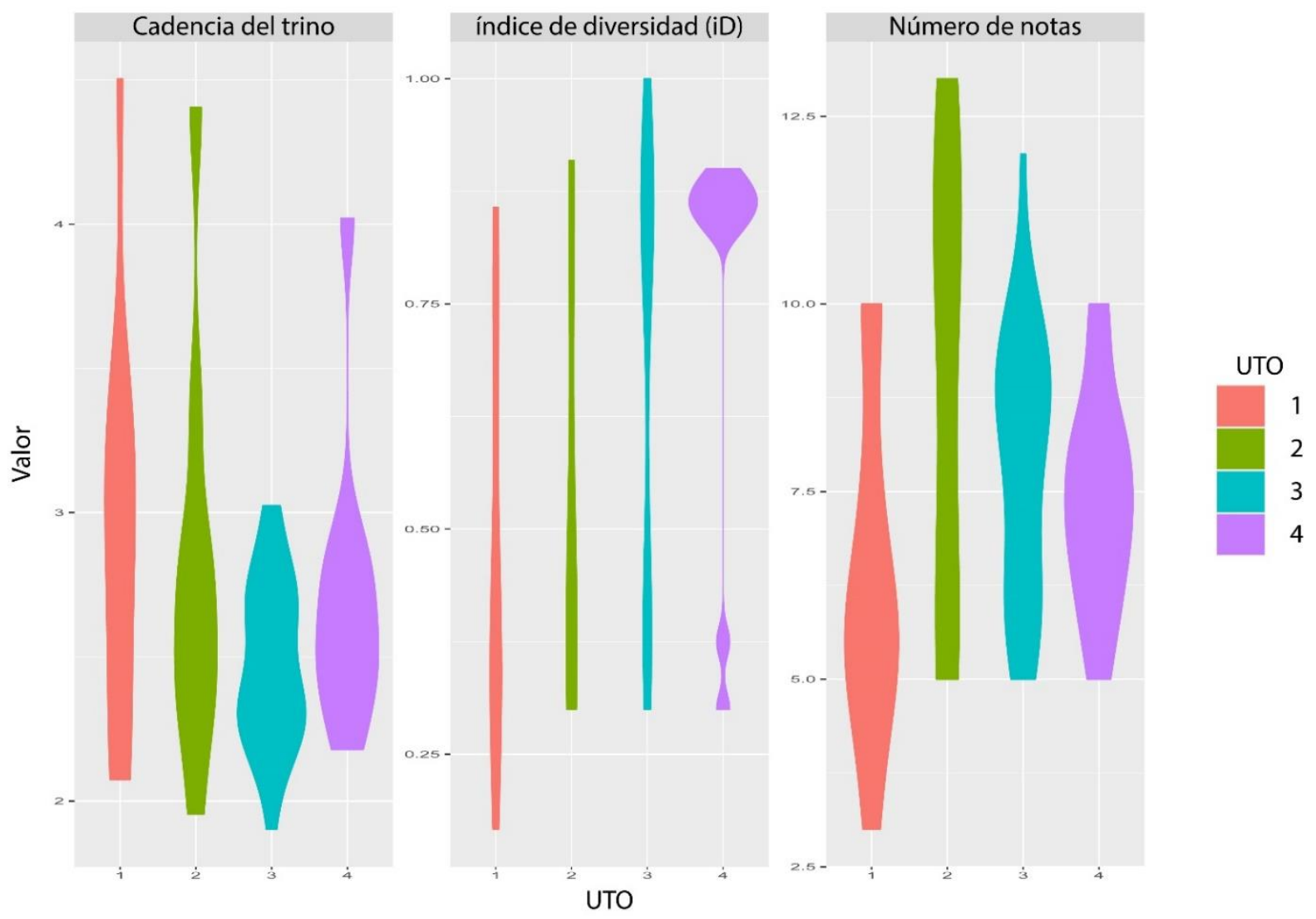

Figura 29. Distribución de los datos de cadencia de trino, índice de diversidad y número de notas de las UTO. 


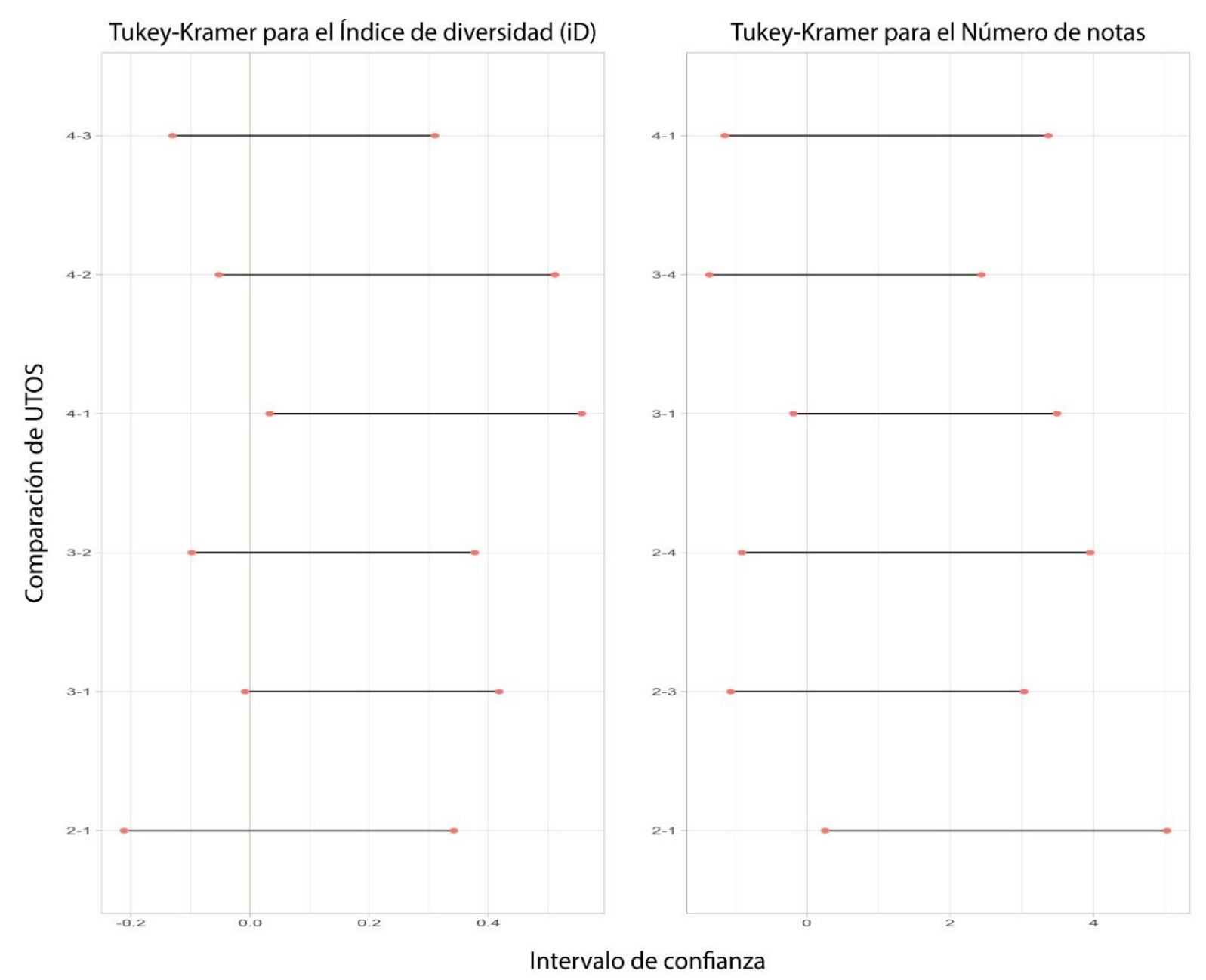

Figura 30. Intervalos de confianza de Tukey-Kramer para los atributos del canto de comunicación de las UTO. Se muestran los intervalos de confianza en la diferenciación de las UTO 1 a 4, obtenidos en la prueba de comparación por pares de Tukey-Kramer. La línea roja vertical representa el cero en el intervalo. 

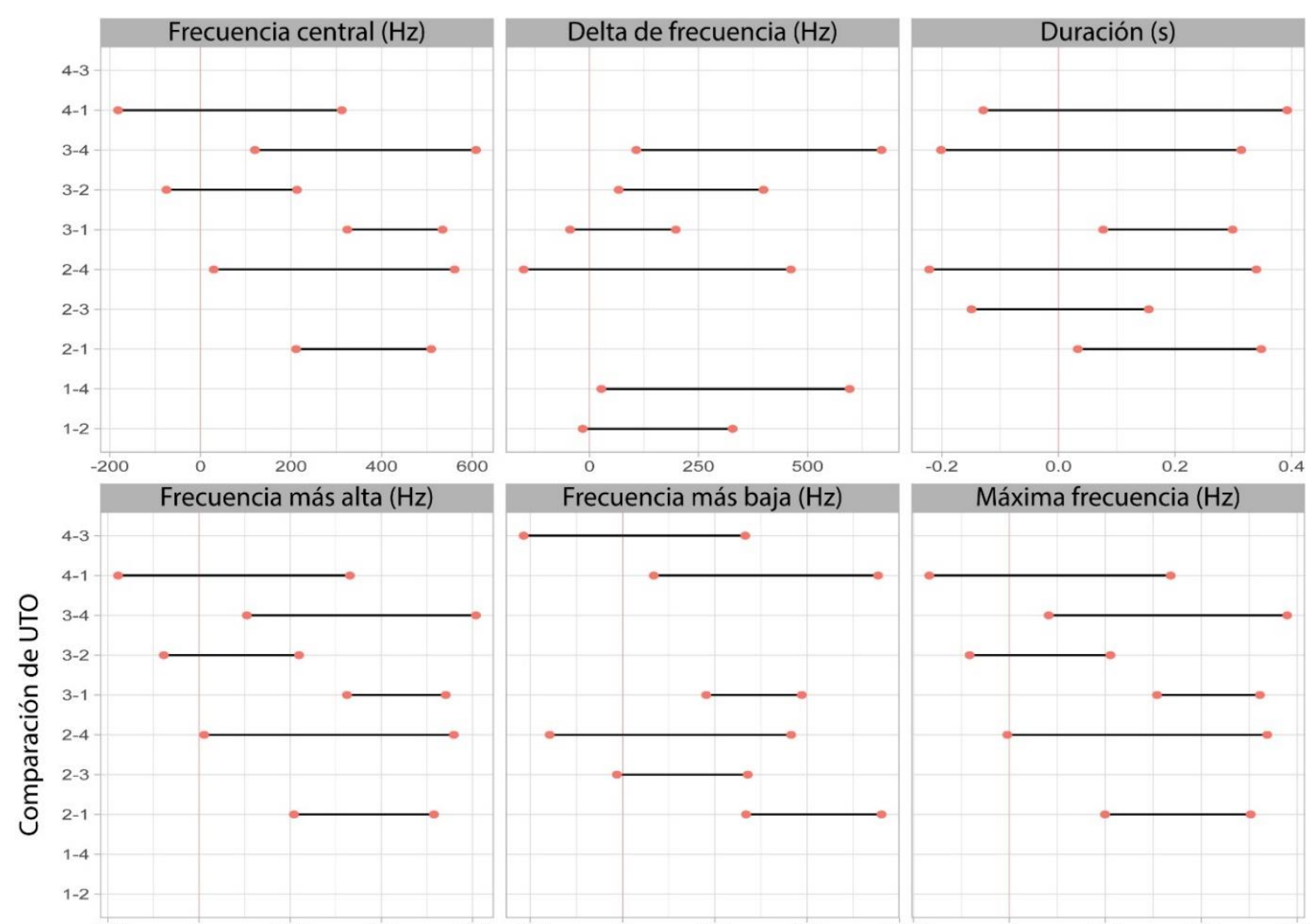

Frecuencia más baja $(\mathrm{Hz})$
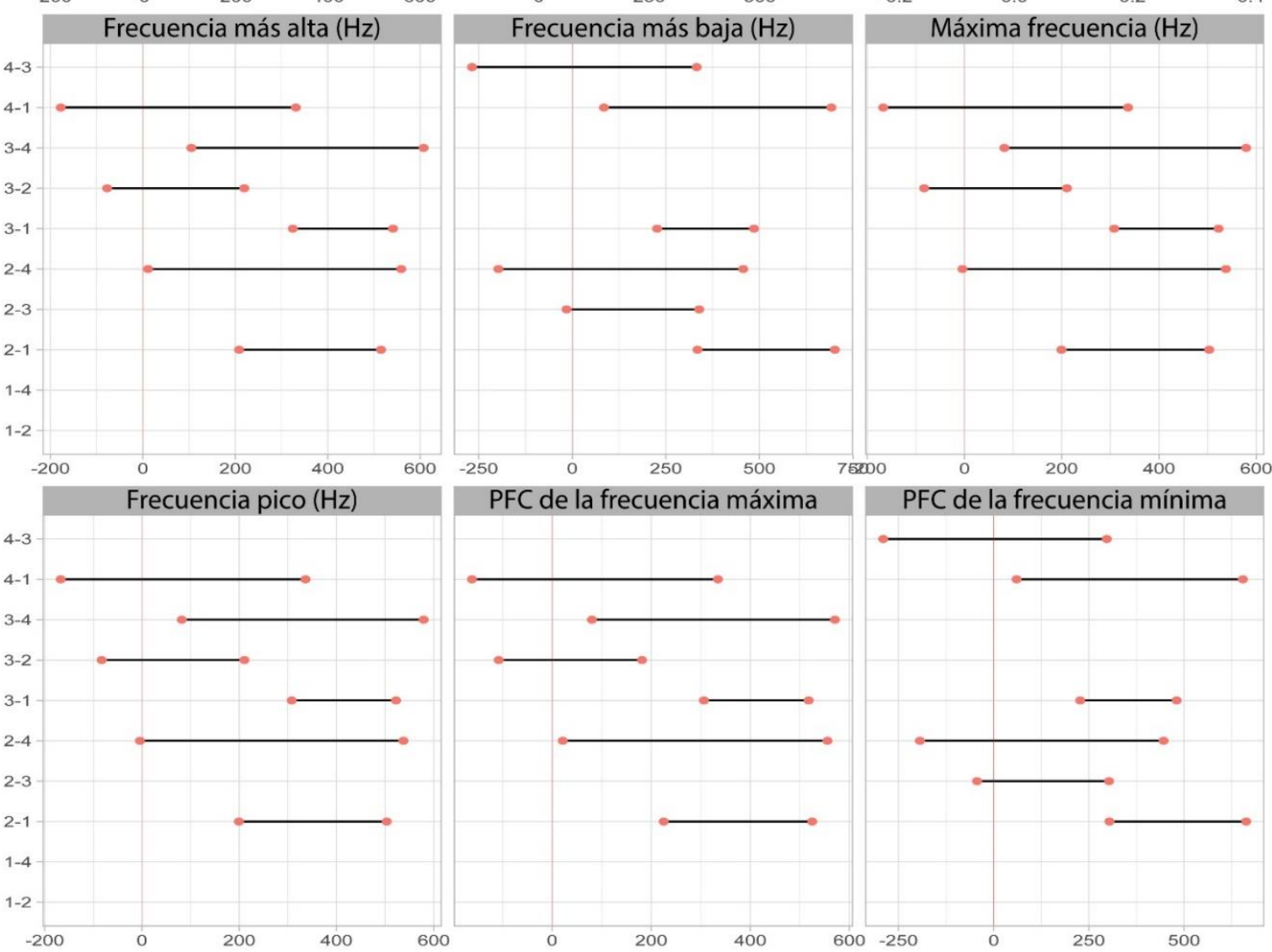

PFC de la frecuencia máxima
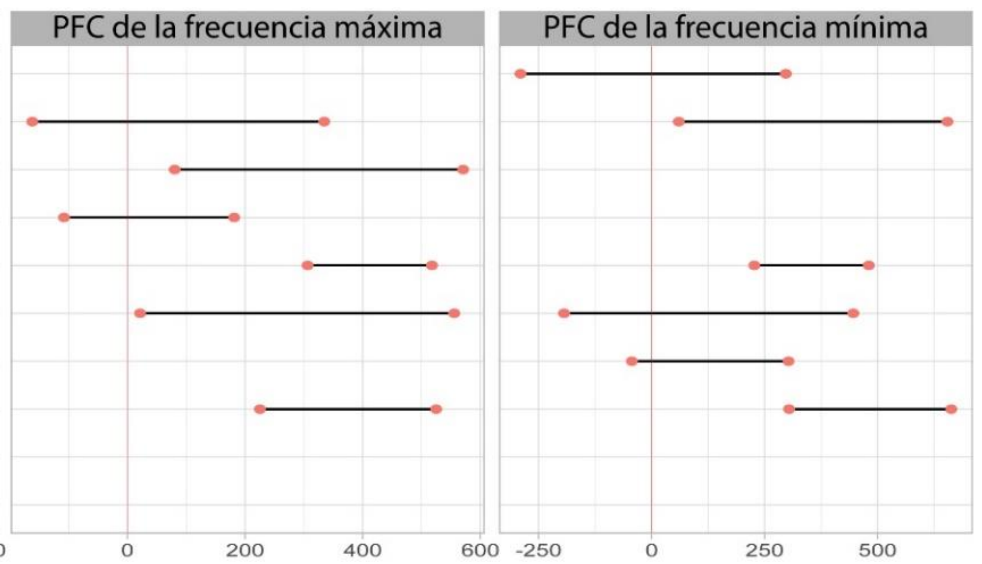

Figura 31. Intervalos de confianza de Tukey-Kramer para los atributos de la nota de tipo D

(canto reproductivo) de las UTO. Se muestran los intervalos de confianza en la diferenciación de las UTO 1 a 4, obtenidos en la prueba de comparación por pares de Tukey-Kramer. La línea roja vertical representa el cero en el intervalo. 


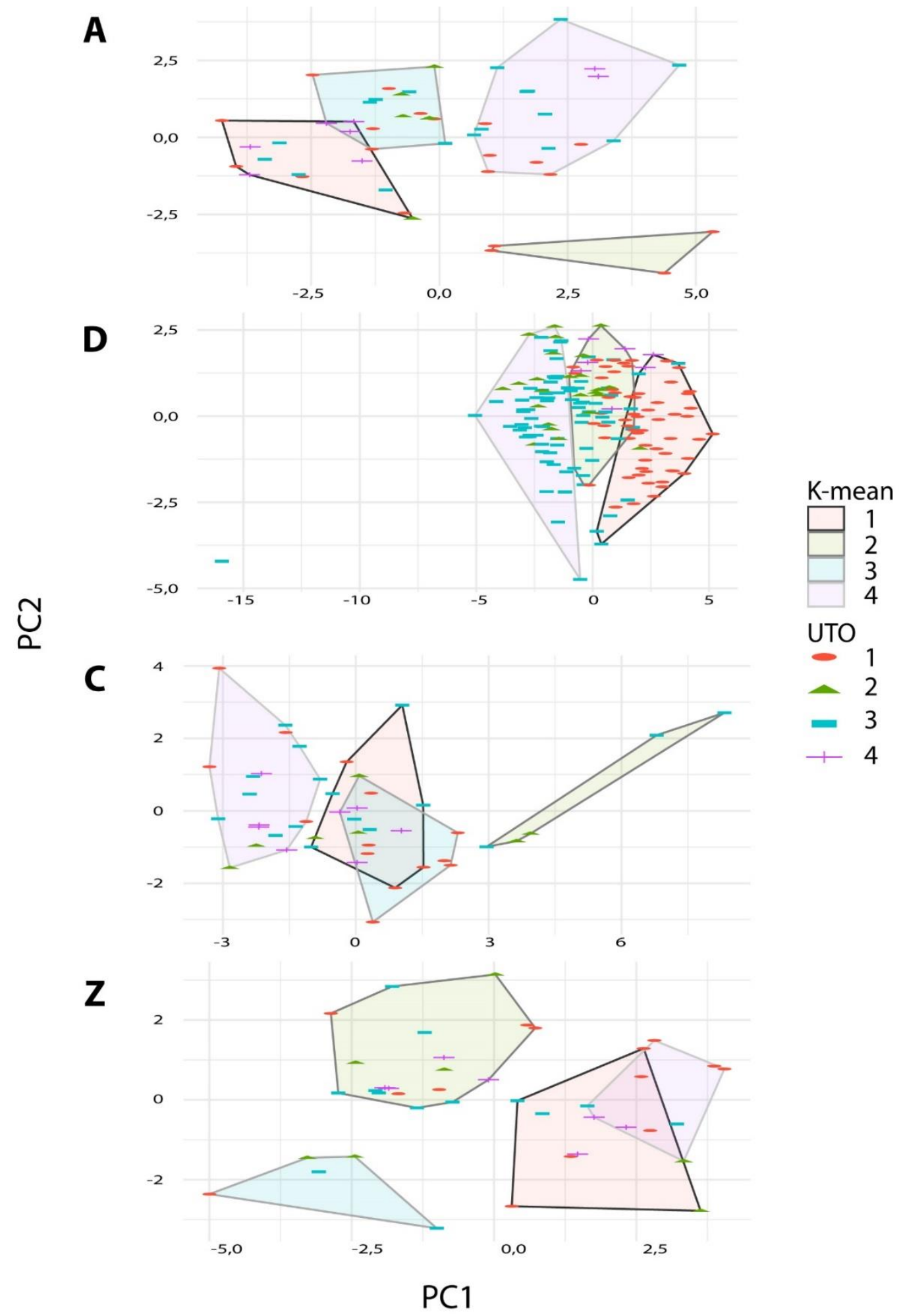

Figura 32. Agrupación supervisada por pares de $k$-means para los tipos de notas de las UTO.

Se muestra la distribución de los elementos de las UTO dentro de las agrupaciones supervisadas de $K$-means para los tipos de nota A, D, C y el canto de comunicación (Z). 


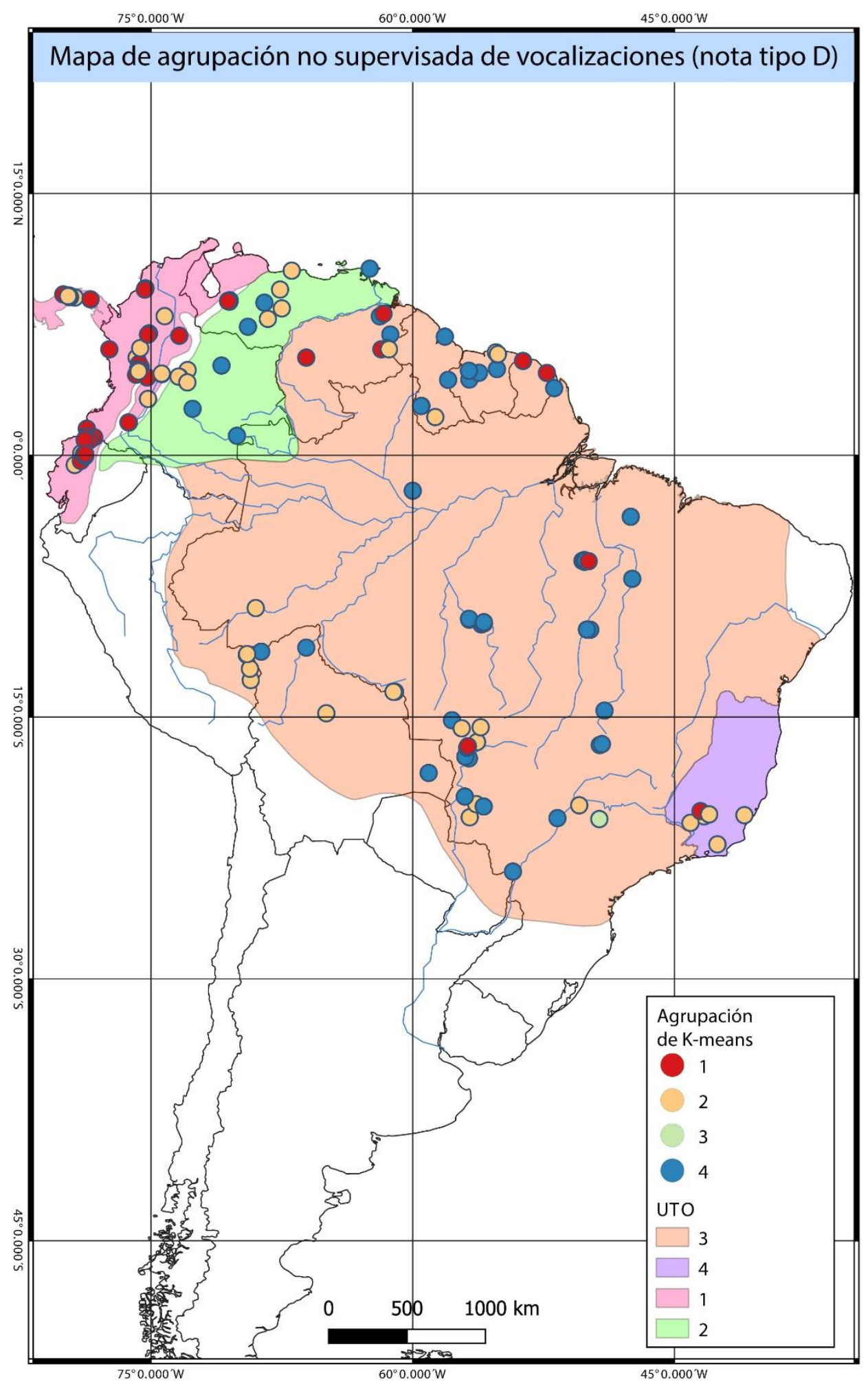

Figura 33. Mapa de agrupación no supervisada por pares de $\boldsymbol{K}$-means para la nota de tipo D de las UTO. Se muestran las agrupaciones de $K$-means para la nota de tipo D y la distribución de las Unidades Taxonómicas Operativas (UTO). 
UTO 1
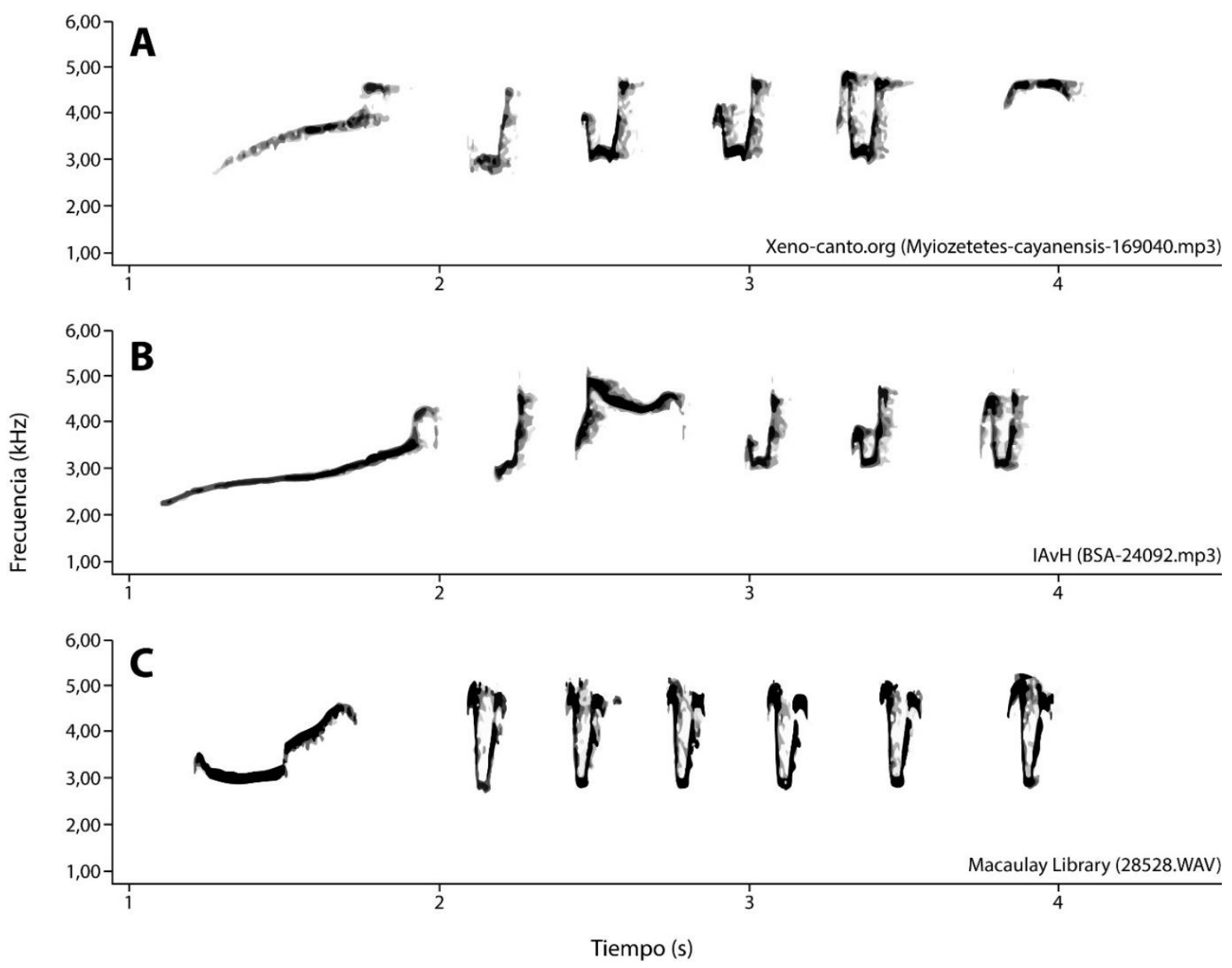

Figura 34. Ejemplos de sonogramas de los individuos pertenecientes a la UTO 1. Se muestran tres ejemplos de sonogramas típicos (A, B y C) de los individuos pertenecientes a la UTO 1. EI área inferior derecha de cada recuadro indica el identificador del archivo de sonido utilizado y la base de datos de referencia. 
UTO 2
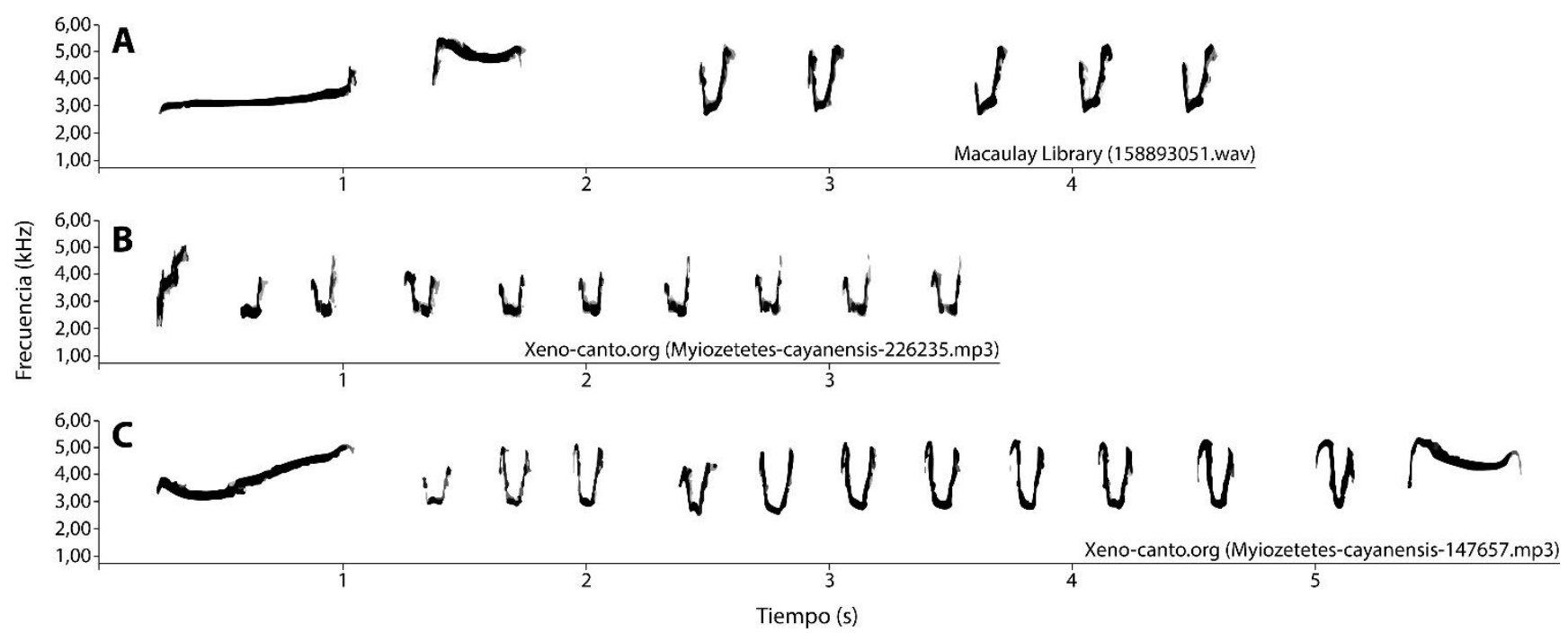

Figura 35. Ejemplos de sonogramas de los individuos pertenecientes a la UTO 2. Se muestran tres ejemplos de sonogramas típicos (A, B y C) de los individuos pertenecientes a la UTO 2. EI área inferior derecha de cada recuadro indica el identificador del archivo de sonido utilizado y la base de datos de referencia. 
UTO 3

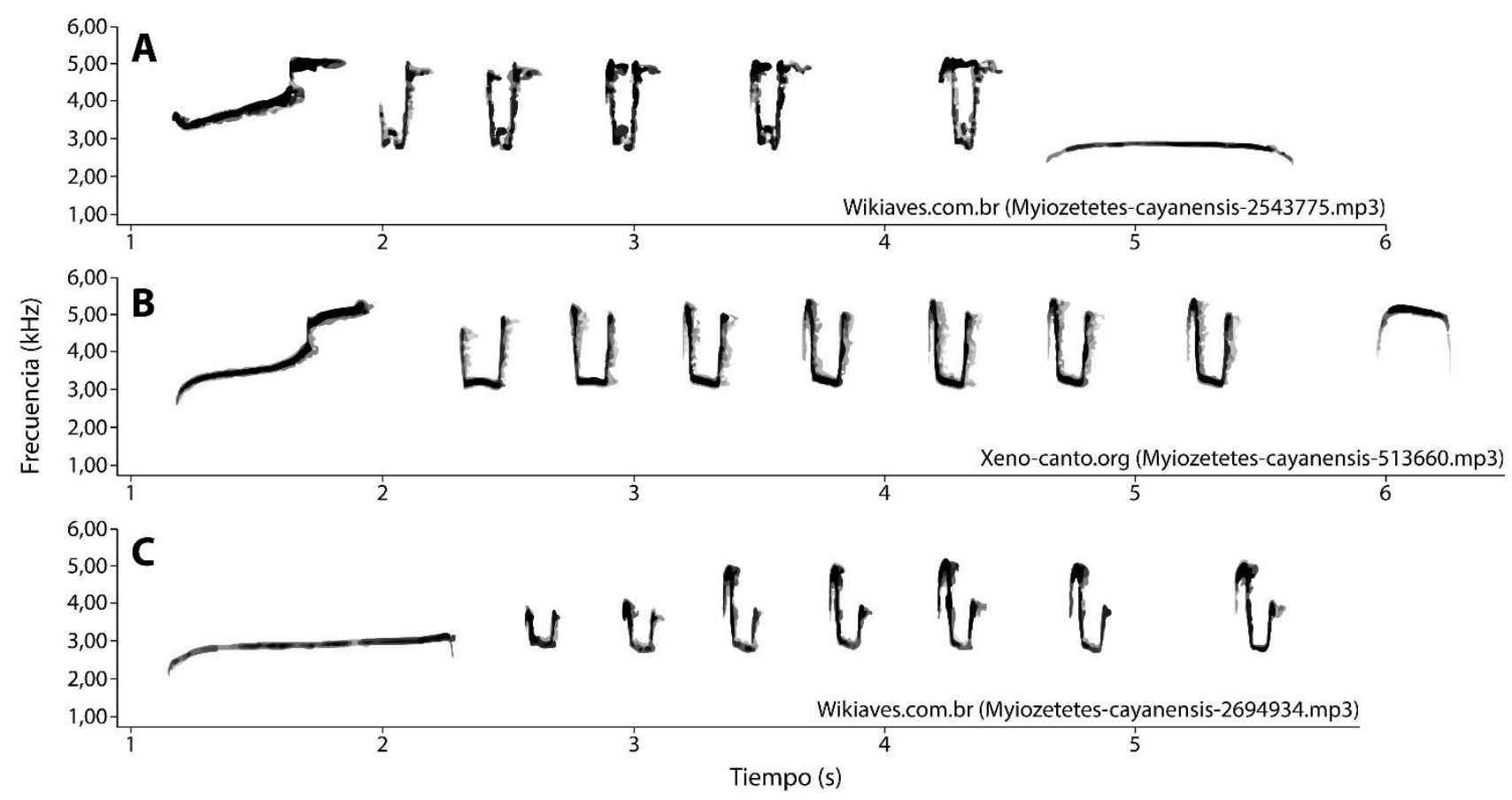

Figura 36. Ejemplos de sonogramas de los individuos pertenecientes a la UTO 3. Se muestran tres ejemplos de sonogramas típicos (A, B y C) de los individuos pertenecientes a la UTO 3 . El área inferior derecha de cada recuadro indica el identificador del archivo de sonido utilizado y la base de datos de referencia. 


\section{UTO 4}

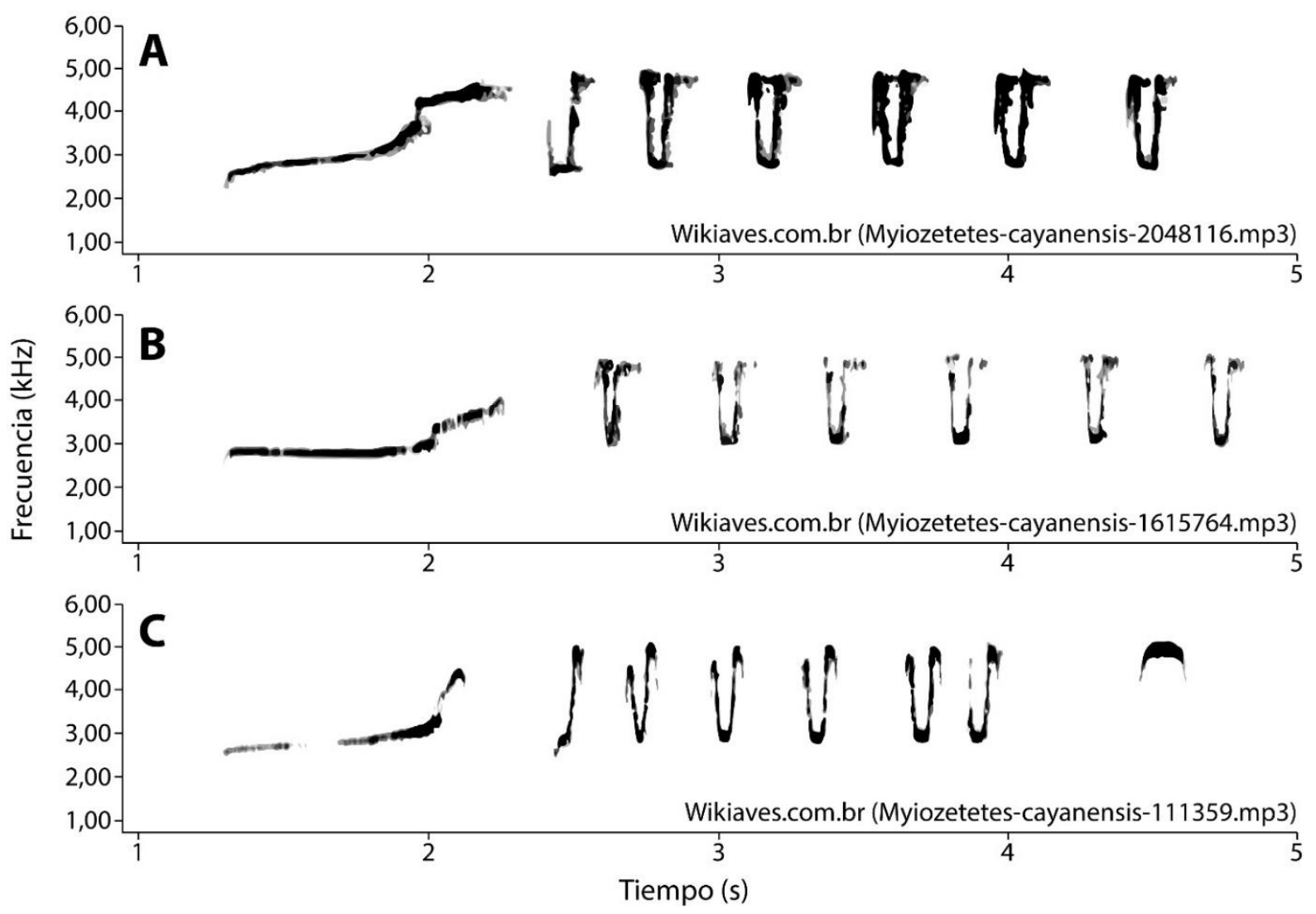

Figura 37. Ejemplos de sonogramas de los individuos pertenecientes a la UTO 4. Se muestran tres ejemplos de sonogramas típicos (A, B y C) de los individuos pertenecientes a la UTO 4 . EI área inferior derecha de cada recuadro indica el identificador del archivo de sonido utilizado y la base de datos de referencia. 


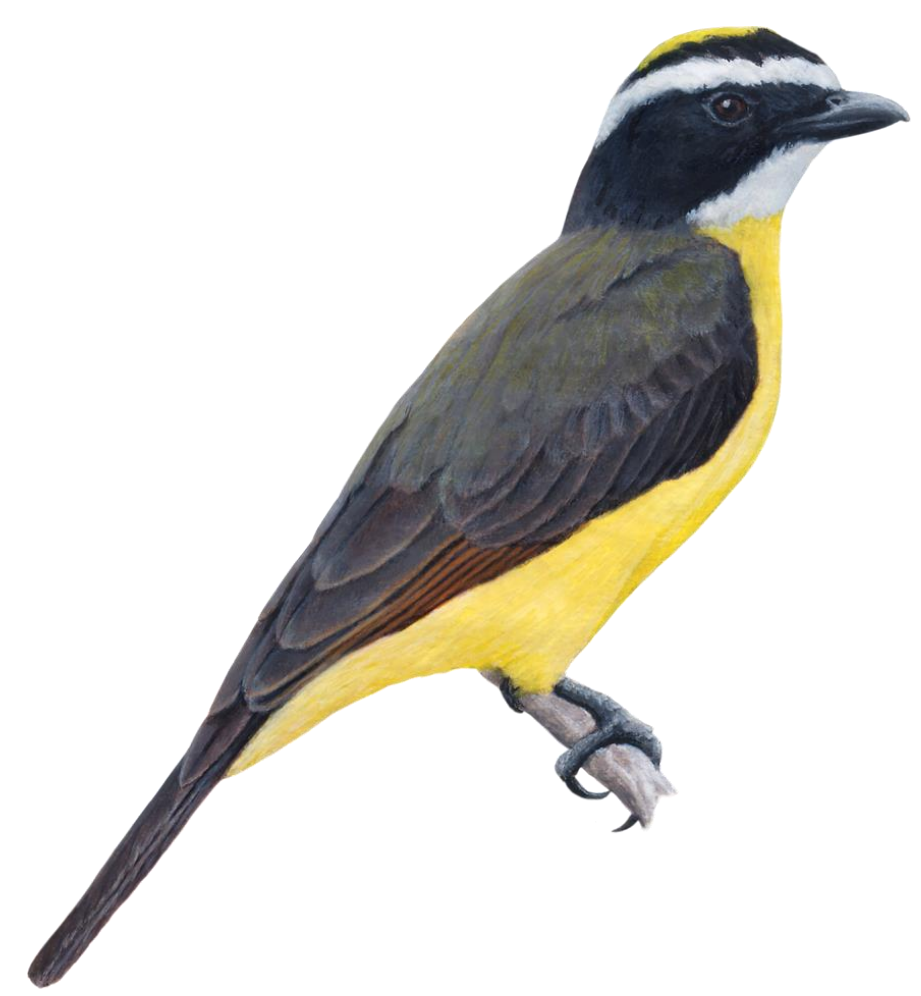

Figura 38. Myiozetetes hellmayri Hartet y Goodson, 1917. Ilustración: Rafael Nascimento. 


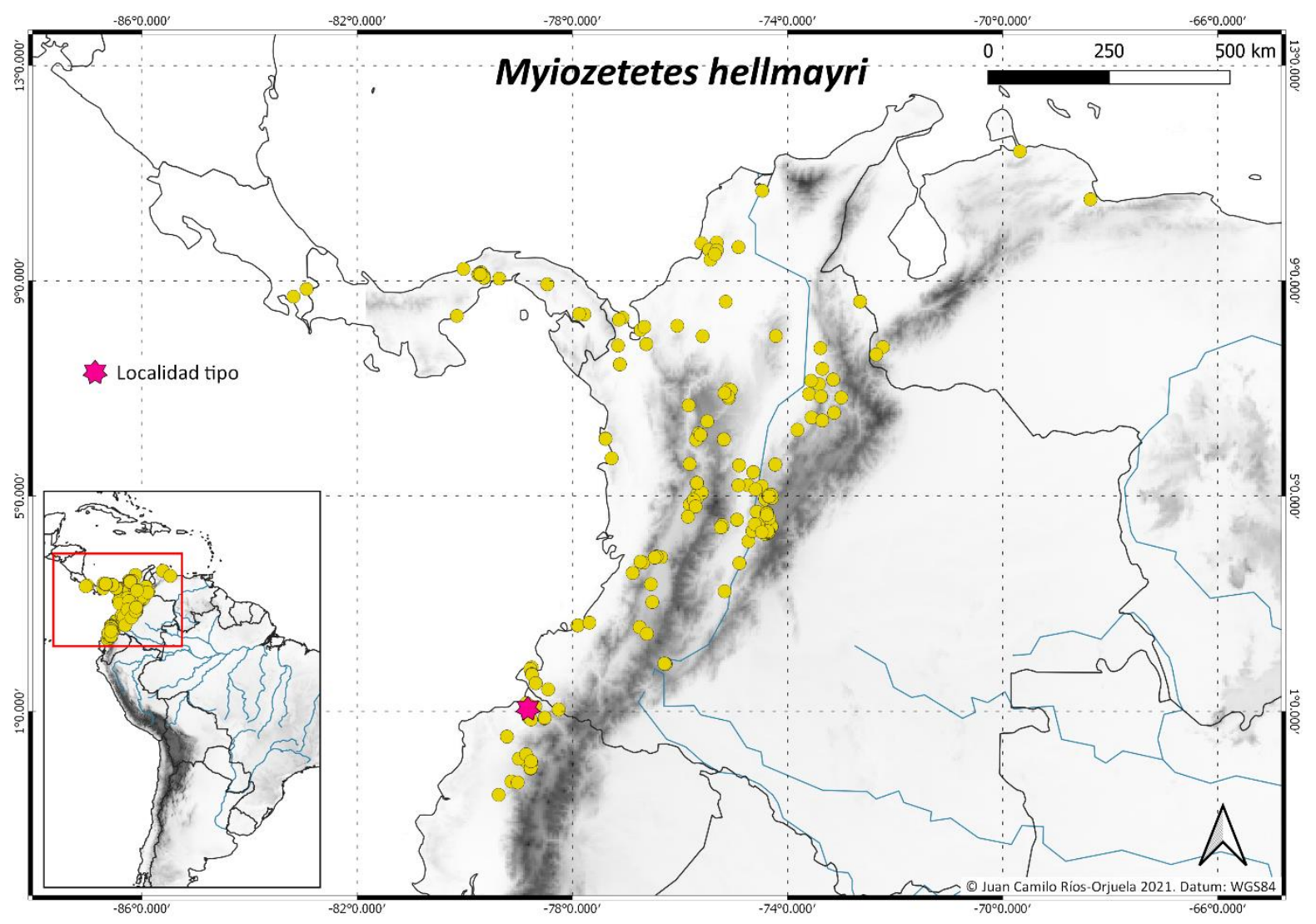

Figura 39. Distribución geográfica de Myiozetetes hellmayri Hartet y Goodson, 1917. Se muestra el rango de ocurrencia de los registros asociados a $M$. hellmayri y su localidad tipo. 


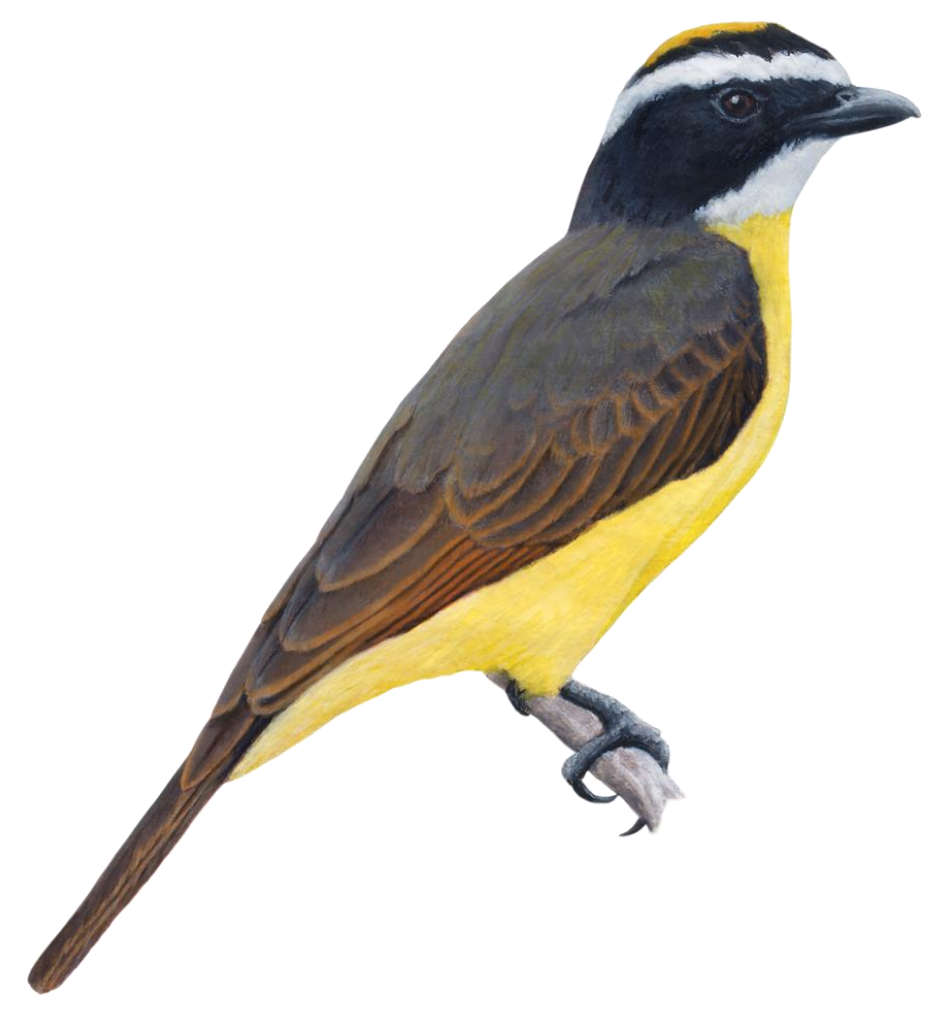

Figura 40. Myiozetetes cayanensis rufipennis Lawrence, 1870. Ilustración: Rafael Nascimento. 


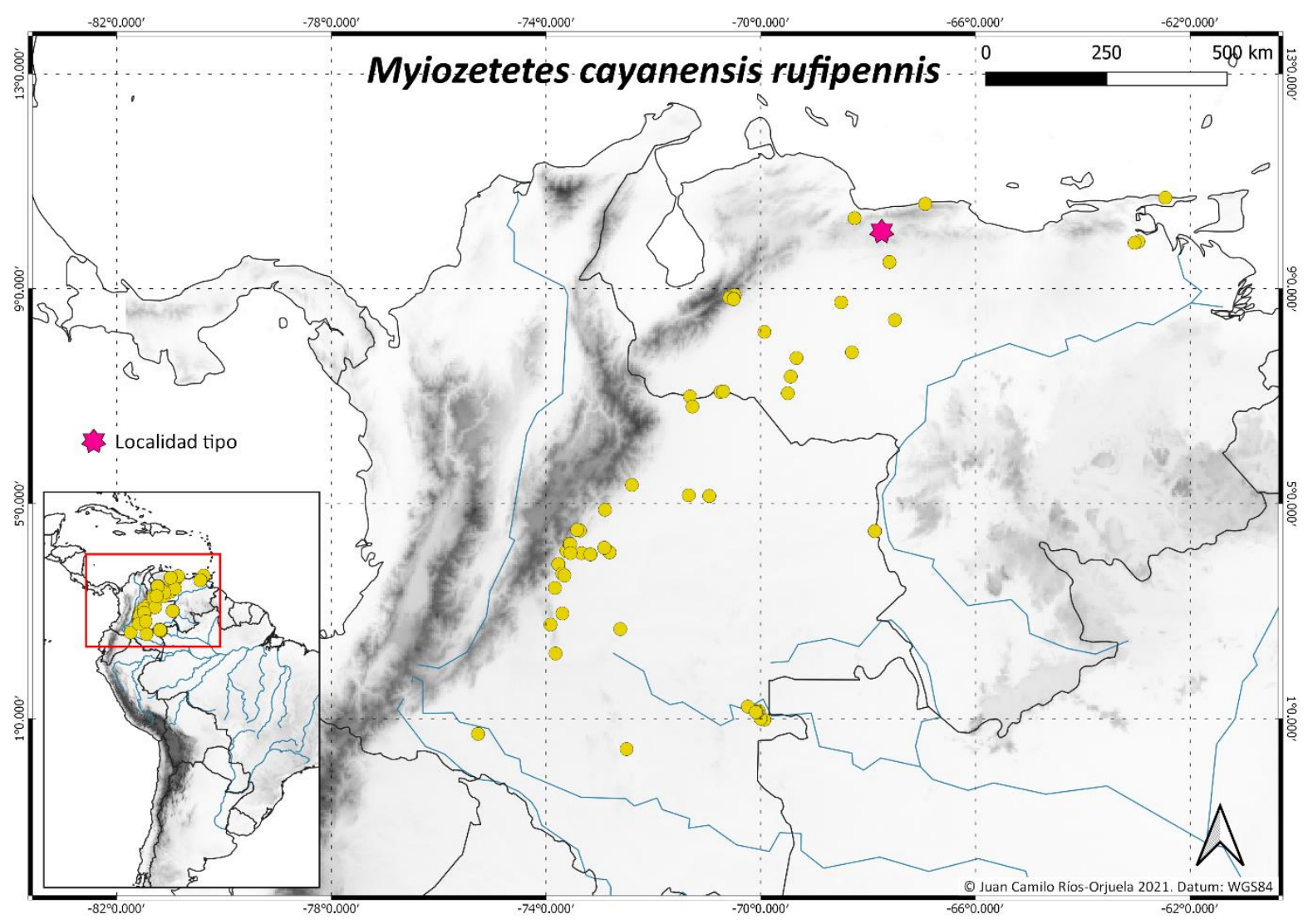

Figura 41. Distribución geográfica de Myiozetetes cayanensis rufipennis Lawrence, 1870 . Se muestra el rango de ocurrencia de los registros asociados a M. c. rufipennis y su localidad tipo. 


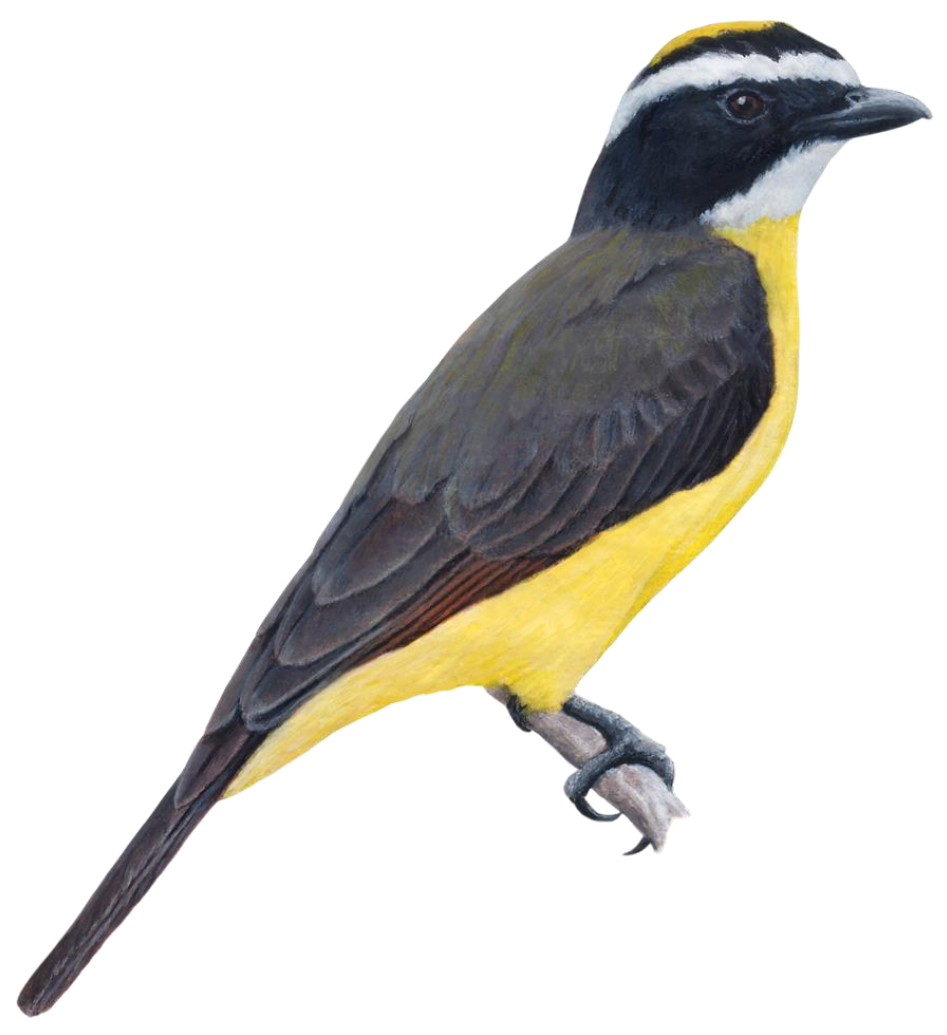

Figura 42. Myiozetetes cayanensis cayanensis (Linnaeus, 1766). Ilustración: Rafael Nascimento. 


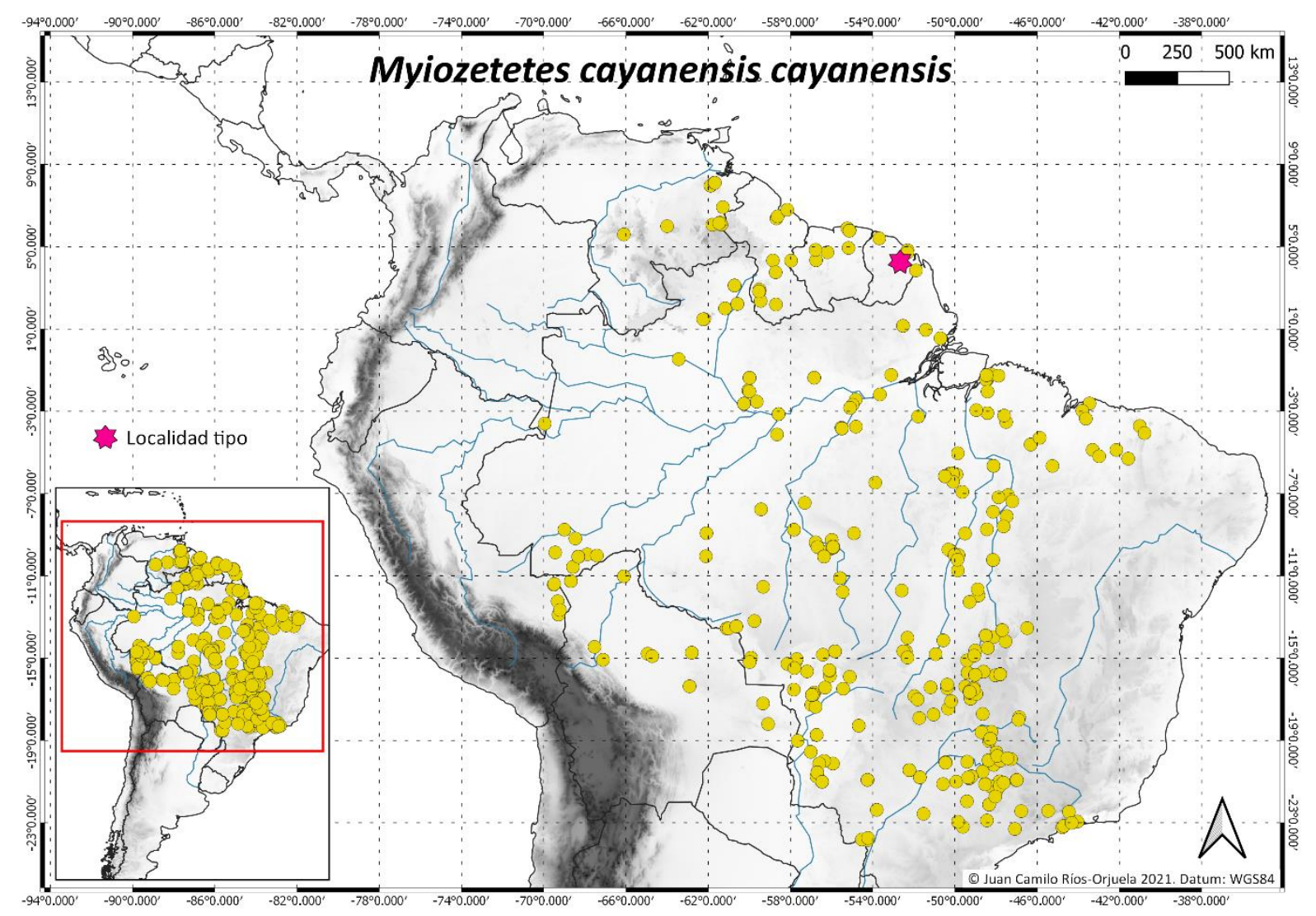

Figura 43. Distribución geográfica de Myiozetetes cayanensis cayanensis (Linnaeus, 1766).

Se muestra el rango de ocurrencia de los registros asociados a M. c. cayanensis y su localidad tipo. 


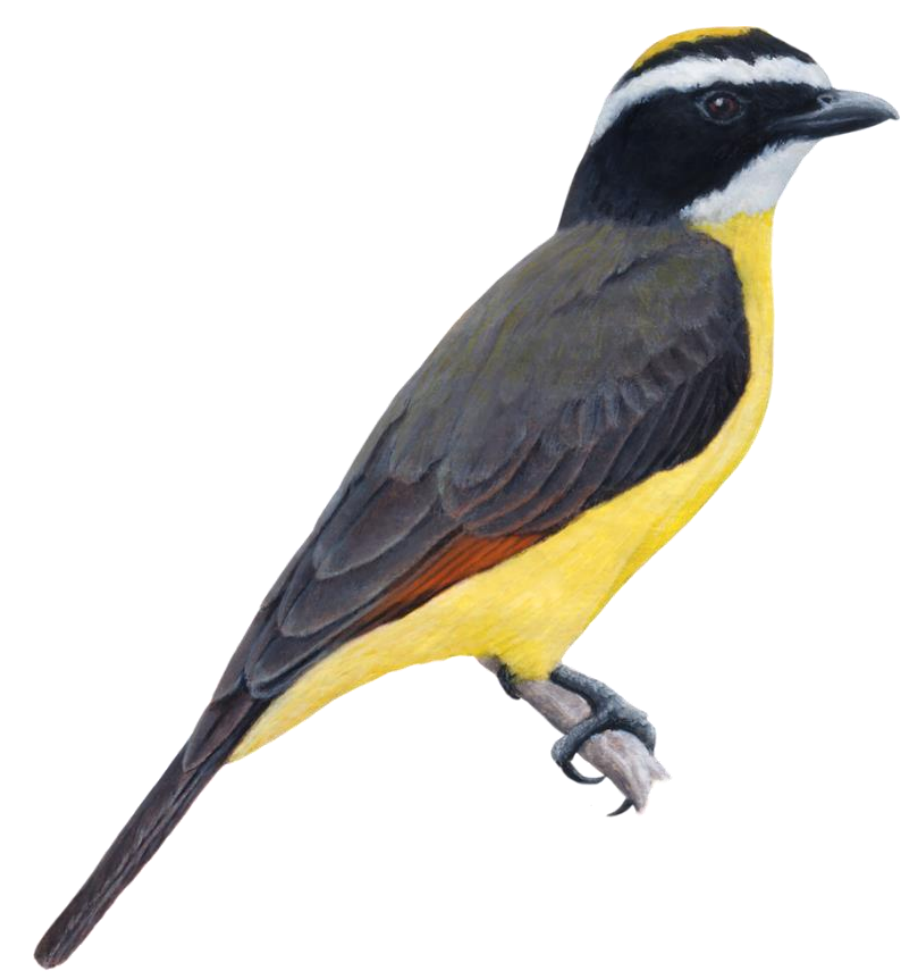

Figura 44. Myiozetetes erythropterus (Lafresnaye, 1853). Ilustración: Rafael Nascimento. 


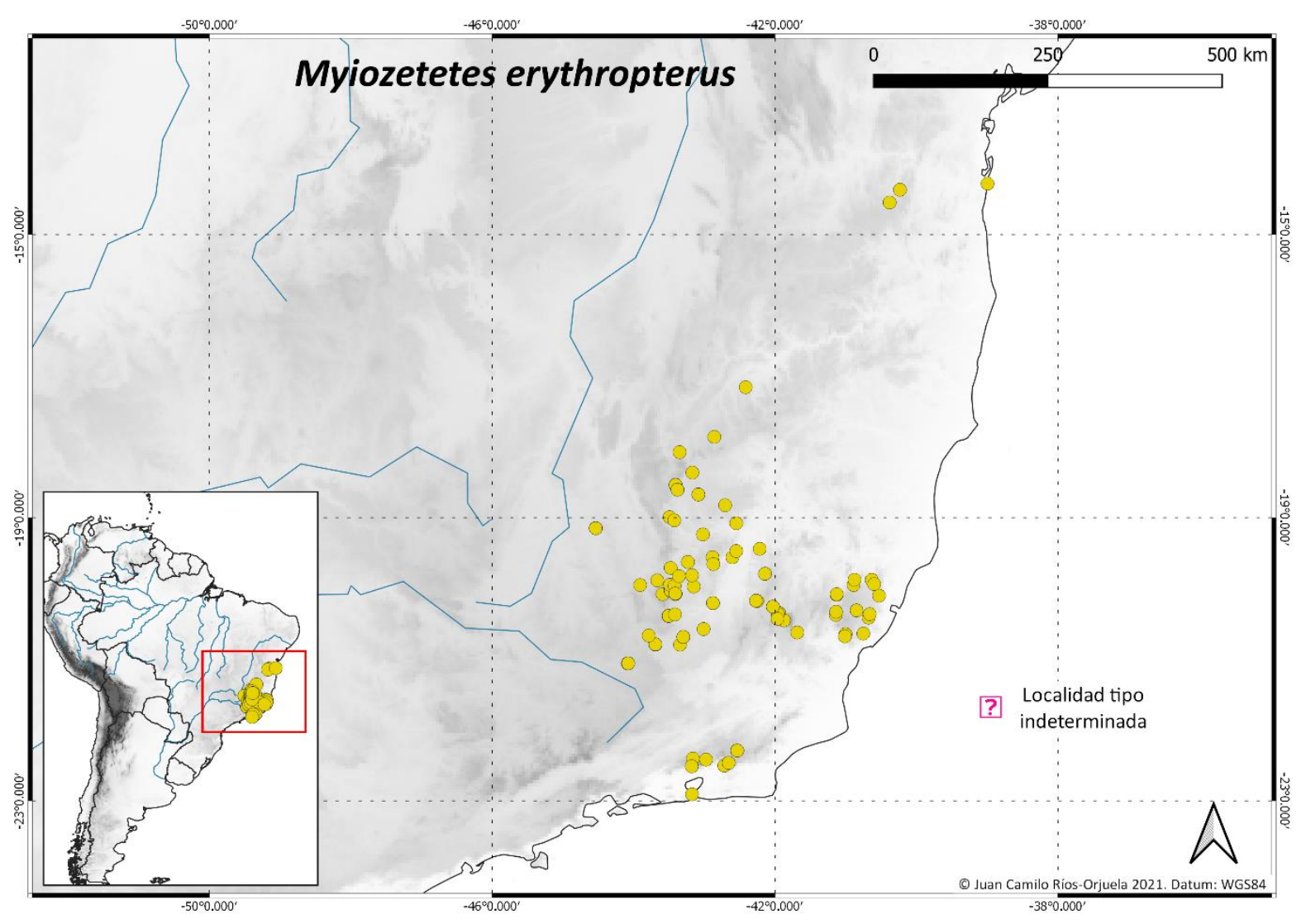

Figura 45. Distribución geográfica de Myiozetetes erythropterus (Lafresnaye, 1853). Se muestra el rango de ocurrencia de los registros asociados a $M$. erythropterus y la ausencia de localidad tipo. 


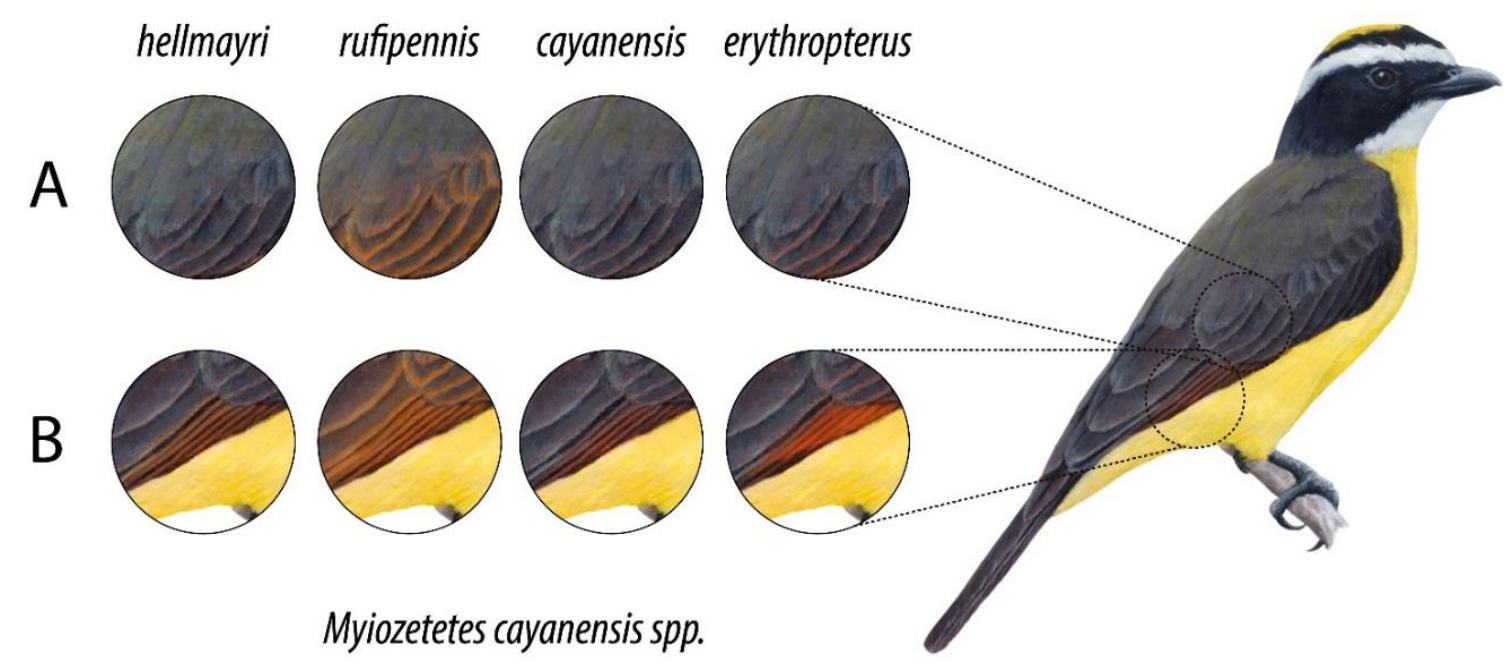

Figura 46. Variación en la coloración del plumaje de las Unidades Taxonómicas Operativas.

Se muestran las diferencias en la coloración del plumaje de (A) las plumas coberteras y (B) el borde de las primarias, en las Unidades Taxonómicas Operativas. Ilustración: Rafael Nascimento. 
132

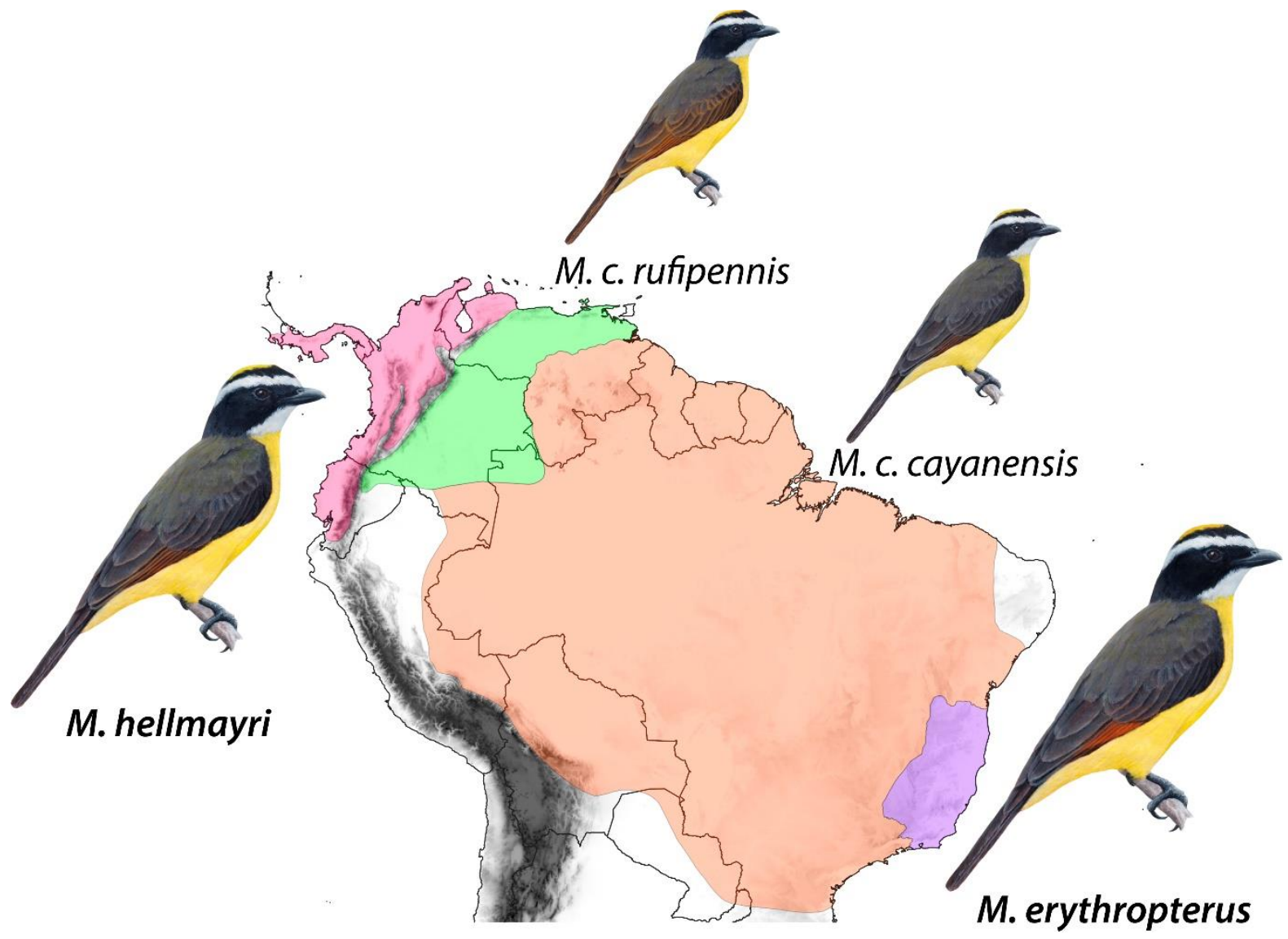

Figura 47. Distribución geográfica de Myiozetetes hellmayri, Myiozetetes cayanensis y Myiozetetes erythropterus. Se muestra la distribución geográfica de Myiozetetes hellmayri, Myiozetetes cayanensis (subespecies rufipennis y cayanensis) y Myiozetetes erythropterus. Ilustraciones: Rafael Nascimento. 


\section{TABLAS}

Tabla 1. Características del plumaje en las UTO del complejo Myiozetetes cayanensis en muda reproductiva.

\begin{tabular}{|c|c|c|c|}
\hline & UTO 2 & UTO 3 & Diferencias \\
\hline Corona & $\begin{array}{l}\text { Fusco uniforme, ligeramente más } \\
\text { en mudas pre-reproductiva }\end{array}$ & $\begin{array}{cc} & \text { Marrón } \\
& \text { grisáceo oscuro } \\
& \text { uniforme, } \\
\text { pálido/grisáceo } & \text { ligeramente } \\
\text { ss (NCS21) } & \text { más } \\
& \text { pálido/fusco en } \\
& \text { mudas pre- } \\
& \text { reproductivas } \\
& \text { (NCS20) }\end{array}$ & $\begin{array}{l}\text { Más oscura en } \\
\text { UTO } 4 \text { que en } \\
\text { UTO } 1 / \text { UTO } \\
\text { 2/UTO } 3\end{array}$ \\
\hline Zona auricular & $\begin{array}{r}\text { Fusco uniforme, ligeramente má } \\
\text { reproductiv }\end{array}$ & $\begin{array}{l}\text { is claro (grisáceo) en mudas pre- } \\
\text { las (NCS21) }\end{array}$ & $\begin{array}{c}\text { Existe una } \\
\text { diferencia } \\
\text { entre el color } \\
\text { de la cabeza y } \\
\text { la cara de UTO } \\
\text { 4, siendo la } \\
\text { cabeza más } \\
\text { oscura que la } \\
\text { cara y el patrón } \\
\text { general del } \\
\text { dorso. Esto no } \\
\text { ocurre en las } \\
\text { otras } 3 \text { UTO }\end{array}$ \\
\hline $\begin{array}{c}\text { Coronilla } \\
\text { (basal y distal) } \\
* *\end{array}$ & $\begin{array}{r}\text { Amarillo intenso, más oscuro en } \\
\text { de la coron }\end{array}$ & $\begin{array}{l}\text { transición hacia la región medial } \\
\text { illa (NCS55) }\end{array}$ & NA \\
\hline $\begin{array}{l}\text { Coronilla } \\
\text { (medial)* }\end{array}$ & $\begin{array}{l}\text { Amarillo } \\
\text { naranja, un } \\
\text { poco más } \\
\text { oscuro que las } \\
\text { regiones basal } \\
\text { y distal de la } \\
\text { coronilla } \\
\text { (NCS18) }\end{array}$ & $\begin{array}{l}\text { o, oscuro y contrastante con las } \\
\text { l y distal de la coronilla (NCS17) }\end{array}$ & $\begin{array}{c}\text { Más pálida } \\
\text { (amarilla) en } \\
\text { UTO } 1 \text { con } \\
\text { respecto a UTO } \\
\text { 2/UTO 3/UTO } 4\end{array}$ \\
\hline Pecho & $\begin{array}{r}\text { Amarillo intenso en mudas pre } \\
\text { (puede ser pálido en algu }\end{array}$ & $\begin{array}{l}\text {-reproductivas y reproductivas } \\
\text { unos individuos) (NCS55) }\end{array}$ & NA \\
\hline $\begin{array}{l}\text { Dorso y } \\
\text { supracaudales } \\
\text { (basal) }\end{array}$ & $\begin{array}{c}\text { Marrón oliva uniforme, } \\
\text { ligeramente más pálido/grisáceo } \\
\text { en mudas pre-reproductivas } \\
\text { (NCS28) }\end{array}$ & $\begin{array}{c}\text { Marrón acanelado uniforme, } \\
\text { ligeramente más pálido/grisáceo } \\
\text { en mudas pre-reproductivas } \\
\text { (NCS33) }\end{array}$ & $\begin{array}{c}\text { Más } \\
\text { canela/rojizo } \\
\text { en UTO 3/UTO } \\
4 \text { con respecto } \\
\text { a UTO 1/UTO } 2\end{array}$ \\
\hline
\end{tabular}




\begin{tabular}{|c|c|c|c|c|c|}
\hline Dorso (distal) & \multicolumn{2}{|c|}{$\begin{array}{c}\text { Citrino, más rojizo o ausente en } \\
\text { individuos pre-reproductivos } \\
\text { (NCS51) }\end{array}$} & $\begin{array}{l}\text { Oliva verdoso, } \\
\text { ausente en } \\
\text { individuos pre- } \\
\text { reproductivos } \\
\text { (NCS49) }\end{array}$ & $\begin{array}{l}\text { Verde oliva, } \\
\text { ausente en } \\
\text { individuos pre- } \\
\text { reproductivos } \\
\text { (NCS48) }\end{array}$ & $\begin{array}{l}\text { Más oscuro en } \\
\text { UTO } 3 \text { con } \\
\text { respecto a UTO } \\
\text { 4. más pálido } \\
\text { en UTO } 1 / \text { UTO } \\
2 \text { con respecto } \\
\text { a UTO } 3 / \text { UTO } 4\end{array}$ \\
\hline $\begin{array}{l}\text { Plumas de } \\
\text { arrastre }\end{array}$ & \multicolumn{2}{|c|}{$\begin{array}{l}\text { Marrón oliva uniforme, } \\
\text { ligeramente más pálido en } \\
\text { mudas pre-reproductivas } \\
\text { (NCS28) }\end{array}$} & $\begin{array}{l}\text { Fusco } \\
\text { uniforme, } \\
\text { ligeramente } \\
\text { más pálido en } \\
\text { mudas pre- } \\
\text { reproductivas } \\
\text { (NCS21) }\end{array}$ & $\begin{array}{l}\text { Marrón oliva } \\
\text { uniforme, } \\
\text { ligeramente } \\
\text { más pálido en } \\
\text { mudas pre- } \\
\text { reproductivas } \\
\text { (NCS28) }\end{array}$ & $\begin{array}{l}\text { más oscuro en } \\
\text { UTO } 3 \text { con } \\
\text { respecto a UTO } \\
\text { 1/UTO 2/UTO } 4\end{array}$ \\
\hline $\begin{array}{c}\text { Borde } \\
\text { primarias }\end{array}$ & $\begin{array}{c}\text { Marrón } \\
\text { antiguo, más } \\
\text { pálido/anteado } \\
\text { en individuos } \\
\text { pre- } \\
\text { reproductivos } \\
\text { (NCS37) }\end{array}$ & $\begin{array}{l}\text { Leonado, rojizo } \\
\text { en individuos } \\
\text { pre- } \\
\text { reproductivos } \\
\text { (NCS38) }\end{array}$ & $\begin{array}{c}\text { Marrón } \\
\text { antiguo, más } \\
\text { pálido/anteado } \\
\text { en individuos } \\
\text { pre- } \\
\text { reproductivos } \\
\text { (NCS37) }\end{array}$ & $\begin{array}{c}\text { Ámbar, más } \\
\text { pálido/anteado } \\
\text { en individuos } \\
\text { pre- } \\
\text { reproductivos } \\
\text { (NCS36) }\end{array}$ & $\begin{array}{l}\text { más oscuro en } \\
\text { UTO 1/UTO } 3 \\
\text { con respecto a } \\
\text { /UTO 2/UTO } 4\end{array}$ \\
\hline $\begin{array}{l}\text { Borde } \\
\text { secundarias y } \\
\text { rectrices }\end{array}$ & $\begin{array}{l}\text { Amarillo- } \\
\text { anteado, más } \\
\text { pálido en } \\
\text { individuos pre- } \\
\text { reproductivos } \\
\text { (NCS53) }\end{array}$ & $\begin{array}{l}\text { Canela, rojizo } \\
\text { en individuos } \\
\text { pre- } \\
\text { reproductivos } \\
\text { (NCS39) }\end{array}$ & $\begin{array}{l}\text { Arcilla, más } \\
\text { pálido/anteado } \\
\text { en individuos } \\
\text { pre- } \\
\text { reproductivos } \\
\text { (NCS26) }\end{array}$ & $\begin{array}{c}\text { Canela, más } \\
\text { pálido/anteado } \\
\text { en individuos } \\
\text { pre- } \\
\text { reproductivos } \\
\text { (NCS39) }\end{array}$ & $\begin{array}{c}\text { Canela en UTO } \\
\text { 2/UTO 4, } \\
\text { anteado/arcilla } \\
\text { en UTO } 1 / \text { UTO } \\
3\end{array}$ \\
\hline $\begin{array}{l}\text { Coberteras } \\
\text { menores y } \\
\text { mayores } \\
\text { (basal) }\end{array}$ & $\begin{array}{r}\text { Marrón oliv } \\
\text { ligeramente } r \\
\text { mudas pre- } r \\
\text { (NCs }\end{array}$ & $\begin{array}{l}\text { a uniforme, } \\
\text { más pálido en } \\
\text { eproductivas } \\
\text { S28) }\end{array}$ & $\begin{array}{c}\text { Marrón } \\
\text { acanelado } \\
\text { uniforme, } \\
\text { ligeramente } \\
\text { más } \\
\text { pálido/grisáceo } \\
\text { en mudas pre- } \\
\text { reproductivas } \\
\text { (NCS33) }\end{array}$ & $\begin{array}{l}\text { Marrón oliva } \\
\text { uniforme, } \\
\text { ligeramente } \\
\text { más pálido en } \\
\text { mudas pre- } \\
\text { reproductivas } \\
\text { (NCS28) }\end{array}$ & $\begin{array}{c}\text { Más } \\
\text { canela/rojizo } \\
\text { en UTO } 3 \text { con } \\
\text { respecto a UTO } \\
\text { 1/UTO 2/UTO } 4\end{array}$ \\
\hline $\begin{array}{l}\text { Coberteras } \\
\text { menores y } \\
\text { mayores } \\
\text { (distal) }\end{array}$ & $\begin{array}{l}\text { Color arcilla, } \\
\text { más pálido y } \\
\text { amplio en } \\
\text { individuos pre- } \\
\text { reproductivos } \\
\text { (NCS123B) }\end{array}$ & $\begin{array}{l}\text { Canela, rojizo y } \\
\text { más amplio en } \\
\text { individuos pre- } \\
\text { reproductivos } \\
\text { (NCS123A) }\end{array}$ & $\begin{array}{l}\text { Citrino, más } \\
\text { rojizo o } \\
\text { ausente en } \\
\text { individuos pre- } \\
\text { reproductivos } \\
\text { (NCS51) }\end{array}$ & $\begin{array}{l}\text { Marrón oliva } \\
\text { uniforme, } \\
\text { ligeramente } \\
\text { más pálido en } \\
\text { mudas pre- } \\
\text { reproductivas } \\
\text { (NCS28) }\end{array}$ & $\begin{array}{l}\text { Todas las UTO } \\
\text { poseen } \\
\text { colores/tonos } \\
\text { diferentes }\end{array}$ \\
\hline $\begin{array}{c}\text { Supracaudales } \\
\text { (distal) }\end{array}$ & $\begin{array}{l}\text { Color arcilla, } \\
\text { más pálido y } \\
\text { amplio en } \\
\text { individuos pre- } \\
\text { reproductivos } \\
\text { (NCS123B) }\end{array}$ & $\begin{array}{l}\text { Leonado, rojizo } \\
\text { en individuos } \\
\text { pre- } \\
\text { reproductivos } \\
\text { (NCS38) }\end{array}$ & $\begin{array}{l}\text { Oliva verdoso, } \\
\text { ausente en } \\
\text { individuos pre- } \\
\text { reproductivos } \\
\text { (NCS49) }\end{array}$ & $\begin{array}{l}\text { Verde oliva, } \\
\text { ausente en } \\
\text { individuos pre- } \\
\text { reproductivos } \\
\text { (NCS48) }\end{array}$ & $\begin{array}{l}\text { Todas las UTO } \\
\text { poseen } \\
\text { colores/tonos } \\
\text { diferentes }\end{array}$ \\
\hline
\end{tabular}




\begin{tabular}{|c|c|c|c|c|}
\hline Rectrices & $\begin{array}{c}\text { Marrón oliva uniforme, } \\
\text { ligeramente más pálido en } \\
\text { mudas pre-reproductivas } \\
\text { (NCS28) }\end{array}$ & $\begin{array}{c}\text { Fusco } \\
\text { uniforme, } \\
\text { ligeramente } \\
\text { más } \\
\text { pálido/grisáceo } \\
\text { en mudas pre- } \\
\text { reproductivas } \\
\text { (NCS21) }\end{array}$ & $\begin{array}{l}\text { Marrón oliva } \\
\text { uniforme, } \\
\text { ligeramente } \\
\text { más pálido en } \\
\text { mudas pre- } \\
\text { reproductivas } \\
\text { (NCS28) }\end{array}$ & $\begin{array}{l}\text { más oscuro en } \\
\text { UTO } 3 \text { con } \\
\text { respecto a UTO } \\
\text { 1/UTO 2/UTO } 4\end{array}$ \\
\hline
\end{tabular}

* No presentes en individuos juveniles 
Tabla 2. Medidas de tendencia central de las variables morfométricas analizadas.

\begin{tabular}{lrrrrrr} 
& $\begin{array}{c}\text { Media } \\
(\mathbf{m m})\end{array}$ & $\begin{array}{r}\text { Desviación } \\
\text { estándar }(\mathbf{m m})\end{array}$ & $\begin{array}{c}\text { Mediana } \\
(\mathbf{m m})\end{array}$ & Varianza & $\begin{array}{c}\text { Mínima } \\
(\mathbf{m m})\end{array}$ & $\begin{array}{c}\text { Máxima } \\
(\mathbf{m m})\end{array}$ \\
\hline Culmen (n) & 9,58 & 0,58 & 9,58 & 0,34 & 8,18 & 11,34 \\
Alto del pico & 5,04 & 0,37 & 5,04 & 0,14 & 4,15 & 6,19 \\
Tarso & 18,46 & 0,86 & 18,46 & 0,74 & 15,78 & 20,45 \\
Ancho distal del tarso & 1,48 & 0,13 & 1,47 & 0,02 & 1,16 & 1,95 \\
Cola & 73,70 & 4,39 & 73,36 & 19,28 & 63,58 & 87,92 \\
Ancho de la rectriz central & 11,84 & 1,36 & 11,84 & 1,85 & 7,73 & 16,22 \\
Cuerda alar & 86,82 & 4,38 & 86,86 & 19,23 & 75,47 & 102,44 \\
Largo de P10 & 76,77 & 4,65 & 76,79 & 21,60 & 56,89 & 92,86 \\
Largo de P1 & 72,14 & 3,15 & 72,14 & 9,94 & 63,73 & 82,60 \\
Largo de S1 & 71,64 & 3,58 & 71,64 & 12,81 & 63,31 & 83,15 \\
\hline
\end{tabular}


Tabla 3. Matriz de correlación de las variables morfométricas seleccionadas para el análisis. Se muestran los valores de $r$ (mitad superior) de correlación de Pearson y los valores $\mathrm{p}$ (mitad inferior) de significancia de colinealidad siguiendo el método de Hollander y Wolfe (1973). Los valores resaltados en negrilla indican valores significativos $(p<0,05)$.

\begin{tabular}{|c|c|c|c|c|c|c|c|c|c|c|}
\hline & $\begin{array}{c}\text { Culmen } \\
\text { (n) }\end{array}$ & $\begin{array}{l}\text { Alto } \\
\text { del } \\
\text { pico }\end{array}$ & Tarso & $\begin{array}{c}\text { Ancho } \\
\text { distal del } \\
\text { tarso }\end{array}$ & Cola & $\begin{array}{l}\text { Ancho } \\
\text { de la } \\
\text { rectriz } \\
\text { central }\end{array}$ & $\begin{array}{l}\text { Cuerda } \\
\text { alar }\end{array}$ & $\begin{array}{l}\text { Largo } \\
\text { de P10 }\end{array}$ & $\begin{array}{l}\text { Largo } \\
\text { de S1 }\end{array}$ & $\begin{array}{l}\text { Largo } \\
\text { de P1 }\end{array}$ \\
\hline Culmen (n) & & $-0,0094$ & 0,2461 & 0,0270 & 0,0913 & $-0,0046$ & 0,1975 & 0,0678 & 0,1925 & 0,1504 \\
\hline Alto del pico & 0,8979 & & $-0,0097$ & 0,0434 & $-0,0156$ & 0,0053 & $\begin{array}{r}- \\
0,0252\end{array}$ & 0,0513 & 0,0615 & 0,0405 \\
\hline Tarso & 0,0003 & 0,8961 & & 0,1390 & 0,3468 & 0,1720 & 0,4380 & 0,3594 & 0,3311 & 0,3713 \\
\hline $\begin{array}{l}\text { Ancho distal } \\
\text { del tarso }\end{array}$ & 0,6983 & 0,5622 & 0,0442 & & 0,2732 & 0,1459 & 0,2228 & 0,1979 & 0,3124 & 0,2834 \\
\hline Cola & 0,185 & 0,833 & $2,2 \mathrm{E}-07$ & $6,8 \mathrm{E}-05$ & & 0,2890 & 0,6233 & 0,4745 & 0,6051 & 0,5491 \\
\hline $\begin{array}{l}\text { Ancho de la } \\
\text { rectriz } \\
\text { central }\end{array}$ & 948 & 0,944 & 0,013 & 0,039 & 2,3E-05 & & 0,3465 & 0,3175 & 0,2805 & 0,3387 \\
\hline Cuerda alar & 0,0036 & 0,7318 & 0,0000 & 0,0012 & 0 & $2,8 \mathrm{E}-07$ & & 0,8178 & 0,8301 & 0,8021 \\
\hline Largo de P10 & 0,3362 & 0,5003 & $1,2 \mathrm{E}-07$ & 0,0050 & 9,7E-13 & $5,2 \mathrm{E}-06$ & 0 & & 0,6730 & 0,6508 \\
\hline Largo de S1 & 0,0056 & 0,4132 & $1,2 \mathrm{E}-06$ & 6,7E-06 & 0 & $6,0 \mathrm{E}-05$ & 0 & 0 & & 0,8298 \\
\hline Largo de P1 & 0,0314 & 0,5929 & $4,6 \mathrm{E}-08$ & $5,0 \mathrm{E}-05$ & 0 & 9,9E-07 & 0 & 0 & 0 & \\
\hline
\end{tabular}

Los valores en negrilla corresponden a $p$ de Hollander y Wolfe $<0,05$ 
Tabla 4. Valores de normalidad de Shapiro-Wilks para las variables morfométricas seleccionadas.

\begin{tabular}{|c|c|c|c|c|c|c|c|c|c|c|}
\hline & $\begin{array}{c}\text { Culmen } \\
\text { (n) }\end{array}$ & $\begin{array}{l}\text { Alto } \\
\text { del } \\
\text { pico }\end{array}$ & Tarso & $\begin{array}{l}\text { Ancho } \\
\text { distal } \\
\text { del } \\
\text { tarso }\end{array}$ & Cola & $\begin{array}{l}\text { Ancho } \\
\text { de la } \\
\text { rectriz } \\
\text { central }\end{array}$ & $\begin{array}{c}\text { Cuerda } \\
\text { alar }\end{array}$ & $\begin{array}{l}\text { Largo } \\
\text { de P10 }\end{array}$ & $\begin{array}{l}\text { Largo } \\
\text { de S1 }\end{array}$ & $\begin{array}{l}\text { Largo } \\
\text { de P1 }\end{array}$ \\
\hline UTO 1 & 0,2508 & 0,0043 & 0,5791 & 0,0398 & 0,2150 & 0,3867 & 0,1122 & 0,1155 & 0,2361 & 0,5410 \\
\hline UTO 2 & 0,4043 & 0,2733 & 0,5308 & 0,8737 & 0,1507 & 0,1885 & 0,5266 & 0,7156 & 0,3809 & 0,1542 \\
\hline UTO 3 & 0,9435 & 0,4428 & 0,7104 & 0,4397 & 0,3228 & 0,6564 & 0,6921 & 0,1730 & 0,7073 & 0,8763 \\
\hline UTO 4 & 0,8967 & 0,2225 & 0,2203 & 0,6761 & 0,7225 & 0,8319 & 0,3517 & 0,3890 & 0,0795 & 0,0608 \\
\hline
\end{tabular}

Los valores en negrilla corresponden a Shapiro-Wilks $<0,05$ 
Tabla 5. Parámetros y evaluación de supuestos de análisis de MANOVA en los modelos 1, 2 y 3.

\begin{tabular}{lccc} 
& Modelo 1 & Modelo 2 & Modelo 3 \\
\hline Numero de variables & 10 & 7 & 4 \\
& $\begin{array}{c}\text { Culmen (n), alto del pico, } \\
\text { tarso, ancho distal del } \\
\text { tarso, cola, ancho de la }\end{array}$ & Culmen total, culmen (n), & Culmen (n), tarso, cola y \\
Variables & alto del pico, tarso, cola, & cuerda alar \\
alar, largo de P1, largo de & cuerda alar y largo de S1 & \\
P10 y largo de S1 & &
\end{tabular}

Normalidad univariada

(Shapiro-Wilks)

SI

SI

SI

Normalidad multivariada

(M Shapiro)

$\begin{array}{ll}0,000000118 & 0,000000118\end{array}$

0,232

Homogeneidad de

covarianzas (Box's M)

$\begin{array}{lll}\mathbf{0 , 0 2 0 6} & \mathbf{0 , 0 2 1 8} & 0,108\end{array}$

Cumple los supuestos

NO

NO

SI

Los valores en negrilla indican $p<0,05$ 
Tabla 6. Resultados de MANOVA para las variables morfométricas del modelo 1.

\begin{tabular}{lrcrrrr} 
& DF & test stat & approx F & num Df & den Df & p value \\
\hline Pillai & 4 & 0,4213688 & 6,240527 & 16 & 848 & $\mathbf{2 , 7 7 E - 1 3}$ \\
Wilks & 4 & 0,6225665 & 6,703021 & 16 & 639,1432 & $\mathbf{3 , 1 6 E - 1 4}$ \\
Hotelling-Lawley & 4 & 0,5369313 & 6,963328 & 16 & 830 & $\mathbf{3 , 3 2 E - 1 5}$ \\
Roy & 4 & 0,3551185 & 18,821279 & 4 & 212 & $\mathbf{2 , 9 5 E - 1 3}$
\end{tabular}

Valores en negrilla indican $p<0,05$ 
Tabla 7. Soporte de diferencias entre los grupos de UTO en las pruebas de comparación por pares. Se muestran los valores $p$ de Tukey-Kramer. El soporte total es el resultado de la sumatoria de las variables por cada prueba de comparación.

\begin{tabular}{|c|c|c|c|c|c|c|c|}
\hline Variables & Grupo 1 & Grupo 2 & Valor $p$ & $\begin{array}{l}\text { Tukey- } \\
\text { Kramer }\end{array}$ & $\begin{array}{l}\text { Games- } \\
\text { Howell }\end{array}$ & Dunnet & $\begin{array}{c}\text { Soporte } \\
\text { total }\end{array}$ \\
\hline Cola & & & $4,62 E-10$ & 1 & 1 & 1 & \\
\hline $\begin{array}{l}\text { Cuerda alar } \\
\text { Culmen (n) }\end{array}$ & UTO 3 & UTO 4 & $\begin{array}{c}\text { 1,36E-06 } \\
0,514\end{array}$ & 1 & 1 & 1 & 8 \\
\hline Tarso & & & 0,0212 & 1 & 1 & & \\
\hline Cola & & & 0,889 & & & & \\
\hline $\begin{array}{l}\text { Cuerda alar } \\
\text { Culmen (n) }\end{array}$ & UTO 3 & UTO 1 & $\begin{array}{r}0,00186 \\
5,06 E-05\end{array}$ & $\begin{array}{l}1 \\
1\end{array}$ & & $\begin{array}{l}1 \\
1\end{array}$ & 4 \\
\hline Tarso & & & 0,337 & & & & \\
\hline Cola & & & 0,963 & & & & \\
\hline $\begin{array}{l}\text { Cuerda alar } \\
\text { Culmen (n) }\end{array}$ & UTO 3 & UTO 2 & $\begin{array}{l}0,921 \\
0,988\end{array}$ & & & & 0 \\
\hline Tarso & & & 0,938 & & & & \\
\hline Cola & & & $9,53 \mathrm{E}-12$ & 1 & & 1 & \\
\hline $\begin{array}{l}\text { Cuerda alar } \\
\text { Culmen (n) }\end{array}$ & UTO 4 & UTO 1 & $\begin{array}{c}1,50 E-11 \\
0,813\end{array}$ & 1 & & 1 & 6 \\
\hline Tarso & & & 0,000794 & 1 & & 1 & \\
\hline Cola & & & $2,73 E-08$ & 1 & & 1 & \\
\hline $\begin{array}{l}\text { Cuerda alar } \\
\text { Culmen (n) }\end{array}$ & UTO 4 & UTO 2 & $\begin{array}{c}\text { 9,84E-07 } \\
0,699\end{array}$ & 1 & & 1 & 6 \\
\hline Tarso & & & 0,0122 & 1 & & 1 & \\
\hline Cola & & & 0,687 & & & 1 & \\
\hline $\begin{array}{l}\text { Cuerda alar } \\
\text { Culmen (n) }\end{array}$ & UTO 1 & UTO 2 & $\begin{array}{c}0,126 \\
\mathbf{0 , 0 0 6 8 7}\end{array}$ & 1 & 1 & $\begin{array}{l}1 \\
1\end{array}$ & 5 \\
\hline Tarso & & & 0,898 & & & & \\
\hline
\end{tabular}

Los valores en negrilla indican $p<0,05$ 
Tabla 8. Valores $p$ de $t$-student para dimorfismo sexual de las Unidades taxonómicas operativas.

\begin{tabular}{|c|c|c|c|c|c|c|c|}
\hline Variable & UTO & Grupo 1 & Grupo 2 & n1 & $\mathrm{n} 2$ & Df & Valor $p$ \\
\hline \multirow{4}{*}{ Alto de pico } & 3 & $F$ & $M$ & 22 & 27 & 4,5195 & 1 \\
\hline & 4 & $\mathrm{~F}$ & M & 4 & 5 & 4,9721 & 1 \\
\hline & 1 & $\mathrm{~F}$ & M & 52 & 60 & 5,3199 & 1 \\
\hline & 2 & $\mathrm{~F}$ & $M$ & 15 & 15 & 2,7902 & 1 \\
\hline \multirow{4}{*}{$\begin{array}{c}\text { Ancho de la rectriz } \\
\text { central }\end{array}$} & 3 & $\mathrm{~F}$ & $M$ & 22 & 27 & 4,6875 & 1 \\
\hline & 4 & $\mathrm{~F}$ & $M$ & 4 & 5 & 6,9008 & 1 \\
\hline & 1 & $\mathrm{~F}$ & $M$ & 52 & 60 & 1,0688 & 1 \\
\hline & 2 & $\mathrm{~F}$ & $M$ & 15 & 15 & 2,4891 & 0,456 \\
\hline \multirow{4}{*}{ Ancho distal del tarso } & 3 & $\mathrm{~F}$ & $M$ & 22 & 27 & 4,2065 & 0,788 \\
\hline & 4 & $\mathrm{~F}$ & $M$ & 4 & 5 & 6,8804 & 1 \\
\hline & 1 & $\mathrm{~F}$ & $M$ & 52 & 60 & 1,0580 & 1 \\
\hline & 2 & $\mathrm{~F}$ & $M$ & 15 & 15 & 2,4291 & 1 \\
\hline \multirow{4}{*}{ Cola } & 3 & $\mathrm{~F}$ & $M$ & 22 & 27 & 4,5025 & 0,1524 \\
\hline & 4 & $\mathrm{~F}$ & $M$ & 4 & 5 & 6,7514 & 1 \\
\hline & 1 & $\mathrm{~F}$ & $M$ & 52 & 60 & 9,8851 & 0,796 \\
\hline & 2 & $\mathrm{~F}$ & $M$ & 15 & 15 & 2,7598 & 0,456 \\
\hline \multirow{4}{*}{ Cuerda alar } & 3 & $\mathrm{~F}$ & $M$ & 22 & 27 & 4,0644 & 0,112 \\
\hline & 4 & $\mathrm{~F}$ & $M$ & 4 & 5 & 6,6191 & 1 \\
\hline & 1 & $\mathrm{~F}$ & $M$ & 52 & 60 & 1,0949 & $1,48 \mathrm{E}-13$ \\
\hline & 2 & $\mathrm{~F}$ & $M$ & 15 & 15 & 2,7910 & 0,0876 \\
\hline \multirow{4}{*}{ Culmen (n) } & 3 & $\mathrm{~F}$ & $M$ & 22 & 27 & 3,9371 & 1 \\
\hline & 4 & $\mathrm{~F}$ & $M$ & 4 & 5 & 6,9685 & 1 \\
\hline & 1 & $\mathrm{~F}$ & $M$ & 52 & 60 & 1,0997 & 1 \\
\hline & 2 & $\mathrm{~F}$ & $M$ & 15 & 15 & 2,1061 & 1 \\
\hline \multirow{4}{*}{ Largo de P1 } & 3 & $\mathrm{~F}$ & $M$ & 22 & 27 & 4,1857 & 1 \\
\hline & 4 & $\mathrm{~F}$ & $M$ & 4 & 5 & 5,5005 & 1 \\
\hline & 1 & $\mathrm{~F}$ & $M$ & 52 & 60 & 1,1000 & 0,00238 \\
\hline & 2 & $\mathrm{~F}$ & $M$ & 15 & 15 & 2,7780 & 1 \\
\hline \multirow{4}{*}{ Largo de P10 } & 3 & $\mathrm{~F}$ & $M$ & 22 & 27 & 4,1455 & 0,00972 \\
\hline & 4 & $\mathrm{~F}$ & $M$ & 4 & 5 & 5,2752 & 0,792 \\
\hline & 1 & $\mathrm{~F}$ & $M$ & 52 & 60 & 1,0951 & $2,96 \mathrm{E}-09$ \\
\hline & 2 & $\mathrm{~F}$ & $M$ & 15 & 15 & 2,7358 & 0,1816 \\
\hline \multirow{4}{*}{ Largo de S1 } & 3 & $\mathrm{~F}$ & $M$ & 22 & 27 & 4,6638 & 1 \\
\hline & 4 & $\mathrm{~F}$ & $M$ & 4 & 5 & 6,0526 & 0,3392 \\
\hline & 1 & $\mathrm{~F}$ & $M$ & 52 & 60 & 1,0733 & $2,20 \mathrm{E}-07$ \\
\hline & 2 & $\mathrm{~F}$ & $M$ & 15 & 15 & 2,7890 & 1 \\
\hline \multirow{4}{*}{ Tarso } & 3 & $\mathrm{~F}$ & $M$ & 22 & 27 & 4,5695 & 0,232 \\
\hline & 4 & $\mathrm{~F}$ & $M$ & 4 & 5 & 4,4521 & 1 \\
\hline & 1 & $\mathrm{~F}$ & M & 52 & 60 & 1,0912 & 0,003824 \\
\hline & 2 & $\mathrm{~F}$ & $M$ & 15 & 15 & 2,8000 & 1 \\
\hline
\end{tabular}

Los valores en negrilla indican $p<0,05 ; D f=$ grados de libertad; $F=$ hembras; $M=$ machos; $n=$ número de observaciones por grupo 
Tabla 9. Resumen descriptivo de las características auditivas de las notas identificadas.

\begin{tabular}{ccccc} 
Nota & Frecuencia pico $(\mathrm{Hz})$ & Frecuencia central $(\mathbf{H z})$ & Ancho de banda al 90\% (Hz) & Duración (s) \\
\hline A & $3715,21 \pm 896,88$ & $3538,14 \pm 804,53$ & $1022,58 \pm 476,89$ & $0,87 \pm 0,2$ \\
B & $4951,41 \pm 1452,23$ & $4764,73 \pm 1324,16$ & $910,25 \pm 264,42$ & $0,56 \pm 0,08$ \\
C & $4094,86 \pm 712,36$ & $3883,7 \pm 643,02$ & $1803,19 \pm 514,12$ & $0,32 \pm 0,19$ \\
D & $2902,21 \pm 309,33$ & $2904,53 \pm 310,39$ & $385,02 \pm 178,44$ & $1,11 \pm 0,27$ \\
F & $4986,05 \pm 108,12$ & $4986,05 \pm 108,12$ & $399 \pm 166,59$ & $0,29 \pm 0,06$ \\
\hline I = Desviación estándar &
\end{tabular}


Tabla 10. Valores $p$ de ANOVA para los parámetros vocales de las UTO.

\begin{tabular}{|c|c|c|}
\hline Tipo de nota & Variable & Valor $p$ corregido \\
\hline \multirow{10}{*}{$A$} & Frecuencia más baja (Hz) & 0,570 \\
\hline & Frecuencia más alta $(\mathrm{Hz})$ & 1 \\
\hline & Frecuencia pico $(\mathrm{Hz})$ & 1 \\
\hline & PFC de la máxima frecuencia $(\mathrm{Hz})$ & 1 \\
\hline & PFC de la mínima frecuencia $(\mathrm{Hz})$ & 1 \\
\hline & Ancho de banda al $90 \%(\mathrm{~Hz})$ & 1 \\
\hline & Delta de frecuencia $(\mathrm{Hz})$ & 0,182 \\
\hline & Duración (s) & 1 \\
\hline & Frecuencia central (Hz) & 1 \\
\hline & Máxima frecuencia $(\mathrm{Hz})$ & 1 \\
\hline \multirow{10}{*}{ C } & Frecuencia más baja (Hz) & 1 \\
\hline & Frecuencia más alta $(\mathrm{Hz})$ & 1 \\
\hline & Frecuencia pico $(\mathrm{Hz})$ & 1 \\
\hline & PFC de la máxima frecuencia $(\mathrm{Hz})$ & 1 \\
\hline & PFC de la mínima frecuencia $(\mathrm{Hz})$ & 1 \\
\hline & Ancho de banda al $90 \%(\mathrm{~Hz})$ & 1 \\
\hline & Delta de frecuencia $(\mathrm{Hz})$ & 1 \\
\hline & Duración (s) & 1 \\
\hline & Frecuencia central (Hz) & 1 \\
\hline & Máxima frecuencia $(\mathrm{Hz})$ & 1 \\
\hline \multirow{10}{*}{ D } & Frecuencia más baja (Hz) & 1,27E-06 \\
\hline & Frecuencia más alta $(\mathrm{Hz})$ & $4,90 \mathrm{E}-12$ \\
\hline & Frecuencia pico $(\mathrm{Hz})$ & $4,00 E-11$ \\
\hline & PFC de la máxima frecuencia $(\mathrm{Hz})$ & $1,21 E-11$ \\
\hline & PFC de la mínima frecuencia $(\mathrm{Hz})$ & 3,21E-06 \\
\hline & Ancho de banda al $90 \%(\mathrm{~Hz})$ & $1,00 E+06$ \\
\hline & Delta de frecuencia $(\mathrm{Hz})$ & $7,84 \mathrm{E}+02$ \\
\hline & Duración (s) & $1,45 E+03$ \\
\hline & Frecuencia central (Hz) & $9,30 E-13$ \\
\hline & Máxima frecuencia $(\mathrm{Hz})$ & $4,00 E-11$ \\
\hline \multirow{10}{*}{ Z } & Frecuencia más baja (Hz) & 1 \\
\hline & Frecuencia más alta $(\mathrm{Hz})$ & 1 \\
\hline & Frecuencia pico $(\mathrm{Hz})$ & 1 \\
\hline & PFC de la máxima frecuencia $(\mathrm{Hz})$ & 1 \\
\hline & PFC de la mínima frecuencia $(\mathrm{Hz})$ & 1 \\
\hline & Ancho de banda al $90 \%(\mathrm{~Hz})$ & 1 \\
\hline & Delta de frecuencia $(\mathrm{Hz})$ & 1 \\
\hline & Duración (s) & 1 \\
\hline & Frecuencia central (Hz) & 1 \\
\hline & Máxima frecuencia $(\mathrm{Hz})$ & 1 \\
\hline
\end{tabular}


Tabla 11. Resultados de ANOVA para los atributos del canto de comunicación.

\begin{tabular}{ccccc} 
Variable & Df & Sum of Squares & Res std error & p value \\
\hline Índice de diversidad (iD) & 3 & 0,650359 & 0,2373292 & $\mathbf{0 , 0 4 1 6 6 0 3 9}$ \\
Número de notas & 3 & 4,020915 & 2,043568 & $\mathbf{0 , 0 4 4 0 7 7 4 8}$ \\
Cadencia del trino & 3 & 1,886876 & 0,4924363 & 0,06099507 \\
\hline
\end{tabular}

Valores en negrilla indican $p<0,05$ 
Tabla 12. Valores de $\mathrm{p}$ de Tukey-Kramer para el índice de diversidad y el número de notas del canto de las UTO.

\begin{tabular}{cccccc} 
Variable & Comparación UTO & Diff & Lwr & Upr & p value \\
\hline & $2-1$ & 0,0652 & $-0,2115$ & 0,3419 & 0,9243 \\
& $3-1$ & 0,2049 & $-0,0085$ & 0,4182 & 0,0642 \\
Índice de & $4-1$ & 0,2949 & 0,0329 & 0,5568 & $\mathbf{0 , 0 2 1 4}$ \\
diversidad (iD) & $3-2$ & 0,1397 & $-0,0979$ & 0,3772 & 0,4124 \\
& $4-2$ & 0,2297 & $-0,0524$ & 0,5117 & 0,1487 \\
& $4-3$ & 0,0900 & $-0,1302$ & 0,3102 & 0,7028 \\
& $4-1$ & 1,1136 & $-1,1416$ & 3,3689 & 0,5632 \\
Número de notas & $3-1$ & 1,6532 & $-0,1836$ & 3,4901 & 0,0923 \\
& $2-1$ & 2,6389 & 0,2565 & 5,0213 & $\mathbf{0 , 0 2 4 3}$ \\
& $3-4$ & 0,5396 & $-1,3565$ & 2,4357 & 0,8753 \\
& $2-4$ & 1,5253 & $-0,9031$ & 3,9536 & 0,3535 \\
& $2-3$ & 0,9857 & $-1,0600$ & 3,0314 & 0,5830
\end{tabular}

Los valores en negrilla indican $p<0,05$ 
Tabla 13. Atributos principales del canto de comunicación de las Unidades Taxonómicas Operativas (UTO).

\begin{tabular}{lcccc}
\multicolumn{1}{c}{ Atributo } & UTO 1 & UTO 2 & UTO 3 & UTO 4 \\
\hline Número de tipos de notas & 4 & 4 & 5 & 3 \\
Tipos de notas & A, B, C, F & A, B, C, F & A, B, C, F, D & A, C, F \\
Índice de diversidad (iD) & $0,52 \pm 0,22$ & $0,46 \pm 0,25$ & $0,32 \pm 0,24$ & $0,23 \pm 0,21$ \\
Longitud (notas) & $6,25 \pm 2,18$ & $8,88 \pm 3,14$ & $7,9 \pm 1,78$ & $7,36 \pm 1,43$ \\
Tiempo (s) & $2,89 \pm 0,82$ & $3,53 \pm 1,22$ & $3,54 \pm 0,82$ & $3,35 \pm 0,88$ \\
Longitud del trino (notas) & $4,41 \pm 2,10$ & $7,22 \pm 3,15$ & $6,45 \pm 1,63$ & $6,18 \pm 1,60$ \\
Cadencia del trino (notas/s) & $2,90 \pm 0,69$ & $2,78 \pm 0,72$ & $2,48 \pm 0,28$ & $2,69 \pm 0,50$ \\
\hline $\mathbf{\pm}=$ Desviación estándar & & & & \\
\hline
\end{tabular}


Tabla 14. Matriz de soporte de diferencias entre taxones. Se muestra la matriz de diferencias en los caracteres de coloración, morfometría y vocalizaciones; en la comparación por pares de taxones (unidades taxonómicas operativas) analizados. Los valores en negrilla indican el número de caracteres de soporte en la diferencia entre comparaciones de las UTO. * ausencia de soporte para la comparación especifica (morfometría = 0 en comparación UTO 2 -3).

\begin{tabular}{|c|c|c|c|c|c|c|c|}
\hline & Comparación (UTO) & $1--2$ & 1--3 & $1--4$ & 2--3 & $2--4$ & 3--4 \\
\hline \multirow{11}{*}{ Coloración } & Corona & 0 & 0 & 1 & 0 & 1 & 1 \\
\hline & Coronilla (medial) & 1 & 1 & 1 & 0 & 0 & 0 \\
\hline & Dorso y supracaudales (basal) & 0 & 1 & 1 & 1 & 1 & 0 \\
\hline & Dorso (distal) & 0 & 1 & 1 & 1 & 1 & 1 \\
\hline & Plumas de arrastre & 0 & 1 & 0 & 1 & 0 & 1 \\
\hline & Borde primarias & 1 & 0 & 1 & 1 & 1 & 1 \\
\hline & Borde secundarias y rectrices & 1 & 1 & 1 & 1 & 0 & 1 \\
\hline & $\begin{array}{l}\text { Coberteras menores y mayores } \\
\text { (basal) }\end{array}$ & 0 & 1 & 0 & 1 & 0 & 1 \\
\hline & $\begin{array}{l}\text { Coberteras menores y mayores } \\
\text { (distal) }\end{array}$ & 1 & 1 & 1 & 1 & 1 & 1 \\
\hline & Supracaudales (distal) & 1 & 1 & 1 & 1 & 1 & 1 \\
\hline & Rectrices & 0 & 1 & 0 & 1 & 0 & 1 \\
\hline \multirow{4}{*}{ Morfometría } & Cola & 0 & 0 & 1 & 0 & 1 & 1 \\
\hline & Cuerda alar & 0 & 1 & 1 & 0 & 1 & 1 \\
\hline & Culmen (n) & 1 & 1 & 0 & 0 & 0 & 0 \\
\hline & Tarso & 0 & 0 & 1 & 0 & 1 & 1 \\
\hline \multirow{11}{*}{ Vocalizaciones } & Índice de diversidad (iD) & 0 & 0 & 1 & 0 & 0 & 0 \\
\hline & Número de notas & 1 & 0 & 0 & 0 & 0 & 0 \\
\hline & Frecuencia central (D) & 1 & 1 & 0 & 0 & 1 & 0 \\
\hline & Delta de frecuencia (D) & 0 & 0 & 1 & 1 & 0 & 1 \\
\hline & Duración (D) & 1 & 1 & 0 & 0 & 0 & 0 \\
\hline & Frecuencia más alta (D) & 1 & 1 & 0 & 0 & 1 & 1 \\
\hline & Frecuencia más baja (D) & 1 & 1 & 1 & 0 & 0 & 0 \\
\hline & Máxima frecuencia (D) & 1 & 1 & 0 & 0 & 0 & 1 \\
\hline & Frecuencia pico (D) & 1 & 1 & 0 & 0 & 0 & 1 \\
\hline & PFC de la máxima frecuencia (D) & 1 & 1 & 0 & 0 & 0 & 1 \\
\hline & PFC de la mínima frecuencia (D) & 1 & 1 & 1 & 0 & 0 & 0 \\
\hline \multicolumn{8}{|c|}{ Sumatoria } \\
\hline & Coloración & 5 & 9 & 8 & 9 & 6 & 9 \\
\hline & Morfometría & 1 & 2 & 3 & $0 *$ & 3 & 3 \\
\hline & Vocalizaciones & 9 & 8 & 4 & 1 & 2 & 5 \\
\hline & Totales & 15 & 19 & 15 & 10 & 11 & 17 \\
\hline
\end{tabular}




\section{ANEXOS}

Anexo A. Distribución del material de Myiozetetes cayanensis analizado. Se muestra la localización del material de colección (pieles), bancos de sonido (archivos de audio) y repositorios virtuales (fotografías), de Myiozetetes cayanensis analizado en el presente estudio.

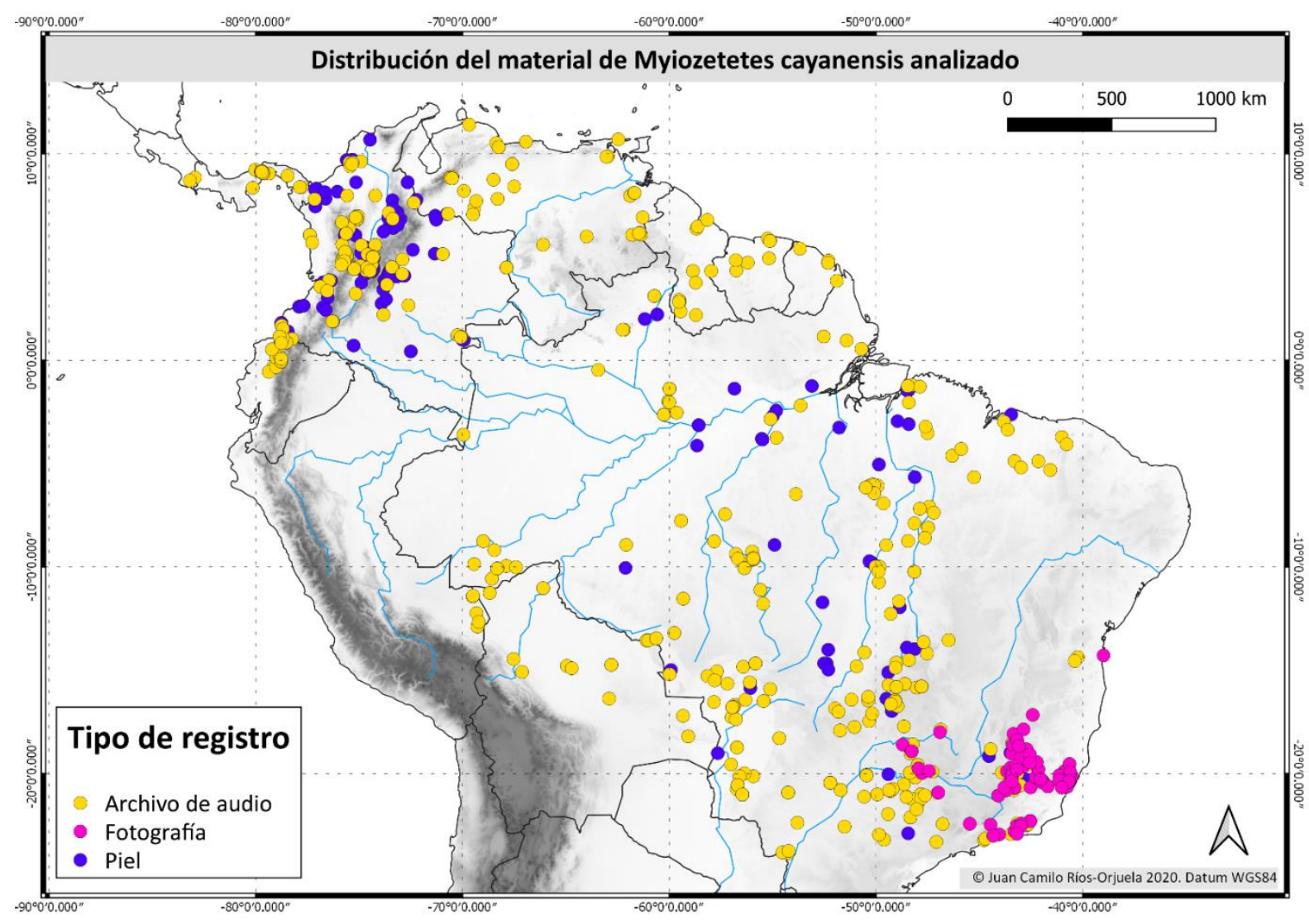

Para ver el mapa interactivo de registros siga el enlace: jcrioso.github.io/Mapa myiozetetes cayanensis/

O el escanee el siguiente codigo QR:

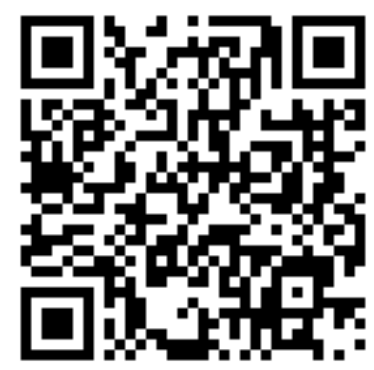


Anexo B. Material de Myiozetetes cayanensis analizado. Se muestra la lista completa del material de colección (pieles), bancos de sonido (archivos de audio) y repositorios virtuales (fotografías), de Myiozetetes cayanensis analizado en el presente estudio, así como su respectiva localidad, coordenadas y número de catálogo. IAvH: Instituto Alexander von Humboldt, ICN: Instituto de Ciencias Naturales de la Universidad Nacional de Colombia, MCN: Museu de Ciências Naturais da PUC-Minas, MZUSP: Museu de Zoologia da Universidade de São Paulo, ML: Macaulay Library del Cornell Lab of Ornithology, WK: wikiaves.com.br, XC: Xeno-canto.org.

Esta información puede ser descargada en formato CSV en el repositorio: https://github.com/jcrioso/Mapa myiozetetes cayanensis

\begin{tabular}{|c|c|c|c|c|c|c|c|c|c|c|}
\hline $\begin{array}{l}\text { Nombre } \\
\text { científico }\end{array}$ & Subespecie & UTO & $\begin{array}{l}\text { Número de } \\
\text { catalogo }\end{array}$ & Colección & País & Estado & Localidad & $\begin{array}{l}\text { Latitud } \\
\text { decimal }\end{array}$ & $\begin{array}{l}\text { Longitud } \\
\text { decimal }\end{array}$ & $\begin{array}{l}\text { Tipo de } \\
\text { registro }\end{array}$ \\
\hline $\begin{array}{l}\text { Myiozetetes } \\
\text { cayanensis }\end{array}$ & hellmayri & 1 & 1352 & MZUSP & Venezuela & Táchira & San Cristobal & 7,771213 & $-72,226289$ & Piel \\
\hline $\begin{array}{l}\text { Myiozetetes } \\
\text { cayanensis }\end{array}$ & cayanensis & 3 & 7188 & MZUSP & Brasil & Maranhão & Miritiba & $-2,616668$ & $-43,450002$ & Piel \\
\hline $\begin{array}{l}\text { Myiozetetes } \\
\text { cayanensis }\end{array}$ & hellmayri & 1 & 13586 & MZUSP & Colombia & Cauca & San Antonio & 3,032479 & $-76,513594$ & Piel \\
\hline $\begin{array}{l}\text { Myiozetetes } \\
\text { cayanensis }\end{array}$ & hellmayri & 1 & 13716 & MZUSP & Colombia & Tolima & $\begin{array}{l}\text { Honda/Rio } \\
\text { Magdalena }\end{array}$ & 5,207129 & $-74,73639$ & Piel \\
\hline $\begin{array}{l}\text { Myiozetetes } \\
\text { cayanensis }\end{array}$ & cayanensis & 3 & 15535 & MZUSP & Brasil & Goiás & $\begin{array}{c}\text { Rio das Almas, } \\
\text { Fazenda Manuel } \\
\text { Peixoto }\end{array}$ & $-15,103258$ & $-49,405328$ & Piel \\
\hline $\begin{array}{l}\text { Myiozetetes } \\
\text { cayanensis }\end{array}$ & cayanensis & 3 & 15536 & MZUSP & Brasil & Goiás & $\begin{array}{c}\text { Inhumas, antigo } \\
\text { Goiabeiras }\end{array}$ & $-16,373117$ & $-49,491677$ & Piel \\
\hline $\begin{array}{l}\text { Myiozetetes } \\
\text { cayanensis }\end{array}$ & cayanensis & 3 & 17204 & MZUSP & Brasil & Mato Grosso & $\begin{array}{c}\text { Cuiabá, Santo } \\
\text { Antônio, Fazenda } \\
\text { Maravilha }\end{array}$ & $-15,86145$ & $-56,076138$ & Piel \\
\hline $\begin{array}{l}\text { Myiozetetes } \\
\text { cayanensis }\end{array}$ & cayanensis & 3 & 17958 & MZUSP & Brasil & Amazonas & $\begin{array}{c}\text { Rio Amazonas } \\
\text { (Norte), Itacoatiara }\end{array}$ & $-3,134267$ & $-58,574731$ & Piel \\
\hline $\begin{array}{l}\text { Myiozetetes } \\
\text { cayanensis }\end{array}$ & cayanensis & 3 & 17985 & MZUSP & Brasil & Mato Grosso & Vale do Araguaia & $-14,00197$ & $-52,307231$ & Piel \\
\hline $\begin{array}{l}\text { Myiozetetes } \\
\text { cayanensis }\end{array}$ & cayanensis & 3 & 19789 & MZUSP & Brasil & Amazonas & $\begin{array}{c}\text { Rio Amazonas } \\
\text { (Norte), Itacoatiara }\end{array}$ & $-3,134267$ & $-58,574731$ & Piel \\
\hline $\begin{array}{l}\text { Myiozetetes } \\
\text { cayanensis }\end{array}$ & cayanensis & 3 & 19797 & MZUSP & Brasil & Amazonas & $\begin{array}{c}\text { Rio Amazonas } \\
\text { (Norte), Itacoatiara }\end{array}$ & $-3,134267$ & $-58,574731$ & Piel \\
\hline $\begin{array}{l}\text { Myiozetetes } \\
\text { cayanensis }\end{array}$ & cayanensis & 3 & 19804 & MZUSP & Brasil & Amazonas & $\begin{array}{c}\text { Rio Amazonas } \\
\text { (Norte), Itacoatiara }\end{array}$ & $-3,134267$ & $-58,574731$ & Piel \\
\hline $\begin{array}{l}\text { Myiozetetes } \\
\text { cayanensis }\end{array}$ & cayanensis & 3 & 20219 & MZUSP & Brasil & Amazonas & $\begin{array}{l}\text { Rio Amazonas } \\
\text { (Norte), Itacoatiara }\end{array}$ & $-3,134267$ & $-58,574731$ & Piel \\
\hline $\begin{array}{l}\text { Myiozetetes } \\
\text { cayanensis }\end{array}$ & cayanensis & 3 & 22649 & MZUSP & Brasil & Pará & $\begin{array}{c}\text { Rio Amazonas } \\
\text { (Norte), Igarapé } \\
\text { Bravo }\end{array}$ & $-1,235994$ & $-53,097461$ & Piel \\
\hline $\begin{array}{l}\text { Myiozetetes } \\
\text { cayanensis }\end{array}$ & cayanensis & 3 & 22674 & MZUSP & Brasil & Pará & $\begin{array}{c}\text { Boca do rio } \\
\text { Tapajós, Santarém }\end{array}$ & $-2,429683$ & $-54,822907$ & Piel \\
\hline $\begin{array}{l}\text { Myiozetetes } \\
\text { cayanensis }\end{array}$ & erythropterus & 4 & 25355 & MZUSP & Brasil & Minas Gerais & $\begin{array}{c}\text { São José da Lagôa, } \\
\text { Fazenda Boa } \\
\text { Esperança }\end{array}$ & $-19,14704$ & $-44,535456$ & Piel \\
\hline $\begin{array}{l}\text { Myiozetetes } \\
\text { cayanensis }\end{array}$ & erythropterus & 4 & 25356 & MZUSP & Brasil & Minas Gerais & $\begin{array}{c}\text { São José da Lagôa, } \\
\text { Fazenda Boa } \\
\text { Esperança }\end{array}$ & $-19,14704$ & $-44,535456$ & Piel \\
\hline
\end{tabular}




\begin{tabular}{|c|c|c|c|c|c|c|c|c|c|c|}
\hline $\begin{array}{l}\text { Myiozetetes } \\
\text { cayanensis }\end{array}$ & erythropterus & 4 & 25357 & MZUSP & Brasil & Minas Gerais & $\begin{array}{c}\text { São José da Lagôa, } \\
\text { Fazenda Boa } \\
\text { Esperança }\end{array}$ & $-19,14704$ & $-44,535456$ & Piel \\
\hline $\begin{array}{l}\text { Myiozetetes } \\
\text { cayanensis }\end{array}$ & erythropterus & 4 & 25358 & MZUSP & Brasil & Minas Gerais & $\begin{array}{c}\text { São José da Lagôa, } \\
\text { Fazenda Boa } \\
\text { Esperança }\end{array}$ & $-19,14704$ & $-44,535456$ & Piel \\
\hline $\begin{array}{l}\text { Myiozetetes } \\
\text { cayanensis }\end{array}$ & erythropterus & 4 & 25359 & MZUSP & Brasil & Minas Gerais & $\begin{array}{c}\text { Rio Doce, Baixo Rio } \\
\text { Piracicaba } \\
\text { (margem } \\
\text { esquerda) }\end{array}$ & $-20,204504$ & $-42,876961$ & Piel \\
\hline $\begin{array}{l}\text { Myiozetetes } \\
\text { cayanensis }\end{array}$ & erythropterus & 4 & 25360 & MZUSP & Brasil & Minas Gerais & $\begin{array}{c}\text { Rio Doce, Baixo Rio } \\
\text { Piracicaba } \\
\text { (margem } \\
\text { esquerda) }\end{array}$ & $-20,204504$ & $-42,876961$ & Piel \\
\hline $\begin{array}{l}\text { Myiozetetes } \\
\text { cayanensis }\end{array}$ & erythropterus & 4 & 25361 & MZUSP & Brasil & Minas Gerais & $\begin{array}{c}\text { Rio Doce, Baixo Rio } \\
\text { Piracicaba } \\
\text { (margem } \\
\text { esquerda) }\end{array}$ & $-20,204504$ & $-42,876961$ & Piel \\
\hline $\begin{array}{l}\text { Myiozetetes } \\
\text { cayanensis }\end{array}$ & cayanensis & 3 & 30290 & MZUSP & Brasil & $\begin{array}{c}\text { Mato Grosso do } \\
\text { Sul }\end{array}$ & Corumbá & $-19,010606$ & $-57,65084$ & Piel \\
\hline $\begin{array}{l}\text { Myiozetetes } \\
\text { cayanensis }\end{array}$ & cayanensis & 3 & 30291 & MZUSP & Brasil & Mato Grosso & Cuiabá & $-15,600483$ & $-56,095926$ & Piel \\
\hline $\begin{array}{l}\text { Myiozetetes } \\
\text { cayanensis }\end{array}$ & cayanensis & 3 & 32500 & MZUSP & Brasil & Mato Grosso & Chavantina & $-14,66504$ & $-52,384392$ & Piel \\
\hline $\begin{array}{l}\text { Myiozetetes } \\
\text { cayanensis }\end{array}$ & cayanensis & 3 & 32715 & MZUSP & Brasil & Pará & $\begin{array}{c}\text { Rio Tapajós, } \\
\text { Aramanaí }\end{array}$ & $-2,678354$ & $-54,974773$ & Piel \\
\hline $\begin{array}{l}\text { Myiozetetes } \\
\text { cayanensis }\end{array}$ & cayanensis & 3 & 35218 & MZUSP & Brasil & Mato Grosso & $\begin{array}{l}\text { Rio das Mortes, } \\
\text { São Domingos }\end{array}$ & $-14,66302$ & $-52,511103$ & Piel \\
\hline $\begin{array}{l}\text { Myiozetetes } \\
\text { cayanensis }\end{array}$ & cayanensis & 3 & 35219 & MZUSP & Brasil & Mato Grosso & $\begin{array}{c}\text { Molha Saco, } \\
\text { Pindaíba São } \\
\text { Domingos }\end{array}$ & $-14,96454$ & $-52,30278$ & Piel \\
\hline $\begin{array}{l}\text { Myiozetetes } \\
\text { cayanensis }\end{array}$ & cayanensis & 3 & 36256 & MZUSP & Brasil & Pará & $\begin{array}{l}\text { Utinga, arredores } \\
\text { de Belém }\end{array}$ & $-1,426068$ & $-48,442564$ & Piel \\
\hline $\begin{array}{l}\text { Myiozetetes } \\
\text { cayanensis }\end{array}$ & cayanensis & 3 & 37789 & MZUSP & Brasil & Pará & $\begin{array}{l}\text { Utinga, arredores } \\
\text { de Belém }\end{array}$ & $-1,426068$ & $-48,442564$ & Piel \\
\hline $\begin{array}{l}\text { Myiozetetes } \\
\text { cayanensis }\end{array}$ & cayanensis & 3 & 37790 & MZUSP & Brasil & Pará & $\begin{array}{l}\text { Murutucu, } \\
\text { arredores de } \\
\text { Belém }\end{array}$ & $-1,443162$ & $-48,418921$ & Piel \\
\hline $\begin{array}{l}\text { Myiozetetes } \\
\text { cayanensis }\end{array}$ & cayanensis & 3 & 38547 & MZUSP & Brasil & Pará & Cachimbo & $-8,927258$ & $-54,905308$ & Piel \\
\hline $\begin{array}{l}\text { Myiozetetes } \\
\text { cayanensis }\end{array}$ & cayanensis & 3 & 45536 & MZUSP & Brasil & Pará & Belém, Utinga & $-1,426068$ & $-48,442564$ & Piel \\
\hline $\begin{array}{l}\text { Myiozetetes } \\
\text { cayanensis }\end{array}$ & cayanensis & 3 & 47050 & MZUSP & Brasil & Pará & $\begin{array}{c}\text { Rio Tapajós (leste) } \\
\text { Fordlândia }\end{array}$ & $-3,829163$ & $-55,494504$ & Piel \\
\hline $\begin{array}{l}\text { Myiozetetes } \\
\text { cayanensis }\end{array}$ & cayanensis & 3 & 47051 & MZUSP & Brasil & Pará & $\begin{array}{c}\text { Rio Tapajós (leste) } \\
\text { Fordlândia }\end{array}$ & $-3,829163$ & $-55,494504$ & Piel \\
\hline $\begin{array}{l}\text { Myiozetetes } \\
\text { cayanensis }\end{array}$ & cayanensis & 3 & 52801 & MZUSP & Brasil & Tocantins & Araguatins & $-5,648427$ & $-48,11944$ & Piel \\
\hline $\begin{array}{l}\text { Myiozetetes } \\
\text { cayanensis }\end{array}$ & cayanensis & 3 & 52802 & MZUSP & Brasil & Tocantins & Araguatins & $-5,648427$ & $-48,11944$ & Piel \\
\hline $\begin{array}{l}\text { Myiozetetes } \\
\text { cayanensis }\end{array}$ & cayanensis & 3 & 52803 & MZUSP & Brasil & Tocantins & Araguatins & $-5,648427$ & $-48,11944$ & Piel \\
\hline $\begin{array}{l}\text { Myiozetetes } \\
\text { cayanensis }\end{array}$ & cayanensis & 3 & 52804 & MZUSP & Brasil & Tocantins & Araguatins & $-5,648427$ & $-48,11944$ & Piel \\
\hline $\begin{array}{l}\text { Myiozetetes } \\
\text { cayanensis }\end{array}$ & cayanensis & 3 & 54299 & MZUSP & Brasil & São Paulo & $\begin{array}{c}\text { Rio Grande, Paulo } \\
\text { de Faria }\end{array}$ & $-20,030277$ & $-49,400415$ & Piel \\
\hline $\begin{array}{l}\text { Myiozetetes } \\
\text { cayanensis }\end{array}$ & cayanensis & 3 & 56096 & MZUSP & Brasil & Roraima & $\begin{array}{c}\text { Rio Água Boa do } \\
\text { Mucajaí , afluente } \\
\text { ocidental do Rio } \\
\text { Branco }\end{array}$ & 2,005382 & $-61,182323$ & Piel \\
\hline $\begin{array}{l}\text { Myiozetetes } \\
\text { cayanensis }\end{array}$ & cayanensis & 3 & 58963 & MZUSP & Brasil & Pará & $\begin{array}{c}\text { Rio Tapajós (leste) } \\
\text { Fordlândia }\end{array}$ & $-3,829163$ & $-55,494504$ & Piel \\
\hline $\begin{array}{l}\text { Myiozetetes } \\
\text { cayanensis }\end{array}$ & cayanensis & 3 & 58964 & MZUSP & Brasil & Pará & $\begin{array}{c}\text { Rio Tapajós (leste) } \\
\text { Fordlândia }\end{array}$ & $-3,829163$ & $-55,494504$ & Piel \\
\hline $\begin{array}{l}\text { Myiozetetes } \\
\text { cayanensis }\end{array}$ & cayanensis & 3 & 58965 & MZUSP & Brasil & Pará & $\begin{array}{c}\text { Rio Tapajós (leste) } \\
\text { Fordlândia }\end{array}$ & $-3,829163$ & $-55,494504$ & Piel \\
\hline
\end{tabular}




\begin{tabular}{|c|c|c|c|c|c|c|c|c|c|c|}
\hline $\begin{array}{l}\text { Myiozetetes } \\
\text { cayanensis }\end{array}$ & cayanensis & 3 & 58968 & MZUSP & Brasil & Pará & $\begin{array}{l}\text { Rio Tapajós } \\
\text { (Oeste), } \\
\text { Urucurituba }\end{array}$ & $-3,792585$ & $-55,521189$ & Piel \\
\hline $\begin{array}{l}\text { Myiozetetes } \\
\text { cayanensis }\end{array}$ & cayanensis & 3 & 58969 & MZUSP & Brasil & Pará & $\begin{array}{c}\text { Rio Tapajós (leste) } \\
\text { Fordlândia }\end{array}$ & $-3,829163$ & $-55,494504$ & Piel \\
\hline $\begin{array}{l}\text { Myiozetetes } \\
\text { cayanensis }\end{array}$ & cayanensis & 3 & 58970 & MZUSP & Brasil & Pará & $\begin{array}{c}\text { Rio Tapajós (leste) } \\
\text { Fordlândia }\end{array}$ & $-3,829163$ & $-55,494504$ & Piel \\
\hline $\begin{array}{l}\text { Myiozetetes } \\
\text { cayanensis }\end{array}$ & cayanensis & 3 & 58971 & MZUSP & Brasil & Pará & $\begin{array}{l}\text { Rio Tapajós (leste) } \\
\text { Fordlândia }\end{array}$ & $-3,829163$ & $-55,494504$ & Piel \\
\hline $\begin{array}{l}\text { Myiozetetes } \\
\text { cayanensis }\end{array}$ & cayanensis & 3 & 59594 & MZUSP & Brasil & Pará & Belém do Pará & $-1,455763$ & $-48,490205$ & Piel \\
\hline $\begin{array}{l}\text { Myiozetetes } \\
\text { cayanensis }\end{array}$ & cayanensis & 3 & 60041 & MZUSP & Brasil & Pará & $\begin{array}{l}\text { Jacaré, Rio } \\
\text { Trombetas }\end{array}$ & $-1,366919$ & $-56,841994$ & Piel \\
\hline $\begin{array}{l}\text { Myiozetetes } \\
\text { cayanensis }\end{array}$ & cayanensis & 3 & 60042 & MZUSP & Brasil & Pará & $\begin{array}{l}\text { Rio Trombetas, } \\
\text { Oriximina, Jacaré }\end{array}$ & $-1,366919$ & $-56,841994$ & Piel \\
\hline $\begin{array}{l}\text { Myiozetetes } \\
\text { cayanensis }\end{array}$ & cayanensis & 3 & 63075 & MZUSP & Brasil & Pará & $\begin{array}{l}\text { Rio Tapajós (leste) } \\
\text { Fordlândia }\end{array}$ & $-3,829163$ & $-55,494504$ & Piel \\
\hline $\begin{array}{l}\text { Myiozetetes } \\
\text { cayanensis }\end{array}$ & cayanensis & 3 & 65449 & MZUSP & Brasil & Goiás & Goiania & $-16,686891$ & $-49,264794$ & Piel \\
\hline $\begin{array}{l}\text { Myiozetetes } \\
\text { cayanensis }\end{array}$ & cayanensis & 3 & 65450 & MZUSP & Brasil & Goiás & Goiania & $-16,686891$ & $-49,264794$ & Piel \\
\hline $\begin{array}{l}\text { Myiozetetes } \\
\text { cayanensis }\end{array}$ & cayanensis & 3 & 65451 & MZUSP & Brasil & Goiás & Hidrolândia & $-16,965475$ & $-49,231456$ & Piel \\
\hline $\begin{array}{l}\text { Myiozetetes } \\
\text { cayanensis }\end{array}$ & cayanensis & 3 & 65452 & MZUSP & Brasil & Goiás & Hidrolândia & $-16,965475$ & $-49,231456$ & Piel \\
\hline $\begin{array}{l}\text { Myiozetetes } \\
\text { cayanensis }\end{array}$ & cayanensis & 3 & 66002 & MZUSP & Brasil & Rondônia & $\begin{array}{c}\text { Rio Anari, Pedra } \\
\text { Branca }\end{array}$ & $-10,041718$ & $-62,11251$ & Piel \\
\hline $\begin{array}{l}\text { Myiozetetes } \\
\text { cayanensis }\end{array}$ & cayanensis & 3 & 66684 & MZUSP & Brasil & Pará & $\begin{array}{l}\text { Rio Tapajós, } \\
\text { Fordlândia }\end{array}$ & $-3,829163$ & $-55,494504$ & Piel \\
\hline $\begin{array}{l}\text { Myiozetetes } \\
\text { cayanensis }\end{array}$ & cayanensis & 3 & 68722 & MZUSP & Brasil & Goiás & Goiania & $-16,686891$ & $-49,264794$ & Piel \\
\hline $\begin{array}{l}\text { Myiozetetes } \\
\text { cayanensis }\end{array}$ & cayanensis & 3 & 68723 & MZUSP & Brasil & Goiás & Inhumas & $-16,373117$ & $-49,491677$ & Piel \\
\hline $\begin{array}{l}\text { Myiozetetes } \\
\text { cayanensis }\end{array}$ & cayanensis & 3 & 69330 & MZUSP & Brasil & Mato Grosso & Rio Suiá-Miçú & $-11,710405$ & $-52,582794$ & Piel \\
\hline $\begin{array}{l}\text { Myiozetetes } \\
\text { cayanensis }\end{array}$ & cayanensis & 3 & 70098 & MZUSP & Brasil & Pará & $\begin{array}{l}\text { Rio Tocantins, } \\
\text { Itupiranga }\end{array}$ & $-5,037753$ & $-49,854727$ & Piel \\
\hline $\begin{array}{l}\text { Myiozetetes } \\
\text { cayanensis }\end{array}$ & cayanensis & 3 & 70100 & MZUSP & Brasil & Goiás & Hidrolândia & $-16,965475$ & $-49,231456$ & Piel \\
\hline $\begin{array}{l}\text { Myiozetetes } \\
\text { cayanensis }\end{array}$ & cayanensis & 3 & 73413 & MZUSP & Brasil & Roraima & $\begin{array}{c}\text { Rio Branco } \\
\text { (margem leste), } \\
\text { oposto a Boa Vista, } \\
\text { Fazenda Santa } \\
\text { Cecília }\end{array}$ & 2,220882 & $-60,581866$ & Piel \\
\hline $\begin{array}{l}\text { Myiozetetes } \\
\text { cayanensis }\end{array}$ & cayanensis & 3 & 73414 & MZUSP & Brasil & Roraima & $\begin{array}{c}\text { Rio Branco } \\
\text { (margem leste), } \\
\text { oposto a Boa Vista, } \\
\text { Fazenda Santa } \\
\text { Cecília }\end{array}$ & 2,220882 & $-60,581866$ & Piel \\
\hline $\begin{array}{l}\text { Myiozetetes } \\
\text { cayanensis }\end{array}$ & cayanensis & 3 & 74124 & MZUSP & Brasil & Goiás & $\begin{array}{c}\text { Serra da Mesa, } \\
\text { Colinas do Sul, Rio } \\
\text { Tocantins(margem } \\
\text { esquerda) }\end{array}$ & $-13,971729$ & $-48,120575$ & Piel \\
\hline $\begin{array}{l}\text { Myiozetetes } \\
\text { cayanensis }\end{array}$ & cayanensis & 3 & 74534 & MZUSP & Brasil & São Paulo & $\begin{array}{l}\text { Rio Paraná } \\
\text { (margem } \\
\text { esquerda), Alfredo } \\
\text { de Castilho }\end{array}$ & $-22,886272$ & $-48,440606$ & Piel \\
\hline $\begin{array}{l}\text { Myiozetetes } \\
\text { cayanensis }\end{array}$ & cayanensis & 3 & 75739 & MZUSP & Brasil & Tocantins & Fazenda Sapezal & $-3,0833333$ & $\begin{array}{c}- \\
48,4166667\end{array}$ & Piel \\
\hline $\begin{array}{l}\text { Myiozetetes } \\
\text { cayanensis }\end{array}$ & cayanensis & 3 & 75740 & MZUSP & Brasil & Tocantins & Fazenda Sapezal & $-3,0833333$ & $\begin{array}{c}- \\
48,4166667\end{array}$ & Piel \\
\hline $\begin{array}{l}\text { Myiozetetes } \\
\text { cayanensis }\end{array}$ & cayanensis & 3 & 76992 & MZUSP & Brasil & Amazonas & $\begin{array}{c}\text { Rio Abacaxis } \\
\text { (margem direita), } \\
\text { Paca }\end{array}$ & $-4,123478$ & $-58,652369$ & Piel \\
\hline $\begin{array}{l}\text { Myiozetetes } \\
\text { cayanensis }\end{array}$ & cayanensis & 3 & 77497 & MZUSP & Brasil & Pará & $\begin{array}{l}\text { Reserva Florestal } \\
\text { da Agropalma, } \\
\text { Tailândia }\end{array}$ & $-2,940168$ & $-48,954731$ & Piel \\
\hline
\end{tabular}




\begin{tabular}{|c|c|c|c|c|c|c|c|c|c|c|}
\hline $\begin{array}{l}\text { Myiozetetes } \\
\text { cayanensis }\end{array}$ & cayanensis & 3 & 80118 & MZUSP & Brasil & Tocantins & $\begin{array}{l}\text { Sucupira, Fazenda } \\
\text { São Luís }\end{array}$ & $-11,95$ & $-48,85$ & Piel \\
\hline $\begin{array}{l}\text { Myiozetetes } \\
\text { cayanensis }\end{array}$ & cayanensis & 3 & 80119 & MZUSP & Brasil & Tocantins & $\begin{array}{c}\text { Sucupira, Fazenda } \\
\text { São Luís }\end{array}$ & $-11,95$ & $-48,85$ & Piel \\
\hline $\begin{array}{l}\text { Myiozetetes } \\
\text { cayanensis }\end{array}$ & cayanensis & 3 & 88213 & MZUSP & Brasil & Pará & $\begin{array}{c}\text { Santana do } \\
\text { Araguaia, Fazenda } \\
\text { Fartura }\end{array}$ & $-9,7833333$ & $-50,2$ & Piel \\
\hline $\begin{array}{l}\text { Myiozetetes } \\
\text { cayanensis }\end{array}$ & cayanensis & 3 & 88213 & MZUSP & Brasil & Mato Grosso & $\begin{array}{c}\text { Santana do } \\
\text { Araguaia, Fazenda } \\
\text { Fartura }\end{array}$ & $-9,7833333$ & $-50,2$ & Piel \\
\hline $\begin{array}{l}\text { Myiozetetes } \\
\text { cayanensis }\end{array}$ & cayanensis & 3 & 94679 & MZUSP & Brasil & Goiás & Campinaçu & $-13,9$ & $-48,5$ & Piel \\
\hline $\begin{array}{l}\text { Myiozetetes } \\
\text { cayanensis }\end{array}$ & cayanensis & 3 & 94680 & MZUSP & Brasil & Goiás & Campinaçu & $-13,9$ & $-48,5$ & Piel \\
\hline $\begin{array}{l}\text { Myiozetetes } \\
\text { cayanensis }\end{array}$ & cayanensis & 3 & 94681 & MZUSP & Brasil & Goiás & Campinaçu & $-13,9$ & $-48,5$ & Piel \\
\hline $\begin{array}{l}\text { Myiozetetes } \\
\text { cayanensis }\end{array}$ & cayanensis & 3 & 95499 & MZUSP & Brasil & Pará & $\begin{array}{l}\text { Rio Xingu (margem } \\
\text { esquerda), Vitória } \\
\text { do Xingu, T13 }\end{array}$ & $-3,25$ & 51,7833333 & Piel \\
\hline $\begin{array}{l}\text { Myiozetetes } \\
\text { cayanensis }\end{array}$ & cayanensis & 3 & 98581 & MZUSP & Brasil & Mato Grosso & $\begin{array}{l}\text { Vila Bela da } \\
\text { Santíssima } \\
\text { Trindade }\end{array}$ & $-14,983333$ & $\begin{array}{c}- \\
59,9166667\end{array}$ & Piel \\
\hline $\begin{array}{l}\text { Myiozetetes } \\
\text { cayanensis }\end{array}$ & cayanensis & 3 & 107220 & MZUSP & Brasil & Pará & $\begin{array}{c}\text { Santana do } \\
\text { Araguaia, Fazenda } \\
\text { Fartura }\end{array}$ & $-9,7166667$ & $\begin{array}{c}- \\
50,3166667\end{array}$ & Piel \\
\hline $\begin{array}{l}\text { Myiozetetes } \\
\text { cayanensis }\end{array}$ & hellmayri & 1 & 4458 & ICN & Colombia & Cundinamarca & $\begin{array}{l}\text { Cund., Sasaima, } \\
\text { Vereda de Guane. }\end{array}$ & 4,95035693 & $\begin{array}{c}- \\
74,4175747\end{array}$ & Piel \\
\hline $\begin{array}{l}\text { Myiozetetes } \\
\text { cayanensis }\end{array}$ & rufipennis & 2 & 4639 & ICN & Colombia & Vaupés & Sin datos. & 1,13332999 & 70,0500031 & Piel \\
\hline $\begin{array}{l}\text { Myiozetetes } \\
\text { cayanensis }\end{array}$ & rufipennis & 2 & 4641 & ICN & Colombia & Vaupés & Sin datos. & 1,13332999 & $\begin{array}{c}- \\
70,0500031\end{array}$ & Piel \\
\hline $\begin{array}{l}\text { Myiozetetes } \\
\text { cayanensis }\end{array}$ & rufipennis & 2 & 4642 & ICN & Colombia & Vaupés & Sin datos. & 1,13332999 & 70,0500031 & Piel \\
\hline $\begin{array}{l}\text { Myiozetetes } \\
\text { cayanensis }\end{array}$ & rufipennis & 2 & 4643 & ICN & Colombia & Vaupés & Sin datos. & 1,13332999 & $\begin{array}{c}- \\
70,0500031\end{array}$ & Piel \\
\hline $\begin{array}{l}\text { Myiozetetes } \\
\text { cayanensis }\end{array}$ & rufipennis & 2 & 6848 & ICN & Colombia & Vaupés & Villa Fátima. & 0,98000002 & 69,9300003 & Piel \\
\hline $\begin{array}{l}\text { Myiozetetes } \\
\text { cayanensis }\end{array}$ & hellmayri & 1 & 6900 & ICN & Colombia & Cundinamarca & $\begin{array}{l}\text { Cund., carretera } \\
\text { entre Fusagasugá y } \\
\text { Arbelaéz. }\end{array}$ & 4,29555886 & $-74,409868$ & Piel \\
\hline $\begin{array}{l}\text { Myiozetetes } \\
\text { cayanensis }\end{array}$ & hellmayri & 1 & 7055 & ICN & Colombia & Cauca & Guapi (Cauca) ARL. & 2,5999999 & 77,9000015 & Piel \\
\hline $\begin{array}{l}\text { Myiozetetes } \\
\text { cayanensis }\end{array}$ & hellmayri & 1 & 7056 & ICN & Colombia & Cauca & Guapi (Cauca) ARL. & 2,5999999 & $\begin{array}{c}- \\
77,9000015\end{array}$ & Piel \\
\hline $\begin{array}{l}\text { Myiozetetes } \\
\text { cayanensis }\end{array}$ & hellmayri & 1 & 7057 & ICN & Colombia & Cauca & Guapi (Cauca) ARL. & 2,5999999 & 77,9000015 & Piel \\
\hline $\begin{array}{l}\text { Myiozetetes } \\
\text { cayanensis }\end{array}$ & hellmayri & 1 & 7058 & ICN & Colombia & Cauca & Guapi (Cauca) ARL. & 2,5999999 & $\begin{array}{c}- \\
77,9000015\end{array}$ & Piel \\
\hline $\begin{array}{l}\text { Myiozetetes } \\
\text { cayanensis }\end{array}$ & hellmayri & 1 & 7060 & ICN & Colombia & Cauca & Guapi (Cauca) ARL. & 2,59999999 & $\begin{array}{c}- \\
77,9000015\end{array}$ & Piel \\
\hline $\begin{array}{l}\text { Myiozetetes } \\
\text { cayanensis }\end{array}$ & hellmayri & 1 & 7061 & ICN & Colombia & Cauca & Guapi (Cauca) ARL. & 2,5999999 & $\begin{array}{c}- \\
77,9000015\end{array}$ & Piel \\
\hline $\begin{array}{l}\text { Myiozetetes } \\
\text { cayanensis }\end{array}$ & hellmayri & 1 & 7062 & ICN & Colombia & Cauca & Guapi (Cauca) ARL. & 2,5999999 & 77,9000015 & Piel \\
\hline $\begin{array}{l}\text { Myiozetetes } \\
\text { cayanensis }\end{array}$ & hellmayri & 1 & 7063 & ICN & Colombia & Cauca & $\begin{array}{l}\text { Guapi (Cauca), S. } \\
\text { Antonio de Guajui. }\end{array}$ & 2,6500001 & $\begin{array}{c}- \\
77,6833267\end{array}$ & Piel \\
\hline $\begin{array}{l}\text { Myiozetetes } \\
\text { cayanensis }\end{array}$ & hellmayri & 1 & 7064 & ICN & Colombia & Cauca & $\begin{array}{l}\text { Guapi (Cauca), S. } \\
\text { Antonio de Guajui. }\end{array}$ & 2,6500001 & $\begin{array}{c}- \\
77,6833267\end{array}$ & Piel \\
\hline $\begin{array}{l}\text { Myiozetetes } \\
\text { cayanensis }\end{array}$ & hellmayri & 1 & 7065 & ICN & Colombia & Cundinamarca & $\begin{array}{l}\text { Cund., San } \\
\text { Francisco, } \\
\text { alrededores de la } \\
\text { población. }\end{array}$ & 4,97054825 & $-74,290259$ & Piel \\
\hline $\begin{array}{l}\text { Myiozetetes } \\
\text { cayanensis }\end{array}$ & hellmayri & 1 & 7066 & ICN & Colombia & Cundinamarca & $\begin{array}{l}\text { Cund., Sasaima, } \\
\text { Vereda de Guane. }\end{array}$ & 4,95035693 & $\begin{array}{c}- \\
74,4175747\end{array}$ & Piel \\
\hline $\begin{array}{l}\text { Myiozetetes } \\
\text { cayanensis }\end{array}$ & hellmayri & 1 & 7067 & ICN & Colombia & Antioquia & $\begin{array}{l}\text { Río Guaudalito, SE } \\
\text { Turbo, Antioquia. }\end{array}$ & 8,08993811 & $\begin{array}{c}- \\
76,7309669\end{array}$ & Piel \\
\hline $\begin{array}{l}\text { Myiozetetes } \\
\text { cayanensis }\end{array}$ & hellmayri & 1 & 7068 & ICN & Colombia & Antioquia & $\begin{array}{l}\text { Río Guaudalito, SE } \\
\text { Turbo, Antioquia. }\end{array}$ & 8,08993811 & 76,7309669 & Piel \\
\hline
\end{tabular}




\begin{tabular}{|c|c|c|c|c|c|c|c|c|c|c|}
\hline $\begin{array}{l}\text { Myiozetetes } \\
\text { cayanensis }\end{array}$ & hellmayri & 1 & 7069 & $\mathrm{ICN}$ & Colombia & Antioquia & Sautata, N Chocó. & 7,82671673 & $\begin{array}{c}- \\
76,6271292\end{array}$ & Piel \\
\hline $\begin{array}{l}\text { Myiozetetes } \\
\text { cayanensis }\end{array}$ & hellmayri & 1 & 7070 & $\mathrm{ICN}$ & Colombia & Antioquia & Sautata, N Chocó. & 7,82671673 & $\begin{array}{c}- \\
76,6271292\end{array}$ & Piel \\
\hline $\begin{array}{l}\text { Myiozetetes } \\
\text { cayanensis }\end{array}$ & hellmayri & 1 & 7071 & $\mathrm{ICN}$ & Colombia & Chocó & $\begin{array}{c}\text { Titumate, N Chocó. } \\
\text { Coast. }\end{array}$ & 8,31667042 & $\begin{array}{c}- \\
77,0666733\end{array}$ & Piel \\
\hline $\begin{array}{l}\text { Myiozetetes } \\
\text { cayanensis }\end{array}$ & hellmayri & 1 & 7072 & $\mathrm{ICN}$ & Colombia & Chocó & $\begin{array}{l}\text { Río Tanela, N } \\
\text { Chocó. }\end{array}$ & 8,28140371 & $\begin{array}{c}- \\
77,1334238\end{array}$ & Piel \\
\hline $\begin{array}{l}\text { Myiozetetes } \\
\text { cayanensis }\end{array}$ & hellmayri & 1 & 7073 & $\mathrm{ICN}$ & Colombia & Antioquia & $\begin{array}{c}\text { Río Mulatos, NE } \\
\text { Turbo. }\end{array}$ & 8,1462173 & $\begin{array}{c}- \\
76,6611756\end{array}$ & Piel \\
\hline $\begin{array}{l}\text { Myiozetetes } \\
\text { cayanensis }\end{array}$ & hellmayri & 1 & 7074 & $\mathrm{ICN}$ & Colombia & Caldas & $\begin{array}{l}\text { Caldas, Palestina, } \\
\text { (La Plata). }\end{array}$ & 5,03860998 & $\begin{array}{c}- \\
75,6774979\end{array}$ & Piel \\
\hline $\begin{array}{l}\text { Myiozetetes } \\
\text { cayanensis }\end{array}$ & hellmayri & 1 & 7075 & ICN & Colombia & Caldas & Chinchiná, Caldas. & 4,97409509 & $\begin{array}{c}- \\
75,6789905\end{array}$ & Piel \\
\hline $\begin{array}{l}\text { Myiozetetes } \\
\text { cayanensis }\end{array}$ & hellmayri & 1 & 7076 & $\mathrm{ICN}$ & Colombia & Cundinamarca & Cund., Fusagasugá. & 4,34645621 & $-74,378534$ & Piel \\
\hline $\begin{array}{l}\text { Myiozetetes } \\
\text { cayanensis }\end{array}$ & hellmayri & 1 & 7077 & $\mathrm{ICN}$ & Colombia & Tolima & $\begin{array}{l}\text { Tolima, Mariquita, } \\
\text { (Guayacán). }\end{array}$ & 5,20110989 & $\begin{array}{c}- \\
74,9127808\end{array}$ & Piel \\
\hline $\begin{array}{l}\text { Myiozetetes } \\
\text { cayanensis }\end{array}$ & hellmayri & 1 & 7078 & $\mathrm{ICN}$ & Colombia & Cauca & Cauca, Popayán. & 2,44483599 & $\begin{array}{c}- \\
76,6147132\end{array}$ & Piel \\
\hline $\begin{array}{l}\text { Myiozetetes } \\
\text { cayanensis }\end{array}$ & hellmayri & 1 & 7079 & $\mathrm{ICN}$ & Colombia & Cauca & Cauca, Palacé. & 2,56666994 & $-76,75$ & Piel \\
\hline $\begin{array}{l}\text { Myiozetetes } \\
\text { cayanensis }\end{array}$ & hellmayri & 1 & 7080 & $\mathrm{ICN}$ & Colombia & Cauca & Cauca, Popayán. & 2,44483599 & 76,6147132 & Piel \\
\hline $\begin{array}{l}\text { Myiozetetes } \\
\text { cayanensis }\end{array}$ & hellmayri & 1 & 7081 & $\mathrm{ICN}$ & Colombia & Santander & $\begin{array}{c}\text { Sant., Cordillera La } \\
\text { Paz, Hda. Monte } \\
\text { Bello. }\end{array}$ & 7,08333015 & $\begin{array}{c}- \\
73,4166718\end{array}$ & Piel \\
\hline $\begin{array}{l}\text { Myiozetetes } \\
\text { cayanensis }\end{array}$ & hellmayri & 1 & 7084 & $\mathrm{ICN}$ & Colombia & Santander & $\begin{array}{c}\text { Santander, El } \\
\text { Guadual, Vereda } \\
\text { Palmira. }\end{array}$ & 6,46328695 & 73,5537013 & Piel \\
\hline $\begin{array}{l}\text { Myiozetetes } \\
\text { cayanensis }\end{array}$ & hellmayri & 1 & 7085 & $\mathrm{ICN}$ & Colombia & Santander & $\begin{array}{l}\text { Santander, } \\
\text { Marengo, Vda. } \\
\text { Llano Caliente. }\end{array}$ & 6,9000001 & $\begin{array}{c}- \\
73,5999985\end{array}$ & Piel \\
\hline $\begin{array}{l}\text { Myiozetetes } \\
\text { cayanensis }\end{array}$ & hellmayri & 1 & 7086 & $\mathrm{ICN}$ & Colombia & Huila & San Agustín, Huila. & 1,88332999 & $\begin{array}{c}- \\
76,2666702\end{array}$ & Piel \\
\hline $\begin{array}{l}\text { Myiozetetes } \\
\text { cayanensis }\end{array}$ & rufipennis & 2 & 7087 & $\mathrm{ICN}$ & Colombia & Meta & $\begin{array}{l}\text { Meta, La Macarena } \\
\text { Sur. Camp. } 3 .\end{array}$ & 2,75 & - & Piel \\
\hline $\begin{array}{l}\text { Myiozetetes } \\
\text { cayanensis }\end{array}$ & rufipennis & 2 & 7088 & $\mathrm{ICN}$ & Colombia & Meta & Meta, Peralonso. & 4,08147587 & $\begin{array}{c}- \\
73,3394715\end{array}$ & Piel \\
\hline $\begin{array}{l}\text { Myiozetetes } \\
\text { cayanensis }\end{array}$ & hellmayri & 1 & 7104 & $\mathrm{ICN}$ & Colombia & Tolima & $\begin{array}{c}\text { Tolima, Carmen de } \\
\text { Apicalá. }\end{array}$ & 4,16192124 & $\begin{array}{c}- \\
74,7264553\end{array}$ & Piel \\
\hline $\begin{array}{l}\text { Myiozetetes } \\
\text { cayanensis }\end{array}$ & hellmayri & 1 & 9298 & $\mathrm{ICN}$ & Colombia & Bolívar & $\begin{array}{l}\text { Bolívar, } 20 \text { k west } \\
\text { of El Carmen, } \\
\text { Lázaro. }\end{array}$ & 9,71667004 & 75,3166733 & Piel \\
\hline $\begin{array}{l}\text { Myiozetetes } \\
\text { cayanensis }\end{array}$ & hellmayri & 1 & 9299 & $\mathrm{ICN}$ & Colombia & Sucre & $\begin{array}{l}\text { Bolívar, } 25 \mathrm{k} \text { west } \\
\text { of El Carmen, } \\
\text { Arroyo Tigre. }\end{array}$ & 9,69999981 & $\begin{array}{c}- \\
75,5999985\end{array}$ & Piel \\
\hline $\begin{array}{l}\text { Myiozetetes } \\
\text { cayanensis }\end{array}$ & rufipennis & 2 & 9944 & $\mathrm{ICN}$ & Colombia & Vaupés & $\begin{array}{l}\text { Vaupés, Sabanas } \\
\text { del Cubiyú. }\end{array}$ & 1 & -70 & Piel \\
\hline $\begin{array}{l}\text { Myiozetetes } \\
\text { cayanensis }\end{array}$ & rufipennis & 2 & 9945 & ICN & Colombia & Vaupés & $\begin{array}{l}\text { Vaupés, Sabanas } \\
\text { del Cubiyú. }\end{array}$ & 1 & -70 & Piel \\
\hline $\begin{array}{l}\text { Myiozetetes } \\
\text { cayanensis }\end{array}$ & hellmayri & 1 & 13485 & ICN & Colombia & Santander & $\begin{array}{l}\text { Santander, Río } \\
\text { Chicamocha, } \\
\text { Pescadero. }\end{array}$ & 6,83333015 & -73 & Piel \\
\hline $\begin{array}{l}\text { Myiozetetes } \\
\text { cayanensis }\end{array}$ & rufipennis & 2 & 13589 & $\mathrm{ICN}$ & Colombia & Caquetá & $\begin{array}{c}\text { Río Orteguaza, Tres } \\
\text { Esquinas. }\end{array}$ & 0,71666002 & $\begin{array}{c}- \\
75,2666702\end{array}$ & Piel \\
\hline $\begin{array}{l}\text { Myiozetetes } \\
\text { cayanensis }\end{array}$ & rufipennis & 2 & 13590 & $\mathrm{ICN}$ & Colombia & Caquetá & $\begin{array}{c}\text { Río Orteguaza, Tres } \\
\text { Esquinas. }\end{array}$ & 0,71666002 & $\begin{array}{c}- \\
75,2666702\end{array}$ & Piel \\
\hline $\begin{array}{l}\text { Myiozetetes } \\
\text { cayanensis }\end{array}$ & hellmayri & 1 & 14064 & $\mathrm{ICN}$ & Colombia & Cundinamarca & $\begin{array}{l}\text { Cund. Utica, Grnaja } \\
\text { de la U. Nacional. }\end{array}$ & 5,18721425 & $\begin{array}{c}- \\
74,4809404\end{array}$ & Piel \\
\hline $\begin{array}{l}\text { Myiozetetes } \\
\text { cayanensis }\end{array}$ & hellmayri & 1 & 14128 & $\mathrm{ICN}$ & Colombia & Cundinamarca & $\begin{array}{l}\text { Cund., Planicies de } \\
\text { Fusagasugá. }\end{array}$ & 4,3499999 & 74,3666687 & Piel \\
\hline $\begin{array}{l}\text { Myiozetetes } \\
\text { cayanensis }\end{array}$ & hellmayri & 1 & 14129 & $\mathrm{ICN}$ & Colombia & Cundinamarca & $\begin{array}{l}\text { Cund., Planicie de } \\
\text { Fusagasugá. }\end{array}$ & 4,3499999 & $\begin{array}{c}- \\
74,3666687\end{array}$ & Piel \\
\hline $\begin{array}{l}\text { Myiozetetes } \\
\text { cayanensis }\end{array}$ & hellmayri & 1 & 14291 & ICN & Colombia & Cundinamarca & $\begin{array}{l}\text { Cund., Planicie de } \\
\text { Fusagasugá. }\end{array}$ & 4,3499999 & 74,3666687 & Piel \\
\hline
\end{tabular}




\begin{tabular}{|c|c|c|c|c|c|c|c|c|c|c|}
\hline $\begin{array}{l}\text { Myiozetetes } \\
\text { cayanensis }\end{array}$ & hellmayri & 1 & 14360 & ICN & Colombia & Cundinamarca & $\begin{array}{l}\text { Cund., Planicie de } \\
\text { Fusagasugá. }\end{array}$ & 4,31932778 & $\begin{array}{c}- \\
74,3603307\end{array}$ & Piel \\
\hline $\begin{array}{l}\text { Myiozetetes } \\
\text { cayanensis }\end{array}$ & hellmayri & 1 & 14634 & ICN & Colombia & Cundinamarca & $\begin{array}{l}\text { Cund., Mun. de La } \\
\text { Vega, Vda. El } \\
\text { Tabacal. }\end{array}$ & 5,02005673 & $-74,323558$ & Piel \\
\hline $\begin{array}{l}\text { Myiozetetes } \\
\text { cayanensis }\end{array}$ & hellmayri & 1 & 15082 & ICN & Colombia & Cundinamarca & Cund., Fusagasugá. & 4,34645621 & $-74,378534$ & Piel \\
\hline $\begin{array}{l}\text { Myiozetetes } \\
\text { cayanensis }\end{array}$ & hellmayri & 1 & 15265 & ICN & Colombia & $\begin{array}{l}\text { Norte de } \\
\text { Santander }\end{array}$ & $\begin{array}{l}\text { N. de Santander, } \\
\text { Tibú, (entre Tres } \\
\text { Bocas y Petrolea). }\end{array}$ & 8,61666965 & $\begin{array}{c}- \\
72,6500015\end{array}$ & Piel \\
\hline $\begin{array}{l}\text { Myiozetetes } \\
\text { cayanensis }\end{array}$ & hellmayri & 1 & 15266 & $\mathrm{ICN}$ & Colombia & $\begin{array}{l}\text { Norte de } \\
\text { Santander }\end{array}$ & $\begin{array}{l}\text { N. de Santander, } \\
\text { Tibú, (entre Tres } \\
\text { Bocas y Petrolea). }\end{array}$ & 8,61666965 & $\begin{array}{c}- \\
72,6500015\end{array}$ & Piel \\
\hline $\begin{array}{l}\text { Myiozetetes } \\
\text { cayanensis }\end{array}$ & hellmayri & 1 & 15267 & $\mathrm{ICN}$ & Colombia & $\begin{array}{l}\text { Norte de } \\
\text { Santander }\end{array}$ & $\begin{array}{l}\text { N. de Santander, } \\
\text { Tibú, (entre Tres } \\
\text { Bocas y Petrolea). }\end{array}$ & 8,61666965 & $\begin{array}{c}- \\
72,6500015\end{array}$ & Piel \\
\hline $\begin{array}{l}\text { Myiozetetes } \\
\text { cayanensis }\end{array}$ & rufipennis & 2 & 15268 & ICN & Colombia & $\begin{array}{l}\text { Norte de } \\
\text { Santander }\end{array}$ & $\begin{array}{l}\text { N. de Santander, } \\
\text { Catatumbo, Río de } \\
\text { Oro, (Campamento } \\
\text { Colpet, frontera } \\
\text { Col.-Ven.). }\end{array}$ & 7,16666985 & $\begin{array}{c}- \\
73,1500015\end{array}$ & Piel \\
\hline $\begin{array}{l}\text { Myiozetetes } \\
\text { cayanensis }\end{array}$ & hellmayri & 1 & 15269 & $\mathrm{ICN}$ & Colombia & $\begin{array}{l}\text { Norte de } \\
\text { Santander }\end{array}$ & $\begin{array}{l}\text { N. de Santander, } \\
\text { Catatumbo, Río de } \\
\text { Oro, (Campamento } \\
\text { Colpet, frontera } \\
\text { Col.-Ven.). }\end{array}$ & 7,16666985 & 73,1500015 & Piel \\
\hline $\begin{array}{l}\text { Myiozetetes } \\
\text { cayanensis }\end{array}$ & hellmayri & 1 & 15270 & $\mathrm{ICN}$ & Colombia & $\begin{array}{l}\text { Norte de } \\
\text { Santander }\end{array}$ & $\begin{array}{c}\text { N. de Santander, } \\
\text { Catatumbo, Río de } \\
\text { Oro, (Campamento } \\
\text { Colpet, frontera } \\
\text { Col.-Ven.). }\end{array}$ & 7,16666985 & $\begin{array}{c}- \\
73,1500015\end{array}$ & Piel \\
\hline $\begin{array}{l}\text { Myiozetetes } \\
\text { cayanensis }\end{array}$ & hellmayri & 1 & 15271 & ICN & Colombia & $\begin{array}{l}\text { Norte de } \\
\text { Santander }\end{array}$ & $\begin{array}{l}\text { N. de Santander, } \\
\text { Catatumbo, Río de } \\
\text { Oro, (Campamento } \\
\text { Colpet, frontera } \\
\text { Col.-Ven.). }\end{array}$ & 7,16666985 & $\begin{array}{c}- \\
73,1500015\end{array}$ & Piel \\
\hline $\begin{array}{l}\text { Myiozetetes } \\
\text { cayanensis }\end{array}$ & rufipennis & 2 & 15264 & $\mathrm{ICN}$ & Colombia & $\begin{array}{l}\text { Norte de } \\
\text { Santander }\end{array}$ & $\begin{array}{l}\text { N. de Santander, } \\
\text { Tibú, (entre Tres } \\
\text { Bocas y Petrolea). }\end{array}$ & 8,61666965 & $\begin{array}{c}- \\
72,6500015\end{array}$ & Piel \\
\hline $\begin{array}{l}\text { Myiozetetes } \\
\text { cayanensis }\end{array}$ & hellmayri & 1 & 15685 & $\mathrm{ICN}$ & Colombia & Cundinamarca & Cund., La Vega. & 5,00050734 & $\begin{array}{c}- \\
74,3394403\end{array}$ & Piel \\
\hline $\begin{array}{l}\text { Myiozetetes } \\
\text { cayanensis }\end{array}$ & hellmayri & 1 & 15686 & $\mathrm{ICN}$ & Colombia & Cundinamarca & $\begin{array}{l}\text { Cund., La Vega, } \\
\text { Vda. La Cabaña. }\end{array}$ & 5,01965534 & $-74,334101$ & Piel \\
\hline $\begin{array}{l}\text { Myiozetetes } \\
\text { cayanensis }\end{array}$ & hellmayri & 1 & 15687 & $\mathrm{ICN}$ & Colombia & Cundinamarca & $\begin{array}{c}\text { Cund., La Vega, } \\
\text { Escuela de San } \\
\text { Juan. }\end{array}$ & 5 & $\begin{array}{c}- \\
74,3499985\end{array}$ & Piel \\
\hline $\begin{array}{l}\text { Myiozetetes } \\
\text { cayanensis }\end{array}$ & hellmayri & 1 & 15688 & $\mathrm{ICN}$ & Colombia & Cundinamarca & $\begin{array}{l}\text { Cund., La Vega, } \\
\text { Finca El Encanto. }\end{array}$ & 5 & $\begin{array}{c}- \\
74,3499985\end{array}$ & Piel \\
\hline $\begin{array}{l}\text { Myiozetetes } \\
\text { cayanensis }\end{array}$ & hellmayri & 1 & 16051 & $\mathrm{ICN}$ & Colombia & Cundinamarca & $\begin{array}{l}\text { Cund., La Vega, } \\
\text { Vda. El Roble. }\end{array}$ & 4,99182126 & $\begin{array}{c}- \\
74,3496835\end{array}$ & Piel \\
\hline $\begin{array}{l}\text { Myiozetetes } \\
\text { cayanensis }\end{array}$ & hellmayri & 1 & 16052 & ICN & Colombia & Cundinamarca & $\begin{array}{l}\text { Cund., La Vega, } \\
\text { Finca El Encanto. }\end{array}$ & 5 & $\begin{array}{c}- \\
74,3499985\end{array}$ & Piel \\
\hline $\begin{array}{l}\text { Myiozetetes } \\
\text { cayanensis }\end{array}$ & hellmayri & 1 & 16314 & $\mathrm{ICN}$ & Colombia & Cundinamarca & $\begin{array}{l}\text { Cund., La Vega, } \\
\text { Finca El Encanto. }\end{array}$ & 5 & $\begin{array}{c}- \\
74,3499985\end{array}$ & Piel \\
\hline $\begin{array}{l}\text { Myiozetetes } \\
\text { cayanensis }\end{array}$ & hellmayri & 1 & 16340 & $\mathrm{ICN}$ & Colombia & Cundinamarca & Cund., El Ocaso. & 4,71667004 & $\begin{array}{c}- \\
74,4166718\end{array}$ & Piel \\
\hline $\begin{array}{l}\text { Myiozetetes } \\
\text { cayanensis }\end{array}$ & hellmayri & 1 & 16567 & ICN & Colombia & Cundinamarca & $\begin{array}{l}\text { Cund., La Vega, } \\
\text { Laguna El Tabacal } \\
\text { (Bosques } \\
\text { aledaños). }\end{array}$ & 5,03332996 & $\begin{array}{c}- \\
74,3166733\end{array}$ & Piel \\
\hline $\begin{array}{l}\text { Myiozetetes } \\
\text { cayanensis }\end{array}$ & hellmayri & 1 & 16568 & $\mathrm{ICN}$ & Colombia & Cundinamarca & $\begin{array}{l}\text { Cund., La Vega, } \\
\text { Laguna El Tabacal } \\
\text { (Bosques } \\
\text { aledaños). }\end{array}$ & 5,03332996 & $\begin{array}{c}- \\
74,3166733\end{array}$ & Piel \\
\hline $\begin{array}{l}\text { Myiozetetes } \\
\text { cayanensis }\end{array}$ & hellmayri & 1 & 16569 & $\mathrm{ICN}$ & Colombia & Cundinamarca & $\begin{array}{l}\text { Cund., La Vega, } \\
\text { Finca Casa Blanca. }\end{array}$ & 5 & $\begin{array}{c}- \\
74,3499985\end{array}$ & Piel \\
\hline $\begin{array}{l}\text { Myiozetetes } \\
\text { cayanensis }\end{array}$ & hellmayri & 1 & 16570 & ICN & Colombia & Cundinamarca & $\begin{array}{c}\text { Cund., La Vega, } \\
\text { Finca Casa Blanca. }\end{array}$ & 5 & $\begin{array}{c}- \\
74,3499985\end{array}$ & Piel \\
\hline
\end{tabular}




\begin{tabular}{|c|c|c|c|c|c|c|c|c|c|c|}
\hline $\begin{array}{l}\text { Myiozetetes } \\
\text { cayanensis }\end{array}$ & hellmayri & 1 & 16571 & $\mathrm{ICN}$ & Colombia & Cundinamarca & $\begin{array}{l}\text { Cund., La Vega, } \\
\text { Finca Casa Blanca. }\end{array}$ & 5 & $\begin{array}{c}- \\
74,3499985\end{array}$ & Piel \\
\hline $\begin{array}{l}\text { Myiozetetes } \\
\text { cayanensis }\end{array}$ & hellmayri & 1 & 16572 & $\mathrm{ICN}$ & Colombia & Cundinamarca & $\begin{array}{l}\text { Cund., La Vega, } \\
\text { Finca Casa Blanca. }\end{array}$ & 5 & $\begin{array}{c}- \\
74,3499985\end{array}$ & Piel \\
\hline $\begin{array}{l}\text { Myiozetetes } \\
\text { cayanensis }\end{array}$ & hellmayri & 1 & 16573 & $\mathrm{ICN}$ & Colombia & Cundinamarca & $\begin{array}{l}\text { Cund., La Vega, } \\
\text { Finca Casa Blanca. }\end{array}$ & 5 & $\begin{array}{c}- \\
74,3499985\end{array}$ & Piel \\
\hline $\begin{array}{l}\text { Myiozetetes } \\
\text { cayanensis }\end{array}$ & hellmayri & 1 & 16574 & $\mathrm{ICN}$ & Colombia & Cundinamarca & $\begin{array}{l}\text { Cund., La Vega, } \\
\text { Finca Casa Blanca. }\end{array}$ & 5 & $\begin{array}{c}- \\
74,3499985\end{array}$ & Piel \\
\hline $\begin{array}{l}\text { Myiozetetes } \\
\text { cayanensis }\end{array}$ & hellmayri & 1 & 16575 & $\mathrm{ICN}$ & Colombia & Cundinamarca & $\begin{array}{l}\text { Cund., La Vega, } \\
\text { Laguna El Tabacal } \\
\text { (Bosques } \\
\text { aledaños). }\end{array}$ & 5,03332996 & $\begin{array}{c}- \\
74,3166733\end{array}$ & Piel \\
\hline $\begin{array}{l}\text { Myiozetetes } \\
\text { cayanensis }\end{array}$ & hellmayri & 1 & 16752 & $\mathrm{ICN}$ & Colombia & Cundinamarca & $\begin{array}{l}\text { Cund., La Vega, } \\
\text { Vda. El Roble. }\end{array}$ & 4,99182126 & $\begin{array}{c}- \\
74,3496835\end{array}$ & Piel \\
\hline $\begin{array}{l}\text { Myiozetetes } \\
\text { cayanensis }\end{array}$ & hellmayri & 1 & 16753 & $\mathrm{ICN}$ & Colombia & Cundinamarca & $\begin{array}{l}\text { Cund., La Vega, } \\
\text { Vda. El Roble. }\end{array}$ & 4,99182126 & $\begin{array}{c}- \\
74,3496835\end{array}$ & Piel \\
\hline $\begin{array}{l}\text { Myiozetetes } \\
\text { cayanensis }\end{array}$ & hellmayri & 1 & 17118 & $\mathrm{ICN}$ & Colombia & Cundinamarca & $\begin{array}{l}\text { Cund., Planicie de } \\
\text { Fusagasugá, } \\
\text { Quebrada Seca. }\end{array}$ & 4,33399176 & $\begin{array}{c}- \\
74,3528364\end{array}$ & Piel \\
\hline $\begin{array}{l}\text { Myiozetetes } \\
\text { cayanensis }\end{array}$ & hellmayri & 1 & 18384 & $\mathrm{ICN}$ & Colombia & Cundinamarca & $\begin{array}{l}\text { Cund., Puerto } \\
\text { Salgar, Hda. } \\
\text { Vizcaya. }\end{array}$ & 5,44790012 & $\begin{array}{c}- \\
74,6344868\end{array}$ & Piel \\
\hline $\begin{array}{l}\text { Myiozetetes } \\
\text { cayanensis }\end{array}$ & hellmayri & 1 & 18385 & ICN & Colombia & Cundinamarca & $\begin{array}{l}\text { Cund., Puerto } \\
\text { Salgar, Hda. } \\
\text { Vizcaya. }\end{array}$ & 5,44790012 & $\begin{array}{c}- \\
74,6344868\end{array}$ & Piel \\
\hline $\begin{array}{l}\text { Myiozetetes } \\
\text { cayanensis }\end{array}$ & hellmayri & 1 & 18386 & $\mathrm{ICN}$ & Colombia & Cundinamarca & $\begin{array}{l}\text { Cund., Puerto } \\
\text { Salgar, Hda. } \\
\text { Vizcaya. }\end{array}$ & 5,44790012 & $\begin{array}{c}- \\
74,6344868\end{array}$ & Piel \\
\hline $\begin{array}{l}\text { Myiozetetes } \\
\text { cayanensis }\end{array}$ & rufipennis & 2 & 18707 & $\mathrm{ICN}$ & Colombia & Meta & $\begin{array}{c}\text { Meta, } \\
\text { Pachaquiaro. }\end{array}$ & 4,05770614 & $-73,17237$ & Piel \\
\hline $\begin{array}{l}\text { Myiozetetes } \\
\text { cayanensis }\end{array}$ & rufipennis & 2 & 18708 & $\mathrm{ICN}$ & Colombia & Meta & $\begin{array}{c}\text { Meta, } \\
\text { Pachaquiaro. }\end{array}$ & 4,05770614 & $-73,17237$ & Piel \\
\hline $\begin{array}{l}\text { Myiozetetes } \\
\text { cayanensis }\end{array}$ & rufipennis & 2 & 18878 & $\mathrm{ICN}$ & Colombia & Meta & Meta, Villavicencio. & 4,11973666 & $\begin{array}{c}- \\
73,6179619\end{array}$ & Piel \\
\hline $\begin{array}{l}\text { Myiozetetes } \\
\text { cayanensis }\end{array}$ & rufipennis & 2 & 18897 & $\mathrm{ICN}$ & Colombia & Meta & Meta, Guamal. & 3,87587898 & $-73,769593$ & Piel \\
\hline $\begin{array}{l}\text { Myiozetetes } \\
\text { cayanensis }\end{array}$ & rufipennis & 2 & 18898 & $\mathrm{ICN}$ & Colombia & Meta & Meta, Guamal. & 3,87587898 & $-73,769593$ & Piel \\
\hline $\begin{array}{l}\text { Myiozetetes } \\
\text { cayanensis }\end{array}$ & rufipennis & 2 & 18899 & $\mathrm{ICN}$ & Colombia & Meta & Meta, Guamal. & 3,87587898 & $-73,769593$ & Piel \\
\hline $\begin{array}{l}\text { Myiozetetes } \\
\text { cayanensis }\end{array}$ & hellmayri & 1 & 19026 & $\mathrm{ICN}$ & Colombia & Antioquia & Antioquia. & 6,16666985 & $\begin{array}{c}- \\
75,6500015\end{array}$ & Piel \\
\hline $\begin{array}{l}\text { Myiozetetes } \\
\text { cayanensis }\end{array}$ & hellmayri & 1 & 19028 & ICN & Colombia & Antioquia & $\begin{array}{l}\text { Antioquia, La } \\
\text { Estrella. }\end{array}$ & 6,16666985 & $\begin{array}{c}- \\
75,6500015\end{array}$ & Piel \\
\hline $\begin{array}{l}\text { Myiozetetes } \\
\text { cayanensis }\end{array}$ & hellmayri & 1 & 19226 & $\mathrm{ICN}$ & Colombia & Cundinamarca & $\begin{array}{l}\text { Cund., Apulo, Río } \\
\text { Calandaima. }\end{array}$ & 4,47565277 & $\begin{array}{c}- \\
74,5423425\end{array}$ & Piel \\
\hline $\begin{array}{l}\text { Myiozetetes } \\
\text { cayanensis }\end{array}$ & hellmayri & 1 & 19227 & ICN & Colombia & Cundinamarca & $\begin{array}{l}\text { Cund., Apulo, Río } \\
\text { Calandaima. }\end{array}$ & 4,47565277 & $\begin{array}{c}- \\
74,5423425\end{array}$ & Piel \\
\hline $\begin{array}{l}\text { Myiozetetes } \\
\text { cayanensis }\end{array}$ & hellmayri & 1 & 19228 & $\mathrm{ICN}$ & Colombia & Cundinamarca & $\begin{array}{l}\text { Cund., Apulo, Río } \\
\text { Calandaima. }\end{array}$ & 4,47565277 & $\begin{array}{c}- \\
74,5423425\end{array}$ & Piel \\
\hline $\begin{array}{l}\text { Myiozetetes } \\
\text { cayanensis }\end{array}$ & hellmayri & 1 & 19229 & $\mathrm{ICN}$ & Colombia & Cundinamarca & $\begin{array}{c}\text { Cund., Apulo, Río } \\
\text { Calandaima. }\end{array}$ & 4,47565277 & $\begin{array}{c}- \\
74,5423425\end{array}$ & Piel \\
\hline $\begin{array}{l}\text { Myiozetetes } \\
\text { cayanensis }\end{array}$ & hellmayri & 1 & 19448 & ICN & Colombia & Cundinamarca & $\begin{array}{l}\text { Cund., El Peñón, } \\
\text { Hda. Belalcázar. }\end{array}$ & 4,43333006 & $\begin{array}{c}- \\
74,3000031\end{array}$ & Piel \\
\hline $\begin{array}{l}\text { Myiozetetes } \\
\text { cayanensis }\end{array}$ & hellmayri & 1 & 19449 & ICN & Colombia & Cundinamarca & $\begin{array}{l}\text { Cund., El Peñón, } \\
\text { Hda. Belalcázar. }\end{array}$ & 4,43333006 & $\begin{array}{c}- \\
74,3000031\end{array}$ & Piel \\
\hline $\begin{array}{l}\text { Myiozetetes } \\
\text { cayanensis }\end{array}$ & hellmayri & 1 & 19450 & $\mathrm{ICN}$ & Colombia & Cundinamarca & $\begin{array}{l}\text { Cund., El Peñón, } \\
\text { Hda. Belalcázar. }\end{array}$ & 4,43333006 & $\begin{array}{c}- \\
74,3000031\end{array}$ & Piel \\
\hline $\begin{array}{l}\text { Myiozetetes } \\
\text { cayanensis }\end{array}$ & hellmayri & 1 & 19451 & $\mathrm{ICN}$ & Colombia & Cundinamarca & $\begin{array}{l}\text { Cund., El Peñón, } \\
\text { Hda. Belalcázar. }\end{array}$ & 4,43333006 & $\begin{array}{c}- \\
74,3000031\end{array}$ & Piel \\
\hline $\begin{array}{l}\text { Myiozetetes } \\
\text { cayanensis }\end{array}$ & hellmayri & 1 & 19452 & $\mathrm{ICN}$ & Colombia & Cundinamarca & $\begin{array}{l}\text { Cund., El Peñón, } \\
\text { Hda. Belalcázar. }\end{array}$ & 4,43333006 & $\begin{array}{c}- \\
74,3000031\end{array}$ & Piel \\
\hline $\begin{array}{l}\text { Myiozetetes } \\
\text { cayanensis }\end{array}$ & rufipennis & 2 & 19550 & $\mathrm{ICN}$ & Colombia & Meta & Meta, Guamal. & 3,87587898 & $-73,769593$ & Piel \\
\hline $\begin{array}{l}\text { Myiozetetes } \\
\text { cayanensis }\end{array}$ & hellmayri & 1 & 19918 & ICN & Colombia & Santander & $\begin{array}{l}\text { Sant. Simacota - } \\
\text { Chima, (Vda. S. } \\
\text { Pascual, Picurales). }\end{array}$ & 6,40860987 & $\begin{array}{c}- \\
73,3541718\end{array}$ & Piel \\
\hline
\end{tabular}




\begin{tabular}{|c|c|c|c|c|c|c|c|c|c|c|}
\hline $\begin{array}{l}\text { Myiozetetes } \\
\text { cayanensis }\end{array}$ & hellmayri & 1 & 20086 & ICN & Colombia & Cundinamarca & $\begin{array}{l}\text { Cund., Apulo (R. } \\
\text { Reyes), Río } \\
\text { Calandaima. }\end{array}$ & 4,47565277 & 74,5423425 & Piel \\
\hline $\begin{array}{l}\text { Myiozetetes } \\
\text { cayanensis }\end{array}$ & hellmayri & 1 & 20162 & ICN & Colombia & Antioquia & Ant., Amagá. & 6,05000019 & $\begin{array}{c}- \\
75,6999969\end{array}$ & Piel \\
\hline $\begin{array}{l}\text { Myiozetetes } \\
\text { cayanensis }\end{array}$ & hellmayri & 1 & 20283 & ICN & Colombia & Santander & $\begin{array}{l}\text { Sant., Simacota } \\
\text { (Vda. San Pascual). }\end{array}$ & 6,40860987 & 73,3541718 & Piel \\
\hline $\begin{array}{l}\text { Myiozetetes } \\
\text { cayanensis }\end{array}$ & hellmayri & 1 & 20381 & ICN & Colombia & Cundinamarca & $\begin{array}{l}\text { Cund., Laguna de } \\
\text { Pedro Palo. }\end{array}$ & 4,68599832 & $\begin{array}{c}- \\
74,3865976\end{array}$ & Piel \\
\hline $\begin{array}{l}\text { Myiozetetes } \\
\text { cayanensis }\end{array}$ & hellmayri & 1 & 20837 & ICN & Colombia & Cundinamarca & $\begin{array}{c}\text { Cund., } \\
\text { Santandercito, } \\
\text { Finca Monterrey. }\end{array}$ & 4,59301489 & $\begin{array}{c}- \\
74,3470495\end{array}$ & Piel \\
\hline $\begin{array}{l}\text { Myiozetetes } \\
\text { cayanensis }\end{array}$ & hellmayri & 1 & 20838 & ICN & Colombia & Cundinamarca & $\begin{array}{l}\text { Cund., } \\
\text { Santandercito, } \\
\text { Finca Monterrey. }\end{array}$ & 4,59301489 & $\begin{array}{c}- \\
74,3470495\end{array}$ & Piel \\
\hline $\begin{array}{l}\text { Myiozetetes } \\
\text { cayanensis }\end{array}$ & hellmayri & 1 & 20839 & ICN & Colombia & Cundinamarca & $\begin{array}{l}\text { Cund., } \\
\text { Santandercito, } \\
\text { Monterredonde. }\end{array}$ & 4,59003862 & $\begin{array}{c}- \\
74,3429123\end{array}$ & Piel \\
\hline $\begin{array}{l}\text { Myiozetetes } \\
\text { cayanensis }\end{array}$ & rufipennis & 2 & 20971 & ICN & Colombia & Meta & $\begin{array}{c}\text { Meta, San Juan de } \\
\text { Arama, Hato } \\
\text { Mirolindo. }\end{array}$ & 3,43333006 & $\begin{array}{c}- \\
73,8333282\end{array}$ & Piel \\
\hline $\begin{array}{l}\text { Myiozetetes } \\
\text { cayanensis }\end{array}$ & hellmayri & 1 & 21114 & ICN & Colombia & Cundinamarca & $\begin{array}{l}\text { Cund., Planicie de } \\
\text { Fusagasugá, } \\
\text { (Quebrada Seca). }\end{array}$ & 4,33399176 & $\begin{array}{c}- \\
74,3528364\end{array}$ & Piel \\
\hline $\begin{array}{l}\text { Myiozetetes } \\
\text { cayanensis }\end{array}$ & hellmayri & 1 & 21115 & ICN & Colombia & Cundinamarca & $\begin{array}{l}\text { Cund., Planicie de } \\
\text { Fusagasugá, } \\
\text { (Quebrada Seca). }\end{array}$ & 4,33399176 & $\begin{array}{c}- \\
74,3528364\end{array}$ & Piel \\
\hline $\begin{array}{l}\text { Myiozetetes } \\
\text { cayanensis }\end{array}$ & hellmayri & 1 & 21116 & ICN & Colombia & Cundinamarca & $\begin{array}{l}\text { Cund., Planicie de } \\
\text { Fusagasugá, } \\
\text { (Quebrada Seca). }\end{array}$ & 4,33399176 & $\begin{array}{c}- \\
74,3528364\end{array}$ & Piel \\
\hline $\begin{array}{l}\text { Myiozetetes } \\
\text { cayanensis }\end{array}$ & hellmayri & 1 & 21117 & ICN & Colombia & Cundinamarca & $\begin{array}{c}\text { Cund., Planicies de } \\
\text { Fusagasugá, } \\
\text { (Quebrada Seca). }\end{array}$ & 4,333999176 & $\begin{array}{c}- \\
74,3528364\end{array}$ & Piel \\
\hline $\begin{array}{l}\text { Myiozetetes } \\
\text { cayanensis }\end{array}$ & hellmayri & 1 & 21257 & ICN & Colombia & Cundinamarca & $\begin{array}{c}\text { Cund., Planicies de } \\
\text { Fusagasugá, } \\
\text { Quebrada Seca. }\end{array}$ & 4,33399176 & 74,3528364 & Piel \\
\hline $\begin{array}{l}\text { Myiozetetes } \\
\text { cayanensis }\end{array}$ & hellmayri & 1 & 21361 & ICN & Colombia & Cundinamarca & $\begin{array}{l}\text { Cund., Planicies de } \\
\text { Fusagasugá, (La } \\
\text { Serena). }\end{array}$ & 4,3499999 & $\begin{array}{c}- \\
74,3666687\end{array}$ & Piel \\
\hline $\begin{array}{l}\text { Myiozetetes } \\
\text { cayanensis }\end{array}$ & hellmayri & 1 & 21476 & ICN & Colombia & Cundinamarca & $\begin{array}{l}\text { Cund., Plan. de } \\
\text { Fusagasugá. }\end{array}$ & 4,3499999 & $\begin{array}{c}- \\
74,3666687\end{array}$ & Piel \\
\hline $\begin{array}{l}\text { Myiozetetes } \\
\text { cayanensis }\end{array}$ & hellmayri & 1 & 21819 & ICN & Colombia & Huila & Huila, San Agustín. & 1,88332999 & $\begin{array}{c}- \\
76,2666702\end{array}$ & Piel \\
\hline $\begin{array}{l}\text { Myiozetetes } \\
\text { cayanensis }\end{array}$ & hellmayri & 1 & 21820 & ICN & Colombia & Huila & Huila, San Agustín. & 1,88332999 & $\begin{array}{c}- \\
76,2666702\end{array}$ & Piel \\
\hline $\begin{array}{l}\text { Myiozetetes } \\
\text { cayanensis }\end{array}$ & hellmayri & 1 & 22485 & ICN & Colombia & Santander & $\begin{array}{l}\text { Sant. Simacota, } \\
\text { (Vda. San Pascual, } \\
\text { Finca Picurales). }\end{array}$ & 6,40860987 & $\begin{array}{c}- \\
73,3541718\end{array}$ & Piel \\
\hline $\begin{array}{l}\text { Myiozetetes } \\
\text { cayanensis }\end{array}$ & rufipennis & 2 & 23159 & ICN & Colombia & Meta & $\begin{array}{l}\text { Hacienda Los } \\
\text { Guaduales. }\end{array}$ & 3,70000005 & 73,6999969 & Piel \\
\hline $\begin{array}{l}\text { Myiozetetes } \\
\text { cayanensis }\end{array}$ & rufipennis & 2 & 23727 & ICN & Colombia & Meta & $\begin{array}{l}\text { Meta, Piñalito } \\
\text { (Serranía de La } \\
\text { Macarena). }\end{array}$ & 2,95698539 & $\begin{array}{c}- \\
73,6968959\end{array}$ & Piel \\
\hline $\begin{array}{l}\text { Myiozetetes } \\
\text { cayanensis }\end{array}$ & hellmayri & 1 & 24328 & ICN & Colombia & Valle del Cauca & $\begin{array}{l}\text { Laguna de Sonso, } \\
\text { Buga, Valle. }\end{array}$ & 3,86666989 & 76,3499985 & Piel \\
\hline $\begin{array}{l}\text { Myiozetetes } \\
\text { cayanensis }\end{array}$ & hellmayri & 1 & 24367 & ICN & Colombia & Santander & $\begin{array}{l}\text { Santander, San Gil, } \\
\text { Finca Zaragoza. }\end{array}$ & 6,55000019 & 73,1333313 & Piel \\
\hline $\begin{array}{l}\text { Myiozetetes } \\
\text { cayanensis }\end{array}$ & rufipennis & 2 & 25175 & ICN & Colombia & Meta & $\begin{array}{c}\text { Dept. Meta, Munc. } \\
\text { Restrepo, Estación } \\
\text { Pisicultura U. del } \\
\text { Llano. }\end{array}$ & 4,25 & 73,5500031 & Piel \\
\hline $\begin{array}{l}\text { Myiozetetes } \\
\text { cayanensis }\end{array}$ & hellmayri & 1 & 25681 & ICN & Colombia & Cesar & Cesar, San Alberto. & 7,75250006 & $\begin{array}{c}- \\
73,3891678\end{array}$ & Piel \\
\hline
\end{tabular}




\begin{tabular}{|c|c|c|c|c|c|c|c|c|c|c|}
\hline $\begin{array}{l}\text { Myiozetetes } \\
\text { cayanensis }\end{array}$ & hellmayri & 1 & 26511 & ICN & Colombia & Valle del Cauca & $\begin{array}{c}\text { Valle del Cauca, } \\
\text { Dagua, } \\
\text { Corregimiento } \\
\text { Loboguerrero, } \\
\text { carretera Cali - } \\
\text { Buenaventura, } \\
\text { margen izquierda } \\
\text { del Río Bitaco, Finc. } \\
\text { La Querella. }\end{array}$ & 3,76666999 & $\begin{array}{c}- \\
76,6833267\end{array}$ & Piel \\
\hline $\begin{array}{l}\text { Myiozetetes } \\
\text { cayanensis }\end{array}$ & hellmayri & 1 & 26537 & ICN & Colombia & Valle del Cauca & $\begin{array}{c}\text { Valle del Cauca, } \\
\text { Dagua, } \\
\text { Corregimiento } \\
\text { Loboguerrero, } \\
\text { carretera Cali - } \\
\text { Buenaventura } \\
\text { margen izquierda } \\
\text { del Río Bitaco, Finc. } \\
\text { La Querella. }\end{array}$ & 3,76666999 & $\begin{array}{c}- \\
76,6833267\end{array}$ & Piel \\
\hline $\begin{array}{l}\text { Myiozetetes } \\
\text { cayanensis }\end{array}$ & hellmayri & 1 & 26538 & ICN & Colombia & Valle del Cauca & $\begin{array}{c}\text { Valle del Cauca, } \\
\text { Dagua, } \\
\text { Corregimiento } \\
\text { Loboguerrero, } \\
\text { carretera Cali - } \\
\text { Buenaventura, } \\
\text { margen izquierda } \\
\text { del Río Bitaco, Finc. } \\
\text { La Querella. }\end{array}$ & 3,76666999 & $\begin{array}{c}- \\
76,6833267\end{array}$ & Piel \\
\hline $\begin{array}{l}\text { Myiozetetes } \\
\text { cayanensis }\end{array}$ & hellmayri & 1 & 26636 & ICN & Colombia & Valle del Cauca & $\begin{array}{c}\text { Valle del Cauca, } \\
\text { Dagua, } \\
\text { Corregimiento El } \\
\text { Naranjo, carretera } \\
\text { Cali - } \\
\text { Buenaventura, } \\
\text { Quebrada Los } \\
\text { Indios, margen } \\
\text { derecha del Río } \\
\text { Dagua. }\end{array}$ & 3,78332996 & $\begin{array}{c}- \\
76,7333298\end{array}$ & Piel \\
\hline $\begin{array}{l}\text { Myiozetetes } \\
\text { cayanensis }\end{array}$ & hellmayri & 1 & 27126 & ICN & Colombia & Santander & $\begin{array}{l}\text { Río Lebrijá, } \\
\text { Chuspas, F. } \\
\text { Pradera }\end{array}$ & 7,36667013 & $\begin{array}{c}- \\
73,3499985\end{array}$ & Piel \\
\hline $\begin{array}{l}\text { Myiozetetes } \\
\text { cayanensis }\end{array}$ & hellmayri & 1 & 27321 & ICN & Colombia & Tolima & $\begin{array}{l}\text { Tolima, Prado, } \\
\text { Represa del Prado. }\end{array}$ & 3,75584691 & $\begin{array}{c}- \\
74,8939346\end{array}$ & Piel \\
\hline $\begin{array}{l}\text { Myiozetetes } \\
\text { cayanensis }\end{array}$ & rufipennis & 2 & 28264 & ICN & Colombia & Arauca & $\begin{array}{l}\text { Arauca, Arauca, } \\
\text { casco urbano. }\end{array}$ & 7,08333015 & $-70,75$ & Piel \\
\hline $\begin{array}{l}\text { Myiozetetes } \\
\text { cayanensis }\end{array}$ & rufipennis & 2 & 28504 & ICN & Colombia & Meta & $\begin{array}{c}\text { Meta, Puerto } \\
\text { López, Vereda } \\
\text { Menegua, Finca } \\
\text { Lagunazo. }\end{array}$ & 4,09594031 & $\begin{array}{c}- \\
72,8155735\end{array}$ & Piel \\
\hline $\begin{array}{l}\text { Myiozetetes } \\
\text { cayanensis }\end{array}$ & rufipennis & 2 & 28515 & ICN & Colombia & Meta & $\begin{array}{c}\text { Meta, Puerto } \\
\text { López, Vereda } \\
\text { Menegua, Finca } \\
\text { Lagunazo. }\end{array}$ & 4,09594031 & $\begin{array}{c}- \\
72,8155735\end{array}$ & Piel \\
\hline $\begin{array}{l}\text { Myiozetetes } \\
\text { cayanensis }\end{array}$ & rufipennis & 2 & 28539 & ICN & Colombia & Meta & $\begin{array}{c}\text { Meta, Puerto } \\
\text { López, Vereda } \\
\text { Menegua, Finca } \\
\text { Lagunazo. }\end{array}$ & 4,09594031 & $\begin{array}{c}- \\
72,8155735\end{array}$ & Piel \\
\hline $\begin{array}{l}\text { Myiozetetes } \\
\text { cayanensis }\end{array}$ & hellmayri & 1 & 28601 & ICN & Colombia & Santander & $\begin{array}{l}\text { Santander, } \\
\text { Landázuri. }\end{array}$ & 6,23332977 & $\begin{array}{c}- \\
73,8166733\end{array}$ & Piel \\
\hline $\begin{array}{l}\text { Myiozetetes } \\
\text { cayanensis }\end{array}$ & hellmayri & 1 & 28703 & ICN & Colombia & Cundinamarca & $\begin{array}{l}\text { Cundinamarca, } \\
\text { Quipile, La Botica, } \\
\text { Vereda La Hoya. }\end{array}$ & 4,71248313 & $\begin{array}{c}- \\
74,5733134\end{array}$ & Piel \\
\hline $\begin{array}{l}\text { Myiozetetes } \\
\text { cayanensis }\end{array}$ & hellmayri & 1 & 30146 & ICN & Colombia & Antioquia & $\begin{array}{c}\text { Antioquia, } \\
\text { Cocorná, Vereda La } \\
\text { Veta. }\end{array}$ & 6,05653488 & $\begin{array}{c}- \\
75,1824297\end{array}$ & Piel \\
\hline $\begin{array}{l}\text { Myiozetetes } \\
\text { cayanensis }\end{array}$ & hellmayri & 1 & 30147 & ICN & Colombia & Antioquia & $\begin{array}{c}\text { Antioquia, } \\
\text { Cocorná, Vereda La } \\
\text { Veta. }\end{array}$ & 6,05653488 & $\begin{array}{c}- \\
75,1824297\end{array}$ & Piel \\
\hline
\end{tabular}




\begin{tabular}{|c|c|c|c|c|c|c|c|c|c|c|}
\hline $\begin{array}{l}\text { Myiozetetes } \\
\text { cayanensis }\end{array}$ & hellmayri & 1 & 30177 & $\mathrm{ICN}$ & Colombia & Antioquia & $\begin{array}{c}\text { Antioquia, } \\
\text { Cocorná, Vereda La } \\
\text { Veta, } 2 \text { km arriba } \\
\text { del cruce del } \\
\text { puente sobre el Río } \\
\text { Calderas. }\end{array}$ & 6,05653488 & $\begin{array}{c}- \\
75,1824297\end{array}$ & Piel \\
\hline $\begin{array}{l}\text { Myiozetetes } \\
\text { cayanensis }\end{array}$ & hellmayri & 1 & 30180 & ICN & Colombia & Antioquia & $\begin{array}{c}\text { Antioquia, } \\
\text { Cocorná, Vereda La } \\
\text { Veta, } 2 \text { km arriba } \\
\text { del curce del } \\
\text { puente sobre el Río } \\
\text { Calderas. }\end{array}$ & 6,05653488 & $\begin{array}{c}- \\
75,1824297\end{array}$ & Piel \\
\hline $\begin{array}{l}\text { Myiozetetes } \\
\text { cayanensis }\end{array}$ & hellmayri & 1 & 30237 & ICN & Colombia & Antioquia & $\begin{array}{c}\text { Antioquia, } \\
\text { Cocorná, Vereda La } \\
\text { Veta. }\end{array}$ & 6,05653488 & $\begin{array}{c}- \\
75,1824297\end{array}$ & Piel \\
\hline $\begin{array}{l}\text { Myiozetetes } \\
\text { cayanensis }\end{array}$ & rufipennis & 2 & 30605 & $\mathrm{ICN}$ & Colombia & Meta & $\begin{array}{l}\text { Meta, Restrepo, } \\
\text { Universidad } \\
\text { Tecnológica del } \\
\text { Llano. }\end{array}$ & 4,26239703 & $\begin{array}{c}- \\
73,5671178\end{array}$ & Piel \\
\hline $\begin{array}{l}\text { Myiozetetes } \\
\text { cayanensis }\end{array}$ & rufipennis & 2 & 30607 & ICN & Colombia & Meta & $\begin{array}{l}\text { Meta, Restrepo, } \\
\text { Universidad } \\
\text { Tecnológica del } \\
\text { Llano. }\end{array}$ & 4,26239703 & $\begin{array}{c}- \\
73,5671178\end{array}$ & Piel \\
\hline $\begin{array}{l}\text { Myiozetetes } \\
\text { cayanensis }\end{array}$ & hellmayri & 1 & 30673 & $\mathrm{ICN}$ & Colombia & Chocó & $\begin{array}{l}\text { Chocó, municipio } \\
\text { de Riosucio, } \\
\text { Campamento La } \\
\text { Balsa. }\end{array}$ & 7,44999981 & $\begin{array}{c}- \\
77,1166687\end{array}$ & Piel \\
\hline $\begin{array}{l}\text { Myiozetetes } \\
\text { cayanensis }\end{array}$ & hellmayri & 1 & 30674 & ICN & Colombia & Chocó & $\begin{array}{l}\text { Chocó, municipio } \\
\text { de Riosucio, } \\
\text { Campamento La } \\
\text { Balsa. }\end{array}$ & 7,44999981 & $\begin{array}{c}- \\
77,1166687\end{array}$ & Piel \\
\hline $\begin{array}{l}\text { Myiozetetes } \\
\text { cayanensis }\end{array}$ & hellmayri & 1 & 30675 & $\mathrm{ICN}$ & Colombia & Chocó & $\begin{array}{l}\text { Chocó, municipio } \\
\text { de Riosucio, } \\
\text { Campamento La } \\
\text { Balsa. }\end{array}$ & 7,44999981 & $\begin{array}{c}- \\
77,1166687\end{array}$ & Piel \\
\hline $\begin{array}{l}\text { Myiozetetes } \\
\text { cayanensis }\end{array}$ & rufipennis & 2 & 30826 & ICN & Colombia & Meta & $\begin{array}{l}\text { Meta, Restrepo, } \\
\text { alrededores Cread. }\end{array}$ & 4,25 & $\begin{array}{c}- \\
73,5500031\end{array}$ & Piel \\
\hline $\begin{array}{l}\text { Myiozetetes } \\
\text { cayanensis }\end{array}$ & rufipennis & 2 & 31072 & ICN & Colombia & Arauca & $\begin{array}{l}\text { Departamento de } \\
\text { Arauca, } 50 \text { km SW } \\
\text { de Arauca, Campo } \\
\text { Caño Limón. }\end{array}$ & 7 & $\begin{array}{c}- \\
71,3166733\end{array}$ & Piel \\
\hline $\begin{array}{l}\text { Myiozetetes } \\
\text { cayanensis }\end{array}$ & hellmayri & 1 & 31495 & ICN & Colombia & Córdoba & $\begin{array}{c}\text { Córdoba, Tierralta, } \\
\text { Caserío Frasquillo - } \\
\text { El Remolino, } \\
\text { Quebrada Gaita. }\end{array}$ & 8,16666985 & $\begin{array}{c}- \\
76,0500031\end{array}$ & Piel \\
\hline $\begin{array}{c}\text { Myiozetetes } \\
\text { cayanensis }\end{array}$ & rufipennis & 2 & 32004 & $\mathrm{ICN}$ & Colombia & Caquetá & $\begin{array}{c}\text { Río Cuñare, raudal } \\
\text { El Tubo. }\end{array}$ & 0,43333 & $-72,5$ & Piel \\
\hline $\begin{array}{l}\text { Myiozetetes } \\
\text { cayanensis }\end{array}$ & rufipennis & 2 & 32149 & ICN & Colombia & Meta & $\begin{array}{l}\text { Dpto. Meta, Mpo. } \\
\text { Restrepo, } 0.5 \mathrm{~km} \mathrm{E} \\
\quad \text { Restrepo. }\end{array}$ & 4,25 & $\begin{array}{c}- \\
73,5500031\end{array}$ & Piel \\
\hline $\begin{array}{l}\text { Myiozetetes } \\
\text { cayanensis }\end{array}$ & hellmayri & 1 & 32431 & ICN & Colombia & Magdalena & $\begin{array}{l}\text { Magdalena, } \\
\text { Municipios de } \\
\text { Pivijay y Remolino, } \\
\text { SFF Ciénaga } \\
\text { Grande, Playón, } \\
\text { Río Fundación. }\end{array}$ & 10,6775054 & $\begin{array}{c}- \\
74,4702443\end{array}$ & Piel \\
\hline $\begin{array}{l}\text { Myiozetetes } \\
\text { cayanensis }\end{array}$ & hellmayri & 1 & 32432 & ICN & Colombia & Magdalena & $\begin{array}{l}\text { Magdalena, } \\
\text { Municipios de } \\
\text { Pivijay y Remolino, } \\
\text { SFF Ciénaga } \\
\text { Grande, Playón, } \\
\text { Río Fundación. }\end{array}$ & 10,6775054 & $\begin{array}{c}- \\
74,4702443\end{array}$ & Piel \\
\hline $\begin{array}{l}\text { Myiozetetes } \\
\text { cayanensis }\end{array}$ & rufipennis & 2 & 32765 & ICN & Colombia & Casanare & $\begin{array}{l}\text { Casanare, Yopal, } \\
\text { Vereda El Guineo, } \\
\text { Finca Namasté. }\end{array}$ & 5,3499999 & $\begin{array}{c}- \\
72,4000015\end{array}$ & Piel \\
\hline
\end{tabular}




\begin{tabular}{|c|c|c|c|c|c|c|c|c|c|c|}
\hline $\begin{array}{l}\text { Myiozetetes } \\
\text { cayanensis }\end{array}$ & hellmayri & 1 & 34262 & ICN & Colombia & Nariño & $\begin{array}{l}\text { Nariño, Mpio. } \\
\text { Tumaco, Isla El } \\
\text { Morro. }\end{array}$ & 1,81888998 & 78,7566681 & Piel \\
\hline $\begin{array}{l}\text { Myiozetetes } \\
\text { cayanensis }\end{array}$ & hellmayri & 1 & 35136 & ICN & Colombia & Sucre & $\begin{array}{l}\text { Sucre: San } \\
\text { Marcos:Vda. La } \\
\text { Florida: Granja } \\
\text { Crocodylia. }\end{array}$ & 8,61638927 & $\begin{array}{c}- \\
75,1500015\end{array}$ & Piel \\
\hline $\begin{array}{l}\text { Myiozetetes } \\
\text { cayanensis }\end{array}$ & hellmayri & 1 & 35631 & ICN & Colombia & Nariño & $\begin{array}{l}\text { Nariño, Mpio. } \\
\text { Tumaco, B. } \\
\text { Ciudadela. Sede } \\
\text { Universidad de } \\
\text { Nariño }\end{array}$ & 1,78776181 & $\begin{array}{c}- \\
78,7815812\end{array}$ & Piel \\
\hline $\begin{array}{l}\text { Myiozetetes } \\
\text { cayanensis }\end{array}$ & rufipennis & 2 & 37763 & ICN & Colombia & Casanare & $\begin{array}{c}\text { Balquereña, } \\
\text { hacienda La Villa } \\
\text { Laura. }\end{array}$ & 5,16 & $-71,34$ & Piel \\
\hline $\begin{array}{l}\text { Myiozetetes } \\
\text { cayanensis }\end{array}$ & rufipennis & 2 & 38417 & ICN & Colombia & Meta & $\begin{array}{c}\text { Meta, Villavicencio, } \\
\text { Vereda la llanerita, } \\
\text { Finca Villa Franca } \\
\text { de Oria }\end{array}$ & 4,08250723 & $\begin{array}{c}- \\
73,5440338\end{array}$ & Piel \\
\hline $\begin{array}{l}\text { Myiozetetes } \\
\text { cayanensis }\end{array}$ & rufipennis & 2 & 38822 & ICN & Colombia & Arauca & $\begin{array}{c}\text { Arauca, Arauca, } \\
\text { Vereda Panamá, } \\
\text { Estero Lipa Doña } \\
\text { Juana }\end{array}$ & 6,80795784 & $\begin{array}{c}- \\
71,2721443\end{array}$ & Piel \\
\hline $\begin{array}{l}\text { Myiozetetes } \\
\text { cayanensis }\end{array}$ & hellmayri & 1 & 39488 & ICN & Colombia & Nariño & $\begin{array}{l}\text { Nariño. Mpio. } \\
\text { Tumaco, FCA mar } \\
\text { agricola, km22 } \\
\text { carretera junin }\end{array}$ & 1,41 & $-78,45$ & Piel \\
\hline $\begin{array}{l}\text { Myiozetetes } \\
\text { cayanensis }\end{array}$ & hellmayri & 1 & 39596 & ICN & Colombia & Nariño & $\begin{array}{l}\text { Nariño. Mpio. } \\
\text { Tumaco, FCA mar } \\
\text { agricola }\end{array}$ & 1,69635252 & $\begin{array}{c}- \\
78,7539273\end{array}$ & Piel \\
\hline $\begin{array}{l}\text { Myiozetetes } \\
\text { cayanensis }\end{array}$ & erythropterus & 4 & 1831 & MCN & Brasil & Minas Gerais & $\begin{array}{l}\text { Santa Barbara, } \\
\text { Fazenda Bocaina }\end{array}$ & $-19,999722$ & $\begin{array}{c}- \\
43,4708333\end{array}$ & Piel \\
\hline $\begin{array}{l}\text { Myiozetetes } \\
\text { cayanensis }\end{array}$ & erythropterus & 4 & 1832 & $\mathrm{MCN}$ & Brasil & Minas Gerais & $\begin{array}{l}\text { Santa Barbara, } \\
\text { Fazenda Bocaina }\end{array}$ & $-19,999722$ & $\begin{array}{c}- \\
43,4708333\end{array}$ & Piel \\
\hline $\begin{array}{l}\text { Myiozetetes } \\
\text { cayanensis }\end{array}$ & erythropterus & 4 & 1962 & $\mathrm{MCN}$ & Brasil & Minas Gerais & $\begin{array}{c}\text { Santa Barbara, } \\
\text { Conceicao do Rio } \\
\text { Acima }\end{array}$ & $-20,078333$ & $\begin{array}{c}- \\
43,5905556\end{array}$ & Piel \\
\hline $\begin{array}{l}\text { Myiozetetes } \\
\text { cayanensis }\end{array}$ & erythropterus & 4 & 4547 & $\mathrm{MCN}$ & Brasil & Minas Gerais & $\begin{array}{l}\text { Conceicao do Mato } \\
\text { Dentro, Piraquara, } \\
\text { margem do Rio } \\
\text { Parauninha }\end{array}$ & $-18,991111$ & $-43,49$ & Piel \\
\hline $\begin{array}{l}\text { Myiozetetes } \\
\text { cayanensis }\end{array}$ & hellmayri & 1 & BSA-4696 & IAvH & Colombia & Bolívar & Córdoba & 9,6300001 & $\begin{array}{c}- \\
74,9122238\end{array}$ & $\begin{array}{l}\text { Archivo } \\
\text { de audio }\end{array}$ \\
\hline $\begin{array}{l}\text { Myiozetetes } \\
\text { cayanensis }\end{array}$ & hellmayri & 1 & BSA-8236 & IAvH & Colombia & Caldas & $\begin{array}{c}\text { Neira, Vereda Los } \\
\text { Bohíos, Hacienda } \\
\text { Tintiná, Cuenca río } \\
\text { Tapias }\end{array}$ & 5,2461109 & $\begin{array}{c}- \\
75,6852798\end{array}$ & $\begin{array}{l}\text { Archivo } \\
\text { de audio }\end{array}$ \\
\hline $\begin{array}{l}\text { Myiozetetes } \\
\text { cayanensis }\end{array}$ & hellmayri & 1 & BSA-8471 & IAvH & Colombia & Caldas & $\begin{array}{c}\text { Neira, Vereda Los } \\
\text { Bohíos, Hacienda } \\
\text { Tintiná, Cuenca río } \\
\text { Tapias }\end{array}$ & 5,2461109 & $\begin{array}{c}- \\
75,6852798\end{array}$ & $\begin{array}{l}\text { Archivo } \\
\text { de audio }\end{array}$ \\
\hline $\begin{array}{l}\text { Myiozetetes } \\
\text { cayanensis }\end{array}$ & hellmayri & 1 & BSA-8483 & IAvH & Colombia & Caldas & $\begin{array}{c}\text { Neira, Vereda Los } \\
\text { Bohíos, Hacienda } \\
\text { Tintiná, Cuenca río } \\
\text { Tapias }\end{array}$ & 5,2461109 & $\begin{array}{c}- \\
75,6852798\end{array}$ & $\begin{array}{l}\text { Archivo } \\
\text { de audio }\end{array}$ \\
\hline $\begin{array}{l}\text { Myiozetetes } \\
\text { cayanensis }\end{array}$ & hellmayri & 1 & BSA-10690 & IAvH & Colombia & Chocó & $\begin{array}{l}\text { Bahía Solano, PNN } \\
\text { Ensenada de Utría }\end{array}$ & 6,0666666 & $\begin{array}{c}- \\
77,3833313\end{array}$ & $\begin{array}{l}\text { Archivo } \\
\text { de audio }\end{array}$ \\
\hline $\begin{array}{l}\text { Myiozetetes } \\
\text { cayanensis }\end{array}$ & hellmayri & 1 & BSA-10912 & IAvH & Colombia & Caldas & $\begin{array}{c}\text { Norcasia, } \\
\text { Corregimiento de } \\
\text { Norcasia-La Acacia- } \\
\text { Campamento Miel } \\
1\end{array}$ & 5,5741668 & $\begin{array}{c}- \\
74,9000015\end{array}$ & $\begin{array}{l}\text { Archivo } \\
\text { de audio }\end{array}$ \\
\hline $\begin{array}{l}\text { Myiozetetes } \\
\text { cayanensis }\end{array}$ & hellmayri & 1 & BSA-11279 & IAvH & Colombia & Cundinamarca & $\begin{array}{l}\text { Yacopí, El Monte } \\
\text { del Diablo }\end{array}$ & 5,5886946 & $\begin{array}{c}- \\
74,2283325\end{array}$ & $\begin{array}{l}\text { Archivo } \\
\text { de audio }\end{array}$ \\
\hline
\end{tabular}




\begin{tabular}{|c|c|c|c|c|c|c|c|c|c|c|}
\hline $\begin{array}{l}\text { Myiozetetes } \\
\text { cayanensis }\end{array}$ & hellmayri & 1 & BSA-11386 & $\mathrm{IAvH}$ & Colombia & Huila & San Agustín & 1,882536 & $-76,268832$ & $\begin{array}{l}\text { Archivo } \\
\text { de audio }\end{array}$ \\
\hline $\begin{array}{l}\text { Myiozetetes } \\
\text { cayanensis }\end{array}$ & hellmayri & 1 & BSA-11989 & IAvH & Colombia & Valle del Cauca & $\begin{array}{c}\text { Yotoco, Reserva } \\
\text { privada Yotoco }\end{array}$ & 3,8775001 & $\begin{array}{c}- \\
76,4319458\end{array}$ & $\begin{array}{l}\text { Archivo } \\
\text { de audio }\end{array}$ \\
\hline $\begin{array}{l}\text { Myiozetetes } \\
\text { cayanensis }\end{array}$ & hellmayri & 1 & BSA-11990 & $\mathrm{IAvH}$ & Colombia & Valle del Cauca & $\begin{array}{c}\text { Yotoco, Reserva } \\
\text { privada Yotoco }\end{array}$ & 3,8775001 & $\begin{array}{c}- \\
76,4319458\end{array}$ & $\begin{array}{l}\text { Archivo } \\
\text { de audio }\end{array}$ \\
\hline $\begin{array}{l}\text { Myiozetetes } \\
\text { cayanensis }\end{array}$ & hellmayri & 1 & BSA-12883 & $\mathrm{IAvH}$ & Colombia & Valle del Cauca & $\begin{array}{c}\text { Yotoco, Reserva } \\
\text { privada Yotoco }\end{array}$ & 3,8775001 & $\begin{array}{c}- \\
76,4319458\end{array}$ & $\begin{array}{l}\text { Archivo } \\
\text { de audio }\end{array}$ \\
\hline $\begin{array}{l}\text { Myiozetetes } \\
\text { cayanensis }\end{array}$ & hellmayri & 1 & BSA-12890 & $\mathrm{IAvH}$ & Colombia & Valle del Cauca & $\begin{array}{c}\text { Yotoco, Reserva } \\
\text { privada Yotoco }\end{array}$ & 3,8775001 & $\begin{array}{c}- \\
76,4319458\end{array}$ & $\begin{array}{l}\text { Archivo } \\
\text { de audio }\end{array}$ \\
\hline $\begin{array}{l}\text { Myiozetetes } \\
\text { cayanensis }\end{array}$ & hellmayri & 1 & BSA-12923 & $\mathrm{IAvH}$ & Colombia & Valle del Cauca & $\begin{array}{c}\text { Yotoco, Reserva } \\
\text { privada Yotoco }\end{array}$ & 3,8775001 & $\begin{array}{c}- \\
76,4319458\end{array}$ & $\begin{array}{l}\text { Archivo } \\
\text { de audio }\end{array}$ \\
\hline $\begin{array}{l}\text { Myiozetetes } \\
\text { cayanensis }\end{array}$ & hellmayri & 1 & BSA-13002 & $\mathrm{IAvH}$ & Colombia & Valle del Cauca & $\begin{array}{c}\text { Yotoco, Hacienda } \\
\text { La Gloria, Bosque } \\
\text { de Chatas }\end{array}$ & 3,8594444 & $\begin{array}{c}- \\
76,4692764\end{array}$ & $\begin{array}{l}\text { Archivo } \\
\text { de audio }\end{array}$ \\
\hline $\begin{array}{l}\text { Myiozetetes } \\
\text { cayanensis }\end{array}$ & hellmayri & 1 & BSA-17367 & $\mathrm{IAvH}$ & Colombia & Cundinamarca & $\begin{array}{c}\text { Tena, Reserva } \\
\text { Forestal } \\
\text { Protectora, } \\
\text { Productora Laguna } \\
\text { de Pedro Palo, } \\
\text { Vereda } \\
\text { Catalamonte }\end{array}$ & 4,6971002 & $\begin{array}{c}- \\
74,3835831\end{array}$ & $\begin{array}{l}\text { Archivo } \\
\text { de audio }\end{array}$ \\
\hline $\begin{array}{l}\text { Myiozetetes } \\
\text { cayanensis }\end{array}$ & hellmayri & 1 & BSA-19152 & $\mathrm{IAvH}$ & Colombia & Antioquia & $\begin{array}{c}\text { Amalfi, Vereda } \\
\text { Salazar, Bodega } \\
\text { Vieja, Orilla del Rio } \\
\text { Riachón }\end{array}$ & 6,9715834 & $\begin{array}{c}- \\
75,0568085\end{array}$ & $\begin{array}{l}\text { Archivo } \\
\text { de audio }\end{array}$ \\
\hline $\begin{array}{l}\text { Myiozetetes } \\
\text { cayanensis }\end{array}$ & hellmayri & 1 & BSA-19293 & $\mathrm{IAvH}$ & Colombia & Antioquia & $\begin{array}{c}\text { Amalfi, Vereda } \\
\text { Cajamarca, Finca } \\
\text { Canales }\end{array}$ & 6,8235002 & $\begin{array}{c}- \\
75,0935288\end{array}$ & $\begin{array}{l}\text { Archivo } \\
\text { de audio }\end{array}$ \\
\hline $\begin{array}{l}\text { Myiozetetes } \\
\text { cayanensis }\end{array}$ & hellmayri & 1 & BSA-24092 & $\mathrm{IAvH}$ & Colombia & Huila & San Agustín & 1,882536 & $-76,268832$ & $\begin{array}{l}\text { Archivo } \\
\text { de audio }\end{array}$ \\
\hline $\begin{array}{l}\text { Myiozetetes } \\
\text { cayanensis }\end{array}$ & hellmayri & 1 & BSA-24093 & $\mathrm{IAvH}$ & Colombia & Huila & San Agustín & 1,882536 & $-76,268832$ & $\begin{array}{l}\text { Archivo } \\
\text { de audio }\end{array}$ \\
\hline $\begin{array}{l}\text { Myiozetetes } \\
\text { cayanensis }\end{array}$ & rufipennis & 2 & BSA-28717 & $\mathrm{IAvH}$ & Colombia & Vichada & $\begin{array}{c}\text { Cumaribo, } \\
\text { Resguardo } \\
\text { Unificado Selva de } \\
\text { Matavén, Cuenca } \\
\text { baja del caño } \\
\text { Matavén, sector } \\
\text { Matavén-Fruta }\end{array}$ & 4,4902778 & $\begin{array}{c}- \\
67,8719482\end{array}$ & $\begin{array}{l}\text { Archivo } \\
\text { de audio }\end{array}$ \\
\hline $\begin{array}{l}\text { Myiozetetes } \\
\text { cayanensis }\end{array}$ & rufipennis & 2 & BSA-28722 & $\mathrm{IAvH}$ & Colombia & Vichada & $\begin{array}{c}\text { Cumaribo, } \\
\text { Resguardo } \\
\text { Unificado Selva de } \\
\text { Matavén, Cuenca } \\
\text { baja del caño } \\
\text { Matavén, sector } \\
\text { Matavén-Fruta }\end{array}$ & 4,4902778 & $\begin{array}{c}- \\
67,8719482\end{array}$ & $\begin{array}{l}\text { Archivo } \\
\text { de audio }\end{array}$ \\
\hline $\begin{array}{l}\text { Myiozetetes } \\
\text { cayanensis }\end{array}$ & rufipennis & 2 & BSA-28934 & IAvH & Colombia & Vichada & $\begin{array}{c}\text { Cumaribo, } \\
\text { Resguardo } \\
\text { Unificado Selva de } \\
\text { Matavén, Cuenca } \\
\text { baja del caño } \\
\text { Matavén, sector } \\
\text { Matavén-Fruta }\end{array}$ & 4,4902778 & $\begin{array}{c}- \\
67,8719482\end{array}$ & $\begin{array}{l}\text { Archivo } \\
\text { de audio }\end{array}$ \\
\hline $\begin{array}{l}\text { Myiozetetes } \\
\text { cayanensis }\end{array}$ & rufipennis & 2 & BSA-28936 & IAvH & Colombia & Vichada & $\begin{array}{c}\text { Cumaribo, } \\
\text { Resguardo } \\
\text { Unificado Selva de } \\
\text { Matavén, Cuenca } \\
\text { baja del caño } \\
\text { Matavén, sector } \\
\text { Matavén-Fruta }\end{array}$ & 4,4902778 & $\begin{array}{c}- \\
67,8719482\end{array}$ & $\begin{array}{l}\text { Archivo } \\
\text { de audio }\end{array}$ \\
\hline $\begin{array}{l}\text { Myiozetetes } \\
\text { cayanensis }\end{array}$ & hellmayri & 1 & BSA-31568 & IAvH & Colombia & Sucre & $\begin{array}{l}\text { Tolú, Montaña de } \\
\text { los Navas, Bosque } \\
\text { húmedo }\end{array}$ & 9,5775003 & $-75,461113$ & $\begin{array}{l}\text { Archivo } \\
\text { de audio }\end{array}$ \\
\hline $\begin{array}{l}\text { Myiozetetes } \\
\text { cayanensis }\end{array}$ & hellmayri & 1 & BSA-31638 & IAvH & Colombia & Sucre & $\begin{array}{l}\text { Tolú, Montaña de } \\
\text { los Navas, Bosque } \\
\text { húmedo }\end{array}$ & 9,5775003 & $-75,461113$ & $\begin{array}{l}\text { Archivo } \\
\text { de audio }\end{array}$ \\
\hline
\end{tabular}




\begin{tabular}{|c|c|c|c|c|c|c|c|c|c|c|}
\hline $\begin{array}{l}\text { Myiozetetes } \\
\text { cayanensis }\end{array}$ & hellmayri & 1 & 7828 & $\mathrm{ML}$ & Panamá & Colón & Colón & 9,14454 & $-79,702428$ & $\begin{array}{l}\text { Archivo } \\
\text { de audio }\end{array}$ \\
\hline $\begin{array}{l}\text { Myiozetetes } \\
\text { cayanensis }\end{array}$ & hellmayri & 1 & 7829 & $\mathrm{ML}$ & Panamá & Colón & Colón & 9,16181 & $-79,697002$ & $\begin{array}{l}\text { Archivo } \\
\text { de audio }\end{array}$ \\
\hline $\begin{array}{l}\text { Myiozetetes } \\
\text { cayanensis }\end{array}$ & cayanensis & 3 & 24132 & $\mathrm{ML}$ & Perú & Madre de Dios & $\begin{array}{c}\text { Reserva Nacional } \\
\text { Tambopata }\end{array}$ & $-12,88$ & $-69,28$ & $\begin{array}{l}\text { Archivo } \\
\text { de audio }\end{array}$ \\
\hline $\begin{array}{l}\text { Myiozetetes } \\
\text { cayanensis }\end{array}$ & cayanensis & 3 & 24171 & $\mathrm{ML}$ & Perú & Madre de Dios & $\begin{array}{c}\text { Reserva Nacional } \\
\text { Tambopata }\end{array}$ & $-12,88$ & $-69,28$ & $\begin{array}{l}\text { Archivo } \\
\text { de audio }\end{array}$ \\
\hline $\begin{array}{l}\text { Myiozetetes } \\
\text { cayanensis }\end{array}$ & cayanensis & 3 & 25266 & $\mathrm{ML}$ & Surinam & Sipaliwini & Kabalebo & 4,732311 & $-56,198035$ & $\begin{array}{l}\text { Archivo } \\
\text { de audio }\end{array}$ \\
\hline $\begin{array}{l}\text { Myiozetetes } \\
\text { cayanensis }\end{array}$ & hellmayri & 1 & 25601 & $\mathrm{ML}$ & Panamá & Panamá & Panamá & 9,043028 & $-79,358043$ & $\begin{array}{l}\text { Archivo } \\
\text { de audio }\end{array}$ \\
\hline $\begin{array}{l}\text { Myiozetetes } \\
\text { cayanensis }\end{array}$ & hellmayri & 1 & 25633 & $\mathrm{ML}$ & Panamá & Panamá & Panamá & 9,043028 & $-79,358043$ & $\begin{array}{l}\text { Archivo } \\
\text { de audio }\end{array}$ \\
\hline $\begin{array}{l}\text { Myiozetetes } \\
\text { cayanensis }\end{array}$ & cayanensis & 3 & 26112 & $\mathrm{ML}$ & Surinam & Bokopondo & NA & 4,943367 & $-55,170701$ & $\begin{array}{l}\text { Archivo } \\
\text { de audio }\end{array}$ \\
\hline $\begin{array}{l}\text { Myiozetetes } \\
\text { cayanensis }\end{array}$ & hellmayri & 1 & 28433 & $\mathrm{ML}$ & Panamá & Panamá & Panamá & 9,1 & $-79,65$ & $\begin{array}{l}\text { Archivo } \\
\text { de audio }\end{array}$ \\
\hline $\begin{array}{l}\text { Myiozetetes } \\
\text { cayanensis }\end{array}$ & hellmayri & 1 & 28528 & $\mathrm{ML}$ & Ecuador & $\begin{array}{l}\text { Santo Domingo } \\
\text { de los Tsachilas }\end{array}$ & Santo Domingo & $-0,3$ & $-79,1333$ & $\begin{array}{l}\text { Archivo } \\
\text { de audio }\end{array}$ \\
\hline $\begin{array}{l}\text { Myiozetetes } \\
\text { cayanensis }\end{array}$ & cayanensis & 3 & 30545 & $\mathrm{ML}$ & Venezuela & Bolívar & Sifontes & 6,08 & $-61,36$ & $\begin{array}{l}\text { Archivo } \\
\text { de audio }\end{array}$ \\
\hline $\begin{array}{l}\text { Myiozetetes } \\
\text { cayanensis }\end{array}$ & cayanensis & 3 & 34839 & $\mathrm{ML}$ & Brasil & Mato Grosso & Poconé & $-17,2667$ & $-56,9833$ & $\begin{array}{l}\text { Archivo } \\
\text { de audio }\end{array}$ \\
\hline $\begin{array}{l}\text { Myiozetetes } \\
\text { cayanensis }\end{array}$ & rufipennis & 2 & 35307 & $\mathrm{ML}$ & Venezuela & Cojedes & Girardot & 8,75 & $-68,5$ & $\begin{array}{l}\text { Archivo } \\
\text { de audio }\end{array}$ \\
\hline $\begin{array}{l}\text { Myiozetetes } \\
\text { cayanensis }\end{array}$ & cayanensis & 3 & 38580 & $\mathrm{ML}$ & Bolivia & Pando & Porvenir & $-11,25$ & $-68,68$ & $\begin{array}{l}\text { Archivo } \\
\text { de audio }\end{array}$ \\
\hline $\begin{array}{l}\text { Myiozetetes } \\
\text { cayanensis }\end{array}$ & cayanensis & 3 & 38835 & $\mathrm{ML}$ & Bolivia & Pando & Porvenir & $-11,25$ & $-68,68$ & $\begin{array}{l}\text { Archivo } \\
\text { de audio }\end{array}$ \\
\hline $\begin{array}{l}\text { Myiozetetes } \\
\text { cayanensis }\end{array}$ & rufipennis & 2 & 40413 & $\mathrm{ML}$ & Venezuela & Barinas & Bolívar & 8,8333 & $-70,5$ & $\begin{array}{l}\text { Archivo } \\
\text { de audio }\end{array}$ \\
\hline $\begin{array}{l}\text { Myiozetetes } \\
\text { cayanensis }\end{array}$ & cayanensis & 3 & 47943 & $\mathrm{ML}$ & Brasil & Pará & Placas & $-3,743695$ & $-54,812329$ & $\begin{array}{l}\text { Archivo } \\
\text { de audio }\end{array}$ \\
\hline $\begin{array}{l}\text { Myiozetetes } \\
\text { cayanensis }\end{array}$ & cayanensis & 3 & 48064 & $\mathrm{ML}$ & Brasil & Mato Grosso & Alta Floresta & $-9,686402$ & $-56,065542$ & $\begin{array}{l}\text { Archivo } \\
\text { de audio }\end{array}$ \\
\hline $\begin{array}{l}\text { Myiozetetes } \\
\text { cayanensis }\end{array}$ & cayanensis & 3 & 49725 & $\mathrm{ML}$ & Venezuela & Bolívar & Raúl Leoni & 6 & -64 & $\begin{array}{l}\text { Archivo } \\
\text { de audio }\end{array}$ \\
\hline $\begin{array}{l}\text { Myiozetetes } \\
\text { cayanensis }\end{array}$ & cayanensis & 3 & 51813 & $\mathrm{ML}$ & Bolivia & Beni & Rurrenabaque & $-14,45$ & $-67,53$ & $\begin{array}{l}\text { Archivo } \\
\text { de audio }\end{array}$ \\
\hline $\begin{array}{l}\text { Myiozetetes } \\
\text { cayanensis }\end{array}$ & cayanensis & 3 & 52176 & $\mathrm{ML}$ & Brasil & Mato Grosso & Paranaíta & $-9,417138$ & $-56,758759$ & $\begin{array}{l}\text { Archivo } \\
\text { de audio }\end{array}$ \\
\hline $\begin{array}{l}\text { Myiozetetes } \\
\text { cayanensis }\end{array}$ & rufipennis & 2 & 64807 & $\mathrm{ML}$ & Venezuela & Apure & Achaguas & 7,82 & $-68,3$ & $\begin{array}{l}\text { Archivo } \\
\text { de audio }\end{array}$ \\
\hline $\begin{array}{l}\text { Myiozetetes } \\
\text { cayanensis }\end{array}$ & rufipennis & 2 & 66515 & $\mathrm{ML}$ & Venezuela & La Guaira & Vargas & 10,5833 & $-66,9333$ & $\begin{array}{l}\text { Archivo } \\
\text { de audio }\end{array}$ \\
\hline $\begin{array}{l}\text { Myiozetetes } \\
\text { cayanensis }\end{array}$ & rufipennis & 2 & 66516 & $\mathrm{ML}$ & Venezuela & Guárico & Ortíz & 9,5 & $-67,6$ & $\begin{array}{l}\text { Archivo } \\
\text { de audio }\end{array}$ \\
\hline $\begin{array}{l}\text { Myiozetetes } \\
\text { cayanensis }\end{array}$ & rufipennis & 2 & 66517 & $\mathrm{ML}$ & Venezuela & Guárico & Ortíz & 9,5 & $-67,6$ & $\begin{array}{l}\text { Archivo } \\
\text { de audio }\end{array}$ \\
\hline $\begin{array}{l}\text { Myiozetetes } \\
\text { cayanensis }\end{array}$ & rufipennis & 2 & 66518 & $\mathrm{ML}$ & Venezuela & Guárico & Ortíz & 9,5 & $-67,6$ & $\begin{array}{l}\text { Archivo } \\
\text { de audio }\end{array}$ \\
\hline $\begin{array}{l}\text { Myiozetetes } \\
\text { cayanensis }\end{array}$ & rufipennis & 2 & 66519 & $\mathrm{ML}$ & Venezuela & Guárico & Ortíz & 9,5 & $-67,6$ & $\begin{array}{l}\text { Archivo } \\
\text { de audio }\end{array}$ \\
\hline $\begin{array}{l}\text { Myiozetetes } \\
\text { cayanensis }\end{array}$ & rufipennis & 2 & 66520 & $\mathrm{ML}$ & Venezuela & Guárico & Ortíz & 9,5 & $-67,6$ & $\begin{array}{l}\text { Archivo } \\
\text { de audio }\end{array}$ \\
\hline $\begin{array}{l}\text { Myiozetetes } \\
\text { cayanensis }\end{array}$ & rufipennis & 2 & 66521 & $\mathrm{ML}$ & Venezuela & La Guaira & Vargas & 10,5833 & $-66,9333$ & $\begin{array}{l}\text { Archivo } \\
\text { de audio }\end{array}$ \\
\hline $\begin{array}{l}\text { Myiozetetes } \\
\text { cayanensis }\end{array}$ & rufipennis & 2 & 66522 & $\mathrm{ML}$ & Venezuela & Guárico & $\begin{array}{c}\text { San Gerónimo de } \\
\text { Guayabal }\end{array}$ & 8,42 & $-67,5$ & $\begin{array}{l}\text { Archivo } \\
\text { de audio }\end{array}$ \\
\hline $\begin{array}{l}\text { Myiozetetes } \\
\text { cayanensis }\end{array}$ & cayanensis & 3 & 66523 & $\mathrm{ML}$ & Venezuela & Bolívar & Padre Pedro Chien & 7,9667 & $-61,8833$ & $\begin{array}{l}\text { Archivo } \\
\text { de audio }\end{array}$ \\
\hline $\begin{array}{l}\text { Myiozetetes } \\
\text { cayanensis }\end{array}$ & rufipennis & 2 & 66524 & $\mathrm{ML}$ & Venezuela & Barinas & Barinas & 8,2 & $-69,93$ & $\begin{array}{l}\text { Archivo } \\
\text { de audio }\end{array}$ \\
\hline $\begin{array}{l}\text { Myiozetetes } \\
\text { cayanensis }\end{array}$ & cayanensis & 3 & 66525 & $\mathrm{ML}$ & Venezuela & Bolívar & Padre Pedro Chien & 7,9667 & $-61,8833$ & $\begin{array}{l}\text { Archivo } \\
\text { de audio }\end{array}$ \\
\hline $\begin{array}{l}\text { Myiozetetes } \\
\text { cayanensis }\end{array}$ & cayanensis & 3 & 66526 & $\mathrm{ML}$ & Guyana & $\begin{array}{l}\text { Cuyuni- } \\
\text { Mazaruni }\end{array}$ & Bartica & 6,38 & $-58,68$ & $\begin{array}{l}\text { Archivo } \\
\text { de audio }\end{array}$ \\
\hline
\end{tabular}




\begin{tabular}{|c|c|c|c|c|c|c|c|c|c|c|}
\hline $\begin{array}{l}\text { Myiozetetes } \\
\text { cayanensis }\end{array}$ & cayanensis & 3 & 80488 & $\mathrm{ML}$ & Bolivia & Santa Cruz & $\begin{array}{l}\text { Reserva Ríos } \\
\text { Blanco y Negro }\end{array}$ & $-14,73$ & $-62,8$ & $\begin{array}{l}\text { Archivo } \\
\text { de audio }\end{array}$ \\
\hline $\begin{array}{l}\text { Myiozetetes } \\
\text { cayanensis }\end{array}$ & cayanensis & 3 & 80493 & $\mathrm{ML}$ & Bolivia & Santa Cruz & $\begin{array}{l}\text { Reserva Ríos } \\
\text { Blanco y Negro }\end{array}$ & $-14,73$ & $-62,8$ & $\begin{array}{l}\text { Archivo } \\
\text { de audio }\end{array}$ \\
\hline $\begin{array}{l}\text { Myiozetetes } \\
\text { cayanensis }\end{array}$ & cayanensis & 3 & 85019 & $\mathrm{ML}$ & Guyana & $\begin{array}{l}\text { Cuyuni- } \\
\text { Mazaruni }\end{array}$ & Bartica & 6,481696 & $-58,618898$ & $\begin{array}{l}\text { Archivo } \\
\text { de audio }\end{array}$ \\
\hline $\begin{array}{l}\text { Myiozetetes } \\
\text { cayanensis }\end{array}$ & hellmayri & 1 & 86005 & $\mathrm{ML}$ & Ecuador & Esmeraldas & San Lorenzo & 0,87 & $-78,52$ & $\begin{array}{l}\text { Archivo } \\
\text { de audio }\end{array}$ \\
\hline $\begin{array}{l}\text { Myiozetetes } \\
\text { cayanensis }\end{array}$ & hellmayri & 1 & 86827 & $\mathrm{ML}$ & Ecuador & Los Ríos & Buena Fé & $-0,55$ & $-79,37$ & $\begin{array}{l}\text { Archivo } \\
\text { de audio }\end{array}$ \\
\hline $\begin{array}{l}\text { Myiozetetes } \\
\text { cayanensis }\end{array}$ & cayanensis & 3 & 88169 & $\mathrm{ML}$ & Venezuela & Amazonas & $\begin{array}{l}\text { Autónomo } \\
\text { Manapiare }\end{array}$ & 5,6 & $-66,1$ & $\begin{array}{l}\text { Archivo } \\
\text { de audio }\end{array}$ \\
\hline $\begin{array}{l}\text { Myiozetetes } \\
\text { cayanensis }\end{array}$ & cayanensis & 3 & 89031 & $\mathrm{ML}$ & Brasil & Pará & Jacareacanga & $-9,3647$ & $-56,772604$ & $\begin{array}{l}\text { Archivo } \\
\text { de audio }\end{array}$ \\
\hline $\begin{array}{l}\text { Myiozetetes } \\
\text { cayanensis }\end{array}$ & cayanensis & 3 & 90647 & $\mathrm{ML}$ & Guyana & $\begin{array}{l}\text { Uppertakutu- } \\
\text { Upperessequibo }\end{array}$ & Dadanawa & 2,9 & $-59,5$ & $\begin{array}{l}\text { Archivo } \\
\text { de audio }\end{array}$ \\
\hline $\begin{array}{l}\text { Myiozetetes } \\
\text { cayanensis }\end{array}$ & cayanensis & 3 & 101750 & $\mathrm{ML}$ & Bolivia & Beni & Trinidad & $-14,78$ & $-64,95$ & $\begin{array}{l}\text { Archivo } \\
\text { de audio }\end{array}$ \\
\hline $\begin{array}{l}\text { Myiozetetes } \\
\text { cayanensis }\end{array}$ & cayanensis & 3 & 106216 & $\mathrm{ML}$ & Guyana & $\begin{array}{l}\text { Uppertakutu- } \\
\text { Upperessequibo }\end{array}$ & Dadanawa & 2,8333 & $-59,5$ & $\begin{array}{l}\text { Archivo } \\
\text { de audio }\end{array}$ \\
\hline $\begin{array}{l}\text { Myiozetetes } \\
\text { cayanensis }\end{array}$ & cayanensis & 3 & 106242 & $\mathrm{ML}$ & Guyana & $\begin{array}{l}\text { Uppertakutu- } \\
\text { Upperessequibo }\end{array}$ & Dadanawa & 2,918 & $-59,5105$ & $\begin{array}{l}\text { Archivo } \\
\text { de audio }\end{array}$ \\
\hline $\begin{array}{l}\text { Myiozetetes } \\
\text { cayanensis }\end{array}$ & cayanensis & 3 & 106358 & $\mathrm{ML}$ & Guyana & $\begin{array}{l}\text { Uppertakutu- } \\
\text { Upperessequibo }\end{array}$ & Aishalton & 2,193 & $-58,7038$ & $\begin{array}{l}\text { Archivo } \\
\text { de audio }\end{array}$ \\
\hline $\begin{array}{l}\text { Myiozetetes } \\
\text { cayanensis }\end{array}$ & cayanensis & 3 & 126654 & $\mathrm{ML}$ & Brasil & Pará & Parauapebas & $-6,0443$ & $-50,269$ & $\begin{array}{l}\text { Archivo } \\
\text { de audio }\end{array}$ \\
\hline $\begin{array}{l}\text { Myiozetetes } \\
\text { cayanensis }\end{array}$ & cayanensis & 3 & 134347 & $\mathrm{ML}$ & Guyana & $\begin{array}{l}\text { Uppertakutu- } \\
\text { Upperessequibo }\end{array}$ & Dadanawa & 2,826013 & $-59,512613$ & $\begin{array}{l}\text { Archivo } \\
\text { de audio }\end{array}$ \\
\hline $\begin{array}{l}\text { Myiozetetes } \\
\text { cayanensis }\end{array}$ & cayanensis & 3 & 134613 & $\mathrm{ML}$ & Surinam & Sipaliwini & Kabalebo & 4,345386 & $-56,750076$ & $\begin{array}{l}\text { Archivo } \\
\text { de audio }\end{array}$ \\
\hline $\begin{array}{l}\text { Myiozetetes } \\
\text { cayanensis }\end{array}$ & cayanensis & 3 & 134743 & $\mathrm{ML}$ & Bolivia & Santa Cruz & Boqui & $-18,198478$ & $-59,08421$ & $\begin{array}{l}\text { Archivo } \\
\text { de audio }\end{array}$ \\
\hline $\begin{array}{l}\text { Myiozetetes } \\
\text { cayanensis }\end{array}$ & cayanensis & 3 & 134820 & $\mathrm{ML}$ & Guyana & $\begin{array}{l}\text { Eastberbice- } \\
\text { Corentyne }\end{array}$ & NA & 4,326077 & $-57,969274$ & $\begin{array}{l}\text { Archivo } \\
\text { de audio }\end{array}$ \\
\hline $\begin{array}{l}\text { Myiozetetes } \\
\text { cayanensis }\end{array}$ & cayanensis & 3 & 144545 & $\mathrm{ML}$ & Guyana & Potaro-Siparuni & NA & 4,3333 & $-58,85$ & $\begin{array}{l}\text { Archivo } \\
\text { de audio }\end{array}$ \\
\hline $\begin{array}{l}\text { Myiozetetes } \\
\text { cayanensis }\end{array}$ & cayanensis & 3 & 144974 & $\mathrm{ML}$ & Guyana & $\begin{array}{l}\text { Uppertakutu- } \\
\text { Upperessequibo }\end{array}$ & Aishalton & 2,3667 & $-59,45$ & $\begin{array}{l}\text { Archivo } \\
\text { de audio }\end{array}$ \\
\hline $\begin{array}{l}\text { Myiozetetes } \\
\text { cayanensis }\end{array}$ & cayanensis & 3 & 145552 & $\mathrm{ML}$ & Guyana & Georgetown & Georgetown & 6,801272 & $-58,155125$ & $\begin{array}{l}\text { Archivo } \\
\text { de audio }\end{array}$ \\
\hline $\begin{array}{l}\text { Myiozetetes } \\
\text { cayanensis }\end{array}$ & hellmayri & 1 & 165955 & $\mathrm{ML}$ & Colombia & Antioquia & Anorí, El retiro. & 6,9848 & $-75,1117$ & $\begin{array}{l}\text { Archivo } \\
\text { de audio }\end{array}$ \\
\hline $\begin{array}{l}\text { Myiozetetes } \\
\text { cayanensis }\end{array}$ & hellmayri & 1 & 165958 & $\mathrm{ML}$ & Colombia & Antioquia & Anorí, El retiro. & 6,9848 & $-75,1117$ & $\begin{array}{l}\text { Archivo } \\
\text { de audio }\end{array}$ \\
\hline $\begin{array}{l}\text { Myiozetetes } \\
\text { cayanensis }\end{array}$ & cayanensis & 3 & 168800 & $\mathrm{ML}$ & Perú & Madre de Dios & Iberia & $-11,41$ & $-69,5$ & $\begin{array}{l}\text { Archivo } \\
\text { de audio }\end{array}$ \\
\hline $\begin{array}{l}\text { Myiozetetes } \\
\text { cayanensis }\end{array}$ & cayanensis & 3 & 172502 & $\mathrm{ML}$ & Venezuela & Bolívar & Sifontes & 6,1819 & $-61,4192$ & $\begin{array}{l}\text { Archivo } \\
\text { de audio }\end{array}$ \\
\hline $\begin{array}{l}\text { Myiozetetes } \\
\text { cayanensis }\end{array}$ & cayanensis & 3 & 172523 & $\mathrm{ML}$ & Venezuela & Bolívar & Sifontes & 6,075431 & $-61,793379$ & $\begin{array}{l}\text { Archivo } \\
\text { de audio }\end{array}$ \\
\hline $\begin{array}{l}\text { Myiozetetes } \\
\text { cayanensis }\end{array}$ & rufipennis & 2 & 177343 & $\mathrm{ML}$ & Venezuela & Sucre & Arismendi & 10,6985 & $-62,4597$ & $\begin{array}{l}\text { Archivo } \\
\text { de audio }\end{array}$ \\
\hline $\begin{array}{l}\text { Myiozetetes } \\
\text { cayanensis }\end{array}$ & cayanensis & 3 & 190756 & $\mathrm{ML}$ & Brasil & Mato Grosso & Poconé & $-16,7575$ & $-56,8764$ & $\begin{array}{l}\text { Archivo } \\
\text { de audio }\end{array}$ \\
\hline $\begin{array}{l}\text { Myiozetetes } \\
\text { cayanensis }\end{array}$ & rufipennis & 2 & 221655 & $\mathrm{ML}$ & Venezuela & Monagas & Maturín & 9,8818 & $-62,9593$ & $\begin{array}{l}\text { Archivo } \\
\text { de audio }\end{array}$ \\
\hline $\begin{array}{l}\text { Myiozetetes } \\
\text { cayanensis }\end{array}$ & cayanensis & 3 & 221694 & $\mathrm{ML}$ & Venezuela & Delta Amacuro & Antonio Díaz & 8,1103 & $-61,6955$ & $\begin{array}{l}\text { Archivo } \\
\text { de audio }\end{array}$ \\
\hline $\begin{array}{l}\text { Myiozetetes } \\
\text { cayanensis }\end{array}$ & cayanensis & 3 & 227518 & $\mathrm{ML}$ & Brasil & Mato Grosso & Poconé & $-17,364594$ & $-56,774851$ & $\begin{array}{l}\text { Archivo } \\
\text { de audio }\end{array}$ \\
\hline $\begin{array}{l}\text { Myiozetetes } \\
\text { cayanensis }\end{array}$ & cayanensis & 3 & 227762 & $\mathrm{ML}$ & Brasil & Mato Grosso & Novo Mundo & $-9,625478$ & $-55,913034$ & $\begin{array}{l}\text { Archivo } \\
\text { de audio }\end{array}$ \\
\hline $\begin{array}{l}\text { Myiozetetes } \\
\text { cayanensis }\end{array}$ & hellmayri & 1 & 242446 & $\mathrm{ML}$ & Ecuador & Esmeraldas & San Lorenzo & 0,883 & $-78,514$ & $\begin{array}{l}\text { Archivo } \\
\text { de audio }\end{array}$ \\
\hline $\begin{array}{l}\text { Myiozetetes } \\
\text { cayanensis }\end{array}$ & hellmayri & 1 & 245426 & $\mathrm{ML}$ & Ecuador & Esmeraldas & San Lorenzo & 1,0914 & $-78,6882$ & $\begin{array}{l}\text { Archivo } \\
\text { de audio }\end{array}$ \\
\hline $\begin{array}{l}\text { Myiozetetes } \\
\text { cayanensis }\end{array}$ & hellmayri & 1 & 245445 & $\mathrm{ML}$ & Ecuador & Esmeraldas & San Lorenzo & 1,154 & $-78,851$ & $\begin{array}{l}\text { Archivo } \\
\text { de audio }\end{array}$ \\
\hline
\end{tabular}




\begin{tabular}{|c|c|c|c|c|c|c|c|c|c|c|}
\hline $\begin{array}{l}\text { Myiozetetes } \\
\text { cayanensis }\end{array}$ & hellmayri & 1 & 247946 & $\mathrm{ML}$ & Ecuador & Esmeraldas & Eloy Alfaro & 0,533 & $-79,217$ & $\begin{array}{l}\text { Archivo } \\
\text { de audio }\end{array}$ \\
\hline $\begin{array}{l}\text { Myiozetetes } \\
\text { cayanensis }\end{array}$ & hellmayri & 1 & 258041 & $\mathrm{ML}$ & Colombia & Caldas & Neira, Tapias. & 5,2461 & $-75,6853$ & $\begin{array}{l}\text { Archivo } \\
\text { de audio }\end{array}$ \\
\hline $\begin{array}{l}\text { Myiozetetes } \\
\text { cayanensis }\end{array}$ & hellmayri & 1 & 258271 & $\mathrm{ML}$ & Colombia & Caldas & Neira, Tapias. & 5,2461 & $-75,6853$ & $\begin{array}{l}\text { Archivo } \\
\text { de audio }\end{array}$ \\
\hline $\begin{array}{l}\text { Myiozetetes } \\
\text { cayanensis }\end{array}$ & hellmayri & 1 & 258283 & $\mathrm{ML}$ & Colombia & Caldas & Neira, Tapias. & 5,2461 & $-75,6853$ & $\begin{array}{l}\text { Archivo } \\
\text { de audio }\end{array}$ \\
\hline $\begin{array}{l}\text { Myiozetetes } \\
\text { cayanensis }\end{array}$ & hellmayri & 1 & 260486 & $\mathrm{ML}$ & Colombia & Chocó & Bahia Solano & 6,0667 & $-77,3833$ & $\begin{array}{l}\text { Archivo } \\
\text { de audio }\end{array}$ \\
\hline $\begin{array}{l}\text { Myiozetetes } \\
\text { cayanensis }\end{array}$ & hellmayri & 1 & 260708 & $\mathrm{ML}$ & Colombia & Caldas & $\begin{array}{l}\text { Norcasia, } \\
\text { Moscovita. }\end{array}$ & 5,5742 & $-74,9$ & $\begin{array}{l}\text { Archivo } \\
\text { de audio }\end{array}$ \\
\hline $\begin{array}{l}\text { Myiozetetes } \\
\text { cayanensis }\end{array}$ & hellmayri & 1 & 261075 & $\mathrm{ML}$ & Colombia & Boyacá & Quípama, Cormal. & 5,5886 & $-74,2283$ & $\begin{array}{l}\text { Archivo } \\
\text { de audio }\end{array}$ \\
\hline $\begin{array}{l}\text { Myiozetetes } \\
\text { cayanensis }\end{array}$ & hellmayri & 1 & 261182 & $\mathrm{ML}$ & Colombia & Chocó & $\begin{array}{l}\text { Riosucio, Pueblo } \\
\text { regado }\end{array}$ & 7,8 & $-77,15$ & $\begin{array}{l}\text { Archivo } \\
\text { de audio }\end{array}$ \\
\hline $\begin{array}{l}\text { Myiozetetes } \\
\text { cayanensis }\end{array}$ & hellmayri & 1 & 261785 & $\mathrm{ML}$ & Colombia & Valle del Cauca & $\begin{array}{c}\text { Yotoco, } \\
\text { Mediacanoa. }\end{array}$ & 3,8775 & $-76,4319$ & $\begin{array}{l}\text { Archivo } \\
\text { de audio }\end{array}$ \\
\hline $\begin{array}{l}\text { Myiozetetes } \\
\text { cayanensis }\end{array}$ & hellmayri & 1 & 261786 & $\mathrm{ML}$ & Colombia & Valle del Cauca & $\begin{array}{c}\text { Yotoco, } \\
\text { Mediacanoa. }\end{array}$ & 3,8775 & $-76,4319$ & $\begin{array}{l}\text { Archivo } \\
\text { de audio }\end{array}$ \\
\hline $\begin{array}{l}\text { Myiozetetes } \\
\text { cayanensis }\end{array}$ & hellmayri & 1 & 261952 & $\mathrm{ML}$ & Colombia & Valle del Cauca & $\begin{array}{c}\text { Yotoco, } \\
\text { Mediacanoa. }\end{array}$ & 3,8775 & $-76,4319$ & $\begin{array}{l}\text { Archivo } \\
\text { de audio }\end{array}$ \\
\hline $\begin{array}{l}\text { Myiozetetes } \\
\text { cayanensis }\end{array}$ & hellmayri & 1 & 262679 & $\mathrm{ML}$ & Colombia & Valle del Cauca & $\begin{array}{c}\text { Yotoco, } \\
\text { Mediacanoa. }\end{array}$ & 3,8775 & $-76,4319$ & $\begin{array}{l}\text { Archivo } \\
\text { de audio }\end{array}$ \\
\hline $\begin{array}{l}\text { Myiozetetes } \\
\text { cayanensis }\end{array}$ & hellmayri & 1 & 262686 & $\mathrm{ML}$ & Colombia & Valle del Cauca & $\begin{array}{c}\text { Yotoco, } \\
\text { Mediacanoa. }\end{array}$ & 3,8775 & $-76,4319$ & $\begin{array}{l}\text { Archivo } \\
\text { de audio }\end{array}$ \\
\hline $\begin{array}{l}\text { Myiozetetes } \\
\text { cayanensis }\end{array}$ & hellmayri & 1 & 262719 & $\mathrm{ML}$ & Colombia & Valle del Cauca & $\begin{array}{c}\text { Yotoco, } \\
\text { Mediacanoa. }\end{array}$ & 3,8775 & $-76,4319$ & $\begin{array}{l}\text { Archivo } \\
\text { de audio }\end{array}$ \\
\hline $\begin{array}{l}\text { Myiozetetes } \\
\text { cayanensis }\end{array}$ & hellmayri & 1 & 262798 & $\mathrm{ML}$ & Colombia & Valle del Cauca & $\begin{array}{l}\text { Yotoco, } \\
\text { Cordobitas. }\end{array}$ & 3,8594 & $-76,4692$ & $\begin{array}{l}\text { Archivo } \\
\text { de audio }\end{array}$ \\
\hline $\begin{array}{l}\text { Myiozetetes } \\
\text { cayanensis }\end{array}$ & hellmayri & 1 & 267024 & $\mathrm{ML}$ & Colombia & Cundinamarca & Bojacá, Chilcal. & 4,6972 & $-74,3836$ & $\begin{array}{l}\text { Archivo } \\
\text { de audio }\end{array}$ \\
\hline $\begin{array}{l}\text { Myiozetetes } \\
\text { cayanensis }\end{array}$ & hellmayri & 1 & 267740 & $\mathrm{ML}$ & Colombia & Santander & $\begin{array}{c}\text { Barrancabermeja, } \\
\text { La Fortuna. }\end{array}$ & 7,1465 & $-73,5615$ & $\begin{array}{l}\text { Archivo } \\
\text { de audio }\end{array}$ \\
\hline $\begin{array}{l}\text { Myiozetetes } \\
\text { cayanensis }\end{array}$ & hellmayri & 1 & 268029 & $\mathrm{ML}$ & Colombia & Antioquia & $\begin{array}{l}\text { Amalfi, Los } \\
\text { Mangos. }\end{array}$ & 6,9717 & $-75,0569$ & $\begin{array}{l}\text { Archivo } \\
\text { de audio }\end{array}$ \\
\hline $\begin{array}{l}\text { Myiozetetes } \\
\text { cayanensis }\end{array}$ & hellmayri & 1 & 268169 & $\mathrm{ML}$ & Colombia & Antioquia & Amalfi, Guayabito. & 6,8236 & $-75,0936$ & $\begin{array}{l}\text { Archivo } \\
\text { de audio }\end{array}$ \\
\hline $\begin{array}{l}\text { Myiozetetes } \\
\text { cayanensis }\end{array}$ & hellmayri & 1 & 269199 & $\mathrm{ML}$ & Colombia & Huila & San Agustín & 1,879912 & $-76,270209$ & $\begin{array}{l}\text { Archivo } \\
\text { de audio }\end{array}$ \\
\hline $\begin{array}{l}\text { Myiozetetes } \\
\text { cayanensis }\end{array}$ & hellmayri & 1 & 269200 & $\mathrm{ML}$ & Colombia & Huila & San Agustín & 1,879912 & $-76,270209$ & $\begin{array}{l}\text { Archivo } \\
\text { de audio }\end{array}$ \\
\hline $\begin{array}{l}\text { Myiozetetes } \\
\text { cayanensis }\end{array}$ & rufipennis & 2 & 270609 & $\mathrm{ML}$ & Colombia & Vichada & $\begin{array}{c}\text { Cumaribo, } \\
\text { Resguardo Selva de } \\
\text { Matavén. }\end{array}$ & 4,4903 & $-67,8719$ & $\begin{array}{l}\text { Archivo } \\
\text { de audio }\end{array}$ \\
\hline $\begin{array}{l}\text { Myiozetetes } \\
\text { cayanensis }\end{array}$ & rufipennis & 2 & 270614 & $\mathrm{ML}$ & Colombia & Vichada & $\begin{array}{c}\text { Cumaribo, } \\
\text { Resguardo Selva de } \\
\text { Matavén. }\end{array}$ & 4,4903 & $-67,8719$ & $\begin{array}{l}\text { Archivo } \\
\text { de audio }\end{array}$ \\
\hline $\begin{array}{l}\text { Myiozetetes } \\
\text { cayanensis }\end{array}$ & rufipennis & 2 & 270824 & $\mathrm{ML}$ & Colombia & Vichada & $\begin{array}{c}\text { Cumaribo, } \\
\text { Resguardo Selva de } \\
\text { Matavén. }\end{array}$ & 4,4903 & $-67,8719$ & $\begin{array}{l}\text { Archivo } \\
\text { de audio }\end{array}$ \\
\hline $\begin{array}{l}\text { Myiozetetes } \\
\text { cayanensis }\end{array}$ & rufipennis & 2 & 270826 & $\mathrm{ML}$ & Colombia & Vichada & $\begin{array}{c}\text { Cumaribo, } \\
\text { Resguardo Selva de } \\
\text { Matavén. }\end{array}$ & 4,4903 & $-67,8719$ & $\begin{array}{l}\text { Archivo } \\
\text { de audio }\end{array}$ \\
\hline $\begin{array}{l}\text { Myiozetetes } \\
\text { cayanensis }\end{array}$ & cayanensis & 3 & 518390 & $\mathrm{ML}$ & Bolivia & Santa Cruz & Guapasa & $-17,2$ & $-59,3333$ & $\begin{array}{l}\text { Archivo } \\
\text { de audio }\end{array}$ \\
\hline $\begin{array}{l}\text { Myiozetetes } \\
\text { cayanensis }\end{array}$ & hellmayri & 1 & 30160321 & $\mathrm{ML}$ & Costa Rica & Puntarenas & Coto Brus & 8,8499 & $-82,9405$ & $\begin{array}{l}\text { Archivo } \\
\text { de audio }\end{array}$ \\
\hline $\begin{array}{l}\text { Myiozetetes } \\
\text { cayanensis }\end{array}$ & hellmayri & 1 & 33303091 & $\mathrm{ML}$ & Panamá & $\begin{array}{l}\text { Comarca } \\
\text { Emberá } \\
\text { Wounaán }\end{array}$ & Cémaco & 8,378 & $-77,7759$ & $\begin{array}{l}\text { Archivo } \\
\text { de audio }\end{array}$ \\
\hline $\begin{array}{l}\text { Myiozetetes } \\
\text { cayanensis }\end{array}$ & hellmayri & 1 & 37125911 & $\mathrm{ML}$ & Colombia & Antioquia & Amalfi, El Tapiro. & 6,8575 & $-75,1015$ & $\begin{array}{l}\text { Archivo } \\
\text { de audio }\end{array}$ \\
\hline $\begin{array}{l}\text { Myiozetetes } \\
\text { cayanensis }\end{array}$ & hellmayri & 1 & 51635671 & $\mathrm{ML}$ & Colombia & Sucre & Chalán, La Ceiba. & 9,5696 & $-75,3144$ & $\begin{array}{l}\text { Archivo } \\
\text { de audio }\end{array}$ \\
\hline $\begin{array}{l}\text { Myiozetetes } \\
\text { cayanensis }\end{array}$ & hellmayri & 1 & 51848081 & $\mathrm{ML}$ & Colombia & Sucre & Chalán, La Ceiba. & 9,5696 & $-75,3144$ & $\begin{array}{l}\text { Archivo } \\
\text { de audio }\end{array}$ \\
\hline
\end{tabular}




\begin{tabular}{|c|c|c|c|c|c|c|c|c|c|c|}
\hline $\begin{array}{l}\text { Myiozetetes } \\
\text { cayanensis }\end{array}$ & cayanensis & 3 & 52332001 & $\mathrm{ML}$ & Brasil & Distrito Federal & Brasília & $-15,8321$ & $-48,0303$ & $\begin{array}{l}\text { Archivo } \\
\text { de audio }\end{array}$ \\
\hline $\begin{array}{l}\text { Myiozetetes } \\
\text { cayanensis }\end{array}$ & cayanensis & 3 & 66008521 & $\mathrm{ML}$ & Guyana & $\begin{array}{l}\text { Uppertakutu- } \\
\text { Upperessequibo }\end{array}$ & Dadanawa & 2,8279 & $-59,528$ & $\begin{array}{l}\text { Archivo } \\
\text { de audio }\end{array}$ \\
\hline $\begin{array}{l}\text { Myiozetetes } \\
\text { cayanensis }\end{array}$ & hellmayri & 1 & 67209471 & $\mathrm{ML}$ & Panamá & Colón & Gamboa & 9,1178 & $-79,6986$ & $\begin{array}{l}\text { Archivo } \\
\text { de audio }\end{array}$ \\
\hline $\begin{array}{l}\text { Myiozetetes } \\
\text { cayanensis }\end{array}$ & hellmayri & 1 & 67415281 & $\mathrm{ML}$ & Panamá & Colón & Gamboa & 9,1178 & $-79,6986$ & $\begin{array}{l}\text { Archivo } \\
\text { de audio }\end{array}$ \\
\hline $\begin{array}{l}\text { Myiozetetes } \\
\text { cayanensis }\end{array}$ & hellmayri & 1 & 67664041 & $\mathrm{ML}$ & Panamá & Colón & Colón & 9,1409 & $-79,7281$ & $\begin{array}{l}\text { Archivo } \\
\text { de audio }\end{array}$ \\
\hline $\begin{array}{l}\text { Myiozetetes } \\
\text { cayanensis }\end{array}$ & hellmayri & 1 & 68100511 & $\mathrm{ML}$ & Panamá & Colón & Gamboa & 9,1178 & $-79,6986$ & $\begin{array}{l}\text { Archivo } \\
\text { de audio }\end{array}$ \\
\hline $\begin{array}{l}\text { Myiozetetes } \\
\text { cayanensis }\end{array}$ & hellmayri & 1 & 69499791 & $\mathrm{ML}$ & Panamá & Colón & Gamboa & 9,1178 & $-79,6986$ & $\begin{array}{l}\text { Archivo } \\
\text { de audio }\end{array}$ \\
\hline $\begin{array}{l}\text { Myiozetetes } \\
\text { cayanensis }\end{array}$ & hellmayri & 1 & 71253061 & $\mathrm{ML}$ & Panamá & Colón & Gamboa & 9,1178 & $-79,6986$ & $\begin{array}{l}\text { Archivo } \\
\text { de audio }\end{array}$ \\
\hline $\begin{array}{l}\text { Myiozetetes } \\
\text { cayanensis }\end{array}$ & hellmayri & 1 & 72187411 & $\mathrm{ML}$ & Ecuador & Carchi & Tulcan & 1,0366 & $-78,2546$ & $\begin{array}{l}\text { Archivo } \\
\text { de audio }\end{array}$ \\
\hline $\begin{array}{l}\text { Myiozetetes } \\
\text { cayanensis }\end{array}$ & cayanensis & 3 & 85883181 & $\mathrm{ML}$ & Brasil & Mato Grosso & Poconé & $-16,7581$ & $-56,8772$ & $\begin{array}{l}\text { Archivo } \\
\text { de audio }\end{array}$ \\
\hline $\begin{array}{l}\text { Myiozetetes } \\
\text { cayanensis }\end{array}$ & cayanensis & 3 & 85885271 & $\mathrm{ML}$ & Brasil & Mato Grosso & Poconé & $-16,7581$ & $-56,8772$ & $\begin{array}{l}\text { Archivo } \\
\text { de audio }\end{array}$ \\
\hline $\begin{array}{l}\text { Myiozetetes } \\
\text { cayanensis }\end{array}$ & hellmayri & 1 & 90549611 & $\mathrm{ML}$ & Panamá & Colón & Colón & 9,1196 & $-79,7128$ & $\begin{array}{l}\text { Archivo } \\
\text { de audio }\end{array}$ \\
\hline $\begin{array}{l}\text { Myiozetetes } \\
\text { cayanensis }\end{array}$ & hellmayri & 1 & 92382441 & $\mathrm{ML}$ & Panamá & Colón & Colón & 9,1178 & $-79,6986$ & $\begin{array}{l}\text { Archivo } \\
\text { de audio }\end{array}$ \\
\hline $\begin{array}{l}\text { Myiozetetes } \\
\text { cayanensis }\end{array}$ & hellmayri & 1 & 92382631 & $\mathrm{ML}$ & Panamá & Colón & Colón & 9,1178 & $-79,6986$ & $\begin{array}{l}\text { Archivo } \\
\text { de audio }\end{array}$ \\
\hline $\begin{array}{l}\text { Myiozetetes } \\
\text { cayanensis }\end{array}$ & rufipennis & 2 & 96366891 & $\mathrm{ML}$ & Colombia & Cundinamarca & $\begin{array}{l}\text { Medina, } \\
\text { Mesanegra. }\end{array}$ & 4,502 & $-73,3687$ & $\begin{array}{l}\text { Archivo } \\
\text { de audio }\end{array}$ \\
\hline $\begin{array}{l}\text { Myiozetetes } \\
\text { cayanensis }\end{array}$ & rufipennis & 2 & 96412721 & $\mathrm{ML}$ & Colombia & Cundinamarca & Medina, Periquito. & 4,512 & $-73,4196$ & $\begin{array}{l}\text { Archivo } \\
\text { de audio }\end{array}$ \\
\hline $\begin{array}{l}\text { Myiozetetes } \\
\text { cayanensis }\end{array}$ & hellmayri & 1 & 101590191 & $\mathrm{ML}$ & Colombia & Córdoba & $\begin{array}{l}\text { Puerto Libertador, } \\
\text { Torno rojo. }\end{array}$ & 7,9734 & $-75,5789$ & $\begin{array}{l}\text { Archivo } \\
\text { de audio }\end{array}$ \\
\hline $\begin{array}{l}\text { Myiozetetes } \\
\text { cayanensis }\end{array}$ & cayanensis & 3 & 101637061 & $\mathrm{ML}$ & Brasil & São Paulo & Taiaçu & $-21,111$ & $-48,5233$ & $\begin{array}{l}\text { Archivo } \\
\text { de audio }\end{array}$ \\
\hline $\begin{array}{l}\text { Myiozetetes } \\
\text { cayanensis }\end{array}$ & cayanensis & 3 & 102631571 & $\mathrm{ML}$ & Brasil & Mato Grosso & Alta Floresta & $-9,5973$ & $-55,9311$ & $\begin{array}{l}\text { Archivo } \\
\text { de audio }\end{array}$ \\
\hline $\begin{array}{l}\text { Myiozetetes } \\
\text { cayanensis }\end{array}$ & rufipennis & 2 & 103958391 & $\mathrm{ML}$ & Colombia & Meta & $\begin{array}{l}\text { San Martin, } \\
\text { Guaranana. }\end{array}$ & 3,6654 & $-73,6578$ & $\begin{array}{l}\text { Archivo } \\
\text { de audio }\end{array}$ \\
\hline $\begin{array}{l}\text { Myiozetetes } \\
\text { cayanensis }\end{array}$ & hellmayri & 1 & 104565251 & $\mathrm{ML}$ & Colombia & Nariño & $\begin{array}{c}\text { Tumaco, } \\
\text { Corregimiento } \\
\text { Corredor Espriella. }\end{array}$ & 1,527 & $-78,6794$ & $\begin{array}{l}\text { Archivo } \\
\text { de audio }\end{array}$ \\
\hline $\begin{array}{l}\text { Myiozetetes } \\
\text { cayanensis }\end{array}$ & hellmayri & 1 & 104683671 & $\mathrm{ML}$ & Colombia & Nariño & $\begin{array}{c}\text { Tumaco, } \\
\text { Corregimiento } \\
\text { Rescate Las Varas. }\end{array}$ & 1,6833 & $-78,75$ & $\begin{array}{l}\text { Archivo } \\
\text { de audio }\end{array}$ \\
\hline $\begin{array}{l}\text { Myiozetetes } \\
\text { cayanensis }\end{array}$ & rufipennis & 2 & 106623731 & $\mathrm{ML}$ & Colombia & Casanare & $\begin{array}{c}\text { Monterrey, Tierra } \\
\text { grata. }\end{array}$ & 4,8873 & $-72,9009$ & $\begin{array}{l}\text { Archivo } \\
\text { de audio }\end{array}$ \\
\hline $\begin{array}{l}\text { Myiozetetes } \\
\text { cayanensis }\end{array}$ & rufipennis & 2 & 107633871 & $\mathrm{ML}$ & Colombia & Vaupés & Mitú, Ozirpa. & 1,1191 & $-70,0826$ & $\begin{array}{l}\text { Archivo } \\
\text { de audio }\end{array}$ \\
\hline $\begin{array}{l}\text { Myiozetetes } \\
\text { cayanensis }\end{array}$ & rufipennis & 2 & 108698861 & $\mathrm{ML}$ & Colombia & Vaupés & Mitú, Ozirpa. & 1,2242 & $-70,2384$ & $\begin{array}{l}\text { Archivo } \\
\text { de audio }\end{array}$ \\
\hline $\begin{array}{l}\text { Myiozetetes } \\
\text { cayanensis }\end{array}$ & cayanensis & 3 & 112100441 & $\mathrm{ML}$ & Brasil & $\begin{array}{l}\text { Mato Grosso do } \\
\text { Sul }\end{array}$ & Bodoquena & $-20,7397$ & $-56,731$ & $\begin{array}{l}\text { Archivo } \\
\text { de audio }\end{array}$ \\
\hline $\begin{array}{l}\text { Myiozetetes } \\
\text { cayanensis }\end{array}$ & cayanensis & 3 & 114199571 & $\mathrm{ML}$ & Perú & Madre de Dios & Iberia & $-11,4151$ & $-69,4993$ & $\begin{array}{l}\text { Archivo } \\
\text { de audio }\end{array}$ \\
\hline $\begin{array}{l}\text { Myiozetetes } \\
\text { cayanensis }\end{array}$ & cayanensis & 3 & 114201421 & $\mathrm{ML}$ & Perú & Madre de Dios & Iberia & $-11,4284$ & $-69,5098$ & $\begin{array}{l}\text { Archivo } \\
\text { de audio }\end{array}$ \\
\hline $\begin{array}{l}\text { Myiozetetes } \\
\text { cayanensis }\end{array}$ & hellmayri & 1 & 116015041 & $\mathrm{ML}$ & Colombia & Caldas & $\begin{array}{l}\text { Manizales, La } \\
\text { China. }\end{array}$ & 5,0647 & $-75,5922$ & $\begin{array}{l}\text { Archivo } \\
\text { de audio }\end{array}$ \\
\hline $\begin{array}{l}\text { Myiozetetes } \\
\text { cayanensis }\end{array}$ & hellmayri & 1 & 116015511 & $\mathrm{ML}$ & Colombia & Caldas & $\begin{array}{l}\text { Manizales, La } \\
\text { China. }\end{array}$ & 5,0647 & $-75,5922$ & $\begin{array}{l}\text { Archivo } \\
\text { de audio }\end{array}$ \\
\hline $\begin{array}{l}\text { Myiozetetes } \\
\text { cayanensis }\end{array}$ & hellmayri & 1 & 129667121 & $\mathrm{ML}$ & Colombia & Santander & $\begin{array}{c}\text { San Vicente de } \\
\text { Chucurí, Zona } \\
\text { urbana. }\end{array}$ & 6,8522 & $-73,3768$ & $\begin{array}{l}\text { Archivo } \\
\text { de audio }\end{array}$ \\
\hline $\begin{array}{l}\text { Myiozetetes } \\
\text { cayanensis }\end{array}$ & hellmayri & 1 & 133928841 & $\mathrm{ML}$ & Ecuador & Pichincha & $\begin{array}{c}\text { San Miguel de los } \\
\text { Bancos }\end{array}$ & $-0,051$ & $-78,7784$ & $\begin{array}{l}\text { Archivo } \\
\text { de audio }\end{array}$ \\
\hline
\end{tabular}




\begin{tabular}{|c|c|c|c|c|c|c|c|c|c|c|}
\hline $\begin{array}{l}\text { Myiozetetes } \\
\text { cayanensis }\end{array}$ & hellmayri & 1 & 141554391 & $M L$ & Colombia & Risaralda & Marsella, Caracas. & 4,9299 & $-75,7456$ & $\begin{array}{l}\text { Archivo } \\
\text { de audio }\end{array}$ \\
\hline $\begin{array}{l}\text { Myiozetetes } \\
\text { cayanensis }\end{array}$ & hellmayri & 1 & 142152131 & $M L$ & Colombia & Risaralda & Pereira, Caimalito. & 4,843 & $-75,8186$ & $\begin{array}{l}\text { Archivo } \\
\text { de audio }\end{array}$ \\
\hline $\begin{array}{l}\text { Myiozetetes } \\
\text { cayanensis }\end{array}$ & hellmayri & 1 & 142552281 & $M L$ & Panamá & Darién & Pinogana & 8,3874 & $-77,8732$ & $\begin{array}{l}\text { Archivo } \\
\text { de audio }\end{array}$ \\
\hline $\begin{array}{l}\text { Myiozetetes } \\
\text { cayanensis }\end{array}$ & hellmayri & 1 & 148352761 & $M L$ & Panamá & Colón & Colón & 9,1311 & $-79,7259$ & $\begin{array}{l}\text { Archivo } \\
\text { de audio }\end{array}$ \\
\hline $\begin{array}{l}\text { Myiozetetes } \\
\text { cayanensis }\end{array}$ & hellmayri & 1 & 150240731 & $M L$ & Ecuador & Pichincha & $\begin{array}{c}\text { San Miguel de los } \\
\text { Bancos }\end{array}$ & $-0,0628$ & $-78,7743$ & $\begin{array}{l}\text { Archivo } \\
\text { de audio }\end{array}$ \\
\hline $\begin{array}{l}\text { Myiozetetes } \\
\text { cayanensis }\end{array}$ & rufipennis & 2 & 158373241 & $\mathrm{ML}$ & Colombia & Vaupés & Mitú, Alubva. & 1,1481 & $-70,0398$ & $\begin{array}{l}\text { Archivo } \\
\text { de audio }\end{array}$ \\
\hline $\begin{array}{l}\text { Myiozetetes } \\
\text { cayanensis }\end{array}$ & rufipennis & 2 & 158891691 & $M L$ & Colombia & Vaupés & Mitú, Alubva. & 1,1219 & $-70,069$ & $\begin{array}{l}\text { Archivo } \\
\text { de audio }\end{array}$ \\
\hline $\begin{array}{l}\text { Myiozetetes } \\
\text { cayanensis }\end{array}$ & rufipennis & 2 & 158893051 & $M L$ & Colombia & Vaupés & Mitú, Ozirpa. & 1,1222 & $-70,0913$ & $\begin{array}{l}\text { Archivo } \\
\text { de audio }\end{array}$ \\
\hline $\begin{array}{l}\text { Myiozetetes } \\
\text { cayanensis }\end{array}$ & cayanensis & 3 & 169781981 & $M L$ & Venezuela & Bolívar & Sifontes & 6,9244 & $-61,2838$ & $\begin{array}{l}\text { Archivo } \\
\text { de audio }\end{array}$ \\
\hline $\begin{array}{l}\text { Myiozetetes } \\
\text { cayanensis }\end{array}$ & cayanensis & 3 & 170068851 & $M L$ & Venezuela & Bolívar & Sifontes & 6,15 & $-61,4694$ & $\begin{array}{l}\text { Archivo } \\
\text { de audio }\end{array}$ \\
\hline $\begin{array}{l}\text { Myiozetetes } \\
\text { cayanensis }\end{array}$ & hellmayri & 1 & 171388601 & $M L$ & Costa Rica & Puntarenas & Golfito & 8,7086 & $-83,1841$ & $\begin{array}{l}\text { Archivo } \\
\text { de audio }\end{array}$ \\
\hline $\begin{array}{l}\text { Myiozetetes } \\
\text { cayanensis }\end{array}$ & hellmayri & 1 & 178221061 & $M L$ & Colombia & Caldas & $\begin{array}{l}\text { Manizales, La } \\
\text { China. }\end{array}$ & 5,0647 & $-75,5922$ & $\begin{array}{l}\text { Archivo } \\
\text { de audio }\end{array}$ \\
\hline $\begin{array}{l}\text { Myiozetetes } \\
\text { cayanensis }\end{array}$ & hellmayri & 1 & 178982701 & $M L$ & Colombia & Nariño & $\begin{array}{c}\text { Tumaco, } \\
\text { Corregimiento } \\
\text { Corredor Espriella. }\end{array}$ & 1,5254 & $-78,6835$ & $\begin{array}{l}\text { Archivo } \\
\text { de audio }\end{array}$ \\
\hline $\begin{array}{l}\text { Myiozetetes } \\
\text { cayanensis }\end{array}$ & hellmayri & 1 & 183211111 & $M L$ & Colombia & Tolima & Ibagué & 4,4283 & $-75,2142$ & $\begin{array}{l}\text { Archivo } \\
\text { de audio }\end{array}$ \\
\hline $\begin{array}{l}\text { Myiozetetes } \\
\text { cayanensis }\end{array}$ & cayanensis & 3 & 183366491 & $M L$ & Brasil & São Paulo & $\begin{array}{l}\text { São José do Rio } \\
\text { Preto }\end{array}$ & $-20,8417$ & $-49,2964$ & $\begin{array}{l}\text { Archivo } \\
\text { de audio }\end{array}$ \\
\hline $\begin{array}{l}\text { Myiozetetes } \\
\text { cayanensis }\end{array}$ & hellmayri & 1 & 183602401 & $M L$ & Venezuela & Falcón & Miranda & 11,41 & $-69,6795$ & $\begin{array}{l}\text { Archivo } \\
\text { de audio }\end{array}$ \\
\hline $\begin{array}{l}\text { Myiozetetes } \\
\text { cayanensis }\end{array}$ & hellmayri & 1 & 185147471 & $M L$ & Panamá & Coclé & Antón & 8,3517 & $-80,1471$ & $\begin{array}{l}\text { Archivo } \\
\text { de audio }\end{array}$ \\
\hline $\begin{array}{l}\text { Myiozetetes } \\
\text { cayanensis }\end{array}$ & hellmayri & 1 & 186428601 & $M L$ & Panamá & Panamá & Panamá & 9,0558 & $-79,6403$ & $\begin{array}{l}\text { Archivo } \\
\text { de audio }\end{array}$ \\
\hline $\begin{array}{l}\text { Myiozetetes } \\
\text { cayanensis }\end{array}$ & cayanensis & 3 & 190876591 & $M L$ & Brasil & Amazonas & $\begin{array}{l}\text { Presidente } \\
\text { Figueiredo }\end{array}$ & $-1,9803$ & $-60,0514$ & $\begin{array}{l}\text { Archivo } \\
\text { de audio }\end{array}$ \\
\hline $\begin{array}{l}\text { Myiozetetes } \\
\text { cayanensis }\end{array}$ & hellmayri & 1 & 195311741 & $M L$ & Colombia & Antioquia & Jardín & 5,5998 & $-75,8188$ & $\begin{array}{l}\text { Archivo } \\
\text { de audio }\end{array}$ \\
\hline $\begin{array}{l}\text { Myiozetetes } \\
\text { cayanensis }\end{array}$ & hellmayri & 1 & 200438641 & $M L$ & Colombia & Tolima & $\begin{array}{c}\text { Alvarado, Cabecera } \\
\text { Municipal. }\end{array}$ & 4,5628 & $-74,9422$ & $\begin{array}{l}\text { Archivo } \\
\text { de audio }\end{array}$ \\
\hline $\begin{array}{l}\text { Myiozetetes } \\
\text { cayanensis }\end{array}$ & cayanensis & 3 & 203695961 & $M L$ & Brasil & Pará & Novo Progresso & $-9,2467$ & $-55,988$ & $\begin{array}{l}\text { Archivo } \\
\text { de audio }\end{array}$ \\
\hline $\begin{array}{l}\text { Myiozetetes } \\
\text { cayanensis }\end{array}$ & cayanensis & 3 & 203897451 & $M L$ & Brasil & Tocantins & Lagoa da Confusão & $-10,7347$ & $-49,8567$ & $\begin{array}{l}\text { Archivo } \\
\text { de audio }\end{array}$ \\
\hline $\begin{array}{l}\text { Myiozetetes } \\
\text { cayanensis }\end{array}$ & cayanensis & 3 & 203899711 & $M L$ & Brasil & Tocantins & Pium & $-9,99$ & $-49,8215$ & $\begin{array}{l}\text { Archivo } \\
\text { de audio }\end{array}$ \\
\hline $\begin{array}{l}\text { Myiozetetes } \\
\text { cayanensis }\end{array}$ & hellmayri & 1 & 203900701 & $M L$ & Panamá & Colón & Colón & 9,1192 & $-79,6979$ & $\begin{array}{l}\text { Archivo } \\
\text { de audio }\end{array}$ \\
\hline $\begin{array}{l}\text { Myiozetetes } \\
\text { cayanensis }\end{array}$ & hellmayri & 1 & 203922511 & $M L$ & Colombia & Valle del Cauca & $\begin{array}{l}\text { Buenaventura, } \\
\text { Llano Bajo. }\end{array}$ & 3,5747 & $-76,8783$ & $\begin{array}{l}\text { Archivo } \\
\text { de audio }\end{array}$ \\
\hline $\begin{array}{l}\text { Myiozetetes } \\
\text { cayanensis }\end{array}$ & hellmayri & 1 & 203922521 & $M L$ & Colombia & Valle del Cauca & $\begin{array}{l}\text { Buenaventura, } \\
\text { Llano Bajo. }\end{array}$ & 3,5747 & $-76,8783$ & $\begin{array}{l}\text { Archivo } \\
\text { de audio }\end{array}$ \\
\hline $\begin{array}{l}\text { Myiozetetes } \\
\text { cayanensis }\end{array}$ & erythropterus & 4 & 212488401 & $M L$ & Brasil & Minas Gerais & Belo Horizonte & $-19,95$ & $-43,9069$ & $\begin{array}{l}\text { Archivo } \\
\text { de audio }\end{array}$ \\
\hline $\begin{array}{l}\text { Myiozetetes } \\
\text { cayanensis }\end{array}$ & cayanensis & 3 & 215335381 & $M L$ & Bolivia & Beni & Riveralta & $-11,023$ & $-66,0987$ & $\begin{array}{l}\text { Archivo } \\
\text { de audio }\end{array}$ \\
\hline $\begin{array}{l}\text { Myiozetetes } \\
\text { cayanensis }\end{array}$ & cayanensis & 3 & 218559061 & $M L$ & Brasil & Roraima & NA & 1,4831 & $-62,1916$ & $\begin{array}{l}\text { Archivo } \\
\text { de audio }\end{array}$ \\
\hline $\begin{array}{l}\text { Myiozetetes } \\
\text { cayanensis }\end{array}$ & cayanensis & 3 & 537847 & $\mathrm{XC}$ & Brasil & Amazonas & $\begin{array}{l}\text { Purus River, Boca } \\
\text { do Acre, State of } \\
\text { Amazonas }\end{array}$ & $-8,7512$ & $-68,9938$ & $\begin{array}{l}\text { Archivo } \\
\text { de audio }\end{array}$ \\
\hline $\begin{array}{l}\text { Myiozetetes } \\
\text { cayanensis }\end{array}$ & cayanensis & 3 & 536761 & $\mathrm{XC}$ & Brasil & Amazonas & $\begin{array}{l}\text { Purus River, Boca } \\
\text { do Acre, State of } \\
\text { Amazonas }\end{array}$ & $-8,7512$ & $-68,9938$ & $\begin{array}{l}\text { Archivo } \\
\text { de audio }\end{array}$ \\
\hline
\end{tabular}




\begin{tabular}{|c|c|c|c|c|c|c|c|c|c|c|}
\hline $\begin{array}{l}\text { Myiozetetes } \\
\text { cayanensis }\end{array}$ & hellmayri & 1 & 533974 & $\mathrm{XC}$ & Colombia & Cundinamarca & $\begin{array}{l}\text { Mana Dulce, } \\
\text { Cundinamarca }\end{array}$ & 4,351 & $-74,652$ & $\begin{array}{l}\text { Archivo } \\
\text { de audio }\end{array}$ \\
\hline $\begin{array}{l}\text { Myiozetetes } \\
\text { cayanensis }\end{array}$ & cayanensis & 3 & 521301 & $\mathrm{XC}$ & Brasil & Mato Grosso & $\begin{array}{c}\text { Cristalino Jungle } \\
\text { Lodge, MT }\end{array}$ & $-9,5981$ & $-55,932$ & $\begin{array}{l}\text { Archivo } \\
\text { de audio }\end{array}$ \\
\hline $\begin{array}{l}\text { Myiozetetes } \\
\text { cayanensis }\end{array}$ & cayanensis & 3 & 508234 & $\mathrm{XC}$ & $\begin{array}{l}\text { Guyana } \\
\text { Francesa }\end{array}$ & Beauséjour & Roura, Beauséjour & 4,7216 & $-52,3073$ & $\begin{array}{l}\text { Archivo } \\
\text { de audio }\end{array}$ \\
\hline $\begin{array}{l}\text { Myiozetetes } \\
\text { cayanensis }\end{array}$ & cayanensis & 3 & 508233 & $\mathrm{XC}$ & $\begin{array}{l}\text { Guyana } \\
\text { Francesa }\end{array}$ & Beauséjour & Roura, Beauséjour & 4,7216 & $-52,3073$ & $\begin{array}{l}\text { Archivo } \\
\text { de audio }\end{array}$ \\
\hline $\begin{array}{l}\text { Myiozetetes } \\
\text { cayanensis }\end{array}$ & cayanensis & 3 & 484431 & $\mathrm{XC}$ & $\begin{array}{l}\text { Guyana } \\
\text { Francesa }\end{array}$ & La Levée & Matoury, La Levée & 4,8313 & $-52,2973$ & $\begin{array}{l}\text { Archivo } \\
\text { de audio }\end{array}$ \\
\hline $\begin{array}{l}\text { Myiozetetes } \\
\text { cayanensis }\end{array}$ & hellmayri & 1 & 468186 & $\mathrm{XC}$ & Colombia & Cundinamarca & $\begin{array}{l}\text { Mana Dulce, } \\
\text { Cundinamarca }\end{array}$ & 4,351 & $-74,652$ & $\begin{array}{l}\text { Archivo } \\
\text { de audio }\end{array}$ \\
\hline $\begin{array}{l}\text { Myiozetetes } \\
\text { cayanensis }\end{array}$ & hellmayri & 1 & 456638 & $\mathrm{XC}$ & Panamá & Colón & Achiote, Colón & 9,222 & $-80,024$ & $\begin{array}{l}\text { Archivo } \\
\text { de audio }\end{array}$ \\
\hline $\begin{array}{l}\text { Myiozetetes } \\
\text { cayanensis }\end{array}$ & cayanensis & 3 & 456415 & $\mathrm{XC}$ & $\begin{array}{l}\text { Guyana } \\
\text { Francesa }\end{array}$ & Saint-Georges & $\begin{array}{l}\text { piste Armontabo', } \\
\text { piste de Saut } \\
\text { Maripa, Saint- } \\
\text { Georges }\end{array}$ & 3,8518 & $-51,8894$ & $\begin{array}{l}\text { Archivo } \\
\text { de audio }\end{array}$ \\
\hline $\begin{array}{l}\text { Myiozetetes } \\
\text { cayanensis }\end{array}$ & hellmayri & 1 & 454266 & $\mathrm{XC}$ & Panamá & Colón & $\begin{array}{c}\text { Gamboa, Colon } \\
\text { province }\end{array}$ & 9,1223 & $-79,7501$ & $\begin{array}{l}\text { Archivo } \\
\text { de audio }\end{array}$ \\
\hline $\begin{array}{l}\text { Myiozetetes } \\
\text { cayanensis }\end{array}$ & cayanensis & 3 & 410473 & $\mathrm{XC}$ & $\begin{array}{l}\text { Guyana } \\
\text { Francesa }\end{array}$ & Beauséjour & Roura, Beauséjour & 4,7216 & $-52,3073$ & $\begin{array}{l}\text { Archivo } \\
\text { de audio }\end{array}$ \\
\hline $\begin{array}{l}\text { Myiozetetes } \\
\text { cayanensis }\end{array}$ & hellmayri & 1 & 390712 & $\mathrm{XC}$ & Ecuador & Pichincha & $\begin{array}{l}\text { Mashpi Road, } \\
\text { Pichincha }\end{array}$ & 0,1612 & $-78,8492$ & $\begin{array}{l}\text { Archivo } \\
\text { de audio }\end{array}$ \\
\hline $\begin{array}{l}\text { Myiozetetes } \\
\text { cayanensis }\end{array}$ & cayanensis & 3 & 147694 & $\mathrm{xC}$ & Brasil & Río de Janeiro & $\begin{array}{l}\text { Paraty, Rio de } \\
\text { Janeiro }\end{array}$ & $-23,2179$ & $-44,77$ & $\begin{array}{l}\text { Archivo } \\
\text { de audio }\end{array}$ \\
\hline $\begin{array}{l}\text { Myiozetetes } \\
\text { cayanensis }\end{array}$ & hellmayri & 1 & 520902 & $\mathrm{XC}$ & Colombia & Tolima & $\begin{array}{l}\text { Parque Nacional } \\
\text { del Arroz (La } \\
\text { Guaira)Estación } \\
\text { Ferrocarril Caldas } \\
\text { Viejo, Alvarado, } \\
\text { Tolima }\end{array}$ & 4,5646 & $-74,9392$ & $\begin{array}{l}\text { Archivo } \\
\text { de audio }\end{array}$ \\
\hline $\begin{array}{l}\text { Myiozetetes } \\
\text { cayanensis }\end{array}$ & cayanensis & 3 & 513660 & $\mathrm{XC}$ & Brasil & São Paulo & $\begin{array}{l}\text { Córrego da Onça, } \\
\text { São José do Rio } \\
\text { Preto, São Paulo }\end{array}$ & $-20,8413$ & $-49,303$ & $\begin{array}{l}\text { Archivo } \\
\text { de audio }\end{array}$ \\
\hline $\begin{array}{l}\text { Myiozetetes } \\
\text { cayanensis }\end{array}$ & hellmayri & 1 & 495469 & $\mathrm{XC}$ & Ecuador & Pichincha & $\begin{array}{c}\text { Un Poco del Choco, } \\
\text { Pichincha }\end{array}$ & 0,0736 & $-78,7685$ & $\begin{array}{l}\text { Archivo } \\
\text { de audio }\end{array}$ \\
\hline $\begin{array}{l}\text { Myiozetetes } \\
\text { cayanensis }\end{array}$ & hellmayri & 1 & 495468 & $\mathrm{XC}$ & Ecuador & Pichincha & $\begin{array}{l}\text { Andes, Distrito } \\
\text { Metropolitano de } \\
\text { Quito, Pichincha }\end{array}$ & 0,053 & $-78,8422$ & $\begin{array}{l}\text { Archivo } \\
\text { de audio }\end{array}$ \\
\hline $\begin{array}{l}\text { Myiozetetes } \\
\text { cayanensis }\end{array}$ & cayanensis & 3 & 466586 & $\mathrm{XC}$ & Bolivia & Santa Cruz & $\begin{array}{c}\text { Guarayos, } \\
\text { Departamento de } \\
\text { Santa Cruz }\end{array}$ & $-16,3658$ & $-62,9065$ & $\begin{array}{l}\text { Archivo } \\
\text { de audio }\end{array}$ \\
\hline $\begin{array}{l}\text { Myiozetetes } \\
\text { cayanensis }\end{array}$ & hellmayri & 1 & 459230 & $\mathrm{XC}$ & Colombia & Risaralda & $\begin{array}{l}\text { Reserva Natural } \\
\text { Cauquitá }\end{array}$ & 4,843 & $-75,8187$ & $\begin{array}{l}\text { Archivo } \\
\text { de audio }\end{array}$ \\
\hline $\begin{array}{l}\text { Myiozetetes } \\
\text { cayanensis }\end{array}$ & hellmayri & 1 & 457252 & $\mathrm{XC}$ & Colombia & Risaralda & $\begin{array}{c}\text { Eco Hotel Los } \\
\text { Lagos, Marsella, } \\
\text { Risaralda }\end{array}$ & 4,9308 & $-75,7451$ & $\begin{array}{l}\text { Archivo } \\
\text { de audio }\end{array}$ \\
\hline $\begin{array}{l}\text { Myiozetetes } \\
\text { cayanensis }\end{array}$ & hellmayri & 1 & 439770 & $\mathrm{XC}$ & Colombia & Tolima & $\begin{array}{l}\text { Reserva Natural } \\
\text { Gaia, Ibagué, } \\
\text { Tolima }\end{array}$ & 4,4611 & $-75,2151$ & $\begin{array}{l}\text { Archivo } \\
\text { de audio }\end{array}$ \\
\hline $\begin{array}{l}\text { Myiozetetes } \\
\text { cayanensis }\end{array}$ & cayanensis & 3 & 437961 & $\mathrm{XC}$ & Brasil & Mato Grosso & $\begin{array}{l}\text { Serra Nova, } \\
\text { Cristalino Jungle } \\
\text { Lodge, Mato } \\
\text { Grosso }\end{array}$ & $-9,5627$ & $-55,9207$ & $\begin{array}{l}\text { Archivo } \\
\text { de audio }\end{array}$ \\
\hline $\begin{array}{l}\text { Myiozetetes } \\
\text { cayanensis }\end{array}$ & hellmayri & 1 & 437830 & $\mathrm{XC}$ & Colombia & Valle del Cauca & $\begin{array}{c}\text { Zanjón del Burro, } \\
\text { Cali, Valle del } \\
\text { Cauca }\end{array}$ & 3,3641 & $-76,5402$ & $\begin{array}{l}\text { Archivo } \\
\text { de audio }\end{array}$ \\
\hline $\begin{array}{l}\text { Myiozetetes } \\
\text { cayanensis }\end{array}$ & hellmayri & 1 & 432827 & $\mathrm{XC}$ & Colombia & Antioquia & $\begin{array}{c}\text { El Reodo, } \\
\text { Copacabana, } \\
\text { Antioquia }\end{array}$ & 6,3921 & $-75,4914$ & $\begin{array}{l}\text { Archivo } \\
\text { de audio }\end{array}$ \\
\hline $\begin{array}{l}\text { Myiozetetes } \\
\text { cayanensis }\end{array}$ & cayanensis & 3 & 429281 & $\mathrm{XC}$ & Bolivia & Beni & $\begin{array}{c}\text { Puerto Hamburgo, } \\
\text { Riberalta, Beni }\end{array}$ & $-11,0189$ & $-66,0995$ & $\begin{array}{l}\text { Archivo } \\
\text { de audio }\end{array}$ \\
\hline $\begin{array}{l}\text { Myiozetetes } \\
\text { cayanensis }\end{array}$ & cayanensis & 3 & 427665 & $\mathrm{XC}$ & Brasil & Maranhão & $\begin{array}{l}\text { Serra da } \\
\text { Curiosidade, } \\
\text { Carolina-MA, } \\
\text { Maranhão }\end{array}$ & $-7,07401$ & $-47,4166$ & $\begin{array}{l}\text { Archivo } \\
\text { de audio }\end{array}$ \\
\hline
\end{tabular}




\begin{tabular}{|c|c|c|c|c|c|c|c|c|c|c|}
\hline $\begin{array}{l}\text { Myiozetetes } \\
\text { cayanensis }\end{array}$ & cayanensis & 3 & 427664 & $x C$ & Brasil & Espírito Santo & $\begin{array}{l}\text { Mata dos Caetés, } \\
\text { Vargem Alta-ES, } \\
\text { Espírito Santo }\end{array}$ & $-20,618021$ & $-40,986854$ & $\begin{array}{l}\text { Archivo } \\
\text { de audio }\end{array}$ \\
\hline $\begin{array}{l}\text { Myiozetetes } \\
\text { cayanensis }\end{array}$ & hellmayri & 1 & 423695 & $x c$ & Colombia & Tolima & $\begin{array}{l}\text { Reserva Natural } \\
\text { Agua fría, } \\
\text { Martinica parte } \\
\text { baja, Ibagué, } \\
\text { Tolima }\end{array}$ & 4,4216 & $-75,2387$ & $\begin{array}{l}\text { Archivo } \\
\text { de audio }\end{array}$ \\
\hline $\begin{array}{l}\text { Myiozetetes } \\
\text { cayanensis }\end{array}$ & cayanensis & 3 & 419092 & $x C$ & Brasil & Goiás & $\begin{array}{c}\text { Alta Floresta } \\
\text { Eventos - Av. } \\
\text { Samambaia } \\
\text { Campus UFGO. } \\
\text { Goiânia-GO }\end{array}$ & $-16,6869$ & $-49,2648$ & $\begin{array}{l}\text { Archivo } \\
\text { de audio }\end{array}$ \\
\hline $\begin{array}{l}\text { Myiozetetes } \\
\text { cayanensis }\end{array}$ & cayanensis & 3 & 407709 & $x C$ & Brasil & Goiás & $\begin{array}{l}\text { Santa Rita do Novo } \\
\text { Destino, Goiás, } \\
\text { Chácara Jayrson }\end{array}$ & $-14,625$ & $-49,0051$ & $\begin{array}{l}\text { Archivo } \\
\text { de audio }\end{array}$ \\
\hline $\begin{array}{l}\text { Myiozetetes } \\
\text { cayanensis }\end{array}$ & cayanensis & 3 & 407706 & $x C$ & Brasil & Goiás & $\begin{array}{c}\text { Parque Estadual } \\
\text { Altamiro de Moura } \\
\text { Pacheco, } \\
\text { Goianápolis, Goiás }\end{array}$ & $-16,5456$ & $-49,1572$ & $\begin{array}{l}\text { Archivo } \\
\text { de audio }\end{array}$ \\
\hline
\end{tabular}

\begin{tabular}{|c|c|c|c|c|c|c|c|c|c|c|}
\hline $\begin{array}{l}\text { Myiozetetes } \\
\text { cayanensis }\end{array}$ & cayanensis & 3 & 393146 & $x C$ & Brasil & Mato Grosso & $\begin{array}{c}\text { Parque Mae } \\
\text { Bonifacia, Cuiabá, } \\
\text { Mato Grosso }\end{array}$ & $-15,5805$ & $-56,1045$ & $\begin{array}{l}\text { Archivo } \\
\text { de audio }\end{array}$ \\
\hline $\begin{array}{l}\text { Myiozetetes } \\
\text { cayanensis }\end{array}$ & hellmayri & 1 & 374701 & $x C$ & Panamá & Torti & $\begin{array}{c}\text { San Francisco } \\
\text { Nature Reserve, } \\
\text { Torti }\end{array}$ & 8,939 & $-78,465$ & $\begin{array}{l}\text { Archivo } \\
\text { de audio }\end{array}$ \\
\hline $\begin{array}{l}\text { Myiozetetes } \\
\text { cayanensis }\end{array}$ & cayanensis & 3 & 323862 & $\mathrm{XC}$ & Brasil & Pará & $\begin{array}{l}\text { Serra dos Carajás. } \\
\text { Rio Parauapebas }\end{array}$ & $-6,0667$ & $-49,9167$ & $\begin{array}{l}\text { Archivo } \\
\text { de audio }\end{array}$ \\
\hline $\begin{array}{l}\text { Myiozetetes } \\
\text { cayanensis }\end{array}$ & cayanensis & 3 & 301611 & $X C$ & Brasil & Goiás & $\begin{array}{c}\text { Setor Samambaia, } \\
\text { mata seca, perto } \\
\text { da UFG- } \\
\text { Agronomia, } \\
\text { Goiânia-Goiás }\end{array}$ & $-16,6003$ & $-49,2802$ & $\begin{array}{l}\text { Archivo } \\
\text { de audio }\end{array}$ \\
\hline $\begin{array}{l}\text { Myiozetetes } \\
\text { cayanensis }\end{array}$ & cayanensis & 3 & 288547 & XC & Brasil & Goiás & $\begin{array}{c}\text { Caiapônia, } \\
\text { Caiapônia, Goiás }\end{array}$ & $-16,8453$ & $-51,9712$ & $\begin{array}{l}\text { Archivo } \\
\text { de audio }\end{array}$ \\
\hline $\begin{array}{l}\text { Myiozetetes } \\
\text { cayanensis }\end{array}$ & hellmayri & 1 & 275464 & $X C$ & Ecuador & Esmeraldas & $\begin{array}{l}\text { Esmeraldas: Playa } \\
\text { de Oro, village and } \\
\text { vicinity, Río } \\
\text { Santiago }\end{array}$ & 0,883 & $-78,8$ & $\begin{array}{l}\text { Archivo } \\
\text { de audio }\end{array}$ \\
\hline $\begin{array}{l}\text { Myiozetetes } \\
\text { cayanensis }\end{array}$ & cayanensis & 3 & 272497 & XC & Surinam & Paramaribo & $\begin{array}{c}\text { Weg naar Zee, } \\
\text { Paramaribo }\end{array}$ & 5,9 & $-55,23$ & $\begin{array}{l}\text { Archivo } \\
\text { de audio }\end{array}$ \\
\hline $\begin{array}{l}\text { Myiozetetes } \\
\text { cayanensis }\end{array}$ & cayanensis & 3 & 272429 & $X C$ & Surinam & Commewijne & $\begin{array}{l}\text { Peperpot, } \\
\text { Commewijne }\end{array}$ & 5,78 & $-55,13$ & $\begin{array}{l}\text { Archivo } \\
\text { de audio }\end{array}$ \\
\hline $\begin{array}{l}\text { Myiozetetes } \\
\text { cayanensis }\end{array}$ & cayanensis & 3 & 272062 & $X C$ & Surinam & W Suriname & $\begin{array}{l}\text { Mozeskreek area, } \\
\text { W Suriname }\end{array}$ & 4,848194 & $-56,776361$ & $\begin{array}{l}\text { Archivo } \\
\text { de audio }\end{array}$ \\
\hline $\begin{array}{l}\text { Myiozetetes } \\
\text { cayanensis }\end{array}$ & hellmayri & 1 & 262245 & $X C$ & Ecuador & Esmeraldas & $\begin{array}{l}\text { Esmeraldas: Playa } \\
\text { de Oro, village and } \\
\text { vicinity, Río } \\
\text { Santiago }\end{array}$ & 0,883 & $-78,8$ & $\begin{array}{l}\text { Archivo } \\
\text { de audio }\end{array}$ \\
\hline $\begin{array}{l}\text { Myiozetetes } \\
\text { cayanensis }\end{array}$ & hellmayri & 1 & 262244 & XC & Ecuador & Esmeraldas & $\begin{array}{l}\text { Esmeraldas: Playa } \\
\text { de Oro, village and } \\
\text { vicinity, Río } \\
\text { Santiago }\end{array}$ & 0,883 & $-78,8$ & $\begin{array}{l}\text { Archivo } \\
\text { de audio }\end{array}$ \\
\hline $\begin{array}{l}\text { Myiozetetes } \\
\text { cayanensis }\end{array}$ & hellmayri & 1 & 262243 & XC & Ecuador & Esmeraldas & $\begin{array}{l}\text { Esmeraldas: Playa } \\
\text { de Oro, village and } \\
\text { vicinity, Río } \\
\text { Santiago }\end{array}$ & 0,883 & $-78,8$ & $\begin{array}{l}\text { Archivo } \\
\text { de audio }\end{array}$ \\
\hline $\begin{array}{l}\text { Myiozetetes } \\
\text { cayanensis }\end{array}$ & hellmayri & 1 & 262242 & $x C$ & Ecuador & Esmeraldas & $\begin{array}{l}\text { Esmeraldas: Playa } \\
\text { de Oro, village and } \\
\text { vicinity, Río } \\
\text { Santiago }\end{array}$ & 0,883 & $-78,8$ & $\begin{array}{l}\text { Archivo } \\
\text { de audio }\end{array}$ \\
\hline $\begin{array}{l}\text { Myiozetetes } \\
\text { cayanensis }\end{array}$ & hellmayri & 1 & 259003 & XC & Ecuador & Pichincha & $\begin{array}{c}\text { Pichincha: Mindo } \\
\text { area }\end{array}$ & $-0,051$ & $-78,778$ & $\begin{array}{l}\text { Archivo } \\
\text { de audio }\end{array}$ \\
\hline $\begin{array}{l}\text { Myiozetetes } \\
\text { cayanensis }\end{array}$ & hellmayri & 1 & 259002 & $\mathrm{XC}$ & Ecuador & $\begin{array}{l}\text { Santo Domingo } \\
\text { de los Tsachilas }\end{array}$ & $\begin{array}{l}\text { Santo Domingo: } \\
\text { 'Tinalandia' }\end{array}$ & $-0,32$ & $-79,018$ & $\begin{array}{l}\text { Archivo } \\
\text { de audio }\end{array}$ \\
\hline $\begin{array}{l}\text { Myiozetetes } \\
\text { cayanensis }\end{array}$ & hellmayri & 1 & 248260 & XC & Ecuador & Esmeraldas & $\begin{array}{l}\text { Esmeraldas: } 7 \text { km } \\
\text { WNW Lita }\end{array}$ & 0,883 & $-78,514$ & $\begin{array}{l}\text { Archivo } \\
\text { de audio }\end{array}$ \\
\hline
\end{tabular}




\begin{tabular}{|c|c|c|c|c|c|c|c|c|c|c|}
\hline $\begin{array}{l}\text { Myiozetetes } \\
\text { cayanensis }\end{array}$ & hellmayri & 1 & 248259 & $\mathrm{XC}$ & Ecuador & Esmeraldas & $\begin{array}{c}\text { Esmeraldas: } 7 \text { km } \\
\text { WNW Lita }\end{array}$ & 0,883 & $-78,514$ & $\begin{array}{l}\text { Archivo } \\
\text { de audio }\end{array}$ \\
\hline $\begin{array}{l}\text { Myiozetetes } \\
\text { cayanensis }\end{array}$ & hellmayri & 1 & 248173 & $\mathrm{XC}$ & Ecuador & Esmeraldas & $\begin{array}{l}\text { Esmeraldas: ca.26 } \\
\text { km W Alto Tambo, } \\
800 \text { m S main road }\end{array}$ & 1,0914 & $-78,6882$ & $\begin{array}{l}\text { Archivo } \\
\text { de audio }\end{array}$ \\
\hline $\begin{array}{l}\text { Myiozetetes } \\
\text { cayanensis }\end{array}$ & hellmayri & 1 & 248166 & $\mathrm{XC}$ & Ecuador & Esmeraldas & $\begin{array}{c}\text { Esmeraldas: } \\
\text { Humedal de Yalare }\end{array}$ & 1,154 & $-78,851$ & $\begin{array}{l}\text { Archivo } \\
\text { de audio }\end{array}$ \\
\hline $\begin{array}{l}\text { Myiozetetes } \\
\text { cayanensis }\end{array}$ & rufipennis & 2 & 244839 & $X C$ & Colombia & Casanare & $\begin{array}{c}\text { Finca La Bretaña, } \\
\text { Vereda } \\
\text { Guaracuara, San } \\
\text { Luis de Palenque, } \\
\text { Casanare }\end{array}$ & 5,145 & $-70,9575$ & $\begin{array}{l}\text { Archivo } \\
\text { de audio }\end{array}$ \\
\hline $\begin{array}{l}\text { Myiozetetes } \\
\text { cayanensis }\end{array}$ & cayanensis & 3 & 226230 & $\mathrm{XC}$ & Brasil & Mato Grosso & $\begin{array}{l}\text { Pantanal, Pixaim } \\
\text { area, Mato Grosso }\end{array}$ & $-16,666667$ & $\begin{array}{c}- \\
56,8333333\end{array}$ & $\begin{array}{l}\text { Archivo } \\
\text { de audio }\end{array}$ \\
\hline $\begin{array}{l}\text { Myiozetetes } \\
\text { cayanensis }\end{array}$ & rufipennis & 2 & 226229 & $\mathrm{XC}$ & Venezuela & Carabobo & $\begin{array}{c}\text { Casa Maria, } \\
\text { Carabobo }\end{array}$ & 10,3166667 & $-68,25$ & $\begin{array}{l}\text { Archivo } \\
\text { de audio }\end{array}$ \\
\hline $\begin{array}{l}\text { Myiozetetes } \\
\text { cayanensis }\end{array}$ & hellmayri & 1 & 217484 & $\mathrm{XC}$ & Ecuador & Pichincha & $\begin{array}{c}\text { Reserva } \\
\text { Mangaloma, } \\
\text { Pichincha }\end{array}$ & 0,1228 & $-78,9942$ & $\begin{array}{l}\text { Archivo } \\
\text { de audio }\end{array}$ \\
\hline $\begin{array}{l}\text { Myiozetetes } \\
\text { cayanensis }\end{array}$ & cayanensis & 3 & 210835 & $\mathrm{XC}$ & Brasil & Pará & $\begin{array}{l}\text { Serra dos Carajás. } \\
\text { Road to dam at } \\
\text { iron mine }\end{array}$ & $-6,1001$ & $-50,1501$ & $\begin{array}{l}\text { Archivo } \\
\text { de audio }\end{array}$ \\
\hline $\begin{array}{l}\text { Myiozetetes } \\
\text { cayanensis }\end{array}$ & rufipennis & 2 & 197492 & $\mathrm{XC}$ & Venezuela & Apure & Modulos de Apure & 7,3682 & $-69,4407$ & $\begin{array}{l}\text { Archivo } \\
\text { de audio }\end{array}$ \\
\hline $\begin{array}{l}\text { Myiozetetes } \\
\text { cayanensis }\end{array}$ & cayanensis & 3 & 174029 & $\mathrm{XC}$ & Brasil & Mato Grosso & $\begin{array}{l}\text { Rosário Oeste, } \\
\text { Mato Grosso }\end{array}$ & $-14,8264$ & $-56,4241$ & $\begin{array}{l}\text { Archivo } \\
\text { de audio }\end{array}$ \\
\hline $\begin{array}{l}\text { Myiozetetes } \\
\text { cayanensis }\end{array}$ & hellmayri & 1 & 169040 & $\mathrm{XC}$ & Ecuador & Pichincha & Mindo, Pichincha & $-0,0523$ & $-78,7764$ & $\begin{array}{l}\text { Archivo } \\
\text { de audio }\end{array}$ \\
\hline $\begin{array}{l}\text { Myiozetetes } \\
\text { cayanensis }\end{array}$ & hellmayri & 1 & 158448 & $\mathrm{XC}$ & Ecuador & Pichincha & Mindo, Pichincha & $-0,0523$ & $-78,7764$ & $\begin{array}{l}\text { Archivo } \\
\text { de audio }\end{array}$ \\
\hline $\begin{array}{l}\text { Myiozetetes } \\
\text { cayanensis }\end{array}$ & hellmayri & 1 & 148307 & $X C$ & Colombia & Huila & Huila, San Agustin & 1,8888 & $-76,2888$ & $\begin{array}{l}\text { Archivo } \\
\text { de audio }\end{array}$ \\
\hline $\begin{array}{l}\text { Myiozetetes } \\
\text { cayanensis }\end{array}$ & rufipennis & 2 & 127205 & $\mathrm{XC}$ & Colombia & Meta & $\begin{array}{l}\text { Puerto Lopez, } \\
\text { Meta }\end{array}$ & 4,1809 & $-72,9136$ & $\begin{array}{l}\text { Archivo } \\
\text { de audio }\end{array}$ \\
\hline $\begin{array}{l}\text { Myiozetetes } \\
\text { cayanensis }\end{array}$ & cayanensis & 3 & 123674 & $\mathrm{XC}$ & Brasil & Mato Grosso & $\begin{array}{c}\text { Pantanal Wildlife } \\
\text { Center, MT }\end{array}$ & $-16,7581$ & $-56,8764$ & $\begin{array}{l}\text { Archivo } \\
\text { de audio }\end{array}$ \\
\hline $\begin{array}{l}\text { Myiozetetes } \\
\text { cayanensis }\end{array}$ & cayanensis & 3 & 118868 & $\mathrm{XC}$ & Brasil & Tocantins & $\begin{array}{c}\text { Estação de } \\
\text { Pesquisa do } \\
\text { Canguçu - Pium - } \\
\text { Tocantins State }\end{array}$ & $-9,9826$ & $-50,0151$ & $\begin{array}{l}\text { Archivo } \\
\text { de audio }\end{array}$ \\
\hline $\begin{array}{l}\text { Myiozetetes } \\
\text { cayanensis }\end{array}$ & hellmayri & 1 & 118234 & $\mathrm{XC}$ & Ecuador & Pichincha & Mindo, Pichincha & $-0,0523$ & $-78,7764$ & $\begin{array}{l}\text { Archivo } \\
\text { de audio }\end{array}$ \\
\hline $\begin{array}{l}\text { Myiozetetes } \\
\text { cayanensis }\end{array}$ & cayanensis & 3 & 108643 & $\mathrm{XC}$ & Brasil & $\begin{array}{l}\text { Mato Grosso do } \\
\text { Sul }\end{array}$ & $\begin{array}{l}\text { Eldorado, Mato } \\
\text { Grosso do Sul state }\end{array}$ & $-23,8501$ & $-54,2537$ & $\begin{array}{l}\text { Archivo } \\
\text { de audio }\end{array}$ \\
\hline $\begin{array}{l}\text { Myiozetetes } \\
\text { cayanensis }\end{array}$ & rufipennis & 2 & 105365 & $\mathrm{XC}$ & Venezuela & Barinas & $\begin{array}{l}\text { La Soledad Tunnel, } \\
20 \text { km NW } \\
\text { Barinitas, Barinas }\end{array}$ & 8,8526 & $-70,5806$ & $\begin{array}{l}\text { Archivo } \\
\text { de audio }\end{array}$ \\
\hline $\begin{array}{l}\text { Myiozetetes } \\
\text { cayanensis }\end{array}$ & cayanensis & 3 & 86748 & $\mathrm{XC}$ & Brasil & Pará & $\begin{array}{c}\text { Paragominas, PA, } \\
\text { Brazil, Bacia } 423\end{array}$ & $-3,519$ & $-47,504$ & $\begin{array}{l}\text { Archivo } \\
\text { de audio }\end{array}$ \\
\hline $\begin{array}{l}\text { Myiozetetes } \\
\text { cayanensis }\end{array}$ & cayanensis & 3 & 58405 & $\mathrm{XC}$ & Colombia & Amazonas & $\begin{array}{l}\text { Ome Ecological } \\
\text { Station, Purite } \\
\text { River, Amazonas } \\
\text { dep. } 70 \mathrm{Km} \mathrm{N} \text { of } \\
\text { Leticia }\end{array}$ & $-3,594416$ & $-69,949365$ & $\begin{array}{l}\text { Archivo } \\
\text { de audio }\end{array}$ \\
\hline $\begin{array}{l}\text { Myiozetetes } \\
\text { cayanensis }\end{array}$ & hellmayri & 1 & 54523 & $\mathrm{XC}$ & Colombia & Cundinamarca & $\begin{array}{c}\text { Laguna de Pedro } \\
\text { Palo, Tena, } \\
\text { Cundinamarca }\end{array}$ & 4,683487 & $-74,384744$ & $\begin{array}{l}\text { Archivo } \\
\text { de audio }\end{array}$ \\
\hline $\begin{array}{l}\text { Myiozetetes } \\
\text { cayanensis }\end{array}$ & cayanensis & 3 & 45178 & $\mathrm{XC}$ & Brasil & São Paulo & Turmalina, SP & $-20,052$ & $-50,455$ & $\begin{array}{l}\text { Archivo } \\
\text { de audio }\end{array}$ \\
\hline $\begin{array}{l}\text { Myiozetetes } \\
\text { cayanensis }\end{array}$ & rufipennis & 2 & 11621 & $\mathrm{XC}$ & Venezuela & Monagas & Maturin-La Pica & 9,857 & $-63,035$ & $\begin{array}{l}\text { Archivo } \\
\text { de audio }\end{array}$ \\
\hline $\begin{array}{l}\text { Myiozetetes } \\
\text { cayanensis }\end{array}$ & hellmayri & 1 & 2820 & $\mathrm{XC}$ & Panamá & Colón & $\begin{array}{c}\text { Gamboa, Colon } \\
\text { province }\end{array}$ & 9,11023 & $-79,6845$ & $\begin{array}{l}\text { Archivo } \\
\text { de audio }\end{array}$ \\
\hline $\begin{array}{l}\text { Myiozetetes } \\
\text { cayanensis }\end{array}$ & cayanensis & 3 & 2329 & $\mathrm{XC}$ & Bolivia & Santa Cruz & $\begin{array}{l}\text { Mouth of the Río } \\
\text { Paucerna, Noel } \\
\text { Kempff Mercado } \\
\text { NP, Santa Cruz }\end{array}$ & $-13,55$ & $-61,1$ & $\begin{array}{l}\text { Archivo } \\
\text { de audio }\end{array}$ \\
\hline
\end{tabular}




\begin{tabular}{|c|c|c|c|c|c|c|c|c|c|c|}
\hline $\begin{array}{l}\text { Myiozetetes } \\
\text { cayanensis }\end{array}$ & cayanensis & 3 & 2265 & $x C$ & Bolivia & Santa Cruz & $\begin{array}{l}\text { Flor d'Oro, Noel } \\
\text { Kempff Mercado } \\
\text { NP, Santa Cruz }\end{array}$ & $-13,55$ & $-61,01$ & $\begin{array}{l}\text { Archivo } \\
\text { de audio }\end{array}$ \\
\hline $\begin{array}{l}\text { Myiozetetes } \\
\text { cayanensis }\end{array}$ & hellmayri & 1 & 532376 & $x C$ & Colombia & Caldas & $\begin{array}{l}\text { Vereda Los Bohíos, } \\
\text { Hacienda Tintiná, } \\
\text { Cuenca río Tapias, } \\
\text { Guadual y borde } \\
\text { de cañada }\end{array}$ & 5,2461111 & $\begin{array}{c}- \\
75,6852778\end{array}$ & $\begin{array}{l}\text { Archivo } \\
\text { de audio }\end{array}$ \\
\hline $\begin{array}{l}\text { Myiozetetes } \\
\text { cayanensis }\end{array}$ & hellmayri & 1 & 532375 & $x C$ & Colombia & Caldas & $\begin{array}{l}\text { Vereda Los Bohíos, } \\
\text { Hacienda Tintiná, } \\
\text { Cuenca río Tapias, } \\
\text { Guadual y borde } \\
\text { de cañada }\end{array}$ & 5,2461111 & $\begin{array}{c}- \\
75,6852778\end{array}$ & $\begin{array}{l}\text { Archivo } \\
\text { de audio }\end{array}$ \\
\hline $\begin{array}{l}\text { Myiozetetes } \\
\text { cayanensis }\end{array}$ & hellmayri & 1 & 532374 & $x C$ & Colombia & Caldas & $\begin{array}{l}\text { Vereda Los Bohíos, } \\
\text { Hacienda Tintiná, } \\
\text { Cuenca río Tapias, } \\
\text { Guadual y borde } \\
\text { de cañada }\end{array}$ & 5,2461111 & $\begin{array}{c}- \\
75,6852778\end{array}$ & $\begin{array}{l}\text { Archivo } \\
\text { de audio }\end{array}$ \\
\hline $\begin{array}{l}\text { Myiozetetes } \\
\text { cayanensis }\end{array}$ & hellmayri & 1 & 461014 & $x c$ & Colombia & Risaralda & $\begin{array}{l}\text { Corredor Ecológico } \\
\text { La Dulcera // } \\
\text { Sector La } \\
\text { Bombonera, } \\
\text { Pereira, Risaralda }\end{array}$ & 4,8101 & $-75,71$ & $\begin{array}{l}\text { Archivo } \\
\text { de audio }\end{array}$ \\
\hline $\begin{array}{l}\text { Myiozetetes } \\
\text { cayanensis }\end{array}$ & hellmayri & 1 & 461013 & $x C$ & Colombia & Risaralda & $\begin{array}{l}\text { Corredor Ecológico } \\
\text { La Dulcera // } \\
\text { Sector La } \\
\text { Bombonera, } \\
\text { Pereira, Risaralda }\end{array}$ & 4,8101 & $-75,71$ & $\begin{array}{l}\text { Archivo } \\
\text { de audio }\end{array}$ \\
\hline $\begin{array}{l}\text { Myiozetetes } \\
\text { cayanensis }\end{array}$ & cayanensis & 3 & 433185 & $x C$ & Brasil & Mato Grosso & $\begin{array}{c}\text { Rio Teles Pires. Ilha } \\
\text { Ariosto }\end{array}$ & $-9,6296$ & $-55,9504$ & $\begin{array}{l}\text { Archivo } \\
\text { de audio }\end{array}$ \\
\hline $\begin{array}{l}\text { Myiozetetes } \\
\text { cayanensis }\end{array}$ & cayanensis & 3 & 401274 & $x C$ & Surinam & Commewijne & $\begin{array}{c}\text { Meerzorg, } \\
\text { Meerzorg, } \\
\text { Commewijne }\end{array}$ & 5,7985 & $-55,1179$ & $\begin{array}{l}\text { Archivo } \\
\text { de audio }\end{array}$ \\
\hline $\begin{array}{l}\text { Myiozetetes } \\
\text { cayanensis }\end{array}$ & hellmayri & 1 & 388413 & $x C$ & Colombia & Cundinamarca & $\begin{array}{c}\text { Guaduas, } \\
\text { Cundinamarca }\end{array}$ & 5,1266 & $-74,5924$ & $\begin{array}{l}\text { Archivo } \\
\text { de audio }\end{array}$ \\
\hline $\begin{array}{l}\text { Myiozetetes } \\
\text { cayanensis }\end{array}$ & cayanensis & 3 & 387956 & $x C$ & Brasil & Mato Grosso & $\begin{array}{c}\text { Porto Estrela, Mato } \\
\text { Grosso }\end{array}$ & $-15,6421$ & $-57,1899$ & $\begin{array}{l}\text { Archivo } \\
\text { de audio }\end{array}$ \\
\hline $\begin{array}{l}\text { Myiozetetes } \\
\text { cayanensis }\end{array}$ & rufipennis & 2 & 387068 & $x C$ & Venezuela & Barinas & Bolívar, Barinas & 8,8085 & $-70,5049$ & $\begin{array}{l}\text { Archivo } \\
\text { de audio }\end{array}$ \\
\hline $\begin{array}{l}\text { Myiozetetes } \\
\text { cayanensis }\end{array}$ & cayanensis & 3 & 377335 & $x C$ & Brasil & Mato Grosso & $\begin{array}{c}\text { Near Faz } \\
\text { Araçatuba, Lambari } \\
\text { D'Oeste, Mato } \\
\text { Grosso }\end{array}$ & $-15,1789$ & $-57,7467$ & $\begin{array}{l}\text { Archivo } \\
\text { de audio }\end{array}$ \\
\hline $\begin{array}{l}\text { Myiozetetes } \\
\text { cayanensis }\end{array}$ & hellmayri & 1 & 374407 & $x C$ & Colombia & Chocó & $\begin{array}{c}\text { El Valle-Utría } \\
\text { National Park Trail }\end{array}$ & 6,0747 & $-77,3868$ & $\begin{array}{l}\text { Archivo } \\
\text { de audio }\end{array}$ \\
\hline $\begin{array}{l}\text { Myiozetetes } \\
\text { cayanensis }\end{array}$ & hellmayri & 1 & 360727 & $x C$ & Colombia & Sucre & Tolú Viejo, Sucre & 9,3955 & $-75,4304$ & $\begin{array}{l}\text { Archivo } \\
\text { de audio }\end{array}$ \\
\hline $\begin{array}{l}\text { Myiozetetes } \\
\text { cayanensis }\end{array}$ & hellmayri & 1 & 360378 & $x C$ & Colombia & Sucre & Colosó, Sucre & 9,4975 & $-75,3507$ & $\begin{array}{l}\text { Archivo } \\
\text { de audio }\end{array}$ \\
\hline $\begin{array}{l}\text { Myiozetetes } \\
\text { cayanensis }\end{array}$ & cayanensis & 3 & 339479 & $x C$ & Brasil & Acre & $\begin{array}{c}\text { Parque } \\
\text { Zoobotanico/UFAC, } \\
\text { Rio Branco, Acre }\end{array}$ & $-9,9491$ & $-67,8809$ & $\begin{array}{l}\text { Archivo } \\
\text { de audio }\end{array}$ \\
\hline $\begin{array}{l}\text { Myiozetetes } \\
\text { cayanensis }\end{array}$ & hellmayri & 1 & 333230 & $x C$ & Ecuador & Pichincha & $\begin{array}{l}\text { Pichincha: turnoff } \\
\text { to Mindo }\end{array}$ & $-0,028$ & $-78,761$ & $\begin{array}{l}\text { Archivo } \\
\text { de audio }\end{array}$ \\
\hline $\begin{array}{l}\text { Myiozetetes } \\
\text { cayanensis }\end{array}$ & hellmayri & 1 & 332146 & $x C$ & Colombia & Antioquia & $\begin{array}{l}\text { vereda Pajonal, } \\
\text { Anorí, Antioquia }\end{array}$ & 6,9189 & $-75,1709$ & $\begin{array}{l}\text { Archivo } \\
\text { de audio }\end{array}$ \\
\hline $\begin{array}{l}\text { Myiozetetes } \\
\text { cayanensis }\end{array}$ & hellmayri & 1 & 332145 & $x C$ & Colombia & Antioquia & $\begin{array}{l}\text { vereda Pajonal, } \\
\text { Anorí, Antioquia }\end{array}$ & 6,9189 & $-75,1709$ & $\begin{array}{l}\text { Archivo } \\
\text { de audio }\end{array}$ \\
\hline $\begin{array}{l}\text { Myiozetetes } \\
\text { cayanensis }\end{array}$ & cayanensis & 3 & 304401 & $x C$ & Brasil & $\begin{array}{c}\text { Mato Grosso do } \\
\text { Sul }\end{array}$ & $\begin{array}{l}\text { Três Lagoas, Mato } \\
\text { Grosso do Sul }\end{array}$ & $-20,7882$ & $-51,703$ & $\begin{array}{l}\text { Archivo } \\
\text { de audio }\end{array}$ \\
\hline $\begin{array}{l}\text { Myiozetetes } \\
\text { cayanensis }\end{array}$ & cayanensis & 3 & 304400 & $x C$ & Brasil & $\begin{array}{l}\text { Mato Grosso do } \\
\text { Sul }\end{array}$ & $\begin{array}{l}\text { Três Lagoas, Mato } \\
\text { Grosso do Sul }\end{array}$ & $-20,7882$ & $-51,703$ & $\begin{array}{l}\text { Archivo } \\
\text { de audio }\end{array}$ \\
\hline $\begin{array}{l}\text { Myiozetetes } \\
\text { cayanensis }\end{array}$ & cayanensis & 3 & 272428 & $x C$ & Surinam & Commewijne & $\begin{array}{l}\text { Peperpot, } \\
\text { Commewijne }\end{array}$ & 5,78 & $-55,13$ & $\begin{array}{l}\text { Archivo } \\
\text { de audio }\end{array}$ \\
\hline $\begin{array}{l}\text { Myiozetetes } \\
\text { cayanensis }\end{array}$ & hellmayri & 1 & 248165 & $x C$ & Ecuador & Esmeraldas & $\begin{array}{l}\text { Esmeraldas: } \\
\text { Humedal de Yalare }\end{array}$ & 1,154 & $-78,851$ & $\begin{array}{l}\text { Archivo } \\
\text { de audio }\end{array}$ \\
\hline
\end{tabular}


Myiozetetes cayanensis

Myiozetetes Myiozetetes

Myiozetetes

cayanensis

Myiozetetes

cayanensis

Myiozetetes

cayanensis

Myiozetetes

cayanensis

Myiozetetes

cayanensis

Myiozetetes

cayanensis

Myiozetetes

cayanensis

Myiozetetes

cayanensis

Myiozetetes

cayanensis

Myiozetetes

cayanensis rufipennis

$2 \quad 244949$

XC

Finca La Bretaña,

$$
\text { Vereda }
$$

Guaracuara, San

$5,145 \quad-70,9575$

Archivo Luis de Palenque,

Casanare

\begin{tabular}{|c|c|c|c|c|c|c|c|c|c|}
\hline hellmayri & 1 & 226236 & $x C$ & Venezuela & Carabobo & $\begin{array}{c}\text { San Pablo marsh, } \\
\text { Carabobo }\end{array}$ & 10,5166667 & $\begin{array}{c}- \\
68,3666667\end{array}$ & $\begin{array}{l}\text { Archivo } \\
\text { de audio }\end{array}$ \\
\hline rufipennis & 2 & 226235 & $\mathrm{XC}$ & Venezuela & Carabobo & $\begin{array}{c}\text { Casa Maria, } \\
\text { Carabobo }\end{array}$ & 10,3166667 & $-68,25$ & $\begin{array}{l}\text { Archivo } \\
\text { de audio }\end{array}$ \\
\hline rufipennis & 2 & 226234 & $x C$ & Venezuela & Carabobo & $\begin{array}{c}\text { Casa Maria, } \\
\text { Carabobo }\end{array}$ & 10,3166667 & $-68,25$ & $\begin{array}{l}\text { Archivo } \\
\text { de audio }\end{array}$ \\
\hline hellmayri & 1 & 226233 & $\mathrm{XC}$ & Venezuela & Táchira & $\begin{array}{c}\text { La Petrolea area, } \\
\text { Tachira }\end{array}$ & 7,63333333 & $-72,35$ & $\begin{array}{l}\text { Archivo } \\
\text { de audio }\end{array}$ \\
\hline cayanensis & 3 & 226232 & $\mathrm{XC}$ & Brasil & Pará & $\begin{array}{l}\text { Carajás, Airport } \\
\text { area, Pará }\end{array}$ & -6 & $\begin{array}{c}- \\
50,1666667\end{array}$ & $\begin{array}{l}\text { Archivo } \\
\text { de audio }\end{array}$ \\
\hline cayanensis & 3 & 226231 & $\mathrm{XC}$ & Brasil & Pará & $\begin{array}{c}\text { Carajás, Airport } \\
\text { area, Pará }\end{array}$ & -6 & $\begin{array}{c}- \\
50,1666667\end{array}$ & $\begin{array}{l}\text { Archivo } \\
\text { de audio }\end{array}$ \\
\hline rufipennis & 2 & 210836 & $\mathrm{XC}$ & Colombia & Meta & $\begin{array}{c}\text { Serrania de la } \\
\text { Macarena. Caño } \\
\text { Cristales }\end{array}$ & 2,213 & $-73,823$ & $\begin{array}{l}\text { Archivo } \\
\text { de audio }\end{array}$ \\
\hline rufipennis & 2 & 197488 & $\mathrm{XC}$ & Venezuela & Apure & $\begin{array}{c}\text { Los Llanos - Hato el } \\
\text { Cedral }\end{array}$ & 7,059 & $-69,494$ & $\begin{array}{l}\text { Archivo } \\
\text { de audio }\end{array}$ \\
\hline cayanensis & 3 & 167312 & $\mathrm{XC}$ & Brasil & Rondônia & $\begin{array}{c}\text { Floresta Estadual } \\
\text { Rio Preto-Jacundá, } \\
\text { Machadinho } \\
\text { d'Oeste, Rondônia }\end{array}$ & $-8,9244$ & $-62,0836$ & $\begin{array}{l}\text { Archivo } \\
\text { de audio }\end{array}$ \\
\hline hellmayri & 1 & 156308 & $\mathrm{XC}$ & Colombia & Antioquia & $\begin{array}{c}\text { Cortijo de San José, } \\
\text { Sabaneta, } \\
\text { Antioquia }\end{array}$ & 6,141 & $-75,6168$ & $\begin{array}{l}\text { Archivo } \\
\text { de audio }\end{array}$ \\
\hline hellmayri & 1 & 156306 & $x C$ & Colombia & Antioquia & $\begin{array}{c}\text { Cortijo de San José, } \\
\text { Sabaneta, } \\
\text { Antioquia }\end{array}$ & 6,141 & $-75,6168$ & $\begin{array}{l}\text { Archivo } \\
\text { de audio }\end{array}$ \\
\hline
\end{tabular}

Chocó, Bahía

Solano, PNN

Myiozetetes hellmayri $1 \quad 148074 \quad$ XC $\quad$ Colombia

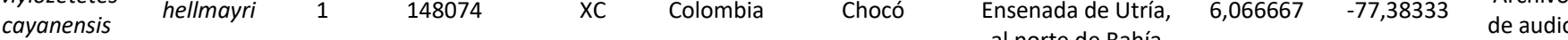
al norte de Bahía Solano

\begin{tabular}{|c|c|c|c|c|c|c|c|c|c|c|}
\hline $\begin{array}{l}\text { Myiozetetes } \\
\text { cayanensis }\end{array}$ & rufipennis & 2 & 147657 & $\mathrm{XC}$ & Venezuela & Apure & Munoz, Apure & 7,7143 & $-69,3326$ & $\begin{array}{l}\text { Archivo } \\
\text { de audio }\end{array}$ \\
\hline $\begin{array}{c}\text { Myiozetetes } \\
\text { cayanensis }\end{array}$ & rufipennis & 2 & 147613 & $\mathrm{XC}$ & Venezuela & Barinas & $\begin{array}{c}\text { Barinitas, Bolivar, } \\
\text { Barinas }\end{array}$ & 8,8863 & $-70,4877$ & $\begin{array}{l}\text { Archivo } \\
\text { de audio }\end{array}$ \\
\hline $\begin{array}{l}\text { Myiozetetes } \\
\text { cayanensis }\end{array}$ & rufipennis & 2 & 147588 & $\mathrm{XC}$ & Venezuela & Barinas & $\begin{array}{c}\text { Barinitas, Bolivar, } \\
\text { Barinas }\end{array}$ & 8,8863 & $-70,4877$ & $\begin{array}{l}\text { Archivo } \\
\text { de audio }\end{array}$ \\
\hline $\begin{array}{l}\text { Myiozetetes } \\
\text { cayanensis }\end{array}$ & rufipennis & 2 & 127204 & $\mathrm{XC}$ & Colombia & Meta & $\begin{array}{l}\text { Puerto Lopez, } \\
\text { Meta }\end{array}$ & 4,1809 & $-72,9136$ & $\begin{array}{l}\text { Archivo } \\
\text { de audio }\end{array}$ \\
\hline $\begin{array}{l}\text { Myiozetetes } \\
\text { cayanensis }\end{array}$ & cayanensis & 3 & 123644 & $\mathrm{XC}$ & Brasil & Mato Grosso & $\begin{array}{c}\text { Pantanal Wildlife } \\
\text { Center, MT }\end{array}$ & $-16,7581$ & $-56,8764$ & $\begin{array}{l}\text { Archivo } \\
\text { de audio }\end{array}$ \\
\hline $\begin{array}{l}\text { Myiozetetes } \\
\text { cayanensis }\end{array}$ & cayanensis & 3 & 113473 & $\mathrm{XC}$ & Brasil & Mato Grosso & $\begin{array}{c}\text { Poconé, Mato } \\
\text { Grosso }\end{array}$ & $-16,4277$ & $-56,3132$ & $\begin{array}{l}\text { Archivo } \\
\text { de audio }\end{array}$ \\
\hline $\begin{array}{l}\text { Myiozetetes } \\
\text { cayanensis }\end{array}$ & hellmayri & 1 & 97906 & $\mathrm{XC}$ & Ecuador & Pichincha & Mindo, Pichincha & $-0,0523$ & $-78,7764$ & $\begin{array}{l}\text { Archivo } \\
\text { de audio }\end{array}$ \\
\hline $\begin{array}{l}\text { Myiozetetes } \\
\text { cayanensis }\end{array}$ & hellmayri & 1 & 92252 & $\mathrm{XC}$ & Colombia & Cundinamarca & $\begin{array}{l}\text { La Vega, Vereda La } \\
\text { Libertad } \\
\text { (Cundinamarca- } \\
\text { Colombia) }\end{array}$ & 5,014 & $-74,35$ & $\begin{array}{l}\text { Archivo } \\
\text { de audio }\end{array}$ \\
\hline $\begin{array}{l}\text { Myiozetetes } \\
\text { cayanensis }\end{array}$ & cayanensis & 3 & 91319 & $\mathrm{XC}$ & Perú & Madre de Dios & $\begin{array}{l}\text { Iberia, Madre de } \\
\text { Dios }\end{array}$ & $-11,389$ & $-69,489$ & $\begin{array}{l}\text { Archivo } \\
\text { de audio }\end{array}$ \\
\hline $\begin{array}{l}\text { Myiozetetes } \\
\text { cayanensis }\end{array}$ & cayanensis & 3 & 82269 & $\mathrm{XC}$ & Brasil & $\begin{array}{l}\text { Mato Grosso do } \\
\text { Sul }\end{array}$ & $\begin{array}{c}\text { Aquidauana, MS. } \\
\text { Road to Barra } \\
\text { Mansa }\end{array}$ & $-20,1213$ & $-55,9297$ & $\begin{array}{l}\text { Archivo } \\
\text { de audio }\end{array}$ \\
\hline $\begin{array}{l}\text { Myiozetetes } \\
\text { cayanensis }\end{array}$ & hellmayri & 1 & 82050 & $X C$ & Colombia & Cundinamarca & $\begin{array}{c}\text { Laguna de Pedro } \\
\text { Palo, } \\
\text { Cundinamarca }\end{array}$ & 4,6834 & $-74,3834$ & $\begin{array}{l}\text { Archivo } \\
\text { de audio }\end{array}$ \\
\hline $\begin{array}{l}\text { Myiozetetes } \\
\text { cayanensis }\end{array}$ & cayanensis & 3 & 75351 & $\mathrm{XC}$ & $\begin{array}{l}\text { Guyana } \\
\text { Francesa }\end{array}$ & Mana & Angoulème, Mana & 5,4092 & $-53,6773$ & $\begin{array}{l}\text { Archivo } \\
\text { de audio }\end{array}$ \\
\hline $\begin{array}{l}\text { Myiozetetes } \\
\text { cayanensis }\end{array}$ & rufipennis & 2 & 65918 & $X C$ & Venezuela & Barinas & $\begin{array}{c}\text { San Isidro quarry, } \\
\text { Barinas }\end{array}$ & 8,8323 & $-70,5801$ & $\begin{array}{l}\text { Archivo } \\
\text { de audio }\end{array}$ \\
\hline
\end{tabular}




\begin{tabular}{|c|c|c|c|c|c|c|c|c|c|c|}
\hline $\begin{array}{l}\text { Myiozetetes } \\
\text { cayanensis }\end{array}$ & hellmayri & 1 & 57826 & $\mathrm{XC}$ & Ecuador & Pichincha & $\begin{array}{l}\text { Mashpi Reserve } \\
\text { turnoff, } 9 \mathrm{~km} \text { past } \\
\text { La Delicia, } \\
\text { Pichincha }\end{array}$ & 0,203173 & $-78,861809$ & $\begin{array}{l}\text { Archivo } \\
\text { de audio }\end{array}$ \\
\hline $\begin{array}{l}\text { Myiozetetes } \\
\text { cayanensis }\end{array}$ & hellmayri & 1 & 56459 & $\mathrm{XC}$ & Panamá & Colón & $\begin{array}{c}\text { Gamboa, Colon } \\
\text { province }\end{array}$ & 9,1223 & $-79,7501$ & $\begin{array}{l}\text { Archivo } \\
\text { de audio }\end{array}$ \\
\hline $\begin{array}{l}\text { Myiozetetes } \\
\text { cayanensis }\end{array}$ & hellmayri & 1 & 56458 & $\mathrm{XC}$ & Panamá & Panamá & $\begin{array}{c}\text { Torti, San Francisco } \\
\text { Nature Reserve, } \\
\text { Panama }\end{array}$ & 8,9389 & $-78,4651$ & $\begin{array}{l}\text { Archivo } \\
\text { de audio }\end{array}$ \\
\hline $\begin{array}{l}\text { Myiozetetes } \\
\text { cayanensis }\end{array}$ & hellmayri & 1 & 54522 & $\mathrm{XC}$ & Colombia & Cundinamarca & $\begin{array}{l}\text { Laguna de Pedro } \\
\text { Palo, Tena, } \\
\text { Cundinamarca }\end{array}$ & 4,683487 & $-74,384744$ & $\begin{array}{l}\text { Archivo } \\
\text { de audio }\end{array}$ \\
\hline $\begin{array}{l}\text { Myiozetetes } \\
\text { cayanensis }\end{array}$ & hellmayri & 1 & 54521 & $\mathrm{XC}$ & Colombia & Cundinamarca & $\begin{array}{l}\text { Laguna de Pedro } \\
\text { Palo, Tena, } \\
\text { Cundinamarca }\end{array}$ & 4,683487 & $-74,384744$ & $\begin{array}{l}\text { Archivo } \\
\text { de audio }\end{array}$ \\
\hline $\begin{array}{l}\text { Myiozetetes } \\
\text { cayanensis }\end{array}$ & cayanensis & 3 & 52761 & $\mathrm{XC}$ & Brasil & $\begin{array}{l}\text { Mato Grosso do } \\
\text { Sul }\end{array}$ & $\begin{array}{l}\text { Pantanal do } \\
\text { Miranda - MS }\end{array}$ & $-19,5512$ & $-57,012$ & $\begin{array}{l}\text { Archivo } \\
\text { de audio }\end{array}$ \\
\hline $\begin{array}{l}\text { Myiozetetes } \\
\text { cayanensis }\end{array}$ & rufipennis & 2 & 43258 & $\mathrm{XC}$ & Venezuela & Barinas & $\begin{array}{l}\text { Altamira Edo } \\
\text { Barinas }\end{array}$ & 8,821126 & $-70,501328$ & $\begin{array}{l}\text { Archivo } \\
\text { de audio }\end{array}$ \\
\hline $\begin{array}{l}\text { Myiozetetes } \\
\text { cayanensis }\end{array}$ & cayanensis & 3 & 39773 & $x C$ & Perú & Madre de Dios & $\begin{array}{l}\text { Tipishca Lodge, } \\
\text { Madre de Dios }\end{array}$ & $-12,2292$ & $-69,3256$ & $\begin{array}{l}\text { Archivo } \\
\text { de audio }\end{array}$ \\
\hline $\begin{array}{l}\text { Myiozetetes } \\
\text { cayanensis }\end{array}$ & erythropterus & 4 & 26132 & $\mathrm{XC}$ & Brasil & Río de Janeiro & $\begin{array}{l}\text { Reserva Ecológica } \\
\text { de Guapiaçu, RJ }\end{array}$ & $-22,5001$ & $-42,7167$ & $\begin{array}{l}\text { Archivo } \\
\text { de audio }\end{array}$ \\
\hline $\begin{array}{l}\text { Myiozetetes } \\
\text { cayanensis }\end{array}$ & erythropterus & 4 & 26131 & $\mathrm{XC}$ & Brasil & Río de Janeiro & $\begin{array}{l}\text { Reserva Ecológica } \\
\text { de Guapiaçu, RJ }\end{array}$ & $-22,5001$ & $-42,7167$ & $\begin{array}{l}\text { Archivo } \\
\text { de audio }\end{array}$ \\
\hline $\begin{array}{l}\text { Myiozetetes } \\
\text { cayanensis }\end{array}$ & hellmayri & 1 & 16060 & $\mathrm{XC}$ & Panamá & Panamá & $\begin{array}{l}\text { Old Gamboa Road, } \\
\text { Panama Province }\end{array}$ & 9,0617 & $-79,6498$ & $\begin{array}{l}\text { Archivo } \\
\text { de audio }\end{array}$ \\
\hline $\begin{array}{l}\text { Myiozetetes } \\
\text { cayanensis }\end{array}$ & hellmayri & 1 & 15714 & $\mathrm{XC}$ & Panamá & Panamá & Summit Gardens & 9,057516 & $-79,636288$ & $\begin{array}{l}\text { Archivo } \\
\text { de audio }\end{array}$ \\
\hline $\begin{array}{l}\text { Myiozetetes } \\
\text { cayanensis }\end{array}$ & hellmayri & 1 & 12843 & $\mathrm{XC}$ & Colombia & Cundinamarca & $\begin{array}{c}\text { Tena, Laguna de } \\
\text { pedro palo, } \\
\text { Cundinamarca }\end{array}$ & 4,6501 & $-74,3834$ & $\begin{array}{l}\text { Archivo } \\
\text { de audio }\end{array}$ \\
\hline $\begin{array}{l}\text { Myiozetetes } \\
\text { cayanensis }\end{array}$ & hellmayri & 1 & 11283 & $\mathrm{XC}$ & Ecuador & Pichincha & Mindo, Pichincha & $-0,0523$ & $-78,7764$ & $\begin{array}{l}\text { Archivo } \\
\text { de audio }\end{array}$ \\
\hline $\begin{array}{l}\text { Myiozetetes } \\
\text { cayanensis }\end{array}$ & hellmayri & 1 & 10091 & $\mathrm{XC}$ & Panamá & Panamá & $\begin{array}{l}\text { Old Gamboa Road, } \\
\text { Panama Province }\end{array}$ & 9,0617 & $-79,6498$ & $\begin{array}{l}\text { Archivo } \\
\text { de audio }\end{array}$ \\
\hline $\begin{array}{l}\text { Myiozetetes } \\
\text { cayanensis }\end{array}$ & cayanensis & 3 & 9196 & $\mathrm{XC}$ & Venezuela & Bolívar & $\begin{array}{l}\text { Rio Grande, Sierra } \\
\text { de Imataca, Bolivar }\end{array}$ & 8,1101 & $-61,6714$ & $\begin{array}{l}\text { Archivo } \\
\text { de audio }\end{array}$ \\
\hline $\begin{array}{l}\text { Myiozetetes } \\
\text { cayanensis }\end{array}$ & hellmayri & 1 & 464412 & $x C$ & Panamá & Colón & $\begin{array}{l}\text { Panama Rainforest } \\
\text { Discovery Center, } \\
\text { Corregimiento de } \\
\text { Cristóbal, Colón, } \\
\text { Provincia de Colón }\end{array}$ & 9,1307 & $-79,7283$ & $\begin{array}{l}\text { Archivo } \\
\text { de audio }\end{array}$ \\
\hline $\begin{array}{l}\text { Myiozetetes } \\
\text { cayanensis }\end{array}$ & hellmayri & 1 & 433513 & $\mathrm{XC}$ & Colombia & Antioquia & Liborina, Antioquia & 6,6887 & $-75,8375$ & $\begin{array}{l}\text { Archivo } \\
\text { de audio }\end{array}$ \\
\hline $\begin{array}{l}\text { Myiozetetes } \\
\text { cayanensis }\end{array}$ & hellmayri & 1 & 433202 & $\mathrm{XC}$ & Colombia & Cundinamarca & $\begin{array}{c}\text { Tocaima, } \\
\text { Cundinamarca }\end{array}$ & 4,4796 & $-74,6082$ & $\begin{array}{l}\text { Archivo } \\
\text { de audio }\end{array}$ \\
\hline $\begin{array}{l}\text { Myiozetetes } \\
\text { cayanensis }\end{array}$ & hellmayri & 1 & 420112 & $x C$ & Colombia & Chocó & $\begin{array}{l}\text { Airport, Nuquí, } \\
\text { Chocó }\end{array}$ & 5,7059 & $-77,2703$ & $\begin{array}{l}\text { Archivo } \\
\text { de audio }\end{array}$ \\
\hline $\begin{array}{l}\text { Myiozetetes } \\
\text { cayanensis }\end{array}$ & hellmayri & 1 & 406578 & $\mathrm{XC}$ & Panamá & Colón & $\begin{array}{l}\text { Ammo Dump } \\
\text { Ponds, Colon } \\
\text { Province }\end{array}$ & 9,124 & $-79,7104$ & $\begin{array}{l}\text { Archivo } \\
\text { de audio }\end{array}$ \\
\hline $\begin{array}{l}\text { Myiozetetes } \\
\text { cayanensis }\end{array}$ & cayanensis & 3 & 394781 & $x C$ & Brasil & Acre & $\begin{array}{l}\text { Floresta Estadual } \\
\text { do Antimary - Sena } \\
\text { Madureira, Acre }\end{array}$ & $-9,1795$ & $-68,4627$ & $\begin{array}{l}\text { Archivo } \\
\text { de audio }\end{array}$ \\
\hline $\begin{array}{l}\text { Myiozetetes } \\
\text { cayanensis }\end{array}$ & rufipennis & 2 & 387881 & $\mathrm{XC}$ & Venezuela & Barinas & Bolívar, Barinas & 8,8085 & $-70,5049$ & $\begin{array}{l}\text { Archivo } \\
\text { de audio }\end{array}$ \\
\hline $\begin{array}{l}\text { Myiozetetes } \\
\text { cayanensis }\end{array}$ & cayanensis & 3 & 304402 & $x C$ & Brasil & $\begin{array}{l}\text { Mato Grosso do } \\
\text { Sul }\end{array}$ & $\begin{array}{l}\text { Três Lagoas, Mato } \\
\text { Grosso do Sul }\end{array}$ & $-20,7882$ & $-51,703$ & $\begin{array}{l}\text { Archivo } \\
\text { de audio }\end{array}$ \\
\hline $\begin{array}{l}\text { Myiozetetes } \\
\text { cayanensis }\end{array}$ & cayanensis & 3 & 303294 & $\mathrm{XC}$ & Brasil & Pará & $\begin{array}{c}\text { Parauapebas, } \\
\text { Parauapebas, Pará }\end{array}$ & $-6,0313$ & $-50,0895$ & $\begin{array}{l}\text { Archivo } \\
\text { de audio }\end{array}$ \\
\hline $\begin{array}{l}\text { Myiozetetes } \\
\text { cayanensis }\end{array}$ & cayanensis & 3 & 303194 & $\mathrm{XC}$ & Brasil & Roraima & Caracaraí, Roraima & 1,4787 & $-62,2446$ & $\begin{array}{l}\text { Archivo } \\
\text { de audio }\end{array}$ \\
\hline $\begin{array}{l}\text { Myiozetetes } \\
\text { cayanensis }\end{array}$ & hellmayri & 1 & 251202 & $\mathrm{XC}$ & Ecuador & Esmeraldas & $\begin{array}{l}\text { Esmeraldas: } \\
\text { Canandé }\end{array}$ & 0,533 & $-79,217$ & $\begin{array}{l}\text { Archivo } \\
\text { de audio }\end{array}$ \\
\hline
\end{tabular}




\begin{tabular}{|c|c|c|c|c|c|c|c|c|c|c|}
\hline $\begin{array}{l}\text { Myiozetetes } \\
\text { cayanensis }\end{array}$ & hellmayri & 1 & 153170 & $\mathrm{XC}$ & Colombia & Antioquia & $\begin{array}{l}\text { Sabaneta, } \\
\text { Antioquia }\end{array}$ & 6,1413 & $-75,6174$ & $\begin{array}{l}\text { Archivo } \\
\text { de audio }\end{array}$ \\
\hline $\begin{array}{l}\text { Myiozetetes } \\
\text { cayanensis }\end{array}$ & cayanensis & 3 & 145414 & $\mathrm{XC}$ & Brasil & $\begin{array}{l}\text { Mato Grosso do } \\
\text { Sul }\end{array}$ & $\begin{array}{l}\text { Miranda, Mato } \\
\text { Grosso do Sul }\end{array}$ & $-19,9873$ & $-56,3585$ & $\begin{array}{l}\text { Archivo } \\
\text { de audio }\end{array}$ \\
\hline $\begin{array}{l}\text { Myiozetetes } \\
\text { cayanensis }\end{array}$ & cayanensis & 3 & 129961 & $\mathrm{XC}$ & Brasil & Mato Grosso & $\begin{array}{l}\text { Cristalino Jungle } \\
\text { Lodge, MT }\end{array}$ & $-9,5981$ & $-55,932$ & $\begin{array}{l}\text { Archivo } \\
\text { de audio }\end{array}$ \\
\hline $\begin{array}{l}\text { Myiozetetes } \\
\text { cayanensis }\end{array}$ & hellmayri & 1 & 128468 & $\mathrm{XC}$ & Colombia & Quindío & $\begin{array}{l}\text { Reserva Natural La } \\
\text { Montaña del } \\
\text { Ocaso, Quimbaya, } \\
\text { Quindío }\end{array}$ & 4,6197 & $-75,851$ & $\begin{array}{l}\text { Archivo } \\
\text { de audio }\end{array}$ \\
\hline $\begin{array}{l}\text { Myiozetetes } \\
\text { cayanensis }\end{array}$ & hellmayri & 1 & 128451 & $\mathrm{XC}$ & Ecuador & Pichincha & $\begin{array}{c}\text { Un Poco del Choco, } \\
\text { Pichincha }\end{array}$ & 0,0736 & $-78,7685$ & $\begin{array}{l}\text { Archivo } \\
\text { de audio }\end{array}$ \\
\hline $\begin{array}{l}\text { Myiozetetes } \\
\text { cayanensis }\end{array}$ & hellmayri & 1 & 120669 & $\mathrm{XC}$ & Ecuador & Esmeraldas & $\begin{array}{c}\text { Playa de Oro, } \\
\text { Esmeraldas } \\
\text { Province, Ecuador }\end{array}$ & 0,8501 & $-78,7667$ & $\begin{array}{l}\text { Archivo } \\
\text { de audio }\end{array}$ \\
\hline $\begin{array}{l}\text { Myiozetetes } \\
\text { cayanensis }\end{array}$ & rufipennis & 2 & 109016 & $\mathrm{XC}$ & Colombia & Arauca & $\begin{array}{c}\text { Arauca, Laguna } \\
\text { Rodriguera }\end{array}$ & 7,092 & $-70,697$ & $\begin{array}{l}\text { Archivo } \\
\text { de audio }\end{array}$ \\
\hline $\begin{array}{l}\text { Myiozetetes } \\
\text { cayanensis }\end{array}$ & rufipennis & 2 & 106295 & $\mathrm{XC}$ & Colombia & Guaviare & $\begin{array}{l}\text { San Jose de } \\
\text { Guaviare - } \\
\text { Departamento del } \\
\text { Guaviare }\end{array}$ & 2,667 & $-72,6148$ & $\begin{array}{l}\text { Archivo } \\
\text { de audio }\end{array}$ \\
\hline $\begin{array}{l}\text { Myiozetetes } \\
\text { cayanensis }\end{array}$ & hellmayri & 1 & 104450 & $\mathrm{XC}$ & Colombia & Bolívar & $\begin{array}{c}\text { Santa Cecilia, } \\
\text { Serrania de San } \\
\text { Lucas }\end{array}$ & 7,9759 & $-74,2153$ & $\begin{array}{l}\text { Archivo } \\
\text { de audio }\end{array}$ \\
\hline $\begin{array}{l}\text { Myiozetetes } \\
\text { cayanensis }\end{array}$ & hellmayri & 1 & 92253 & $\mathrm{XC}$ & Colombia & Cundinamarca & $\begin{array}{c}\text { La Vega, Vereda La } \\
\text { Libertad } \\
\text { (Cundinamarca- } \\
\text { Colombia) }\end{array}$ & 5,014 & $-74,35$ & $\begin{array}{l}\text { Archivo } \\
\text { de audio }\end{array}$ \\
\hline $\begin{array}{l}\text { Myiozetetes } \\
\text { cayanensis }\end{array}$ & hellmayri & 1 & 92251 & $\mathrm{XC}$ & Colombia & Cundinamarca & $\begin{array}{c}\text { La Vega, Vereda La } \\
\text { Libertad } \\
\text { (Cundinamarca- } \\
\text { Colombia) }\end{array}$ & 5,014 & $-74,35$ & $\begin{array}{l}\text { Archivo } \\
\text { de audio }\end{array}$ \\
\hline $\begin{array}{l}\text { Myiozetetes } \\
\text { cayanensis }\end{array}$ & hellmayri & 1 & 78844 & $\mathrm{XC}$ & Colombia & Huila & $\begin{array}{l}\text { desierto de la } \\
\text { Tatacoa_Huila }\end{array}$ & 3,232375 & $-75,168795$ & $\begin{array}{l}\text { Archivo } \\
\text { de audio }\end{array}$ \\
\hline $\begin{array}{l}\text { Myiozetetes } \\
\text { cayanensis }\end{array}$ & cayanensis & 3 & 74385 & $\mathrm{XC}$ & Brasil & Amazonas & $\begin{array}{l}\text { Presidente } \\
\text { Figueiredo, } \\
\text { Amazonas }\end{array}$ & $-2,028$ & $-59,997$ & $\begin{array}{l}\text { Archivo } \\
\text { de audio }\end{array}$ \\
\hline $\begin{array}{l}\text { Myiozetetes } \\
\text { cayanensis }\end{array}$ & cayanensis & 3 & 46162 & $\mathrm{XC}$ & Guyana & Guyana & Rewa, Guyana & 3,759602 & $-58,716345$ & $\begin{array}{l}\text { Archivo } \\
\text { de audio }\end{array}$ \\
\hline $\begin{array}{l}\text { Myiozetetes } \\
\text { cayanensis }\end{array}$ & hellmayri & 1 & 42370 & $\mathrm{XC}$ & Colombia & Cundinamarca & $\begin{array}{l}\text { Vereda Cumaca, } \\
\text { Municipio de } \\
\text { Tibacuy, } \\
\text { Cundinamarca }\end{array}$ & 4,3331 & $-74,4764$ & $\begin{array}{l}\text { Archivo } \\
\text { de audio }\end{array}$ \\
\hline $\begin{array}{l}\text { Myiozetetes } \\
\text { cayanensis }\end{array}$ & hellmayri & 1 & 37321 & $\mathrm{XC}$ & Colombia & Cundinamarca & $\begin{array}{c}\text { La Vega, } \\
\text { inmediaciones } \\
\text { Laguna del Tabacal }\end{array}$ & 5,020922 & $-74,302082$ & $\begin{array}{l}\text { Archivo } \\
\text { de audio }\end{array}$ \\
\hline $\begin{array}{l}\text { Myiozetetes } \\
\text { cayanensis }\end{array}$ & hellmayri & 1 & 37286 & $\mathrm{XC}$ & Colombia & Cundinamarca & La Vega & 4,996554 & $-74,340363$ & $\begin{array}{l}\text { Archivo } \\
\text { de audio }\end{array}$ \\
\hline $\begin{array}{l}\text { Myiozetetes } \\
\text { cayanensis }\end{array}$ & cayanensis & 3 & 4292 & $\mathrm{XC}$ & Bolivia & Beni & $\begin{array}{c}\text { Río Dartagnan, } \\
\text { Pilón Lajas } \\
\text { Biosphere Reserve, } \\
\text { Beni }\end{array}$ & $-15,0751$ & $-67,1167$ & $\begin{array}{l}\text { Archivo } \\
\text { de audio }\end{array}$ \\
\hline $\begin{array}{l}\text { Myiozetetes } \\
\text { cayanensis }\end{array}$ & cayanensis & 3 & 2752 & $\mathrm{XC}$ & Bolivia & Beni & $\begin{array}{l}\text { Near Trinidad, } \\
\text { along the road to } \\
\text { Santa Cruz, Beni }\end{array}$ & $-14,9001$ & $-64,7167$ & $\begin{array}{l}\text { Archivo } \\
\text { de audio }\end{array}$ \\
\hline $\begin{array}{l}\text { Myiozetetes } \\
\text { cayanensis }\end{array}$ & cayanensis & 3 & 188843 & $x C$ & Perú & Madre de Dios & $\begin{array}{c}\text { Puerto Maldonado, } \\
\text { Tambopata, Madre } \\
\text { de Dios }\end{array}$ & $-12,6579$ & $-69,2097$ & $\begin{array}{l}\text { Archivo } \\
\text { de audio }\end{array}$ \\
\hline $\begin{array}{l}\text { Myiozetetes } \\
\text { cayanensis }\end{array}$ & erythropterus & 4 & 22144 & WK & Brasil & Río de Janeiro & $\begin{array}{c}\text { Cachoeiras de } \\
\text { Macacu/RJ }\end{array}$ & $-22,462291$ & $-42,654436$ & $\begin{array}{l}\text { Archivo } \\
\text { de audio }\end{array}$ \\
\hline $\begin{array}{l}\text { Myiozetetes } \\
\text { cayanensis }\end{array}$ & cayanensis & 3 & 26272 & WK & Brasil & Tocantins & Palmas/TO & $-10,224231$ & $\begin{array}{c}- \\
48,1422328\end{array}$ & $\begin{array}{l}\text { Archivo } \\
\text { de audio }\end{array}$ \\
\hline $\begin{array}{l}\text { Myiozetetes } \\
\text { cayanensis }\end{array}$ & cayanensis & 3 & 42496 & WK & Brasil & Amazonas & Manaus/AM & $-2,625919$ & $\begin{array}{c}- \\
60,2590208\end{array}$ & $\begin{array}{l}\text { Archivo } \\
\text { de audio }\end{array}$ \\
\hline $\begin{array}{l}\text { Myiozetetes } \\
\text { cayanensis }\end{array}$ & cayanensis & 3 & 44686 & WK & Brasil & Amazonas & Manaus/AM & $-2,625919$ & $\begin{array}{c}- \\
60,2590208\end{array}$ & $\begin{array}{l}\text { Archivo } \\
\text { de audio }\end{array}$ \\
\hline
\end{tabular}




\begin{tabular}{|c|c|c|c|c|c|c|c|c|c|c|}
\hline $\begin{array}{l}\text { Myiozetetes } \\
\text { cayanensis }\end{array}$ & erythropterus & 4 & 60847 & WK & Brasil & Río de Janeiro & Petrópolis/RJ & $-22,401721$ & 43,1609786 & $\begin{array}{l}\text { Archivo } \\
\text { de audio }\end{array}$ \\
\hline $\begin{array}{l}\text { Myiozetetes } \\
\text { cayanensis }\end{array}$ & cayanensis & 3 & 62949 & WK & Brasil & Amazonas & Manaus/AM & $-2,625919$ & 60,2590208 & $\begin{array}{l}\text { Archivo } \\
\text { de audio }\end{array}$ \\
\hline $\begin{array}{l}\text { Myiozetetes } \\
\text { cayanensis }\end{array}$ & cayanensis & 3 & 74218 & WK & Brasil & Tocantins & Pium/TO & $-10,210032$ & $-49,872287$ & $\begin{array}{l}\text { Archivo } \\
\text { de audio }\end{array}$ \\
\hline $\begin{array}{l}\text { Myiozetetes } \\
\text { cayanensis }\end{array}$ & cayanensis & 3 & 77295 & WK & Brasil & $\begin{array}{l}\text { Mato Grosso do } \\
\text { Sul }\end{array}$ & Três Lagoas/MS & $-20,438274$ & $\begin{array}{c}- \\
52,2110981\end{array}$ & $\begin{array}{l}\text { Archivo } \\
\text { de audio }\end{array}$ \\
\hline $\begin{array}{l}\text { Myiozetetes } \\
\text { cayanensis }\end{array}$ & cayanensis & 3 & 103307 & WK & Brasil & São Paulo & Turmalina/SP & $-20,083148$ & $\begin{array}{c}- \\
50,4565403\end{array}$ & $\begin{array}{l}\text { Archivo } \\
\text { de audio }\end{array}$ \\
\hline $\begin{array}{l}\text { Myiozetetes } \\
\text { cayanensis }\end{array}$ & erythropterus & 4 & 111359 & WK & Brasil & Minas Gerais & Catas Altas/MG & $-20,075438$ & $-43,408135$ & $\begin{array}{l}\text { Archivo } \\
\text { de audio }\end{array}$ \\
\hline $\begin{array}{l}\text { Myiozetetes } \\
\text { cayanensis }\end{array}$ & erythropterus & 4 & 126465 & WK & Brasil & Río de Janeiro & Nova Friburgo/RJ & $-22,288644$ & $-42,534183$ & $\begin{array}{l}\text { Archivo } \\
\text { de audio }\end{array}$ \\
\hline $\begin{array}{l}\text { Myiozetetes } \\
\text { cayanensis }\end{array}$ & cayanensis & 3 & 136089 & WK & Brasil & Minas Gerais & $\begin{array}{c}\text { Conceição das } \\
\text { Alagoas/MG }\end{array}$ & $-19,959005$ & 48,3608266 & $\begin{array}{l}\text { Archivo } \\
\text { de audio }\end{array}$ \\
\hline $\begin{array}{l}\text { Myiozetetes } \\
\text { cayanensis }\end{array}$ & erythropterus & 4 & 136462 & WK & Brasil & Minas Gerais & Ouro Preto/MG & $-20,386474$ & $-43,504737$ & $\begin{array}{l}\text { Archivo } \\
\text { de audio }\end{array}$ \\
\hline $\begin{array}{l}\text { Myiozetetes } \\
\text { cayanensis }\end{array}$ & cayanensis & 3 & 150823 & WK & Brasil & $\begin{array}{l}\text { Mato Grosso do } \\
\text { Sul }\end{array}$ & Coxim/MS & $-18,280368$ & $\begin{array}{c}- \\
54,6743747\end{array}$ & $\begin{array}{l}\text { Archivo } \\
\text { de audio }\end{array}$ \\
\hline $\begin{array}{l}\text { Myiozetetes } \\
\text { cayanensis }\end{array}$ & cayanensis & 3 & 158209 & WK & Brasil & Goiás & Acreúna/GO & $-17,451892$ & $-50,325547$ & $\begin{array}{l}\text { Archivo } \\
\text { de audio }\end{array}$ \\
\hline $\begin{array}{l}\text { Myiozetetes } \\
\text { cayanensis }\end{array}$ & cayanensis & 3 & 158220 & WK & Brasil & Goiás & Jandaia/GO & $-17,091646$ & $\begin{array}{c}- \\
50,1819663\end{array}$ & $\begin{array}{l}\text { Archivo } \\
\text { de audio }\end{array}$ \\
\hline $\begin{array}{l}\text { Myiozetetes } \\
\text { cayanensis }\end{array}$ & cayanensis & 3 & 198082 & WK & Brasil & São Paulo & Dourado/SP & $-22,116567$ & $\begin{array}{c}- \\
48,3329623\end{array}$ & $\begin{array}{l}\text { Archivo } \\
\text { de audio }\end{array}$ \\
\hline $\begin{array}{l}\text { Myiozetetes } \\
\text { cayanensis }\end{array}$ & cayanensis & 3 & 219956 & WK & Brasil & Goiás & Jaraguá/GO & $-15,704836$ & $\begin{array}{c}- \\
49,3849404\end{array}$ & $\begin{array}{l}\text { Archivo } \\
\text { de audio }\end{array}$ \\
\hline $\begin{array}{l}\text { Myiozetetes } \\
\text { cayanensis }\end{array}$ & cayanensis & 3 & 256249 & WK & Brasil & Distrito Federal & Brasília/DF & $-15,780741$ & $\begin{array}{c}- \\
47,7967907\end{array}$ & $\begin{array}{l}\text { Archivo } \\
\text { de audio }\end{array}$ \\
\hline $\begin{array}{l}\text { Myiozetetes } \\
\text { cayanensis }\end{array}$ & cayanensis & 3 & 257982 & WK & Brasil & Goiás & Cavalcante/GO & $-13,634762$ & $\begin{array}{c}- \\
47,6957015\end{array}$ & $\begin{array}{l}\text { Archivo } \\
\text { de audio }\end{array}$ \\
\hline $\begin{array}{l}\text { Myiozetetes } \\
\text { cayanensis }\end{array}$ & cayanensis & 3 & 282009 & WK & Brasil & Ceará & Tianguá/CE & $-3,7151441$ & $\begin{array}{c}- \\
41,0027397\end{array}$ & $\begin{array}{l}\text { Archivo } \\
\text { de audio }\end{array}$ \\
\hline $\begin{array}{l}\text { Myiozetetes } \\
\text { cayanensis }\end{array}$ & cayanensis & 3 & 283861 & WK & Brasil & Maranhão & Morros/MA & $-2,9715093$ & $-43,802628$ & $\begin{array}{l}\text { Archivo } \\
\text { de audio }\end{array}$ \\
\hline $\begin{array}{l}\text { Myiozetetes } \\
\text { cayanensis }\end{array}$ & cayanensis & 3 & 286740 & WK & Brasil & Roraima & Boa Vista/RR & 3,11827869 & $\begin{array}{c}- \\
60,7174232\end{array}$ & $\begin{array}{l}\text { Archivo } \\
\text { de audio }\end{array}$ \\
\hline $\begin{array}{l}\text { Myiozetetes } \\
\text { cayanensis }\end{array}$ & cayanensis & 3 & 287096 & WK & Brasil & Tocantins & Palmeirante/TO & $-7,8844905$ & $-48,139129$ & $\begin{array}{l}\text { Archivo } \\
\text { de audio }\end{array}$ \\
\hline $\begin{array}{l}\text { Myiozetetes } \\
\text { cayanensis }\end{array}$ & cayanensis & 3 & 287368 & WK & Brasil & Amazonas & $\begin{array}{l}\text { Rio Preto da } \\
\text { Eva/AM }\end{array}$ & $-2,5295133$ & $\begin{array}{c}- \\
59,6379196\end{array}$ & $\begin{array}{l}\text { Archivo } \\
\text { de audio }\end{array}$ \\
\hline $\begin{array}{l}\text { Myiozetetes } \\
\text { cayanensis }\end{array}$ & cayanensis & 3 & 316300 & WK & Brasil & Mato Grosso & Juína/MT & $-11,530767$ & $\begin{array}{c}- \\
59,3229921\end{array}$ & $\begin{array}{l}\text { Archivo } \\
\text { de audio }\end{array}$ \\
\hline $\begin{array}{l}\text { Myiozetetes } \\
\text { cayanensis }\end{array}$ & cayanensis & 3 & 318034 & WK & Brasil & Pará & $\begin{array}{c}\text { Santa Bárbara do } \\
\text { Pará/PA }\end{array}$ & $-1,1940019$ & $\begin{array}{c}- \\
48,2501179\end{array}$ & $\begin{array}{l}\text { Archivo } \\
\text { de audio }\end{array}$ \\
\hline $\begin{array}{l}\text { Myiozetetes } \\
\text { cayanensis }\end{array}$ & cayanensis & 3 & 318670 & WK & Brasil & Amazonas & $\begin{array}{c}\text { Presidente } \\
\text { Figueiredo/AM }\end{array}$ & $-1,3720793$ & $\begin{array}{c}- \\
59,9884392\end{array}$ & $\begin{array}{l}\text { Archivo } \\
\text { de audio }\end{array}$ \\
\hline $\begin{array}{l}\text { Myiozetetes } \\
\text { cayanensis }\end{array}$ & cayanensis & 3 & 346728 & WK & Brasil & Amapá & Macapá/AP & 0,55442463 & $\begin{array}{c}- \\
50,7022237\end{array}$ & $\begin{array}{l}\text { Archivo } \\
\text { de audio }\end{array}$ \\
\hline $\begin{array}{l}\text { Myiozetetes } \\
\text { cayanensis }\end{array}$ & erythropterus & 4 & 350190 & WK & Brasil & Minas Gerais & Santa Bárbara/MG & $-20,029107$ & $\begin{array}{c}- \\
43,4753397\end{array}$ & $\begin{array}{l}\text { Archivo } \\
\text { de audio }\end{array}$ \\
\hline $\begin{array}{l}\text { Myiozetetes } \\
\text { cayanensis }\end{array}$ & cayanensis & 3 & 373926 & WK & Brasil & Roraima & Boa Vista/RR & 3,11827869 & $\begin{array}{c}- \\
60,7174232\end{array}$ & $\begin{array}{l}\text { Archivo } \\
\text { de audio }\end{array}$ \\
\hline $\begin{array}{l}\text { Myiozetetes } \\
\text { cayanensis }\end{array}$ & cayanensis & 3 & 383471 & WK & Brasil & Distrito Federal & Brasília/DF & $-15,780741$ & $\begin{array}{c}- \\
47,7967907\end{array}$ & $\begin{array}{l}\text { Archivo } \\
\text { de audio }\end{array}$ \\
\hline $\begin{array}{l}\text { Myiozetetes } \\
\text { cayanensis }\end{array}$ & cayanensis & 3 & 414997 & WK & Brasil & Amazonas & Manaus/AM & $-2,625919$ & $\begin{array}{c}- \\
60,2590208\end{array}$ & $\begin{array}{l}\text { Archivo } \\
\text { de audio }\end{array}$ \\
\hline $\begin{array}{l}\text { Myiozetetes } \\
\text { cayanensis }\end{array}$ & cayanensis & 3 & 424041 & WK & Brasil & Mato Grosso & Poconé/MT & $-16,78447$ & $\begin{array}{c}- \\
56,9485054\end{array}$ & $\begin{array}{l}\text { Archivo } \\
\text { de audio }\end{array}$ \\
\hline $\begin{array}{l}\text { Myiozetetes } \\
\text { cayanensis }\end{array}$ & cayanensis & 3 & 427765 & WK & Brasil & Maranhão & Buriticupu/MA & $-4,6128163$ & $\begin{array}{c}- \\
46,3117152\end{array}$ & $\begin{array}{l}\text { Archivo } \\
\text { de audio }\end{array}$ \\
\hline $\begin{array}{l}\text { Myiozetetes } \\
\text { cayanensis }\end{array}$ & cayanensis & 3 & 427766 & WK & Brasil & Maranhão & Buriticupu/MA & $-4,6128163$ & $\begin{array}{c}- \\
46,3117152\end{array}$ & $\begin{array}{l}\text { Archivo } \\
\text { de audio }\end{array}$ \\
\hline $\begin{array}{l}\text { Myiozetetes } \\
\text { cayanensis }\end{array}$ & cayanensis & 3 & 481217 & WK & Brasil & $\begin{array}{l}\text { Mato Grosso do } \\
\text { Sul }\end{array}$ & Japorã/MS & $-23,813281$ & $\begin{array}{c}- \\
54,5247225\end{array}$ & $\begin{array}{l}\text { Archivo } \\
\text { de audio }\end{array}$ \\
\hline $\begin{array}{l}\text { Myiozetetes } \\
\text { cayanensis }\end{array}$ & cayanensis & 3 & 481613 & WK & Brasil & Distrito Federal & Brasília/DF & $-15,780741$ & $\begin{array}{c}- \\
47,7967907\end{array}$ & $\begin{array}{l}\text { Archivo } \\
\text { de audio }\end{array}$ \\
\hline
\end{tabular}




\begin{tabular}{|c|c|c|c|c|c|c|c|c|c|c|}
\hline $\begin{array}{l}\text { Myiozetetes } \\
\text { cayanensis }\end{array}$ & cayanensis & 3 & 509627 & WK & Brasil & Goiás & Aruanã/GO & $-14,805158$ & $\begin{array}{c}- \\
50,9367461\end{array}$ & $\begin{array}{l}\text { Archivo } \\
\text { de audio }\end{array}$ \\
\hline $\begin{array}{l}\text { Myiozetetes } \\
\text { cayanensis }\end{array}$ & cayanensis & 3 & 518365 & WK & Brasil & Acre & Rio Branco/AC & $-10,067291$ & $\begin{array}{c}- \\
68,3192194\end{array}$ & $\begin{array}{l}\text { Archivo } \\
\text { de audio }\end{array}$ \\
\hline $\begin{array}{l}\text { Myiozetetes } \\
\text { cayanensis }\end{array}$ & cayanensis & 3 & 518943 & WK & Brasil & Acre & Rio Branco/AC & $-10,067291$ & $\begin{array}{c}- \\
68,3192194\end{array}$ & $\begin{array}{l}\text { Archivo } \\
\text { de audio }\end{array}$ \\
\hline $\begin{array}{l}\text { Myiozetetes } \\
\text { cayanensis }\end{array}$ & cayanensis & 3 & 534989 & WK & Brasil & $\begin{array}{l}\text { Mato Grosso do } \\
\text { Sul }\end{array}$ & Bonito/MS & $-21,007472$ & $\begin{array}{c}- \\
56,4597222\end{array}$ & $\begin{array}{l}\text { Archivo } \\
\text { de audio }\end{array}$ \\
\hline $\begin{array}{l}\text { Myiozetetes } \\
\text { cayanensis }\end{array}$ & cayanensis & 3 & 587404 & WK & Brasil & Acre & Rio Branco/AC & $-10,067291$ & $\begin{array}{c}- \\
68,3192194\end{array}$ & $\begin{array}{l}\text { Archivo } \\
\text { de audio }\end{array}$ \\
\hline $\begin{array}{l}\text { Myiozetetes } \\
\text { cayanensis }\end{array}$ & cayanensis & 3 & 596477 & WK & Brasil & Pará & Parauapebas/PA & $-6,1544368$ & $\begin{array}{c}- \\
50,4860742\end{array}$ & $\begin{array}{l}\text { Archivo } \\
\text { de audio }\end{array}$ \\
\hline $\begin{array}{l}\text { Myiozetetes } \\
\text { cayanensis }\end{array}$ & cayanensis & 3 & 600727 & WK & Brasil & Mato Grosso & $\begin{array}{l}\text { Santo Antônio do } \\
\text { Leverger/MT }\end{array}$ & $-16,482519$ & $\begin{array}{c}- \\
55,4364492\end{array}$ & $\begin{array}{l}\text { Archivo } \\
\text { de audio }\end{array}$ \\
\hline $\begin{array}{l}\text { Myiozetetes } \\
\text { cayanensis }\end{array}$ & cayanensis & 3 & 608114 & WK & Brasil & $\begin{array}{l}\text { Mato Grosso do } \\
\text { Sul }\end{array}$ & Corumbá/MS & $-18,730961$ & $\begin{array}{c}- \\
56,7232711\end{array}$ & $\begin{array}{l}\text { Archivo } \\
\text { de audio }\end{array}$ \\
\hline $\begin{array}{l}\text { Myiozetetes } \\
\text { cayanensis }\end{array}$ & cayanensis & 3 & 642643 & WK & Brasil & Minas Gerais & Uberlândia/MG & $-19,027452$ & $\begin{array}{c}- \\
48,3298488\end{array}$ & $\begin{array}{l}\text { Archivo } \\
\text { de audio }\end{array}$ \\
\hline $\begin{array}{l}\text { Myiozetetes } \\
\text { cayanensis }\end{array}$ & cayanensis & 3 & 643089 & WK & Brasil & Tocantins & Palmas/TO & $-10,224231$ & $\begin{array}{c}- \\
48,1422328\end{array}$ & $\begin{array}{l}\text { Archivo } \\
\text { de audio }\end{array}$ \\
\hline $\begin{array}{l}\text { Myiozetetes } \\
\text { cayanensis }\end{array}$ & cayanensis & 3 & 650624 & WK & Brasil & Mato Grosso & Alta Floresta/MT & $-10,055008$ & $\begin{array}{c}- \\
56,3660622\end{array}$ & $\begin{array}{l}\text { Archivo } \\
\text { de audio }\end{array}$ \\
\hline $\begin{array}{l}\text { Myiozetetes } \\
\text { cayanensis }\end{array}$ & cayanensis & 3 & 678002 & WK & Brasil & Tocantins & Palmas/TO & $-10,224231$ & $\begin{array}{c}- \\
48,1422328\end{array}$ & $\begin{array}{l}\text { Archivo } \\
\text { de audio }\end{array}$ \\
\hline $\begin{array}{l}\text { Myiozetetes } \\
\text { cayanensis }\end{array}$ & cayanensis & 3 & 681685 & WK & Brasil & $\begin{array}{l}\text { Mato Grosso do } \\
\text { Sul }\end{array}$ & Bonito/MS & $-21,007472$ & $\begin{array}{c}- \\
56,4597222\end{array}$ & $\begin{array}{l}\text { Archivo } \\
\text { de audio }\end{array}$ \\
\hline $\begin{array}{l}\text { Myiozetetes } \\
\text { cayanensis }\end{array}$ & cayanensis & 3 & 704913 & WK & Brasil & Minas Gerais & Araguari/MG & $-18,575335$ & $-48,221858$ & $\begin{array}{l}\text { Archivo } \\
\text { de audio }\end{array}$ \\
\hline $\begin{array}{l}\text { Myiozetetes } \\
\text { cayanensis }\end{array}$ & cayanensis & 3 & 706149 & WK & Brasil & Mato Grosso & Comodoro/MT & $-13,188712$ & $\begin{array}{c}- \\
59,7517942\end{array}$ & $\begin{array}{l}\text { Archivo } \\
\text { de audio }\end{array}$ \\
\hline $\begin{array}{l}\text { Myiozetetes } \\
\text { cayanensis }\end{array}$ & cayanensis & 3 & 722109 & WK & Brasil & $\begin{array}{l}\text { Mato Grosso do } \\
\text { Sul }\end{array}$ & Eldorado/MS & $-23,737442$ & $\begin{array}{c}- \\
54,2244384\end{array}$ & $\begin{array}{l}\text { Archivo } \\
\text { de audio }\end{array}$ \\
\hline $\begin{array}{l}\text { Myiozetetes } \\
\text { cayanensis }\end{array}$ & erythropterus & 4 & 722695 & WK & Brasil & Río de Janeiro & Nova Friburgo/RJ & $-22,288644$ & $-42,534183$ & $\begin{array}{l}\text { Archivo } \\
\text { de audio }\end{array}$ \\
\hline $\begin{array}{l}\text { Myiozetetes } \\
\text { cayanensis }\end{array}$ & cayanensis & 3 & 723884 & WK & Brasil & $\begin{array}{l}\text { Mato Grosso do } \\
\text { Sul }\end{array}$ & Miranda/MS & $-20,119777$ & $\begin{array}{c}- \\
56,5975499\end{array}$ & $\begin{array}{l}\text { Archivo } \\
\text { de audio }\end{array}$ \\
\hline $\begin{array}{l}\text { Myiozetetes } \\
\text { cayanensis }\end{array}$ & cayanensis & 3 & 755857 & WK & Brasil & Río de Janeiro & Rio de Janeiro/RJ & $-22,927629$ & $\begin{array}{c}- \\
43,4691378\end{array}$ & $\begin{array}{l}\text { Archivo } \\
\text { de audio }\end{array}$ \\
\hline $\begin{array}{l}\text { Myiozetetes } \\
\text { cayanensis }\end{array}$ & cayanensis & 3 & 825378 & WK & Brasil & Mato Grosso & Jaciara/MT & $-15,902872$ & $\begin{array}{c}- \\
55,1104369\end{array}$ & $\begin{array}{l}\text { Archivo } \\
\text { de audio }\end{array}$ \\
\hline $\begin{array}{l}\text { Myiozetetes } \\
\text { cayanensis }\end{array}$ & cayanensis & 3 & 830615 & WK & Brasil & Mato Grosso & $\begin{array}{l}\text { Vila Bela da } \\
\text { Santíssima } \\
\text { Trindade/MT }\end{array}$ & $-15,187472$ & $\begin{array}{c}- \\
59,9830146\end{array}$ & $\begin{array}{l}\text { Archivo } \\
\text { de audio }\end{array}$ \\
\hline $\begin{array}{l}\text { Myiozetetes } \\
\text { cayanensis }\end{array}$ & cayanensis & 3 & 924089 & WK & Brasil & Pará & Altamira/PA & $-6,4636149$ & $\begin{array}{c}- \\
53,8729712\end{array}$ & $\begin{array}{l}\text { Archivo } \\
\text { de audio }\end{array}$ \\
\hline $\begin{array}{l}\text { Myiozetetes } \\
\text { cayanensis }\end{array}$ & cayanensis & 3 & 925991 & WK & Brasil & Distrito Federal & Brasília/DF & $-15,780741$ & $\begin{array}{c}- \\
47,7967907\end{array}$ & $\begin{array}{l}\text { Archivo } \\
\text { de audio }\end{array}$ \\
\hline $\begin{array}{l}\text { Myiozetetes } \\
\text { cayanensis }\end{array}$ & erythropterus & 4 & 936364 & WK & Brasil & Río de Janeiro & $\begin{array}{c}\text { Cachoeiras de } \\
\text { Macacu/RJ }\end{array}$ & $-22,462291$ & $-42,654436$ & $\begin{array}{l}\text { Archivo } \\
\text { de audio }\end{array}$ \\
\hline $\begin{array}{l}\text { Myiozetetes } \\
\text { cayanensis }\end{array}$ & erythropterus & 4 & 938394 & WK & Brasil & Espírito Santo & Afonso Cláudio/ES & $-20,088187$ & $-41,127739$ & $\begin{array}{l}\text { Archivo } \\
\text { de audio }\end{array}$ \\
\hline $\begin{array}{l}\text { Myiozetetes } \\
\text { cayanensis }\end{array}$ & erythropterus & 4 & 966001 & WK & Brasil & Minas Gerais & Antônio Dias/MG & $-19,557906$ & $\begin{array}{c}- \\
42,8824248\end{array}$ & $\begin{array}{l}\text { Archivo } \\
\text { de audio }\end{array}$ \\
\hline $\begin{array}{l}\text { Myiozetetes } \\
\text { cayanensis }\end{array}$ & cayanensis & 3 & 970814 & WK & Brasil & Goiás & $\begin{array}{c}\text { São Luís de Montes } \\
\text { Belos/GO }\end{array}$ & $-16,454709$ & $\begin{array}{c}- \\
50,3740456\end{array}$ & $\begin{array}{l}\text { Archivo } \\
\text { de audio }\end{array}$ \\
\hline $\begin{array}{l}\text { Myiozetetes } \\
\text { cayanensis }\end{array}$ & cayanensis & 3 & 984851 & WK & Brasil & São Paulo & Miguelópolis/SP & $-20,184742$ & $\begin{array}{c}- \\
48,1032023\end{array}$ & $\begin{array}{l}\text { Archivo } \\
\text { de audio }\end{array}$ \\
\hline $\begin{array}{l}\text { Myiozetetes } \\
\text { cayanensis }\end{array}$ & erythropterus & 4 & 1001510 & WK & Brasil & Minas Gerais & Rio Piracicaba/MG & $-19,971256$ & $-43,149615$ & $\begin{array}{l}\text { Archivo } \\
\text { de audio }\end{array}$ \\
\hline $\begin{array}{l}\text { Myiozetetes } \\
\text { cayanensis }\end{array}$ & cayanensis & 3 & 1004367 & WK & Brasil & São Paulo & Miguelópolis/SP & $-20,184742$ & $\begin{array}{c}- \\
48,1032023\end{array}$ & $\begin{array}{l}\text { Archivo } \\
\text { de audio }\end{array}$ \\
\hline $\begin{array}{l}\text { Myiozetetes } \\
\text { cayanensis }\end{array}$ & cayanensis & 3 & 1017922 & WK & Brasil & Acre & Rio Branco/AC & $-10,067291$ & $\begin{array}{c}- \\
68,3192194\end{array}$ & $\begin{array}{l}\text { Archivo } \\
\text { de audio }\end{array}$ \\
\hline $\begin{array}{l}\text { Myiozetetes } \\
\text { cayanensis }\end{array}$ & cayanensis & 3 & 1020153 & WK & Brasil & Acre & Xapuri/AC & $-10,563501$ & $\begin{array}{c}- \\
68,5774728\end{array}$ & $\begin{array}{l}\text { Archivo } \\
\text { de audio }\end{array}$ \\
\hline $\begin{array}{c}\text { Myiozetetes } \\
\text { cayanensis }\end{array}$ & cayanensis & 3 & 1020317 & WK & Brasil & Acre & Rio Branco/AC & $-10,067291$ & $\begin{array}{c}- \\
68,3192194\end{array}$ & $\begin{array}{l}\text { Archivo } \\
\text { de audio }\end{array}$ \\
\hline
\end{tabular}




\begin{tabular}{|c|c|c|c|c|c|c|c|c|c|c|}
\hline $\begin{array}{l}\text { Myiozetetes } \\
\text { cayanensis }\end{array}$ & cayanensis & 3 & 1021920 & WK & Brasil & Acre & Xapuri/AC & $-10,563501$ & $\begin{array}{c}- \\
68,5774728\end{array}$ & $\begin{array}{l}\text { Archivo } \\
\text { de audio }\end{array}$ \\
\hline $\begin{array}{l}\text { Myiozetetes } \\
\text { cayanensis }\end{array}$ & cayanensis & 3 & 1026286 & WK & Brasil & Goiás & Jaraguá/GO & $-15,704836$ & $\begin{array}{c}- \\
49,3849404\end{array}$ & $\begin{array}{l}\text { Archivo } \\
\text { de audio }\end{array}$ \\
\hline $\begin{array}{l}\text { Myiozetetes } \\
\text { cayanensis }\end{array}$ & cayanensis & 3 & 1027335 & WK & Brasil & Mato Grosso & Apiacás/MT & $-8,7529015$ & $\begin{array}{c}- \\
57,8272477\end{array}$ & $\begin{array}{l}\text { Archivo } \\
\text { de audio }\end{array}$ \\
\hline $\begin{array}{l}\text { Myiozetetes } \\
\text { cayanensis }\end{array}$ & cayanensis & 3 & 1044189 & WK & Brasil & Mato Grosso & Rosário Oeste/MT & $-14,662774$ & $\begin{array}{c}- \\
55,8277569\end{array}$ & $\begin{array}{l}\text { Archivo } \\
\text { de audio }\end{array}$ \\
\hline $\begin{array}{l}\text { Myiozetetes } \\
\text { cayanensis }\end{array}$ & cayanensis & 3 & 1045263 & WK & Brasil & Mato Grosso & Rosário Oeste/MT & $-14,662774$ & $\begin{array}{c}- \\
55,8277569\end{array}$ & $\begin{array}{l}\text { Archivo } \\
\text { de audio }\end{array}$ \\
\hline $\begin{array}{l}\text { Myiozetetes } \\
\text { cayanensis }\end{array}$ & cayanensis & 3 & 1058476 & WK & Brasil & Amazonas & $\begin{array}{c}\text { Presidente } \\
\text { Figueiredo/AM }\end{array}$ & $-1,3720793$ & 59,9884392 & $\begin{array}{l}\text { Archivo } \\
\text { de audio }\end{array}$ \\
\hline $\begin{array}{l}\text { Myiozetetes } \\
\text { cayanensis }\end{array}$ & cayanensis & 3 & 1066900 & WK & Brasil & São Paulo & Planalto/SP & $-21,000777$ & $\begin{array}{c}- \\
49,9356571\end{array}$ & $\begin{array}{l}\text { Archivo } \\
\text { de audio }\end{array}$ \\
\hline $\begin{array}{l}\text { Myiozetetes } \\
\text { cayanensis }\end{array}$ & cayanensis & 3 & 1069629 & WK & Brasil & Maranhão & Barra do Corda/MA & $-5,6537886$ & $\begin{array}{c}- \\
45,2541548\end{array}$ & $\begin{array}{l}\text { Archivo } \\
\text { de audio }\end{array}$ \\
\hline $\begin{array}{l}\text { Myiozetetes } \\
\text { cayanensis }\end{array}$ & cayanensis & 3 & 1071968 & WK & Brasil & $\begin{array}{l}\text { Mato Grosso do } \\
\text { Sul }\end{array}$ & Três Lagoas/MS & $-20,438274$ & $\begin{array}{c}- \\
52,2110981\end{array}$ & $\begin{array}{l}\text { Archivo } \\
\text { de audio }\end{array}$ \\
\hline $\begin{array}{l}\text { Myiozetetes } \\
\text { cayanensis }\end{array}$ & cayanensis & 3 & 1071969 & WK & Brasil & $\begin{array}{l}\text { Mato Grosso do } \\
\text { Sul }\end{array}$ & Três Lagoas/MS & $-20,438274$ & $\begin{array}{c}- \\
52,2110981\end{array}$ & $\begin{array}{l}\text { Archivo } \\
\text { de audio }\end{array}$ \\
\hline $\begin{array}{l}\text { Myiozetetes } \\
\text { cayanensis }\end{array}$ & cayanensis & 3 & 1071970 & WK & Brasil & $\begin{array}{l}\text { Mato Grosso do } \\
\text { Sul }\end{array}$ & Três Lagoas/MS & $-20,438274$ & $\begin{array}{c}- \\
52,2110981\end{array}$ & $\begin{array}{l}\text { Archivo } \\
\text { de audio }\end{array}$ \\
\hline $\begin{array}{l}\text { Myiozetetes } \\
\text { cayanensis }\end{array}$ & cayanensis & 3 & 1121858 & WK & Brasil & Amazonas & Apuí/AM & $-7,7628722$ & $\begin{array}{c}- \\
59,4278823\end{array}$ & $\begin{array}{l}\text { Archivo } \\
\text { de audio }\end{array}$ \\
\hline $\begin{array}{l}\text { Myiozetetes } \\
\text { cayanensis }\end{array}$ & cayanensis & 3 & 1131207 & WK & Brasil & Rondônia & Cabixi/RO & $-13,450123$ & $\begin{array}{c}- \\
60,6293352\end{array}$ & $\begin{array}{l}\text { Archivo } \\
\text { de audio }\end{array}$ \\
\hline $\begin{array}{l}\text { Myiozetetes } \\
\text { cayanensis }\end{array}$ & cayanensis & 3 & 1131240 & WK & Brasil & Rondônia & Cabixi/RO & $-13,450123$ & 60,6293352 & $\begin{array}{l}\text { Archivo } \\
\text { de audio }\end{array}$ \\
\hline $\begin{array}{l}\text { Myiozetetes } \\
\text { cayanensis }\end{array}$ & erythropterus & 4 & 1138922 & WK & Brasil & Minas Gerais & Prados/MG & $-21,057568$ & $-44,079945$ & $\begin{array}{l}\text { Archivo } \\
\text { de audio }\end{array}$ \\
\hline $\begin{array}{l}\text { Myiozetetes } \\
\text { cayanensis }\end{array}$ & cayanensis & 3 & 1154534 & WK & Brasil & Distrito Federal & Brasília/DF & $-15,780741$ & 47,7967907 & $\begin{array}{l}\text { Archivo } \\
\text { de audio }\end{array}$ \\
\hline $\begin{array}{l}\text { Myiozetetes } \\
\text { cayanensis }\end{array}$ & cayanensis & 3 & 1189275 & WK & Brasil & Pará & Paragominas/PA & $-3,2028794$ & $\begin{array}{c}- \\
47,6024228\end{array}$ & $\begin{array}{l}\text { Archivo } \\
\text { de audio }\end{array}$ \\
\hline $\begin{array}{l}\text { Myiozetetes } \\
\text { cayanensis }\end{array}$ & cayanensis & 3 & 1197378 & WK & Brasil & Pará & Jacareacanga/PA & $-7,4449748$ & $\begin{array}{c}- \\
57,3022118\end{array}$ & $\begin{array}{l}\text { Archivo } \\
\text { de audio }\end{array}$ \\
\hline $\begin{array}{l}\text { Myiozetetes } \\
\text { cayanensis }\end{array}$ & erythropterus & 4 & 1201579 & WK & Brasil & Minas Gerais & Piranga/MG & $-20,68389$ & $-43,296731$ & $\begin{array}{l}\text { Archivo } \\
\text { de audio }\end{array}$ \\
\hline $\begin{array}{l}\text { Myiozetetes } \\
\text { cayanensis }\end{array}$ & cayanensis & 3 & 1204652 & WK & Brasil & São Paulo & Barretos/SP & $-20,513609$ & $-48,651553$ & $\begin{array}{l}\text { Archivo } \\
\text { de audio }\end{array}$ \\
\hline $\begin{array}{l}\text { Myiozetetes } \\
\text { cayanensis }\end{array}$ & cayanensis & 3 & 1208036 & WK & Brasil & Minas Gerais & $\begin{array}{c}\text { Conceição das } \\
\text { Alagoas/MG }\end{array}$ & $-19,959005$ & $\begin{array}{c}- \\
48,3608266\end{array}$ & $\begin{array}{l}\text { Archivo } \\
\text { de audio }\end{array}$ \\
\hline $\begin{array}{l}\text { Myiozetetes } \\
\text { cayanensis }\end{array}$ & cayanensis & 3 & 1209814 & WK & Brasil & Minas Gerais & $\begin{array}{l}\text { Conceição das } \\
\text { Alagoas/MG }\end{array}$ & $-19,959005$ & $\begin{array}{c}- \\
48,3608266\end{array}$ & $\begin{array}{l}\text { Archivo } \\
\text { de audio }\end{array}$ \\
\hline $\begin{array}{l}\text { Myiozetetes } \\
\text { cayanensis }\end{array}$ & cayanensis & 3 & 1214471 & WK & Brasil & $\begin{array}{l}\text { Mato Grosso do } \\
\text { Sul }\end{array}$ & Campo Grande/MS & $-20,912673$ & $\begin{array}{c}- \\
54,2493924\end{array}$ & $\begin{array}{l}\text { Archivo } \\
\text { de audio }\end{array}$ \\
\hline $\begin{array}{l}\text { Myiozetetes } \\
\text { cayanensis }\end{array}$ & cayanensis & 3 & 1220749 & WK & Brasil & Pará & Santarém/PA & $-2,8289151$ & $\begin{array}{c}- \\
55,0952449\end{array}$ & $\begin{array}{l}\text { Archivo } \\
\text { de audio }\end{array}$ \\
\hline $\begin{array}{l}\text { Myiozetetes } \\
\text { cayanensis }\end{array}$ & cayanensis & 3 & 1233223 & WK & Brasil & Tocantins & Babaçulândia/TO & $-7,1784066$ & $\begin{array}{c}- \\
47,8771722\end{array}$ & $\begin{array}{l}\text { Archivo } \\
\text { de audio }\end{array}$ \\
\hline $\begin{array}{l}\text { Myiozetetes } \\
\text { cayanensis }\end{array}$ & cayanensis & 3 & 1238128 & WK & Brasil & Mato Grosso & Apiacás/MT & $-8,7529015$ & $\begin{array}{c}- \\
57,8272477\end{array}$ & $\begin{array}{l}\text { Archivo } \\
\text { de audio }\end{array}$ \\
\hline $\begin{array}{l}\text { Myiozetetes } \\
\text { cayanensis }\end{array}$ & erythropterus & 4 & 1243586 & WK & Brasil & Minas Gerais & Piranga/MG & $-20,68389$ & $-43,296731$ & $\begin{array}{l}\text { Archivo } \\
\text { de audio }\end{array}$ \\
\hline $\begin{array}{l}\text { Myiozetetes } \\
\text { cayanensis }\end{array}$ & cayanensis & 3 & 1256191 & WK & Brasil & Pará & Prainha/PA & $-2,1796108$ & 53,6480702 & $\begin{array}{l}\text { Archivo } \\
\text { de audio }\end{array}$ \\
\hline $\begin{array}{l}\text { Myiozetetes } \\
\text { cayanensis }\end{array}$ & cayanensis & 3 & 1257149 & WK & Brasil & Pará & Belém/PA & $-1,2405368$ & $\begin{array}{c}- \\
48,4591789\end{array}$ & $\begin{array}{l}\text { Archivo } \\
\text { de audio }\end{array}$ \\
\hline $\begin{array}{l}\text { Myiozetetes } \\
\text { cayanensis }\end{array}$ & cayanensis & 3 & 1258246 & WK & Brasil & Maranhão & Santa Luzia/MA & $-4,2844556$ & $\begin{array}{c}- \\
45,8742734\end{array}$ & $\begin{array}{l}\text { Archivo } \\
\text { de audio }\end{array}$ \\
\hline $\begin{array}{l}\text { Myiozetetes } \\
\text { cayanensis }\end{array}$ & cayanensis & 3 & 1272169 & WK & Brasil & São Paulo & Itapira/SP & $-22,431174$ & $\begin{array}{c}- \\
46,7715907\end{array}$ & $\begin{array}{l}\text { Archivo } \\
\text { de audio }\end{array}$ \\
\hline $\begin{array}{l}\text { Myiozetetes } \\
\text { cayanensis }\end{array}$ & cayanensis & 3 & 1294341 & WK & Brasil & $\begin{array}{l}\text { Mato Grosso do } \\
\text { Sul }\end{array}$ & Corumbá/MS & $-18,730961$ & $\begin{array}{c}- \\
56,7232711\end{array}$ & $\begin{array}{l}\text { Archivo } \\
\text { de audio }\end{array}$ \\
\hline $\begin{array}{l}\text { Myiozetetes } \\
\text { cayanensis }\end{array}$ & cayanensis & 3 & 1330478 & WK & Brasil & Acre & Rio Branco/AC & $-10,067291$ & $\begin{array}{c}- \\
68,3192194\end{array}$ & $\begin{array}{l}\text { Archivo } \\
\text { de audio }\end{array}$ \\
\hline $\begin{array}{l}\text { Myiozetetes } \\
\text { cayanensis }\end{array}$ & cayanensis & 3 & 1344380 & WK & Brasil & Goiás & $\begin{array}{c}\text { Terezópolis de } \\
\text { Goiás/GO }\end{array}$ & $-16,442571$ & $\begin{array}{c}- \\
49,0739285\end{array}$ & $\begin{array}{l}\text { Archivo } \\
\text { de audio }\end{array}$ \\
\hline
\end{tabular}




\begin{tabular}{|c|c|c|c|c|c|c|c|c|c|c|}
\hline $\begin{array}{l}\text { Myiozetetes } \\
\text { cayanensis }\end{array}$ & erythropterus & 4 & 1344622 & WK & Brasil & Minas Gerais & Prados/MG & $-21,057568$ & $-44,079945$ & $\begin{array}{l}\text { Archivo } \\
\text { de audio }\end{array}$ \\
\hline $\begin{array}{l}\text { Myiozetetes } \\
\text { cayanensis }\end{array}$ & cayanensis & 3 & 1345371 & WK & Brasil & Goiás & Nova Crixás/GO & $-14,133547$ & $-50,561348$ & $\begin{array}{l}\text { Archivo } \\
\text { de audio }\end{array}$ \\
\hline $\begin{array}{l}\text { Myiozetetes } \\
\text { cayanensis }\end{array}$ & cayanensis & 3 & 1356035 & WK & Brasil & Mato Grosso & Rosário Oeste/MT & $-14,662774$ & $\begin{array}{c}- \\
55,8277569\end{array}$ & $\begin{array}{l}\text { Archivo } \\
\text { de audio }\end{array}$ \\
\hline $\begin{array}{l}\text { Myiozetetes } \\
\text { cayanensis }\end{array}$ & cayanensis & 3 & 1366785 & WK & Brasil & Acre & $\begin{array}{c}\text { Senador } \\
\text { Guiomard/AC }\end{array}$ & $-10,010938$ & $\begin{array}{c}- \\
67,4088237\end{array}$ & $\begin{array}{l}\text { Archivo } \\
\text { de audio }\end{array}$ \\
\hline $\begin{array}{l}\text { Myiozetetes } \\
\text { cayanensis }\end{array}$ & erythropterus & 4 & 1404914 & WK & Brasil & Espírito Santo & Santa Teresa/ES & $-19,876807$ & $\begin{array}{c}- \\
40,6324805\end{array}$ & $\begin{array}{l}\text { Archivo } \\
\text { de audio }\end{array}$ \\
\hline $\begin{array}{l}\text { Myiozetetes } \\
\text { cayanensis }\end{array}$ & cayanensis & 3 & 1411341 & WK & Brasil & Goiás & $\begin{array}{l}\text { Terezópolis de } \\
\text { Goiás/GO }\end{array}$ & $-16,442571$ & $\begin{array}{c}- \\
49,0739285\end{array}$ & $\begin{array}{l}\text { Archivo } \\
\text { de audio }\end{array}$ \\
\hline $\begin{array}{l}\text { Myiozetetes } \\
\text { cayanensis }\end{array}$ & cayanensis & 3 & 1432458 & WK & Brasil & Goiás & São Domingos/GO & $-13,536621$ & $\begin{array}{c}- \\
46,4864452\end{array}$ & $\begin{array}{l}\text { Archivo } \\
\text { de audio }\end{array}$ \\
\hline $\begin{array}{l}\text { Myiozetetes } \\
\text { cayanensis }\end{array}$ & cayanensis & 3 & 1438765 & WK & Brasil & Amazonas & Manaus/AM & $-2,625919$ & $\begin{array}{c}- \\
60,2590208\end{array}$ & $\begin{array}{l}\text { Archivo } \\
\text { de audio }\end{array}$ \\
\hline $\begin{array}{l}\text { Myiozetetes } \\
\text { cayanensis }\end{array}$ & cayanensis & 3 & 1448045 & WK & Brasil & $\begin{array}{l}\text { Mato Grosso do } \\
\text { Sul }\end{array}$ & Ivinhema/MS & $-22,370951$ & $\begin{array}{c}- \\
53,7985963\end{array}$ & $\begin{array}{l}\text { Archivo } \\
\text { de audio }\end{array}$ \\
\hline $\begin{array}{l}\text { Myiozetetes } \\
\text { cayanensis }\end{array}$ & erythropterus & 4 & 1449594 & WK & Brasil & Minas Gerais & Caputira/MG & $-20,175974$ & $\begin{array}{c}- \\
42,2524008\end{array}$ & $\begin{array}{l}\text { Archivo } \\
\text { de audio }\end{array}$ \\
\hline $\begin{array}{l}\text { Myiozetetes } \\
\text { cayanensis }\end{array}$ & cayanensis & 3 & 1456982 & WK & Brasil & Distrito Federal & Brasília/DF & $-15,780741$ & $\begin{array}{c}- \\
47,7967907\end{array}$ & $\begin{array}{l}\text { Archivo } \\
\text { de audio }\end{array}$ \\
\hline $\begin{array}{l}\text { Myiozetetes } \\
\text { cayanensis }\end{array}$ & erythropterus & 4 & 1459838 & WK & Brasil & Minas Gerais & $\begin{array}{l}\text { Senhora de } \\
\text { Oliveira/MG }\end{array}$ & $-20,792733$ & $-43,343401$ & $\begin{array}{l}\text { Archivo } \\
\text { de audio }\end{array}$ \\
\hline $\begin{array}{l}\text { Myiozetetes } \\
\text { cayanensis }\end{array}$ & cayanensis & 3 & 1461579 & WK & Brasil & Pará & Santarém/PA & $-2,8289151$ & $\begin{array}{c}- \\
55,0952449\end{array}$ & $\begin{array}{l}\text { Archivo } \\
\text { de audio }\end{array}$ \\
\hline $\begin{array}{l}\text { Myiozetetes } \\
\text { cayanensis }\end{array}$ & erythropterus & 4 & 1467548 & WK & Brasil & Espírito Santo & Itarana/ES & $-19,94903$ & $\begin{array}{c}- \\
40,8881887\end{array}$ & $\begin{array}{l}\text { Archivo } \\
\text { de audio }\end{array}$ \\
\hline $\begin{array}{l}\text { Myiozetetes } \\
\text { cayanensis }\end{array}$ & erythropterus & 4 & 1516812 & WK & Brasil & Minas Gerais & Prados/MG & $-21,057568$ & $-44,079945$ & $\begin{array}{l}\text { Archivo } \\
\text { de audio }\end{array}$ \\
\hline $\begin{array}{l}\text { Myiozetetes } \\
\text { cayanensis }\end{array}$ & cayanensis & 3 & 1521160 & WK & Brasil & São Paulo & Guapiaçu/SP & $-20,742678$ & $\begin{array}{c}- \\
49,1896805\end{array}$ & $\begin{array}{l}\text { Archivo } \\
\text { de audio }\end{array}$ \\
\hline $\begin{array}{l}\text { Myiozetetes } \\
\text { cayanensis }\end{array}$ & cayanensis & 3 & 1530099 & WK & Brasil & Goiás & Caiapônia/GO & $-17,007212$ & $\begin{array}{c}- \\
51,8152413\end{array}$ & $\begin{array}{l}\text { Archivo } \\
\text { de audio }\end{array}$ \\
\hline $\begin{array}{l}\text { Myiozetetes } \\
\text { cayanensis }\end{array}$ & cayanensis & 3 & 1532281 & WK & Brasil & Amazonas & Manaus/AM & $-2,625919$ & $\begin{array}{c}- \\
60,2590208\end{array}$ & $\begin{array}{l}\text { Archivo } \\
\text { de audio }\end{array}$ \\
\hline $\begin{array}{l}\text { Myiozetetes } \\
\text { cayanensis }\end{array}$ & erythropterus & 4 & 1554273 & WK & Brasil & Río de Janeiro & Nova Friburgo/RJ & $-22,288644$ & $-42,534183$ & $\begin{array}{l}\text { Archivo } \\
\text { de audio }\end{array}$ \\
\hline $\begin{array}{l}\text { Myiozetetes } \\
\text { cayanensis }\end{array}$ & erythropterus & 4 & 1575264 & WK & Brasil & Minas Gerais & Antônio Dias/MG & $-19,557906$ & $\begin{array}{c}- \\
42,8824248\end{array}$ & $\begin{array}{l}\text { Archivo } \\
\text { de audio }\end{array}$ \\
\hline $\begin{array}{l}\text { Myiozetetes } \\
\text { cayanensis }\end{array}$ & erythropterus & 4 & 1591042 & WK & Brasil & Bahía & Boa Nova/BA & $-14,364257$ & $\begin{array}{c}- \\
40,2324546\end{array}$ & $\begin{array}{l}\text { Archivo } \\
\text { de audio }\end{array}$ \\
\hline $\begin{array}{l}\text { Myiozetetes } \\
\text { cayanensis }\end{array}$ & erythropterus & 4 & 1592123 & WK & Brasil & Minas Gerais & Ouro Preto/MG & $-20,386474$ & $-43,504737$ & $\begin{array}{l}\text { Archivo } \\
\text { de audio }\end{array}$ \\
\hline $\begin{array}{l}\text { Myiozetetes } \\
\text { cayanensis }\end{array}$ & cayanensis & 3 & 1609727 & WK & Brasil & $\begin{array}{l}\text { Mato Grosso do } \\
\text { Sul }\end{array}$ & Três Lagoas/MS & $-20,438274$ & $\begin{array}{c}- \\
52,2110981\end{array}$ & $\begin{array}{l}\text { Archivo } \\
\text { de audio }\end{array}$ \\
\hline $\begin{array}{l}\text { Myiozetetes } \\
\text { cayanensis }\end{array}$ & cayanensis & 3 & 1609728 & WK & Brasil & $\begin{array}{l}\text { Mato Grosso do } \\
\text { Sul }\end{array}$ & Três Lagoas/MS & $-20,438274$ & $\begin{array}{c}- \\
52,2110981\end{array}$ & $\begin{array}{l}\text { Archivo } \\
\text { de audio }\end{array}$ \\
\hline $\begin{array}{l}\text { Myiozetetes } \\
\text { cayanensis }\end{array}$ & cayanensis & 3 & 1609729 & WK & Brasil & $\begin{array}{l}\text { Mato Grosso do } \\
\text { Sul }\end{array}$ & Três Lagoas/MS & $-20,438274$ & $\begin{array}{c}- \\
52,2110981\end{array}$ & $\begin{array}{l}\text { Archivo } \\
\text { de audio }\end{array}$ \\
\hline $\begin{array}{l}\text { Myiozetetes } \\
\text { cayanensis }\end{array}$ & erythropterus & 4 & 1615764 & WK & Brasil & Minas Gerais & Caputira/MG & $-20,175974$ & $\begin{array}{c}- \\
42,2524008\end{array}$ & $\begin{array}{l}\text { Archivo } \\
\text { de audio }\end{array}$ \\
\hline $\begin{array}{l}\text { Myiozetetes } \\
\text { cayanensis }\end{array}$ & erythropterus & 4 & 1627313 & WK & Brasil & Minas Gerais & Piranga/MG & $-20,68389$ & $-43,296731$ & $\begin{array}{l}\text { Archivo } \\
\text { de audio }\end{array}$ \\
\hline $\begin{array}{l}\text { Myiozetetes } \\
\text { cayanensis }\end{array}$ & cayanensis & 3 & 1639752 & WK & Brasil & São Paulo & Pirajuí/SP & $-21,956588$ & $\begin{array}{c}- \\
49,4131559\end{array}$ & $\begin{array}{l}\text { Archivo } \\
\text { de audio }\end{array}$ \\
\hline $\begin{array}{l}\text { Myiozetetes } \\
\text { cayanensis }\end{array}$ & erythropterus & 4 & 1655851 & WK & Brasil & Minas Gerais & Guaraciaba/MG & $-20,571981$ & $-43,009998$ & $\begin{array}{l}\text { Archivo } \\
\text { de audio }\end{array}$ \\
\hline $\begin{array}{l}\text { Myiozetetes } \\
\text { cayanensis }\end{array}$ & erythropterus & 4 & 1659206 & WK & Brasil & Minas Gerais & Ouro Preto/MG & $-20,386474$ & $-43,504737$ & $\begin{array}{l}\text { Archivo } \\
\text { de audio }\end{array}$ \\
\hline $\begin{array}{l}\text { Myiozetetes } \\
\text { cayanensis }\end{array}$ & erythropterus & 4 & 1666565 & WK & Brasil & Minas Gerais & Catas Altas/MG & $-20,075438$ & $-43,408135$ & $\begin{array}{l}\text { Archivo } \\
\text { de audio }\end{array}$ \\
\hline $\begin{array}{l}\text { Myiozetetes } \\
\text { cayanensis }\end{array}$ & cayanensis & 3 & 1684971 & WK & Brasil & Mato Grosso & $\begin{array}{c}\text { Barra do } \\
\text { Bugres/MT }\end{array}$ & $-15,043295$ & $\begin{array}{c}- \\
57,6917836\end{array}$ & $\begin{array}{l}\text { Archivo } \\
\text { de audio }\end{array}$ \\
\hline $\begin{array}{l}\text { Myiozetetes } \\
\text { cayanensis }\end{array}$ & cayanensis & 3 & 1704606 & WK & Brasil & Mato Grosso & Paranaíta/MT & $-9,5943941$ & $\begin{array}{c}- \\
56,6821428\end{array}$ & $\begin{array}{l}\text { Archivo } \\
\text { de audio }\end{array}$ \\
\hline $\begin{array}{l}\text { Myiozetetes } \\
\text { cayanensis }\end{array}$ & cayanensis & 3 & 1708514 & WK & Brasil & Río de Janeiro & Paraty/RJ & $-23,148698$ & $\begin{array}{c}- \\
44,7057584\end{array}$ & $\begin{array}{l}\text { Archivo } \\
\text { de audio }\end{array}$ \\
\hline
\end{tabular}




\begin{tabular}{|c|c|c|c|c|c|c|c|c|c|c|}
\hline $\begin{array}{l}\text { Myiozetetes } \\
\text { cayanensis }\end{array}$ & cayanensis & 3 & 1735810 & WK & Brasil & Mato Grosso & Poconé/MT & $-16,78447$ & $\begin{array}{c}- \\
56,9485054\end{array}$ & $\begin{array}{l}\text { Archivo } \\
\text { de audio }\end{array}$ \\
\hline $\begin{array}{l}\text { Myiozetetes } \\
\text { cayanensis }\end{array}$ & erythropterus & 4 & 1767207 & WK & Brasil & Minas Gerais & Serro/MG & $-18,534932$ & $\begin{array}{c}- \\
43,4059307\end{array}$ & $\begin{array}{l}\text { Archivo } \\
\text { de audio }\end{array}$ \\
\hline $\begin{array}{l}\text { Myiozetetes } \\
\text { cayanensis }\end{array}$ & erythropterus & 4 & 1767220 & WK & Brasil & Minas Gerais & Serro/MG & $-18,534932$ & $\begin{array}{c}- \\
43,4059307\end{array}$ & $\begin{array}{l}\text { Archivo } \\
\text { de audio }\end{array}$ \\
\hline $\begin{array}{l}\text { Myiozetetes } \\
\text { cayanensis }\end{array}$ & cayanensis & 3 & 1770063 & WK & Brasil & Goiás & Rio Verde/GO & $-17,739135$ & $\begin{array}{c}- \\
51,0378468\end{array}$ & $\begin{array}{l}\text { Archivo } \\
\text { de audio }\end{array}$ \\
\hline $\begin{array}{l}\text { Myiozetetes } \\
\text { cayanensis }\end{array}$ & cayanensis & 3 & 1777560 & WK & Brasil & Pará & Parauapebas/PA & $-6,1544368$ & $\begin{array}{c}- \\
50,4860742\end{array}$ & $\begin{array}{l}\text { Archivo } \\
\text { de audio }\end{array}$ \\
\hline $\begin{array}{l}\text { Myiozetetes } \\
\text { cayanensis }\end{array}$ & cayanensis & 3 & 1786968 & WK & Brasil & Amazonas & $\begin{array}{c}\text { Presidente } \\
\text { Figueiredo/AM }\end{array}$ & $-1,3720793$ & 59,9884392 & $\begin{array}{l}\text { Archivo } \\
\text { de audio }\end{array}$ \\
\hline $\begin{array}{l}\text { Myiozetetes } \\
\text { cayanensis }\end{array}$ & cayanensis & 3 & 1790156 & WK & Brasil & Río de Janeiro & Angra dos Reis/RJ & $-23,00667$ & $-44,31806$ & $\begin{array}{l}\text { Archivo } \\
\text { de audio }\end{array}$ \\
\hline $\begin{array}{l}\text { Myiozetetes } \\
\text { cayanensis }\end{array}$ & cayanensis & 3 & 1797213 & WK & Brasil & Maranhão & Caxias/MA & $-4,871664$ & $\begin{array}{c}- \\
43,2967965\end{array}$ & $\begin{array}{l}\text { Archivo } \\
\text { de audio }\end{array}$ \\
\hline $\begin{array}{l}\text { Myiozetetes } \\
\text { cayanensis }\end{array}$ & cayanensis & 3 & 1798615 & WK & Brasil & São Paulo & Timburi/SP & $-23,194877$ & $\begin{array}{c}- \\
49,6100158\end{array}$ & $\begin{array}{l}\text { Archivo } \\
\text { de audio }\end{array}$ \\
\hline $\begin{array}{l}\text { Myiozetetes } \\
\text { cayanensis }\end{array}$ & cayanensis & 3 & 1805533 & WK & Brasil & Mato Grosso & Alta Floresta/MT & $-10,055008$ & 56,3660622 & $\begin{array}{l}\text { Archivo } \\
\text { de audio }\end{array}$ \\
\hline $\begin{array}{l}\text { Myiozetetes } \\
\text { cayanensis }\end{array}$ & cayanensis & 3 & 1840380 & WK & Brasil & Piauí & Campo Maior/PI & $-4,8774848$ & $\begin{array}{c}- \\
42,1467673\end{array}$ & $\begin{array}{l}\text { Archivo } \\
\text { de audio }\end{array}$ \\
\hline $\begin{array}{l}\text { Myiozetetes } \\
\text { cayanensis }\end{array}$ & cayanensis & 3 & 1843196 & WK & Brasil & Goiás & Goiânia/GO & $-16,642076$ & $\begin{array}{c}- \\
49,2750915\end{array}$ & $\begin{array}{l}\text { Archivo } \\
\text { de audio }\end{array}$ \\
\hline $\begin{array}{l}\text { Myiozetetes } \\
\text { cayanensis }\end{array}$ & cayanensis & 3 & 1860614 & WK & Brasil & Goiás & Sanclerlândia/GO & $-16,281869$ & $\begin{array}{c}- \\
50,3716197\end{array}$ & $\begin{array}{l}\text { Archivo } \\
\text { de audio }\end{array}$ \\
\hline $\begin{array}{l}\text { Myiozetetes } \\
\text { cayanensis }\end{array}$ & cayanensis & 3 & 1878981 & WK & Brasil & Goiás & $\begin{array}{c}\text { Cocalzinho de } \\
\text { Goiás/GO }\end{array}$ & $-15,655204$ & $\begin{array}{c}- \\
48,6024671\end{array}$ & $\begin{array}{l}\text { Archivo } \\
\text { de audio }\end{array}$ \\
\hline $\begin{array}{l}\text { Myiozetetes } \\
\text { cayanensis }\end{array}$ & cayanensis & 3 & 1893874 & WK & Brasil & Distrito Federal & Brasília/DF & $-15,780741$ & $\begin{array}{c}- \\
47,7967907\end{array}$ & $\begin{array}{l}\text { Archivo } \\
\text { de audio }\end{array}$ \\
\hline $\begin{array}{l}\text { Myiozetetes } \\
\text { cayanensis }\end{array}$ & cayanensis & 3 & 1921181 & WK & Brasil & Goiás & Niquelândia/GO & $-14,498258$ & $\begin{array}{c}- \\
48,4012427\end{array}$ & $\begin{array}{l}\text { Archivo } \\
\text { de audio }\end{array}$ \\
\hline $\begin{array}{l}\text { Myiozetetes } \\
\text { cayanensis }\end{array}$ & cayanensis & 3 & 1929456 & WK & Brasil & Goiás & Caldazinha/GO & $-16,735222$ & $\begin{array}{c}- \\
48,9515444\end{array}$ & $\begin{array}{l}\text { Archivo } \\
\text { de audio }\end{array}$ \\
\hline $\begin{array}{l}\text { Myiozetetes } \\
\text { cayanensis }\end{array}$ & cayanensis & 3 & 1942966 & WK & Brasil & $\begin{array}{l}\text { Mato Grosso do } \\
\text { Sul }\end{array}$ & Campo Grande/MS & $-20,912673$ & $\begin{array}{c}- \\
54,2493924\end{array}$ & $\begin{array}{l}\text { Archivo } \\
\text { de audio }\end{array}$ \\
\hline $\begin{array}{l}\text { Myiozetetes } \\
\text { cayanensis }\end{array}$ & cayanensis & 3 & 1943103 & WK & Brasil & Minas Gerais & $\begin{array}{l}\text { São Sebastião do } \\
\text { Paraíso/MG }\end{array}$ & $-20,923655$ & $-47,005879$ & $\begin{array}{l}\text { Archivo } \\
\text { de audio }\end{array}$ \\
\hline $\begin{array}{l}\text { Myiozetetes } \\
\text { cayanensis }\end{array}$ & cayanensis & 3 & 1962656 & WK & Brasil & Tocantins & Goiatins/TO & $-8,0934534$ & 47,4807736 & $\begin{array}{l}\text { Archivo } \\
\text { de audio }\end{array}$ \\
\hline $\begin{array}{l}\text { Myiozetetes } \\
\text { cayanensis }\end{array}$ & cayanensis & 3 & 1963339 & WK & Brasil & Tocantins & Goiatins/TO & $-8,0934534$ & $\begin{array}{c}- \\
47,4807736\end{array}$ & $\begin{array}{l}\text { Archivo } \\
\text { de audio }\end{array}$ \\
\hline $\begin{array}{l}\text { Myiozetetes } \\
\text { cayanensis }\end{array}$ & cayanensis & 3 & 1980888 & WK & Brasil & Goiás & Niquelândia/GO & $-14,498258$ & $\begin{array}{c}- \\
48,4012427\end{array}$ & $\begin{array}{l}\text { Archivo } \\
\text { de audio }\end{array}$ \\
\hline $\begin{array}{l}\text { Myiozetetes } \\
\text { cayanensis }\end{array}$ & cayanensis & 3 & 2014825 & WK & Brasil & Tocantins & Itacajá/TO & $-8,5917713$ & $\begin{array}{c}- \\
47,6305039\end{array}$ & $\begin{array}{l}\text { Archivo } \\
\text { de audio }\end{array}$ \\
\hline $\begin{array}{l}\text { Myiozetetes } \\
\text { cayanensis }\end{array}$ & cayanensis & 3 & 2034013 & WK & Brasil & Mato Grosso & Sinop/MT & $-11,773518$ & $\begin{array}{c}- \\
55,4551592\end{array}$ & $\begin{array}{l}\text { Archivo } \\
\text { de audio }\end{array}$ \\
\hline $\begin{array}{l}\text { Myiozetetes } \\
\text { cayanensis }\end{array}$ & cayanensis & 3 & 2042155 & WK & Brasil & Acre & Rio Branco/AC & $-10,067291$ & $\begin{array}{c}- \\
68,3192194\end{array}$ & $\begin{array}{l}\text { Archivo } \\
\text { de audio }\end{array}$ \\
\hline $\begin{array}{l}\text { Myiozetetes } \\
\text { cayanensis }\end{array}$ & erythropterus & 4 & 2048116 & WK & Brasil & Minas Gerais & Serro/MG & $-18,534932$ & $\begin{array}{c}- \\
43,4059307\end{array}$ & $\begin{array}{l}\text { Archivo } \\
\text { de audio }\end{array}$ \\
\hline $\begin{array}{l}\text { Myiozetetes } \\
\text { cayanensis }\end{array}$ & cayanensis & 3 & 2079231 & WK & Brasil & Goiás & Pirenópolis/GO & $-15,807197$ & $\begin{array}{c}- \\
49,0100159\end{array}$ & $\begin{array}{l}\text { Archivo } \\
\text { de audio }\end{array}$ \\
\hline $\begin{array}{l}\text { Myiozetetes } \\
\text { cayanensis }\end{array}$ & cayanensis & 3 & 2088163 & WK & Brasil & Maranhão & Carolina/MA & $-7,3717932$ & $\begin{array}{c}- \\
47,2033999\end{array}$ & $\begin{array}{l}\text { Archivo } \\
\text { de audio }\end{array}$ \\
\hline $\begin{array}{l}\text { Myiozetetes } \\
\text { cayanensis }\end{array}$ & cayanensis & 3 & 2106449 & WK & Brasil & São Paulo & Turmalina/SP & $-20,083148$ & $\begin{array}{c}- \\
50,4565403\end{array}$ & $\begin{array}{l}\text { Archivo } \\
\text { de audio }\end{array}$ \\
\hline $\begin{array}{l}\text { Myiozetetes } \\
\text { cayanensis }\end{array}$ & cayanensis & 3 & 2124174 & WK & Brasil & $\begin{array}{l}\text { Mato Grosso do } \\
\text { Sul }\end{array}$ & Campo Grande/MS & $-20,912673$ & $\begin{array}{c}- \\
54,2493924\end{array}$ & $\begin{array}{l}\text { Archivo } \\
\text { de audio }\end{array}$ \\
\hline $\begin{array}{l}\text { Myiozetetes } \\
\text { cayanensis }\end{array}$ & cayanensis & 3 & 2132911 & WK & Brasil & Tocantins & Guaraí/TO & $-8,7385547$ & $\begin{array}{c}- \\
48,4409762\end{array}$ & $\begin{array}{l}\text { Archivo } \\
\text { de audio }\end{array}$ \\
\hline $\begin{array}{l}\text { Myiozetetes } \\
\text { cayanensis }\end{array}$ & cayanensis & 3 & 2141114 & WK & Brasil & Mato Grosso & Rio Branco/MT & $-15,273788$ & $\begin{array}{c}- \\
58,1457838\end{array}$ & $\begin{array}{l}\text { Archivo } \\
\text { de audio }\end{array}$ \\
\hline $\begin{array}{l}\text { Myiozetetes } \\
\text { cayanensis }\end{array}$ & erythropterus & 4 & 2158454 & WK & Brasil & Minas Gerais & $\begin{array}{l}\text { Santana dos } \\
\text { Montes/MG }\end{array}$ & $-20,790302$ & $-43,691326$ & $\begin{array}{l}\text { Archivo } \\
\text { de audio }\end{array}$ \\
\hline $\begin{array}{l}\text { Myiozetetes } \\
\text { cayanensis }\end{array}$ & erythropterus & 4 & 2185521 & WK & Brasil & Minas Gerais & $\begin{array}{l}\text { Santana dos } \\
\text { Montes/MG }\end{array}$ & $-20,790302$ & $-43,691326$ & $\begin{array}{l}\text { Archivo } \\
\text { de audio }\end{array}$ \\
\hline
\end{tabular}




\begin{tabular}{|c|c|c|c|c|c|c|c|c|c|c|}
\hline $\begin{array}{l}\text { Myiozetetes } \\
\text { cayanensis }\end{array}$ & cayanensis & 3 & 2194179 & WK & Brasil & $\begin{array}{c}\text { Mato Grosso do } \\
\text { Sul }\end{array}$ & Coxim/MS & $-18,280368$ & $\begin{array}{c}- \\
54,6743747\end{array}$ & $\begin{array}{l}\text { Archivo } \\
\text { de audio }\end{array}$ \\
\hline $\begin{array}{l}\text { Myiozetetes } \\
\text { cayanensis }\end{array}$ & erythropterus & 4 & 2199235 & WK & Brasil & Minas Gerais & $\begin{array}{l}\text { Santana dos } \\
\text { Montes/MG }\end{array}$ & $-20,790302$ & $-43,691326$ & $\begin{array}{l}\text { Archivo } \\
\text { de audio }\end{array}$ \\
\hline $\begin{array}{l}\text { Myiozetetes } \\
\text { cayanensis }\end{array}$ & cayanensis & 3 & 2230846 & WK & Brasil & Mato Grosso & Alta Floresta/MT & $-10,055008$ & $\begin{array}{c}- \\
56,3660622\end{array}$ & $\begin{array}{l}\text { Archivo } \\
\text { de audio }\end{array}$ \\
\hline $\begin{array}{l}\text { Myiozetetes } \\
\text { cayanensis }\end{array}$ & cayanensis & 3 & 2234294 & WK & Brasil & Distrito Federal & Brasília/DF & $-15,780741$ & $\begin{array}{c}- \\
47,7967907\end{array}$ & $\begin{array}{l}\text { Archivo } \\
\text { de audio }\end{array}$ \\
\hline $\begin{array}{l}\text { Myiozetetes } \\
\text { cayanensis }\end{array}$ & cayanensis & 3 & 2234297 & WK & Brasil & Distrito Federal & Brasília/DF & $-15,780741$ & $\begin{array}{c}- \\
47,7967907\end{array}$ & $\begin{array}{l}\text { Archivo } \\
\text { de audio }\end{array}$ \\
\hline $\begin{array}{l}\text { Myiozetetes } \\
\text { cayanensis }\end{array}$ & erythropterus & 4 & 2284699 & WK & Brasil & Espírito Santo & Vargem Alta/ES & $-20,644973$ & $\begin{array}{c}- \\
41,0027139\end{array}$ & $\begin{array}{l}\text { Archivo } \\
\text { de audio }\end{array}$ \\
\hline $\begin{array}{l}\text { Myiozetetes } \\
\text { cayanensis }\end{array}$ & cayanensis & 3 & 2289736 & WK & Brasil & Amazonas & Manaus/AM & $-2,625919$ & $\begin{array}{c}- \\
60,2590208\end{array}$ & $\begin{array}{l}\text { Archivo } \\
\text { de audio }\end{array}$ \\
\hline $\begin{array}{l}\text { Myiozetetes } \\
\text { cayanensis }\end{array}$ & cayanensis & 3 & 2297654 & WK & Brasil & Amazonas & Manaus/AM & $-2,625919$ & $\begin{array}{c}- \\
60,2590208\end{array}$ & $\begin{array}{l}\text { Archivo } \\
\text { de audio }\end{array}$ \\
\hline $\begin{array}{l}\text { Myiozetetes } \\
\text { cayanensis }\end{array}$ & cayanensis & 3 & 2347434 & WK & Brasil & Tocantins & Figueirópolis/TO & $-12,263883$ & $\begin{array}{c}- \\
49,2827575\end{array}$ & $\begin{array}{l}\text { Archivo } \\
\text { de audio }\end{array}$ \\
\hline $\begin{array}{l}\text { Myiozetetes } \\
\text { cayanensis }\end{array}$ & cayanensis & 3 & 2373106 & WK & Brasil & Mato Grosso & Poconé/MT & $-16,78447$ & $\begin{array}{c}- \\
56,9485054\end{array}$ & $\begin{array}{l}\text { Archivo } \\
\text { de audio }\end{array}$ \\
\hline $\begin{array}{l}\text { Myiozetetes } \\
\text { cayanensis }\end{array}$ & cayanensis & 3 & 2400955 & WK & Brasil & Piauí & Castelo do Piauí/PI & $-5,2999607$ & $\begin{array}{c}- \\
41,5688869\end{array}$ & $\begin{array}{l}\text { Archivo } \\
\text { de audio }\end{array}$ \\
\hline $\begin{array}{l}\text { Myiozetetes } \\
\text { cayanensis }\end{array}$ & cayanensis & 3 & 2408563 & WK & Brasil & Goiás & Caldas Novas/GO & $-17,70872$ & $\begin{array}{c}- \\
48,6454223\end{array}$ & $\begin{array}{l}\text { Archivo } \\
\text { de audio }\end{array}$ \\
\hline $\begin{array}{l}\text { Myiozetetes } \\
\text { cayanensis }\end{array}$ & cayanensis & 3 & 2435216 & WK & Brasil & São Paulo & Dourado/SP & $-22,116567$ & $\begin{array}{c}- \\
48,3329623\end{array}$ & $\begin{array}{l}\text { Archivo } \\
\text { de audio }\end{array}$ \\
\hline $\begin{array}{l}\text { Myiozetetes } \\
\text { cayanensis }\end{array}$ & cayanensis & 3 & 2445874 & WK & Brasil & São Paulo & Cabreúva/SP & $-23,300533$ & $\begin{array}{c}- \\
47,0795288\end{array}$ & $\begin{array}{l}\text { Archivo } \\
\text { de audio }\end{array}$ \\
\hline $\begin{array}{l}\text { Myiozetetes } \\
\text { cayanensis }\end{array}$ & erythropterus & 4 & 2449199 & WK & Brasil & Espírito Santo & $\begin{array}{l}\text { Domingos } \\
\text { Martins/ES }\end{array}$ & $-20,308461$ & $\begin{array}{c}- \\
40,8461865\end{array}$ & $\begin{array}{l}\text { Archivo } \\
\text { de audio }\end{array}$ \\
\hline $\begin{array}{l}\text { Myiozetetes } \\
\text { cayanensis }\end{array}$ & cayanensis & 3 & 2450286 & WK & Brasil & Distrito Federal & Brasília/DF & $-15,780741$ & $\begin{array}{c}- \\
47,7967907\end{array}$ & $\begin{array}{l}\text { Archivo } \\
\text { de audio }\end{array}$ \\
\hline $\begin{array}{l}\text { Myiozetetes } \\
\text { cayanensis }\end{array}$ & cayanensis & 3 & 2450669 & WK & Brasil & Minas Gerais & Curvelo/MG & $-18,820364$ & $\begin{array}{c}- \\
44,4484785\end{array}$ & $\begin{array}{l}\text { Archivo } \\
\text { de audio }\end{array}$ \\
\hline $\begin{array}{l}\text { Myiozetetes } \\
\text { cayanensis }\end{array}$ & cayanensis & 3 & 2465934 & WK & Brasil & Amapá & $\begin{array}{c}\text { Pedra Branca do } \\
\text { Amaparí/AP }\end{array}$ & 1,15836321 & $\begin{array}{c}- \\
52,5233215\end{array}$ & $\begin{array}{l}\text { Archivo } \\
\text { de audio }\end{array}$ \\
\hline $\begin{array}{l}\text { Myiozetetes } \\
\text { cayanensis }\end{array}$ & cayanensis & 3 & 2509470 & WK & Brasil & $\begin{array}{c}\text { Mato Grosso do } \\
\text { Sul }\end{array}$ & Corumbá/MS & $-18,730961$ & $\begin{array}{c}- \\
56,7232711\end{array}$ & $\begin{array}{l}\text { Archivo } \\
\text { de audio }\end{array}$ \\
\hline $\begin{array}{l}\text { Myiozetetes } \\
\text { cayanensis }\end{array}$ & cayanensis & 3 & 2535675 & WK & Brasil & Minas Gerais & Uberaba/MG & $-19,580173$ & $\begin{array}{c}- \\
47,9764517\end{array}$ & $\begin{array}{l}\text { Archivo } \\
\text { de audio }\end{array}$ \\
\hline $\begin{array}{l}\text { Myiozetetes } \\
\text { cayanensis }\end{array}$ & erythropterus & 4 & 2535863 & WK & Brasil & Minas Gerais & Serro/MG & $-18,534932$ & $\begin{array}{c}- \\
43,4059307\end{array}$ & $\begin{array}{l}\text { Archivo } \\
\text { de audio }\end{array}$ \\
\hline $\begin{array}{l}\text { Myiozetetes } \\
\text { cayanensis }\end{array}$ & cayanensis & 3 & 2542469 & WK & Brasil & Distrito Federal & Brasília/DF & $-15,780741$ & $\begin{array}{c}- \\
47,7967907\end{array}$ & $\begin{array}{l}\text { Archivo } \\
\text { de audio }\end{array}$ \\
\hline $\begin{array}{l}\text { Myiozetetes } \\
\text { cayanensis }\end{array}$ & cayanensis & 3 & 2543775 & WK & Brasil & Distrito Federal & Brasília/DF & $-15,780741$ & $\begin{array}{c}- \\
47,7967907\end{array}$ & $\begin{array}{l}\text { Archivo } \\
\text { de audio }\end{array}$ \\
\hline $\begin{array}{l}\text { Myiozetetes } \\
\text { cayanensis }\end{array}$ & cayanensis & 3 & 2545706 & WK & Brasil & Amapá & $\begin{array}{c}\text { Pedra Branca do } \\
\text { Amaparí/AP }\end{array}$ & 1,15836321 & $\begin{array}{c}- \\
52,5233215\end{array}$ & $\begin{array}{l}\text { Archivo } \\
\text { de audio }\end{array}$ \\
\hline $\begin{array}{l}\text { Myiozetetes } \\
\text { cayanensis }\end{array}$ & cayanensis & 3 & 2548359 & WK & Brasil & São Paulo & Ourinhos/SP & $-22,952436$ & $\begin{array}{c}- \\
49,8519088\end{array}$ & $\begin{array}{l}\text { Archivo } \\
\text { de audio }\end{array}$ \\
\hline $\begin{array}{l}\text { Myiozetetes } \\
\text { cayanensis }\end{array}$ & erythropterus & 4 & 2550030 & WK & Brasil & Minas Gerais & Santa Bárbara/MG & $-20,029107$ & $\begin{array}{c}- \\
43,4753397\end{array}$ & $\begin{array}{l}\text { Archivo } \\
\text { de audio }\end{array}$ \\
\hline $\begin{array}{l}\text { Myiozetetes } \\
\text { cayanensis }\end{array}$ & erythropterus & 4 & 2554155 & WK & Brasil & Espírito Santo & $\begin{array}{l}\text { Domingos } \\
\text { Martins/ES }\end{array}$ & $-20,308461$ & $\begin{array}{c}- \\
40,8461865\end{array}$ & $\begin{array}{l}\text { Archivo } \\
\text { de audio }\end{array}$ \\
\hline $\begin{array}{l}\text { Myiozetetes } \\
\text { cayanensis }\end{array}$ & cayanensis & 3 & 2563597 & WK & Brasil & Distrito Federal & Brasília/DF & $-15,780741$ & $\begin{array}{c}- \\
47,7967907\end{array}$ & $\begin{array}{l}\text { Archivo } \\
\text { de audio }\end{array}$ \\
\hline $\begin{array}{l}\text { Myiozetetes } \\
\text { cayanensis }\end{array}$ & cayanensis & 3 & 2599442 & WK & Brasil & Minas Gerais & Uberaba/MG & $-19,580173$ & $\begin{array}{c}- \\
47,9764517\end{array}$ & $\begin{array}{l}\text { Archivo } \\
\text { de audio }\end{array}$ \\
\hline $\begin{array}{l}\text { Myiozetetes } \\
\text { cayanensis }\end{array}$ & erythropterus & 4 & 2599823 & WK & Brasil & Minas Gerais & Prados/MG & $-21,057568$ & $-44,079945$ & $\begin{array}{l}\text { Archivo } \\
\text { de audio }\end{array}$ \\
\hline $\begin{array}{l}\text { Myiozetetes } \\
\text { cayanensis }\end{array}$ & cayanensis & 3 & 2608380 & WK & Brasil & Goiás & Jataí/GO & $-17,909435$ & $-51,731508$ & $\begin{array}{l}\text { Archivo } \\
\text { de audio }\end{array}$ \\
\hline $\begin{array}{l}\text { Myiozetetes } \\
\text { cayanensis }\end{array}$ & cayanensis & 3 & 2609587 & WK & Brasil & Goiás & $\begin{array}{c}\text { Alto Paraíso de } \\
\text { Goiás/GO }\end{array}$ & $-14,182647$ & $\begin{array}{c}- \\
47,5347111\end{array}$ & $\begin{array}{l}\text { Archivo } \\
\text { de audio }\end{array}$ \\
\hline $\begin{array}{l}\text { Myiozetetes } \\
\text { cayanensis }\end{array}$ & cayanensis & 3 & 2612531 & WK & Brasil & São Paulo & Guatapará/SP & $-21,452855$ & $\begin{array}{c}- \\
47,9795328\end{array}$ & $\begin{array}{l}\text { Archivo } \\
\text { de audio }\end{array}$ \\
\hline $\begin{array}{l}\text { Myiozetetes } \\
\text { cayanensis }\end{array}$ & cayanensis & 3 & 2612534 & WK & Brasil & São Paulo & Barrinha/SP & $-21,231844$ & $\begin{array}{c}- \\
48,0945833\end{array}$ & $\begin{array}{l}\text { Archivo } \\
\text { de audio }\end{array}$ \\
\hline
\end{tabular}




\begin{tabular}{|c|c|c|c|c|c|c|c|c|c|c|}
\hline $\begin{array}{l}\text { Myiozetetes } \\
\text { cayanensis }\end{array}$ & cayanensis & 3 & 2612536 & WK & Brasil & São Paulo & Dumont/SP & $-21,24667$ & $\begin{array}{c}- \\
47,9794073\end{array}$ & $\begin{array}{l}\text { Archivo } \\
\text { de audio }\end{array}$ \\
\hline $\begin{array}{l}\text { Myiozetetes } \\
\text { cayanensis }\end{array}$ & cayanensis & 3 & 2621509 & WK & Brasil & Goiás & Goianápolis/GO & $-16,514142$ & $\begin{array}{c}- \\
49,0683076\end{array}$ & $\begin{array}{l}\text { Archivo } \\
\text { de audio }\end{array}$ \\
\hline $\begin{array}{l}\text { Myiozetetes } \\
\text { cayanensis }\end{array}$ & erythropterus & 4 & 2632407 & WK & Brasil & Minas Gerais & Santa Bárbara/MG & $-20,029107$ & $\begin{array}{c}- \\
43,4753397\end{array}$ & $\begin{array}{l}\text { Archivo } \\
\text { de audio }\end{array}$ \\
\hline $\begin{array}{l}\text { Myiozetetes } \\
\text { cayanensis }\end{array}$ & erythropterus & 4 & 2632973 & WK & Brasil & Minas Gerais & Manhumirim/MG & $-20,347464$ & $\begin{array}{c}- \\
41,9395311\end{array}$ & $\begin{array}{l}\text { Archivo } \\
\text { de audio }\end{array}$ \\
\hline $\begin{array}{l}\text { Myiozetetes } \\
\text { cayanensis }\end{array}$ & erythropterus & 4 & 2632975 & WK & Brasil & Minas Gerais & Manhumirim/MG & $-20,347464$ & $\begin{array}{c}- \\
41,9395311\end{array}$ & $\begin{array}{l}\text { Archivo } \\
\text { de audio }\end{array}$ \\
\hline $\begin{array}{l}\text { Myiozetetes } \\
\text { cayanensis }\end{array}$ & erythropterus & 4 & 2644509 & WK & Brasil & Minas Gerais & Prados/MG & $-21,057568$ & $-44,079945$ & $\begin{array}{l}\text { Archivo } \\
\text { de audio }\end{array}$ \\
\hline $\begin{array}{l}\text { Myiozetetes } \\
\text { cayanensis }\end{array}$ & cayanensis & 3 & 2661668 & WK & Brasil & Mato Grosso & Poconé/MT & $-16,78447$ & $\begin{array}{c}- \\
56,9485054\end{array}$ & $\begin{array}{l}\text { Archivo } \\
\text { de audio }\end{array}$ \\
\hline $\begin{array}{l}\text { Myiozetetes } \\
\text { cayanensis }\end{array}$ & cayanensis & 3 & 2675284 & WK & Brasil & Mato Grosso & Alta Floresta/MT & $-10,055008$ & $\begin{array}{c}- \\
56,3660622\end{array}$ & $\begin{array}{l}\text { Archivo } \\
\text { de audio }\end{array}$ \\
\hline $\begin{array}{l}\text { Myiozetetes } \\
\text { cayanensis }\end{array}$ & cayanensis & 3 & 2689032 & WK & Brasil & Mato Grosso & $\begin{array}{c}\text { Lambari } \\
\text { d'Oeste/MT }\end{array}$ & $-15,483007$ & $\begin{array}{c}- \\
57,8020869\end{array}$ & $\begin{array}{l}\text { Archivo } \\
\text { de audio }\end{array}$ \\
\hline $\begin{array}{l}\text { Myiozetetes } \\
\text { cayanensis }\end{array}$ & cayanensis & 3 & 2694934 & WK & Brasil & Distrito Federal & Brasília/DF & $-15,780741$ & 47,7967907 & $\begin{array}{l}\text { Archivo } \\
\text { de audio }\end{array}$ \\
\hline $\begin{array}{l}\text { Myiozetetes } \\
\text { cayanensis }\end{array}$ & erythropterus & 4 & 2721389 & WK & Brasil & Bahía & Poções/BA & $-14,544352$ & $\begin{array}{c}- \\
40,3785766\end{array}$ & $\begin{array}{l}\text { Archivo } \\
\text { de audio }\end{array}$ \\
\hline $\begin{array}{l}\text { Myiozetetes } \\
\text { cayanensis }\end{array}$ & erythropterus & 4 & 2728908 & WK & Brasil & Espírito Santo & $\begin{array}{l}\text { Venda Nova do } \\
\text { Imigrante/ES }\end{array}$ & $-20,370322$ & $-41,139141$ & $\begin{array}{l}\text { Archivo } \\
\text { de audio }\end{array}$ \\
\hline $\begin{array}{l}\text { Myiozetetes } \\
\text { cayanensis }\end{array}$ & cayanensis & 3 & 2733111 & WK & Brasil & Mato Grosso & Poconé/MT & $-16,78447$ & $\begin{array}{c}- \\
56,9485054\end{array}$ & $\begin{array}{l}\text { Archivo } \\
\text { de audio }\end{array}$ \\
\hline $\begin{array}{l}\text { Myiozetetes } \\
\text { cayanensis }\end{array}$ & cayanensis & 3 & 2750106 & WK & Brasil & Mato Grosso & Poconé/MT & $-16,78447$ & 56,9485054 & $\begin{array}{l}\text { Archivo } \\
\text { de audio }\end{array}$ \\
\hline $\begin{array}{l}\text { Myiozetetes } \\
\text { cayanensis }\end{array}$ & cayanensis & 3 & 2794614 & WK & Brasil & Tocantins & Araguacema/TO & $-8,9391673$ & $\begin{array}{c}- \\
49,5043575\end{array}$ & $\begin{array}{l}\text { Archivo } \\
\text { de audio }\end{array}$ \\
\hline $\begin{array}{l}\text { Myiozetetes } \\
\text { cayanensis }\end{array}$ & cayanensis & 3 & 2795922 & WK & Brasil & Goiás & $\begin{array}{c}\text { Santa Rita do Novo } \\
\text { Destino/GO }\end{array}$ & $-14,876282$ & $\begin{array}{c}- \\
49,0557963\end{array}$ & $\begin{array}{l}\text { Archivo } \\
\text { de audio }\end{array}$ \\
\hline $\begin{array}{l}\text { Myiozetetes } \\
\text { cayanensis }\end{array}$ & cayanensis & 3 & 2800583 & WK & Brasil & Acre & $\begin{array}{c}\text { Sena } \\
\text { Madureira/AC }\end{array}$ & $-9,8572575$ & $\begin{array}{c}- \\
69,4376171\end{array}$ & $\begin{array}{l}\text { Archivo } \\
\text { de audio }\end{array}$ \\
\hline $\begin{array}{l}\text { Myiozetetes } \\
\text { cayanensis }\end{array}$ & cayanensis & 3 & 2811565 & WK & Brasil & Amapá & Ferreira Gomes/AP & 0,95937753 & $\begin{array}{c}- \\
51,4185846\end{array}$ & $\begin{array}{l}\text { Archivo } \\
\text { de audio }\end{array}$ \\
\hline $\begin{array}{l}\text { Myiozetetes } \\
\text { cayanensis }\end{array}$ & cayanensis & 3 & 2824248 & WK & Brasil & São Paulo & Jardinópolis/SP & $-20,991452$ & $\begin{array}{c}- \\
47,8259975\end{array}$ & $\begin{array}{l}\text { Archivo } \\
\text { de audio }\end{array}$ \\
\hline $\begin{array}{l}\text { Myiozetetes } \\
\text { cayanensis }\end{array}$ & cayanensis & 3 & 2824249 & WK & Brasil & São Paulo & Jardinópolis/SP & $-20,991452$ & $\begin{array}{c}- \\
47,8259975\end{array}$ & $\begin{array}{l}\text { Archivo } \\
\text { de audio }\end{array}$ \\
\hline $\begin{array}{l}\text { Myiozetetes } \\
\text { cayanensis }\end{array}$ & erythropterus & 4 & 2850220 & WK & Brasil & Espírito Santo & Vargem Alta/ES & $-20,644973$ & $\begin{array}{c}- \\
41,0027139\end{array}$ & $\begin{array}{l}\text { Archivo } \\
\text { de audio }\end{array}$ \\
\hline $\begin{array}{l}\text { Myiozetetes } \\
\text { cayanensis }\end{array}$ & cayanensis & 3 & 2860318 & WK & Brasil & Tocantins & Gurupi/TO & $-11,655801$ & $\begin{array}{c}- \\
48,8888046\end{array}$ & $\begin{array}{l}\text { Archivo } \\
\text { de audio }\end{array}$ \\
\hline $\begin{array}{l}\text { Myiozetetes } \\
\text { cayanensis }\end{array}$ & cayanensis & 3 & 2863733 & WK & Brasil & São Paulo & Guapiaçu/SP & $-20,742678$ & $\begin{array}{c}- \\
49,1896805\end{array}$ & $\begin{array}{l}\text { Archivo } \\
\text { de audio }\end{array}$ \\
\hline $\begin{array}{l}\text { Myiozetetes } \\
\text { cayanensis }\end{array}$ & cayanensis & 3 & 2888529 & WK & Brasil & Mato Grosso & Poconé/MT & $-16,78447$ & $\begin{array}{c}- \\
56,9485054\end{array}$ & $\begin{array}{l}\text { Archivo } \\
\text { de audio }\end{array}$ \\
\hline $\begin{array}{l}\text { Myiozetetes } \\
\text { cayanensis }\end{array}$ & cayanensis & 3 & 2908062 & WK & Brasil & Pará & Acará/PA & $-2,0351419$ & $\begin{array}{c}- \\
48,4110393\end{array}$ & $\begin{array}{l}\text { Archivo } \\
\text { de audio }\end{array}$ \\
\hline $\begin{array}{l}\text { Myiozetetes } \\
\text { cayanensis }\end{array}$ & cayanensis & 3 & 2912401 & WK & Brasil & Minas Gerais & Vazante/MG & $-17,868617$ & $\begin{array}{c}- \\
46,8670001\end{array}$ & $\begin{array}{l}\text { Archivo } \\
\text { de audio }\end{array}$ \\
\hline $\begin{array}{l}\text { Myiozetetes } \\
\text { cayanensis }\end{array}$ & cayanensis & 3 & 2914388 & WK & Brasil & São Paulo & Jaboticabal/SP & $-21,217551$ & $-48,2875$ & $\begin{array}{l}\text { Archivo } \\
\text { de audio }\end{array}$ \\
\hline $\begin{array}{l}\text { Myiozetetes } \\
\text { cayanensis }\end{array}$ & cayanensis & 3 & 2920021 & WK & Brasil & São Paulo & Araçatuba/SP & $-21,113287$ & 50,5741822 & $\begin{array}{l}\text { Archivo } \\
\text { de audio }\end{array}$ \\
\hline $\begin{array}{l}\text { Myiozetetes } \\
\text { cayanensis }\end{array}$ & cayanensis & 3 & 2924475 & WK & Brasil & Pará & $\begin{array}{l}\text { Canaã dos } \\
\text { Carajás/PA }\end{array}$ & $-6,4239482$ & $\begin{array}{c}- \\
50,0851486\end{array}$ & $\begin{array}{l}\text { Archivo } \\
\text { de audio }\end{array}$ \\
\hline $\begin{array}{l}\text { Myiozetetes } \\
\text { cayanensis }\end{array}$ & cayanensis & 3 & 2926812 & WK & Brasil & Mato Grosso & Cáceres/MT & $-16,527764$ & $\begin{array}{c}- \\
57,8365125\end{array}$ & $\begin{array}{l}\text { Archivo } \\
\text { de audio }\end{array}$ \\
\hline $\begin{array}{l}\text { Myiozetetes } \\
\text { cayanensis }\end{array}$ & cayanensis & 3 & 2938330 & WK & Brasil & Roraima & Boa Vista/RR & 3,11827869 & $\begin{array}{c}- \\
60,7174232\end{array}$ & $\begin{array}{l}\text { Archivo } \\
\text { de audio }\end{array}$ \\
\hline $\begin{array}{l}\text { Myiozetetes } \\
\text { cayanensis }\end{array}$ & cayanensis & 3 & 2942367 & WK & Brasil & Minas Gerais & Sacramento/MG & $-19,904732$ & $-47,266174$ & $\begin{array}{l}\text { Archivo } \\
\text { de audio }\end{array}$ \\
\hline $\begin{array}{l}\text { Myiozetetes } \\
\text { cayanensis }\end{array}$ & cayanensis & 3 & 2968547 & WK & Brasil & Amazonas & Barcelos/AM & $-0,4630249$ & $\begin{array}{c}- \\
63,4336557\end{array}$ & $\begin{array}{l}\text { Archivo } \\
\text { de audio }\end{array}$ \\
\hline $\begin{array}{l}\text { Myiozetetes } \\
\text { cayanensis }\end{array}$ & cayanensis & 3 & 2980586 & WK & Brasil & São Paulo & Taiaçu/SP & $-21,131504$ & $-48,529844$ & $\begin{array}{l}\text { Archivo } \\
\text { de audio }\end{array}$ \\
\hline
\end{tabular}




\begin{tabular}{|c|c|c|c|c|c|c|c|c|c|c|}
\hline $\begin{array}{l}\text { Myiozetetes } \\
\text { cayanensis }\end{array}$ & cayanensis & 3 & 2996827 & WK & Brasil & Goiás & Goiânia/GO & $-16,642076$ & $\begin{array}{c}- \\
49,2750915\end{array}$ & $\begin{array}{l}\text { Archivo } \\
\text { de audio }\end{array}$ \\
\hline $\begin{array}{l}\text { Myiozetetes } \\
\text { cayanensis }\end{array}$ & cayanensis & 3 & 3005359 & WK & Brasil & Pará & Xinguara/PA & $-6,9174418$ & $\begin{array}{c}- \\
49,6347139\end{array}$ & $\begin{array}{l}\text { Archivo } \\
\text { de audio }\end{array}$ \\
\hline $\begin{array}{l}\text { Myiozetetes } \\
\text { cayanensis }\end{array}$ & cayanensis & 3 & 3035530 & WK & Brasil & $\begin{array}{l}\text { Mato Grosso do } \\
\text { Sul }\end{array}$ & Bodoquena/MS & $-20,55305$ & $\begin{array}{c}- \\
56,6919394\end{array}$ & $\begin{array}{l}\text { Archivo } \\
\text { de audio }\end{array}$ \\
\hline $\begin{array}{l}\text { Myiozetetes } \\
\text { cayanensis }\end{array}$ & cayanensis & 3 & 3055303 & WK & Brasil & $\begin{array}{l}\text { Mato Grosso do } \\
\text { Sul }\end{array}$ & Bodoquena/MS & $-20,55305$ & $\begin{array}{c}- \\
56,6919394\end{array}$ & $\begin{array}{l}\text { Archivo } \\
\text { de audio }\end{array}$ \\
\hline $\begin{array}{l}\text { Myiozetetes } \\
\text { cayanensis }\end{array}$ & cayanensis & 3 & 3078818 & WK & Brasil & Mato Grosso & Poconé/MT & $-16,78447$ & $\begin{array}{c}- \\
56,9485054\end{array}$ & $\begin{array}{l}\text { Archivo } \\
\text { de audio }\end{array}$ \\
\hline $\begin{array}{l}\text { Myiozetetes } \\
\text { cayanensis }\end{array}$ & erythropterus & 4 & 3080642 & WK & Brasil & Minas Gerais & Timóteo/MG & $-19,557489$ & $\begin{array}{c}- \\
42,6024491\end{array}$ & $\begin{array}{l}\text { Archivo } \\
\text { de audio }\end{array}$ \\
\hline $\begin{array}{l}\text { Myiozetetes } \\
\text { cayanensis }\end{array}$ & cayanensis & 3 & 3106649 & WK & Brasil & Pará & Parauapebas/PA & $-6,1544368$ & $\begin{array}{c}- \\
50,4860742\end{array}$ & $\begin{array}{l}\text { Archivo } \\
\text { de audio }\end{array}$ \\
\hline $\begin{array}{l}\text { Myiozetetes } \\
\text { cayanensis }\end{array}$ & cayanensis & 3 & 3110394 & WK & Brasil & Goiás & Cavalcante/GO & $-13,634762$ & $\begin{array}{c}- \\
47,6957015\end{array}$ & $\begin{array}{l}\text { Archivo } \\
\text { de audio }\end{array}$ \\
\hline $\begin{array}{l}\text { Myiozetetes } \\
\text { cayanensis }\end{array}$ & cayanensis & 3 & 3142667 & WK & Brasil & $\begin{array}{l}\text { Mato Grosso do } \\
\text { Sul }\end{array}$ & Bonito/MS & $-21,007472$ & $\begin{array}{c}- \\
56,4597222\end{array}$ & $\begin{array}{l}\text { Archivo } \\
\text { de audio }\end{array}$ \\
\hline $\begin{array}{l}\text { Myiozetetes } \\
\text { cayanensis }\end{array}$ & cayanensis & 3 & 3143823 & WK & Brasil & $\begin{array}{l}\text { Mato Grosso do } \\
\text { Sul }\end{array}$ & Bodoquena/MS & $-20,55305$ & $\begin{array}{c}- \\
56,6919394\end{array}$ & $\begin{array}{l}\text { Archivo } \\
\text { de audio }\end{array}$ \\
\hline $\begin{array}{l}\text { Myiozetetes } \\
\text { cayanensis }\end{array}$ & cayanensis & 3 & 3182710 & WK & Brasil & Pará & Belém/PA & $-1,2405368$ & $\begin{array}{c}- \\
48,4591789\end{array}$ & $\begin{array}{l}\text { Archivo } \\
\text { de audio }\end{array}$ \\
\hline $\begin{array}{l}\text { Myiozetetes } \\
\text { cayanensis }\end{array}$ & cayanensis & 3 & 3186909 & WK & Brasil & Mato Grosso & Poconé/MT & $-16,78447$ & $\begin{array}{c}- \\
56,9485054\end{array}$ & $\begin{array}{l}\text { Archivo } \\
\text { de audio }\end{array}$ \\
\hline $\begin{array}{l}\text { Myiozetetes } \\
\text { cayanensis }\end{array}$ & cayanensis & 3 & 3191246 & WK & Brasil & Maranhão & Timon/MA & $-5,1846962$ & $\begin{array}{c}- \\
42,9771408\end{array}$ & $\begin{array}{l}\text { Archivo } \\
\text { de audio }\end{array}$ \\
\hline $\begin{array}{l}\text { Myiozetetes } \\
\text { cayanensis }\end{array}$ & cayanensis & 3 & 3254013 & WK & Brasil & Distrito Federal & Brasília/DF & $-15,780741$ & $\begin{array}{c}- \\
47,7967907\end{array}$ & $\begin{array}{l}\text { Archivo } \\
\text { de audio }\end{array}$ \\
\hline $\begin{array}{l}\text { Myiozetetes } \\
\text { cayanensis }\end{array}$ & erythropterus & 4 & 3268730 & WK & Brasil & Minas Gerais & Catas Altas/MG & $-20,075438$ & $-43,408135$ & $\begin{array}{l}\text { Archivo } \\
\text { de audio }\end{array}$ \\
\hline $\begin{array}{l}\text { Myiozetetes } \\
\text { cayanensis }\end{array}$ & erythropterus & 4 & 3273311 & WK & Brasil & Minas Gerais & Catas Altas/MG & $-20,075438$ & $-43,408135$ & $\begin{array}{l}\text { Archivo } \\
\text { de audio }\end{array}$ \\
\hline $\begin{array}{l}\text { Myiozetetes } \\
\text { cayanensis }\end{array}$ & erythropterus & 4 & 3273313 & WK & Brasil & Minas Gerais & Catas Altas/MG & $-20,075438$ & $-43,408135$ & $\begin{array}{l}\text { Archivo } \\
\text { de audio }\end{array}$ \\
\hline $\begin{array}{l}\text { Myiozetetes } \\
\text { cayanensis }\end{array}$ & cayanensis & 3 & 3275256 & WK & Brasil & Pará & Parauapebas/PA & $-6,1544368$ & $\begin{array}{c}- \\
50,4860742\end{array}$ & $\begin{array}{l}\text { Archivo } \\
\text { de audio }\end{array}$ \\
\hline $\begin{array}{l}\text { Myiozetetes } \\
\text { cayanensis }\end{array}$ & cayanensis & 3 & 3293590 & WK & Brasil & $\begin{array}{l}\text { Mato Grosso do } \\
\text { Sul }\end{array}$ & Bodoquena/MS & $-20,55305$ & $\begin{array}{c}- \\
56,6919394\end{array}$ & $\begin{array}{l}\text { Archivo } \\
\text { de audio }\end{array}$ \\
\hline $\begin{array}{l}\text { Myiozetetes } \\
\text { cayanensis }\end{array}$ & cayanensis & 3 & 3328682 & WK & Brasil & São Paulo & Narandiba/SP & $-22,563317$ & $\begin{array}{c}- \\
51,5211584\end{array}$ & $\begin{array}{l}\text { Archivo } \\
\text { de audio }\end{array}$ \\
\hline $\begin{array}{l}\text { Myiozetetes } \\
\text { cayanensis }\end{array}$ & erythropterus & 4 & 3332969 & WK & Brasil & Minas Gerais & Santa Bárbara/MG & $-20,029107$ & $\begin{array}{c}- \\
43,4753397\end{array}$ & $\begin{array}{l}\text { Archivo } \\
\text { de audio }\end{array}$ \\
\hline $\begin{array}{l}\text { Myiozetetes } \\
\text { cayanensis }\end{array}$ & cayanensis & 3 & 3374857 & WK & Brasil & Mato Grosso & Itaúba/MT & $-11,103965$ & $\begin{array}{c}- \\
55,5877252\end{array}$ & $\begin{array}{l}\text { Archivo } \\
\text { de audio }\end{array}$ \\
\hline $\begin{array}{l}\text { Myiozetetes } \\
\text { cayanensis }\end{array}$ & cayanensis & 3 & 3376233 & WK & Brasil & São Paulo & Ribeirão Preto/SP & $-21,210454$ & $\begin{array}{c}- \\
47,8208587\end{array}$ & $\begin{array}{l}\text { Archivo } \\
\text { de audio }\end{array}$ \\
\hline $\begin{array}{l}\text { Myiozetetes } \\
\text { cayanensis }\end{array}$ & cayanensis & 3 & 3400593 & WK & Brasil & Mato Grosso & Itaúba/MT & $-11,103965$ & $\begin{array}{c}- \\
55,5877252\end{array}$ & $\begin{array}{l}\text { Archivo } \\
\text { de audio }\end{array}$ \\
\hline $\begin{array}{l}\text { Myiozetetes } \\
\text { cayanensis }\end{array}$ & erythropterus & 4 & 3415114 & WK & Brasil & Minas Gerais & $\begin{array}{l}\text { Santana dos } \\
\text { Montes/MG }\end{array}$ & $-20,790302$ & $-43,691326$ & $\begin{array}{l}\text { Archivo } \\
\text { de audio }\end{array}$ \\
\hline $\begin{array}{l}\text { Myiozetetes } \\
\text { cayanensis }\end{array}$ & cayanensis & 3 & 3424323 & WK & Brasil & $\begin{array}{l}\text { Mato Grosso do } \\
\text { Sul }\end{array}$ & Bonito/MS & $-21,007472$ & $\begin{array}{c}- \\
56,4597222\end{array}$ & $\begin{array}{l}\text { Archivo } \\
\text { de audio }\end{array}$ \\
\hline $\begin{array}{l}\text { Myiozetetes } \\
\text { cayanensis }\end{array}$ & cayanensis & 3 & 3439417 & WK & Brasil & Pará & Castanhal/PA & $-1,2681343$ & $\begin{array}{c}- \\
47,8775761\end{array}$ & $\begin{array}{l}\text { Archivo } \\
\text { de audio }\end{array}$ \\
\hline $\begin{array}{l}\text { Myiozetetes } \\
\text { cayanensis }\end{array}$ & cayanensis & 3 & 3441875 & WK & Brasil & $\begin{array}{l}\text { Mato Grosso do } \\
\text { Sul }\end{array}$ & Bonito/MS & $-21,007472$ & $\begin{array}{c}- \\
56,4597222\end{array}$ & $\begin{array}{l}\text { Archivo } \\
\text { de audio }\end{array}$ \\
\hline $\begin{array}{l}\text { Myiozetetes } \\
\text { cayanensis }\end{array}$ & cayanensis & 3 & 3455675 & WK & Brasil & Goiás & Iporá/GO & $-16,415556$ & $\begin{array}{c}- \\
51,1802879\end{array}$ & $\begin{array}{l}\text { Archivo } \\
\text { de audio }\end{array}$ \\
\hline $\begin{array}{l}\text { Myiozetetes } \\
\text { cayanensis }\end{array}$ & erythropterus & 4 & 3464634 & WK & Brasil & Espírito Santo & Santa Teresa/ES & $-19,876807$ & $\begin{array}{c}- \\
40,6324805\end{array}$ & $\begin{array}{l}\text { Archivo } \\
\text { de audio }\end{array}$ \\
\hline $\begin{array}{l}\text { Myiozetetes } \\
\text { cayanensis }\end{array}$ & cayanensis & 3 & 3490213 & WK & Brasil & São Paulo & Brodowski/SP & $-21,048937$ & $\begin{array}{c}- \\
47,6275625\end{array}$ & $\begin{array}{l}\text { Archivo } \\
\text { de audio }\end{array}$ \\
\hline $\begin{array}{l}\text { Myiozetetes } \\
\text { cayanensis }\end{array}$ & cayanensis & 3 & 3502096 & WK & Brasil & Maranhão & $\begin{array}{l}\text { São Benedito do } \\
\text { Rio Preto/MA }\end{array}$ & $-3,3556474$ & $\begin{array}{c}- \\
43,6278778\end{array}$ & $\begin{array}{l}\text { Archivo } \\
\text { de audio }\end{array}$ \\
\hline $\begin{array}{l}\text { Myiozetetes } \\
\text { cayanensis }\end{array}$ & cayanensis & 3 & 3544321 & WK & Brasil & São Paulo & $\begin{array}{c}\text { São José do Rio } \\
\text { Preto/SP }\end{array}$ & $-20,796786$ & $\begin{array}{c}- \\
49,3582146\end{array}$ & $\begin{array}{l}\text { Archivo } \\
\text { de audio }\end{array}$ \\
\hline $\begin{array}{l}\text { Myiozetetes } \\
\text { cayanensis }\end{array}$ & cayanensis & 3 & 3554845 & WK & Brasil & Goiás & Pirenópolis/GO & $-15,807197$ & $\begin{array}{c}- \\
49,0100159\end{array}$ & $\begin{array}{l}\text { Archivo } \\
\text { de audio }\end{array}$ \\
\hline
\end{tabular}




\begin{tabular}{|c|c|c|c|c|c|c|c|c|c|c|}
\hline $\begin{array}{l}\text { Myiozetetes } \\
\text { cayanensis }\end{array}$ & cayanensis & 3 & 3563016 & WK & Brasil & Ceará & Graça/CE & $-4,0556905$ & $\begin{array}{c}- \\
40,7719238\end{array}$ & $\begin{array}{l}\text { Archivo } \\
\text { de audio }\end{array}$ \\
\hline $\begin{array}{l}\text { Myiozetetes } \\
\text { cayanensis }\end{array}$ & cayanensis & 3 & 3574995 & WK & Brasil & Pará & Belém/PA & $-1,2405368$ & $\begin{array}{c}- \\
48,4591789\end{array}$ & $\begin{array}{l}\text { Archivo } \\
\text { de audio }\end{array}$ \\
\hline $\begin{array}{l}\text { Myiozetetes } \\
\text { cayanensis }\end{array}$ & cayanensis & 3 & 3591137 & WK & Brasil & Amazonas & $\begin{array}{c}\text { Presidente } \\
\text { Figueiredo/AM }\end{array}$ & $-1,3720793$ & $\begin{array}{c}- \\
59,9884392\end{array}$ & $\begin{array}{l}\text { Archivo } \\
\text { de audio }\end{array}$ \\
\hline $\begin{array}{l}\text { Myiozetetes } \\
\text { cayanensis }\end{array}$ & cayanensis & 3 & 3607968 & WK & Brasil & São Paulo & $\begin{array}{c}\text { Américo } \\
\text { Brasiliense/SP }\end{array}$ & $-21,721353$ & $\begin{array}{c}- \\
48,0320387\end{array}$ & $\begin{array}{l}\text { Archivo } \\
\text { de audio }\end{array}$ \\
\hline $\begin{array}{l}\text { Myiozetetes } \\
\text { cayanensis }\end{array}$ & erythropterus & 4 & 3646085 & WK & Brasil & Minas Gerais & $\begin{array}{l}\text { Santana dos } \\
\text { Montes/MG }\end{array}$ & $-20,790302$ & $-43,691326$ & $\begin{array}{l}\text { Archivo } \\
\text { de audio }\end{array}$ \\
\hline $\begin{array}{l}\text { Myiozetetes } \\
\text { cayanensis }\end{array}$ & cayanensis & 3 & 3657965 & WK & Brasil & Amazonas & Manaus/AM & $-2,625919$ & 60,2590208 & $\begin{array}{l}\text { Archivo } \\
\text { de audio }\end{array}$ \\
\hline $\begin{array}{l}\text { Myiozetetes } \\
\text { cayanensis }\end{array}$ & cayanensis & 3 & 3660947 & WK & Brasil & Goiás & $\begin{array}{l}\text { Cocalzinho de } \\
\text { Goiás/GO }\end{array}$ & $-15,655204$ & $\begin{array}{c}- \\
48,6024671\end{array}$ & $\begin{array}{l}\text { Archivo } \\
\text { de audio }\end{array}$ \\
\hline $\begin{array}{l}\text { Myiozetetes } \\
\text { cayanensis }\end{array}$ & cayanensis & 3 & 3688792 & WK & Brasil & Goiás & Pirenópolis/GO & $-15,807197$ & $\begin{array}{c}- \\
49,0100159\end{array}$ & $\begin{array}{l}\text { Archivo } \\
\text { de audio }\end{array}$ \\
\hline $\begin{array}{l}\text { Myiozetetes } \\
\text { cayanensis }\end{array}$ & erythropterus & 4 & 3696896 & WK & Brasil & Río de Janeiro & Nova Friburgo/RJ & $-22,288644$ & $-42,534183$ & $\begin{array}{l}\text { Archivo } \\
\text { de audio }\end{array}$ \\
\hline $\begin{array}{l}\text { Myiozetetes } \\
\text { cayanensis }\end{array}$ & erythropterus & 4 & OBS295496987 & $x C$ & Brasil & Bahía & Itacare & $-14,2785$ & $-38,9934$ & Fotografía \\
\hline $\begin{array}{l}\text { Myiozetetes } \\
\text { cayanensis }\end{array}$ & erythropterus & 4 & WA1006170 & WK & Brasil & Minas Gerais & $\begin{array}{c}\text { Conceição do Mato } \\
\text { Dentro }\end{array}$ & $-19,03554$ & $-43,424645$ & Fotografía \\
\hline $\begin{array}{l}\text { Myiozetetes } \\
\text { cayanensis }\end{array}$ & cayanensis & 3 & WA100844 & WK & Brasil & Minas Gerais & Araponga & $-20,66661$ & $-42,522017$ & Fotografía \\
\hline $\begin{array}{l}\text { Myiozetetes } \\
\text { cayanensis }\end{array}$ & erythropterus & 4 & WA1065827 & WK & Brasil & Minas Gerais & Antônio Dias & $-19,653716$ & $-42,872245$ & Fotografía \\
\hline $\begin{array}{l}\text { Myiozetetes } \\
\text { cayanensis }\end{array}$ & erythropterus & 4 & WA1123715 & WK & Brasil & Minas Gerais & $\begin{array}{c}\text { São Gonçalo do Rio } \\
\text { Preto }\end{array}$ & $-18,072522$ & $-43,348776$ & Fotografía \\
\hline $\begin{array}{l}\text { Myiozetetes } \\
\text { cayanensis }\end{array}$ & erythropterus & 4 & WA1298883 & WK & Brasil & Espírito Santo & Alfredo Chaves & $-20,636201$ & $-40,750071$ & Fotografía \\
\hline $\begin{array}{l}\text { Myiozetetes } \\
\text { cayanensis }\end{array}$ & erythropterus & 4 & WA1303014 & WK & Brasil & Minas Gerais & Prados & $-21,056602$ & $-44,076454$ & Fotografía \\
\hline $\begin{array}{l}\text { Myiozetetes } \\
\text { cayanensis }\end{array}$ & erythropterus & 4 & WA1456015 & WK & Brasil & Minas Gerais & Caputira & $-20,170828$ & $-42,268719$ & Fotografía \\
\hline $\begin{array}{l}\text { Myiozetetes } \\
\text { cayanensis }\end{array}$ & erythropterus & 4 & WA1467562 & WK & Brasil & Espírito Santo & Itarana & $-19,87565$ & $-40,875477$ & Fotografía \\
\hline $\begin{array}{l}\text { Myiozetetes } \\
\text { cayanensis }\end{array}$ & erythropterus & 4 & WA1472691 & WK & Brasil & Minas Gerais & Manhumirim & $-20,359639$ & $-41,958907$ & Fotografía \\
\hline $\begin{array}{l}\text { Myiozetetes } \\
\text { cayanensis }\end{array}$ & erythropterus & 4 & WA1496554 & WK & Brasil & Espírito Santo & Afonso Cláudio & $-20,078315$ & $-41,126451$ & Fotografía \\
\hline $\begin{array}{l}\text { Myiozetetes } \\
\text { cayanensis }\end{array}$ & erythropterus & 4 & WA152168 & WK & Brasil & Minas Gerais & Santa Bárbara & $-19,963398$ & $-43,420686$ & Fotografía \\
\hline $\begin{array}{l}\text { Myiozetetes } \\
\text { cayanensis }\end{array}$ & erythropterus & 4 & WA1548234 & WK & Brasil & Río de Janeiro & Nova Friburgo & $-22,288761$ & $-42,53416$ & Fotografía \\
\hline $\begin{array}{l}\text { Myiozetetes } \\
\text { cayanensis }\end{array}$ & erythropterus & 4 & WA1548507 & WK & Brasil & Minas Gerais & Piranga & $-20,683243$ & $-43,297661$ & Fotografía \\
\hline $\begin{array}{l}\text { Myiozetetes } \\
\text { cayanensis }\end{array}$ & erythropterus & 4 & WA156482 & WK & Brasil & Minas Gerais & Barão de Cocais & $-19,946815$ & $-43,484553$ & Fotografía \\
\hline $\begin{array}{l}\text { Myiozetetes } \\
\text { cayanensis }\end{array}$ & erythropterus & 4 & WA1654662 & WK & Brasil & Minas Gerais & Ouro Preto & $-20,385403$ & $-43,502832$ & Fotografía \\
\hline $\begin{array}{l}\text { Myiozetetes } \\
\text { cayanensis }\end{array}$ & erythropterus & 4 & WA1674119 & WK & Brasil & Minas Gerais & Serro & $-18,603301$ & $-43,378988$ & Fotografía \\
\hline $\begin{array}{l}\text { Myiozetetes } \\
\text { cayanensis }\end{array}$ & cayanensis & 3 & WA1684499 & WK & Brasil & Río de Janeiro & Duque de Caxias & $-22,786375$ & $-43,305661$ & Fotografía \\
\hline $\begin{array}{l}\text { Myiozetetes } \\
\text { cayanensis }\end{array}$ & erythropterus & 4 & WA1779169 & WK & Brasil & Minas Gerais & Alto Caparaó & $-20,447991$ & $-41,873238$ & Fotografía \\
\hline $\begin{array}{l}\text { Myiozetetes } \\
\text { cayanensis }\end{array}$ & erythropterus & 4 & WA1791691 & WK & Brasil & Minas Gerais & Santa Bárbara & $-19,963398$ & $-43,420686$ & Fotografía \\
\hline $\begin{array}{l}\text { Myiozetetes } \\
\text { cayanensis }\end{array}$ & erythropterus & 4 & WA1797488 & WK & Brasil & Minas Gerais & Catas Altas & $-20,070273$ & $-43,410064$ & Fotografía \\
\hline $\begin{array}{l}\text { Myiozetetes } \\
\text { cayanensis }\end{array}$ & erythropterus & 4 & WA1828821 & WK & Brasil & Minas Gerais & Caputira & $-20,170828$ & $-42,268719$ & Fotografía \\
\hline $\begin{array}{l}\text { Myiozetetes } \\
\text { cayanensis }\end{array}$ & erythropterus & 4 & WA1830028 & WK & Brasil & Minas Gerais & Manhumirim & $-20,359639$ & $-41,958907$ & Fotografía \\
\hline $\begin{array}{l}\text { Myiozetetes } \\
\text { cayanensis }\end{array}$ & erythropterus & 4 & WA1833162 & WK & Brasil & Minas Gerais & Caeté & $-19,885146$ & $-43,664282$ & Fotografía \\
\hline $\begin{array}{l}\text { Myiozetetes } \\
\text { cayanensis }\end{array}$ & erythropterus & 4 & WA1881797 & WK & Brasil & Minas Gerais & Alto Caparaó & $-20,447991$ & $-41,873238$ & Fotografía \\
\hline
\end{tabular}




\begin{tabular}{|c|c|c|c|c|c|c|c|c|c|c|}
\hline $\begin{array}{l}\text { Myiozetetes } \\
\text { cayanensis }\end{array}$ & erythropterus & 4 & WA1913271 & WK & Brasil & Espírito Santo & Marechal Floriano & $-20,411045$ & $-40,676148$ & Fotografía \\
\hline $\begin{array}{l}\text { Myiozetetes } \\
\text { cayanensis }\end{array}$ & cayanensis & 3 & WA1965496 & WK & Brasil & Espírito Santo & Colatina & $-19,526284$ & $-40,63512$ & Fotografía \\
\hline $\begin{array}{l}\text { Myiozetetes } \\
\text { cayanensis }\end{array}$ & cayanensis & 3 & WA1972001 & WK & Brasil & Río de Janeiro & Resende & $-22,471414$ & $-44,451727$ & Fotografía \\
\hline $\begin{array}{l}\text { Myiozetetes } \\
\text { cayanensis }\end{array}$ & cayanensis & 3 & WA1986574 & WK & Brasil & Minas Gerais & $\begin{array}{c}\text { São Sebastião do } \\
\text { Paraíso }\end{array}$ & $-20,916902$ & $-46,983905$ & Fotografía \\
\hline $\begin{array}{l}\text { Myiozetetes } \\
\text { cayanensis }\end{array}$ & erythropterus & 4 & WA1999779 & WK & Brasil & Minas Gerais & Virginópolis & $-18,821928$ & $-42,705039$ & Fotografía \\
\hline $\begin{array}{l}\text { Myiozetetes } \\
\text { cayanensis }\end{array}$ & cayanensis & 3 & WA2024848 & WK & Brasil & Minas Gerais & Tupaciguara & $-18,593831$ & $-48,704322$ & Fotografía \\
\hline $\begin{array}{l}\text { Myiozetetes } \\
\text { cayanensis }\end{array}$ & cayanensis & 3 & WA2081680 & WK & Brasil & Minas Gerais & Uberaba & $-19,747163$ & $-47,939289$ & Fotografía \\
\hline $\begin{array}{l}\text { Myiozetetes } \\
\text { cayanensis }\end{array}$ & erythropterus & 4 & WA2092327 & WK & Brasil & Espírito Santo & Santa Leopoldina & $-20,100769$ & $-40,527556$ & Fotografía \\
\hline $\begin{array}{l}\text { Myiozetetes } \\
\text { cayanensis }\end{array}$ & erythropterus & 4 & WA2150782 & WK & Brasil & Minas Gerais & Serro & $-18,603301$ & $-43,378988$ & Fotografía \\
\hline $\begin{array}{l}\text { Myiozetetes } \\
\text { cayanensis }\end{array}$ & erythropterus & 4 & WA2157498 & WK & Brasil & Minas Gerais & $\begin{array}{l}\text { Santana dos } \\
\text { Montes }\end{array}$ & $-20,787423$ & $-43,691299$ & Fotografía \\
\hline $\begin{array}{l}\text { Myiozetetes } \\
\text { cayanensis }\end{array}$ & cayanensis & 3 & WA2202187 & WK & Brasil & Minas Gerais & Uberlândia & $-18,913876$ & $-48,275033$ & Fotografía \\
\hline $\begin{array}{l}\text { Myiozetetes } \\
\text { cayanensis }\end{array}$ & erythropterus & 4 & WA2242805 & WK & Brasil & Minas Gerais & $\begin{array}{l}\text { Bom Jesus do } \\
\text { Amparo }\end{array}$ & $-19,708385$ & $-43,475783$ & Fotografía \\
\hline $\begin{array}{l}\text { Myiozetetes } \\
\text { cayanensis }\end{array}$ & erythropterus & 4 & WA2243707 & WK & Brasil & Minas Gerais & Prados & $-21,056602$ & $-44,076454$ & Fotografía \\
\hline $\begin{array}{l}\text { Myiozetetes } \\
\text { cayanensis }\end{array}$ & erythropterus & 4 & WA2286193 & WK & Brasil & Río de Janeiro & Nova Friburgo & $-22,288761$ & $-42,53416$ & Fotografía \\
\hline $\begin{array}{l}\text { Myiozetetes } \\
\text { cayanensis }\end{array}$ & cayanensis & 3 & WA248117 & WK & Brasil & Minas Gerais & Delta & $-19,97246$ & $-47,772847$ & Fotografía \\
\hline $\begin{array}{l}\text { Myiozetetes } \\
\text { cayanensis }\end{array}$ & erythropterus & 4 & WA2533999 & WK & Brasil & Minas Gerais & Itamarandiba & $-17,857298$ & $-42,858391$ & Fotografía \\
\hline $\begin{array}{l}\text { Myiozetetes } \\
\text { cayanensis }\end{array}$ & erythropterus & 4 & WA2610635 & WK & Brasil & Minas Gerais & Manhumirim & $-20,359639$ & $-41,958907$ & Fotografía \\
\hline $\begin{array}{l}\text { Myiozetetes } \\
\text { cayanensis }\end{array}$ & erythropterus & 4 & WA2728904 & WK & Brasil & Espírito Santo & $\begin{array}{c}\text { Venda Nova do } \\
\text { Imigrante }\end{array}$ & $-20,327709$ & $-41,135861$ & Fotografía \\
\hline $\begin{array}{l}\text { Myiozetetes } \\
\text { cayanensis }\end{array}$ & cayanensis & 3 & WA2841240 & WK & Brasil & Minas Gerais & Vazante & $-17,992947$ & $-46,90412$ & Fotografía \\
\hline $\begin{array}{l}\text { Myiozetetes } \\
\text { cayanensis }\end{array}$ & cayanensis & 3 & WA2918290 & WK & Brasil & Minas Gerais & Uberlândia & $-18,913876$ & $-48,275033$ & Fotografía \\
\hline $\begin{array}{l}\text { Myiozetetes } \\
\text { cayanensis }\end{array}$ & erythropterus & 4 & WA2923187 & WK & Brasil & Espírito Santo & Santa Teresa & $-19,93727$ & $-40,598137$ & Fotografía \\
\hline $\begin{array}{l}\text { Myiozetetes } \\
\text { cayanensis }\end{array}$ & erythropterus & 4 & WA2965540 & WK & Brasil & Minas Gerais & Piranga & $-20,683243$ & $-43,297661$ & Fotografía \\
\hline $\begin{array}{l}\text { Myiozetetes } \\
\text { cayanensis }\end{array}$ & erythropterus & 4 & WA3029779 & WK & Brasil & Minas Gerais & Alto Jequitibá & $-20,426664$ & $-41,964066$ & Fotografía \\
\hline $\begin{array}{l}\text { Myiozetetes } \\
\text { cayanensis }\end{array}$ & erythropterus & 4 & WA3045281 & WK & Brasil & Minas Gerais & Ferros & $-19,234789$ & $-43,019636$ & Fotografía \\
\hline $\begin{array}{l}\text { Myiozetetes } \\
\text { cayanensis }\end{array}$ & cayanensis & 3 & WA3059823 & WK & Brasil & Minas Gerais & Uberaba & $-19,747163$ & $-47,939289$ & Fotografía \\
\hline $\begin{array}{l}\text { Myiozetetes } \\
\text { cayanensis }\end{array}$ & erythropterus & 4 & WA3099142 & WK & Brasil & Minas Gerais & Chapada do Norte & $-17,155883$ & $-42,41355$ & Fotografía \\
\hline $\begin{array}{l}\text { Myiozetetes } \\
\text { cayanensis }\end{array}$ & cayanensis & 3 & WA3102447 & WK & Brasil & Minas Gerais & Itajubá & $-22,422832$ & $-45,46014$ & Fotografía \\
\hline $\begin{array}{l}\text { Myiozetetes } \\
\text { cayanensis }\end{array}$ & erythropterus & 4 & WA3110598 & WK & Brasil & Minas Gerais & $\begin{array}{l}\text { São Gonçalo do Rio } \\
\text { Abaixo }\end{array}$ & $-19,824582$ & $-43,35875$ & Fotografía \\
\hline $\begin{array}{l}\text { Myiozetetes } \\
\text { cayanensis }\end{array}$ & erythropterus & 4 & WA3168284 & WK & Brasil & Minas Gerais & Caratinga & $-19,790491$ & $-42,141489$ & Fotografía \\
\hline $\begin{array}{l}\text { Myiozetetes } \\
\text { cayanensis }\end{array}$ & erythropterus & 4 & WA3230159 & WK & Brasil & Minas Gerais & Catas Altas & $-20,070273$ & $-43,410064$ & Fotografía \\
\hline $\begin{array}{l}\text { Myiozetetes } \\
\text { cayanensis }\end{array}$ & erythropterus & 4 & WA3250101 & WK & Brasil & Minas Gerais & Caratinga & $-19,790491$ & $-42,141489$ & Fotografía \\
\hline $\begin{array}{l}\text { Myiozetetes } \\
\text { cayanensis }\end{array}$ & erythropterus & 4 & WA3301774 & WK & Brasil & Minas Gerais & lapu & $-19,439142$ & $-42,215296$ & Fotografía \\
\hline $\begin{array}{l}\text { Myiozetetes } \\
\text { cayanensis }\end{array}$ & erythropterus & 4 & WA3337898 & WK & Brasil & Minas Gerais & Catas Altas & $-20,070273$ & $-43,410064$ & Fotografía \\
\hline $\begin{array}{l}\text { Myiozetetes } \\
\text { cayanensis }\end{array}$ & erythropterus & 4 & WA3381098 & WK & Brasil & Minas Gerais & $\begin{array}{l}\text { Santana dos } \\
\text { Montes }\end{array}$ & $-20,787423$ & $-43,691299$ & Fotografía \\
\hline
\end{tabular}




\begin{tabular}{|c|c|c|c|c|c|c|c|c|c|c|}
\hline $\begin{array}{c}\text { Myiozetetes } \\
\text { cayanensis }\end{array}$ & erythropterus & 4 & WA3454348 & WK & Brasil & Espírito Santo & $\begin{array}{c}\text { Divino de São } \\
\text { Lourenço }\end{array}$ & $-20,620538$ & $-41,685188$ & Fotografía \\
\hline $\begin{array}{c}\text { Myiozetetes } \\
\text { cayanensis }\end{array}$ & erythropterus & 4 & WA3461813 & WK & Brasil & Minas Gerais & $\begin{array}{c}\text { Conselheiro } \\
\text { Lafaiete }\end{array}$ & $-20,663871$ & $-43,785009$ & Fotografía \\
\hline $\begin{array}{c}\text { Myiozetetes } \\
\text { cayanensis }\end{array}$ & erythropterus & 4 & WA3473310 & WK & Brasil & Río de Janeiro & Petrópolis & $-22,511198$ & $-43,17778$ & Fotografía \\
\hline $\begin{array}{c}\text { Myiozetetes } \\
\text { cayanensis }\end{array}$ & cayanensis & 3 & WA3569859 & WK & Brasil & Río de Janeiro & Mangaratiba & $-22,940931$ & $-44,035744$ & Fotografía \\
\hline $\begin{array}{c}\text { Myiozetetes } \\
\text { cayanensis }\end{array}$ & erythropterus & 4 & WA36182 & WK & Brasil & Minas Gerais & Açucena & $-19,076427$ & $-42,547897$ & Fotografía \\
\hline $\begin{array}{c}\text { Myiozetetes } \\
\text { cayanensis }\end{array}$ & erythropterus & 4 & WA3642144 & WK & Brasil & Espírito Santo & Domingos Martins & $-20,360799$ & $-40,659817$ & Fotografía \\
\hline $\begin{array}{c}\text { Myiozetetes } \\
\text { cayanensis }\end{array}$ & erythropterus & 4 & WA3657758 & WK & Brasil & Minas Gerais & Mariana & $-20,365272$ & $-43,415007$ & Fotografía \\
\hline $\begin{array}{c}\text { Myiozetetes } \\
\text { cayanensis }\end{array}$ & erythropterus & 4 & WA3666012 & WK & Brasil & Minas Gerais & Itabira & $-19,624258$ & $-43,230936$ & Fotografía \\
\hline $\begin{array}{c}\text { Myiozetetes } \\
\text { cayanensis }\end{array}$ & cayanensis & 3 & WA3736885 & WK & Brasil & Minas Gerais & Sacramento & $-19,865494$ & $-47,4399$ & Fotografía \\
\hline $\begin{array}{c}\text { Myiozetetes } \\
\text { cayanensis }\end{array}$ & erythropterus & 4 & WA3748316 & WK & Brasil & Minas Gerais & Manhuaçu & $-20,255665$ & $-42,02956$ & Fotografía \\
\hline $\begin{array}{c}\text { Myiozetetes } \\
\text { cayanensis }\end{array}$ & erythropterus & 4 & WA3760130 & WK & Brasil & Minas Gerais & Ipatinga & $-19,46909$ & $-42,548457$ & Fotografía \\
\hline $\begin{array}{c}\text { Myiozetetes } \\
\text { cayanensis }\end{array}$ & erythropterus & 4 & WA3790087 & WK & Brasil & Río de Janeiro & Teresópolis & $-22,416504$ & $-42,975557$ & Fotografía \\
\hline $\begin{array}{c}\text { Myiozetetes } \\
\text { cayanensis }\end{array}$ & erythropterus & 4 & WA3829272 & WK & Brasil & Minas Gerais & Manhuaçu & $-20,255665$ & $-42,02956$ & Fotografía \\
\hline $\begin{array}{c}\text { Myiozetetes } \\
\text { cayanensis }\end{array}$ & erythropterus & 4 & WA3869615 & WK & Brasil & Espírito Santo & Vargem Alta & $-20,673934$ & $-41,00977$ & Fotografía \\
\hline $\begin{array}{c}\text { Myiozetetes } \\
\text { cayanensis }\end{array}$ & erythropterus & 4 & WA436373 & WK & Brasil & Minas Gerais & $\begin{array}{c}\text { Serra Azul de } \\
\text { Minas }\end{array}$ & $-18,361064$ & $-43,168508$ & Fotografía \\
\hline $\begin{array}{c}\text { Myiozetetes } \\
\text { cayanensis }\end{array}$ & erythropterus & 4 & WA585156 & WK & Brasil & Minas Gerais & Sabinópolis & $-18,670731$ & $-43,083636$ & Fotografía \\
\hline $\begin{array}{c}\text { Myiozetetes } \\
\text { cayanensis }\end{array}$ & cayanensis & 3 & WA636031 & WK & Brasil & Minas Gerais & Uberlândia & $-18,913876$ & $-48,275033$ & Fotografía \\
\hline $\begin{array}{c}\text { Myiozetetes } \\
\text { cayanensis }\end{array}$ & erythropterus & 4 & WA697119 & WK & Brasil & Minas Gerais & João Monlevade & $-19,813037$ & $-43,173854$ & Fotografía \\
\hline $\begin{array}{c}\text { Myiozetetes } \\
\text { cayanensis }\end{array}$ & erythropterus & 4 & WA801609 & WK & Brasil & Minas Gerais & Sabinópolis & $-18,670731$ & $-43,083636$ & Fotografía \\
\hline $\begin{array}{c}\text { Myiozetetes } \\
\text { cayanensis }\end{array}$ & cayanensis & 3 & WA828346 & WK & Brasil & Río de Janeiro & Guapimirim & $-22,536681$ & $-42,991809$ & Fotografía \\
\hline $\begin{array}{c}\text { Myiozetetes } \\
\text { cayanensis }\end{array}$ & erythropterus & 4 & WA879519 & WK & Brasil & Río de Janeiro & Rio de Janeiro & $-22,906824$ & $-43,172962$ & Fotografía \\
\hline $\begin{array}{c}\text { Myiozetetes } \\
\text { cayanensis }\end{array}$ & cayanensis & 3 & WA99588 & WK & Brasil & Río de Janeiro & Angra dos Reis & $-23,005735$ & $-44,315759$ & Fotografía \\
\hline
\end{tabular}


Anexo C. Registro de cantos negativos. Se muestra la lista de archivos de audio excluidos del análisis, así como su identificador, la colección/base de datos de origen, el tipo de error identificado y los comentarios acerca de su exclusión. IAvH: Instituto Alexander von Humboldt, ML: Macaulay Library del Cornell Lab of Ornithology, WK: wikiaves.com.br, XC: Xeno-canto.org.

Esta información puede ser descargada en formato CSV en el repositorio:

https://github.com/icrioso/Mapa myiozetetes cayanensis

\begin{tabular}{|c|c|c|c|}
\hline Colección & $\begin{array}{l}\text { Número de } \\
\text { catálogo }\end{array}$ & Tipo de error & Observaciones \\
\hline $\mathrm{IAvH}$ & BSA-4696 & (4) Sonidos no medibles & \\
\hline $\mathrm{IAvH}$ & BSA-4739 & (1) Registro de otra especie & Myiozetetes similis \\
\hline $\mathrm{IAvH}$ & BSA-8329 & (4) Sonidos no medibles & \\
\hline $\mathrm{IAvH}$ & BSA-1175 & (1) Registro de otra especie & Myiozetetes similis \\
\hline $\mathrm{IAvH}$ & BSA-1177 & (1) Registro de otra especie & Myiozetetes similis \\
\hline $\mathrm{IAvH}$ & BSA-12890 & (4) Sonidos no medibles & \\
\hline $\mathrm{IAvH}$ & BSA-14011 & (1) Registro de otra especie & Vocalización desconocida \\
\hline $\mathrm{IAvH}$ & BSA-19152 & (2) Ruido de fondo (micrófono, golpes, elementos externos) & \\
\hline $\mathrm{IAvH}$ & BSA-19293 & (2) Ruido de fondo (micrófono, golpes, elementos externos) & \\
\hline $\mathrm{IAvH}$ & BSA-28717 & (4) Sonidos no medibles & \\
\hline $\mathrm{IAvH}$ & BSA-28722 & (4) Sonidos no medibles & \\
\hline $\mathrm{IAvH}$ & BSA-31568 & (1) Registro de otra especie & Ara ararauna/Pitangus sulphuratus \\
\hline $\mathrm{IAvH}$ & BSA-31638 & (1) Registro de otra especie & Glaucidium brasilianum/Trogon sp. \\
\hline $\mathrm{xC}$ & 10091 & (2) Ruido de fondo (micrófono, golpes, elementos externos) & \\
\hline $\mathrm{XC}$ & 37321 & (4) Sonidos no medibles & Ruido de fondo/datos inexactos \\
\hline $\mathrm{XC}$ & 42370 & (2) Ruido de fondo (micrófono, golpes, elementos externos) & \\
\hline$x C$ & 43258 & (5) Errores técnicos & No reconocido por Software de análisis \\
\hline XC & 46162 & (1) Registro de otra especie & \\
\hline $\mathrm{XC}$ & 54521 & (3) Ruido de fondo (otros cantos) & \\
\hline $\mathrm{XC}$ & 54522 & (4) Sonidos no medibles & Reclamos \\
\hline XC & 56458 & (1) Registro de otra especie & Sin certeza en la identificación \\
\hline$x C$ & 57826 & (5) Errores técnicos & No hay vocalización \\
\hline$x C$ & 58405 & (1) Registro de otra especie & \\
\hline $\mathrm{XC}$ & 92252 & (4) Sonidos no medibles & \\
\hline$x C$ & 92253 & (4) Sonidos no medibles & \\
\hline$x C$ & 109016 & (3) Ruido de fondo (otros cantos) & \\
\hline $\mathrm{XC}$ & 129961 & (4) Sonidos no medibles & Señales poco perceptibles (volumen muy bajo) \\
\hline $\mathrm{XC}$ & 147694 & (1) Registro de otra especie & Sin certeza en la identificación \\
\hline$x C$ & 167312 & (4) Sonidos no medibles & \\
\hline$x C$ & 197488 & (4) Sonidos no medibles & \\
\hline $\mathrm{XC}$ & 226233 & (1) Registro de otra especie & Sin certeza en la identificación \\
\hline $\mathrm{XC}$ & 226234 & (1) Registro de otra especie & Sin certeza en la identificación \\
\hline$x C$ & 248165 & (4) Sonidos no medibles & \\
\hline$x C$ & 248166 & (4) Sonidos no medibles & \\
\hline
\end{tabular}


XC

$\mathrm{XC}$

$\mathrm{XC}$

$x C$

$x C$

$x C$

$X C$

$x C$

$x C$

$x C$

$x C$

$x C$

$x C$

$X C$

$X C$

$x C$

$X C$

$x C$

$X C$

$x C$

$x C$

$X C$

$X C$

$X C$

WK

WK

WK

WK

WK

WK

WK

WK

WK

WK

WK

WK

WK

WK

WK

WK

WK

WK

WK

WK

WK

WK

WK

WK
248259

262242

262243

272428

303194

339479

360727

388413

390712

394781

419092

420112

423695

433202

433513

437830

459230

461014

484431

521301

522374

532375

532376

537847

60847

136089

158209

257982

282009

350190

373926

427765

678002

722695

825378

966001

970814

1004367

1020153

1026286

1058476

1131207

1208036

1214471

1272169

1356035

1411341

1459838
(4) Sonidos no medibles

(3) Ruido de fondo (otros cantos)

(4) Sonidos no medibles

(4) Sonidos no medibles

(1) Registro de otra especie

(4) Sonidos no medibles

(4) Sonidos no medibles

(3) Ruido de fondo (otros cantos)

(1) Registro de otra especie

Sin certeza en la identificación

(4) Sonidos no medibles

(1) Registro de otra especie

(1) Registro de otra especie

Sin certeza en el tipo de nota

Sin certeza en el tipo de nota

(4) Sonidos no medibles

(4) Sonidos no medibles

(2) Ruido de fondo (micrófono, golpes, elementos externos)

(4) Sonidos no medibles

(4) Sonidos no medibles

(4) Sonidos no medibles

(1) Registro de otra especie

Sin certeza en el tipo de nota

(2) Ruido de fondo (micrófono, golpes, elementos externos)

(1) Registro de otra especie

(1) Registro de otra especie

(1) Registro de otra especie

(4) Sonidos no medibles

(5) Errores técnicos

(5) Errores técnicos

reconocido por Software de análisis No reconocido por Software de análisis

(1) Registro de otra especie

(5) Errores técnicos

No reconocido por Software de análisis

(5) Errores técnicos

No reconocido por Software de análisis

(2) Ruido de fondo (micrófono, golpes, elementos externos)

(5) Errores técnicos

No reconocido por Software de análisis

(1) Registro de otra especie

(5) Errores técnicos

No reconocido por Software de análisis

(4) Sonidos no medibles

(2) Ruido de fondo (micrófono, golpes, elementos externos)

(4) Sonidos no medibles

(2) Ruido de fondo (micrófono, golpes, elementos externos)

(2) Ruido de fondo (micrófono, golpes, elementos externos)

(5) Errores técnicos

(5) Errores técnicos

(5) Errores técnicos

No reconocido por Software de análisis

No reconocido por Software de análisis

No reconocido por Software de análisis

(4) Sonidos no medibles

(4) Sonidos no medibles

(4) Sonidos no medibles

(4) Sonidos no medibles

(1) Registro de otra especie

Sin certeza en el tipo de nota

(4) Sonidos no medibles

(4) Sonidos no medibles 


\begin{tabular}{|c|c|c|c|}
\hline WK & 1575664 & (4) Sonidos no medibles & \\
\hline WK & 1591042 & (2) Ruido de fondo (micrófono, golpes, elementos externos) & \\
\hline WK & 1627313 & (5) Errores técnicos & No reconocido por Software de análisis \\
\hline WK & 1659206 & (1) Registro de otra especie & \\
\hline WK & 1790156 & (2) Ruido de fondo (micrófono, golpes, elementos externos) & \\
\hline WK & 1798615 & (4) Sonidos no medibles & \\
\hline WK & 1840380 & (4) Sonidos no medibles & \\
\hline WK & 1921181 & (5) Errores técnicos & No reconocido por Software de análisis \\
\hline WK & 1942966 & (5) Errores técnicos & No reconocido por Software de análisis \\
\hline WK & 1943103 & (2) Ruido de fondo (micrófono, golpes, elementos externos) & \\
\hline WK & 1963339 & (2) Ruido de fondo (micrófono, golpes, elementos externos) & \\
\hline WK & 1980888 & (5) Errores técnicos & No reconocido por Software de análisis \\
\hline WK & 2014825 & (5) Errores técnicos & No reconocido por Software de análisis \\
\hline WK & 2034013 & (3) Ruido de fondo (otros cantos) & \\
\hline WK & 2079231 & (5) Errores técnicos & No reconocido por Software de análisis \\
\hline WK & 2132911 & (4) Sonidos no medibles & \\
\hline WK & 2158454 & (4) Sonidos no medibles & \\
\hline WK & 2185521 & (4) Sonidos no medibles & \\
\hline WK & 2199235 & (4) Sonidos no medibles & \\
\hline WK & 2289736 & (2) Ruido de fondo (micrófono, golpes, elementos externos) & \\
\hline WK & 2297654 & (2) Ruido de fondo (micrófono, golpes, elementos externos) & \\
\hline WK & 2445874 & (1) Registro de otra especie & Sin certeza en la identificación \\
\hline WK & 2450669 & (2) Ruido de fondo (micrófono, golpes, elementos externos) & \\
\hline WK & 2535675 & (4) Sonidos no medibles & \\
\hline WK & 2542469 & (4) Sonidos no medibles & \\
\hline WK & 2550030 & (1) Registro de otra especie & Sin certeza en la identificación \\
\hline WK & 2563597 & (5) Errores técnicos & No reconocido por Software de análisis \\
\hline WK & 2599829 & (1) Registro de otra especie & Sin certeza en el tipo de nota \\
\hline WK & 2609587 & (4) Sonidos no medibles & \\
\hline WK & 2612531 & (4) Sonidos no medibles & \\
\hline WK & 2632975 & (4) Sonidos no medibles & \\
\hline WK & 2675284 & (2) Ruido de fondo (micrófono, golpes, elementos externos) & \\
\hline WK & 2800583 & (4) Sonidos no medibles & \\
\hline WK & 2824248 & (4) Sonidos no medibles & \\
\hline WK & 2850220 & (4) Sonidos no medibles & \\
\hline WK & 2863733 & (2) Ruido de fondo (micrófono, golpes, elementos externos) & \\
\hline WK & 2908062 & (1) Registro de otra especie & Sin certeza en el tipo de nota \\
\hline WK & 2912401 & (5) Errores técnicos & No reconocido por Software de análisis \\
\hline WK & 2914388 & (2) Ruido de fondo (micrófono, golpes, elementos externos) & \\
\hline WK & 2938330 & (2) Ruido de fondo (micrófono, golpes, elementos externos) & \\
\hline WK & 2942367 & (2) Ruido de fondo (micrófono, golpes, elementos externos) & \\
\hline WK & 2980586 & (4) Sonidos no medibles & \\
\hline WK & 2996827 & (1) Registro de otra especie & Sin certeza en el tipo de nota \\
\hline WK & 3080642 & (2) Ruido de fondo (micrófono, golpes, elementos externos) & \\
\hline WK & 3110394 & (1) Registro de otra especie & Sin certeza en la identificación \\
\hline WK & 3142667 & (2) Ruido de fondo (micrófono, golpes, elementos externos) & \\
\hline WK & 3143823 & (2) Ruido de fondo (micrófono, golpes, elementos externos) & \\
\hline WK & 3182710 & (2) Ruido de fondo (micrófono, golpes, elementos externos) & \\
\hline
\end{tabular}




\begin{tabular}{|c|c|c|c|}
\hline WK & 3273311 & (4) Sonidos no medibles & \\
\hline WK & 3275256 & (2) Ruido de fondo (micrófono, golpes, elementos externos) & \\
\hline WK & 3328682 & (4) Sonidos no medibles & \\
\hline WK & 3374857 & (4) Sonidos no medibles & \\
\hline WK & 3400593 & (4) Sonidos no medibles & \\
\hline WK & 3439417 & (4) Sonidos no medibles & \\
\hline WK & 3455675 & (4) Sonidos no medibles & \\
\hline WK & 3563016 & (4) Sonidos no medibles & \\
\hline WK & 3574995 & (5) Errores técnicos & No reconocido por Software de análisis \\
\hline WK & 3646085 & (4) Sonidos no medibles & \\
\hline WK & 3696896 & (2) Ruido de fondo (micrófono, golpes, elementos externos) & Señales poco perceptibles (volumen muy bajo) \\
\hline ML & 24132 & (3) Ruido de fondo (otros cantos) & Anhima cornuta en primer plano \\
\hline ML & 38580 & (3) Ruido de fondo (otros cantos) & \\
\hline ML & 66518 & (1) Registro de otra especie & Sin certeza en el tipo de nota \\
\hline ML & 66519 & (1) Registro de otra especie & Sin certeza en el tipo de nota \\
\hline ML & 66520 & (4) Sonidos no medibles & \\
\hline ML & 66523 & (1) Registro de otra especie & Sin certeza en el tipo de nota \\
\hline ML & 66524 & (1) Registro de otra especie & Sin certeza en el tipo de nota \\
\hline ML & 66526 & (1) Registro de otra especie & Sin certeza en el tipo de nota \\
\hline ML & 80488 & (4) Sonidos no medibles & \\
\hline ML & 80493 & (4) Sonidos no medibles & \\
\hline ML & 85019 & (1) Registro de otra especie & Sin certeza en el tipo de nota \\
\hline ML & 106242 & (4) Sonidos no medibles & \\
\hline ML & 165958 & (4) Sonidos no medibles & \\
\hline ML & 172502 & (1) Registro de otra especie & Sin certeza en el tipo de nota \\
\hline ML & 247946 & (2) Ruido de fondo (micrófono, golpes, elementos externos) & \\
\hline ML & 258271 & (1) Registro de otra especie & Registro duplicado con IAvH BSA-8471 \\
\hline ML & 258283 & (1) Registro de otra especie & Registro duplicado con IAvH BSA-8483 \\
\hline ML & 260486 & (1) Registro de otra especie & Registro duplicado con IAvH BSA-10690 \\
\hline ML & 260708 & (1) Registro de otra especie & Registro duplicado con IAvH BSA-10912 \\
\hline ML & 261075 & (1) Registro de otra especie & Registro duplicado con IAvH BSA-11279 \\
\hline ML & 261182 & (1) Registro de otra especie & Registro duplicado con IAvH BSA-11386 \\
\hline ML & 261785 & (1) Registro de otra especie & Registro duplicado con IAvH BSA-11989 \\
\hline ML & 261786 & (1) Registro de otra especie & Registro duplicado con IAvH BSA-11990 \\
\hline ML & 261952 & (1) Registro de otra especie & Registro duplicado con IAvH BSA-12156 \\
\hline ML & 262679 & (1) Registro de otra especie & Registro duplicado con IAvH BSA-12883 \\
\hline ML & 262686 & (1) Registro de otra especie & Registro duplicado con IAvH BSA-12890 \\
\hline ML & 262719 & (1) Registro de otra especie & Registro duplicado con IAvH BSA-12923 \\
\hline ML & 262798 & (1) Registro de otra especie & Registro duplicado con IAvH BSA-13002 \\
\hline ML & 267024 & (1) Registro de otra especie & Registro duplicado con IAvH BSA-17367 \\
\hline $\mathrm{ML}$ & 267740 & (1) Registro de otra especie & Registro duplicado con IAvH BSA-18860 \\
\hline ML & 268029 & (1) Registro de otra especie & Registro duplicado con IAvH BSA-19152 \\
\hline ML & 268169 & (1) Registro de otra especie & Registro duplicado con IAvH BSA-19293 \\
\hline ML & 270609 & (1) Registro de otra especie & Registro duplicado con IAvH BSA-28717 \\
\hline ML & 270614 & (1) Registro de otra especie & Registro duplicado con IAvH BSA-28722 \\
\hline ML & 270824 & (1) Registro de otra especie & Registro duplicado con IAvH BSA-28934 \\
\hline $\mathrm{ML}$ & 270826 & (1) Registro de otra especie & Registro duplicado con IAvH BSA-28936 \\
\hline ML & 518390 & (4) Sonidos no medibles & \\
\hline
\end{tabular}




\begin{tabular}{|c|c|c|c|}
\hline ML & 33303091 & (5) Errores técnicos & No tiene forma de onda \\
\hline ML & 37125911 & (4) Sonidos no medibles & \\
\hline ML & 51848081 & (4) Sonidos no medibles & \\
\hline ML & 67209471 & (5) Errores técnicos & No tiene forma de onda \\
\hline ML & 67415281 & (5) Errores técnicos & No tiene forma de onda \\
\hline ML & 67664041 & (5) Errores técnicos & No tiene forma de onda \\
\hline ML & 68100511 & (5) Errores técnicos & No tiene forma de onda \\
\hline ML & 69499791 & (5) Errores técnicos & No tiene forma de onda \\
\hline ML & 71253061 & (5) Errores técnicos & No tiene forma de onda \\
\hline ML & 85885271 & (4) Sonidos no medibles & \\
\hline ML & 90549611 & (2) Ruido de fondo (micrófono, golpes, elementos externos) & \\
\hline ML & 92382411 & (5) Errores técnicos & No tiene forma de onda \\
\hline ML & 101590191 & (5) Errores técnicos & No reconocido por Software de análisis \\
\hline ML & 101637061 & (4) Sonidos no medibles & \\
\hline ML & 103958391 & (1) Registro de otra especie & \\
\hline ML & 104683671 & (4) Sonidos no medibles & \\
\hline ML & 107633871 & (4) Sonidos no medibles & \\
\hline ML & 108698861 & (1) Registro de otra especie & Sin certeza en la identificación \\
\hline ML & 142152131 & (4) Sonidos no medibles & \\
\hline ML & 142552281 & (4) Sonidos no medibles & \\
\hline ML & 178982701 & (5) Errores técnicos & No tiene forma de onda \\
\hline ML & 183211111 & (4) Sonidos no medibles & \\
\hline ML & 183602401 & (4) Sonidos no medibles & \\
\hline ML & 185147471 & (4) Sonidos no medibles & \\
\hline ML & 186428601 & (2) Ruido de fondo (micrófono, golpes, elementos externos) & \\
\hline ML & 203695961 & (1) Registro de otra especie & Sin certeza en el tipo de nota \\
\hline ML & 203922511 & (4) Sonidos no medibles & \\
\hline ML & 203922521 & (4) Sonidos no medibles & \\
\hline ML & 212488401 & (2) Ruido de fondo (micrófono, golpes, elementos externos) & \\
\hline ML & 215335381 & (5) Errores técnicos & No tiene forma de onda \\
\hline ML & 218559061 & (2) Ruido de fondo (micrófono, golpes, elementos externos) & \\
\hline
\end{tabular}

